\title{
Internationale Klassifikation von Kopfschmerzerkrankungen
}

\author{
3. Auflage
}

\section{COPYRIGHT}

Die 3. Auflage der Internationalen Klassifikation von Kopfschmerzerkrankungen (ICHD-3) darf zu wissenschaftlichen, Weiterbildungs- oder klinischen Zwecken von Einrichtungen, Fachgesellschaften oder Einzelpersonen frei vervielfältigt werden. Ansonsten liegt das Urheberrecht ausschließlich bei der International Headache Society. Jede vollständige oder auszugsweise Wiedergabe welcher Art auch immer zu kommerziellen Zwecken bedarf der Genehmigung der IHS, die gegen Entrichtung einer Gebühr gewährt wird. Bitte kontaktieren Sie den Herausgeber unter der untenstehenden Anschrift.

CInternational Headache Society 2013-2018. Anträge auf urheberrechtliche Genehmigungen sind einzureichen bei Sage Publications Ltd, 1 Oliver's Yard, 55 City Road, London EC1Y 1SP, United Kingdom (Tel.: + 44 (0) 207324 8500; Fax:+44(0) 2073248600; permissions@sagepub.co.uk) (www. uksagepub.com).

\section{ÜBERSETZUNGEN}

Die Internationale Kopfschmerzgesellschaft (International Headache Society, IHS) erlaubt ausdrücklich die gesamte oder auszugsweise Übersetzung der ICHD-3 zu klinischen Anwendungs- und Weiterbildungszwecken, für Feldstudien oder sonstige Forschungszwecke. Diese Genehmigung erfolgt mit der Auflage, dass alle Übersetzungen bei der IHS anzumelden sind. Bevor eine Übersetzung in Angriff genommen wird, wird den potenziellen Übersetzerinnen und Übersetzern geraten, sich zu erkundigen, ob bereits eine Übersetzung in die anvisierte Sprache vorliegt.

Alle Übersetzerinnen und Übersetzer sollen sich gewahr sein, dass bei der Übersetzung strenge Richtlinien einzuhalten sind. Veröffentlichungen, die über Studien berichten, die auf komplette oder teilweise Übersetzungen der ICHD-3 zurückgreifen, sollten auch eine kurze Beschreibung des Übersetzungsprozesses beinhalten, einschließlich der Identität der Übersetzerinnen und Übersetzer (die Übersetzung sollte immer von mehr als einer Person erfolgen).

Die IHS erteilt keine nachträglichen Zustimmungen zu Übersetzungen. Solche Zustimmungen können von den nationalen Mitgliedsgesellschaften erteilt werden; sofern solche existieren, sollte um eine solche Zustimmung ersucht werden.

\section{Bibliografie}

DOI https://doi.org/10.1055/a-0930-5739

Nervenheilkunde 2019; 38: S3-S182

(c) Georg Thieme Verlag KG Stuttgart · New York

ISSN 0722-1541

\section{EDITORIAL}

Zum dritten Mal wird nun seit 1988 die komplette Kopfschmerzklassifikation der International Hedache Society vorgelegt. Es hat sich diese Mal um einen sehr langwierigen Prozess gehandelt, der schon vor 2013 begann und mit einer ersten beta-Version (publiziert in 2013) seine erste Stufe erreichte. In den Jahren danach wurden erstmals sogenannte Feldstudien durchgeführt, die in einige Revisionen der Kriterien auf Grundlage dieser wissenschaftlichen Ergebnisse mündeten. Dies führte zur Publikationen der endgültigen 3. Auflage der Kopfschmerzklassifikation im Januar 2018. Bereits davor, aber insbesondere danach folgte eine sehr detaillierter, professionell unterstützter Übersetzungsprozess, der sich eng an den Originaltext hielt, aber auch einige Besonderheiten der deutschen Sprache mit berücksichtigte. Ich bin sehr stolz darauf, dass ich diesen Prozess organisieren durfte, sodass nun ein für den medizinischen und wissenschaftlichen Gebrauch hilfreicher Text vorliegt.

\section{Stefan Evers}

Generalsekretär der International Headache Society

Erstes Kopfschmerzklassifikationskomitee

Jes Olesen, Dänemark (Vorsitzender)

André Bes, France (verstorben)

Robert Kunkel, USA

James W Lance, Australien

Giuseppe Nappi, Italien

Volker Pfaffenrath, Deutschland

Frank Clifford Rose, Großbritannien (verstorben)

Bruce S Schoenberg, USA (verstorben)

Dieter Soyka, Deutschland (verstorben)

Peer Tfelt-Hansen, Dänemark (Sekretär)

K Michael A Welch, USA

Marica Wilkinson, Großbritannien (verstorben)

Zweites Kopfschmerzklassifikationskomitee

Jes Olesen, Dänemark (Vorsitzender)

Marie-Germaine Bousser, Frankreich

Hans-Christoph Diener, Deutschland

David Dodick, USA

Michael First, USA

Peter J Goadsby, Großbritannien

Hartmut Göbel, Deutschland

Miguel JA Lainez, Spanien

James W Lance, Australien

Richard B Lipton, USA

Giuseppe Nappi, Italien

Fumihiko Sakai, Japan

Jean Schoenen, Belgien

Stephen D Silberstein, USA

Timothy J Steiner, Großbritannien (Sekretär) 
Drittes Kopfschmerzklassifikationskomitee

Jes Olesen, Dänemark (Vorsitzender)

Lars Bendtsen, Dänemark

David Dodick, USA

Anne Ducros, Frankreich

Stefan Evers, Deutschland

Michael First, USA

Peter ] Goadsby, USA/Großbritannien

Andrew Hershey, USA

Zaza Katsarava, Deutschland

Morris Levin, USA

Julio Pascual, Spanien

Michael B Russell, Norwegen

Todd Schwedt, USA

Timothy J Steiner, Großbritannien (Sekretär)

Cristina Tassorelli, Italien

Gisela M Terwindt, Niederlande

Maurice Vincent, Brasilien

Shuu-jiun Wang, Taiwan

\section{MITGLIEDER DER ARBEITSGRUPPEN ZUR DRITTEN KOPFSCHMERZKLASSIFIKATION}

Arbeitsgruppe Migräne:

J Olesen, Dänemark (Vorsitzender)

(jes.olesen@regionh.dk)

H Bolay, Türkei; A Charles, USA; S Evers, Deutschland; M First, USA; A. Hershey, USA; M Lantéri-Minet, Frankreich; R Lipton, USA; EA MacGregor, Großbritannien; HW Schytz, Dänemark, T Takeshima, Japan.

Arbeitsgruppe Kopfschmerz von Spannungstyp:

\section{Bendtsen, Dänemark (Vorsitzender)}

(lars.bendtsen@regionh.dk)

S Ashina, USA; MT Goicochea, Argentinien; K Hirata, Japan; K Holroyd, USA; C Lampl, Österreich; RB Lipton, USA; DD Mitsikostas, Griechenland; J Schoenen, Belgien.

Arbeitsgruppe Trigemino-autonome Kopfschmerzerkrankungen:

\section{P Goadsby, USA (Vorsitzender)}

(peter.goadsby@kcl.ac.uk)

C Boes, USA; C Bordini, Brasilien; E Cittadini, Großbritannien; A Cohen, Großbritannien; M Leone, Italien; A May, Deutschland; L Newman, USA; J-W Park, Südkorea; T Rozen, USA; E Waldenlind, Schweden.

Arbeitgruppe Andere primäre Kopfschm]erzen:

\section{S-J Wang, Taiwan (Vorsitzender)}

(sjwang@vghtpe.gov.tw)

A Ducros, Frankreich; S Evers, Deutschland; J-L Fuh, Taiwan; A

Özge, Türkei; JA Pareja, Spanien; ] Pascual, Spanien; M Peres, Brasilien; W Young, USA; S-Y Yu, China.
Arbeitsgruppe Kopfschmerz zurückzuführen auf eine Verletzung oder ein Trauma des Kopfes und/oder der HWS:

\section{T Schwedt, USA (Vorsitzender)}

(Schwedt.Todd@mayo.edu)

I Abu-Arafeh, Großbritannien; J Gladstone, Kanada; S-J Huang, Taiwan; R Jensen, Dänemark; JMA Lainez, Spanien; D Obelieniene, Litauen; P Sandor, Schweiz; AI Scher, USA.

Arbeitsgruppe Kopfschmerz zurückzuführen auf Gefäßstörungen im Bereich des Kopfes und/oder des Halses:

\section{A Ducros, Frankreich (Vorsitzende)}

(a-ducros@chu-montpellier.fr)

M Arnold, Schweiz; M Dichgans, Deutschland; J Ferro, Portugal; E Houdart, Frankreich; E Leroux, Kanada; Y-S Li, China; A Singhal, USA; G Tietjen, USA.

Arbeitsgruppe Kopfschmerz zurückzuführen auf nichtvaskuläre intrakranielle Störungen:

\section{DW Dodick, USA (Vorsitzender)}

(Dodick.David@mayo.edu)

S Evers, Deutschland; D Friedman, USA; E Leroux, Kanada; B Mokri, USA; J Pascual, Spanien; M Peres, Brasilien; A Purdy, Kanada; K Ravishankar, Indien; W Schievink, USA; R Stark, Australien; ] VanderPluym, USA.

Arbeitsgruppe Kopfschmerz zurückzuführen auf eine Substanz oder deren Entzug:

\section{MB Russell, Norwegen (Vorsitzender)}

(m.b.russell@medisin.uio.no)

L Bendtsen, Dänemark; J-L Fuh, Taiwan; Z Katsarava, Deutschland; AV Krymchantowski, Brasilien; M Leone, Italien; K Ravishankar, Indien; A Tugrul, Türkei; NJ Wiendels, Niederlande.

Arbeitsgruppe Kopfschmerz zurückzuführen auf eine Infektion:

\section{Tassorelli, Italy (Vorsitzende)}

(cristina.tassorelli@mondino.it)

JR Berger, USA, E Marchioni, Italien; V Osipova, Russland; K Ravishankar, Indien; F Sakai, Japan; L Savi, Italien.

Arbeitsgruppe Kopfschmerz zurückzuführen auf Störungen der Homöostase:

\section{J Pascual, Spanien (Vorsitzender)}

(juliopascualgomez@gmail.com)

M Bigal, Brasilien; C Bordini, Brasilien; J González Menacho, Spanien; F Mainardi, Italien; A Özge, Türkei; J Pereira-Monteiro, Portugal; M Serrano-Dueñas, Ecuador.

Arbeitsgruppe Kopf- oder Gesichtsschmerz zurückzuführen auf Erkrankungen des Schädels sowie von Hals, Augen, Ohren, Nase, Nebenhöhlen, Zähnen, Mund oder anderen Gesichts- oder Schädelstrukturen:

\section{Levin, USA (Vorsitzender)}

(mo.levin@hitchcock.org)

R Cady, USA; C Fernandez de las Peñas, Spanien; D Friedman, USA; V Guidetti, Italien; J Lance, Australien; P Svensson, Dänemark. 
Arbeitsgruppe Kopfschmerz zurückzuführen auf eine psychiatrische Störung:

\section{Vincent, Brasilien (Vorsitzender)}

(maurice.vincent@me.com)

J-I Escobar, USA; M First, USA; A-E Lake III, USA; E Loder, USA; F Radat, Frankreich.

Arbeitsgruppe Schmerzhafte Läsionen der Hirnnerven und andere Gesichtsschmerzen:

Z Katsarava, Germany und T Nurmikko, UK (Co-Vorsitzende) (zaza.katsarava@uni-due.de)

R Benoliel, Israel; Giorgio Cruccu, Italien; R-D Treede, Deutschland; C Sommer, Deutschland.
Arbeitsgruppe Anhang zu Erkrankungen und Kriterien:

GM Terwindt, Niederlande (Vorsitzende)

(G.M.Terwindt@lumc.nl)

\section{DANKSAGUNGEN}

Die Arbeit des Kopfschmerzklassifikationskomitees der IHS wird ausschließlich von der IHS finanziell unterstützt. Es sind keine kommerziellen Sponsorengelder in die Internationale Kopfschmerzklassifikation, 3. Auflage, geflossen.

Wir danken Timothy Steiner für seine Unterstützung, erstens für seine Bemühungen als ehrenamtlicher Sekretär des Klassifikationskomitees und zweitens für seine Arbeit am Lektorat und der Erstellung dieses Manuskripts.

\section{Inhalte}

Vorworte

Anleitung zum Gebrauch der Klassifikation

Klassifikation

\section{Teil eins: Primäre Kopfschmerzerkrankungen}

1. Migräne

2. Kopfschmerz vom Spannungstyp

3. Trigemino-autonome Kopfschmerzerkrankungen

4. Andere primäre Kopfschmerzerkrankungen

Teil zwei: Sekundäre Kopfschmerzerkrankungen

Einleitung

5. Kopfschmerz zurückzuführen auf eine Verletzung oder ein Trauma des Kopfes und/oder der HWS

6. Kopfschmerz zurückzuführen auf Gefäßstörungen im Bereich des Kopfes und/oder des Halses

7. Kopfschmerz zurückzuführen auf nichtvaskuläre intrakranielle Störungen

8. Kopfschmerz zurückzuführen auf eine Substanz oder deren Entzug

9. Kopfschmerz zurückzuführen auf eine Infektion

10. Kopfschmerz zurückzuführen auf eine Störung der Homöeostase

11. Kopf- oder Gesichtsschmerz zurückzuführen auf Erkrankungendes Schädels sowie von Hals, Augen, Ohren, Nase, Nebenhöhlen, Zähnen, Mund oder anderen Gesichts- oder Schädelstrukturen

12. Kopfschmerz zurückzuführen auf psychiatrische Störungen

\section{Teil drei: Schmerzhafte Läsionen der Hirnnerven} und andere Gesichtsschmerzen

13. Schmerzhafte Läsionen der Hirnnerven und andere Gesichtsschmerzen

14. Andere Kopfschmerzerkrankungen

Anhang

Glossar

\section{Vorwort der Präsidentin und Präsidenten der drei deutschsprachigen Kopfschmerz- gesellschaften zur 3. Auflage}

Im Januar 2018 wurde die endgültige 3. Auflage der Internationalen Kopfschmerzklassifikation (ICHD-3) publiziert. Für die DMKG war es eine Selbstverständlichkeit nun rasch für eine offizielle Übersetzung ins Deutsche zu sorgen. Herr Prof. Dr. Dr. Stefan Evers hat alle nötigen Rechte für die offizielle Übersetzung bei der IHS beantragt und er hat nach Genehmigung durch die IHS im Weiteren auch die Koordination der Übersetzungsarbeiten und deren Publikation übernommen. Herrn Prof. Dr. Dr. Stefan Evers gebührt hierfür höchster Dank. Mit dem Präsidium der DMKG wurde entschieden, die Hauptarbeit der Übersetzung in die Hände einer anerkannten Übersetzerin zu legen. Frau Silvia Autenrieth wurde damit beauftragt, den englischen Originaltext ins Deutsche zu bringen und dabei die deutsche Nomenklatur der 2. Auflage zu berücksichtigen. Für die Orthographie der medizinischen Fachausdrücke erfolgte die Festlegung auf die Schreibweise nach Pschyrembel. Danach wurde diese erste Version von Mitgliedern aus dem DMKG Präsidium auf inhaltliche Besonderheiten gegen gelesen und fachlich abgestimmt. Namentlich erwähnt seien hier PD Dr. Charly Gaul, PD Dr. Jan Hoffmann, Prof. Dr. Martin Marziniak, PD Dr. Ruth Ruscheweyh und PD Dr. Stefanie Förderreuther. Übergeordnetes Ziel war basierend auf der deutschen Übersetzung der 2. Auflage nun wiederum einen in sich schlüssigen Text mit einheitlichem Duktus zu schaffen. Die diagnostischen Begriffe der 2. Auflage wurden so weit wie möglich übernommen, um eine sprachliche Kontinuität zu gewährleisten. Es wurde aber auch entschieden, neben der deutschen Nomenklatur zugleich die inzwischen vielerorts etablierten Anglizismen zu nennen und international wie national etablierte $A b$ kürzungen, die auf die englische Nomenklatur zurückgehen, ohne Anpassung an die Deutsche Nomenklatur zu belassen.

Wie schon in der 2. Auflage zeichnen die drei deutschsprachigen wissenschaftlichen Fachgesellschaften aus Deutschland, Österreich und der Schweiz gemeinsam für die Inhalte der Übersetzung verantwortlich. Inhaltliche Fragen, die im Zusammenhang mit der deutschen Übersetzung stehen, sind daher an eine der 3 nationalen Fachgesellschaften zu richten. Das Copyright für die deutsche Übersetzung liegt bei der DMKG, die auch die Kosten für die Über- 
setzung übernommen hat. Dem Thieme Verlag danken wir dafür, dass er für die deutsche Übersetzung ein Sonderheft der Nervenheilkunde herausgeben hat und den Text in ein ansprechendes Layout gebracht hat.

Wir hoffen, dass die offizielle Übersetzung der ICHD-3 weite Verbreitung findet und Ihnen die klinische und wissenschaftliche Arbeit erleichtert. Dies umso mehr, als die im Vergleich zur 2. Auflage deutlich umfassendere und zum Teil detailliertere Klassifikation nichts von ihrer praktischen Anwendbarkeit verloren hat. So bleibt uns nur zu wünschen, dass die 3. Auflage der Klassifikation so wie ihre Vorversionen weiter entwickelt wird, zu einer noch besseren Versorgung der Patienten beiträgt und vor allem auch neue Impulse für wissenschaftliche Kopf- und Gesichtsschmerzprojekte liefert.

\section{Stefanie Förderreuther}

Präsidentin der Deutschen Migräne und Kopfschmerzgesellschaft

\section{Gregor Brössner}

Präsident der Österreichischen Kopfschmerzgesellschaft

\section{Andreas Gantenbein}

Präsident der Schweizerischen Kopfwehgesellschaft

\section{Vorwort}

Ich bin stolz darauf, im Namen des Klassifikationskomitees der Internationalen Kopfschmerzgesellschaft die 3. Auflage der Internationalen Klassifikation von Kopfschmerzerkrankungen (ICHD-3) präsentieren zu können.

Diese folgt auf die Veröffentlichung der ICHD-3 beta 2013. Die Idee hinter der Beta-Version war, vor der Vorlage der endgültigen ICHD-3 eine weitere Validierung in der Praxis zu fördern, was gut funktioniert hat. Es wurden mittlerweile hervorragende Feldstudien veröffentlicht, u. a. zur Migräne mit Aura, zum Clusterkopfschmerz, zur idiopathischen intrakraniellen Druckerhöhung und zur Trigeminusneuralgie. Es wurde zum Beispiel dokumentiert, dass die im Anhang aufgeführten Kriterien für eine A1.2 Migräne mit Aura den Kriterien für eine 1.2 Migräne mit Aura im Haupttext der ICHD-3 beta zur besseren Abgrenzung dieser Erkrankung von transitorischen ischämischen Attacken überlegen waren. Feldversuche zu den neuen assoziierten Merkmalen unter Kriterium C1 für einen 3. Clusterkopfschmerz, Gesichtsrötung und ein Völlegefühl ergaben, dass diese nicht zur Diagnose beitrugen. Dementsprechend werden diese Symptome nur in den Anhang zur ICHD-3 aufgenommen, wo sie zu weiteren Untersuchungen einladen. Dies sind Beispiele für den evidenzbasierten Prozess der Klassifikation von Erkrankungen, der nun das Grundprinzip bei allen künftigen Änderungen an der Internationalen Klassifikation von Kopfschmerzerkrankungen darstellt.

Ein Grund für die Beta-Version war, so unsere Vorstellung, dass in die ICHD-3 die Verschlüsselungen der Internationalen Klassifikation der Krankheiten, 11. Fassung (ICD-11) der Weltgesundheitsorganisation (WHO) mit aufgenommen werden könnten, sobald diese veröffentlicht wären. Wir gingen davon aus, dass die ICD-11 2016 vorliegen würde, leider jedoch gab es lange und unerwartete Verzögerungen, so dass die endgültigen Verschlüsselungen der
Diagnosen noch immer nicht verfügbar sind. Also müssen wir die ICHD-3 ohne sie veröffentlichen.

Die ICHD-3 wird als 1. Ausgabe der Zeitschrift Cephalalgia 2018 veröffentlicht, exakt 30 Jahre nach der 1. Fassung der Internationalen Klassifikation von Kopfschmerzerkrankungen, ICHD-1, wie wir sie heute nennen. Diese 1. Fassung stützte sich primär auf Expertenmeinungen, erwies jedoch nichtsdestotrotz in großen Teilen ihre Gültigkeit. In die ICHD-2, veröffentlicht 2004, wurde eine Reihe von Veränderungen aufgenommen, die teils durch neue $\mathrm{Er}$ kenntnisse veranlasst und teils auf mittlerweile revidierte Expertenmeinungen zurückgingen. Neue wissenschaftliche Erkenntnisse spielten bei den an der ICHD-3 beta vorgenommenen Änderungen eine vergleichsweise größere Rolle, und alle weiteren in die ICHD-3 aufgenommenen Neuerungen basieren auf derartigen Erkenntnissen. Die Kopfschmerzklassifikation stützt sich also heute voll und ganz auf die Forschung und wird dies auch künftig tun.

Mit der Veröffentlichung der ICHD-3 geht eine lange Reise zu Ende, die 2010 begann, doch auf das gegenwärtige Komitee wartet auch in den nächsten Jahren noch einiges an Arbeit. Die ICHD3 beta wurde in viele Sprachen übersetzt, und diese Übersetzungen gilt es zu aktualisieren, bevor die ICHD-3 bald in den entsprechenden Sprachen veröffentlicht werden kann. Es steht zu hoffen, dass noch viele weitere Übersetzungen herauskommen, so dass die ICHD-3 in allen geläufigen und sogar in vielen weniger gängigen Sprachen zugänglich ist. Eine elektronische Version der ICHD-3, die bereits unter der Leitung von Professor Hartmut Göbel erstellt wurde, wird auf ICHD-3 aktualisiert werden. In Planung ist ferner in Zusammenarbeit zwischen Prof. Morris Levin und Prof. Jes Olesen die Herausgabe eines Fallbuchs. Und dann wird schließlich von Prof. Timothy Steiner und Prof. Jes Olesen noch eine Brücke zwischen der ICHD-3 und der ICD-11 der WHO hergestellt werden, sobald die Verschlüsselungen für die ICD-11 zur Verfügung stehen.

Wie sieht dann also die Zukunft der Kopfschmerzklassifikation aus? Eine Klassifikation muss im Prinzip konservativ ausgerichtet sein. Sobald an einer Klassifikation größere Veränderungen vorgenommen werden, müssen alle vorangegangenen Untersuchungen, bei denen auf diese nun geänderten Teile der Klassifikation zurückgegriffen wurde, noch einmal aufgegriffen werden. Medikamentenstudien zum Beispiel, die nach den vorherigen diagnostischen Kriterien durchgeführt wurden, müssen wiederholt werden, falls sich bei den diagnostischen Kriterien größere Veränderungen ergeben, da die Patienten, die unter die neue Diagnose fallen, sich von denen unterscheiden werden, die unter die vorherige Diagnose fielen. Meine Hoffnung ist, dass die aktiven Feldstudien und die wissenschaftliche Analyse, die für die ICHD-3 vorgenommen wurden weitergehen und es so ermöglichen, dass künftige Änderungen voll und ganz evidenzbasiert sein werden. Der Tradition entsprechend werden bis zur ICHD-4 10-15 Jahre ins Land gehen, doch werden unterdessen etliche Feldstudien erstellt werden. Modifizierte diagnostische Kriterien der ICHD-II für eine 1.3 chronische Migräne wurden in der Zeitschrift Cephalalgia veröffentlicht, das Klassifikationskomitee stimmte diesen Änderungen zu und bat um ihre sofortige Anwendung, obwohl sie noch nicht in die Internationale Klassifikation von Kopfschmerzerkrankungen aufgenommen worden waren, bevor Jahre später die ICHD-3 beta erschien. Ein künftiges Kopfschmerzklassifikationskomitee sollte auf ähnliche Weise in der Lage sein, der Festlegung neuer oder überarbeiteter diagnos- 
tischer Kriterien vor der Veröffentlichung der ICHD-4 zuzustimmen und diese zu unterstützen, wenn diese durch gute in der Zeitschrift Cephalalgia veröffentlichte Feldstudien untermauert werden.

Mit der Kopfschmerzklassifikation in der ICHD-1 wandelten sich Kopfschmerzen von den mit am schlechtesten klassifizierten neurologischen Erkrankungen zu den bestklassifizierten. Wir haben diese Dynamik 30 Jahre lang beibehalten, und die Überlegenheit unserer Klassifikation zeigte sich neulich im Rahmen der Arbeit des Komitees in Genf am neurologischen Teil der ICD-11. Keine andere Fachrichtung innerhalb der Neurologie verfügt über eine derartige systematische Klassifikation samt expliziten Diagnosekriterien für jede Krankheitsentität. Ich hoffe aufrichtig, dass sich diese Tradition in der Zukunft aufrechterhalten lässt und dass die Kopfschmerzklassifikation so zur Wegbereiterin für die Klassifikation neurologischer Erkrankungen schlechthin wird.

\section{Jes Olesen}

Vorsitzender des

Kopfschmerzklassifikationskomitees der

Internationalen Kopfschmerzgesellschaft

\section{Vorwort zur 1. Auflage (1988)}

Das vorliegende Dokument ist das Ergebnis sehr intensiver Bemühungen. Die Arbeit benötigte fast drei Jahre. An ihr waren nicht nur die Mitglieder des Klassifikationskomitees selbst beteiligt, sondern auch die vielen Mitglieder der zwölf Subkomitees. Die Beratungen erfolgten öffentlich, so dass alle Entwürfe für jeden zugänglich waren, der dies wünschte. Im März 1987 hatten wir ein zweitägiges Treffen über die Kopfschmerzklassifikation durchgeführt, das für jeden interessierten Kollegen offenstand. Am Ende des 3. Internationalen Kopfschmerzkongresses in Florenz im September 1987 wurde der Entwurf der Klassifikation öffentlich vorgestellt und diskutiert. Eine weitere öffentliche Tagung fand am 20. und 21. Februar 1988 in San Diego statt.

Trotz aller Anstrengungen sind Fehler wohl nicht zu vermeiden. Sie werden bei Benutzung der Klassifikation auftauchen und müssen in folgenden Auflagen korrigiert werden. Auch sollte darauf aufmerksam gemacht werden, dass viele Teile der Arbeit wegen des Fehlens hinlänglicher veröffentlichter Befunde auf der Erfahrung der im Komitee arbeitenden Experten basieren. Es kann jedoch die Erwartung geäußert werden, dass das Vorhandensein praktikabler diagnostischer Kriterien, wie sie in dieser Arbeit nun vorgelegt werden, eine zunehmende nosologische und epidemiologische Forschungsaktivität anregen wird.

Wir bitten alle Wissenschaftler, die sich mit Kopfschmerz beschäftigen, sich aktiv an der Testung und Weiterentwicklung der Klassifikation zu beteiligen. Senden Sie bitte Ihre Meinungen, Argumente und Sonderdrucke an den Vorsitzenden des Klassifikationskomitees. Eine zweite Auflage der Klassifikation ist für das Jahr 1993 geplant. Wir hoffen, dass die revidierte Auflage dann auf neuen Befunden aufbauen kann.

Die International Headache Society sieht die Klassifikation und die Erstellung von diagnostischen Kriterien als eine sehr wichtige Aufgabe an. Auch wenn das Dokument noch weiterer Erprobung und Veränderung bedarf, empfehlen wir die umgehende Anwendung bei wissenschaftlichen Vorhaben. Dies gilt nicht nur für Me- dikamentenversuche, sondern auch biochemische und physiologische Studien.

\author{
James W. Lance \\ Präsident der \\ Internationalen Kopfschmerzgesellschaft
}

\section{Jes Olesen}

Vorsitzender des

Kopfschmerzklassifikationskomitees

\section{Vorwort zur 2. Auflage}

Klassifikationen von Erkrankungen und ihre diagnostischen Kriterien werden von der ärztlichen Gemeinde oftmals mit Skepsis aufgenommen und häufig kaum genutzt. Es war daher eine freudige Überraschung zu sehen, wie wohlwollend die erste Auflage der Internationalen Klassifikation von Kopfschmerzerkrankungen aufgenommen wurde. Sie wurde praktisch sofort weltweit für Forschungszwecke akzeptiert. So wurde die große Welle der Triptanstudien mit Patienten durchgeführt, die nach den Kriterien der Internationalen Klassifikation von Kopfschmerzerkrankungen diagnostiziert worden waren. Langsam, aber sicher haben die Prinzipien der Klassifikation auch die klinische Praxis verändert. Viel unnötige Fragen zur Einordnung von primären Kopfschmerzen werden in der Anamnese nicht mehr gestellt, wohingegen neue Kriterien wie eine Verstärkung durch körperliche Aktivität allmählich in den klinischen Alltag Einzug finden. Die Klassifikation wurde in mehr als 20 Sprachen übersetzt und steht damit der Mehrzahl der Mediziner auf der ganzen Welt zur Verfügung.

Als die erste Auflage der Internationalen Klassifikation von Kopfschmerzerkrankungen veröffentlicht wurde, erwartete man, dass eine zweite Auflage innerhalb von 5 Jahren folgen würde, da große Teile eher auf der Meinung der Experten als auf veröffentlichten Daten beruhten. Dennoch dauerte es 15 Jahre bis zur Präsentation der zweiten Auflage. Dafür gibt es mehrere gute Gründe: Es gab relativ wenig Kritik, die eine Revision erforderlich gemacht hätte. Nosographische Untersuchungen, die eine bessere Beschreibung der klinischen Merkmale verschiedener Kopfschmerzerkrankungen erlaubt hätten, erschienen nur langsam und waren nicht ausreichend, um eine vollständig evidenzbasierte Klassifikation zu ermöglichen. Die Verbreitung der englischen Fassung der ersten Auflage und ihre Übersetzung in mehr als 20 verschiedene Sprachen dauerte länger als erwartet. Langsam mehrten sich jedoch Vorschläge für eine Revision und nosographisches Wissen wuchs in dem Maße, dass es sinnvoll erschien, die Arbeiten für eine zweite Auflage zu beginnen.

Wie bei der ersten Auflage, hatte ich auch diesmal das Privileg des Vorsitzenden, die Mitglieder des Komitees zu benennen. Obwohl das erste Komitee seine Arbeit hervorragend gemacht hatte, war ich der Meinung, dass ein größerer Austausch der Mitglieder des Klassifikationskomitees stattfinden sollte, um sicherzustellen, dass die nächste Generation von Kopfschmerzforschern ausreichend repräsentiert wäre. Daher waren Guiseppe Nappi, James W. Lance und ich selbst die einzigen im ersten und zweiten Komitee vertretenen Mitglieder. Wir waren damit für die Kontinuität verantwortlich. Bei der Benennung der neuen Mitglieder habe ich in erster Linie die persönliche Qualifikation berücksichtigt. Geographi- 
sche Repräsentation und der Wunsch, Personen einzuschließen, die konstruktive Kritik an der ersten Auflage geübt hatten, wurden ebenso berücksichtigt. Ich glaube sagen zu können, dass sich die Rekrutierung nach diesen Prinzipien als erfolgreich erwiesen hat. Jedes Mitglied hat lebhaftes Interesse gezeigt, war freimütig und hat gut argumentiert. Vor dem Hintergrund der vom Komitee der ersten Auflage geleisteten gewaltigen Arbeit wurde vom Komitee der zweiten Auflage über jeden Aspekt der Kopfschmerzklassifikation offen debattiert. Aufgrund der sorgfältigen Arbeit und der vielen fruchtbaren Diskussionen brauchte die zweite Auflage deutlich länger als erwartet. Jeder einzelne Satz von Kriterien, jede Nummer und jedes Wort wurden sorgfältig abgewogen, enorme Bemühungen und Gedanken wurden in die vorliegende Veröffentlichung eingebracht. Alle Meinungen jedes einzelnen Mitgliedes konnten nicht berücksichtigt werden, aber jedes Mitglied hatte einen beträchtlichen Einfluss auf die Klassifikation.

Für jeden Bereich der Medizin ist es wichtig, eine allgemein akzeptierte Klassifikation zu haben, die weltweit genutzt wird. Dies ist insbesondere wichtig für Kopfschmerzen als junges und sich noch entwickelndes Forschungsgebiet, gibt es doch noch viele Vorurteile gegenüber Kopfschmerzerkrankungen. Deshalb ist es extrem wichtig, dass die Kopfschmerzgemeinde im allgemeinen und die Kopfschmerzforscher im speziellen den Einsatz von Die Internationale Klassifikation von Kopfschmerzerkrankungen, 2. Auflage unterstützen. Keine Zeitschrift sollte eine Arbeit über Kopfschmerzen veröffentlichen, die nicht diese Klassifikation und die damit verbundenen Kriterien nutzt oder untersucht. Auf der anderen Seite ist es nicht unsere Absicht, die Kopfschmerzforschung einzuengen. Wir richten daher die große Bitte an die Kopfschmerzforscher dieser Welt, diese zweite Auflage wissenschaftlich zu überprüfen. Um solche Untersuchungen anzuregen, haben wir einen Anhang aufgenommen, in dem eine Zahl stiefmütterlich behandelter Erkrankungen beschrieben sind, die einer Validierung benötigen. Wir haben auch einige alternative Kriterien aufgeführt, die im Vergleich zu den offiziellen getestet werden können.

Ich hoffe aufrichtig, dass diese zweite Auflage der Klassifikation von der Kopfschmerzgemeinde in der ganzen Welt positiv angenommen wird und dass sie in noch mehr Sprachen als die erste Version übersetzt wird. Ich hoffe auch, dass sie Grundlage für die Lehre der Kopfschmerzklassifikation und Kopfschmerzdiagnostik weltweit wird und dass damit auch die Kopfschmerzbehandlung profitiert. Die International Headache Society hat das Ziel, die Diagnostik, die Behandlung und die Versorgung von Kopfschmerzpatienten weltweit zu verbessern. Sie arbeitet aber auch daran, Kopfschmerzpatienten zu entstigmatisieren und die Anerkennung dieser Erkrankung als neurobiologische Störung zu bewirken - eine Erkrankung, die den Patienten, ihren Verwandten und der Gesellschaft eine große Last auferlegt. Für den Erfolg dieser Bemühungen ist es unerlässlich, dass Forscher und Kliniker ein einziges diagnostisches System nutzen und dass dieses System so präzise wie möglich ist. Auf diesem Weg hat die erste Auflage der Internationalen Klassifikation von Kopfschmerzerkrankungen ein beträchtliches Stück zurückgelegt. Diese zweite Auflage wird hoffentlich die Einheit noch weiter fördern, mit der wir Kopfschmerzen weltweit klassifizieren, diagnostizieren und behandeln.
Jes Olesen

Vorsitzender des Kopfschmerzklassifikationskomitees der Internationalen Kopfschmerzgesellschaft

\section{Vorwort der Präsidenten der drei deutsch- sprachigen Kopfschmerzgesellschaften zur}

\section{Auflage}

Mit der deutschen Übersetzung der 2. Auflage der Internationalen Klassifikation von Kopfschmerzerkrankungen wird einer guten Tradition gefolgt, nachdem bereits die erste Auflage der Klassifikation in einem Sonderheft der Nervenheilkunde 1989 publiziert worden war.

Neu ist jedoch, dass nunmehr die drei deutschsprachigen wissenschaftlichen Fachgesellschaften aus Deutschland, Österreich und der Schweiz gemeinsam für diese Übersetzung verantwortlich zeichnen. Als nationale Mitgliedsgesellschaften der International Headache Society haben die Deutsche Migräne- und Kopfschmerzgesellschaft, die Österreichische Kopfschmerzgesellschaft und die Schweizerische Kopfwehgesellschaft das Copyright für diese Übersetzung übernommen. Alle Anfragen in Zusammenhang mit der deutschen Übersetzung sind jeweils an eine dieser drei zuständigen nationalen Fachgesellschaften zu richten.

Die Hauptübersetzungsarbeit wurde von Herrn Prof. Dr. H. Göbel zusammen mit seiner Mitarbeiterin Frau Dr. K. Heinze-Kuhn und seinem Mitarbeiter Herrn Dr. A. Heinze geleistet. Die Organisation des Übersetzungs- und Publikationsablaufs wurde von Herrn PD Dr. Dr. S. Evers koordiniert. Ihnen gebührt insbesondere der Dank der drei Fachgesellschaften.

Ziel war es, einen einheitlichen deutschen Text zu entwickeln, der es den in der Kopfschmerzbehandlung und Kopfschmerzforschung Tätigen ermöglicht, anhand operationalisierter Kriterien exakte Diagnosen zu stellen. Dabei konnten regionale oder umgangssprachliche Besonderheiten in der Bezeichnung verschiedener Kopfschmerzformen keine Berücksichtigung finden; in der Orthographie wurde der jüngsten Rechtschreibreform nach dem Duden gefolgt. Ziel war es auch, die diagnostischen Begriffe der ersten Auflage so weit wie möglich zu übernehmen, d. h. keine unnötigen Änderungen einzuführen. Änderungen der originalen englischen Begriffe wurden jedoch nachvollzogen.

Wir wünschen uns eine weite Verbreitung dieser deutschen Übersetzung zum Nutzen der Patientinnen und Patienten, die an den vielen verschiedenen Kopfschmerzarten leiden, und zur Weiterentwicklung der Kopfschmerzforschung, die letztlich ja wiederum diesen Patientinnen und Patienten nutzen soll.

Dem Schattauer Verlag sei für seine unkomplizierte Kooperation und Hilfsbereitschaft bei der Erstellung dieses Sonderhefts der Nervenheilkunde gedankt.

\section{Prof. Dr. G. Haag}

Präsident der Deutschen Migräne- und Kopfschmerzgesellschaft

Univ.-Prof. Dr. Peter Wessely

Präsident der Österreichischen Kopfschmerzgesellschaft

Dr. C. Meyer

Präsident der Schweizerischen Kopfwehgesellschaft 
Die deutschsprachige Übersetzung der 2. Auflage der Kopfschmerzklassifikation der International Headache Society - Einleitung

\author{
Autoren \\ Stefan Evers ${ }^{1}$ \\ Hartmut Göbel ${ }^{2}$ \\ Institute \\ 1 Klinik und Poliklinik für Neurologie, \\ Universitätsklinikum Münster \\ 2 Neurologisch-verhaltensmedizinische Schmerzklinik Kiel
}

Die International Headache Society (IHS) hat auf ihrem Kongress im September 2003 in Rom die neue Klassifikation der Kopfschmerzerkrankungen vorgestellt. Sie folgt der ersten Fassung aus dem Jahr 1988 (Cephalalgia 1988; 8 Suppl. 7: 1-92), die 1989 in ihrer deutschen Version erschienen ist (Nervenheilkunde 1989; 8: 161-203) und 1999 erstmalig in adaptierter Form offiziell von der Weltgesundheitsorganisation in deutscher Sprache publiziert worden ist (ICD-10-Richtlinien für die Klassifikation und Diagnostik on Kopfschmerzen. Berlin: Springer-Verlag 1999). Die Klassifikation ist auch unter Beteiligung von Kollegen aus Deutschland, Österreich und der Schweiz entstanden, diese sind namentlich in der Einleitung zum Hauptteil aufgeführt.

Zeitnah wird nun auch von der zweiten Fassung eine deutschsprachige Übersetzung veröffentlicht, die sich direkt an der englischsprachigen Originalfassung orientiert, die in der Zeitschrift Cephalalgia veröffentlicht worden ist. In dieser Einleitung sollen die wichtigsten Aspekte, die bei dieser Übersetzung eine Rolle gespielt haben, skizziert werden.

In der deutschen Fassung der Kopfschmerzklassifikation ist in größtmöglicher Übereinstimmung mit dem englischsprachigen Originaltext erarbeitet worden. Bei einigen Begriffen wurde auf deutsche Umschreibungen zurückgegriffen, die besser als eine wörtliche Übersetzung des englischsprachigen Begriffs in der Lage sind, das entsprechende Kopfschmerzsyndrom zu bezeichnen. An manchen Stellen wurde dagegen die englischsprachige Originalbezeichnung beibehalten (insbesondere bei Akronymen), da sich keine adäquate deutschsprachige Übersetzung finden ließ. Im Aufbau wurde keine Änderung im Vergleich zur Originalversion vorgenommen.

Das grundsätzliche Ziel der Klassifikation ist es, diagnostische Bezeichnungen und explizite Kriterien zur Verfügung zu stellen, mit denen international verbindlich eindeutige Diagnosen formuliert werden können und mit denen die Kopfschmerzforschung möglichst einheitliche Untersuchungsgruppen rekrutieren kann. Gerade Letzteres bedeutet, dass im Konfliktfall der Spezifität einer Diagnose mehr Gewicht verliehen worden ist als der Sensitivität. Die Systematik der Diagnosen folgt auch weiterhin der Semiologie der Kopfschmerzen, d.h. sie ist phänomenologisch und nicht ätiologisch orientiert. Auch die deutschsprachige Übersetzung hat diesen Grundprinzipien der Klassifikation Rechnung getragen. Dies macht eine sorgfältige Formulierung der Kriterien umso wichtiger, da die Diagnosestellung entscheidend von den Angaben der Patienten und nicht von objektivierbaren Untersuchungsergebnissen abhängt. An einigen Stellen fordert die Klassifikation jedoch apparative Zusatzdiagnostik (bei primären Kopfschmerzen zum Ausschluss anderer Ursachen, bei sekundären Kopfschmerzen zum Nachweis der Erkrankung, auf die die Kopfschmerzen zurückgeführt werden können).

Wie auch in der ersten Fassung teilt die überarbeitete Klassifikation in primäre und sekundäre Kopfschmerzformen ein. Die sprachliche Unterteilung in idiopathische und symptomatische Kopfschmerzen wurde aufgegeben zugunsten einer konsequenten Verwendung der Bezeichnungen primär versus sekundär. Dem ist in der deutschsprachigen Übersetzung gefolgt worden. Die sekundären Kopfschmerzen werden in der englischsprachigen Version als „attributed to “ bezeichnet; dies löst die Formulierung „associated with “ ab. Damit soll zum Ausdruck gebracht werden, dass eine pathophysiologische Beziehung zwischen dem Kopfschmerz und der zugrundeliegenden Erkrankung, Störung oder Bedingung vorliegen muss. Der Zusammenhang muss also über eine einfache Assoziation (z. B. rein zeitlicher Natur) hinausgehen. In der deutschsprachigen Fassung wird dies durch den Begriff „zurückzuführen auf“ zum Ausdruck gebracht. Damit ist gemeint, dass eine direktionale Beziehung zwischen Kopfschmerzsymptom und zugrundeliegender Erkrankung, Störung oder Bedingung vorliegen muss. Bei den meisten sekundären Kopfschmerzen ist die ursächliche Verknüpfung zwischen einer durch die klinischen oder durch die weiterführenden Untersuchungsergebnisse fassbaren Erkrankung und den bestehenden Kopfschmerzen ausreichend gut nachgewiesen. Die Übersetzer und die herausgebenden Fachgesellschaften sind sich darüber im Klaren, dass die Formulierung ?zurückzuführen auf“ im klinischen Alltag umständlich und manchmal wenig praktikabel ist. Formulierungen wie „aufgrund“ oder „bedingt durch“, die im klinischen Alltag verwendet werden mögen, sind aber weniger geeignet, die Intention des englischsprachigen Originaltextes wiederzugeben.

In der Strukturierung der einzelnen Kapitel wurden als neue Gruppe die Kopfschmerzen eingeführt, die auf eine psychiatrische Störung zurückzuführen sind. Die Definition der psychiatrischen Störung folgt dabei dem DSMIV und nicht dem sonst in Deutschland üblichen ICD10. Die Gesichtsneuralgien und zentralen Ursachen von Kopfschmerzen sind in einen eigenständigen dritten Abschnitt übernommen worden und nicht mehr Teil der sekundären bzw. symptomatischen Kopfschmerzen. Außerdem wurde neu ein Anhang formuliert, in dem zum einen Kopfschmerzdiagnosen übernommen worden sind, die zwar in der ersten Fassung der Klassifikation verzeichnet waren, für deren Entität es aber nicht genügend wissenschaftliche Belege gibt. Zum anderen finden sich darin Kopfschmerzdiagnosen, für die es noch nicht genügend wissenschaftliche Evidenz gibt, die aber möglicherweise in einer zukünftigen Klassifikation zu berücksichtigen sind. Außerdem werden alternative diagnostische Kriterien vorgestellt, die vor allem für Forschungszwecke gedacht sind.

Die IHS stellt das Copyright der Übersetzung ihren jeweiligen nationalen Mitgliedsgesellschaften zur Verfügung. Die Deutsche Migräne- und Kopfschmerzgesellschaft (DMKG) hat dies für Deutschland übernommen. In Absprache mit der Österreichischen Kopfschmerzgesellschaft und der Schweizerischen Kopfwehgesellschaft wurde eine einheitliche Übersetzung angefertigt, die im gesamten deutschsprachigen Raum verwendet werden soll. Das Copyright für die einzelnen Länder liegt somit bei den jeweiligen nationalen 
Fachgesellschaften. Es ist zu hoffen, dass die anderen medizinischen Fachgesellschaften sich an dieser Übersetzung orientieren werden. Nicht zuletzt dafür ist eine Synopsis mit dem ICD10 ebenfalls Bestandteil der Klassifikation.

Die vollständige Übersetzung der englischsprachigen Originalversion erfolgte durch Dr. Katja Heinze-Kuhn und Dr. Axel Heinze aus der Schmerzklinik Kiel und Leitung von Prof. Dr. H. Göbel, der auch Mitglied des Klassifikationskomitees der IHS ist. Die Hintergründe verschiedener Formulierungen, deren Zielrichtung und die Konsensbildung während der insgesamt vierjährigen Arbeit des Klassifikationskomitees konnten dadurch bei der deutschen Übersetzung zusätzlich berücksichtigt werden. Diese immense Arbeitsleistung hat erst die deutschsprachige Veröffentlichung unmittelbar nach der englischsprachigen Erstpublikation ermöglicht.

Von der DMKG wurde PD Dr. Dr. S. Evers beauftragt, die notwendigen organisatorischen Voraussetzungen für eine endgültige Publikation zu schaffen. Der abschließende Text der Übersetzung ist in den zuständigen Gremien der Fachgesellschaften zirkuliert und von diesen akzeptiert worden.

In der Einleitung zur englischsprachigen Version wird vielen Menschen, die zum Gelingen der neuen Klassifikation beigetragen haben, Dankt gesagt. Diesem Dank können sich die Bearbeiter der deutschsprachigen Version nur anschließen. Zusätzlich seien hier aber auch diejenigen Personen genannt, die zur Publikation der deutschsprachigen Übersetzung beigetragen haben. Hierzu gehören Prof. Dr. D. Soyka, der als ehemaliger Präsident der DMKG, als Ehrenpräsident der IHS und als Herausgeber der Nervenheilkunde dem Projekt seine volle Unterstützung gegeben hat. Der Schattauer-Verlag, namentlich Frau S. Friedel, hat das Projekt sehr kooperativ begleitet und eine Publikation innerhalb kürzester Zeit möglich gemacht.

Korrespondenzadresse

Prof. Dr. med. Dr. phil. Stefan Evers

Klinik und Poliklinik für Neurologie

Universitätsklinikum Münster

Albert-Schweitzer-Str. 33

48129 Münster

Tel. + 49-251-8348016

Fax $+49-251-8348181$

Email everss@uni-muenster.de

\section{Anleitung zum Gebrauch der Klassifikation}

Dieses umfassende Werk ist nicht zum Auswendiglernen gedacht. Selbst die Mitglieder des Kopfschmerzklassifikationskomitees dürften wohl kaum in der Lage sein, sich an alle Details zu erinnern. Vielmehr versteht es sich als Standardwerk, das bei Bedarf immer wieder konsultiert werden kann. Auf diese Weise wird man Stück für Stück die diagnostischen Kriterien für eine 1.1 Migräne ohne Aura, eine 1.2 Migräne mit Aura, die wichtigsten Subtypen des 2. Kopfschmerzes vom Spannungstyp und des 3.1 Clusterkopfschmerzes sowie einiger anderen Kopfschmerztypen kennenlernen. Die übrigen Kopfschmerztypen wird man immer nachschlagen müssen. Im klinischen Alltag wird sich die Klassifikation etwa bei einer eindeutigen Migräne oder einem eindeutigen Kopfschmerz vom Spannungstyp erübrigen. Nützlich ist sie aber stets dann, wenn die Diagnose unsicher ist. Unentbehrlich ist die Klassifikation für wissenschaftliche Zwecke. Jeder Patient, der in ein Studienprojekt aufgenommen werden soll, sei es in eine Medikamentenstudie, eine pathophysiologische Untersuchung oder eine Studie zur Biochemie von Kopfschmerzen, muss bestimmte diagnostische Kriterien erfüllen.

Die Klassifikation ist hierarchisch gegliedert und der Anwender muss im Einzelfall selbst entscheiden, wie detailliert seine Diagnose ausfallen soll - von der ersten Stelle bis zur fünften. Die erste Stelle bietet eine grobe Einordnung des Kopfschmerzes in eine bestimmte Diagnosekategorie. Handelt es sich z. B. um eine 1. Migräne, einen 2. Kopfschmerz vom Spannungstyp oder einen 3. Clusterkopfschmerz bzw. einen anderen trigemino-autonomen Kopfschmerz? Die weiteren Stellen beinhalten dann detailliertere Informationen zur Diagnose. Die gewünschte Detailtiefe hängt vom Zweck ab. In der Praxis des Allgemeinmediziners werden in der Regel nur Diagnosen benötigt werden, die auf die 1. oder 2. Stelle exakt sind, während spezielle Fachpraxen oder Kopfschmerzzentren Diagnosen auf der Ebene der 4. oder 5. Stelle verwenden werden.

Die Patienten erhalten eine Diagnose entsprechend der Kopfschmerzphänomenologie, die aktuell auftritt oder im Verlauf des letzten Jahres bestand. Für genetische und andere Zwecke werden auch Kopfschmerzen herangezogen, die im Laufe des Lebens aufgetreten sind.

Jeder einzelne bei einem Patienten bestehende Kopfschmerztyp muss diagnostiziert und kodiert werden. So erhalten schwer betroffene Patienten eines spezialisierten Kopfschmerzzentrums häufig drei Diagnosen: 1.1 Migräne ohne Aura, 1.2 Migräne mit Aura und 8.2 Kopfschmerzen zurückzuführen auf einen Medikamentenübergebrauch.

Falls ein Patient mehr als eine Diagnose erhält, sollten diese in der Reihenfolge ihrer Bedeutung für den Patienten aufgelistet werden.

Falls der Kopfschmerz eines Patienten die diagnostischen Kriterien für zwei verschiedene Kopfschmerzentitäten erfüllt, sollten alle verfügbaren Informationen hinzugezogen werden, um zu entscheiden, welche der beiden Diagnosen die tatsächliche oder zumindest wahrscheinlichere ist. Von Interesse kann der Verlauf der Kopfschmerzerkrankung sein: Wie begannen die Kopfschmerzen? Aber auch die Familienanamnese, die Wirksamkeit von Medikamenten, die Beziehung zur Menstruation, das Alter, Geschlecht und eine Reihe andere Merkmale sollten berücksichtigt werden. Sind die Kriterien einer 1. Migräne, eines 2. Kopfschmerzes vom Spannungstyp, einer 3. trigemino-autonomen Kopfschmerzerkrankung oder einer ihrer Subtypen vollständig erfüllt, stechen diese Diagnosen immer die am Ende der betreffenden Kapitel angeführten wahrscheinlichen diagnostischen Kategorien. Falls ein Patient z. B. einen Kopfschmerz aufweist, der sowohl die Kriterien für eine 1.5 wahrscheinliche Migräne als auch für einen 2.1 selten auftretenden episodischen Kopfschmerz vom Spannungstyp erfüllt, sollte eine Kodierung unter letzterer Diagnose erfolgen. Es sollte jedoch auch bedacht werden, dass einige Attacken die Kriterien einer Kopfschmerzform und andere Attacken die Kriterien einer anderen erfüllen können. In diesem Fall sollten zwei Diagnosen vergeben werden.

Um eine Kopfschmerzdiagnose zu erhalten, muss der Patient in vielen Fällen bereits eine bestimmte Anzahl an Attacken (oder 
Tagen) mit diesem Kopfschmerz gehabt haben. Die genaue Anzahl ist für jeden Kopfschmerztyp bzw. -subtyp in den diagnostischen Kriterien definiert. Die Kopfschmerzen müssen weiter eine Reihe von Bedingungen erfüllen, die unter alphabetischen Gliederungspunkten beschrieben sind: A, B, C, etc. Hinter einigen dieser Gliederungspunkte verbirgt sich eine einzelne Bedingung, die zutreffen muss, hinter anderen findet sich eine Auflistung von Punkten, von denen eine bestimmte Anzahl erfüllt sein muss, z. B. zwei von vier Charakteristika.

Ein vollständiger Kriterienkatalog findet sich bei einigen Kopfschmerzformen nur bis zur Ebene der ersten oder zweiten Stelle. Die diagnostischen Kriterien der dritten, vierten und gelegentlich fünften Stelle fordern dann als Kriterium A, dass die Kriterien der Ebenen 1 und/oder 2 erfüllt sind, um ab Kriterium B die weiteren Kriterien zu spezifizieren, die erfüllt sein müssen.

Die Frequenz primärer Kopfschmerzen kann von einer Attacke in einem oder zwei Jahren bis zum täglichen Auftreten variieren. Auch die Intensität der Kopfschmerzen kann sehr unterschiedlich sein. Die Internationale Klassifikation von Kopfschmerzerkrankungen, 3. Auflage bietet keine grundsätzliche Möglichkeit, die Frequenz oder Intensität der Kopfschmerzen zu kodieren, empfiehlt aber, dass Frequenz und Intensität im freien Text spezifiziert werden.

Primärer und/oder sekundärer Kopfschmerz: Tritt ein neuer Kopfschmerz erstmals in engem zeitlichen Zusammenhang zu einer bekannten Kopfschmerzursache auf, sollte dieser Kopfschmerz der ursächlichen Erkrankung entsprechend als sekundärer Kopfschmerz kodiert werden. Dies ist auch der Fall, wenn der Kopfschmerz das klinische Bild eines primären Kopfschmerzes (einer Migräne, eines Kopfschmerzes vom Spannungstyp oder eines Clusterkopfschmerzes bzw. einer anderen trigemino-autonomen Kopfschmerzerkrankung) aufweist. Wenn ein vorbestehender primärer Kopfschmerz in engem zeitlichen Zusammenhang zu einer bekannten Kopfschmerzursache chronisch wird, sollte der Patient die Diagnose des vorbestehenden primären Kopfschmerzes wie auch die des sekundären Kopfschmerzes erhalten. Wenn sich ein vorbestehender primärer Kopfschmerz in engem zeitlichem Zusammenhang zu einer bekannten Kopfschmerzursache deutlich verschlechtert (üblicherweise definiert als eine mindestens zweifache Steigerung der Häufigkeit und/oder Intensität), sollte sowohl die Diagnose des vorbestehenden primären Kopfschmerzes als auch die des sekundären Kopfschmerzes vergeben werden, sofern gute Hinweise darauf bestehen, dass die verdächtigte Störung Kopfschmerzen hervorrufen kann.

Das letzte Kriterium für fast alle Kopfschmerzerkrankungen lautet: „Nicht besser erklärt durch eine andere ICHD-3-Diagnose”. Die Berücksichtigung anderer möglicher Diagnosen (die Differenzialdiagnose) ist routinemäßig Bestandteil der klinischen Diagnostik. Wenn ein Kopfschmerz die Kriterien für eine bestimmte Kopfschmerzerkrankung zu erfüllen scheint, erinnert dieses letzte Kriterium daran, immer andere Diagnosen in Betracht zu ziehen, die den Kopfschmerz besser erklären könnten.

Dies gilt vor allem für die Beurteilung, ob ein sekundärer oder ein primärer Kopfschmerz vorliegt. Es kann auch für alternative ursächliche Störungen gelten: zum Beispiel kann ein Kopfschmerz, der in engem zeitlichen Zusammenhang mit einem akuten ischämischen Infarkt auftritt, nicht Folge des Hirninfarkts sein, sondern seiner Ursache (z. B. Dissektion).
Viele Patienten, die die diagnostischen Kriterien einer Kopfschmerzerkrankung erfüllen, kennen in der Regel auch ähnliche Kopfschmerzen, die die Kriterien nicht ganz erfüllen. Dies kann u. a. auf eine Behandlung zurückzuführen sein, aber auch auf die Unfähigkeit, Symptome genau zu erinnern oder andere Faktoren. Man sollte den Patienten bitten, eine typische unbehandelte oder unzureichend behandelte Attacke zu beschreiben und man sollte sicherstellen, dass eine ausreichende Anzahl davon abgelaufen ist, um eine Diagnose stellen zu können. Die weniger typischen Attacken können dann bei der Beschreibung der Attackenhäufigkeit mitgezählt werden.

Falls der Verdacht besteht, dass ein Patient mehr als nur einen Kopfschmerz oder eine Kopfschmerzform aufweist, ist das Führen eines diagnostischen Kopfschmerzkalenders unbedingt empfehlenswert, in dem für jede Kopfschmerzepisode die wichtigsten Merkmale vermerkt werden. Es konnte gezeigt werden, dass Kopfschmerzkalender die diagnostische Genauigkeit erhöhen und auch eine genauere Beurteilung des Medikamentenkonsums erlauben. Schließlich hilft dieses Tagebuch, die genaue Häufigkeit von zwei oder mehr verschiedenen Kopfschmerzformen oder -subtypen zu beurteilen, und es erleichtert dem Patienten, zwischen den verschiedenen Kopfschmerzformen, z. B. einer Migräne ohne Aura und einem episodischen Kopfschmerz vom Spannungstyp, zu unterscheiden.

In jedem Kapitel zu sekundären Kopfschmerzen werden die am besten bekannten und anerkannten Ursachen erwähnt und entsprechende Kriterien aufgeführt. In vielen Kapiteln gibt es jedoch eine schier unendliche Zahl an möglichen Ursachen, so z. B. bei 9. Kopfschmerzen zurückzuführen auf eine Infektion. Um hier sehr lange Ursachenlisten zu vermeiden, sind nur die wichtigsten erwähnt. Bei diesem Beispiel werden seltenere ursächliche Infektionen der Diagnose 9.2.3 Kopfschmerzen zurückzuführen auf eine andere systemische Infektion zugeordnet. Dasselbe System wird auch in anderen Kapiteln mit sekundären Kopfschmerzen angewandt

Die diagnostischen Kriterien für sekundäre Kopfschmerzerkrankungen fordern nicht mehr die Remission oder deutliche Besserung der ursächlichen Grunderkrankung, bevor die Kopfschmerzdiagnose gestellt werden kann. Die diagnostischen Kriterien der ICHD-3 können bereits bei Auftreten des Kopfschmerzes oder unmittelbar nach Bestätigung der Grunderkrankung angewandt werden. Kriterium A ist das Vorliegen des Kopfschmerzes; Kriterium B ist das Vorliegen der ursächlichen Störung; Kriterium C ist der Nachweis des ursächlichen Zusammenhangs. Im Akutfall reicht oft ein enger zeitlicher Zusammenhang zwischen dem Beginn des Kopfschmerzes und dem Beginn der verdächtigten ursächlichen Störung, während in weniger akuten Fällen üblicherweise weitere ursächliche Nachweise erforderlich sind. In allen Fällen muss das letzte Kriterium geprüft werden: „Nicht besser erklärt durch eine andere ICHD-3-Diagnose“.

In einigen Fällen von sekundären Kopfschmerzen, der 5.2 anhaltender Kopfschmerz zurückzuführen auf eine traumatische Verletzung des Kopfes ist hier ein gutes Beispiel, wird das Auftreten von anhaltenden Kopfschmerztypen oder -subtypen anerkannt; das heißt, ein Kopfschmerz, für den zunächst eine andere Störung ursächlich war, verschwindet nicht, nachdem diese Erkrankung nicht mehr vorliegt. In solchen Fällen verändert sich die Diagnose nach 
einem spezifizierten Zeitraum (bei diesem Beispiel 3 Monate) vom akuten Typ (d. h. 5.1 akuter Kopfschmerz zurückzuführen auf eine traumatische Verletzung des Kopfes) zum anhaltenden Typ (5.2 anhaltender Kopfschmerz zurückzuführen auf eine traumatische Verletzung des Kopfes). Der Nachweis eines ursächlichen Zusammenhangs hängt von der ehemaligen Erfüllung der Kriterien für eine Diagnose des akuten Typs ab und vom Fortbestehen der gleichen Kopfschmerzen.

Die meisten derartigen Diagnosen finden sich im Anhang, da ihre Existenz nur unzureichend belegt ist. Sie werden nicht häufig gebraucht, sollen aber zur wissenschaftlichen Erforschung ursächlicher Zusammenhänge und Ermittlung besserer diagnostischer Kriterien anregen.

Der Anhang ist zu Forschungszwecken beigefügt. Er hilft klinischen Wissenschaftlern, seltene Krankheitsentitäten für eine spätere Einbeziehung in (oder in einigen Fällen einen Ausschluss aus) dem Hauptteil der Klassifikation zu untersuchen. Die meisten Diagnosen und diagnostischen Kriterien im Anhang sind entweder neu oder Alternativen zu Kriterien im Hauptteil. Einige sind alte Entitäten, die noch nicht ausreichend validiert sind; diese werden aller Voraussicht nach in der nächsten Überarbeitung der ICHD gelöscht werden, sofern kein diesbezüglicher Nachweis erbracht wird.

\section{Klassifikation}

\begin{tabular}{|c|c|}
\hline $\begin{array}{l}\text { ICHD-3 } \\
\text { Code }\end{array}$ & Diagnose \\
\hline 1. & Migräne \\
\hline 1.1 & Migräne ohne Aura \\
\hline 1.2 & Migräne mit Aura \\
\hline 1.2 .1 & Migräne mit typischer Aura \\
\hline 1.2.1.1 & Typische Aura mit Kopfschmerz \\
\hline 1.2.1.2 & Typische Aura ohne Kopfschmerz \\
\hline 1.2 .2 & Migräne mit Hirnstammaura \\
\hline 1.2 .3 & Hemiplegische Migräne \\
\hline 1.2.3.1 & Familiäre hemiplegische Migräne (FHM) \\
\hline 1.2.3.1.1 & Familiäre hemiplegische Migräne Typ 1 (FHM1) \\
\hline 1.2.3.1.2 & Familiäre hemiplegische Migräne Typ 2 (FHM2) \\
\hline 1.2.3.1.3 & Familiäre hemiplegische Migräne Typ 3 (FHM3) \\
\hline 1.2.3.1.4 & Familiäre hemiplegische Migräne, andere Genloci \\
\hline 1.2.3.2 & Sporadische hemiplegische Migräne (SHM) \\
\hline 1.2 .4 & Retinale Migräne \\
\hline 1.3 & Chronische Migräne \\
\hline 1.4 & Migränekomplikationen \\
\hline 1.4.1 & Status migränosus \\
\hline 1.4 .2 & Anhaltende Aura ohne Hirninfarkt \\
\hline 1.4 .3 & Migränöser Infarkt \\
\hline 1.4.4 & Epileptischer Anfall, durch Migräneaura getriggert \\
\hline 1.5 & Wahrscheinliche Migräne \\
\hline 1.5.1 & Wahrscheinliche Migräne ohne Aura \\
\hline 1.5 .2 & Wahrscheinliche Migräne mit Aura \\
\hline 1.6 & $\begin{array}{l}\text { Episodische Syndrome, die mit einer Migräne einhergehen } \\
\text { können }\end{array}$ \\
\hline 1.6.1 & Rezidivierende gastrointestinale Störungen \\
\hline
\end{tabular}

\begin{tabular}{|c|c|}
\hline $\begin{array}{l}\text { ICHD-3 } \\
\text { Code }\end{array}$ & Diagnose \\
\hline 1.6.1.1 & Zyklisches Erbrechen \\
\hline 1.6.1.2 & Abdominelle Migräne \\
\hline 1.6 .2 & Gutartiger paroxysmaler Schwindel \\
\hline 1.6 .3 & Gutartiger paroxysmaler Tortikollis \\
\hline 2. & Kopfschmerz vom Spannungstyp \\
\hline 2.1 & $\begin{array}{l}\text { Selten auftretender episodischer Kopfschmerz vom } \\
\text { Spannungstyp }\end{array}$ \\
\hline 2.1 .1 & $\begin{array}{l}\text { Selten auftretender episodischer Kopfschmerz vom } \\
\text { Spannungstyp mit perikranieller Schmerzempfindlichkeit }\end{array}$ \\
\hline 2.1 .2 & $\begin{array}{l}\text { Selten auftretender episodischer Kopfschmerz vom } \\
\text { Spannungstyp ohne perikranielle Schmerzempfindlichkeit }\end{array}$ \\
\hline 2.2 & $\begin{array}{l}\text { Häufig auftretender episodischer Kopfschmerz vom } \\
\text { Spannungstyp }\end{array}$ \\
\hline 2.2 .1 & $\begin{array}{l}\text { Häufig auftretender episodischer Kopfschmerz vom } \\
\text { Spannungstyp mit perikranieller Schmerzempfindlichkeit }\end{array}$ \\
\hline 2.2 .2 & $\begin{array}{l}\text { Häufig auftretender episodischer Kopfschmerz vom } \\
\text { Spannungstyp ohne perikranielle Schmerzempfindlichkeit }\end{array}$ \\
\hline 2.3 & Chronischer Kopfschmerz vom Spannungstyp \\
\hline 2.3 .1 & $\begin{array}{l}\text { Chronischer Kopfschmerz vom Spannungstyp mit peri- } \\
\text { kranieller Schmerzempfindlichkeit }\end{array}$ \\
\hline 2.3 .2 & $\begin{array}{l}\text { Chronischer Kopfschmerz vom Spannungstyp ohne peri- } \\
\text { kranielle Schmerzempfindlichkeit }\end{array}$ \\
\hline 2.4 & Wahrscheinlicher Kopfschmerz vom Spannungstyp \\
\hline 2.4 .1 & $\begin{array}{l}\text { Wahrscheinlicher selten auftretender, episodischer Kopf- } \\
\text { schmerz vom Spannungstyp }\end{array}$ \\
\hline 2.4 .2 & $\begin{array}{l}\text { Wahrscheinlicher häufig auftretender episodischer Kopf- } \\
\text { schmerz vom Spannungstyp }\end{array}$ \\
\hline 2.4 .3 & $\begin{array}{l}\text { Wahrscheinlicher chronischer Kopfschmerz vom Span- } \\
\text { nungstyp }\end{array}$ \\
\hline 3. & Trigemino-autonome Kopfschmerzerkrankungen(TAK) \\
\hline 3.1 & Clusterkopfschmerz \\
\hline 3.1 .1 & Episodischer Clusterkopfschmerz \\
\hline 3.1 .2 & Chronischer Clusterkopfschmerz \\
\hline 3.2 & Paroxysmale Hemikranie \\
\hline 3.2 .1 & Episodische paroxysmale Hemikranie \\
\hline 3.2 .2 & Chronische paroxysmale Hemikranie \\
\hline 3.3 & Short-lasting unilateral neuralgiform headache attacks \\
\hline 3.3 .1 & $\begin{array}{l}\text { Short-lasting unilateral neuralgiform headache attacks } \\
\text { with conjunctival injection and tearing (SUNCT-Syndrom) }\end{array}$ \\
\hline 3.3.1.1 & Episodisches SUNCT-Syndrom \\
\hline 3.3.1.2 & Chronisches SUNCT-Syndrom \\
\hline 3.3 .2 & $\begin{array}{l}\text { Short-lasting unilateral neuralgiform headache attacks } \\
\text { with cranial autonomic symptoms (SUNA-Syndrom) }\end{array}$ \\
\hline 3.3.2.1 & Episodisches SUNA-Syndrom \\
\hline 3.3.2.2 & Chronisches SUNA-Syndrom \\
\hline 3.4 & Hemicrania continua \\
\hline 3.5 & $\begin{array}{l}\text { Wahrscheinliche trigemino-autonome Kopfschmerz- } \\
\text { erkrankung }\end{array}$ \\
\hline 3.5 .1 & Wahrscheinlicher Clusterkopfschmerz \\
\hline 3.5 .2 & Wahrscheinliche paroxysmale Hemikranie \\
\hline 3.5 .3 & $\begin{array}{l}\text { Wahrscheinliche Short-lasting unilateral neuralgiform } \\
\text { headache attacks }\end{array}$ \\
\hline 3.5 .4 & Wahrscheinliche Hemicrania continua \\
\hline
\end{tabular}




\begin{tabular}{|c|c|c|c|}
\hline $\begin{array}{l}\text { ICHD-3 } \\
\text { Code }\end{array}$ & Diagnose & $\begin{array}{l}\text { ICHD-3 } \\
\text { Code }\end{array}$ & Diagnose \\
\hline 4. & Andere primäre Kopfschmerzen & \multirow[t]{2}{*}{5.4} & \multirow{2}{*}{$\begin{array}{l}\text { Anhaltender Kopfschmerz zurückzuführen auf ein HWS- } \\
\text { Beschleunigungstrauma }\end{array}$} \\
\hline 4.1 & Primärer Hustenkopfschmerz & & \\
\hline 4.1 .1 & Wahrscheinlicher primärer Hustenkopfschmerz & 5.5 & Akuter Kopfschmerz zurückzuführen auf eine Kraniotomie \\
\hline 4.2 & Primärer Anstrengungskopfschmerz & \multirow[t]{2}{*}{5.6} & \multirow{2}{*}{$\begin{array}{l}\text { Anhaltender Kopfschmerz zurückzuführen auf eine } \\
\text { Kraniotomie }\end{array}$} \\
\hline 4.2 .1 & Wahrscheinlicher primärer Anstrengungskopfschmerz & & \\
\hline 4.3 & Primärer Sexualkopfschmerz & 6. & $\begin{array}{l}\text { Kopfschmerz zurückzuführen auf Gefäßstörungen im } \\
\text { Bereich des Kopfes und/oder des Halses }\end{array}$ \\
\hline 4.3 .1 & Wahrscheinlicher primärer Sexualkopfschmerz & \multirow[t]{2}{*}{6.1} & \multirow{2}{*}{$\begin{array}{l}\text { Kopfschmerz zurückzuführen auf ein zerebrales ischämi- } \\
\text { sches Ereignis }\end{array}$} \\
\hline 4.4 & Primärer Donnerschlagkopfschmerz & & \\
\hline 4.5 & Kältebedingter Kopfschmerz & \multirow[t]{2}{*}{6.1 .1} & \multirow{2}{*}{$\begin{array}{l}\text { Kopfschmerz zurückzuführen auf einen ischämischen } \\
\text { Infarkt (zerebraler Infarkt) }\end{array}$} \\
\hline \multirow[t]{2}{*}{4.5 .1} & \multirow{2}{*}{$\begin{array}{l}\text { Kopfschmerzen zurückzuführen auf einen äußeren } \\
\text { Kältereiz }\end{array}$} & & \\
\hline & & \multirow[t]{2}{*}{6.1 .1 .1} & \multirow{2}{*}{$\begin{array}{l}\text { Akuter Kopfschmerz zurückzuführen auf einen ischämi- } \\
\text { schen Infarkt (zerebraler Infarkt) }\end{array}$} \\
\hline \multirow[t]{2}{*}{4.5 .2} & \multirow{2}{*}{$\begin{array}{l}\text { Kopfschmerzen zurückzuführen auf Einnahme oder } \\
\text { Inhalation eines Kältereizes }\end{array}$} & & \\
\hline & & \multirow[t]{2}{*}{6.1 .1 .2} & \multirow{2}{*}{$\begin{array}{l}\text { Anhaltender Kopfschmerz zurückzuführen auf einen } \\
\text { früheren ischämischen Infarkt (zerebraler Infarkt) }\end{array}$} \\
\hline 4.5 .3 & Wahrscheinlicher kältebedingter Kopfschmerz & & \\
\hline 4.5.3.1 & $\begin{array}{l}\text { Kopfschmerzen wahrscheinlich zurückzuführen auf einen } \\
\text { äußeren Kältereiz }\end{array}$ & 6.1 .2 & $\begin{array}{l}\text { Kopfschmerz zurückzuführen auf eine transitorische } \\
\text { ischämische Attacke (TIA) }\end{array}$ \\
\hline 4.5.3.2 & $\begin{array}{l}\text { Kopfschmerzen wahrscheinlich zurückzuführen auf Ein- } \\
\text { nahme oder Inhalation eines Kältereizes }\end{array}$ & 6.2 & $\begin{array}{l}\text { Kopfschmerz zurückzuführen auf eine nicht-traumatische } \\
\text { intrakranielle Blutung }\end{array}$ \\
\hline 4.6 & $\begin{array}{l}\text { Kopfschmerz durch Einwirkung von Druck oder Zug auf } \\
\text { den Kopf }\end{array}$ & 6.2 .1 & $\begin{array}{l}\text { Akuter Kopfschmerz zurückzuführen auf eine nicht- } \\
\text { traumatische intrazerebrale Blutung }\end{array}$ \\
\hline 4.6 .1 & Kopfschmerz durch äußeren Druck & 6.2 .2 & Kopfschmerz zurückzuführen auf eine nicht-traumatische \\
\hline 4.6 .2 & Kopfschmerz durch äußeren Zug & & \\
\hline 4.6 .3 & $\begin{array}{l}\text { Wahrscheinlicher Kopfschmerz durch Einwirkung von } \\
\text { Druck oder Zug auf den Kopf }\end{array}$ & 6.2 .3 & $\begin{array}{l}\text { Akuter Kopfschmerz zurückzuführen auf ein nicht- } \\
\text { traumatisches akutes Subduralhämatom (aSDH) }\end{array}$ \\
\hline 4.6.3.1 & Wahrscheinlicher Kopfschmerz durch äußeren Druck & 6.2 .4 & $\begin{array}{l}\text { Anhaltender Kopfschmerz zurückzuführen auf eine } \\
\text { frühere nicht-traumatische intrakranielle Blutung }\end{array}$ \\
\hline 4.6.3.2 & Wahrscheinlicher Kopfschmerz durch äußeren Zug & 6.3 & Kopfschmerz zurückzuführen auf eine nicht-rupturierte \\
\hline 4.7 & Primärer stechender Kopfschmerz & & Gefäßfehlbildung \\
\hline 4.7 .1 & Wahrscheinlicher primärer stechender Kopfschmerz & 6.3 .1 & Kopfschmerz zurückzuführen auf ein nicht-rupturiertes \\
\hline 4.8 & Münzkopfschmerz (engl. Nummular headache) & & sackförmiges Aneurysma \\
\hline 4.8 .1 & Wahrscheinlicher Münzkopfschmerz & 6.3 .2 & Kopfschmerz zurückzuführen auf eine arterio-venöse \\
\hline 4.9 & Schlafgebundener Kopfschmerz (engl. Hypnic headache) & & Malformation (AVM) \\
\hline 4.9 .1 & Wahrscheinlicher schlafgebundener Kopfschmerz & 6.3 .3 & $\begin{array}{l}\text { Kopfschmerz zurückzuführen auf eine durale arterio- } \\
\text { venöse Fistel (dAVF) }\end{array}$ \\
\hline 4.10 & $\begin{array}{l}\text { Neu aufgetretener täglicher anhaltender Kopfschmerz } \\
\text { (engl. New daily persistent headache) }\end{array}$ & 6.3 .4 & Kopfschmerz zurückzuführen auf ein kavernöses Angiom \\
\hline 4.10 .1 & $\begin{array}{l}\text { Wahrscheinlicher neu aufgetretener täglicher anhaltender } \\
\text { Kopfschmerz }\end{array}$ & 6.3 .5 & $\begin{array}{l}\text { Kopfschmerz zurückzuführen auf eine enzephalo-trige- } \\
\text { minale oder leptomeningeale Angiomatose (Sturge- } \\
\text { Weber-Syndrom) }\end{array}$ \\
\hline 5. & $\begin{array}{l}\text { Kopfschmerz zurückzuführen auf eine Verletzung oder } \\
\text { ein Trauma des Kopfes und/oder der HWS }\end{array}$ & 6.4 & Kopfschmerz zurückzuführen auf eine Arteriitis \\
\hline 5.1 & $\begin{array}{l}\text { Akuter Kopfschmerz zurückzuführen auf eine traumati- } \\
\text { sche Verletzung des Kopfes }\end{array}$ & 6.4 .1 & $\begin{array}{l}\text { Kopfschmerz zurückzuführen auf eine Riesenzellarteriitis } \\
\text { (RZA) }\end{array}$ \\
\hline 5.1 .1 & $\begin{array}{l}\text { Akuter Kopfschmerz zurückzuführen auf eine mittlere } \\
\text { oder schwere traumatische Verletzung des Kopfes }\end{array}$ & 6.4 .2 & $\begin{array}{l}\text { Kopfschmerz zurückzuführen auf eine primäre Vaskulitis } \\
\text { des ZNS }\end{array}$ \\
\hline 5.1 .2 & $\begin{array}{l}\text { Akuter Kopfschmerz zurückzuführen auf eine leichte } \\
\text { traumatische Verletzung des Kopfes }\end{array}$ & 6.4 .3 & $\begin{array}{l}\text { Kopfschmerz zurückzuführen auf eine sekundäre Vasku- } \\
\text { litis des ZNS }\end{array}$ \\
\hline 5.2 & $\begin{array}{l}\text { Anhaltender Kopfschmerz zurückzuführen auf eine } \\
\text { traumatische Verletzung des Kopfes }\end{array}$ & 6.5 & $\begin{array}{l}\text { Kopfschmerz zurückzuführen auf eine Störung der } \\
\text { A. carotis oder A. vertebralis }\end{array}$ \\
\hline 5.2 .1 & $\begin{array}{l}\text { Anhaltender Kopfschmerz zurückzuführen auf eine } \\
\text { mittlere oder schwere traumatische Verletzuna des }\end{array}$ & 6.5 .1 & $\begin{array}{l}\text { Kopf-, Gesichts- oder Halsschmerz zurückzuführen auf } \\
\text { eine Dissektion der A. carotis oder vertebralis }\end{array}$ \\
\hline & Kopfes & 6.5.1.1 & Akuter Kopf-, Gesichts- oder Halsschmerz zurückzuführen \\
\hline 5.2 .2 & Anhaltender Kopfschmerz zurückzuführen auf eine leichte & & auf eine arterielle Dissektion \\
\hline & traumatische Verletzung des Kopfes & 6.5 .1 .2 & Anhaltender Kopf-, Gesichts- oder Halsschmerz zurückzu- \\
\hline 5.3 & Akuter Kopfschmerz zurückzuführen auf ein HWS- & & führen auf eine frühere ar \\
\hline & Beschleunigungstrauma & 6.5 .2 & Kopfschmerz zurückzuführen auf eine Endarteriektomie \\
\hline
\end{tabular}




\begin{tabular}{|c|c|}
\hline $\begin{array}{l}\text { ICHD-3 } \\
\text { Code }\end{array}$ & Diagnose \\
\hline 6.5 .3 & $\begin{array}{l}\text { Kopfschmerz zurückzuführen auf eine Carotis- oder Verte- } \\
\text { bralis-Angioplastie oder Stentimplantation }\end{array}$ \\
\hline 6.6 & $\begin{array}{l}\text { Kopfschmerz zurückzuführen auf eine Hirnvenen- } \\
\text { erkrankung }\end{array}$ \\
\hline 6.6 .1 & $\begin{array}{l}\text { Kopfschmerz zurückzuführen auf eine Hirnvenenthrom- } \\
\text { bose }\end{array}$ \\
\hline 6.6 .2 & $\begin{array}{l}\text { Kopfschmerz zurückzuführen auf Stentimplantation eines } \\
\text { venösen Hirnsinus }\end{array}$ \\
\hline 6.7 & $\begin{array}{l}\text { Kopfschmerz zurückzuführen auf eine sonstige akute } \\
\text { intrakranielle Störung }\end{array}$ \\
\hline 6.7 .1 & $\begin{array}{l}\text { Kopfschmerz zurückzuführen auf einen intrakraniellen } \\
\text { endarteriellen Eingriff }\end{array}$ \\
\hline 6.7 .2 & Kopfschmerz zurückzuführen auf Angiographie \\
\hline 6.7 .3 & $\begin{array}{l}\text { Kopfschmerz zurückzuführen auf ein reversibles zereb- } \\
\text { rales Vasokonstriktionsyndrom (engl. Reversible cerebral } \\
\text { vasoconstriction syndrome - RCVS) }\end{array}$ \\
\hline 6.7.3.1 & $\begin{array}{l}\text { Akuter Kopfschmerz zurückzuführen auf ein reversibles } \\
\text { zerebrales Vasokonstriktionsyndrom (RCVS) }\end{array}$ \\
\hline 6.7.3.2 & $\begin{array}{l}\text { Akuter Kopfschmerz wahrscheinlich zurückzuführen } \\
\text { auf ein reversibles zerebrales Vasokonstriktionsyndrom } \\
\text { (RCVS) }\end{array}$ \\
\hline 6.7.3.3 & $\begin{array}{l}\text { Anhaltender Kopfschmerz zurückzuführen auf ein } \\
\text { früheres reversibles zerebrales Vasokonstriktionsyndrom } \\
\text { (RCVS) }\end{array}$ \\
\hline 6.7 .4 & Kopfschmerz zurückzuführen auf eine arterielle Dissektion \\
\hline 6.8 & $\begin{array}{l}\text { Kopfschmerz und/oder migräneartige Aura zurückzu- } \\
\text { führen auf eine chronische intrakranielle Vaskulopathie }\end{array}$ \\
\hline 6.8 .1 & $\begin{array}{l}\text { Kopfschmerz zurückzuführen auf eine zerebrale auto- } \\
\text { somal dominante Arteriopathie mit subkortikalen Infark- } \\
\text { ten und Leukenzephalopathie (engl. Cerebral autosomal } \\
\text { dominant arteriopathy with subcortical infarcts and } \\
\text { leukoencephalopathy - CADASIL) }\end{array}$ \\
\hline 6.8 .2 & $\begin{array}{l}\text { Kopfschmerz zurückzuführen auf eine mitochondriale En- } \\
\text { zephalopathie, Laktatazidose und Schlaganfall-ähnlichen } \\
\text { Episoden (engl. mitochondrial encephalopathy, lactic } \\
\text { acidosis and stroke-like episodes - MELAS) }\end{array}$ \\
\hline 6.8 .3 & $\begin{array}{l}\text { Kopfschmerz zurückzuführen auf eine Moyamoya-Angi- } \\
\text { opathie }\end{array}$ \\
\hline 6.8 .4 & $\begin{array}{l}\text { Migräneartige Aura zurückzuführen auf eine zerebrale } \\
\text { Amyloidangiopathie }\end{array}$ \\
\hline 6.8 .5 & $\begin{array}{l}\text { Kopfschmerz zurückzuführen auf ein retinales } \\
\text { Vaskulopathie-Syndrom mit zerebraler Leukenzephalo- } \\
\text { pathie und systemischen Manifestationen (engl. Retinal } \\
\text { vasculopathy with } \\
\text { cerebral leukoencephalopathy and systemic manifesta- } \\
\text { tions) }\end{array}$ \\
\hline 6.8 .6 & $\begin{array}{l}\text { Kopfschmerz zurückzuführen auf eine sonstige genetisch } \\
\text { bedingte Vaskulopathie }\end{array}$ \\
\hline 6.9 & $\begin{array}{l}\text { Kopfschmerz zurückzuführen auf einen Hypophysenin- } \\
\text { farkt }\end{array}$ \\
\hline 7. & $\begin{array}{l}\text { Kopfschmerz zurückzuführen auf nichtvaskuläre intra- } \\
\text { kranielle Störungen }\end{array}$ \\
\hline
\end{tabular}

\begin{tabular}{|c|c|}
\hline $\begin{array}{l}\text { ICHD-3 } \\
\text { Code }\end{array}$ & Diagnose \\
\hline 7.1 & $\begin{array}{l}\text { Kopfschmerz zurückzuführen auf eine Liquordruck- } \\
\text { steigerung }\end{array}$ \\
\hline 7.1.1 & $\begin{array}{l}\text { Kopfschmerz zurückzuführen auf eine idiopathische intra- } \\
\text { kranielle Hypertension (IIH) }\end{array}$ \\
\hline 7.1 .2 & $\begin{array}{l}\text { Kopfschmerz zurückzuführen auf eine sekundäre Liquor- } \\
\text { drucksteigerung metabolischer, toxischer oder hormo- } \\
\text { neller Genese }\end{array}$ \\
\hline 7.1 .3 & $\begin{array}{l}\text { Kopfschmerz zurückzuführen auf eine sekundäre Liquor- } \\
\text { drucksteigerung infolge einer Chromosomenstörung }\end{array}$ \\
\hline 7.1.4 & $\begin{array}{l}\text { Kopfschmerz zurückzuführen auf eine sekundäre Liquor- } \\
\text { drucksteigerung bei Hydrozephalus }\end{array}$ \\
\hline 7.2 & Kopfschmerz zurückzuführen auf einen Liquorunterdruck \\
\hline 7.2 .1 & Postpunktioneller Kopfschmerz \\
\hline 7.2 .2 & Kopfschmerz bei Liquorfistel \\
\hline 7.2 .3 & $\begin{array}{l}\text { Kopfschmerz zurückzuführen auf ein spontanes Liquor- } \\
\text { unterdrucksyndrom }\end{array}$ \\
\hline 7.3 & $\begin{array}{l}\text { Kopfschmerz zurückzuführen auf nichtinfektiöse entzünd- } \\
\text { liche Erkrankungen }\end{array}$ \\
\hline 7.3 .1 & Kopfschmerz zurückzuführen auf eine Neurosarkoidose \\
\hline 7.3 .2 & $\begin{array}{l}\text { Kopfschmerz zurückzuführen auf eine aseptische (nicht- } \\
\text { infektiöse) Meningitis }\end{array}$ \\
\hline 7.3 .3 & $\begin{array}{l}\text { Kopfschmerz zurückzuführen auf eine andere nicht- } \\
\text { infektiöse entzündliche Erkrankung }\end{array}$ \\
\hline 7.3.4 & $\begin{array}{l}\text { Kopfschmerz zurückzuführen auf eine lymphozytäre } \\
\text { Hypophysitis }\end{array}$ \\
\hline 7.3 .5 & $\begin{array}{l}\text { Syndrom der vorübergehenden Kopfschmerzen mit } \\
\text { neurologischen Defiziten und Liquorlymphozytose (engl. } \\
\text { Headache and neurological deficits with cerebrospinal } \\
\text { fluid lymphocytosis - HaNDL) }\end{array}$ \\
\hline 7.4 & $\begin{array}{l}\text { Kopfschmerz zurückzuführen auf ein intrakranielles } \\
\text { Neoplasma }\end{array}$ \\
\hline 7.4 .1 & $\begin{array}{l}\text { Kopfschmerz zurückzuführen auf ein intrakranielles } \\
\text { Neoplasma }\end{array}$ \\
\hline 7.4.1.1 & $\begin{array}{l}\text { Kopfschmerz zurückzuführen auf eine Kolloidzyste des } \\
\text { dritten Hirnventrikels }\end{array}$ \\
\hline 7.4 .2 & $\begin{array}{l}\text { Kopfschmerz zurückzuführen auf eine Meningeosis } \\
\text { carcinomatosa }\end{array}$ \\
\hline 7.4 .3 & $\begin{array}{l}\text { Kopfschmerz zurückzuführen auf eine hypothalamische } \\
\text { oder hypophysäre Über- oder Unterfunktion }\end{array}$ \\
\hline 7.5 & $\begin{array}{l}\text { Kopfschmerz zurückzuführen auf eine intrathekale } \\
\text { Injektion }\end{array}$ \\
\hline 7.6 & $\begin{array}{l}\text { Kopfschmerz zurückzuführen auf einen epileptischen } \\
\text { Anfall }\end{array}$ \\
\hline 7.6.1 & Iktaler Kopfschmerz bei Epilepsie \\
\hline 7.6.2 & Postiktaler Kopfschmerz \\
\hline 7.7 & $\begin{array}{l}\text { Kopfschmerz zurückzuführen auf eine Chiari-Malforma- } \\
\text { tion Typ I (CM1) }\end{array}$ \\
\hline 7.8 & $\begin{array}{l}\text { Kopfschmerz zurückzuführen auf eine andere nichtvasku- } \\
\text { läre intrakranielle Störung }\end{array}$ \\
\hline 8. & $\begin{array}{l}\text { Kopfschmerz zurückzuführen auf eine Substanz oder } \\
\text { deren Entzug }\end{array}$ \\
\hline
\end{tabular}




\begin{tabular}{|c|c|}
\hline $\begin{array}{l}\text { ICHD-3 } \\
\text { Code }\end{array}$ & Diagnose \\
\hline 8.1 & $\begin{array}{l}\text { Kopfschmerz zurückzuführen auf Substanzgebrauch oder } \\
\text { Substanzexposition }\end{array}$ \\
\hline 8.1 .1 & Kopfschmerz induziert durch Stickoxid (NO)-Donatoren \\
\hline 8.1.1.1 & $\begin{array}{l}\text { Sofortiger Kopfschmerz induziert durch Stickoxid (NO)- } \\
\text { Donatoren }\end{array}$ \\
\hline 8.1 .1 .2 & $\begin{array}{l}\text { Verzögerter Kopfschmerz induziert durch Stickoxid } \\
\text { (NO)-Donatoren }\end{array}$ \\
\hline 8.1 .2 & $\begin{array}{l}\text { Kopfschmerz induziert durch Phosphodiesterase } \\
\text { (PDE)-Hemmer }\end{array}$ \\
\hline 8.1 .3 & Kopfschmerz induziert durch Kohlenmonoxid (CO) \\
\hline 8.1 .4 & Kopfschmerz induziert durch Alkohol \\
\hline 8.1.4.1 & Sofortiger Kopfschmerz induziert durch Alkohol \\
\hline 8.1 .4 .2 & Verzögerter Kopfschmerz induziert durch Alkohol \\
\hline 8.1 .5 & Kopfschmerz induziert durch Kokain \\
\hline 8.1 .6 & Kopfschmerz induziert durch Histamin \\
\hline 8.1.6.1 & Sofortiger Kopfschmerz induziert durch Histamin \\
\hline 8.1 .6 .2 & Verzögerter Kopfschmerz induziert durch Histamin \\
\hline 8.1 .7 & $\begin{array}{l}\text { Kopfschmerz induziert durch Calcitonin-Gene-Related } \\
\text { Peptide (CGRP) }\end{array}$ \\
\hline 8.1.7.1 & Sofortiger Kopfschmerz induziert durch CGRP \\
\hline 8.1.7.2 & Verzögerter Kopfschmerz induziert durch CGRP \\
\hline 8.1 .8 & $\begin{array}{l}\text { Kopfschmerz zurückzuführen auf einen akuten Blutdruck- } \\
\text { anstieg durch eine exogene Substanz }\end{array}$ \\
\hline 8.1 .9 & $\begin{array}{l}\text { Kopfschmerz zurückzuführen auf den gelegentlichen } \\
\text { Gebrauch einer nicht gegen Kopfschmerz eingesetzten } \\
\text { Medikation }\end{array}$ \\
\hline 8.1 .10 & $\begin{array}{l}\text { Kopfschmerz zurückzuführen auf eine nicht gegen Kopf- } \\
\text { schmerz eingesetzte Dauermedikation }\end{array}$ \\
\hline 8.1 .11 & $\begin{array}{l}\text { Kopfschmerz zurückzuführen auf den Gebrauch oder } \\
\text { Exposition einer anderen Substanz }\end{array}$ \\
\hline 8.2 & $\begin{array}{l}\text { Kopfschmerz zurückzuführen auf einen Medikamenten- } \\
\text { übergebrauch }\end{array}$ \\
\hline 8.2 .1 & $\begin{array}{l}\text { Kopfschmerz zurückzuführen auf einen Ergotaminüber- } \\
\text { gebrauch }\end{array}$ \\
\hline 8.2 .2 & $\begin{array}{l}\text { Kopfschmerz zurückzuführen auf einen Triptanüberge- } \\
\text { brauch }\end{array}$ \\
\hline 8.2 .3 & $\begin{array}{l}\text { Kopfschmerz zurückzuführen auf einen Übergebrauch von } \\
\text { Nicht-Opioid-Analgetika }\end{array}$ \\
\hline 8.2.3.1 & $\begin{array}{l}\text { Kopfschmerz zurückzuführen auf einen Übergebrauch von } \\
\text { Paracetamol }\end{array}$ \\
\hline 8.2.3.2 & $\begin{array}{l}\text { Kopfschmerz zurückzuführen auf einen Übergebrauch von } \\
\text { nicht-steroidalen Antirheumatika (NSAR) }\end{array}$ \\
\hline 8.2.3.2.1 & $\begin{array}{l}\text { Kopfschmerz zurückzuführen auf einen Übergebrauch von } \\
\text { Acetylsalicylsäure }\end{array}$ \\
\hline 8.2.3.3 & $\begin{array}{l}\text { Kopfschmerz zurückzuführen auf einen Übergebrauch von } \\
\text { anderen Nicht-Opioid-Analgetika }\end{array}$ \\
\hline 8.2 .4 & $\begin{array}{l}\text { Kopfschmerz zurückzuführen auf einen Opioidüberge- } \\
\text { brauch }\end{array}$ \\
\hline 8.2 .5 & $\begin{array}{l}\text { Kopfschmerz zurückzuführen auf einen Übergebrauch von } \\
\text { Schmerzmittelmischpräparaten }\end{array}$ \\
\hline
\end{tabular}

\begin{tabular}{|l|l|}
$\begin{array}{l}\text { ICHD-3 } \\
\text { Code }\end{array}$ & Diagnose \\
\hline
\end{tabular}

8.2.6 Kopfschmerz zurückzuführen auf einen Übergebrauch von Medikamenten aus mehreren Klassen, ohne Übergebrauch der Einzelsubstanzen

8.2.7 Kopfschmerz zurückzuführen auf einen unspezifizierten oder unbestätigten Übergebrauch von Medikamenten aus mehreren Klassen

8.2.8 Kopfschmerz zurückzuführen auf einen Übergebrauch einer anderen Medikation

8.3 Kopfschmerz zurückzuführen auf den Entzug einer Substanz

\begin{tabular}{|l|l|}
\hline 8.3.1 & Koffeinentzugskopfschmerz \\
\hline 8.3.2 & Opioidentzugskopfschmerz \\
\hline
\end{tabular}

8.3.3 Östrogenentzugskopfschmerz

8.3.4 Kopfschmerz zurückzuführen auf den Entzug anderer dauerhaft eingenommener Substanzen

\begin{tabular}{l|l}
\hline 9. & Kopfschmerz zurückzuführen auf eine Infektion \\
\hline 9.1 & $\begin{array}{l}\text { Kopfschmerz zurückzuführen auf eine intrakranielle } \\
\text { Infektion }\end{array}$ \\
\hline
\end{tabular}

9.1.1 Kopfschmerz zurückzuführen auf eine bakterielle Meningitis oder Meningoenzephalitis

9.1.1.1 Akuter Kopfschmerz zurückzuführen auf eine bakterielle Meningitis oder Meningoenzephalitis

9.1.1.2 Chronischer Kopfschmerz zurückzuführen auf eine bakterielle Meningitis oder Meningoenzephalitis

9.1.1.3 Anhaltender Kopfschmerz zurückzuführen auf eine frühere bakterielle Meningitis oder Meningoenzephalitis

9.1.2 Kopfschmerz zurückzuführen auf eine virale Meningitis oder Enzephalitis

9.1.2.1 Kopfschmerz zurückzuführen auf eine virale Meningitis

9.1.2.2 Kopfschmerz zurückzuführen auf eine virale Enzephalitis

9.1.3 Kopfschmerz zurückzuführen auf eine intrakranielle Pilzoder andere parasitäre Infektion

9.1.3.1 Akuter Kopfschmerz zurückzuführen auf eine intrakranielle Pilz- oder andere parasitäre Infektion

9.1.3.2 Chronischer Kopfschmerz zurückzuführen auf eine intrakranielle Pilz- oder andere parasitäre Infektion

9.1.4 Kopfschmerz zurückzuführen auf eine lokalisierte Hirninfektion

9.2 Kopfschmerz zurückzuführen auf eine systemische Infektion

9.2.1 Kopfschmerz zurückzuführen auf eine systemische bakterielle Infektion

9.2.1.1 Akuter Kopfschmerz zurückzuführen auf eine systemische bakterielle Infektion

9.2.1.2 Chronischer Kopfschmerz zurückzuführen auf eine systemische bakterielle Infektion

9.2.2 Kopfschmerz zurückzuführen auf eine systemische virale Infektion

9.2.2.1 Akuter Kopfschmerz zurückzuführen auf eine systemische virale Infektion

9.2.2.2 Chronischer Kopfschmerz zurückzuführen auf eine systemische virale Infektion

9.2.3 Kopfschmerz zurückzuführen auf eine andere systemische Infektion 


\begin{tabular}{|c|c|}
\hline $\begin{array}{l}\text { ICHD-3 } \\
\text { Code }\end{array}$ & Diagnose \\
\hline 9.2 .3 .1 & $\begin{array}{l}\text { Akuter Kopfschmerz zurückzuführen auf eine andere } \\
\text { systemische Infektion }\end{array}$ \\
\hline 9.2 .3 .2 & $\begin{array}{l}\text { Chronischer Kopfschmerz zurückzuführen auf eine andere } \\
\text { systemische Infektion }\end{array}$ \\
\hline 10. & $\begin{array}{l}\text { Kopfschmerz zurückzuführen eine Störung der } \\
\text { Homöostase }\end{array}$ \\
\hline 10.1 & $\begin{array}{l}\text { Kopfschmerz zurückzuführen auf eine Hypoxie und/oder } \\
\text { Hyperkapnie }\end{array}$ \\
\hline 10.1 .1 & Höhenkopfschmerz \\
\hline 10.1 .2 & Kopfschmerz zurückzuführen auf eine Flugreise \\
\hline 10.1 .3 & Taucherkopfschmerz \\
\hline 10.1 .4 & $\begin{array}{l}\text { Kopfschmerz zurückzuführen auf ein Schlafapnoe- } \\
\text { Syndrom }\end{array}$ \\
\hline 10.2 & Dialysekopfschmerz \\
\hline 10.3 & $\begin{array}{l}\text { Kopfschmerz zurückzuführen auf eine arterielle } \\
\text { Hypertonie }\end{array}$ \\
\hline 10.3 .1 & Kopfschmerz zurückzuführen auf ein Phäochromozytom \\
\hline 10.3 .2 & $\begin{array}{l}\text { Kopfschmerz zurückzuführen auf eine hypertensive Krise } \\
\text { ohne hypertensive Enzephalopathie }\end{array}$ \\
\hline 10.3 .3 & $\begin{array}{l}\text { Kopfschmerz zurückzuführen auf eine hypertensive } \\
\text { Enzephalopathie }\end{array}$ \\
\hline 10.3.4 & $\begin{array}{l}\text { Kopfschmerz zurückzuführen auf eine Präeklampsie oder } \\
\text { Eklampsie }\end{array}$ \\
\hline 10.3 .5 & $\begin{array}{l}\text { Kopfschmerz zurückzuführen auf eine autonome } \\
\text { Dysreflexie }\end{array}$ \\
\hline 10.4 & Kopfschmerz zurückzuführen auf eine Hypothyreose \\
\hline 10.5 & Kopfschmerz zurückzuführen auf Fasten \\
\hline 10.6 & Kopfschmerz zurückzuführen auf eine kardiale Erkrankung \\
\hline 10.7 & $\begin{array}{l}\text { Kopfschmerz zurückzuführen auf eine andere Störung der } \\
\text { Homöostase }\end{array}$ \\
\hline 11. & $\begin{array}{l}\text { Kopf- oder Gesichtsschmerzen zurückzuführen auf } \\
\text { Erkrankungen des Schädels sowie von Hals, Augen, } \\
\text { Ohren, Nase, Nebenhöhlen, Zähnen, Mund und ande- } \\
\text { ren Gesichts- oder Schädelstrukturen }\end{array}$ \\
\hline 11.1 & $\begin{array}{l}\text { Kopfschmerz zurückzuführen auf Erkrankungen der } \\
\text { Schädelknochen }\end{array}$ \\
\hline 11.2 & $\begin{array}{l}\text { Kopfschmerz zurückzuführen auf Erkrankungen des } \\
\text { Halses }\end{array}$ \\
\hline 11.2 .1 & Zervikogener Kopfschmerz \\
\hline 11.2 .2 & $\begin{array}{l}\text { Kopfschmerz zurückzuführen auf eine retropharyngeale } \\
\text { Tendinitis }\end{array}$ \\
\hline 11.2 .3 & $\begin{array}{l}\text { Kopfschmerz zurückzuführen auf eine kraniozervikale } \\
\text { Dystonie }\end{array}$ \\
\hline 11.3 & $\begin{array}{l}\text { Kopfschmerz zurückzuführen auf Erkrankungen der } \\
\text { Augen }\end{array}$ \\
\hline 11.3.1 & $\begin{array}{l}\text { Kopfschmerz zurückzuführen auf ein akutes Engwinkel- } \\
\text { glaukom }\end{array}$ \\
\hline 11.3.2 & Kopfschmerz zurückzuführen auf einen Brechungsfehler \\
\hline 11.3 .3 & $\begin{array}{l}\text { Kopfschmerz zurückzuführen auf eine entzündliche } \\
\text { Erkrankung des Auges }\end{array}$ \\
\hline 11.3.4 & Trochlearis-Kopfschmerz \\
\hline 11.4 & Kopfschmerz zurückzuführen auf Erkrankungen der Ohren \\
\hline
\end{tabular}

\section{ICHD-3 Diagnose
Code}

11.5 Kopfschmerz zurückzuführen auf Erkrankungen der Nase oder der Nasennebenhöhlen

11.5.1 Kopfschmerz zurückzuführen auf eine akute Rhinosinusitis

11.5.2 Kopfschmerz zurückzuführen auf eine chronische oder rezidivierende Rhinosinusitis

11.6 Kopfschmerz zurückzuführen auf Erkrankungen der Zähne

\begin{tabular}{l|l}
11.7 & Kopfschmerz zurückzuführen auf Erkrankungen des
\end{tabular} Kiefergelenkes

\begin{tabular}{l|l}
11.8 & Kopf- oder Gesichtsschmerz zurückzuführen auf Entzün-
\end{tabular} dungen des Ligamentum stylohyoideum

11.9 Kopf- oder Gesichtsschmerz zurückzuführen auf andere Erkrankungen des Schädels sowie von Hals, Augen, Ohren, Nase, Nebenhöhlen, Zähnen, Mund oder anderen Gesichts- oder Schädelstrukturen

\begin{tabular}{|c|l|}
\hline 12. & $\begin{array}{l}\text { Kopfschmerz zurückzuführen auf psychiatrische } \\
\text { Störungen }\end{array}$ \\
\hline 12.1 & $\begin{array}{l}\text { Kopfschmerz zurückzuführen auf eine Somatisierungs- } \\
\text { störung }\end{array}$ \\
\hline 12.2 & $\begin{array}{l}\text { Kopfschmerz zurückzuführen auf eine psychotische } \\
\text { Störung }\end{array}$ \\
\hline
\end{tabular}

13. Schmerzhafte Läsionen der Hirnnerven und andere Gesichtsschmerzen

13.1 Schmerzen zurückzuführen auf eine Läsion oder Erkrankung des N. trigeminus

13.1.1 Trigeminusneuralgie

13.1.1.1 Klassische Trigeminusneuralgie

13.1.1.1.1 Klassische Trigeminusneuralgie, rein paroxysmal

13.1.1.1.2 Klassische Trigeminusneuralgie mit Dauerschmerz

13.1.1.2 Sekundäre Trigeminusneuralgie

13.1.1.2.1 Trigeminusneuralgie zurückzuführen auf Multiple Sklerose

13.1.1.2.2 Trigeminusneuralgie zurückzuführen auf eine raumfordernde Läsion

13.1.1.2.3 Trigeminusneuralgie zurückzuführen auf eine andere Ursache

13.1.1.3 Idiopathische Trigeminusneuralgie

13.1.1.3.1 Idiopathische Trigeminusneuralgie, rein paroxysmal

13.1.1.3.2 Idiopathische Trigeminusneuralgie mit Dauerschmerz

13.1.2 Schmerzhafte Trigeminusneuropathie

13.1.2.1 Schmerzhafte Trigeminusneuropathie zurückzuführen auf Herpes zoster

13.1.2.2 Postherpetische Trigeminusneuralgie

13.1.2.3 Schmerzhafte posttraumatische Trigeminusneuropathie

13.1.2.4 Schmerzhafte Trigeminusneuropathie zurückzuführen auf eine andere Erkrankung

13.1.2.5 Idiopathische schmerzhafte Trigeminusneuropathie

13.2 Schmerz zurückführen auf eine Läsion oder Erkrankung des N. glossopharyngeus

13.2.1 Glossopharyngeusneuralgie

13.2.1.1 Klassische Glossopharyngeusneuralgie

13.2.1.2 Sekundäre Glossopharyngeusneuralgie

13.2.1.3 Idiopathische Glossopharyngeusneuralgie

13.2.2 Schmerzhafte Glossopharyngeusneuropathie 


\begin{tabular}{|c|c|}
\hline $\begin{array}{l}\text { ICHD-3 } \\
\text { Code }\end{array}$ & Diagnose \\
\hline 13.2.2.1 & $\begin{array}{l}\text { Schmerzhafte Glossopharyngeusneuropathie zurück- } \\
\text { zuführen auf eine bekannte Ursache }\end{array}$ \\
\hline 13.2.2.2 & $\begin{array}{l}\text { Idiopathische schmerzhafte Glossopharyngeusneuro- } \\
\text { pathie }\end{array}$ \\
\hline 13.3 & $\begin{array}{l}\text { Schmerz zurückzuführen auf eine Läsion oder Erkrankung } \\
\text { des N. intermedius }\end{array}$ \\
\hline 13.3.1 & Intermediusneuralgie \\
\hline 13.3.1.1 & Klassische Intermediusneuralgie \\
\hline 13.3.1.2 & Sekundäre Intermediusneuralgie \\
\hline 13.3.1.3 & Idiopathische Intermediusneuralgie \\
\hline 13.3 .2 & Schmerzhafte Intermediusneuropathie \\
\hline 13.3.2.1 & $\begin{array}{l}\text { Schmerzhafte Intermediusneuropathie zurückzuführen } \\
\text { auf einen Herpes zoster }\end{array}$ \\
\hline 13.3.2.2 & Postherpetische Intermediusneuralgie \\
\hline 13.3.2.3 & $\begin{array}{l}\text { Schmerzhafte Intermediusneuropathie zurückzuführen } \\
\text { auf eine andere Erkrankung }\end{array}$ \\
\hline 13.3.2.4 & Idiopathische schmerzhafte Intermediusneuropathie \\
\hline 13.4 & Okzipitalisneuralgie \\
\hline 13.5 & Nacken-Zungen-Syndrom (engl. Neck-tongue-syndrome) \\
\hline 13.6 & Schmerzhafte Optikusneuritis \\
\hline 13.7 & $\begin{array}{l}\text { Kopfschmerz zurückzuführen auf eine ischämische } \\
\text { Lähmung des N. oculomotorius }\end{array}$ \\
\hline 13.8 & Tolosa-Hunt-Syndrom \\
\hline 13.9 & Paratrigeminales okulosympathisches (Raeder-) Syndrom \\
\hline 13.10 & $\begin{array}{l}\text { Rezidivierende schmerzhafte ophthalmoplegische Neuro- } \\
\text { pathie }\end{array}$ \\
\hline 13.11 & $\begin{array}{l}\text { Syndrom des brennenden Mundes (engl. Burning mouth } \\
\text { syndrome - BMS) }\end{array}$ \\
\hline 13.12 & $\begin{array}{l}\text { Anhaltender idiopathischer Gesichtsschmerz (engl. Persis- } \\
\text { tent idiopathic facial pain - PIFP) }\end{array}$ \\
\hline 13.13 & Zentraler neuropathischer Schmerz \\
\hline 13.13 .1 & $\begin{array}{l}\text { Zentraler neuropathischer Schmerz zurückzuführen auf } \\
\text { Multiple Sklerose (MS) }\end{array}$ \\
\hline 13.13 .2 & $\begin{array}{l}\text { Zentraler neuropathischer Schmerz nach Hirninfarkt } \\
\text { (engl. Central post stroke pain - CPSP) }\end{array}$ \\
\hline 14. & Andere Kopfschmerzerkrankungen \\
\hline 14.1 & Kopfschmerz nicht anderweitig klassifiziert \\
\hline 14.2 & Kopfschmerz nicht spezifiziert \\
\hline A. & Anhang \\
\hline A1. & Migräne \\
\hline A1.1 & Migräne ohne Aura \\
\hline A1.1.1 & Rein menstruelle Migräne ohne Aura \\
\hline A1.1.2 & Menstruationsassoziierte Migräne ohne Aura \\
\hline A1.1.3 & Nicht-menstruationsassoziierte Migräne ohne Aura \\
\hline A1.2 & Migräne mit Aura \\
\hline A1.2.0.1 & Rein menstruelle Migräne mit Aura \\
\hline A1.2.0.2 & Menstruationsassoziierte Migräne mit Aura \\
\hline A1.2.0.3 & Nicht menstruationsassoziierte Migräne mit Aura \\
\hline A1.3 & Chronische Migräne (alternative Kriterien) \\
\hline A1.3.1 & Chronische Migräne mit schmerzfreien Perioden \\
\hline A1.3.2 & Chronische Migräne mit Dauerschmerz \\
\hline
\end{tabular}

\begin{tabular}{|c|c|}
\hline $\begin{array}{l}\text { ICHD-3 } \\
\text { Code }\end{array}$ & Diagnose \\
\hline A1.4 & Migränekomplikationen \\
\hline A1.4.5 & Migräneaurastatus \\
\hline A1.4.6 & Visual Snow \\
\hline A1.6 & $\begin{array}{l}\text { Episodische Syndrome, die potenziell mit Migräne einher- } \\
\text { gehen }\end{array}$ \\
\hline A1.6.4 & Infantile Koliken \\
\hline A1.6.5 & Alternierende Hemiplegie des Kindesalters \\
\hline A1.6.6 & Vestibuläre Migräne \\
\hline A2. & Kopfschmerz vom Spannungstyp (alternative Kriterien) \\
\hline A3. & Trigemino-autonome Kopfschmerzerkrankungen (TAK) \\
\hline A3.1 & Clusterkopfschmerz (alternative Kriterien) \\
\hline A3.2 & Paroxysmale Hemikranie (alternative Kriterien) \\
\hline A3.3 & SUNA-Syndrom (alternative Kriterien) \\
\hline A3.4 & Hemicrania continua (alternative Kriterien) \\
\hline A3.6 & $\begin{array}{l}\text { Undifferenzierte trigemino-autonome Kopfschmerz- } \\
\text { erkrankung }\end{array}$ \\
\hline A4. & Andere primäre Kopfschmerzen \\
\hline A4.11 & Epicrania fugax \\
\hline A5. & $\begin{array}{l}\text { Kopfschmerz zurückzuführen auf ein Trauma oder eine } \\
\text { Verletzung des Kopfes und/oder der HWS }\end{array}$ \\
\hline A5.1 & $\begin{array}{l}\text { Akuter Kopfschmerz zurückzuführen auf eine trauma- } \\
\text { tische Verletzung des Kopfes }\end{array}$ \\
\hline A5.1.1.1 & $\begin{array}{l}\text { Verzögert auftretender akuter Kopfschmerz zurückzu- } \\
\text { führen auf eine mittelschwere oder schwere traumatische } \\
\text { Verletzung des Kopfes }\end{array}$ \\
\hline A5.1.2.1 & $\begin{array}{l}\text { Verzögert auftretender akuter Kopfschmerz zurückzu- } \\
\text { führen auf eine leichte traumatische Verletzung des } \\
\text { Kopfes }\end{array}$ \\
\hline A5.2 & $\begin{array}{l}\text { Anhaltender Kopfschmerz zurückzuführen auf eine trau- } \\
\text { matische Verletzung des Kopfes }\end{array}$ \\
\hline A5.2.1.1 & $\begin{array}{l}\text { Verzögert auftretender anhaltender Kopfschmerz zurück- } \\
\text { zuführen auf eine mittelschwere oder schwere traumati- } \\
\text { sche Verletzung des Kopfes }\end{array}$ \\
\hline A5.2.2.1 & $\begin{array}{l}\text { Verzögert auftretender anhaltender Kopfschmerz zurück- } \\
\text { zuführen auf eine leichte traumatische Verletzung des } \\
\text { Kopfes }\end{array}$ \\
\hline A5.7 & $\begin{array}{l}\text { Kopfschmerz zurückzuführen auf einen radiochirurgischer } \\
\text { Eingriff am Gehirn }\end{array}$ \\
\hline A5.8 & $\begin{array}{l}\text { Akuter Kopfschmerz zurückzuführen auf ein anderes } \\
\text { Kopf- und/oder HWS-Trauma oder eine andere Kopf- und/ } \\
\text { oder HWS-Verletzung }\end{array}$ \\
\hline A5.9 & $\begin{array}{l}\text { Anhaltender Kopfschmerz zurückzuführen auf ein anderes } \\
\text { Kopf- und/oder HWS-Trauma oder eine andere Kopf- und/ } \\
\text { oder HWS-Verletzung }\end{array}$ \\
\hline A6. & $\begin{array}{l}\text { Kopfschmerz zurückzuführen auf Gefäßstörungen im } \\
\text { Bereich des Kopfes und/oder des Halses }\end{array}$ \\
\hline A6.10 & $\begin{array}{l}\text { Anhaltender Kopfschmerz zurückzuführen auf frühere } \\
\text { Gefäßstörungen im Bereich des Kopfes und/oder des } \\
\text { Halses }\end{array}$ \\
\hline A7. & $\begin{array}{l}\text { Kopfschmerz zurückzuführen auf nichtvaskuläre intra- } \\
\text { kranielle Störungen }\end{array}$ \\
\hline A7.6 & $\begin{array}{l}\text { Kopfschmerz zurückzuführen auf einen epileptischen } \\
\text { Anfall }\end{array}$ \\
\hline A7.6.3 & Kopfschmerz nach Elektrokrampftherapie \\
\hline
\end{tabular}




\begin{tabular}{|c|c|}
\hline $\begin{array}{l}\text { ICHD-3 } \\
\text { Code }\end{array}$ & Diagnose \\
\hline A7.9 & $\begin{array}{l}\text { Anhaltender Kopfschmerz zurückzuführen auf eine } \\
\text { frühere nichtvaskuläre intrakranielle Störung }\end{array}$ \\
\hline A8. & $\begin{array}{l}\text { Kopfschmerz zurückzuführen auf eine Substanz oder } \\
\text { deren Entzug }\end{array}$ \\
\hline A8.4 & $\begin{array}{l}\text { Anhaltender Kopfschmerz zurückzuführen auf früheren } \\
\text { Substanzgebrauch oder frühere Substanzexposition }\end{array}$ \\
\hline A9. & Kopfschmerz zurückzuführen auf eine Infektion \\
\hline A9.1 & $\begin{array}{l}\text { Kopfschmerz zurückzuführen auf eine intrakranielle } \\
\text { Infektion }\end{array}$ \\
\hline A9.1.3.3 & $\begin{array}{l}\text { Anhaltender Kopfschmerz zurückzuführen auf eine } \\
\text { frühere intrakranielle Pilz- oder andere parasitäre Infek- } \\
\text { tion }\end{array}$ \\
\hline A9.1.6 & $\begin{array}{l}\text { Kopfschmerz zurückzuführen auf eine andere raum- } \\
\text { fordernde infektiöse Läsion }\end{array}$ \\
\hline A9.3 & Kopfschmerz zurückzuführen auf eine HIV-Infektion \\
\hline A10. & $\begin{array}{l}\text { Kopfschmerz zurückzuführen auf eine Störung der } \\
\text { Homöostase }\end{array}$ \\
\hline A10.7 & $\begin{array}{l}\text { Kopf- und/oder Nackenschmerzen zurückzuführen auf } \\
\text { eine orthostatische (posturale) Hypotonie }\end{array}$ \\
\hline A10.8 & $\begin{array}{l}\text { Kopfschmerz zurückzuführen auf eine andere Störung der } \\
\text { Homöostase }\end{array}$ \\
\hline A10.8.1 & $\begin{array}{l}\text { Kopfschmerz zurückzuführen auf einen Aufenthalt im } \\
\text { Weltraum }\end{array}$ \\
\hline A10.8.2 & $\begin{array}{l}\text { Kopfschmerz zurückzuführen auf andere metabolische } \\
\text { oder systemische Störungen }\end{array}$ \\
\hline A10.9 & $\begin{array}{l}\text { Anhaltender Kopfschmerz zurückzuführen auf eine } \\
\text { frühere Störung der Homöostase }\end{array}$ \\
\hline A11. & $\begin{array}{l}\text { Kopf- oder Gesichtsschmerzen zurückzuführen auf Erkran- } \\
\text { kungen des Schädels sowie von Hals, Augen, Ohren, Nase, } \\
\text { Nebenhöhlen, Zähnen, Mund und anderen Gesichts- oder } \\
\text { Schädelstrukturen }\end{array}$ \\
\hline A11.2 & $\begin{array}{l}\text { Kopfschmerz zurückzuführen auf Erkrankungen des } \\
\text { Halses }\end{array}$ \\
\hline
\end{tabular}

\begin{tabular}{|l|l|}
\hline $\begin{array}{l}\text { ICHD-3 } \\
\text { Code }\end{array}$ & Diagnose \\
\hline A11.2.4 & $\begin{array}{l}\text { Kopfschmerz zurückzuführen auf eine Radikulopathie der } \\
\text { oberen HWS }\end{array}$ \\
\hline A11.2.5 & $\begin{array}{l}\text { Kopfschmerz zurückzuführen auf ein zervikales myofas- } \\
\text { ziales Schmerzsyndrom }\end{array}$ \\
\hline A11.3 & $\begin{array}{l}\text { Kopfschmerz zurückzuführen auf Erkrankungen der } \\
\text { Augen }\end{array}$ \\
\hline A11.3.5 & $\begin{array}{l}\text { Kopfschmerz zurückzuführen auf Heterophorie oder } \\
\text { Heterotropie }\end{array}$ \\
\hline A11.5 & $\begin{array}{l}\text { Kopfschmerz zurückzuführen auf Erkrankungen der Nase } \\
\text { oder der Nasennebenhöhlen }\end{array}$ \\
\hline A11.5.3 & $\begin{array}{l}\text { Kopfschmerz zurückzuführen auf Erkrankungen der } \\
\text { Nasenschleimhaut, der Nasenmuscheln oder der Nasen- } \\
\text { scheidewand }\end{array}$ \\
\hline A12. & $\begin{array}{l}\text { Kopfschmerz zurückzuführen auf psychiatrische } \\
\text { Störungen }\end{array}$ \\
\hline A12.3 & $\begin{array}{l}\text { Kopfschmerz zurückzuführen auf eine Somatisierungs- } \\
\text { störung }\end{array}$ \\
\hline A12.4 & $\begin{array}{l}\text { Kopfschmerz zurückzuführen auf eine Störung mit } \\
\text { Trennungsangst }\end{array}$ \\
\hline A12.5 & $\begin{array}{l}\text { Kopfschmerz zurückzuführen auf eine Panikstörung } \\
\text { Kopfschmerz zurückzuführen auf eine spezifische Phobie }\end{array}$ \\
\hline A12.6 & $\begin{array}{l}\text { Kopfschmerz zurückzuführen auf eine soziale Angst- } \\
\text { störung (soziale Phobie) }\end{array}$ \\
\hline A12.7 & $\begin{array}{l}\text { Kopfschmerz zurückzuführen auf eine generalisierte } \\
\text { Kopfschmerz zurückzuführen auf eine posttraumatische } \\
\text { Belastungsstörung (PTBS) }\end{array}$ \\
\hline Kopfschmerz zurückzuführen auf eine akute Belastungs- \\
\hline A12.10
\end{tabular}




\section{TEIL EINS}

\section{PRIMÄRER KOPFSCHMERZ}

1. Migräne

2. Kopfschmerz vom Spannungstyp

3. Trigemino-autonome Kopfschmerzerkrankungen

4. Andere primäre Kopfschmerzen

\section{Migräne}

\begin{tabular}{|c|c|}
\hline 1.1 & Migräne ohne Aura \\
\hline 1.2 & Migräne mit Aura \\
\hline 1.2.1 & Migräne mit typischer Aura \\
\hline 1.2.1.1 & Typische Aura mit Kopfschmerz \\
\hline 1.2.1.2 & Typische Aura ohne Kopfschmerz \\
\hline 1.2 .2 & Migräne mit Hirnstammaura \\
\hline 1.2 .3 & Hemiplegische Migräne \\
\hline 1.2.3.1 & Familiäre hemiplegische Migräne (FHM) \\
\hline 1.2.3.1.1 & Familiäre hemiplegische Migräne Typ 1 (FHM1) \\
\hline 1.2.3.1.2 & Familiäre hemiplegische Migräne Typ 2 (FHM2) \\
\hline 1.2.3.1.3 & Familiäre hemiplegische Migräne Typ 3 (FHM3) \\
\hline 1.2.3.1.4 & Familiäre hemiplegische Migräne, andere Genloci \\
\hline 1.2.3.2 & Sporadische hemiplegische Migräne (SHM) \\
\hline 1.2 .4 & Retinale Migräne \\
\hline 1.3 & Chronische Migräne \\
\hline 1.4 & Migränekomplikationen \\
\hline 1.4.1 & Status migränosus \\
\hline 1.4 .2 & Anhaltende Aura ohne Hirninfarkt \\
\hline 1.4 .3 & Migränöser Infarkt \\
\hline 1.4.4 & Epileptische Anfälle, durch Migräneaura getriggert \\
\hline 1.5 & Wahrscheinliche Migräne \\
\hline 1.5.1 & Wahrscheinliche Migräne ohne Aura \\
\hline 1.5 .2 & Wahrscheinliche Migräne mit Aura \\
\hline 1.6 & $\begin{array}{l}\text { Episodische Syndrome, die potenziell mit Migräne einher- } \\
\text { gehen }\end{array}$ \\
\hline 1.6.1 & Rezidivierende gastrointestinale Störungen \\
\hline 1.6.1.1 & Zyklisches Erbrechen \\
\hline 1.6.1.2 & Abdominelle Migräne \\
\hline 1.6 .2 & Gutartiger paroxysmaler Schwindel \\
\hline 1.6 .3 & Gutartiger paroxysmaler Tortikollis \\
\hline
\end{tabular}

An anderer Stelle kodiert: Migräneartige Kopfschmerzen als sekundäre Folge einer anderen Erkrankung (symptomatische Migräne) werden als auf diese Erkrankung zurückzuführende sekundäre Kopfschmerzen kodiert.

\section{Allgemeiner Kommentar Primärer und/oder sekundärer Kopfschmerz?}

Für migräneartige Kopfschmerzen gelten je nach den Umständen drei Regeln:

1. Tritt ein neuer Kopfschmerz mit den Charakteristika einer Migräne erstmalig in engem zeitlichen Zusammenhang mit einer anderen Erkrankung auf, die als Ursache von Kopf- schmerzen angesehen wird oder sonstige Kriterien erfüllt, die für diese Erkrankung als Ursache sprechen, sollte der neue Kopfschmerz entsprechend der ursächlichen Erkrankung als sekundärer Kopfschmerz kodiert werden.

2. Wenn eine vorbestehende Migräne in engem zeitlichen $\mathrm{Zu}$ sammenhang mit einer solchen Erkrankung, die als Ursache von Kopfschmerzen angesehen wird, chronisch wird, sollte der Patient sowohl die Ausgangsdiagnose einer Migräne erhalten als auch die Diagnose eines sekundären Kopfschmerzes. Ein besonders wichtiges Beispiel hierfür ist 8.2 Kopfschmerz zurückzuführen auf einen Medikamentenübergebrauch: bei vorliegendem Medikamentenübergebrauch sollte der Patient sowohl die Diagnose einer Migräne (episodisch oder chronisch) als auch die Diagnose 8.2 Kopfschmerz zurückzuführen auf einen Medikamentenübergebrauch erhalten.

3. Bei deutlicher Verschlechterung einer vorbestehenden Migräne (gewöhnlich gleichbedeutend mit einer mindestens zweifachen Zunahme der Häufigkeit und/oder der Intensität) in engem zeitlichen Zusammenhang mit einer solchen ursächlichen Erkrankung sollte der Patient sowohl die Ausgangsdiagnose einer Migräne erhalten als auch die Diagnose eines sekundären Kopfschmerzes, vorausgesetzt, es gibt gute Hinweise darauf, dass die betreffende Erkrankung Kopfschmerzen hervorrufen kann.

\section{Einleitung}

Die Migräne ist eine häufige, stark behindernde primäre Kopfschmerzerkrankung. Etliche epidemiologische Studien belegen ihre hohe Prävalenz und die immensen sozioökonomischen und persönlichen Auswirkungen. In einer Studie zur globalen Krankheitslast (Global Burden of Disease Survey 2010, GBD2010) wurde diese als das weltweit dritthäufigste Krankheitsbild eingestuft. In der GBD2015 wurde ihr weltweit bei Männern wie auch bei Frauen unter 50 Jahren der Rang der dritthöchsten Ursache von Behinderungen zuerkannt.

Die Migräne kann in zwei Haupttypen unterteilt werden: Die 1.1 Migräne ohne Aura ist ein klinisches Syndrom, für das ein typisches Kopfschmerzbild und die entsprechenden Begleiterscheinungen charakteristisch sind. Die 1.2 Migräne mit Aura ist vornehmlich durch vorübergehende fokale neurologische Symptome gekennzeichnet, die den Kopfschmerzen meist vorangehen oder sie begleiten. Einige Patienten berichten darüber hinaus über eine Vorbotenphase, die den Kopfschmerzen Stunden oder Tage vorausgehen kann, und/oder einer Erholungsphase nach Verschwinden des Kopfschmerzes. Zu den Symptomen der Vorboten- und Resolutionsphase zählen Hyper- und Hypoaktivität, Depression, Heißhunger auf bestimmte Nahrungsmittel, wiederholtes Gähnen, Müdigkeit und Nackensteifheit und/oder -schmerzen.

Erfüllt ein Patient die Kriterien für mehr als einen Migränetyp bzw. -subtyp, sollten diese alle diagnostiziert und kodiert werden. So sollte ein Patient, der in der Regel Attacken mit Aura aufweist, bei dem es aber auch zu Migräneattacken ohne Aura kommt, unter 1.2 Migräne mit Aura und 1.1 Migräne ohne Aura kodiert werden. Da jedoch die diagnostischen Kriterien für 1.3 chronische Migräne Attacken aller Typen und Subtypen umfassen, ist für episodische Migränesubtypen eine zusätzliche Kodierung erforderlich. 


\subsection{Migräne ohne Aura}

Früher verwendete Begriffe

Einfache Migräne; Hemikranie.

\section{Beschreibung}

Wiederkehrende Kopfschmerzerkrankung, die sich in Attacken von 4 bis 72 Stunden Dauer manifestiert. Typische Kopfschmerzcharakteristika sind einseitige Lokalisation, pulsierender Charakter, mäßige bis starke Intensität, Verstärkung durch körperliche Routineaktivitäten und das begleitende Auftreten von Übelkeit und/ oder Licht- und Lärmüberempfindlichkeit.

\section{Diagnostische Kriterien:}

a. Mindestens fünf Attacken1, welche die Kriterien B bis D erfüllen

b. Kopfschmerzattacken, die (unbehandelt oder erfolglos behandelt) 4 bis72 Stunden 2;3 anhalten

c. Der Kopfschmerz weist mindestens zwei der folgenden vier Charakteristika auf:

d. einseitige Lokalisation

e. pulsierender Charakter

f. mittlere oder starke Schmerzintensität

g. Verstärkung durch körperliche Routineaktivitäten (z. B. Gehen oder Treppensteigen) oder führt zu deren Vermeidung

h. Während des Kopfschmerzes besteht mindestens eines:

i. Übelkeit und/oder Erbrechen

j. Photophobie und Phonophobie

k. Nicht besser erklärt durch eine andere ICHD-3-Diagnose.

Anmerkung:

Die Differenzierung einer einmaligen oder von vereinzelten Migräneattacken von symptomatischen migräneartigen Attacken kann schwierig sein. Darüber hinaus kann die Natur einer einmaligen oder die von vereinzelten Attacken schwer zu erfassen sein. Daher werden mindestens fünf Attacken gefordert. Patienten, die ansonsten die Kriterien für 1.1 Migräne ohne Aura erfüllen, aber bisher weniger als fünf Attacken erlitten haben, sollten unter 1.5.1 Wahrscheinliche Migräne ohne Aura kodiert werden.

Schläft ein Patient während einer Migräne ein und erwacht kopfschmerzfrei, gilt als Attackendauer die Zeit bis zum Erwachen.

Bei Kindern und Jugendlichen (im Alter von unter 18 Jahren) können Migräneattacken 2 bis 72 Stunden dauern (eine unbehandelte Dauer von unter 2 Stunden bei Kindern bedarf noch weiterer wissenschaftlicher Untermauerung).

\section{Kommentar:}

Bei Kindern und Jugendlichen (unter 18 Jahren) sind Migränekopfschmerzen häufiger beidseitig als bei Erwachsenen; das Erscheinungsbild eines einseitigen Kopfschmerzes entwickelt sich meist in späten Jugend- oder frühen Erwachsenenalter. Migränekopfschmerzen sind in der Regel frontotemporal lokalisiert. Okzipitale Kopfschmerzen sind bei Kindern selten und erfordern besondere diagnostische Vorsicht. Bei einer Untergruppe von ansonsten typischen Patienten ist der Schmerz im Gesicht lokalisiert, in der Literatur „faziale Migräne " genannt; es findet sich kein Hinweis darauf, dass diese Patienten eine eigene Untergruppe von Migränepatienten bilden.
Die Prodromalsymptome können Stunden oder ein bis zwei Tage vor den sonstigen Symptomen einer Migräneattacke ohne Aura auftreten. Zu ihnen zählen diverse Kombinationen von Müdigkeit, Konzentrationsschwierigkeiten, Nackensteifheit, Licht- und/oder Geräuschempfindlichkeit, Übelkeit, verschwommenes Sehen, Gähnen und Blässe. Die Postdromalsymptome, unter denen Müdigkeit und ein Stimmungshoch oder -tief am häufigsten sind, können auf das Verschwinden des Kopfschmerzes folgen und bis zu 48 Stunden anhalten; diese müssen noch gründlicher untersucht werden.

Migräneattacken können mit kranio-autonomen Symptomen und mit Symptomen einer kutanen Allodynie verbunden sein.

Bei kleinen Kindern lässt sich Licht- und Geräuschempfindlichkeit aus ihrem Verhalten ableiten.

Eine Minderheit (<10\%) von Frauen erleidet Migräneattacken in Verbindung mit einem Großteil ihrer Menstruationszyklen; die meisten dieser Attacken erfolgen ohne Aura. Attacken während der Menstruation sind tendenziell länger und von heftigerer Übelkeit begleitet als Attacken außerhalb des Menstruationszyklus'. Die ICHD-3 bietet Kriterien an für eine A1.1.1 rein menstruelle Migräne ohne Aura, eine A1.1.2 menstruationsassoziierte Migräne ohne Aura und eine A1.1.3 nicht-menstruelle Migräne ohne Aura, jedoch ausschließlich im Anhang, da weiter Unklarheit darüber besteht, ob es sich um separate Entitäten handelt. Zudem werden Kriterien für eine A1.2.0.1 rein menstruelle Migräne mit Aura, eine A1.2.0.2 menstruationsassoziierte Migräne mit Aura und eine A1.2.0.3 nicht-menstruationsassoziierte Migräne mit Aura angeboten, um zu einer besseren Charakterisierung dieser weniger gängigen Subtypen anzuregen, sofern es sich um separate Entitäten handelt.

Sehr häufig auftretende Migräneattacken werden nun als 1.3 chronische Migräne hervorgehoben. Besteht in Verbindung mit diesen ein Medikamentenübergebrauch, sollten beide Diagnosen, 1.3 chronische Migräne und 8.2 Kopfschmerz zurückzuführen auf einen Medikamentenübergebrauch, zur Anwendung kommen. 1.1 Migräne ohne Aura ist die Erkrankung, die am anfälligsten dafür ist, bei zu häufigem Gebrauch von symptomatischer Medikation zuzunehmen.

Der regionale zerebrale Blutfluss zeigt während Migräneattacken der Kategorie 1.1 Migräne ohne Aura keine Veränderungen, die auf eine „cortical spreading depression (CSD)“ hinweisen, auch wenn Veränderungen des Blutflusses im Hirnstamm ebenso auftreten können wie kortikale Veränderungen als Folge der Schmerzaktivierung. Dies steht im Gegensatz zur pathognomonischen „spreading oligaemia“ bei 1.2 Migräne mit Aura. Während ein Großteil der Literatur zu dem Schluss gelangt, dass eine CSD bei 1.1 Migräne ohne Aura nicht auftritt, widersprechen dem einige neuere Studien. Darüber hinaus wurde die Theorie aufgestellt, dass bei $1.1 \mathrm{Mi}$ gräne ohne Aura gliale Kalziumwellen oder andere kortikale Phänomene beteiligt sein könnten. Dagegen sind die Botenmoleküle Stickoxid (NO), Serotonin (5-Hydroxytryptamin; 5-HT) und Calcitonin-Gene-Related Peptide (CGRP) mit Sicherheit involviert. Galt dieses Krankheitsbild in der Vergangenheit noch als rein vaskulär bedingt, rückte die Wichtigkeit der Sensibilisierung von Schmerzbahnen und die Möglichkeit, dass Attacken im Zentralnervensystem generiert werden, in den letzten Jahrzehnten zunehmend in den Mittelpunkt des Interesses. Zur gleichen Zeit sind die Verschaltungen des Migräneschmerzes, das trigemino-vaskuläre System und diverse Aspekte der Neurotransmission peripher und im Nucleus 
caudatus n. trigemini, dem zentralen mesenzephalischen Höhlengrau und Thalamus aufgedeckt. Hochgradig rezeptorspezifische Akutmedikationen, darunter die 5-HT1B/D-Rezeptoragonisten (Triptane), 5-HT1F Rezeptoragonisten und CGRP-Rezeptorantagonisten haben ihre klinische Wirksamkeit bei der Behandlung akuter Migräneattacken bewiesen. Aufgrund ihrer hohen Rezeptorspezifität erlaubt ihr Wirkmechanismus neue Einsichten in die pathophysiologischen Abläufe der Migräne. Es ist heute unstrittig, dass 1.1 Migräne ohne Aura eine neurobiologische Erkrankung ist. Sowohl die klinische als auch die neurologische Grundlagenforschung erweitern unser Wissen über die Migränemechanismen derzeit mit zunehmender Geschwindigkeit.

\subsection{Migräne mit Aura}

\section{Früher verwendete Begriffe}

Klassische Migräne; ophthalmische, hemiparästhetische, hemiplegische oder aphasische Migräne; migraine accompagnée; komplizierte Migräne.

\section{Beschreibung:}

Wiederkehrende, für Minuten anhaltende Attacken mit einseitigen, komplett reversiblen visuellen, sensorischen oder sonstigen Symptomen des Zentralnervensystems, die sich in der Regel allmählich entwickeln und denen in der Regel Kopfschmerzen und damit verbundene Migränesymptome folgen.

\section{Diagnostische Kriterien:}

A. Mindestens zwei Attacken, die das Kriterium B und C erfüllen

B. Ein oder mehrere der folgenden vollständig reversiblen Aurasymptome:

1. visuell

2. sensorisch

3. Sprechen und/oder Sprache

4. motorisch

5. Hirnstamm

6. retinal

C. Mindestens drei der folgenden sechs Merkmale sind erfüllt:

1. wenigstens ein Aurasymptom entwickelt sich allmählich über $\geq 5$ Minuten hinweg

2. zwei oder mehr Aurasymptome treten nacheinander auf

3. jedes Aurasymptom hält 5 bis 60 Minuten 1 an

4. mindestens ein Aurasymptom ist einseitig2

5. mindestens ein Aurasymptom ist positiv3

6. die Aura wird von Kopfschmerz begleitet, oder dieser folgt ihr innerhalb von 60 Minuten

D. Nicht besser erklärt durch eine andere ICHD-3-Diagnose.

\section{Anmerkung:}

Treten während einer Aura zum Beispiel drei Symptome auf, so beträgt die maximal akzeptable Dauer $3 \times 60$ Minuten. Motorische Symptome können bis zu 72 Stunden anhalten.

Eine Aphasie gilt immer als einseitiges Symptom; bei einer Dysarthrie kann, aber muss es nicht so sein.

Flimmerwahrnehmungen sowie nadelstichartige Parästhesien sind Positivsymptome einer Aura.

\section{Kommentar:}

Viele Patienten, die Migräneattacken mit Aura haben, erleben auch Attacken ohne Aura; diese sollten sowohl als 1.2 Migräne mit Aura als auch als 1.1 Migräne ohne Aura kodiert werden.

In Feldstudien wurden die diagnostischen Kriterien für eine 1.2 Migräne mit Aura im Hauptteil der ICHD-3 beta mit denen für eine A1.2 Migräne mit Aura im Anhang verglichen. Letztere schnitt besser ab, wenn es darum ging, eine Migräne mit Aura von einer transitorischen ischämischen Attacke zu unterscheiden. Diese Unterscheidung wird nun in der ICHD-3 übernommen, die keine im Anhang angeführten Kriterien für diese Erkrankung mehr ausweist.

Die Aura ist ein neurologischer Symptomkomplex, der in der Regel unmittelbar vor dem Kopfschmerz bei 1.2 Migräne mit Aura auftritt, kann jedoch auch nach Beginn der Kopfschmerzphase einsetzen oder sich bis in die Kopfschmerzphase hinein fortsetzen.

Die visuelle Aura ist der häufigste Auratyp, der bei mehr als $90 \%$ der Patienten mit einer 1.2 Migräne mit Aura zumindest bei einigen Attacken auftritt. Häufig stellt sie sich in Form eines Fortifikationsspektrums dar. Man versteht darunter eine Zickzack-Figur nahe dem Fixationspunkt, die sich allmählich nach rechts oder links ausbreitet, eine lateralkonvexe Form mit gezackter flimmernder Randzone annimmt und in ihrem Zentrum ein graduell unterschiedliches absolutes oder relatives Skotom hinterlässt. In anderen Fällen tritt ein Skotom ohne positive visuelle Phänomene auf, dessen Beginn oft akut beschrieben wird, bei genauerer Analyse aber doch eine allmähliche Größenzunahme aufweist. Bei Kindern und Jugendlichen treten weniger typische bilaterale visuelle Symptome auf, die eine Aura darstellen. Es wurde eine Bewertungsskala für die visuelle Aura mit hoher Spezifität und Sensitivität entwickelt und validiert.

Nächsthäufiges Aurasymptom sind Sensibilitätsstörungen in Form von nadelstichartigen Parästhesien, die sich langsam vom Ursprungsort ausbreiten und größere oder kleinere Teile einer Körperhälfte einschließlich des Gesichtes und/oder der Zunge erfassen können. In der Folge dieser Sensibilitätsstörung kann ein sensibles Defizit zurückbleiben, es kann aber auch als alleiniges Symptom auftreten.

Weniger häufig sind Sprachstörungen, üblicherweise aphasische Störungen, die jedoch meist schwer einzuordnen sind.

Systematische Studien konnten zeigen, dass viele Patienten mit einer visuellen Aura gelegentlich auch Aurasymptome im Bereich der Extremitäten und/oder sprachbezogene Symptome haben. Umgekehrt scheinen bei Patienten mit Symptomen in den Extremitäten und/oder Symptomen im Hinblick auf Sprechen oder Sprache fast immer wenigstens bei einigen Attacken visuelle Aurasymptome aufzutreten. Eine Abgrenzung einer Migräne mit visueller Aura, Migräne mit hemiparästhetischer Aura und Migräne mit einer Aura bezogen auf Sprechen und/oder Sprache wäre deshalb wahrscheinlich artifiziell und unterbleibt daher in dieser Klassifikation: sie werden alle als eine 1.2.1 Migräne mit typischer Aura kodiert.

Multiple Aurasymptome folgen gewöhnlich aufeinander, beginnend mit visuellen Symptomen, gefolgt von Sensibilitätsstörungen und gegebenenfalls der Aphasie. Eine umgekehrte Reihenfolge oder eine andere Reihung ist jedoch auch beschrieben. Die anerkannte Dauer beträgt für die meisten Aurasymptome eine Stunde, motorische Symptome halten jedoch häufig länger an.

Patienten mit im Hirnstamm entstehenden Aurasymptomen werden unter 1.2.2 Migräne mit Hirnstammaura kodiert, weisen 
jedoch fast immer zusätzliche typische Aurasymptome auf. Beinhaltet die Aura eine motorische Schwäche, sollte die Erkrankung als 1.2.3 hemiplegische Migräne oder eine ihrer Subtypen kodiert werden. Die 1.2.3 hemiplegische Migräne wird aufgrund genetischer und pathophysiologischer Unterschiede im Vergleich zu einer 1.2.1 Migräne mit typischer Aura als separater Subtyp klassifiziert. Patienten mit einer 1.2.3 hemiplegischen Migräne weisen oft zusätzlich Hirnstammsymptome auf.

Wenn Patienten Schwierigkeiten bei der Beschreibung ihrer Symptome haben, sollten sie angeleitet werden, den Zeitablauf und die Symptome aufzuzeichnen. Übliche Fehler sind ungenaue Angabe über die Lateralisation der Kopfschmerzen, Angaben eines plötzlichen anstatt eines tatsächlichen graduellen Beginns der Aurasymptome, Angabe von monokulären anstatt tatsächlichen homonymen visuellen Störungen, ungenaue Angaben über die Dauer der Aura sowie irrtümliche Annahme einer motorischen Schwäche bei einem tatsächlichem sensiblen Defizit. Nach dem Erstgespräch kann der Gebrauch eines Aurakalenders die Diagnose erhellen.

Mitunter geht die Migräneaura mit einem Kopfschmerz einher, der nicht die Kriterien für eine 1.1 Migräne ohne Aura erfüllt, dieser gilt aufgrund seiner Beziehung zur Aura aber dennoch als Migränekopfschmerz. In anderen Fällen kann eine Migräneaura auch ohne Kopfschmerz auftreten.

Vor oder zeitgleich mit dem Beginn der Aurasymptome ist die regionale Hirndurchblutung in der klinisch betroffenen Region vermindert, schließt aber oft auch größere Areale ein. Die Durchblutungsminderung beginnt üblicherweise im hinteren Kortex und dehnt sich dann nach vorne aus. Sie liegt dabei gewöhnlich über der ischämischen Schwelle. Nach einer oder mehreren Stunden entwickelt sich allmählich in der gleichen Region eine Hyperämie. Leãos Cortical Spreading Depression (CSD) wurde mit diesem Geschehen in Verbindung gebracht.

Die früheren Begriffe einer Migräne mit prolongierter Aura und einer Migräne mit akutem Aurabeginn wurden aufgegeben. Nicht selten hält eine Aura mehr als eine Stunde an, doch in der Mehrzahl der Fälle haben Patienten mit dieser Art von Attacken auch solche, die mindestens zwei weitere Merkmale von Kriterium C erfüllen. Selbst wenn ein Großteil der Attacken, die ein Patient erlebt, Kriterium C nicht erfüllt, verhält es sich in der Regel so, dass andere Attacken Kriterien für eine der anerkannten Subtypen einer 1.2 Migräne mit Aura erfüllen, so dass dies die zu stellende Diagnose wäre. Der Rest sollte unter 1.5.2 mögliche Migräne mit Aura kodiert und dabei die atypischen Besonderheiten (prolongierte Aura oder Migräne mit akutem Aurabeginn) in Klammern beigefügt werden. Die Diagnose erschließt sich gewöhnlich erst nach sorgfältiger Anamnese, obwohl es in seltenen Fällen sekundäre Imitationen der Symptome durch andere Erkrankungen wie eine Dissektion der A. carotis, eine arterio-venöse Malformation oder einen epileptischen Anfall gibt.

Vorbotensymptome der Migräne treten einige Stunden bis zwei Tage vor den sonstigen Symptomen einer Migräneattacke mit Aura auf. Diese beinhalten in unterschiedlicher Kombination Beschwerden wie Müdigkeit, Konzentrationsstörungen, Nackensteifigkeit, Licht- oder Lärmüberempfindlichkeit, Übelkeit, Verschwommensehen, Gähnen oder Blässe. Der Begriff „Prodrom“, der an die Stelle von „Vorwarnphase“ oder „Vorwarnsymptome“ getreten ist, schließt keine Aura mit ein. Der Kopfschmerzresolution können in der Nachphase postdromale Symptome folgen, am häufigsten
Müdigkeit und ein Stimmungshoch oder Stimmungstief. Diese können bis zu 48 Stunden bestehen bleiben und wurden bislang weniger gut untersucht.

\subsubsection{Migräne mit typischer Aura}

\section{Beschreibung:}

Migräne mit Aura, bei der die Aura aus visuellen Symptomen und/ oder Sensibilitätsstörungen und/oder Symptomen rund um Sprechen/Sprache besteht, jedoch ohne motorische Schwäche, und von einer allmählichen Entstehung, einem maximal einstündigen Fortbestand eines jeden Symptoms, einer Mischung aus Positiv- und Negativmerkmalen sowie vollständiger Reversibilität gekennzeichnet ist.

\section{Diagnostische Kriterien:}

A. Die Attacken erfüllen die Kriterien für eine 1.2 Migräne mit Aura und Kriterium B unten

B. Aura mit den beiden folgenden Merkmalen:

1. vollständig reversible visuelle Symptome, Sensibilitätsstörungen und/oder Symptome rund um Sprechen/Sprache

2. keine motorischen, Hirnstamm- oder retinalen Symptome.

\subsubsection{Typische Aura mit Kopfschmerz}

Beschreibung:

Migräne mit typischer Aura, bei der die Aura von einem Kopfschmerz mit oder ohne Migränecharakteristik begleitet wird oder dieser innerhalb von 60 Minuten auf diese folgt.

\section{Diagnostische Kriterien:}

A. Die Attacken erfüllen die Kriterien für eine 1.2.1 Migräne mit typischer Aura und Kriterium B unten

B. Die Aura tritt in Begleitung von Kopfschmerz mit oder ohne Migränecharakteristik auf oder dieser folgt ihr innerhalb von 60 Minuten.

\subsubsection{Typische Aura ohne Kopfschmerz}

\section{Beschreibung:}

Migräne mit typischer Aura, bei der die Aura nicht von Kopfschmerz welcher Art auch immer begleitet ist oder der kein Kopfschmerz welcher Art auch immer folgt.

\section{Diagnostische Kriterien:}

A. Die Attacken erfüllen die Kriterien für eine 1.2.1 Migräne mit typischer Aura und Kriterium B unten

B. Die Aura tritt nicht in Begleitung von Kopfschmerz auf und es folgt ihr auch innerhalb von 60 Minuten kein solcher.

\section{Kommentar:}

Bei einigen Patienten folgen einer typischen Aura immer Migränekopfschmerzen. Viele Patienten weisen jedoch zusätzlich Attacken mit Aura auf, denen Kopfschmerzen folgen, die nicht migränetypisch sind oder bei denen die Kopfschmerzen gänzlich fehlen. Eine Reihe von Patienten weisen ausschließlich eine 1.2.1.2 typische Aura ohne Kopfschmerz auf.

Die Tatsache, dass kein Kopfschmerz vorliegt, der die Kriterien für eine 1.1 Migräne ohne Aura erfüllt, erschwert die genaue Diagnose der Aura und ihre Abgrenzung von Imitationen der Symp- 
tome, die eine ernsthafte Erkrankung anzeigen können (z. B. eine transitorische ischämische Attacke) und kann oft weitere Untersuchungen erforderlich machen. Bei erstmaligem Auftreten der Aura nach dem 40. Lebensjahr, bei ausschließlichen Negativsymptomen (z. B. Hemianopie) oder bei prolongierter oder sehr kurz andauernder Aura sollten andere Ursachen, vor allem transitorische ischämische Attacken, ausgeschlossen werden.

\subsubsection{Migräne mit Hirnstammaura}

Früher verwendete Begriffe

Basilarisarterienmigräne; Basilarismigräne; Migräne vom Basilaristyp.

\section{Beschreibung:}

Eine Migräne, bei der die Aurasymptome eindeutig dem Hirnstamm zuzuordnen sind und keine motorische Schwäche vorhanden ist.

Diagnostische Kriterien:

A. Attacken, die die Kriterien für eine 1.2 Migräne mit Aura und Kriterium B unten erfüllen

B. Aura, bei denen beide untenstehenden Punkte erfüllt sind:

1. Mindestens 2 der folgenden vollständig reversiblen Hirnstammsymptome:
a) Dysarthrie 1
b) Schwindel2
c) Tinnitus
d) Hörminderung3
e) Doppelbilder4
f) Ataxie, die nicht auf ein sensibles Defizit zurückzufüh- ren ist
g) Bewusstseinsstörung (GCS $\leq 13) 5$

2. Keine motorischen6 oder retinalen Symptome.

\section{Anmerkung:}

Es sollte die Abgrenzung einer Dysarthrie von einer Aphasie erfolgen.

Schwindel beinhaltet keine Benommenheit und ist von dieser abzugrenzen.

Dieses Kriterium ist nicht erfüllt, wenn Patienten von einem „Völlegefühl“ im Ohr berichten.

Diplopie umfasst kein Verschwommensehen (oder schließt dieses aus).

Eine Abschätzung der Bewusstseinsstörung nach der Glasgow Coma Scale (GCS) mag bereits bei Aufnahme erfolgt sein; alternativ erlauben eindeutig vom Patienten geschilderte Defizite eine GCS-Einstufung.

Bei Vorliegen motorischer Symptome wird die Erkrankung unter 1.2.3 hemiplegische Migräne kodiert.

\section{Kommentar:}

Ursprünglich wurden die Begriffe Basilarisarterienmigräne oder Basilarismigräne verwendet, da aber eine Beteiligung der Basilarisarterie unwahrscheinlich ist, sollte der Begriff Migräne mit Hirnstammaura bevorzugt werden.

Während der meisten Attacken kommt es zusätzlich zu den Hirnstammsymptomen zu typischen Aurasymptomen. Viele Patienten, die Attacken mit Hirnstammaura haben, berichten auch von anderweitigen Attacken mit typischer Aura. Hier sollte sowohl unter 1.2.1 Migräne mit typischer Aura als auch 1.2.2 Migräne mit Hirnstammaura kodiert werden.

Viele der unter Kriterium B1 aufgelisteten Symptome können fehlinterpretiert werden, da sie auch in Verbindung mit Angst und Hyperventilation auftreten können.

\subsubsection{Hemiplegische Migräne}

\section{Beschreibung:}

Migräne mit Aura, die eine motorische Schwäche einschließt.

\section{Diagnostische Kriterien}

A. Attacken erfüllen die Kriterien für eine 1.2 Migräne mit Aura und Kriterium B unten

B. Aura, bei der die beiden untenstehenden Punkte erfüllt sind:

1. Vollständig reversible motorische Schwäche2

2. Vollständig reversible visuelle, sensible und/oder sprech-/ sprachbezogene Symptome.

\section{Anmerkung:}

1. Der Begriff plegisch bezeichnet in den meisten Sprachen eine Lähmung, die meisten Attacken sind jedoch von motorischer Schwäche gekennzeichnet.

2. Die motorischen Symptome halten im Allgemeinen weniger als 72 Stunden an, bei manchen Patienten kann die motorische Schwäche jedoch über Wochen fortbestehen.

\section{Kommentar:}

Es ist teilweise schwer, zwischen einer muskulären Schwäche und einem Sensibilitätsverlust zu unterscheiden.

\subsubsection{Familiäre hemiplegische Migräne (FHM)}

\section{Beschreibung:}

Migräne mit Aura, die eine motorische Schwäche einschließt. Wenigstens ein Verwandter ersten oder zweiten Grades weist ebenfalls Migräneauren mit einer motorischen Schwäche auf.

\section{Diagnostische Kriterien:}

A. Die Attacken erfüllen die Kriterien für eine 1.2.3 hemiplegische Migräne

B. Wenigstens ein Verwandter ersten oder zweiten Grades hat ebenfalls Attacken gehabt, die die Kriterien für eine 1.2.3 hemiplegische Migräne erfüllen

\section{Kommentar:}

Neuere genetische Daten erlauben heute eine präzisere Definition der 1.2.3.1 familiären hemiplegischen Migräne als zuvor. Spezifische genetische Subtypen konnten identifiziert werden: Bei der FHM1 finden sich Mutationen im CACNA1A Gen (Kodierung für einen Kalziumkanal) auf Chromosom 19 und bei FHM2 Mutationen im ATP1A2 Gen (Kodierung für eine K/Na-ATPase) auf Chromosom 1; und bei der FHM3 liegen Mutationen im SCN1A Gen (Kodierung für einen Natriumkanal) auf Chromosom 2vor. Möglicherweise existieren noch weitere, bislang nicht identifizierte Genloci.

Wurde eine genetische Untersuchung durchgeführt, sollte der genetische Subtyp (sofern ermittelt) an der fünften Stelle angegeben werden. 
Es konnte gezeigt werden, dass bei der 1.2.3.1 familiären hemiplegischen Migräne zusätzlich zu den typischen Aurasymptomen sehr oft Hirnstammsymptome auftreten und dass Kopfschmerzen praktisch immer vorhanden sind. In seltenen Fällen können während einer FHM-Attacke Bewusstseinsstörungen (bis zum Koma), Verwirrtheitszustände, Fieber und eine Liquorpleozytose auftreten.

Die 1.2.3.1 familiäre hemiplegische Migräne wird häufig mit einer Epilepsie verwechselt und als solche (erfolglos) behandelt. FHM1-Attacken können durch (leichte) Schädel-Hirn-Traumen getriggert werden. In ungefähr 50 \% der Familien mit FHM ritt unabhängig von den Migräneattacken eine chronische progressive zerebelläre Ataxie auf.

\subsection{Familiäre hemiplegische Migräne Typ 1 (FHM1)}

Diagnostische Kriterien:
A. Die Attacken erfüllen die Kriterien für eine 1.2.3.1 familiäre hemiplegische Migräne
B. Nachgewiesene Mutation im CACNA1A-Gen.

\subsection{Familiäre hemiplegische Migräne Typ 2 (FHM2)} Diagnostische Kriterien:

A. Die Attacken erfüllen die Kriterien für eine 1.2.3.1 familiäre hemiplegische Migräne

B. Nachgewiesene Mutation im ATP1A2 Gen.

\subsection{Familiäre hemiplegische Migräne Typ 3 (FHM3)}

Diagnostische Kriterien:

A. Die Attacken erfüllen die Kriterien für eine 1.2.3.1 familiäre hemiplegische Migräne

B. Nachgewiesene Mutation im SCN1A Gen.

\subsection{Familiäre hemiplegische Migräne, andere Genloci}

Diagnostische Kriterien:

A. Die Attacken erfüllen die Kriterien für eine 1.2.3.1 familiäre hemiplegische Migräne

$B$. Genetische Untersuchungen ergaben keine Mutation in den CACNA1A, ATP1A2 oder SCN1A Genen.

\subsubsection{Sporadische hemiplegische Migräne (SHM)}

Beschreibung:

Migräne mit Aura, die eine motorische Schwäche einschließt. Kein Verwandter ersten oder zweiten Grades weist ebenfalls Migräneauren mit einer motorischen Schwäche auf.

\section{Diagnostische Kriterien:}

A. Die Attacken erfüllen die Kriterien für eine 1.2.3 hemiplegische Migräne

B. Kein Verwandter ersten oder zweiten Grades erfüllt die Kriterien für eine 1.2.3 hemiplegische Migräne.

Kommentar:

Epidemiologische Untersuchungen konnten zeigen, dass die sporadischen Fälle mit ungefähr der gleichen Häufigkeit wie die familiären Fälle vorkommen.

Bei einer 1.2.3.2 sporadischen hemiplegischen Migräne zeigen die Kopfschmerzattacken die gleichen klinischen Merkmale wie bei der 1.2.3.1 familiären hemiplegischen Migräne. Bei einigen scheinbar sporadischen Fälle finden sich bekannte FHM-Mutationen und, bei einigen ein Verwandter ersten oder zweiten Grades, der später eine hemiplegische Migräne entwickelt, womit die Kriterien für eine 1.2.3.1 familiäre hemiplegische Migräne vervollständig erfüllt sind und eine Änderung der Diagnose erforderlich ist.

Die sporadische Form macht jedoch grundsätzlich eine zerebrale Bildgebung und weitere Untersuchungen zum Ausschluss einer anderen Ursache erforderlich. Eine Lumbalpunktion sollte durchgeführt werden, um ein 7.8 Syndrom der vorübergehenden Kopfschmerzen mit neurologischen Defiziten und Liquorlymphozytose (HaNDL) auszuschließen.

\subsubsection{Retinale Migräne}

\section{Beschreibung:}

Wiederholte Anfälle von monokulären visuellen Phänomenen wie Flimmern, Skotomen oder Erblindung in Verbindung mit Migränekopfschmerzen.

\section{Diagnostische Kriterien:}

A. Attacken, die die Kriterien für eine 1.2 Migräne mit Aura und Kriterium B unten erfüllen

B. Eine Aura, auf welche die beiden folgenden Punkte zutreffen: 1. vollständig reversible, monokuläre, positive und/oder negative visuelle Phänomene (z. B. Flimmern, Skotome oder Blindheit). Der Befund sollte während der Attacke auf einem der beiden folgenden Wege oder durch beide bestätigt werden:

a) ärztliche Untersuchung des Gesichtsfelds

b) Zeichnung eines monokulären Gesichtsfelddefekts durch den Patienten (nach vorangehender genauer Instruktion)

2. Es ist wenigstens einer der folgenden Punkte erfüllt:

a) entwickelt sich allmählich über $\geq 5$ Minuten hinweg

b) Symptome halten 5 bis 60 Minuten an

c) begleitet oder innerhalb von 60 Minuten gefolgt von Kopfschmerzen

C. Nicht besser erklärt durch eine andere ICHD-3-Diagnose. Andere Ursachen einer Amaurosis fugax wurden ausgeschlossen.

Kommentar:

Einige Patienten, die eine monokulare Sehstörung beschreiben, haben in Wirklichkeit eine Hemianopie. Es wurde über einige Fälle ohne begleitende Kopfschmerzen berichtet, bei denen der ursächliche Zusammenhang mit einer Migräne jedoch nicht gesichert ist.

Die 1.2.4 retinale Migräne ist eine außerordentlich seltene Ursache eines vorübergehenden monokularen Sehverlusts. Es wurden auch bereits Fälle eines dauerhaften monokularen Sehverlusts in Verbindung mit Migräne beschrieben. Es sind geeignete Untersuchungen durchzuführen, um andere Ursachen einer transienten monokulären Blindheit auszuschließen. 


\subsection{Chronische Migräne}

Beschreibung:

Kopfschmerz, der über mehr als 3 Monate an 15 oder mehr Tagen/ Monat auftritt und der an mindestens 8 Tagen/Monat die Merkmale eines Migränekopfschmerzes aufweist.

\section{Diagnostische Kriterien:}

A. Kopfschmerz (migräneartig oder spannungstypartig1) an $\geq 15$ Tagen/Monat über $>3$ Monate, welcher Kriterium B und $C$ erfüllt

B. Auftreten bei einem Patienten, der mindestens fünf Attacken gehabt hat, welche die Kriterien B bis D für eine 1.1 Migräne ohne Aura und/oder die Kriterien B und C für eine 1.2 Migräne mit Aura erfüllt

C. An $\geq 8$ Tagen/Monat über $>3$ Monate, wobei einer der folgenden Punkte erfüllt ist2:

1. Kriterium C und D für eine 1.1 Migräne ohne Aura

2. Kriterium $B$ und $C$ für eine 1.2 Migräne mit Aura

3. Der Patient geht bei Kopfschmerzbeginn von einer Migräne aus und der Kopfschmerz lässt sich durch ein Triptanoder Ergotaminderivat lindern

D. Nicht besser erklärt durch eine andere ICHD-3-Diagnose3;4;5.

\section{Anmerkung:}

1. Der Grund, warum die 1.3 chronische Migräne von episodischen Migränetypen abgehoben wird ist der, dass es unmöglich ist, die einzelnen Kopfschmerzepisoden bei Patienten mit derart häufigen oder ständigen Kopfschmerzen voneinander abzugrenzen. Tatsächlich kann sich der Kopfschmerzcharakter nicht nur von einem Tag zum anderen, sondern sogar innerhalb desselben Tages ändern. Bei solchen Patienten ist es außerordentlich schwer, auf eine medikamentöse Behandlung zu verzichten, um die natürliche Kopfschmerzgeschichte zu beobachten. In dieser Situation werden Attacken mit wie auch ohne Aura gezählt, ebenso wie sowohl migräneartige als auch spannungstypartige Kopfschmerzen (nicht jedoch sekundäre Kopfschmerzen).

2. Eine Charakterisierung eines häufig wiederkehrenden Kopfschmerzes erfordert in der Regel das Führen eines Kopfschmerztagebuchs, in dem Informationen zum Schmerz und den mit ihm verbundenen Symptomen mindestens einen Monat lang tagtäglich aufgezeichnet werden.

3. Da Kopfschmerz vom Spannungstyp den diagnostischen Kriterien für eine 1.3 chronische Migräne entspricht, schließt diese Diagnose die Diagnose eines 2. Kopfschmerzes vom Spannungstyp oder seine Typen aus.

4. Ein 4.10 neu aufgetretener täglicher Kopfschmerz kann Merkmale aufweisen, die auf eine 1.3 chronische Migräne hindeuten. Letztere ist ein Krankheitsbild, das sich im Laufe der Zeit aus einer 1.1 Migräne ohne Aura und/oder 1.2 Migräne mit Aura entwickelt. Wenn diese Kriterien A bis C also von einem Kopfschmerz erfüllt sind, der eindeutig täglich auftritt und $a b<24$ Stunden nach seinem erstmaligen Auftreten nicht nachlässt, sollte er unter 4.10 neu aufgetretener täglicher Kopfschmerz kodiert werden. Wenn keine Erinnerung an den Beginn der Erkrankung besteht oder dieser anderweitig ungewiss ist, sollte sie als 1.3 chronische Migräne kodiert werden.
5. Die häufigste Ursache von Symptomen, die auf eine chronische Migräne hindeuten, ist Medikamentenübergebrauch gemäß Definition unter 8.2 Kopfschmerz zurückzuführen auf einen Medikamentenübergebrauch. Rund 50 \% der Patienten, die vermeintlich eine 1.3 chronische Migräne aufweisen, kehren nach dem Entzug zu einem episodischen Migränetyp zurück; bei diesen Patienten wird die gewissermaßen unberechtigte Diagnose einer 1.3 chronischen Migräne gestellt. Gleichermaßen gebessert sich der Zustand vieler Patienten mit offensichtlichem Medikamentenübergebrauch nach dem Entzug nicht; für sie kann die Diagnose eines 8.2 Kopfschmerzes zurückzuführen auf einen Medikamentenübergebrauch unangemessen sein (ausgehend von der Annahme, dass die durch Medikamentenübergebrauch induzierte Chronizität immer reversibel ist). Aus diesen Gründen und weil die allgemeine Regel für alle relevanten Diagnosen gilt, sollte bei Patienten, welche die Kriterien für eine 1.3 chronische Migräne und für einen 8.2 Kopfschmerz zurückzuführen auf einen Medikamentenübergebrauch erfüllen, beides kodiert werden. Nach dem Entzug wird die Migräne entweder zu einem episodischen Typ zurückkehren oder chronisch bleiben und sollte dementsprechend neu diagnostiziert werden; bei Letzterem kann die Diagnose eines 8.2 Kopfschmerzes zurückzuführen auf einen Medikamentenübergebrauch aufgehoben werden.

\subsection{Migränekomplikationen}

\section{Kommentar:}

Der Typ oder Subtyp von Migräne sowie die Komplikation sollten getrennt kodiert werden.

\subsubsection{Status migränosus}

Beschreibung:

Stark beeinträchtigende Migräneattacke, die länger als 72 Stunden andauert.

\section{Diagnostische Kriterien:}

A. Die Kopfschmerzattacke erfüllt die Kriterien B und C

B. Sie tritt bei einem Patienten mit einer 1.1 Migräne ohne Aura und/oder 1.2 Migräne mit Aura auf und ist typisch für frühere Attacken mit Ausnahme der Dauer und Intensität

C. Der Kopfschmerz weist beide folgenden Merkmale auf:

1. Über $>72$ Stunden anhaltend1

2. Der Schmerz und/oder die mit ihm verbundenen Symptome sind stark beeinträchtigend 2

D. Nicht besser erklärt durch eine andere ICHD-3-Diagnose.

Eine Unterbrechung durch Schlaf wird nicht berücksichtigt, das gleiche gilt für kurze Unterbrechungen bedingt durch Medikation.

Anmerkung:

1. Remissionen von bis zu 12 Stunden Dauer aufgrund von Medikamentengabe oder Schlaf werden anerkannt.

2. Leichtere Verläufe, die Kriterium C2 nicht erfüllen, werden unter 1.5.1 wahrscheinliche Migräne ohne Aura kodiert. 


\section{Kommentar:}

Ein Kopfschmerz mit den Merkmalen eines 1.4.1 Status migränosus wird häufig durch einen Medikamentenübergebrauch ausgelöst. Erfüllt der Kopfschmerz unter diesen Umständen die Kriterien für einen 8.2 Kopfschmerz zurückzuführen auf einen Medikamentenübergebrauch, sollte er unter diesen Erkrankungen und dem entsprechenden Migränetyp oder -subtyp kodiert werden, nicht jedoch unter 1.4.1 Status migränosus. Besteht der Medikamentenübergebrauch kürzer als 3 Monate, sollte der Kopfschmerz lediglich unter dem entsprechenden Migränetyp oder dem/den entsprechenden Subtyp(en) kodiert werden.

\subsubsection{Anhaltende Aura ohne Hirninfarkt}

Beschreibung:

Eine Woche oder länger anhaltende Aurasymptome, ohne dass mit bildgebenden Verfahren ein Nachweis eines Hirninfarktes gelingt.

\section{Diagnostische Kriterien:}

A. Die Aura erfüllt das Kriterium B

B. Die aktuelle Attacke bei einem Patienten mit 1.2 Migräne mit Aura ist typisch für frühere Attacken mit der Ausnahme, dass ein oder mehrere Aurasymptom(e) für $\geq 1$ Woche persistieren

C. Mit bildgebenden Verfahren gelingt kein Nachweis eines Hirninfarktes

D. Nicht besser erklärt durch eine andere ICHD-3-Diagnose.

\section{Kommentar:}

Anhaltende Aurasymptome sind selten, aber gut dokumentiert. Häufig sind sie bilateral und halten über Monate bis Jahre an. Die Mindestdauer von 1 Woche bei Kriterium B stützt sich auf die Meinung von Experten und sollte formal untersucht werden.

Die diagnostische Abklärung muss darauf abzielen, eine 1.4.2 anhaltende Aura ohne Hirninfarkt von einem 1.4.3 migränösen Infarkt abzugrenzen sowie eine symptomatische Aura aufgrund eines Hirninfarkts aus anderen Gründen auszuschließen. Attacken mit prolongierter Aura, die weniger als eine Woche anhalten und nicht die Kriterien für eine 1.2.1 Migräne mit typischer Aura erfüllen, sollten unter 1.5.2 wahrscheinliche Migräne mit Aura kodiert werden.

\subsubsection{Migränöser Infarkt}

Beschreibung:

Ein oder mehrere im Laufe einer typischen Migräneattacke mit Aura einsetzende Aurasymptom(e) verbunden mit einer in der zerebralen Bildgebung nachgewiesenen ischämischen Läsion in einem relevanten Areal.

\section{Diagnostische Kriterien:}

A. Migräneattacke, die die Kriterien B und C erfüllt

B. Die aktuelle Attacke bei einem Patienten mit 1.2 Migräne mit Aura ist typisch für frühere Attacken, mit der Ausnahme, dass ein oder mehrere Aurasymptom(e) für $>60$ Minuten persistieren 1

C. Die zerebrale Bildgebung zeigt einen ischämischen Infarkt in einem relevanten Hirnareal

D. Nicht besser erklärt durch eine andere Diagnose.
Anmerkung:

1. Es kann zum Auftreten zusätzlicher, auf den Hirninfarkt zurückzuführender Symptome kommen.

\section{Kommentar:}

Ein ischämischer Infarkt bei Migränepatienten kann definiert sein als Hirninfarkt aus anderen Gründen bei gleichzeitig bestehender 1. Migräne, als Hirninfarkt aus anderen Gründen mit Symptomen, die einer 1.2 Migräne mit Aura ähneln oder als Hirninfarkt im Ablauf einer typischen Attacke einer 1.2 Migräne mit Aura. Nur letzteres erfüllt die Kriterien eines 1.4.3 migränösen Infarkts.

Ein 1.4.3 migränöser Infarkt tritt meist in der posterioren Zirkulation und bei jüngeren Frauen auf.

Ein zweifach erhöhtes Risiko für ischämische Infarkte bei Migränepatienten mit einer 1.2 Migräne mit Aura ließ sich in mehreren bevölkerungsbezogenen Studien nachweisen. Es ist jedoch festzuhalten, dass es sich bei diesen Infarkten nicht um migränöse Infarkte handelt. Die Wirkmechanismen hinter dem erhöhten Risiko eines ischämischen Infarkts bei Migränepatienten bleibt unklar; ebenso ist die Beziehung zwischen erhöhtem Risiko, Aurahäufigkeit und der Natur der Aurasymptome, die das höhere Risiko kennzeichnen, unbekannt. Die meisten Untersuchungen ergaben, dass ein Zusammenhang zwischen einer 1.1 Migräne ohne Aura und einem ischämischen Infarkt fehlt.

\subsubsection{Epileptische Anfälle, durch Migräneaura getriggert}

Beschreibung:

Epileptischer Anfall, der durch eine Migräne mit Aura getriggert wurde.

Diagnostische Kriterien:

A. Ein epileptischer Anfall, der die diagnostischen Kriterien eines Epilepsietyps sowie Kriterum B unten erfüllt

B. Dieser epileptische Anfall ereignet sich bei einem Patienten mit 1.2 Migräne mit Aura während oder innerhalb 1 Stunde nach einer Migräneattacke mit Aura

C. Nicht besser erklärt durch eine andere Diagnose.

\section{Kommentar:}

Migräne und Epilepsie sind Prototypen von paroxysmalen zerebralen Anfallsleiden. Während migräneähnliche Kopfschmerzen in der postiktalen Phase bei Epilepsie relativ häufig sind, können manchmal auch epileptische Anfälle während oder im Anschluss an eine Migräne auftreten. Dieses seltene Phänomen, als Migralepsie bezeichnet, wurde zunächst bei Patienten mit einer 1.2 Migräne mit Aura beschrieben.

Belege für einen Zusammenhang mit einer 1.1 Migräne ohne Aura fehlen. 


\subsection{Wahrscheinliche Migräne}

Früher verwendeter Begriff: Migräneartige Störung.

An anderer Stelle kodiert: igräneartige Kopfschmerzen als Folge einer anderen Erkrankung (symptomatische Migräne) werden entsprechend dieser Erkrankung kodiert.

\section{Beschreibung:}

Migräneartige Attacken, bei denen ein Merkmal fehlt, das erforderlich ist, um die Kriterien eines der oben aufgeführten Migränetypen oder Subtypen vollständig zu erfüllen und die nicht die Kriterien einer anderen Kopfschmerzerkrankung erfüllen.

\section{Diagnostische Kriterien:}

A. Attacken, die mit einer Ausnahme die Kriterien A bis D einer

1.1 Migräne ohne Aura oder mit einer Ausnahme die Kriterien A bis C einer 1.2 Migräne mit Aura erfüllen

B. Attacken, die nicht die ICHD-3-Kriterien einer anderen Kopfschmerzerkrankung erfüllen

C. Nicht besser erklärt durch eine andere ICHD-3-Diagnose.

\section{Kommentar:}

Beim Stellen einer Kopfschmerzdiagnose sollten Attacken, die sowohl die Kriterien eines 2. Kopfschmerzes vom Spannungstyp als auch die für eine 1.5 wahrscheinliche Migräne erfüllen, gemäß der allgemeinen Regel, dass eine definitive Diagnose grundsätzlich Vorrang vor einer wahrscheinlichen Diagnose hat, als Ersteres kodiert werden. Im Fall von Patienten, bei denen jedoch bereits eine Migränediagnose vorliegt und bei denen es darum geht, die Anzahl der erlittenen Attacken zu zählen (z. B. zur Ergebnismessung im Rahmen einer Arzneimittelstudie), sollten Attacken, die die Kriterien für eine 1.5 wahrscheinliche Migräne erfüllen, als Migräne zählen. Der Grund dafür ist der, dass leichtere Migräneattacken oder früh behandelte Attacken oft nicht alle Merkmale aufweisen, die für eine Diagnose der Migräneattacke erforderlich sind, aber dennoch auf bestimmte Migränebehandlungen ansprechen.

\subsubsection{Wahrscheinliche Migräne ohne Aura}

Diagnostische Kriterien:

A. Die Attacken erfüllen mit einer Ausnahme die Kriterien A-D für 1.1 Migräne ohne Aura

B. Die Attacken erfüllen nicht die ICHD-3-Kriterien einer anderen Kopfschmerzerkrankung

C. Nicht besser erklärt durch eine andere ICHD-3-Diagnose.

\subsubsection{Wahrscheinliche Migräne mit Aura}

Diagnostische Kriterien:

A. Die Attacken erfüllen mit einer Ausnahme die Kriterien A-C für 1.2 Migräne mit Aura oder eines Subtyps von dieser

B. Die Attacken erfüllen nicht die ICHD-3-Kriterien einer anderen Kopfschmerzerkrankung

C. Nicht besser erklärt durch eine andere ICHD-3-Diagnose.

1.6 Episodische Syndrome, die mit einer Migräne in Verbindung stehen können

Früher verwendete Begriffe Periodische Syndrome in der Kindheit; periodische Kindheitssyndrome.

\section{Kommentar:}

Diese Gruppe von Krankheitsbilder tritt bei Patienten auf, die auch eine 1.1 Migräne ohne Aura oder 1.2 Migräne mit Aura aufweisen oder bei denen eine erhöhte Wahrscheinlichkeit besteht, dass sie eine dieser beiden Störungen entwickeln. Obwohl die Vorgeschichte deren Auftreten in der Kindheit zeigt, kann beides auch im Erwachsenenalter auftreten.

Zu den weiteren Krankheitsbildern, die bei diesen Patienten auftreten können, gehören episodische Reiseübelkeit und periodische Schlafstörungen wie Schlafwandeln, Sprechen im Schlaf, Pavor nocturnus und Zähneknirschen.

\subsubsection{Rezidivierende gastrointestinale Störungen}

Früher verwendete Begriffe Chronische Bauchschmerzen; funktionelle Bauchschmerzen; funktionelle Dyspepsie; Reizdarmsyndrom; funktionelles Bauchschmerzsyndrom.

\section{Beschreibung:}

Rezidivierende episodische Bauchschmerz- und/oder -beschwerdeattacken, Übelkeit und/oder Erbrechen, die unregelmäßig, chronisch oder in absehbaren Zeitabständen auftreten und mit Migräne in Verbindung stehen können.

\section{Diagnostische Kriterien:}

A. Mindestens 5 Attacken mit ausgeprägten Attacken von Bauchschmerzen und/oder Bauchbeschwerden und/oder Übelkeit und/oder Erbrechen

B. Normale Untersuchung und Beurteilung des Magen-Darm-Traktes

C. Nicht auf eine andere Erkrankung zurückzuführen.

\subsubsection{Zyklische Erbrechen}

Beschreibung:

Episodisch wiederkehrende Attacken mit starker Übelkeit und $\mathrm{Er}$ brechen, üblicherweise mit stereotypischen Ablauf bei dem Betroffenen. Die Attacken können mit Blässe und Lethargie verbunden sein. Vollständige Rückbildung der Symptome zwischen den Attacken.

\section{Diagnostische Kriterien:}

A. Mindestens 5 Attacken mit starker Übelkeit und Erbrechen, welche die Kriterien $B$ und $C$ erfüllen

B. Beim Betroffenen stereotyp ablaufend und mit absehbarer Periodizität wiederkehrend

C. Alles von Folgendem:

1. Übelkeit und Erbrechen treten mindestens viermal pro Stunde auf

2. Attacken dauern $\geq 1$ Stunde, bis zu 10 Tage

3. Attacken treten im Abstand von $\geq 1$ Woche auf

D. Vollständige Symptomfreiheit zwischen den Attacken

E. Nicht auf eine andere Erkrankung zurückzuführen1.

\section{Anmerkung:}

1. Insbesondere ergibt die Vorgeschichte und körperliche Untersuchung keinen Hinweis auf eine gastrointestinale Erkrankung. 


\section{Kommentar:}

Das 1.6.1.1 Zyklische Erbrechen ist typischerweise eine sich selbst limitierende episodische Erkrankung des Kindesalters. Zwischen den einzelnen Episoden besteht völlige Beschwerdefreiheit. Kennzeichnend ist seine zyklische Wiederkehr, und die Attacken sind absehbar.

Diese Erkrankung wurde in der ICHD-2 erstmals als periodisches Syndrom in der Kindheit aufgeführt. Das klinische Bild ähnelt den Begleitsymptomen der Migräne und vielfältige Untersuchungen in den letzten Jahren haben eine Verwandtschaft des 1.6.1.1 Zyklischen Erbrechen mit der Migräne nahegelegt.

\subsubsection{Abdominelle Migräne}

Beschreibung:

Idiopathische, wiederkehrende Störung, die vor sich vor allem bei Kindern in Form von mittellinienbetonten Bauchschmerzen manifestiert, welche 2 bis 72 Stunden anhalten. Der Schmerz ist von mittlerer bis schwerer Intensität und assoziiert mit vasomotorischen Symptomen, Übelkeit und Erbrechen. Kein Kopfschmerz zwischen diesen Episoden.

\section{Diagnostische Kriterien:}

A. Mindestens 5 Attacken mit abdominellen Schmerzen, welche die Kriterien B-D erfüllen

B. Die abdominellen Schmerzen weisen mindestens zwei der drei folgenden Charakteristika auf:

1. Lokalisation im Bereich der Mittellinie, periumbilikal oder diffus

2. dumpfer oder „wunder“ Charakter

3. mittlere oder starke Schmerzintensität

C. Während der Attacken sind mindestens zwei der folgenden Punkte erfüllt:

1. Appetitlosigkeit

2. Übelkeit

3. Erbrechen

4. Blässe

D. Attacken dauern unbehandelt oder erfolglos behandelt 2 bis 72 Stunden

E. Vollständige Symptomfreiheit zwischen den Attacken

F. Nicht auf eine andere Erkrankung zurückzuführen1.

\section{Anmerkung:}

Insbesondere ergibt die Vorgeschichte und körperliche Untersuchung keinen Hinweis auf eine gastrointestinale oder renale $\mathrm{Er}$ krankung oder eine solche konnte durch geeignete Untersuchungen ausgeschlossen werden.

\section{Kommentar:}

Der Schmerz bei einer 1.6.1.2 abdominellen Migräne ist schwer genug, um die normalen Alltagsaktivitäten zu beeinträchtigen.

Bei kleineren Kindern wird das Vorliegen von Kopfschmerzen oft übersehen. Es ist eine sorgfältige Anamnese zum Vorhandensein oder Fehlen von Kopfschmerzen zu erheben. Werden Kopfschmerzen während der Attacken ermittelt, sollte die Diagnose einer 1.1 Migräne ohne Aura in Betracht gezogen werden.
Den Kindern fällt es häufig schwer, zwischen Appetitlosigkeit und Übelkeit zu unterscheiden. Die Blässe ist häufig von dunklen Ringen unter den Augen begleitet. Bei einigen Patienten ist eine Gesichtsrötung das vorherrschende vasomotorische Phänomen.

Die meisten Kinder mit einer abdominellen Migräne entwickeln im Laufe ihres Lebens Migränekopfschmerzen.

\subsubsection{Gutartiger paroxysmaler Schwindel}

\section{Beschreibung:}

Diese Störung ist durch wiederkehrende kurze Schwindelattacken charakterisiert, die ohne Vorwarnung bei ansonsten gesunden Kindern auftreten und sich spontan zurückbilden.

\section{Diagnostische Kriterien:}

A. Mindestens 5 Attacken, welche die Kriterien B und C erfüllen

B. Schwindelattacken1, die ohne Vorwarnung auftreten, mit maximaler Intensität zu Beginn, und die sich ohne Bewusstseinsverlust innerhalb von Minuten bis Stunden spontan zurückbilden

C. Mindestens eines der folgenden assoziierten Symptome oder Symptom:
1. Nystagmus
2. Ataxie
3. Erbrechen
4. Blässe
5. Ängstlichkeit

D. Unauffälliger neurologischer Untersuchungsbefund und unauffällige audiometrische und vestibuläre Funktionen zwischen den Attacken

E. Nicht auf eine andere Erkrankung zurückzuführen2.

Anmerkung:

1. Jüngere Kinder mit Schwindel sind unter Umständen nicht in der Lage, Schwindelsymptome zu beschreiben. Elterlicherseits beobachtete episodische Perioden der Gangunsicherheit können bei jüngeren Kindern als Schwindel interpretiert werden.

2. Insbesondere sind Tumore der hinteren Schädelgrube, epileptische Anfälle und vestibuläre Störungen auszuschließen.

\section{Kommentar:}

Die Beziehung zwischen einem 1.6.2 gutartigen paroxysmalen Schwindel und einer A1.6.6 vestibulären Migräne (siehe Anhang) bedarf näherer Untersuchung.

\subsubsection{Gutartiger paroxysmaler Torticollis}

\section{Beschreibung:}

Episodisch wiederkehrende Schrägneigung des Kopfes zu einer Seite, eventuell mit leichter Rotation, die sich spontan zurückbildet. Die Erkrankung tritt bei Säuglingen und Kleinkindern auf und beginnt im ersten Lebensjahr.

\section{Diagnostische Kriterien:}

A. Wiederkehrende Attacken 1 in der frühen Kindheit, welche die Kriterien B und C erfüllen

B. Schrägneigung des Kopfes zu einer Seite, mit oder ohne leichte Drehung, die nach Minuten bis Tagen zurückgeht 
C. Mindestens eines der folgenden assoziierten Symptome oder Symptom:

1. Blässe

2. Reizbarkeit

3. Unwohlsein

4. Erbrechen

5. Ataxie2

D. Normale neurologische Untersuchung zwischen den Attacken

E. Nicht auf eine andere Erkrankung zurückzuführen3.

\section{Anmerkung:}

1. Attacken treten meist im Monatsabstand wieder auf.

2. Ataxie ist bei älteren Kindern innerhalb der betroffenen Altersgruppe wahrscheinlicher.

3. Die differentialdiagnostische Abklärung umfasst die von gastroösophagealem Reflux, idiopathischer Torsionsdystonie und komplexen partiellen Anfällen, besondere Aufmerksamkeit ist jedoch der hinteren Schädelgrube und dem kraniozervikalen Übergang zuzuwenden, wo angeborene oder erworbene Läsionen einen Torticollis bewirken können.

\section{Kommentar:}

Der Kopf des Kindes lässt sich während der Attacken in die Neutralposition zurückdrehen. Zwar kann ein gewisser Widerstand vorhanden sein, dieser lässt sich jedoch überwinden.

Diese Beobachtungen bedürfen einer weiteren Validierung mit Hilfe von Patiententagebüchern, strukturierten Interviews und einer Erhebung von Längsschnittdaten.

Ein 1.6.3 gutartiger paroxysmaler Torticollis kann sich zu einem 1.6.2 gutartigen paroxysmalen Schwindel oder einer 1.2 Migräne mit Aura entwickeln (vor allem 1.2.2 Migräne mit Hirnstammaura) oder ohne weitere Symptome aufhören.

\section{Literatur}

\section{Migräne allgemein}

Arruda MA, Guidetti V, Galli F, Albuquerque RC, Bigal ME. Primary headaches in childhood - a population-based study. Cephalalgia 2010; 30: 1056-1064.

Diener HC, Silberstein SD. Medication overuse headache. In: Olesen J, Goadsby PJ, Ramadan NM, Tfelt-Hansen P, Welch KM (eds). The Headaches, 3 rd ed. Philadelphia: Lippincott Williams \& Wilkins 2006; 971-979.

GBD 2015 Disease and Injury Incidence and Prevalence Collaborators. Global, regional, and national incidence, prevalence, and years lived with disability for 310 diseases and injuries, 1990-2015: a systematic analysis for the Global Burden of Disease Study 2015. Lancet 2016; 388: 1545-1602.

Gelfand AA, Fullerton HJ, Goadsby PJ. Child neurology: migraine with aura in children. Neurology 2010; 75(5): e16-e19.

Goadsby PJ. Recent advances in the diagnosis and management of migraine. BMJ 2006; 332: 25-29.

Goadsby PJ. Migraine pathophysiology. Headache 2005; 45 Suppl 1: S14-S24.

Katsarava Z, Manack A, Yoon MS, Obermann M, Becker H, Dommes P, et al. Chronic migraine: classification and comparisons. Cephalalgia 2011; 31: $520-529$.
Lipton RB, Bigal ME, Steiner TJ, Silberstein SD, Olesen J. Classification of primary headaches. Neurology 2004; 63: 427-435.

Martelletti P, Haimanot RT, Lainez MJ, Rapoport AM, Ravishankar K, Sakai F, Silberstein SD, Vincent M, Steiner TJ. The Global Campaign (GC) to Reduce the Burden of Headache Worldwide. The International Team for Specialist Education (ITSE). J Headache Pain 2005; 6: 261-263.

Silberstein SD. Migraine. Lancet 2004; 363: 381-391.

Vetvik KG, Macgregor EA, Lundqvist C, Russell MB. Prevalence of menstrual migraine: a population-based study. Cephalalgia 2014; 34: 280-288.

Vetvik KG, Benth JŠ, MacGregor EA, Lundqvist C, Russell MB. Menstrual versus non-menstrual attacks of migraine without aura in women with and without menstrual migraine. Cephalalgia 2015; 35: 1261-1268.

Vos T, Flaxman AD, Naghavi M, Lozano R, Michaud C, Ezzati M et al. Years lived with disability (YLD) for 1160 sequelae of 289 diseases and injuries 1990-2010: a systematic analysis for the global burden of disease study 2010. Lancet 2012; 380: 2163-2196.

\subsection{Migräne mit Aura}

Cao Y, Welch KM, Aurora S, et al. Functional MRI-BOLD of visually triggered headache in patients with migraine. Arch Neurol 1999; 56: 548-554.

Charles A, Brennan K. Cortical spreading depression - new insights and persistent questions. Cephalalgia 2009; 29: 1115-1124.

Cologno D, Torelli P, Manzoni GC. Migraine with aura: a review of 81 patients at 10-20 years' follow-up. Cephalalgia 1998; 18: 690-696.

Cutrer FM, Sorensen AG, Weisskoff RM, et al. Perfusion-weighted imaging defects during spontaneous migrainous aura. Ann Neurol 1998; 43: 25-31.

Eriksen MK, Thomsen LL, Andersen I, et al. Clinical characteristics of 362 patients with familial migraine with aura. Cephalalgia 2004; 24: 564-575.

Eriksen MK, Thomsen LL, Olesen J. The Visual Aura Rating Scale (VARS) for migraine aura diagnosis. Cephalalgia 2005; 25: 801-810.

Giffin NJ, Lipton RB, Silberstein SD, Olesen J, Goadsby PJ. The migraine postdrome: An electronic diary study. Neurology 2016; 87: 309-313.

Hadjikhani N, Sanchez del Rio M, Wu O, et al. Mechanisms of migraine aura revealed by functional MRI in human visual cortex. PNAS 2001; 98 : 4687-4692.

Hansen JM, Lipton R, Dodick D, et al. Migraine headache is present in the aura phase - a prospective study. Neurology 2012; 79: 2044-2049.

Jensen $\mathrm{K}$, Tfelt-Hansen P, Lauritzen M, et al. Classic migraine. A prospective recording of symptoms. Acta Neurol Scand 1986; 73: 359-362.

Kallela M, Wessman M, Farkkila M, et al. Clinical characteristics of migraine in a population-based twin sample: similarities and differences between migraine with and without aura. Cephalalgia 1999; 19: 151-158.

Kelman L. The premonitory symptoms (prodrome): A tertiary care study of 893 migraineurs. Headache 2004; 44: 865-872.

Lauritzen M. Pathophysiology of the migraine aura. The spreading depression theory. Brain 1994; 117 (Pt 1): 199-210.

Leao AAP. Spreading depression of activity in the cerebral cortex. J Neurophysiol 1944; 7: 359-390.

Lebedeva ER, Gurary NM, Gilev DV, Olesen J. Prospective testing of ICHD-3 beta diagnostic criteria for migraine with aura and migraine with typical aura in patients with transient ischemic attacks. Cephalalgia 2018; 38: 561-567.

Li D, Christensen AF, Olesen J. Field-testing of the ICHD-3 beta/proposed ICD-11 diagnostic criteria for migraine with aura. Cephalalgia 2015; 35: 748-756.

Olesen J, Friberg L, Olsen TS, et al. Timing and topography of cerebral blood flow, aura, and headache during migraine attacks. Ann Neurol 1990; 28: 791-798. 
Queiroz LP, Friedman DI, Rapoport AM, et al. Characteristics of migraine visual aura in Southern Brazil and Northern USA. Cephalalgia 2011; 31: 1652-1658.

Rasmussen BK, Olesen J. Migraine with aura and migraine without aura: an epidemiological study. Cephalalgia 1992; 12: 221-228 (discussion 186).

Salhofer-Polanyi S, Frantal S, Brannath W, et al. Prospective analysis of factors related to migraine aura - The PAMINA Study. Headache 2012; 52: 1236-1245.

Schoonman GG, Evers DJ, Terwindt GM, et al. The prevalence of premonitory symptoms in migraine: a questionnaire study in 461 patients. Cephalalgia 2006; 26: 1209-1213.

Ulrich V, Gervil M, Kyvik KO, et al. Evidence of a genetic factor in migraine with aura: A population-based Danish twin study. Ann Neurol 1999; 45: 242-246.

\subsubsection{Migräne mit typischer Aura}

Eriksen MK, Thomsen LL, Olesen J. Implications of clinical subtypes of migraine with aura. Headache 2006; 46: 286-297.

Hansen JM, Goadsby PJ, Charles AC. Variability of clinical features in attacks of migraine with aura. Cephalalgia 2016; 36: 216-224.

Matharu MJ, Goadsby PJ. Post-traumatic chronic paroxysmal hemicrania (CPH) with aura. Neurology 2001; 56: 273-275.

Morrison DP. Abnormal perceptual experiences in migraine. Cephalalgia 1990; 10: 273-277.

Silberstein SD, Niknam R, Rozen TD, et al. Cluster headache with aura. Neurology 2000; 54: 219-221.

Viana M, Linde M, Sances G, Ghiotto N, Guaschino E, Allena M, Terrazzino S, Nappi G, Goadsby PJ, Tassorelli C. Migraine aura symptoms: duration, succession and temporal relationship to headache. Cephalalgia 2016; 36: 413-421.

Wijman CA, Wolf PA, Kase CS, et al. Migrainous visual accompaniments are not rare in late life: the Framingham Study. Stroke 1998; 29: 1539-1543.

\subsubsection{Migräne mit Hirnstammaura}

Ambrosini A, D'Onofrio M, Grieco GS, et al. Familial basilar migraine associated with a new mutation in the ATP1A2 gene. Neurology 2005; 65: 1826-1828.

Bickerstaff ER. Basilar artery migraine. Lancet 1961; i: 15.

Caplan LR. Migraine and vertebrobasilar ischemia. Neurology 1991; 41: 55-61.

Eriksen MK, Thomsen LL, Olesen J. Implications of clinical subtypes of migraine with aura. Headache 2006; 46: 286-297.

Kirchmann M, Thomsen LL, Olesen J. Basilar-type migraine: clinical, epidemiologic, and genetic features. Neurology 2006; 66: 880-886.

Lempert T, Neuhauser H, Daroff RB. Vertigo as a symptom of migraine. Ann NY Acad Sci 2009; 1164: 242-251.

Li D, Christensen AF, Olesen J. Field-testing of the ICHD-3 beta/proposed ICD-11 diagnostic criteria for migraine with aura. Cephalalgia 2015; 35: 748-756.

Sturzenegger $\mathrm{MH}$, Meienberg O. Basilar artery migraine: a follow-up study of 82 cases. Headache 1985; 25: 408-415.

Swanson JW, Vick NA. Basilar artery migraine 12 patients, with an attack recorded electroencephalographically. Neurology 1978; 28: 782-786.

Thomsen LL, Eriksen MK, Roemer SF, et al. A population-based study of familial hemiplegic migraine suggests revised diagnostic criteria. Brain 2002; 125: 1379-1391.

Thomsen LL, Ostergaard E, Olesen J, et al. Evidence for a separate type of migraine with aura: sporadic hemiplegic migraine. Neurology 2003; 60: 595-601.

\subsubsection{Hemiplegische Migräne}

Ambrosini A, D’Onofrio M, Grieco GS, et al. Familial basilar migraine associated with a new mutation in the ATP1A2 gene. Neurology 2005; 65: 1826-1828.

De Fusco M, Marconi R, Silvestri L, et al. Haploinsufficiency of ATP1A2 encoding the $\mathrm{Na}+/ \mathrm{K}+$ pump alpha2 subunit associated with familial hemiplegic migraine type 2. Nat Genet 2003; 33: 192-196.

de Vries B, Frants RR, Ferrari M, et al. Molecular genetics of migraine. Human Genet 2009; 126: 115-132.

Dichgans M, Freilinger T, Eckstein G, et al. Mutation in the neuronal voltage-gated sodium channel SCN1A in familial hemiplegic migraine. Lancet 2005; 366: 371-377.

Dreier JP, Jurkat-Rott K, Petzold GC, et al. Opening of the blood-brain barrier preceding cortical edema in a severe attack of FHM type II. Neurology 2005; 64: 2145-2147.

Eriksen MK, Thomsen LL, Olesen J. Implications of clinical subtypes of migraine with aura. Headache 2006; 46: 286-297.

Hansen JM, Schytz HW, Larsen VA, et al. Hemiplegic migraine aura begins with cerebral hypoperfusion: imaging in the acute phase. Headache 2011; 51: 1289-1296.

Hansen JM, Thomsen LL, Olesen J, et al. Coexisting typical migraine in familial hemiplegic migraine. Neurology 2010; 74: 594-600.

lizuka T, Takahashi Y, Sato M, et al. Neurovascular changes in prolonged migraine aura in FHM with a novel ATP1A2 gene mutation. J Neurol Neurosurg Psychiat 2012; 83: 205-212.

Jurkat-Rott K, Freilinger T, Dreier JP, et al. Variability of familial hemiplegic migraine with novel A1A2 Na + / + - ATPase variants. Neurology 2004; 62: 1857-1861.

Kirchmann M, Thomsen LL, Olesen J. Basilar-type migraine: clinical, epidemiologic, and genetic features. Neurology 2006; 66: 880-886.

Leo L, Gherardini L, Barone V, et al. Increased susceptibility to cortical spreading depression in the mouse model of familial hemiplegic migraine type 2. PloS Genet 2011; 7: e1002129.

Thomsen LL, Eriksen MK, Roemer SF, et al. A population-based study of familial hemiplegic migraine suggests revised diagnostic criteria. Brain 2002; 125: 1379-1391.

Thomsen LL, Kirchmann M, Bjornsson A, et al. The genetic spectrum of a population-based sample of familial hemiplegic migraine. Brain 2007; 130: 346-356.

Thomsen LL, Ostergaard E, Olesen J, et al. Evidence for a separate type of migraine with aura: sporadic hemiplegic migraine. Neurology 2003; 60: 595-601.

Vanmolkot KRJ, Kors EE, Turk U, et al. Two de novo mutations in the $\mathrm{Na}, \mathrm{K}$-ATPase gene ATP1A2 associated with pure familial hemiplegic migraine. Eur J Human Genet 2006; 14: 555-560.

\subsubsection{Retinale Migräne}

Carroll D. Retinal migraine. Headache 1970; 10: 9-13.

Chronicle EP, Mulleners WM. Visual system dysfunction in migraine: a review of clinical and psychophysical findings. Cephalalgia 1996; 16: 525-535.

Hedges TR. Isolated ophthalmic migraine in the differential diagnosis of cerebro-ocular ischemia. Stroke 1976; 7: 379-381.

Grosberg BM, Solomon S, Friedman DI, Lipton RB. Retinal migraine reappraised. Cephalalgia 2006; 26: 1275-1286.

Hill DL, Daroff RB, Ducros A, Newman NJ, Biousse V. Most cases labeled as "retinal migraine" are not migraine. J Neuroophthalmol 2007; 27: 3-8.

Martin TJ, Corbett J]. Disorders of the eye. In Silberstein SD, Lipton RB, Dalessio DJ, editors. Wolff's Headache and Other Head Pain. New York: Oxford University Press 2001; 459-474. 
Troost T, Zagami AS. Ophthalmoplegic migraine and retinal migraine. In Olesen J, Tfelt-Hansen P, Welch KMA, editors. The Headaches. Philadelphia: Lippincott Williams \& Wilkins 2000; 511-516.

\subsection{Chronische Migräne}

Aurora SK. Is chronic migraine one end of a spectrum of migraine or a separate entity? Cephalalgia 2009; 29: 597-605.

Bigal ME, Lipton RB. Concepts and mechanisms of migraine chronification. Headache 2008; 48: 7-15.

Bigal M, Rapoport A, Sheftell F, Tepper S, Lipton R. The International Classification of Headache Disorders revised criteria for chronic migraine - field testing in a headache specialty clinic. Cephalalgia 2007; 27: 230-234.

Bigal ME, Serrano D, Reed M, Lipton RB. Chronic migraine in the population: burden, diagnosis, and satisfaction with treatment. Neurology 2008; 71: 559-566.

Bigal ME, Sheftell FD, Rapoprt AM, Lipton RB, Tepper SJ. Chronic daily headache in a tertiary care population: correlation between the International Headache Society diagnostic criteria and proposed revisions of criteria for chronic daily headache. Cephalalgia 2002; 22: 432-438.

Bloudek LM, Stokes M, Buse DC, Wilcox TK, Lipton RB, Goadsby PJ, Varon SF, Blumenfeld AM, Katsarava Z, Pascual J, Lanteri-Minet M, Cortelli P, Martelletti P. Cost of healthcare for patients with migraine in five European countries: results from the International Burden of Migraine Study (IBMS). J Headache Pain 2012; 13: 361-378.

Buse DC, Manack AN, Fanning KM, Serrano D, Reed ML, Turkel CC, Lipton RB. Chronic migraine prevalence, disability, and sociodemographic factors. Results from the American Migraine Prevalence and Prevention Study. Headache 2012; 52: 1456-1470.

Buse D, Manack A, Serrano D, Reed M, Varon S, Turkel C, Lipton R. Headache impact of chronic and episodic migraine: results from the American Migraine Prevalence and Prevention study. Headache 2012; 52: 3-17.

Diamond S. A view of chronic daily headache. Headache Quart 2000; 11: 177.

Diener HC, Dodick DW, Goadsby PJ, Lipton RB, Olesen J, Silberstein SD. Chronic migraine - classification, characteristics and treatment. Nat Rev Neurol 2012; 8: 162-171.

Goadsby PJ, Hargreaves R. Refractory migraine and chronic migraine: pathophysiological mechanisms. Headache 2008; 48: 799-804.

Katsarava Z, Manack A, Yoon MS, Obermann M, Becker H, Dommes P, Turkel C, Lipton RB, Diener HC. Chronic migraine: classification and comparisons. Cephalalgia 2011; 31: 520-529.

Manzoni GC, Bonavita V, Bussone G, Cortelli P, Narbone MC, Cevoli S, D’Amico D, De Simone R, Torelli P; ANIRCEF (Associazione Neurologica Italiana Ricerca Cefalee). Chronic migraine classification: current knowledge and future perspectives. J. Headache Pain 2001; 12: 585-592.

Mathew NT, Stubits E, Nigam MR. Transformation of migraine into daily headache: analysis of factors. Headache 1982; 22: 66-68.

Mathew NT, Stubits E, Nigam MP. Transformed or evolutive migraine. Headache 1987; 27: 102-106.

Natoli JL, Manack A, Dean B, Butler Q, Turkel CC, Stovner L, Lipton RB. Global prevalence of chronic migraine: a systematic review. Cephalalgia 2010; 30: 599-609.

Scher Al, Stewart WF, Liberman J, Lipton RB. Prevalence of frequent headache in a population sample. Headache 1998; 38: 497-506.

Scher Al, Stewart WF, Ricci JA, Lipton RB. Factors associated with the onset and remission of chronic daily headache in a population-based study. Pain 2003; 106: 81-89.

Silberstein SD, Lipton RB, Sliwinski M. Classification of daily and near-daily headaches: field trial of revised IHS criteria. Neurology 1996; 47: 871-875.
Silberstein SD, Lipton RB, Solomon S, Mathew NT. Classification of daily and near-daily headaches: proposed revisions to the IHS criteria. Headache 1994; 34: 1-7.

Stewart WF, Scher AI, Lipton RB. Stressful life events and risk of chronic daily headache: results from the frequent headache epidemiology study. Cephalalgia 2001; 21: 279.

Yalın OÖ, Uluduz D, Özge A, Sungur MA, Selekler M, Siva A. Phenotypic features of chronic migraine. J Headache Pain 2016; 17: 26.

Zeeberg P, Olesen J, Jensen R. Probable medication-overuse headache: the effect of a 2-month drug-free period. Neurology 2006; 66: 18941898.

Zeeberg P, Olesen J, Jensen R. Medication overuse headache and chronic migraine in a specialized headache centre: field-testing proposed new Anhang criteria. Cephalalgia 2009; 29: 214-220.

\subsubsection{Status migränosus}

Akhtar ND, Murray MA, Rothner AD. Status migrainosus in children and adolescents. Semin Pediatr Neurol 2001; 8: 27-33.

Beltramone M, Donnet A. Status migrainosus and migraine aura status in a French tertiary-care center: An 11-year retrospective analysis. Cephalalgia 2014; 34: 633-637.

Couch JR, Diamond S. Status migrainosus. Causative and therapeutic aspects. Headache 1983; 23: 94-101.

Cure J, Rothrock J. Prolonged status migrainosus complicated by cerebellar infarction. Headache 2007; 47: 1091-1092.

Gentile S, Rainero I, Daniele D, Binello E, Valfrè W, Pinessi L. Reversible MRI abnormalities in a patient with recurrent status migrainosus. Cephalalgia 2009; 29: 687-690.

Lanfranconi S, Corti S, Bersano A, Costa A, Prelle A, Sciacco M, Bresolin N, Ghione I. Aphasic and visual aura with increased vasogenic leakage: an atypical migrainosus status. J Neurol Sci 2009; 285: 227-229.

Perucca P, Terzaghi M, Manni R. Status epilepticus migrainosus: clinical, electrophysiologic, and imaging characteristics. Neurology 2010; 75: 373-374.

Raskin NH. Treatment of status migrainosus: the American experience. Headache 1990; 30 Suppl 2: 550-553.

\subsubsection{Anhaltende Aura ohne Hirninfarkt}

Ambrosini A, de Noordhout AM, Schoenen J. Neuromuscular transmission in migraine patients with prolonged aura. Acta Neurol Belg 2001; 101: 166-170.

Bento MS, Esperanca P. Migraine with prolonged aura. Headache 2000; 40: 52-53.

Chen WT, Fuh JL, Lu SR, Wang SJ. Persistent migrainous visual phenomena might be responsive to lamotrigine. Headache 2001; 41: 823-825.

Chen WT, Lin YY, Fuh JL, Hämäläinen MS, Ko YC, Wang SJ. Sustained visual cortex hyperexcitability in migraine with persistent visual aura. Brain 2011; 134 (Pt 8): 2387-2395.

Evans RW, Lay CL. A persistent migraine aura. Headache 2000; 40: 696-698.

Haan J, Sluis P, Sluis IH, Ferrari MD. Acetazolamide treatment for migraine aura status. Neurology 2000; 55: 1588-1589.

Haas DC. Prolonged migraine aura status. Ann Neurol 1982; 11: 197-199.

Lebedeva ER, Gurary NM, Gilev DV, Olesen J. Prospective testing of ICHD-3 beta diagnostic criteria for migraine with aura and migraine with typical aura in patients with transient ischemic attacks. Cephalalgia 2018; 38: 561-567.

Liu GT, Schatz N], Galetta SK et al. Persistent positive visual phenomena in migraine. Neurology 1995; 45: 664-668.

Luda E, Bo E, Sicuro L et al. Sustained visual aura: a totally new variation of migraine. Headache 1991; 31: 582-583. 
Relja G, Granato A, Ukmar M, Ferretti G, Antonello RM, Zorzon M. Persistent aura without infarction: decription of the first case studied with both brain SPECT and perfusion MRI. Cephalalgia 2005; 25: 56-59.

Rothrock JF. Successful treatment of persistent migraine aura with divalproex sodium. Neurology 1997; 48: 261-262.

San-Juan OD, Zermeño PF. Migraine with persistent aura in a Mexican patient: case report and review of the literature. Cephalalgia 2007; 27: 456-460.

Smith M, Cros D, Sheen V. Hyperperfusion with vasogenic leakage by fMRI in migraine with prolonged aura. Neurology 2002; 58: 1308-1310.

Wang YF, Fuh JL, Chen WT, Wang SJ. The visual aura rating scale as an outcome predictor for persistent visual aura without infarction. Cephalalgia 2008; 28: 1298-1304.

\subsubsection{Migränöser Infarkt}

Bono G, Minonzio G, Mauri M, Clerici AM. Complications of migraine: migrainous infarction. Clin Exp Hypertens 2006; 28: 233-242.

Bousser MG, Conard J, Kittner $S$ et al. Recommendations on the risk of ischemic stroke associated with use of combined oral contraceptives and hormone replacement therapy in women with migraine. The International Headache Society Task Force on Combined Oral Contraceptives \& Hormone Replacement Therapy. Cephalalgia 2000; 20: 155-156.

Chang $\mathrm{CL}$, Donaghy M, Poulter N. Migraine and stroke in young women: case-control study. The World Health Organization Collaborative Study of Cardiovascular Disease and Steroid Hormone Contraception. BM] 1999; 318: 13-18.

Connor CCR. Complicated migraine. A study of permanent neurological and visual defects. Lancet 1962; ii: 1072-1075.

Laurell K, Artto V, Bendtsen L, Hagen K, Kallela M, Meyer EL, Putaala J, Tronvik E, Zwart JA, Linde M. Migrainous infarction: a Nordic multicenter study. Eur J Neurol 2011; 18: 1220-1226.

MacGregor EA, Guillebaud J. Combined oral contraceptives, migraine and ischemic stroke. Clinical and Scientific Committee of the Faculty of Family Planning and Reproductive Health Care and the Family Planning Association. Br J Fam Plann 1998; 24: 55-60.

Olesen J, Friberg L, Olsen TS, Andersen AR, Lassen NA, Hansen PE et al. Ischaemia-induced (symptomatic) migraine attacks may be more frequent than migraine induced ischemic insults. Brain 1993; 116: 187-202.

Rothrock JF, Walicke P, Swenson MR et al. Migrainous stroke. Arch Neurol 1988; 45: 63-67.

Schulz UG, Blamire AM, Davies P, Styles P, Rothwell PM. Normal cortical energy metabolism in migrainous stroke: A 31P-MR spectroscopy study. Stroke. 2009; 40: 3740-3744.

Tietjen GE. The relationship of migraine and stroke. Neuroepidemiology 2000; 19: 13-19.

Tzourio C, Kittner SJ, Bousser MG et al. Migraine and stroke in young women. Cephalalgia 2000; 20: 190-199.

Vollbracht S, Robbins MS, Kister I. Classification challenge in migrainous infarction. Headache 2014; 54: 170-171.

Wolf ME, Szabo K, Griebe M, Förster A, Gass A, Hennerici MG, Kern R. Clinical and MRI characteristics of acute migrainous infarction. Neurology 2011; 76: 1911-1917.

1.4.4 Epileptische Anfälle, durch Migräneaura getriggert

Belcastro V, Striano P, Kasteleijn-Nolst Trenité DG, Villa MP, Parisi P. Migralepsy, hemicrania epileptica, post-ictal headache and "iktaler Kopfschmerz bei Epilepsie": a proposal for terminology and classification revision. J Headache Pain 2011; 12: 289-294.

Davies PT, Panayiotopoulos CP. Migraine triggered seizures and epilepsy triggered headache and migraine attacks: a need for re-assessment. J Headache Pain 2011; 12: 287-288.
Friedenberg S, Dodick DW. Migraine-associated seizure: a case of reversible MRI abnormalities and persistent nondominant hemisphere syndrome. Headache 2000; 40: 487-490.

Maggioni F, Mampreso E, Ruffatti S, Viaro F, Lunardelli V, Zanchin G. Migralepsy: is the current definion too narrow? Headache 2008; 48: 1129-1132.

Marks DA, Ehrenberg BL. Migraine-related seizures in adults with epilepsy, with EEG correlation. Neurology 1993; 43: 2476-2483.

Merlino G, Valente MR, D’Anna S, Gigli GL. Seizures with prolonged EEC abnormalities during an attack of migraine without aura. Headache 2007; 47: 919-922.

Parisi P, Kasteleijn-Nolst Trenitè DGA. "Migralepsy”: a call for revision of the definition. Epilepsia 2010; 51: 932-933.

Rogawski MA. Common pathophysiologic mechanisms in migraine and epilepsy. Arch Neurol 2008; 65: 709-714.

Sances G, Guaschino E, Perucca P, Allena M, Ghiotto N, Manni R. Migralepsy: a call for revision of the definition. Epilepsia 2009; 50: 2487-2496.

Ter Berg HW. Migraine-associated seizure: a case of reversible MRI abnormalities and persistent nondominant hemisphere syndrome. Headache 2001; 41: 326-328.

Velioglu SK, Ozmenoglu M. Migraine-related seizures in an epileptic population. Cephalalgia 1999; 19: 797-801.

Verrotti A, Coppola G, Di Fonzo A, Tozzi E, Spalice A, Aloisi P, Bruschi R, lannetti P, Villa MP, Parisi P. Should "migralepsy" be considered an obsolete concept? A multicenter retrospective clinical/EEG study and review of the literature. Epilepsy Behav 2011; 21: 52-59.

\subsection{Wahrscheinliche Migräne}

Granella F, Alessandro RD, Manzoni GC et al. International Headache Society classification: interobserver reliability in the diagnosis of primary headaches. Cephalalgia 1994; 14: 16-20.

Rains JC, Penzien DB, Lipchik GL et al. Diagnosis of migraine: empirical analysis of a large clinical sample of atypical migraine (IHS 1.7) patients and proposed revision of the IHS criteria. Cephalalgia 2001, 21: 584-595.

Rasmussen BK, Jensen R, Olesen J. A population-based analysis of the diagnostic criteria of the International Headache Society. Cephalalgia 1991; 11: 129-134.

Russell MB, Olesen J. Migrainous disorder and its relation to migraine without aura and migraine with aura. A genetic epidemiological study. Cephalalgia 1996; 16: 431-435.

\subsubsection{Rezidivierende gastrointestinale Störungen}

Abu-Arafeh I, Russel G. Prevalence and clinical features of abdominal migraine compared with those of migraine headache. Arch Dis Child 1995; 72: 413-417.

Al-Twaijri WA, Shevell MI. Pediatric migraine equivalents: occurrence and clinical features in practice. Pediatr Neurol 2002; 26: 365-368.

Dignan F, Abu-Arafeh I, Russell G. The prognosis of childhood abdominal migraine. Arch Dis Child 2001; 84: 415-418.

Drossman DA, Dumitrascu DL. Rome III: New standard for functional gastrointestinal disorders. J Gastrointestin Liver Dis 2006; 15: 237-241.

Farquar HA. Abdominal migraine in children. BMJ 1956; i: 1082-1085.

Fleisher DR. Cyclic vomiting syndrome and migraine. J Pediatr 1999; 134: 533-535.

Haan J, Kors EE, Ferrari MD. Familial cyclic vomiting syndrome. Cephalalgia 2002; 22: 552-554.

Li BU. Cyclic vomiting syndrome: age-old syndrome and new insights. Semin Pediatr Neurol 2001; 8: 13-21.

Li BUK, Lefevre F, Chelimsky GG, Boles RG, Nelson SP, Lewis DW, Linder SL, Issenman RM, Rudolph CD. NASPGHAN Consensus Statement on the Diagnosis and Management of CVS. J Pediatr Gastroenterol Nutr 2008; 47: 379-393. 
Rashed H, Abell TL, Familoni BO et al. Autonomic function in cyclic vomiting syndrome and classic migraine. Dig Dis Sci 1999; 44 Suppl 8: 74S-78S.

Russell G, Abu-Arafeh I, Symon DN. Abdominal migraine: evidence for existence and treatment options. Paediatr Drugs 2002; 4: 1-8.

Thiessen PN. Recurrent abdominal pain. Pediatr Rev 2002; 23: 39-46.

Welch KM. Scientific basis of migraine: speculation on the relationship to cyclic vomiting. Dig Dis Sci 1999; 44 Suppl 8: 26S-30S.

\subsubsection{Gutartiger paroxysmaler Schwindel}

Drigo P, Carli G, Laverda AM. Benign paroxysmal vertigo of childhood. Brain Dev (Netherlands) 2001; 23: 38-41.

Dunn DW, Snyder CH. Benign paroxysmal vertigo of childhood. Am J Dis Child 1976; 130: 1099-1100.

Fenichel GM. Migraine as a cause of benign paroxysmal vertigo of childhood. J Pediatr 1967; 71: 114-115.

1.6.3 Gutartiger paroxysmaler Torticollis

Drigo, P., G. Carli, et al. Benign paroxysmal torticollis of infancy. Brain Dev 2000; 22: 169-172.

Giffin NJ, Benton S, et al. Benign paroxysmal torticollis of infancy: four new cases and linkage to CACNA1A mutation. Dev Med Child Neurol 2002; 44: 490-493.

Rosman NP, Douglass LM, et al. The neurology of benign paroxysmal torticollis of infancy: report of 10 new cases and review of the literature. J Child Neurol 2009; 24: 155-160.

\section{Kopfschmerz vom Spannungstyp}

\begin{tabular}{|c|c|}
\hline 2.1 & $\begin{array}{l}\text { Selten auftretender episodischer Kopfschmerz vom } \\
\text { Spannungstyp }\end{array}$ \\
\hline 2.1 .1 & $\begin{array}{l}\text { Selten auftretender episodischer Kopfschmerz vom } \\
\text { Spannungstyp mit perikranieller Schmerzempfindlichkeit }\end{array}$ \\
\hline 2.1 .2 & $\begin{array}{l}\text { Selten auftretender episodischer Kopfschmerz vom } \\
\text { Spannungstyp ohne perikranielle Schmerzempfindlichkeit }\end{array}$ \\
\hline 2.2 & $\begin{array}{l}\text { Häufig auftretender episodischer Kopfschmerz vom } \\
\text { Spannungstyp }\end{array}$ \\
\hline 2.2 .1 & $\begin{array}{l}\text { Häufig auftretender episodischer Kopfschmerz vom } \\
\text { Spannungstyp mit perikranieller Schmerzempfindlichkeit }\end{array}$ \\
\hline 2.2 .2 & $\begin{array}{l}\text { Häufig auftretender episodischer Kopfschmerz vom } \\
\text { Spannungstyp ohne perikranielle Schmerzempfindlichkeit }\end{array}$ \\
\hline 2.3 & Chronischer Kopfschmerz vom Spannungstyp \\
\hline 2.3.1 & $\begin{array}{l}\text { Chronischer Kopfschmerz vom Spannungstyp mit peri- } \\
\text { kranieller Schmerzempfindlichkeit }\end{array}$ \\
\hline 2.3 .2 & $\begin{array}{l}\text { Chronischer Kopfschmerz vom Spannungstyp ohne peri- } \\
\text { kranielle Schmerzempfindlichkeit }\end{array}$ \\
\hline 2.4 & Wahrscheinlicher Kopfschmerz vom Spannungstyp \\
\hline 2.4.1 & $\begin{array}{l}\text { Wahrscheinlicher selten auftretender, episodischer Kopf- } \\
\text { schmerz vom Spannungstyp }\end{array}$ \\
\hline 2.4 .2 & $\begin{array}{l}\text { Wahrscheinlicher häufig auftretender episodischer Kopf- } \\
\text { schmerz vom Spannungstyp }\end{array}$ \\
\hline 2.4 .3 & $\begin{array}{l}\text { Wahrscheinlicher chronischer Kopfschmerz vom } \\
\text { Spannungstyp }\end{array}$ \\
\hline
\end{tabular}

Früher benutzte Begriffe: Spannungskopfschmerz, Muskelkontraktionsschmerz, psychomyogener Kopfschmerz, Stresskopfschmerz, gemeiner Kopfschmerz, essenzieller Kopfschmerz, idiopathischer Kopfschmerz, psychogener Kopfschmerz.
An anderer Stelle kodiert: Ähnliche Kopfschmerzen wie die vom Spannungstyp als sekundäre Folge einer anderen Erkrankung werden entsprechend dieser anderen Erkrankung kodiert.

Primärer und/oder sekundärer Kopfschmerz?

Je nach Umstand gelten für den Kopfschmerz vom Spannungstyp drei Regeln:

1. Tritt ein neuer Kopfschmerz mit den Charakteristika eines Kopfschmerzes vom Spannungstyp zum ersten Mal in zeitlichem Zusammenhang mit einer anderen Erkrankung auf, von der bekannt ist, dass sie Kopfschmerzen hervorrufen kann oder dieser erfüllt sonstige Kriterien, die für eine Verursachung durch diese andere Erkrankung sprechen, wird dieser neu aufgetretene Kopfschmerz als sekundärer Kopfschmerz zurückzuführen auf die ursächliche Erkrankung kodiert.

2. Wenn aber ein vorbestehender Kopfschmerz vom Spannungstyp im engen zeitlichen Zusammenhang mit einer solchen Erkrankung chronisch wird, sollten beide Diagnosen, die ursprüngliche eines Kopfschmerzes vom Spannungstyp und die sekundäre Kopfschmerzdiagnose, vergeben werden.

3. Wenn ein vorbestehender Kopfschmerz vom Spannungstyp sich deutlich verschlechtert (üblicherweise wird hierunter eine Verdopplung der Häufigkeit und/oder der Intensität verstanden) und dies im engen zeitlichen Zusammenhang mit einer solchen ursächlichen Erkrankung auftritt, wird sowohl der vorbestehende Kopfschmerz vom Spannungstyp als auch der sekundäre Kopfschmerz diagnostiziert, wenn es Anhalt dafür gibt, dass diese Erkrankung Kopfschmerzen hervorrufen kann.

Besteht ein chronischer Kopfschmerz vom Spannungstyp und ein Medikamentenübergebrauch, ist der zeitliche Zusammenhang häufig schwer nachzuweisen. Daher werden in solchen Fällen jeweils beide Diagnosen, 2.3 chronischer Kopfschmerz vom Spannungstyp und 8.2 Kopfschmerz zurückzuführen auf einen Medikamentenübergebrauch vergeben.

\section{Einleitung}

2. Kopfschmerz vom Spannungstyp ist sehr verbreitet, die Lebenszeitprävalenz in der Allgemeinbevölkerung reicht verschiedenen Studien zufolge von $30 \%$ bis $78 \%$. Der Kopfschmerz hat eine hohe sozio-ökonomische Bedeutung.

Auch wenn dieser Kopfschmerz früher als primär psychogen eingeordnet wurde, legt eine Reihe von Studien nach der Publikation der ICHD-1 den Schluss nahe, dass zumindest für schwerere Subtypen des Kopfschmerzes vom Spannungstyp eine neurobiologische Grundlage besteht.

Die Aufteilung des 2. Kopfschmerzes vom Spannungstyp in einen episodischen und einen chronischen Verlaufstyp, welche durch die ICHD-1 vorgenommen wurde, hat sich als ausgesprochen nützlich erwiesen. In der ICHD-2 wurde die episodische Variante noch unterteilt in einen selten auftretenden Subtyp mit Kopfschmerzepisoden, die weniger als einmal im Monat auftreten und einen Subtyp mit häufig auftretenden episodischen Kopfschmerzen vom Spannungstyp. Ein 2.2 häufig auftretender episodischer Kopfschmerz vom Spannungstyp kann mit ausgeprägter Beeinträchtigung einhergehen und gelegentlich eine aufwändige medikamen- 
töse Therapie notwendig machen. Im Gegensatz dazu hat der 2.1 selten auftretende episodische Kopfschmerz vom Spannungstyp, der in der Allgemeinbevölkerung weit verbreitet ist, üblicherweise wenig Beeinträchtigung zur Folge und benötigt meist keine intensive medizinische Evaluation. Die Abgrenzung eines 2.1 selten auftretenden Kopfschmerzes vom Spannungstyp von einem 2.2 häufig auftretenden Kopfschmerz vom Spannungstyp erfasst Individuen, die üblicherweise keine medizinische Behandlung benötigen als eigene Kategorie und verhindert, dass fast die gesamte Bevölkerung eine erhebliche Kopfschmerzerkrankung diagnostiziert bekommt - während es gleichzeitig eine Klassifikation ihrer Kopfschmerzen erlaubt. 2.3 chronischer Kopfschmerz vom Spannungstyp ist eine ernsthafte Erkrankung, die eine erhebliche Einschränkung der Lebensqualität und Beeinträchtigung mit sich bringt.

Die exakten Pathomechanismen bei einem 2. Kopfschmerz vom Spannungstyp sind unbekannt. Periphere Schmerzmechanismen scheinen insbesondere bei einem 2.1 selten auftretenden episodischen Kopfschmerz vom Spannungstyp und einem 2.2 häufig auftretenden episodischen Kopfschmerz vom Spannungstyp relevant zu sein, wohingegen bei 2.3 chronischem Kopfschmerz vom Spannungstyp zentrale Schmerzmechanismen eine bedeutendere Rolle spielen. Erhöhte perikranielle Druckempfindlichkeit bei der manuellen Palpation ist der am häufigsten auffällige Befund bei Patienten mit 2. Kopfschmerz vom Spannungstyp: Sie besteht in der Regel auch zwischen den Attacken, nimmt im Verlauf mit Zunahme der Schmerzintensität und mit der Kopfschmerzhäufigkeit eher zu. Die erhöhte Druckschmerzhaftigkeit dürfte pathophysiologisch von Bedeutung sein. Deshalb unterschied die ICHD-2 zwischen Patienten mit und ohne eine solche Störung der perikraniellen Muskeln - eine Untergliederung, die auch in der ICHD-3 beibehalten wurde, um weitere Forschung auf diesem Gebiet anzuregen.

Das Bestehen einer perikraniellen Druckempfindlichkeit lässt sich durch manuelle Palpation leicht feststellen. Mit kleinen kreisenden Bewegungen des Zeige- und Mittelfingers und durch Ausübung von festem Druck (am besten mit Hilfe eines Palpometers) lässt sich die lokale Druckempfindlichkeit für die Mm. frontalis, temporalis, masseter, pterygoideus, sternocleidomastoideus, splenius and trapezius auf einer Skala von 0 bis 3 ermitteln. Aus diesen Werten kann für jeden Patienten ein Summenscore gebildet werden, aus dem die Druckempfindlichkeit insgesamt hervorgeht. Maßnahmen dieser Art bieten eine nützliche Orientierung für die Therapie und zusätzliche Möglichkeiten, dem Patienten Zusammenhänge aufzuzeigen.

Die diagnostische Schwierigkeit bei den primären Kopfschmerzerkrankungen besteht in der Abgrenzung zwischen einem Kopfschmerz vom Spannungstyp und einer leichten Migräne ohne Aura. Dies wird dadurch noch erschwert, dass Patienten mit häufigen Kopfschmerzen häufig an beiden Krankheitsbildern leiden. Es wurde angeregt, die Diagnosekriterien für den 2. Kopfschmerz vom Spannungstyp stärker einzugrenzen, um die Migräne phänotypisch besser abgrenzen zu können. Kriterien hierfür wurden im Anhang der ICHD-2 als A2, Kopfschmerz vom Spannungstyp, vorgeschlagen. Gleichzeitig reduziert diese Erhöhung der Spezifität jedoch die Sensitivität der Diagnosekriterien und erhöht die Anzahl der Patienten, die lediglich unter 2.4 wahrscheinlicher Kopfschmerz vom Spannungstyp oder 1.5 wahrscheinliche Migräne klassifiziert werden. Das Ergebnis ist ein größerer Prozentsatz von Patienten, deren Kopfschmerz sich lediglich als ein 2.4 wahrscheinlicher Kopf- schmerz vom Spannungstyp oder eine 1.5 wahrscheinliche Migräne klassifizieren lässt. Angesichts dessen, dass bislang noch nicht gezeigt werden konnte, dass diese Veränderung von Vorteil wäre, verbleiben diese strengeren Diagnosekriterien im Anhang, sollten jedoch lediglich zu Forschungszwecken angewendet werden. Das Klassifikationskomitee empfiehlt den Vergleich zwischen Patienten, die jeweils nach diesen Kriterien diagnostiziert wurde - nicht nur zur Charakterisierung klinischer Merkmale, sondern auch, um Einblicke in die pathophysiologischen Mechanismen und das Ansprechen auf Behandlungen zu erhalten.

\subsection{Selten auftretender episodischer Kopfschmerz vom Spannungstyp}

Beschreibung:

Selten auftretender episodischer Kopfschmerz, der typischerweise beidseitig als Einengung oder Druck wahrgenommen wird, in seiner Intensität als leicht bis mittelstark eingeschätzt werden und zwischen Minuten und Tagen anhalten kann. Der Kopfschmerz nimmt bei körperlicher Routineaktivität nicht zu und ist nicht begleitet von Übelkeit, jedoch Licht- oder Geräuschempfindlichkeit können vorhanden sein.

\section{Diagnostische Kriterien:}

A. Mindestens 10 Kopfschmerzattacken, die im Durchschnitt weniger als einmal im Monat auftreten (<12 Kopfschmerztage pro Jahr) und die Kriterien B bis D erfüllen.

B. Die Kopfschmerzdauer liegt zwischen 30 Minuten und 7 Tagen.

C. Der Kopfschmerz weist mindestens zwei der folgenden Charakteristika auf:

1. Beidseitige Lokalisation

2. Schmerzcharakter drückend oder beengend, nicht pulsierend

3. Leichte bis mittlere Schmerzintensität

4. Keine Verstärkung durch körperliche Routineaktivität wie Gehen oder Treppensteigen

D. Beide der folgenden Punkte sind erfüllt:

1. Fehlen von Übelkeit oder Erbrechen

2. Es darf entweder eine Photophobie oder eine Phonophobie, nicht jedoch beides vorhanden sein

E. Nicht besser erklärt durch eine andere ICHD-3-Diagnose1.

Anmerkung:

1. Erfüllt der Kopfschmerz sowohl die Kriterien für eine 1.5 wahrscheinliche Migräne und einen 2.1 selten auftretenden episodischen Kopfschmerz vom Spannungstyp, sollte er gemäß der allgemeinen Regel, dass definitive Diagnosen immer Vorrang haben gegenüber wahrscheinlichen, als 2.1 selten auftretender episodischer Kopfschmerz vom Spannungstyp (oder als der entsprechende Subtyp davon, für den die Kriterien erfüllt sind) kodiert werden. 
2.1.1 Selten auftretender episodischer Kopfschmerz vom Spannungstyp mit perikranieller Schmerzempfindlichkeit

Diagnostische Kriterien:

A. Kopfschmerzepisoden, die Kriterien für einen 2.1 selten auftretenden episodischen Kopfschmerz vom Spannungstyp erfüllen

B. Erhöhte perikranielle Schmerzempfindlichkeit bei manueller Palpation.

2.1.2 Selten auftretender episodischer Kopfschmerz vom Spannungstyp ohne perikranielle Schmerzempfindlichkeit

Diagnostische Kriterien:

A. Kopfschmerzepisoden, die die Kriterien für einen 2.1 selten auftretenden episodischen Kopfschmerz vom Spannungstyp erfüllen

B. Keine erhöhte perikranielle Schmerzempfindlichkeit.

2.2 Häufig auftretender episodischer Kopfschmerz vom Spannungstyp

\section{Beschreibung:}

Häufig auftretende Kopfschmerzepisoden mit einer Dauer von Minuten bis Tagen. Der Schmerz ist typischerweise beidseits lokalisiert und von drückendem, beengenden Charakter. Er erreicht eine leichte bis mäßige Intensität und dauert Minuten bis Tage. Er verstärkt sich nicht durch körperliche Routineaktivitäten. Es besteht keine begleitende Übelkeit, aber Photophobie oder Phonophobie können vorhanden sein.

\section{Diagnostische Kriterien:}

A. Wenigstens 10 Kopfschmerzepisoden, die die Kriterien B bis D erfüllen und durchschnittlich an 1 bis 14 Tagen/Monat für $>3$ Monate ( $\geq 12$ und $<180$ Tage/Jahr) auftreten

B. Die Kopfschmerzdauer liegt zwischen 30 Minuten und 7 Tagen

C. Der Kopfschmerz weist mindestens 2 der folgenden vier Charakteristika auf:

1. beidseitige Lokalisation

2. Schmerzcharakter drückend oder beengend (nicht pulsierend

3. leichte bis mittlere Schmerzintensität

4. keine Verstärkung durch körperliche Routineaktivitäten wie Gehen oder Treppensteigen

D. Beide folgenden Punkte sind erfüllt:

1. keine Übelkeit oder Erbrechen

2. Photophobie oder Phonophobie, nicht jedoch beides kann vorhanden sein

E. Nicht besser erklärt durch eine andere ICHD-3-Diagnose1.

\section{Anmerkung:}

1. Erfüllt der Kopfschmerz sowohl die Kriterien für eine 1.5 wahrscheinliche Migräne und einen 2.2 häufig auftretenden episodischen Kopfschmerz vom Spannungstyp, sollte er unter 2.2 häufig auftretender episodischer Kopfschmerz vom Spannungstyp (oder als den Subtyp hiervon, für den die Kriterien erfüllt sind) kodiert werden, gemäß der allgemeinen Regel, dass definitive Diagnosen immer Vorrang gegenüber wahrscheinlichen Diagnosen haben.

\section{Kommentar:}

Ein 2.2 häufig vorkommender episodischer Kopfschmerz vom Spannungstyp tritt oft gemeinsam mit einer 1.1 Migräne ohne Aura auf. Beide sollten idealerweise in einem Kopfschmerztagebuch aufgezeichnet werden, da ihre Behandlung sich deutlich unterscheidet. Es ist daher von größter Wichtigkeit, Patienten zu schulen, zwischen diesen Kopfschmerztypen zu differenzieren, damit sie jeweils die richtige Behandlung wählen und um damit einen Medikamentenübergebrauch und die nachteiligen Folgen eines 8.2 Kopfschmerzes zurückzuführen auf einen Medikamentenübergebrauch zu verhindern.

\subsubsection{Häufig auftretender episodischer Kopfschmerz vom Spannungstyp mit perikranieller Schmerzempfind- lichkeit \\ Diagnostische Kriterien:}

A. Die Episoden erfüllen die Kriterien für einen 2.2 häufigen episodischen Kopfschmerz vom Spannungstyp

B. Erhöhte Schmerzempfindlichkeit der perikraniellen Muskulatur bei manueller Palpation.

2.2.2 Häufig auftretender episodischer Kopfschmerz vom Spannungstyp ohne perikranielle Schmerzempfindlichkeit

Diagnostische Kriterien:

A. Die Episoden erfüllen die Kriterien für einen 2.2 häufigen episodischen Kopfschmerz vom Spannungstyp

B. Keine erhöhte Schmerzempfindlichkeit der perikraniellen Muskulatur bei manueller Palpation.

\subsection{Chronischer Kopfschmerz vom Spannungstyp}

An anderer Stelle kodiert: 4.10 Neu aufgetretener täglicher Kopfschmerz.

\section{Beschreibung:}

Erkrankung, die sich aus einem episodischen Kopfschmerz vom Spannungstyp entwickelt und mit täglichen oder sehr häufigen Kopfschmerzepisoden mit einer Dauer von Stunden bis Tagen einhergeht oder kontinuierlich vorhanden ist. Der Schmerz ist typischerweise beiderseits lokalisiert und von drückendem, beengendem Charakter. Er erreicht eine leichte bis mittelstarke Intensität und verstärkt sich nicht durch körperliche Routineaktivitäten. Milde Übelkeit, Photophobie oder Phonophobie können vorhanden sein.

\section{Diagnostische Kriterien:}

A. Ein Kopfschmerz, der die Kriterien B bis D erfüllt, tritt an durchschnittlich $\geq 15$ Tagen/Monaten über $>3$ Monate ( $\geq 180$ Tage/Jahr) auf

B. Der Kopfschmerz hält für Stunden bis Tage an oder ist kontinuierlich vorhanden

C. Der Kopfschmerz weist mindestens 2 der folgenden Charakteristika auf:

1. Beidseitige Lokalisation 
2. Schmerzcharakter drückend oder beengend, nicht pulsierend

3. Leichte bis mittelstarke Schmerzintensität

4. Keine Verstärkung durch körperliche Routineaktivitäten wie Gehen oder Treppensteigen

D. Beide folgenden Punkte sind erfüllt:

1. höchstens eines ist vorhanden: leichte Übelkeit oder Photophobie oder Phonophobie

2. weder Erbrechen noch mittelstarke bis starke Übelkeit

E. Nicht besser erklärt durch eine andere ICHD-3-Diagnose 1;2;3.

Anmerkung:

Sowohl die Diagnose eines 2.3 chronischen Kopfschmerzes vom Spannungstyp als auch die einer 1.3 chronischen Migräne setzen voraus, dass diese an 15 oder mehr Tagen/Monaten aufgetreten sind. Bei einem 2.3 chronischen Kopfschmerz vom Spannungstyp muss der Kopfschmerz an mindestens 15 Tagen die Kriterien B bis D für einen 2.2 häufig auftretenden episodischen Kopfschmerz vom Spannungstyp erfüllen; für eine 1.3 chronische Migräne muss der Kopfschmerz an mindestens 8 Tagen die Kriterien B bis D für eine 1.1 Migräne ohne Aura erfüllen. Patienten können daher sämtliche Kriterien für beide Diagnosen erfüllen, etwa indem sie an 25 Tagen/ Monat unter Kopfschmerzen leiden, die an 8 Tagen die Kriterien für eine Migräne erfüllen und an 17 Tagen die Kriterien für einen Kopfschmerz vom Spannungstyp. In solchen Fällen sollte nur die Diagnose lediglich 1.3 chronische Migräne lauten.

Der 2.3 chronische Kopfschmerz vom Spannungstyp entwickelt sich mit der Zeit aus einem 2.2 häufigen episodischen Kopfschmerz vom Spannungstyp; wenn die Kriterien A bis E von einem Kopfschmerz erfüllt werden, der eindeutig täglich auftritt und binnen weniger als 24 Stunden nach seine ersten Auftreten nicht remittiert ist, erfolgt eine Kodierung unter 4.10 neu aufgetretener täglicher Kopfschmerz. Wird die Art und Weise des Kopfschmerzbeginns nicht erinnert oder ist der Beginn unklar, sollte eine Kodierung unter 2.3 chronischer Kopfschmerz vom Spannungstyp erfolgen.

In vielen unklaren Fällen spielt ein Medikamentenübergebrauch eine Rolle. Ist hier Kriterium B einer der Subtypen von 8.2 Kopfschmerz zurückzuführen auf einen Medikamentenübergebrauch und sind gleichzeitig die Kriterien für einen 2.3 chronischen Kopfschmerz vom Spannungstyp erfüllt, ist die Grundregel, sowohl unter 2.3 chronischer Kopfschmerz vom Spannungstyp als auch unter 8.2 Kopfschmerz zurückzuführen auf einen Medikamentenübergebrauch zu kodieren. Nach Medikamentenentzug sollte die Diagnose reevaluiert werden: es kommt nicht selten vor, dass die Kriterien für einen 2.3 chronischen Kopfschmerz vom Spannungstyp dann nicht mehr erfüllt sind und der Patient zu dem einen oder anderen episodischen Kopfschmerztyp zurückkehrt. Bleibt die Erkrankung nach Medikamentenentzug chronisch, entfällt die Diagnose 8.2 Kopfschmerz zurückzuführen auf einen Medikamentenübergebrauch.

\subsubsection{Chronischer Kopfschmerz vom Spannungstyp mit perikranieller Schmerzempfindlichkeit}

Diagnostische Kriterien:

A. Der Kopfschmerz erfüllt die Kriterien A bis E für 2.3 chronischer Kopfschmerz vom Spannungstyp

B. Erhöhte Schmerzempfindlichkeit der perikraniellen Muskulatur bei manueller Palpation.

\subsubsection{Chronischer Kopfschmerz vom Spannungstyp ohne perikranielle Schmerzempfindlichkeit}

Diagnostische Kriterien:

A. Der Kopfschmerz erfüllt die Kriterien für 2.3 chronischer Kopfschmerz vom Spannungstyp

B. Keine erhöhte Schmerzempfindlichkeit der perikraniellen Muskulatur bei manueller Palpation.

\subsection{Wahrscheinlicher Kopfschmerz vom Spannungstyp Beschreibung:}

Kopfschmerz vom Spannungstyp, bei dem eines der Merkmale fehlt, die gegeben sein müssen, um alle Kriterien für einen oben kodierten Typ oder Subtyp eines Kopfschmerzes vom Spannungstyp zu erfüllen und der gleichzeitig nicht die Kriterien für eine andere Kopfschmerzerkrankung erfüllt.

\section{Kommentar:}

Patienten, die einen der untenstehenden Kriteriensätze erfüllen, erfüllen unter Umständen auch die Kriterien für 1.5.1 wahrscheinliche Migräne ohne Aura. In diesen Fällen gilt die allgemeine Grundregel einer Hierarchisierung, bei der die Diagnose 1. Migräne und ihre Typen und Subtypen Vorrang gegenüber der eines 2. Kopfschmerzes vom Spannungstyp und dessen Typen und Subtypen hat.

\subsubsection{Wahrscheinlicher selten auftretender episodischer Kopfschmerz vom Spannungstyp}

\section{Diagnostische Kriterien:}

A. Eine oder mehrere Kopfschmerzepisode(n) erfüllen mit einer Ausnahme alle Kriterien A bis D für einen 2.1 selten auftretenden episodischen Kopfschmerz vom Spannungstyp

B. Die Episoden erfüllen nicht die ICHD-3 Kriterien für eine andere Kopfschmerzerkrankung

C. Nicht besser erklärt durch eine andere ICHD-3-Diagnose.

\subsubsection{Wahrscheinlicher häufig auftretender episodischer Kopfschmerz vom Spannungstyp}

Diagnostische Kriterien:

A. Die Kopfschmerzepisoden erfüllen mit einer Ausnahme alle Kriterien A bis D für einen 2.2 häufig auftretenden episodischen Kopfschmerz vom Spannungstyp

B. Die Episoden erfüllen nicht die ICHD-3 Kriterien für eine andere Kopfschmerzerkrankung

C. Nicht besser erklärt durch eine andere ICHD-3-Diagnose.

\subsubsection{Wahrscheinlicher chronischer Kopfschmerz vom Spannungstyp}

Diagnostische Kriterien:

A. Die Kopfschmerzepisoden erfüllen mit einer Ausnahme alle Kriterien A bis D für einen 2.3 chronischen episodischen Kopfschmerz vom Spannungstyp

B. Die Episoden erfüllen nicht die ICHD-3 Kriterien für eine andere Kopfschmerzerkrankung

C. Nicht besser erklärt durch eine andere ICHD-3-Diagnose. 
Literatur

Ashina M. Nitric oxide synthase inhibitors for the treatment of chronic tension-type headache. Expert Opin Pharmacother 2002; 3: 395-399.

Ashina M. Neurobiology of chronic tension-type headache. Cephalalgia 2004; 24: 161-172.

Ashina S, Babenko L, Jensen R, Ashina M, Magerl W, Bendtsen L. Increased muscular and cutaneous pain sensitivity in cephalic region in patients with chronic tension-type headache. Eur J Neurol 2005; 12: 543-549.

Ashina S, Bendtsen L, Ashina M, Magerl W, Jensen R. Generalized hyperalgesia in patients with chronic tension-type headache. Cephalalgia 2006; 26: $940-948$.

Ashina S, Bendtsen L, Jensen R. Analgesic effect of amitriptyline in chronic tension-type headache is not directly related to serotonin reuptake inhibition. Pain 2004; 108: 108-114.

Ashina M, Bendtsen L, Jensen R, Sakai F, Olesen J. Muscle hardness in patients with chronic tension-type headache: relation to actual headache state. Pain 1999; 79: 201-205.

Ashina M, Bendtsen L, Jensen R, Sakai F, Olesen J. Possible mechanisms of glyceryl-trinitrate-induced immediate headache in patients with chronic tension-type headache. Cephalalgia 2000; 20: 919-924.

Ashina M, Lassen LH, Bendtsen L, Jensen R, Olesen J. Effect of inhibition of nitric oxide synthase on chronic tension-type headache: a randomised crossover trial. Lancet 1999; 353: 287-289.

Ashina M, Stallknecht B, Bendtsen L, Pedersen J, Schifter S, Galbo H, Olesen J. Tender points are not sites of ongoing inflammation - in vivo evidence in patients with chronic tension-type headache. Cephalalgia 2003; 23: $109-116$.

Ashina M, Stallknecht B, Bendtsen L, Pedersen JF, Galbo H, Dalgaard P, Olesen $\mathrm{J}$. In vivo evidence of altered skeletal muscle blood flow in chronic tension-type headache. Brain 2002; 125: 320-326.

Bendtsen L. Central sensitization in tension-type headache - possible pathophysiological mechanisms. Cephalalgia 2000; 20: 486-508.

Bendtsen L, Bigal ME, Cerbo R, Diener HC, Holroyd K, Lampl C, Mitsikostas DD, Steiner TJ, Tfelt-Hansen P. Guidelines for controlled trials of drugs in tension-type headache: second edition. Cephalalgia 2010; 30: 1-16.

Bendtsen L, Evers S, Linde M, Mitsikostas DD, Sandrini G, Schoenen J. EFNS guideline on the treatment of tension-type headache - report of an EFNS task force. Eur J Neurol 2010; 17: 1318-1325.

Bendtsen L, Jensen R. Amitriptyline reduces myofascial tenderness in patients with chronic tension-type headache. Cephalalgia 2000; 20: 603-610.

Bendtsen L, Jensen R. Mirtazapine is effective in the prophylactic treatment of chronic tension-type headache. Neurology 2004; 62: 1706-1711.

Bendtsen L, Jensen R. Tension-type headache: the most common, but also the most neglected, headache disorder. Curr Opin Neurol 2006; 19: 305-309.

Bendtsen L, Jensen R, Jensen NK, Olesen J. Pressure-controlled palpation: a new technique which increases the reliability of manual palpation. Cephalalgia 1995; 15: 205-210.

Bendtsen L, Jensen R, Olesen J. A non-selective (amitriptyline), but not a selective (citalopram), serotonin reuptake inhibitor is effective in the prophylactic treatment of chronic tension-type headache. J Neurol Neurosurg Psychiatry 1996; 61: 285-290.

Bendtsen L, Jensen R, Olesen J. Decreased pain detection and tolerance thresholds in chronic tension-type headache. Arch Neurol 1996; 53: 373-376.

Bendtsen L, Jensen R, Olesen J. Qualitatively altered nociception in chronic myofascial pain. Pain 1996; 65: 259-264.

Buchgreitz L, Egsgaard LL, Jensen R, Arendt-Nielsen L, Bendtsen L. Abnormal pain processing in chronic tension-type headache: a high-density EEG brain mapping study. Brain 2008; 131: 3232-3238.
Buchgreitz L, Lyngberg AC, Bendtsen L, Jensen R. Frequency of headache is related to sensitization: a population study. Pain 2006; 123: 19-27.

Buchgreitz L, Lyngberg AC, Bendtsen L, Jensen R. Increased pain sensitivity is not a risk factor but a consequence of frequent headache: a population-based follow-up study. Pain 2008; 137: 623-630.

Cathcart S, Petkov J, Pritchard D. Effects of induced stress on experimental pain sensitivity in chronic tension-type headache sufferers. Eur J Neurol 2008; 15: 552-558.

Cathcart S, Petkov J, Winefield AH, Lushington K, Rolan P. Central mechanisms of stress-induced headache. Cephalalgia 2010; 30: 285-295.

Cathcart S, Winefield AH, Lushington K, Rolan P. Noxious inhibition of temporal summation is impaired in chronic tension-type headache. Headache 2010; 50: 403-412.

Christensen M, Bendtsen L, Ashina M, Jensen R. Experimental induction of muscle tenderness and headache in tension-type headache patients. Cephalalgia 2005; 25: 1061-1067.

Clark GT, Sakai S, Merrill R, Flack VF, McCreary C. Cross-correlation between stress, pain, psycial activity, and temporalis muscle EMG in tension-type headache. Cephalalgia 1995; 15: 511-518.

Fernandez-de-Las-Penas C, Alonso-Blanco C, Cuadrado ML, Gerwin RD, Pareja JA. Myofascial trigger points and their relationship to headache clinical parameters in chronic tension-type headache. Headache 2006; 46: 1264-1272.

Fernandez-de-Las-Penas C, Alonso-Blanco C, Cuadrado ML, Pareja JA. Myofascial trigger points in the suboccipital muscles in episodic tension-type headache. Man Ther 2006; 11: 225-230.

Fernandez-de-Las-Penas C, Cuadrado ML, Arendt-Nielsen L, Ge HY, Pareja JA. Increased pericranial tenderness, decreased pressure pain threshold, and headache clinical parameters in chronic tension-type headache patients. Clin J Pain 2007; 23: 346-352.

Fernandez-de-Las-Penas C, Cuadrado ML, Arendt-Nielsen L, Simons DG, Pareja JA. Myofascial trigger points and sensitization: an updated pain model for tension-type headache. Cephalalgia 2007; 27: 383-393.

Fernandez-de-Las-Penas C, Cuadrado ML, Pareja JA. Myofascial trigger points, neck mobility, and forward head posture in episodic tension-type headache. Headache 2007; 47: 662-672.

Fernandez-de-Las-Penas C, Schoenen J. Chronic tension-type headache: what is new? Curr Opin Neurol 2009; 22: 254-261.

Fumal A, Schoenen J. Tension-type headache: current research and clinical management. Lancet Neurol 2008; 7: 70-83.

Heckman BD, Holroyd KA. Tension-type headache and psychiatric comorbidity. Curr Pain Headache Rep 2006; 10: 439-447.

Holroyd KA, O’Donnell F], Stensland M, Lipchik GL, Cordingley GE, Carlson BW. Management of chronic tension-type headache with tricyclic antidepressant medication, stress management therapy, and their combination: a randomized controlled trial. JAMA 2001; 285: 2208-2215.

Holroyd KA, Stensland M, Lipchik GL, Hill KR, O’Donnell FS, Cordingley G. Psychosocial correlates and impact of chronic tension-type headaches. Headache 2000; 40: 3-16.

Hubbard DR, Berkoff GM. Myofascial trigger points show spontaneous needle EMG activity. Spine 1993; 18: 1803-1807.

Janke EA, Holroyd KA, Romanek K. Depression increases onset of tension-type headache following laboratory stress. Pain 2004; 111: 230-238.

Jensen R. Mechanisms of spontaneous tension-type headaches: an analysis of tenderness, pain thresholds and EMG. Pain 1996; 64: 251-256.

Jensen R. Pathophysiological mechanisms of tension-type headache: a review of epidemiological and experimental studies. Cephalalgia 1999; 19: 602-621.

Jensen R, Bendtsen L, Olesen J. Muscular factors are of importance in tension-type headache. Headache 1998; 38: 10-17. 
Jensen R, Rasmussen BK, Pedersen B, Lous I, Olesen J. Cephalic muscle tenderness and pressure pain threshold in a general population. Pain 1992; 48: 197-203.

Jensen R, Rasmussen BK, Pedersen B, Olesen J. Muscle tenderness and pressure pain thresholds in headache. A population study. Pain 1993; 52: 193-199.

Jensen R, Stovner LJ. Epidemiology and comorbidity of headache. Lancet Neurol 2008; 7: 354-361.

Jensen R, Zeeberg P, Dehlendorff C, Olesen J. Predictors of outcome of the treatment programme in a multidisciplinary headache centre. Cephalalgia 2010; 30: 1214-1224.

Langemark M, Bach FW, Jensen TS, Olesen J. Decreased nociceptive flexion reflex threshold in chronic tension-type headache. Arch Neurol 1993; 50: 1061-1064.

Langemark M, Jensen K, Jensen TS, Olesen J. Pressure pain thresholds and thermal nociceptive thresholds in chronic tension-type headache. Pain 1989; 38: 203-210.

Langemark M, Olesen J. Pericranial tenderness in tension headache. A blind, controlled study. Cephalalgia 1987; 7: 249-255.

Leistad R, Sand T, Westgaard R, Nilsen K, Stovner L. Stress-induced pain and muscle activity in patients with migraine and tension-type headache. Cephalalgia 2006; 26: 64-73.

Lindelof K, Ellrich J, Jensen R, Bendtsen L. Central pain processing in chronic tension-type headache. Clin Neurophysiol 2009; 120: 1364-1370.

Lindelof K, Jung K, Ellrich J, Jensen R, Bendtsen L. Low-frequency electrical stimulation induces long-term depression in patients with chronic tension-type headache. Cephalalgia 2010; 30: 860-867.

Lyngberg AC, Rasmussen BK, Jorgensen T, Jensen R. Has the prevalence of migraine and tension-type headache changed over a 12-year period? A Danish population survey. Eur J Epidemiol 2005; 20: 243-249.

Mathew NT. Tension-type headache. Curr Neurol Neurosci Rep 2006; 6 : 100-105.

Mork H, Ashina M, Bendtsen L, Olesen J, Jensen R. Induction of prolonged tenderness in patients with tension-type headache by means of a new experimental model of myofascial pain. Eur J Neurol 2003; 10: 249-256.

Mork H, Ashina M, Bendtsen L, Olesen J, Jensen R. Possible mechanisms of pain perception in patients with episodic tension-type headache. A new experimental model of myofascial pain. Cephalalgia 2004; 24: 466-475.

Nestoriuc Y, Rief W, Martin A. Meta-analysis of biofeedback for tension-type headache: efficacy, specificity, and treatment moderators. J Consult Clin Psychol 2008; 76: 379-396.

Olesen J. Clinical and pathophysiological observations in migraine and tension-type headache explained by integration of vascular, supraspinal and myofascial inputs. Pain 1991; 46: 125-132.

Pielsticker A, Haag G, Zaudig M, Lautenbacher S. Impairment of pain inhibition in chronic tension-type headache. Pain 2005; 118 (1-2): 215-223.

Rasmussen BK, Jensen R, Schroll M, Olesen J. Epidemiology of headache in a general population - a prevalence study. J Clin Epidemiol 1991; 44: 1147-1157.

Sandrini G, Rossi P, Milanov I, Serrao M, Cecchini AP, Nappi G. Abnormal modulatory influence of diffuse noxious inhibitory controls in migraine and chronic tension-type headache patients. Cephalalgia 2006; 26: 782-789.

Schmidt-Hansen PT, Svensson P, Bendtsen L, Graven-Nielsen T, Bach FW. Increased muscle pain sensitivity in patients with tension-type headache. Pain 2007; 129: 113-121.

Schmidt-Wilcke T, Leinisch E, Straube A, Kampfe N, Draganski B, Diener HC, Bogdahn U, May A. Gray matter decrease in patients with chronic tension type headache. Neurology 2005; 65: 1483-1486.

Schoenen J, Bottin D, Hardy F, Gerard P. Cephalic and extracephalic pressure pain thresholds in chronic tension-type headache. Pain 1991; 47: 145-149.
Schoenen J, Gerard P, De P, V, Sianard GJ. Multiple clinical and paraclinical analyses of chronic tension-type headache associated or unassociated with disorder of pericranial muscles. Cephalalgia 1991; 11: 135-139.

Schwartz BS, Stewart WF, Simon D, Lipton RB. Epidemiology of tension-type headache. JAMA 1998; 4: 381-383.

\section{Trigemino-autonome Kopfschmerz- erkrankungen (TAK)}

\begin{tabular}{|l|l|}
\hline 3.1 & Clusterkopfschmerz \\
\hline 3.1 .1 & Episodischer Clusterkopfschmerz \\
\hline 3.2 & Chronischer Clusterkopfschmerz \\
\hline 3.2 .1 & Paroxysmale Hemikranie \\
\hline 3.2 .2 & Episodische paroxysmale Hemikranie \\
\hline 3.3 & Short-lasting unilateral neuralgiform headache attacks \\
\hline 3.3.1 & $\begin{array}{l}\text { Short-lasting unilateral neuralgiform headache attacks } \\
\text { with conjunctival injection and tearing (SUNCT-Syndrom) }\end{array}$ \\
\hline 3.3.1.1 & Episodisches SUNCT-Syndrom \\
\hline 3.3.1.2 & Chronisches SUNCT-Syndrom \\
\hline 3.3.2 & $\begin{array}{l}\text { Short-lasting unilateral neuralgiform headache attacks } \\
\text { with cranial autonomic symptoms (SUNA-Syndrom) }\end{array}$ \\
\hline 3.3.2.1 & Episodisches SUNA-Syndrom \\
\hline 3.3.2.2 & Chronisches SUNA-Syndrom \\
\hline 3.4 & Hemicrania continua \\
\hline 3.5 & Wahrscheinliche trigemino-autonome Kopfschmerzer- \\
\hline krankung
\end{tabular}

\section{Allgemeiner Kommentar}

Primärer und/oder sekundärer Kopfschmerz?

Für Kopfschmerzen mit den Merkmalen einer trigemino-autonomen Kopfschmerzerkrankung (TAK) gelten je nach den Umständen drei Grundregeln:

Tritt ein neuer Kopfschmerz mit den Charakteristika einer TAK erstmals in engem zeitlichen Zusammenhang mit einer anderen Erkrankung auf, von der bekannt ist, dass sie Kopfschmerzen auslösen kann oder dieser erfüllt sonstige Kriterien für eine Verursachung durch diese Erkrankung, wird der Kopfschmerz als sekundärer Kopfschmerz zurückzuführen auf die ursächliche Erkrankung kodiert.

Wenn eine vorbestehende TAK in engem zeitlichen Zusammenhang mit einer Erkrankung, die als Ursache von Kopfschmerzen angesehen wird, chronisch wird, sollte sowohl die Ausgangsdiagnose einer TAK als auch die Diagnose eines sekundären Kopfschmerzes gestellt werden.

3. Wenn sich eine vorbestehende TAK in engem zeitlichem Zusammenhang mit einer solchen ursächlichen Erkrankung deutlich verschlechtert (was in der Regel eine mindestens zweifache Zunahme der Häufigkeit und/oder Schwere bedeutet), sollte sowohl die Ausgangsdiagnose einer TAK als auch 
die Diagnose eines sekundären Kopfschmerzes gestellt werden, vorausgesetzt, es gibt überzeugende Belege dafür, dass die Erkrankung Kopfschmerzen auslösen kann.

\section{Einleitung}

Die trigemino-autonomen Kopfschmerzerkrankungen (TAK) teilen die klinischen Merkmale, dass sie einseitig auftreten und in der Regel von parasympathischen autonomen Symptomen im Kopfbereich begleitet werden, die lateralisiert und auf der gleichen Seite wie der Kopfschmerz auftreten. Experimentelle Untersuchungen und Untersuchungen mit funktioneller Bildgebung beim Menschen legen nahe, dass diese Syndrome einen normalen trigemino-parasympathischen Reflex aktivieren, während die klinischen Symptome einer kraniell lokalisierten sympathischen Dysfunktion sekundärer Natur sind.

Eine typische Migräneaura wird in Verbindung mit TAK selten beobachtet.

\subsection{Clusterkopfschmerz}

Früher verwendete Begriffe Ziliare Neuralgie, Erythromelalgie des Kopfes, Bing-Erythroprosopalgie, Hemicrania angioparalytica, Hemicrania periodica neuralgiformis, Histaminkopfschmerz, Horton-Syndrom, Harris-Horton-Syndrom, migränöse Neuralgie nach Harris, Petrosusneuralgie nach Gardner, Sluder-Neuralgie, Neuralgie des Ganglion sphenopalatinum, Vidianusneuralgie

An anderer Stelle kodiert: Ein symptomatischer Clusterkopfschmerz als sekundäre Folge einer anderen Erkrankung wird entsprechend dieser Erkrankung kodiert.

\section{Beschreibung:}

Attacken eines schweren, streng einseitigen Schmerzes orbital, supraorbital, temporal oder in einer Kombination dieser Lokalisationen von 15 bis 180 Minuten Dauer und einer Häufigkeit von einer Attacke jeden zweiten Tag bis zu 8 Attacken/Tag. Eines oder mehrere der nachfolgend genannten Begleitsymptome kommen vor: konjunktivale Injektion, Lakrimation, nasale Kongestion, Rhinorrhoe, vermehrtes Schwitzen im Bereich von Stirn und Gesicht, Miosis, Ptosis und/oder Lidödem und/oder Unruhe oder Agitation.

\section{Diagnostische Kriterien:}

A. Mindestens 5 Attacken, die die Kriterien B bis D erfüllen

B. Starke oder sehr starke einseitig orbital, supraorbital und/ oder temporal lokalisierte Schmerzattacken, die unbehandelt 15 bis 180 Minuten 1 anhalten

C. Einer oder beide der folgenden Punkte ist/sind erfüllt:

1. mindestens eines der folgenden Symptome oder Zeichen, jeweils ipsilateral zum Kopfschmerz:

- konjunktivale Injektion und/oder Lakrimation

- nasale Kongestion und/oder Rhinorrhoe

- Lidödem

- Schwitzen im Bereich der Stirn oder des Gesichtes

- Miosis und/oder Ptosis

2. körperliche Unruhe oder Agitiertheit

D. Die Attackenfrequenz liegt zwischen 1 jeden zweiten Tag und 8 pro Tag 2

E. Nicht besser erklärt durch eine andere ICHD-3-Diagnose.
Anmerkung:

1. Während eines Teils (aber weniger als der Hälfte) des Zeitverlaufes bei einem 3.1 Clusterkopfschmerz können die Attacken weniger schwer sein und/oder kürzer oder länger andauern.

2. Während eines Teils (aber weniger als der Hälfte) des Zeitverlaufes bei einem 3.1 Clusterkopfschmerz können die Attacken seltener auftreten.

\section{Kommentar:}

Die Attacken treten üblicherweise in Serien auf, die Wochen oder Monate andauern (sogenannte Clusterepisoden). Dazwischen liegen Remissionszeiten, die meist Monate oder Jahre anhalten. Bei etwa $10 \%$ bis $15 \%$ der Patienten tritt jedoch ein 3.1.2 chronischer Clusterkopfschmerz ohne derartige Remissionen auf. In einer großen Serie von Verläufen konnte gezeigt werden, dass ein Viertel der Patienten lediglich eine Clusterepisode aufwies. Diese Patienten erfüllen die Kriterien für einen 3.1 Clusterkopfschmerz und sollten entsprechend kodiert werden.

Während einer Clusterepisode des 3.1.1 episodischen Clusterkopfschmerzes und beim 3.1.2 chronischen Clusterkopfschmerz kommt es regelmäßig zu Attacken. Diese können durch Alkohol, Histamin oder Nitroglyzerin ausgelöst werden.

Der Schmerz beim 3.1 Clusterkopfschmerz ist schwerpunktmäBig orbital, supraorbital oder temporal bzw. an einer Kombination dieser Orte lokalisiert, kann aber auch in andere Regionen ausstrahlen. Während der schlimmsten Attacken ist die Schmerzintensität unerträglich. Die Patienten sind in der Regel nicht in der Lage, sich hinzulegen und laufen charakteristischerweise umher. Üblicherweise tritt der Schmerz innerhalb einer Clusterepisode immer auf derselben Seite des Kopfes auf.

Das Erkrankungsalter liegt meist zwischen 20 und 40 Jahren. Aus bisher nicht bekannten Gründen sind Männer dreimal häufiger betroffen als Frauen.

Akute Attacken gehen mit einer Aktivierung des posterioren hypothalamischen Graus einher. Der 3.1 Clusterkopfschmerz kann in etwa $5 \%$ der Fälle autosomal dominant sein.

Es sind einige Patienten beschrieben worden, die sowohl einen 3.1 Clusterkopfschmerz als auch eine 13.1.1 Trigeminusneuralgie (mitunter Cluster-Tic-Syndrom genannt) aufwiesen. Diese Patienten sollten beide Diagnosen erhalten. Die Wichtigkeit dieser Beobachtung liegt darin, dass beide Erkrankungen separat behandelt werden müssen, um Schmerzfreiheit zu erreichen.

\subsubsection{Episodischer Clusterkopfschmerz}

Beschreibung:

Clusterkopfschmerzattacken, die in Episoden mit einer Dauer von 7 Tagen bis 1 Jahr auftreten und von schmerzfreien Episoden von mindestens 3 Monaten Dauer unterbrochen werden.

\section{Diagnostische Kriterien:}

A. Die Attacken erfüllen die Kriterien A bis E für einen 3.1 Clusterkopfschmerz und treten schubweise auf (Clusterepisoden)

B. Wenigstens 2 Clusterepisoden mit einer Dauer von 7 bis 365 Tagen (unbehandelt), die durch schmerzfreie Remissionsphasen von $\geq 3$ Monaten Dauer voneinander getrennt sind. 
Kommentar:

Clusterepisoden halten üblicherweise 2 Wochen bis 3 Monate an.

\subsubsection{Chronischer Clusterkopfschmerz}

Beschreibung:

Clusterkopfschmerzattacken, die ein Jahr oder länger ohne Remissionsphasen auftreten oder mit Remissionsphasen, die weniger als 3 Monate anhalten.

\section{Diagnostische Kriterien:}

A. Die Attacken erfüllen die Kriterien für einen 3.1 Clusterkopfschmerz und Kriterium B unten

B. Die Attacken treten für wenigstens 1 Jahr ohne Remissionsphase auf oder mit Remissionen von <3 Monaten Dauer.

\section{Kommentar:}

Der 3.1.2 chronische Clusterkopfschmerz kann de novo entstehen (früher als primär chronischer Clusterkopfschmerz bezeichnet), oder aus dem 3.1.1 episodischen Clusterkopfschmerz (früher sekundär chronischer Clusterkopfschmerz). Bei einigen Patienten kann sich aus einem 3.1.2 chronischen Clusterkopfschmerz ein 3.1.1 episodischer Clusterkopfschmerz entwickeln.

\subsection{Paroxysmale Hemikranie}

Beschreibung:

Starke einseitig orbital, supraorbital und/oder temporal oder in einer beliebigen Kombination dieser Regionen lokalisierte Schmerzattacken, die 2 bis 30 Minuten anhalten und mehrmals oder vielmals am Tag auftreten. Die Attacken treten gewöhnlich in Begleitung von ipsilateraler konjunktivaler Injektion, Lakrimation, nasaler Kongestion, Rhinorrhoe, Schwitzen im Bereich der Stirn und des Gesichtes, Miosis, Ptosis und/oder Lidödem auf. Sie sprechen zuverlässig auf Indometacin an.

\section{Diagnostische Kriterien:}

A. Mindestens 20 Attacken, die die Kriterien B bis E erfüllen

B. Starke einseitig orbital, supraorbital und/oder temporal lokalisierte Schmerzattacken, die 2 bis 30 Minuten anhalten

C. Einer oder beide der folgenden Punkte:

1. mindestens eines der folgenden Symptome oder Zeichen, jeweils ipsilateral zum Kopfschmerz:

- konjunktivale Injektion und/oder Lakrimation

- nasale Kongestion und/oder Rhinorrhoe

- Lidödem

- Schwitzen im Bereich der Stirn oder des Gesichtes

- Miosis und/oder Ptosis

2. körperliche Unruhe oder Agitiertheit

D. Die Attackenfrequenz liegt bei $>5$ pro Tag 1

E. Attacken kann durch therapeutische Dosen Indometacin2 komplett vorgebeugt werden

F. Nicht besser erklärt durch eine andere ICHD-3-Diagnose.

\section{Anmerkung:}

1. Während eines Teils (aber weniger als der Hälfte) des Zeitverlaufes bei 3.2 paroxysmaler Hemikranie können die Attacken seltener auftreten.
2. Bei Erwachsenen sollte Indometacin anfangs in einer Tagesdosis von $\geq 150 \mathrm{mg}$ oral eingesetzt werden und bei Bedarf auf $\geq 225$ mg täglich erhöht werden. Die Dosis für Injektionen beträgt 100 bis $200 \mathrm{mg}$. Als Erhaltungsdosen sind meist jedoch geringere Mengen erforderlich.

\section{Kommentar:}

Anders als beim Clusterkopfschmerz besteht kein vermehrtes Auftreten bei Männern. Die Erkrankung tritt im Allgemeinen erstmals im Erwachsenenalter auf, allerdings wurden auch Fälle im Kindesalter beschrieben.

\subsubsection{Episodische paroxysmale Hemikranie}

\section{Beschreibung:}

Paroxysmale Hemikranieattacken, die in Episoden mit einer Dauer von 7 Tagen bis 1 Jahr auftreten, die von schmerzfreien Episoden von mindestens 3 Monaten Dauer unterbrochen werden.

\section{Diagnostische Kriterien:}

A. Die Attacken erfüllen die Kriterien für eine 3.2 paroxysmale Hemikranie und treten schubweise auf

B. Wenigstens 2 Episoden mit einer Dauer von 7 bis 365 Tagen (unbehandelt), die durch schmerzfreie Remissionsphasen von $\geq 3$ Monaten Dauer unterbrochen werden.

\subsubsection{Chronische paroxysmale Hemikranie}

\section{Beschreibung:}

Paroxysmale Hemikranieattacken, die über mehr als ein Jahr ohne schmerzfreie Episoden auftreten oder von schmerzfreien Episoden von wenigster als 3 Monaten Dauer unterbrochen werden.

\section{Diagnostische Kriterien:}

A. Die Attacken erfüllen die Kriterien für eine 3.2 paroxysmale Hemikranie sowie Kriterium B unten

B. Die Attacken treten für wenigstens 1 Jahr ohne Remissionsphase auf oder mit Remissionen von <3 Monaten Dauer auf.

\section{Kommentar:}

Patienten, die sowohl die Kriterien für eine 3.2.2 chronische paroxysmale Hemikranie als auch für eine 13.1.1 Trigeminusneuralgie (mitunter CPH-Tic-Syndrom genannt) erfüllen, sollten beide Diagnosen erhalten. Die Unterscheidung ist wichtig, da beide Erkrankungen einer Behandlung bedürfen. Die pathophysiologische Bedeutung dieser Beziehung ist noch unklar.

\subsection{Short-lasting unilateral neuralgiform headache attacks}

Beschreibung:

Sekunden bis Minuten anhaltende, streng einseitige mittelstarke oder starke Kopfschmerzattacken, die mindestens einmal am Tag auftreten. In der Regel gehen sie mit einer deutlichen Lakrimation und Rötung des ipsilateralen Auges einher. 
Diagnostische Kriterien:

A. Mindestens 20 Attacken, die die Kriterien B bis D erfüllen

B. Mäßige oder starke einseitig orbital, supraorbital und/oder temporal lokalisierte Schmerzattacken, die 1 bis 600 Sekunden anhalten und als Einzelstiche, Serien von Stichen oder sägezahnmusterartig auftreten

C. Mindestens eines der folgenden kranioautonomen Symptome oder Zeichen ipsilateral zum Kopfschmerz:

- konjunktivale Injektion und/oder Lakrimation

- nasale Kongestion und/oder Rhinorrhoe

- Lidödem

- Schwitzen im Bereich der Stirn oder des Gesichtes

- Miosis und/oder Ptosis

D. Die Attackenfrequenz liegt bei $>1$ pro Tag 1

E. Nicht besser erklärt durch eine andere ICHD-3-Diagnose.

Anmerkung:

1. Während eines Teils (aber weniger als der Hälfte) des Zeitverlaufes beim 3.3 Short-lasting unilateral neuralgiform headache attacks können die Attacken seltener auftreten.

\section{Kommentar:}

Länger andauernde Attacken sind von diversen Stichen oder einem Sägezahnmuster gekennzeichnet.

Es werden zwei Subtypen des 3.3 Short-lasting unilateral neuralgiform headache attacks anerkannt: 3.3.1 Short-lasting unilateral neuralgiform headache attacks with conjunctival injection and tearing (SUNCT-Syndrom) und 3.3.2 Short-lasting unilateral neuralgiform headache attacks with cranial autonomic symptoms (SUNA-Syndrom). Das 3.3.1 SUNCT-Syndrom könnte ein Subtyp des 3.3.2 SUNA-Syndroms sein, obwohl hier noch nähere Untersuchungen gefragt sind. Bis dahin werden sie jeweils als separater Subtyp klassifiziert wie unten beschrieben.

\subsubsection{SUNCT-Syndrom und 3.3.2 SUNA-Syndrom können} in der Regel ohne Refraktärphase getriggert werden. Hiermit stehen sie im Kontrast zu einer 13.1.1 Trigeminusneuralgie, die gewöhnlich nach jeder Attacke eine Refraktärphase aufweist.

\subsubsection{Short-lasting Unilateral Neuralgiform headache attacks with Conjunctival injection and Tearing (SUNCT-Syndrom)}

Diagnostische Kriterien:

A. Attacken, die die Kriterien für 3.3 Short-lasting unilateral neuralgiform headache attacks und das Kriterium B unten erfüllen

B. Beides von folgendem, ipsilateral zum Schmerz:
1. konjunktivale Injektion
2. Lakrimation (Tränenfluss).

\section{Kommentar:}

Die Literatur lässt darauf schließen, dass das 3.3.1 SUNCT-Syndrom am ehesten von einer Läsion in der hinteren Schädelgrube imitiert wird.

Es wurden Patienten beschrieben, bei denen sich eine Überschneidung zwischen 3.3.1 SUNCT-Syndrom und 13.1.1 Trigemi- nusneuralgie findet. Die Differenzierung ist klinisch komplex. Solche Patienten sollten beide Diagnosen erhalten.

Es gab Berichte über Patienten mit 3.3.1 SUNCT-Syndrom wie auch 3.1 Clusterkopfschmerz; die pathophysiologische Bedeutung dieser Überschneidung ist noch zu ermitteln.

\subsubsection{Episodisches SUNCT-Syndrom}

Beschreibung:

SUNCT-Attacken, die über Zeiträume zwischen 7 Tagen und einem Jahr auftreten und durch schmerzfreie Episoden von weniger als 3 Monaten unterbrochen werden.

\section{Diagnostische Kriterien:}

A. Attacken, die die Kriterien für ein 3.3.1 SUNCT-Syndrom erfüllen und episodisch auftreten

B. Mindestens zwei Episoden von einer Dauer zwischen 7 Tagen und 1 Jahr (unbehandelt) und durch schmerzfreie Remissionsphasen $<3$ Monaten unterbrochen werden.

\subsubsection{Chronisches SUNCT-Syndrom} Beschreibung:

SUNCT-Attacken, die über mehr als ein Jahr ohne Remissionsphase auftreten oder von Remissionsphasen von > 3 Monaten unterbrochen werden.

\section{Diagnostische Kriterien:}

A. Attacken, die die Kriterien für ein 3.3.1 SUNCT-Syndrom und das Kriterium B unten erfüllen

B. Attacken, die ohne Remissionsphase oder mit Remissionsphasen von<3 Monaten für mindestens 1 Jahr auftreten.

3.3.2 Short-lasting unilateral neuralgiform headache attacks with cranial autonomic symptoms (SUNA-Syndrom)

A. Attacken, die die Kriterien für ein 3.3 SUNCT-Syndrom und das Kriterium B unten erfüllen

B. Es ist nicht mehr als einer der folgenden Punkte erfüllt, ipsilateral zum Schmerz:

1. konjunktivale Injektion

2. Lakrimation (Tränenfluss).

\subsubsection{Episodisches SUNA-Syndrom}

Beschreibung:

SUNA-Attacken, die für die Dauer von 7 Tagen bis 1 Jahr auftreten und durch schmerzfreie Episoden von > 3 Monaten unterbrochen werden.

\section{Diagnostische Kriterien:}

A. Attacken, die die Kriterien für ein 3.3.2 SUNA-Syndrom erfüllen und episodisch auftreten

B. Mindestens zwei Episoden für die Dauer von 7 Tagen bis 1 Jahr (unbehandelt,) die durch schmerzfreie Episoden von > 3 Monaten unterbrochen werden.

\subsubsection{Chronisches SUNA-Syndrom} Beschreibung:

SUNA-Attacken, die über mehr als ein Jahr ohne Remissionsphase auftreten oder Remissionsphasen von weniger als 3 Monaten aufweisen. 
Diagnostische Kriterien:

A. Attacken, die die Kriterien für 3.3.2 SUNA-Syndrom und das Kriterium B unten erfüllen

B. Attacken, die für mindestens ein Jahr ohne Remissionsphase auftreten oder Remissionsphasen von<3 Monaten aufweisen.

\subsection{Hemicrania continua}

Beschreibung:

Anhaltender, streng einseitiger Kopfschmerz, der in Begleitung von ipsilateraler konjunktivaler Injektion, Lakrimation, nasaler Kongestion, Rhinorrhoe, Schwitzen im Bereich der Stirn und des Gesichtes, Miosis, Ptosis und/oder Lidödemen und/oder Unruhe oder Agitiertheit auftritt und zuverlässig auf Indometacin anspricht.

Diagnostische Kriterien:

A. Einseitiger Kopfschmerz, der die Kriterien B bis D erfüllt

B. Für>3 Monate vorliegend, mit Verschlechterungen von mäßiger oder stärkerer Intensität

C. Einer oder beide der folgenden Punkte:

1. Mindestens eines der folgenden Symptome oder Zeichen, jeweils ipsilateral zum Kopfschmerz:

- konjunktivale Injektion und/oder Lakrimation

- nasale Kongestion und/oder Rhinorrhoe

- Lidödem

- Schwitzen im Bereich der Stirn oder des Gesichtes

- Miosis und/oder Ptosis

2. Körperliche Unruhe oder Agitiertheit oder Schmerzzunahme durch Bewegung

D. Spricht zuverlässig auf therapeutische Dosen Indometacin an1

E. Nicht besser erklärt durch eine andere ICHD-3-Diagnose.

\section{Anmerkung:}

Bei Erwachsenen sollte Indometacin anfangs in einer Tagesdosis von mindestens $150 \mathrm{mg}$ oral eingesetzt werden und bei Bedarf auf über $225 \mathrm{mg}$ täglich erhöht werden. Die Dosis für Injektionen beträgt 100 bis $200 \mathrm{mg}$. Als Erhaltungsdosen sind oft jedoch geringere Mengen erforderlich

\section{Kommentar:}

Bei einer 3.4 Hemicrania continua werden oft Migränesymptome wie Photophobie und Phonophobie beobachtet.

Die 3.4 Hemicrania continua wird aufgrund der Tatsache, dass der Schmerz typischerweise einseitig auftritt, ebenso wie die gegebenenfalls vorhandenen kranio-autonomen Symptome, in der ICHD-3 unter 3. Trigemino-autonome Kopfschmerzerkrankungen geführt (in der ICHD-2 stand sie unter 4. andere primäre Kopfschmerzen).

Die zerebrale Bildgebung ergab bedeutende Überschneidungen zwischen allen hier einbezogenen Erkrankungen, vor allem die Aktivierung in der Region des posterioren hypothalamischen Graus. Darüber hinaus liegt das zuverlässige Ansprechen einer $3.4 \mathrm{He}$ micrania continua auf Indometacin auch bei einer 3.2 paroxysmalen Hemikranie vor.

\subsubsection{Hemicrania continua mit remittierendem Verlauf}

Beschreibung:

Charakteristisch für diesen Subtyp der Hemicrania continua ist ein Kopfschmerz, der nicht durchgängig anhält, sondern von Remissionsphasen von mindestens 24 Stunden Dauer unterbrochen wird.

\section{Diagnostische Kriterien:}

A. Kopfschmerz, der die Kriterien für eine 3.4 Hemicrania continua und Kriterium B unten erfüllt

B. Der Kopfschmerz tritt nicht täglich oder durchgängig auf, sondern wird (unbehandelt) durch Remissionsphasen von $\geq 24$ Stunden unterbrochen.

\section{Kommentar:}

Die 3.4.1 Hemicrania continua mit remittierendem Verlauf kann de novo oder aus einer 3.4.2 Hemicrania continua mit nicht remittierendem Verlauf entstehen.

\subsubsection{Hemicrania continua mit nicht remittierendem Verlauf}

\section{Beschreibung:}

Charakteristisch für diesen Subtyp der Hemicrania continua ist ein Kopfschmerz, der mindestens ein Jahr lang durchgängig anhält, ohne Remissionsphasen von mindestens einem Tag.

\section{Diagnostische Kriterien:}

A. Kopfschmerz, der die Kriterien für eine 3.4 Hemicrania continua, und Kriterium B unten erfüllt

B. Der Kopfschmerz tritt mindestens 1 Jahr lang täglich und durchgängig auf, ohne Remissionsphasen von $\geq 1$ Tag.

Kommentar:

Die 3.4.2 Hemicrania continua mit nicht remittierendem Verlauf kann de novo oder aus einer 3.4.1 Hemicrania continua mit remittierendem Verlauf entstehen. Die Mehrzahl der Patienten zeigt bereits von Anfang an den nicht remittierenden Verlaufstyp.

\subsection{Wahrscheinliche trigemino-autonome Kopfschmerzerkrankung}

Beschreibung:

Kopfschmerzattacken, die am ehesten den 3. trigemino-autonomen Kopfschmerzerkrankungen zugeordnet werden können, bei denen aber ein Symptom fehlt, welches erforderlich ist, um sämtliche Kriterien eines der oben kodierten Typen oder Subtypen zu erfüllen und die nicht alle Kriterien einer anderen Kopfschmerzerkrankung erfüllen.

\section{Diagnostische Kriterien:}

A. Kopfschmerzattacken, die mit einer Ausnahme alle Kriterien A bis D für einen 3.1 Clusterkopfschmerz erfüllen; die Kriterien A bis E für eine 3.2 paroxysmale Hemikranie; die Kriterien A bis D für 3.3 SUNCT-Syndrom oder die Kriterien A bis D für eine 3.4 Hemicrania continua 
B. Die Episoden erfüllen nicht die ICHD-3 Kriterien für eine andere Kopfschmerzerkrankung

C. Nicht besser erklärt durch eine andere ICHD-3-Diagnose.

\section{Kommentar:}

Die Patienten können unter 3.5.1 wahrscheinlicher Clusterkopfschmerz, 3.5.2 wahrscheinliche paroxysmale Hemikranie, 3.5.3 wahrscheinliches SUNA-Syndrom oder 3.5.4 wahrscheinliche Hemicrania continua kodiert werden. Derartige Patienten haben entweder keine ausreichende Anzahl typischer Anfälle gehabt (zum Beispiel nur einen ersten Clusterkopfschmerzanfall), oder haben diese zwar in ausreichend großer Anzahl erlebt, erfüllen aber andere Kriterien nicht.

\section{Literatur}

Bahra A, May A, Goadsby PJ. Cluster headache: a prospective clinical study in 230 patients with diagnostic implications. Neurology 2002; 58: 354-361.

Benoliel R, Sharav Y. Trigeminal neuralgia with lacrimation or SUNCT syndrome? Cephalalgia 1998; 18: 85-90.

Bing R. Uber traumatische Erythromelalgie und Erthroprosopalgie. Nervenarzt 1930; 3: 506-512.

Boes CJ, Matharu MS, Goadsby PJ. The paroxysmal hemicrania-tic syndrome. Cephalalgia 2003; 23: 24-28.

Bordini C, Antonaci F, Stovner LJ, Schrader H, Sjaastad O. "Hemicrania continua”: a clinical review. Headache 1991; 31: 20-26.

Bouhassira D, Attal N, Esteve M, Chauvin M. SUNCT syndrome. A case of transformation from trigeminal neuralgia. Cephalalgia 1994; 14: 168-170.

Broeske D, Lenn NJ, Cantos E. Chronic paroxysmal hemicrania in a young child: possible relation to ipsilateral occipital infarction. J Child Neurol 1993; 8: 235-236.

Bussone G, Leone M, Volta GD, Strada L, Gasparotti R. Short-lasting unilateral neuralgiform headache attacks with tearing and conjunctival injection: the first symptomatic case. Cephalalgia 1991; 11: 123-127.

Caminero AB, Pareja JA, Dobato JL. Chronic paroxysmal hemicrania-tic syndrome. Cephalalgia 1998; 18: 159-161.

Cittadini E, Goadsby PJ. Hemicrania Continua: A clinical study of 39 patients with diagnostic implications. Brain 2010; 133: 1973-1986.

Cittadini E, Matharu MS, Goadsby PJ. Paroxysmal hemicrania: a prospective clinical study of thirty-one cases. Brain 2008; 131: 1142-1155.

Cohen AS, Matharu MS, Goadsby PJ. Short-lasting Unilateral Neuralgiform Headache Attacks with Conjunctival injection and Tearing (SUNCT) or cranial Autonomic features (SUNA). A prospective clinical study of SUNCT and SUNA. Brain 2006; 129: 2746-2760.

De Benedittis G. SUNCT syndrome associated with cavernous angioma of the brain stem. Cephalalgia 1996; 16: 503-506.

Ekbom K. Ergotamine tartrate orally in Horton's 'histaminic cephalalgia' (also called Harris's ciliary neuralgia). Acta Psychiat Scand 1947; 46: 106-113.

Ekbom K. Nitroglycerin as a provocative agent in cluster headache. Arch Neurol 1968; 19: 487-493.

Empl M, Goadsby PJ, Kaube H. Migraine with aura, episodic cluster headache, and SUNCT syndrome consecutively in a patient: trigemino-vascular trinity. Cephalalgia 2003; 23: 584.

Eulenberg A. Lehrbuch der Nervenkrankheiten. 2nd ed. Berlin: Hirschwald 1878.

Goadsby PJ. Pathophysiology of cluster headache: a trigeminal autonomic cephalgia. Lancet Neurology 2002; 1: 37-43.
Goadsby PJ, Lipton RB. A review of paroxysmal hemicranias, SUNCT syndrome and other short-lasting headaches with autonomic features, including new cases. Brain 1997; 120: 193-209.

Goadsby PJ, Lipton RB. Paroxysmal hemicrania-tic syndrome. Headache 2001; 41: 608-609.

Goadsby PJ, Matharu MS, Boes CJ. SUNCT syndrome or trigeminal neuralgia with lacrimation. Cephalalgia 2001; 21: 82-83.

Hannerz J. Trigeminal neuralgia with chronic paroxysmal hemicrania: the CPH-tic syndrome. Cephalalgia 1993; 13: 361-364.

Hannerz J. The second case of chronic paroxysmal hemicrania-tic syndrome [Editorial comment]. Cephalalgia 1998; 18: 124.

Harris W. Ciliary (migrainous) neuralgia and its treatment. BMJ 1936; 1 : 457-460.

Horton BT. Histaminic cephalgia. Lancet 1952; 72: 92-98.

Irimia P, Cittadini E, Paemeleire K, Cohen AS, Goadsby PJ. Unilateral photophobia or phonophobia in migraine compared with trigeminal autonomic cephalalgias. Cephalalgia 2008; 28: 626-630.

Klimek A. Cluster-tic syndrome. Cephalalgia 1987; 7: 161-162.

Kreiner M. Use of streptomycin-lidocaine injections in the treatment of the cluster-tic syndrome. Clinical perspectives and a case report. J Craniomaxillofac Surg 1996; 24: 289-292.

Kudrow L. Cluster headache: mechanisms and management. Oxford: Oxford University Press 1980.

Kudrow DB, Kudrow L. Successful aspirin prophylaxis in a child with chronic paroxysmal hemicrania. Headache 1989; 29: 280-281.

Manzoni GC. Gender ratio of cluster headache over the years: a possible role of changes in lifestyle. Cephalalgia 1998; 18: 138-142.

Manzoni GC, Micieli G, Granella F, Tassorelli C, Zanferrari C, Cavallini A. Cluster headache- course over ten years in 189 patients. Cephalalgia 1991; 11: 169-174.

Manzoni GC, Terzano MG, Bono G, Micieli G, Martucci N, Nappi G. Cluster headache - clinical findings in 180 patients. Cephalalgia 1983; 3: 21-30.

Martinez-Salio A, Porta-Etessam J, Perez-Martinez D, Balseiro J, Gutierrez-Rivas E. Chronic paroxysmal hemicrania-tic syndrome. Headache 2000; 40: 682-685.

May A, Bahra A, Buchel C, Frackowiak RS, Goadsby PJ. Hypothalamic activation in cluster headache attacks. Lancet 1998; 352: 275-278.

May A, Goadsby PJ. The trigeminovascular system in humans: pathophysiological implications for primary headache syndromes of the neural influences on the cerebral circulation. J Cereb Blood Flow Metab 1999; 19: $115-127$.

Monzillo PH, Sanvito WL, Da Costa AR. Cluster-tic syndrome: report of five new cases. Arq Neuropsiquiatr 2000; 58: 518-521.

Morales F, Mostacero E, Marta J, Sanchez S. Vascular malformation of the cerebellopontine angle associated with SUNCT syndrome. Cephalalgia 1994; 14: 301-302.

Mulleners WM, Verhagen WIM. Cluster-tic syndrome. Neurology 1996; 47: 302.

Newman LC, Lipton RB, Solomon S. Hemicrania continua: ten new cases and a review of the literature. Neurology 1994; 44: 2111-2114.

Obermann M, Yoon M-S, Dommes P, Kuznetsova J, Maschke M, Weimar C, et al. Prevalence of trigeminal autonomic symptoms in migraine: a population-based study. Cephalalgia 2007; 27: 504-509.

Olivarius de Fine B. Hemicrania neuralgiformis chronica (Chronic migrainous neuralgia). Quoted by Sjaastad O, editor. Proceedings of the Scandinavian Migraine Society Annual Meeting 1971; p. 8.

Pascual J, Berciano J. Relief of cluster-tic syndrome by the combination of lithium and carbamazepine. Cephalalgia 1993; 13: 205-206.

Romberg MH. Lehrbuch der Nervenkrankheiten des Menschen. Berlin: Dunker 1840. 
Russell MB, Andersson PG, Thomsen LL, Iselius L. Cluster headache is an autosomal dominantly inherited disorder in some families: a complex segregation analysis. J Med Genetics 1995; 32: 954-956.

Sanahuja J, Vazquez P, Falguera M. Paroxysmal hemicrania-tic syndrome responsive to acetazolamide. Cephalalgia 2005; 25: 547-549.

Silberstein SD, Niknam R, Rozen TD, Young WB. Cluster headache with aura. Neurology 2000; 54: 219-221.

Sjaastad O, Dale I. Evidence for a new (?) treatable headache entity. Headache 1974; 14: 105-108.

Sjaastad O, Saunte C, Salvesen R, Fredriksen TA, Seim A, Roe OD, et al. Shortlasting unilateral neuralgiform headache attacks with conjunctival injection, tearing, sweating, and rhinorrhea. Cephalalgia 1989; 9: 147-156.

Sjostrand C, Waldenlind E, Ekbom K. A follow up study of 60 patients after an assumed first period of cluster headache. Cephalalgia 2000; 20: 653-657.

Sluder G. The syndrome of sphenopalatine ganglion neurosis. Amer ] Med 1910; 140: 868-878.

Solomon S, Apfelbaum RI, Guglielmo KM. The cluster-tic syndrome and its surgical therapy. Cephalalgia 1985; 5: 83-89.

Sprenger T, Goadsby PJ. What has functional neuroimaging done for primary headache....and for the clinical neurologist? J Clin Neuroscience 2010; 17: 547-553.

Vail HH. Vidian neuralgia. Ann Otol Rhinol Laryngol 1932; 41: 837-856.

Watson P, Evans R. Cluster-tic syndrome. Headache 1985; 25: 123-126.

Zukerman E, Peres MFP, Kaup AO, Monzillo PH, Costa AR. Chronic paroxysmal hemicrania-tic syndrome. Neurology 2000; 54: 1524-1526.

\section{Andere primäre Kopfschmerzen}

\begin{tabular}{|l|l|}
\hline 4.1 & Primärer Hustenkopfschmerz \\
\hline 4.1 .1 & Wahrscheinlicher primärer Hustenkopfschmerz \\
\hline 4.2 & Primärer Anstrengungskopfschmerz \\
\hline 4.2 .1 & Wahrscheinlicher primärer Anstrengungskopfschmerz \\
\hline 4.3 & Primärer Sexualkopfschmerz \\
\hline 4.3 .1 & Wahrscheinlicher primärer Sexualkopfschmerz \\
\hline 4.4 & Primärer Donnerschlagkopfschmerz \\
\hline 4.5 & Primärer kältebedingter Kopfschmerz \\
\hline 4.5 .1 & Kopfschmerz zurückzuführen auf einen äußeren Kältereiz \\
\hline 4.5 .2 & Kopfschmerzen zurückzuführen auf Einnahme oder \\
\hline 4.5 .3 & Inhalation eines Kältereizes \\
\hline 4.5 .3 .1 & $\begin{array}{l}\text { Kahrscheinlicher kältebedingter Kopfschmerz } \\
\text { äußeren Kältereiz }\end{array}$ \\
\hline 4.5 .3 .2 & Kopfschmerzen wahrscheinlich zurückzuführen auf Ein- \\
\hline nahme oder Inhalation eines Kältereizes \\
\hline 4.6 & $\begin{array}{l}\text { Kopfschmerz durch Einwirkung von Druck oder Zug auf } \\
\text { den Kopf }\end{array}$ \\
\hline 4.6 .1 & Kopfschmerz durch äußeren Druck \\
\hline 4.6 .2 & Kopfschmerz durch äußeren Zug \\
\hline 4.6 .3 & $\begin{array}{l}\text { Wahrscheinlicher Kopfschmerz durch Einwirkung von } \\
\text { Druck oder Zug auf den Kopf }\end{array}$ \\
\hline 4.6 .3 .1 & Wahrscheinlicher Kopfschmerz durch äußeren Druck \\
\hline 4.6 .3 .2 & Wahrscheinlicher Kopfschmerz durch äußeren Zug \\
\hline 4.7 & Primärer stechender Kopfschmerz \\
\hline 4.7 .1 & Wahrscheinlicher stechender Kopfschmerz \\
\hline &
\end{tabular}

\begin{tabular}{|l|l|}
\hline 4.8 & Münzkopfschmerz \\
\hline 4.8 .1 & Wahrscheinlicher Münzkopfschmerz \\
\hline 4.9 & Schlafgebundener Kopfschmerz \\
\hline 4.9 .1 & Wahrscheinlicher Schlafgebundener Kopfschmerz \\
\hline 4.10 & Neu aufgetretener täglicher Kopfschmerz (NDPH) \\
\hline 4.10 .1 & Wahrscheinlicher neu aufgetretener täglicher Kopfschmerz \\
\hline
\end{tabular}

An anderer Stelle kodiert Primärer und/oder sekundärer Kopfschmerz?

Für 4. Andere primäre Kopfschmerzen gelten je nach den Umständen 2 Regeln:

1. Wenn ein neu aufgetretener Kopfschmerz mit den Charakteristika des Kopfschmerzes vom Spannungstyp zum ersten Mal in einem engen zeitlichen Zusammenhang mit einer anderen Erkrankung auftritt, von der bekannt ist, dass sie Kopfschmerzen hervorrufen kann oder dieser erfüllt sonstige Kriterien, die für eine Verursachung durch diese andere Erkrankung sprechen, wird dieser neue Kopfschmerz als sekundärer Kopfschmerz, zurückzuführen auf die ursächliche Erkrankung kodiert.

2. Wenn aber ein vorbestehender Kopfschmerz mit den Merkmalen einer der hier klassifizierten Erkrankungen in einem engen zeitlichen Zusammenhang mit einer solchen ursächlichen Erkrankung chronisch wird oder sich deutlich verschlechtert (üblicherweise definiert als eine mindestens zweifache Steigerung der Häufigkeit oder Schwere), sollte sowohl die ursprüngliche Kopfschmerzdiagnose als auch die Diagnose eines sekundären Kopfschmerzes vergeben werden, sofern gute Hinweise bestehen, dass diese Erkrankung Kopfschmerzen auslösen kann.

\section{Einleitung}

Dieses Kapitel beinhaltet eine Reihe von primären Kopfschmerzerkrankungen, die klinisch heterogen sind. Sie werden in vier Kategorien eingeteilt und in der entsprechenden Abfolge in der ICHD3 kodiert:

Kopfschmerzen in Verbindung mit körperlicher Anstrengung, darunter 4.1 primärer Hustenkopfschmerz, 4.2 primärer Anstrengungskopfschmerz, 4.3 primärer Sexualkopfschmerz und 4.4 primärer Donnerschlagkopfschmerz.

Kopfschmerzen, die direkten physischen Reizen zugeschrieben werden (diese gelten als primäre Kopfschmerzerkrankungen, da sie durch physiologische [nicht schädigende] Reize entstehen), darunter 4.5 primärer kältebedingter Kopfschmerz und 4.6 Kopfschmerz durch Einwirkung von Druck oder Zug auf den Kopf.

Epikranieller Kopfschmerz (d. h. Kopfschmerzen auf der Kopfhaut), darunter 4.7 primärer stechender Kopfschmerz und 4.8 Münzkopfschmerz (sowie A4.11 Epicrania fugax im Anhang).

Diverse sonstige primäre Kopfschmerzerkrankungen, darunter 4.9 schlafgebundener Kopfschmerz und 4.10 neu aufgetretener täglicher Kopfschmerz.

Die Pathogenese dieser Erkrankungen ist noch unzureichend geklärt, und Behandlungsempfehlungen diesbezüglich stützen sich auf Einzelberichte oder unkontrollierten Studien.

Kopfschmerzen mit ähnlichen Charakteristika wie mehrere dieser Erkrankungen können Symptome einer anderen Erkrankung 
sein (d. h. sekundäre Kopfschmerzen); bei ihrem erstmaligen Auftreten erfordern diese eine sorgfältige Beurteilung durch bildgebende Verfahren und/oder andere angemessene Tests. Der Beginn einiger dieser Kopfschmerzen (etwa 4.2 primärer Anstrengungskopfschmerz, 4.3 primärer Sexualkopfschmerz und 4.4 primärer Donnerschlagkopfschmerz) kann akut sein, und die Betroffenen werden mitunter in Notaufnahmen vorstellig. Eine angemessene und vollständige Untersuchung (vor allem durch zerebrale Bildgebung) ist in diesen Fällen obligatorisch.

\subsection{Primärer Hustenkopfschmerz}

Früher verwendete Begriffe enigner Hustenkopfschmerz; Kopfschmerz bei Valsalva-Manöver.

\section{Beschreibung:}

Durch Husten oder ein anderes Valsalva-Manöver, nicht jedoch durch längere körperliche Anstrengung hervorgerufene Kopfschmerzen in Abwesenheit jeglicher intrakranieller Erkrankung.

\section{Diagnostische Kriterien:}

A. Mindestens 2 Kopfschmerzepisoden, die die Kriterien B bis D erfüllen

B. Der Schmerz wird ausgelöst durch Husten, Pressen und/oder ein anderes Valsalva-Manöver und tritt ausschließlich in Verbindung damit auf1

C. Plötzlicher Beginn2

D. Der Schmerz hält 1 Sekunde bis 2 Stunden lang an2

E. Nicht besser erklärt durch eine andere ICHD-3-Diagnose3.

\section{Anmerkung:}

1. Der Kopfschmerz entsteht wenige Augenblicke nach dem Husten oder sonstigen Reizen

2. Der Kopfschmerz erreicht fast sofort seinen Höhepunkt und legt sich dann binnen einiger Sekunden bis weniger Minuten (obwohl einige Patienten zwei Stunden lang leichte bis mäßige Kopfschmerzen erleben).

3. Das Hustenkopfschmerz-Syndrom ist in etwa $40 \%$ der Fälle symptomatischer Natur. Bei der Mehrzahl der Patienten besteht eine Chiari-Malformation Typ I. Andere Ursachen können eine spontane intrakranielle Hypotonie, Erkrankungen der Aa. carotides, der vertebro-basilären Gefäße, Tumore der mittleren oder hinteren Schädelgrube, eine Mittelhirnzyste, eine basiläre Impression, eine Platybasie, ein subdurales Hämatom, zerebrale Aneurysmen und ein reversibles zerebrales Vasokonstriktionssyndrom sein. Die zerebrale Bildgebung spielt daher eine wichtige Rolle bei der Suche nach möglichen intrakraniellen Läsionen oder Abnormalitäten. Da mehr als $50 \%$ der intrakraniellen raumfordernden Läsionen bei Kindern auf subtentorielle Tumore zurückgingen, sollte ein Hustenkopfschmerz bei pädiatrischen Patienten als symptomatisch erachtet werden, solange kein gegenteiliger Beweis erbracht wurde.

\section{Kommentar:}

Der 4.1 primäre Hustenkopfschmerz ist eine seltene Erkrankung, die maximal $1 \%$ aller Kopfschmerzpatienten betrifft, die in neurologischen Kliniken vorstellig werden. Einem Bericht zufolge wies jedoch ein Fünftel der Patienten mit Husten, die eine Thoraxklinik aufsuchten, Hustenkopfschmerz auf.

Der 4.1 primäre Hustenkopfschmerz ist meist beidseitig und hinten lokalisiert und tritt vor allem bei Patienten auf, die älter als 40 Jahre alt sind. Es besteht eine deutliche Korrelation zwischen der Hustenhäufigkeit und der Schwere des Kopfschmerzes. Bis zu zwei Drittel der Patienten mit 4.1 primärem Hustenkopfschmerz berichteten über Begleitsymptome wie Schwindel, Übelkeit und Schlafanomalien.

Der 4.1 primäre Hustenkopfschmerz spricht üblicherweise auf Indometacin (50 bis $200 \mathrm{mg} / \mathrm{Tag}$ ) an. In Einzelfällen war Indometacin aber auch bei symptomatischen Fällen wirksam.

\subsubsection{Wahrscheinlicher primärer Hustenkopfschmerz Diagnostische Kriterien:}

A. Vorliegen eines der folgenden Punkte:

1. eine einmalige Kopfschmerzepisode, die die Kriterien B bis D erfüllt

2. mindestens 2 Kopfschmerzepisode, die Kriterium B sowie entweder Kriterium C oder Kriterium D erfüllen

B. Der Schmerz wird ausgelöst durch Husten, Pressen und/oder ein anderes Valsalva-Manöver und tritt ausschließlich in Verbindung damit auf

C. Plötzlicher Beginn

D. Der Schmerz hält 1 Sekunde bis 2 Stunden an

E. Die Episoden erfüllen nicht die ICHD-3 Kriterien für eine andere Kopfschmerzerkrankung

F. Nicht besser erklärt durch eine andere ICHD-3-Diagnose.

\subsection{Primärer Anstrengungskopfschmerz}

Früher verwendete Begriffe Primärer Kopfschmerz bei körperlicher Anstrengung; benigner Kopfschmerz bei körperlicher Anstrengung.

An anderer Stelle kodiert: Eine Migräne, die durch körperliche Anstrengung ausgelöst wurde, wird unter 1. Migräne entsprechend dem Typ oder Subtyp kodiert.

\section{Beschreibung:}

Kopfschmerz, hervorgerufen durch jede Form von körperlicher Anstrengung bei fehlender intrakraniellen Erkrankung.

\section{Diagnostische Kriterien:}

A. Mindestens 2 Kopfschmerzepisoden, die die Kriterien B und C erfüllen

B. Der Schmerz wird ausgelöst durch körperliche Anstrengung und tritt ausschließlich während oder nach einer solchen auf

C. Dauer $<48$ Stunden

D. Nicht besser erklärt durch eine andere ICHD-3-Diagnose1.

\section{Anmerkung:}

1. Es kommt zu Fällen symptomatischer Natur. Beim erstmaligen Auftreten eines Kopfschmerzes mit diesen Merkmalen ist der Ausschluss einer Subarachnoidalblutung, einer arteriellen Dissektion und eines reversiblen zerebralen Vasokonstriktionssyndroms obligatorisch. 


\section{Kommentar:}

Der 4.2 primäre Anstrengungskopfschmerz tritt bevorzugt bei hohen Temperaturen oder in großen Höhen auf. Subtypen wie der „Gewichtheber-Kopfschmerz" sind anerkannt, werden jedoch nicht eigenständig klassifiziert. Im Gegensatz zum 4.1 primären Hustenkopfschmerz, der durch kurzzeitige Anstrengungen (z. B. ein Valsalva-Manöver) ausgelöst werden kann, geht dem 4.2 primären Anstrengungskopfschmerz gewöhnlich eine anhaltende körperliche Anstrengung voraus.

Der Kopfschmerz hatte in der Vågå-Studie im Fall der meisten Befragten mit Kopfschmerz bei körperlicher Anstrengung einen pulsierenden Charakter (dies galt weniger für die Heranwachsenden unter den Kopfschmerzpatienten, von denen fast die Hälfte eine Kopfschmerzdauer von weniger als 5 Minuten aufwies).

Es gibt Berichte, dass er bei manchen Patienten durch die Einnahme von Ergotamintartrat verhindert werden kann. Indometacin scheint in der Mehrzahl der Fälle wirksam zu sein.

Die pathophysiologischen Mechanismen, die dem 4.2 primären Anstrengungskopfschmerz zugrunde liegen, sind unbekannt. Die meisten Untersucher gehen davon aus, dass dieser Kopfschmerz vaskulären Ursprungs sei und verfolgen die Hypothese, dass eine Überdehnung der Venen oder Arterien als Sekundärfolge körperlicher Anstrengung der schmerzauslösende Mechanismus sei. Die neuere wissenschaftliche Erkenntnis, dass Patienten mit einem 4.2 primären Anstrengungskopfschmerz eine deutlich höhere Prävalenz einer Klappeninsuffizienz der inneren Jugularvenen aufweisen (70\% gegenüber $20 \%$ aus der Kontrollgruppe) lässt darauf schließen, dass eine durch den venösen Rücktransport aus den Jugularvenen verursachte intrakranielle venöse Stauung bei der Pathophysiologie dieser Erkrankung eine Rolle spielen mag.

\subsubsection{Wahrscheinlicher primärer Anstrengungs- kopfschmerz \\ Diagnostische Kriterien:}

A. Vorliegen eines der folgenden Punkte:

1. Eine einmalige Kopfschmerzepisode, die die Kriterien B und $C$ erfüllt

2. Mindestens 2 Kopfschmerzepisoden, die Kriterium B, nicht aber Kriterium C erfüllen

B. Der Schmerz wird ausgelöst durch körperliche Anstrengung und tritt ausschließlich während oder nach einer solchen auf

C. Dauer $<48$ Stunden

D. Die Episoden erfüllen nicht die ICHD-3 Kriterien für eine andere Kopfschmerzerkrankung

E. Nicht besser erklärt durch eine andere ICHD-3-Diagnose.

\subsection{Primärer Sexualkopfschmerz}

Früher verwendete Begriffe Benigner Orgasmuskopfschmerz, Koituscephalgie, sexueller Kopfschmerz

An anderer Stelle kodiert: Ein postkoital auftretender orthostatischer Kopfschmerz sollte unter 7.2.3 Kopfschmerz zurückzuführen auf ein spontanes Liquorunterdrucksyndrom kodiert werden, da er wahrscheinlich auf ein Liquorleck zurückzuführen ist.
Beschreibung:

Kopfschmerz, der durch sexuelle Aktivität hervorgerufen wird. In der Regel beginnt der Kopfschmerz bei zunehmender sexueller Erregung als dumpfer, bilateraler Schmerz und intensiviert sich schlagartig während des Orgasmus. Intrakranielle Erkrankungen bestehen nicht.

\section{Diagnostische Kriterien:}

A. Mindestens 2 Schmerzepisoden im Kopf und/oder Hals, die die Kriterien B bis D erfüllen

B. Der Schmerz wird ausgelöst durch sexuelle Aktivität und tritt ausschließlich während einer solchen auf

C. Einer oder beide der folgenden Punkte sind erfüllt:

1. zunehmende Intensität mit zunehmender sexueller Erregung

2. schlagartige explosive Intensivierung unmittelbar vor dem oder beim Orgasmus

D. Schmerzdauer zwischen 1 Minute und 24 Stunden bei starker Intensität und/oder bis zu 72 Stunden bei leichterer Intensität

E. Nicht besser erklärt durch eine andere ICHD-3-Diagnose1;2.

Anmerkung:

1. 4.3 primärer Sexualkopfschmerz tritt nicht in Verbindung mit einer Bewusstseinstrübung, Erbrechen oder Sensibilitätsstörungen bzw. visuellen oder motorischen Symptomen auf, während dies bei sexuellem Kopfschmerz symptomatischer Natur durchaus der Fall sein kann. Beim erstmaligen Auftreten eines Kopfschmerzes bei sexueller Aktivität ist der Ausschluss einer Subarachnoidalblutung, einer intra- und extrakraniellen arteriellen Dissektion und eines reversiblen zerebralen Vasokonstriktionssyndroms (RCVS) obligatorisch.

2. Multiple explosive Kopfschmerzen bei sexuellen Aktivitäten sollten als 6.7.3 Kopfschmerz zurückzuführen auf ein reversibles zerebrales Vasokonstriktionsyndrom betrachtet werden, solange angiographische Untersuchungen (einschließlich konventioneller, Magnetresonanz- oder CT-Angiographie) oder die transkranielle Dopplerultrasonographie nichts Gegenteiliges bewiesen haben. Man beachte, dass Vasokonstriktionen im Frühstadium eines RCVS nicht unbedingt festzustellen sind; aus diesem Grund können Folgeuntersuchungen erforderlich sein.

Kommentar:

In die ICHD-1 und ICHD-2 waren zwei Subtypen (Präorgasmuskopfschmerz und Orgasmuskopfschmerz) aufgenommen worden, doch ist es in seitdem durchgeführten klinischen Studien nicht gelungen, diese voneinander abzugrenzen. Aus diesem Grund wird 4.3 primärer Sexualkopfschmerz nun als eine einzige Entität mit unterschiedlichen klinischen Erscheinungsbildern angesehen.

Neuere Untersuchungen haben gezeigt, dass bei bis zu $40 \%$ aller Fälle ein chronischer Verlauf über mehr als ein Jahr vorliegt.

Einige Patienten erleben nur einmal in ihrem Leben einen Anfall von 4.3 primärem Sexualkopfschmerz; bei ihnen sollte die Diagnose 4.3.1 wahrscheinlicher primärer Sexualkopfschmerz gestellt werden. Zur weiteren Erforschung dieses Kopfschmerztyps empfiehlt es sich, nur Patienten mit mindestens zwei Attacken in die Studie aufzunehmen. 
Die epidemiologische Forschung hat ferner gezeigt, dass ein 4.3 primärer Sexualkopfschmerz nur im sexuell aktiven Alter auftreten kann, sich bei Männern häufiger zeigt als bei Frauen (das Verhältnis rangiert zwischen 1,2:1 und 3:1), unabhängig von der Art von sexueller Aktivität ist, in den meisten Fällen nicht in Begleitung autonomer oder vegetativer Symptome auftritt, in zwei Dritteln der Fälle beidseits und in einem Drittel der Fälle einseitig und in $80 \%$ der Fälle diffus oder okzipital lokalisiert ist. Die Häufigkeit der Attacken in Verbindung mit 4.3 primärem Sexualkopfschmerz sollte immer mit der Häufigkeit der sexuellen Aktivität korrelieren.

\subsubsection{Wahrscheinlicher primärer Sexualkopfschmerz}

\section{Diagnostische Kriterien:}

A. Es ist wenigstens einer der folgenden Punkte erfüllt:

1. eine einmalige Kopfschmerzepisode, die die Kriterien B bis D erfüllt

2. mindestens 2 Kopfschmerzepisode, die Kriterium B erfüllen und entweder Kriterium C oder D, nicht jedoch beide

B. Der Schmerz wird ausgelöst durch sexuelle Aktivität und tritt ausschließlich während einer solchen auf

C. Einer oder beide der folgenden Punkte sind erfüllt:

1. zunehmende Intensität mit zunehmender sexueller Erregung

2. schlagartige explosive Intensivierung unmittelbar vor dem oder beim Orgasmus

D. Schmerzdauer zwischen 1 Minute und 24 Stunden bei starker Intensität und/oder bis zu 72 Stunden bei leichterer Intensität

E. Die Episoden erfüllen nicht die ICHD-3 Kriterien für eine andere Kopfschmerzerkrankung

F. Nicht besser erklärt durch eine andere ICHD-3-Diagnose.

\subsection{Primärer Donnerschlagkopfschmerz}

Früher verwendeter Begriff: Benigner Donnerschlagkopfschmerz.

An anderer Stelle kodiert: 4.1 Primärer Hustenkopfschmerz, 4.2 primärer Anstrengungskopfschmerz und 4.3 primärer Sexualkopfschmerz können allesamt das klinische Erscheinungsbild eines Donnerschlagkopfschmerzes aufweisen. Wird ein solcher Kopfschmerz ausschließlich einem dieser Auslöser zugeschrieben, sollte er entsprechend als einer dieser Kopfschmerztypen kodiert werden.

\section{Beschreibung:}

Plötzlich auftretender Kopfschmerz stärkster Intensität, der einem Kopfschmerz bei Ruptur eines intrakraniellen Aneurysmas ähnelt bei fehlender intrakranieller Pathologie.

Diagnostische Kriterien:

A. Heftiger Kopfschmerz, der die Kriterien B und C erfüllt

B. Plötzlicher Beginn; die maximale Intensität wird in $<1$ Minute erreicht

C. Dauer $\geq 5$ Minuten

D. Nicht besser erklärt durch eine andere ICHD-3-Diagnose 1;2.

\section{Anmerkung:}

1. Der Donnerschlagkopfschmerz tritt häufig in Verbindung mit ernsthaften intrakraniellen vaskulären Erkrankungen auf, insbesondere einer Subarachnoidalblutung. Eine solche und andere solche Erkrankungen wie eine intrazerebrale Blutung, zerebrale Venenthrombose, nicht-rupturierte vaskuläre Missbildung (meist Aneurysma), arterielle Dissektion (intra- und extrakraniell) und ein reversibles zerebrales Vasokonstriktionssyndrom (RCVS) sowie ein Hypophyseninfarkt müssen daher ausgeschlossen werden. Andere organische Ursachen eines Donnerschlagkopfschmerzes sind Meningitis, eine Kolloidzyste des dritten Ventrikels, eine spontane intrakranielle Hypotension und akute Sinusitis (besonders in Verbindung mit einem Barotrauma). Die Diagnose eines 4.4 primären Donnerschlagkopfschmerzes sollte nur im äußersten Notfall in Erwägung gezogen werden und nur wenn alle anderen organischen Ursachen nachweislich ausgeschlossen werden konnten. Das bedeutet eine normale zerebrale Bildgebung, darunter auch der Gehirngefäße, und/oder ein normaler Liquorbefund.

2. Vasokonstriktionen sind im Frühstadium eines RCVS nicht unbedingt festzustellen. Aus diesem Grund ist wahrscheinlicher primärer Donnerschlagkopfschmerz keine Diagnose, die gestellt werden sollte, nicht einmal temporär.

\section{Kommentar:}

Die Evidenz dafür, dass ein Donnerschlagkopfschmerz als eigenständige primäre Erkrankung existiert, ist nur schwach. Es sollte daher umgehend und sorgfältig nach einer zugrundeliegenden $\mathrm{Er}$ krankung gefahndet werden.

\subsection{Primärer kältebedingter Kopfschmerz Beschreibung:}

Kopfschmerz, der durch einen Kältereiz auf der Kopfoberfläche bzw. orale Aufnahme kalter Speisen oder Getränke oder Einatmung von etwas Kaltem ausgelöst wird.

\subsubsection{Kopfschmerz zurückzuführen auf einen äußeren Kältereiz}

\section{Beschreibung:}

Kopfschmerz nach Kontakt des ungeschützten Kopfbereichs mit einer sehr niedrigen Umgebungstemperatur.

\section{Diagnostische Kriterien:}

A. Mindestens 2 akute Kopfschmerzepisoden, die die Kriterien B und $C$ erfüllen

B. Der Schmerz wird ausgelöst durch einen äußerlichen Kältereiz am Kopf und tritt ausschließlich während eines solchen auf

C. Der Kopfschmerz legt sich innerhalb von 30 Minuten nach Beseitigung des Kältereizes

D. Nicht besser erklärt durch eine andere ICHD-3-Diagnose.

\section{Kommentar:}

Dieser Kopfschmerz geht auf eine Kühlung des Kopfes von außen zurück, die etwa bei sehr kaltem Wetter, beim Eintauchen in kaltes Wasser oder im Zuge einer Kryotherapie stattfindet. Einige Patienten entwickeln infolgedessen heftige, kurz anhaltende, stechende 
Kopfschmerzen mediofrontal, obwohl der Schmerz unilateral und temporal, frontal oder retro-orbital auftreten kann.

\subsubsection{Kopfschmerzen zurückzuführen auf Einnahme oder Inhalation eines Kältereizes}

Früher verwendete Begriffe Ice-cream Headache; Hirnfrost.

\section{Beschreibung:}

Kurz anhaltender frontaler oder temporaler Schmerz, der intensiv sein kann und bei hierfür anfälligen Personen dadurch ausgelöst wird, dass eine kalte Substanz (fest, flüssig oder gasförmig) in Berührung mit dem Gaumen und/oder der Rachenhinterwand kommt.

\section{Diagnostische Kriterien:}

A. Mindestens 2 Episoden eines akuten frontalen oder temporaIen Kopfschmerzes, der die Kriterien B und C erfüllt

B. Der Schmerz wird ausgelöst durch den Kontakt des Gaumens und/oder der Rachenhinterwand mit kalten Speisen oder Getränken oder das Einatmen von kalter Luft und tritt unmittelbar danach auf

C. Der Kopfschmerz legt sich innerhalb von 10 Minuten nach Beseitigung des Kältereizes

D. Nicht besser erklärt durch eine andere ICHD-3-Diagnose.

\section{Kommentar:}

4.5.2 Kopfschmerzen zurückzuführen auf Einnahme oder Inhalation eines Kältereizes ist in der Allgemeinbevölkerung weit verbreitet, besonders jedoch unter Menschen mit 1. Migräne. Eine besonders hohe Wahrscheinlichkeit dafür, dass dieser Kopfschmerz ausgelöst wird, besteht beim hastigen Verzehr von Slush-Eis, doch selbst der langsame Verzehr von normalem Speiseeis kann diese Wirkung zeigen.

Die Kopfschmerzen sind frontal oder temporal lokalisiert und treten meist beidseitig auf (können jedoch auch auf der Seite auftreten, auf der Personen mit einseitigen Kopfschmerzen als Bestandteil einer 1. Migräne gewöhnlich Migränekopfschmerzen erleben).

\subsubsection{Wahrscheinlicher kältebedingter Kopfschmerz} Diagnostische Kriterien:

A. Eine einmalige Kopfschmerzepisode, die die Kriterien B und C erfüllt

B. Der Schmerz wird ausgelöst durch Einwirkung eines äußeren Kältereizes am Kopf oder durch Kontakt mit einem Kältereiz durch orale Aufnahme oder Einatmung und tritt ausschließlich während oder unmittelbar hiernach auf

C. Der Kopfschmerz legt sich innerhalb von 10 Minuten nach Beseitigung des Kältereizes

D. Die Episoden erfüllen nicht die ICHD-3 Kriterien für eine andere Kopfschmerzerkrankung

E. Nicht besser erklärt durch eine andere ICHD-3-Diagnose.

Kommentar:

Kodierbare Subtypen sind 4.5.3.1 Kopfschmerz wahrscheinlich zurückzuführen auf einen äußeren Kältereiz und 4.5.3.2 Kopfschmerzen wahrscheinlich zurückzuführen auf Einnahme oder Inhalation eines Kältereizes.

\subsection{Kopfschmerz durch Einwirkung von Druck oder Zug auf den Kopf}

Beschreibung:

Kopfschmerz infolge anhaltender Druck- oder Zugeinwirkung auf das perikranielle Weichgewebe.

\section{Kommentar:}

4.6 Kopfschmerz durch Einwirkung von Druck oder Zug auf den Kopf ist eine primäre Kopfschmerzerkrankung, da die Druck- und Zugwirkung zu schwach ausgeprägt sind, um die Kopfhaut zu beschädigen; mit anderen Worten, es handelt sich um physiologische Reize.

\subsubsection{Kopfschmerz durch äußeren Druck Beschreibung:}

Kopfschmerz infolge einer anhaltenden Druckeinwirkung auf das perikranielle Weichgewebe, etwa durch ein enges Stirnband, einen eng sitzenden Hut oder Helm oder eine Schwimm- oder Taucherbrille ohne Schädigung der Kopfhaut.

\section{Diagnostische Kriterien:}

A. Mindestens 2 Kopfschmerzepisoden, die die Kriterien B bis D erfüllen

B. Der Schmerz wird ausgelöst durch anhaltenden äußeren Druck auf die Stirn oder Kopfhaut und tritt innerhalb 1 Stunde danach auf

C. Maximale Intensität an der von der Druckeinwirkung von außen betroffenen Stelle

D. Der Kopfschmerz legt sich innerhalb von 1 Stunde nach Lösen des äußerlichen Drucks

E. Nicht besser erklärt durch eine andere ICHD-3-Diagnose.

\subsubsection{Kopfschmerz durch äußeren Zug}

Früher verwendeter Begriff: Ponytail Headache (Kopfschmerz durch straff gebundenen Pferdeschwanz oder Zopf).

\section{Beschreibung:}

Kopfschmerz infolge einer anhaltenden Zugwirkung auf das perikranielle Weichgewebe ohne Schädigung der Kopfhaut.

Diagnostische Kriterien:

A. Mindestens 2 Kopfschmerzepisoden, die die Kriterien B bis D erfüllen

B. Der Schmerz wird ausgelöst durch anhaltende Zugwirkung auf die Kopfhaut und tritt ausschließlich während einer solchen auf

C. Maximale Intensität an der von der Zugwirkung betroffenen Stelle

D. Verschwindet innerhalb von 1 Stunde nach Entlastung von der Zugwirkung

E. Nicht besser erklärt durch eine andere ICHD-3-Diagnose.

Kommentar:

Die Kopfschmerzdauer variiert je nach Heftigkeit und Dauer der Zugwirkung. Zwar tritt der Kopfschmerz in maximaler Intensität an der Stelle auf, die von der Zugwirkung betroffen ist, doch breitet er sich auch auf andere Regionen des Kopfes aus. 


\subsubsection{Wahrscheinlicher Kopfschmerz durch Einwir- kung von Druck oder Zug auf den Kopf}

Diagnostische Kriterien:

A. Es ist wenigstens einer der folgenden Punkte erfüllt:

1. eine einmalige Kopfschmerzepisode, die die Kriterien B bis D erfüllt

2. mindestens 2 Kopfschmerzepisoden, die Kriterium B und entweder Kriterium C oder D, nicht jedoch beide erfüllen

B. Der Schmerz wird ausgelöst durch einen anhaltenden äußeren Druck auf die Stirn und/oder Kopfhaut oder eine Zugwirkung dort und tritt ausschließlich hierbei auf

C. Maximale Intensität an der von dem Druck oder der Zugwirkung betroffenen Stelle

D. Verschwindet innerhalb von 1 Stunde nach Entlastung von der Druck- oder Zugwirkung

E. Die Episoden erfüllen nicht die ICHD-3 Kriterien für eine andere Kopfschmerzerkrankung

F. Nicht besser erklärt durch eine andere ICHD-3-Diagnose.

Kommentar:

Kodierbare Subtypen sind 4.6.3.1 wahrscheinlicher Kopfschmerz durch äußeren Druck und 4.6.3.2 wahrscheinlicher Kopfschmerz durch äußeren Zug.

\subsection{Primärer stechender Kopfschmerz}

Früher verwendete Begriffe Eispickelschmerz, Jabs-and-joltsSyndrom, Needle-in-the-eye-Syndrom, periodische Ophthalmodynie; kurzzeitiger stechender Kopfschmerz.

\section{Beschreibung:}

Vorübergehende und umschriebene schmerzhafte Stiche im Kopf, die spontan ohne eine organische Erkrankung der betreffenden Strukturen oder eines Hirnnervs auftreten.

\section{Diagnostische Kriterien:}

A. Kopfschmerz in Form einzelner Stiche oder einer Serie von Stichen, die die Kriterien B und C erfüllen

B. Die einzelnen Stiche halten nur wenige Sekunden an1

C. Die Stiche wiederholen sich mit einer unregelmäßigen Frequenz von einem Stich bis zu vielen pro Tag2

D. Keine kranio-autonomen Symptome

E. Nicht besser erklärt durch eine andere ICHD-3-Diagnose.

\section{Anmerkung:}

In Studien hielten 80 \% der Stiche 3 oder weniger Sekunden an; in seltenen Fällen halten die Stiche 10 bis 120 Sekunden lang an.

2. Die Attackenhäufigkeit ist im Allgemeinen gering, eine oder wenige pro Tag. In seltenen Fällen können die Stiche über mehrere Tage hinweg wiederholt auftreten. Ein Status mit einer Dauer der aktiven Periode von bis zu 1 Woche wurde beschrieben.

\section{Kommentar:}

Feldstudien haben die Gültigkeit dieser diagnostischen Kriterien für einen 4.7 primären stechenden Kopfschmerz bestätigt. Diese ermöglichen die Diagnose der meisten von stechendem Schmerz charakterisierten primären Kopfschmerzen, die nicht in der ICHD2 klassifiziert wurden.

Bei einem 4.7 primären stechenden Kopfschmerz sind in $70 \%$ der Fälle extratrigeminale Regionen beteiligt. Er kann von einer Region zu einer anderen weiterwandern. Die Lokalisation der Stiche kann innerhalb der Kopfhälfte und zwischen den Kopfhälften wechseln: bei nur einem Drittel der Patienten hat er eine feste Position. Wenn der Schmerz ausschließlich auf eine bestimmte Region begrenzt ist, setzt die Diagnose den Ausschluss einer strukturellen Veränderung am Orte des Schmerzes bzw. im Versorgungsbereich des betreffenden Hirnnervs voraus.

Bei einigen Patienten kommt es zu Begleitsymptomen, die sich jedoch nicht auf kranio-autonome Symptome erstrecken. Letztere tragen dazu bei, einen 4.7 primären stechenden Kopfschmerz von einem 3.3 SUNA-Syndrom abzugrenzen.

4.7 primärer stechender Kopfschmerz tritt häufiger bei Personen auf, die unter 1 . Migräne (etwa $40 \%$ ) leiden, wobei er dann in der Regel auf der Seite auftritt, auf der auch gewöhnlich die Kopfschmerzen verspürt werden.

\subsubsection{Wahrscheinlicher primärer stechender Kopf- schmerz}

\section{Diagnostische Kriterien:}

A. Spontan in Form einzelner Stiche oder einer Serie von Stichen auftretender Kopfschmerz

B. Nicht mehr als zwei der folgenden Punkte sind erfüllt:

1. die einzelnen Stiche halten nur wenige Sekunden an

2. die Stiche wiederholen sich mit einer unregelmäßigen Frequenz von einem Stich bis zu vielen pro Tag

3. keine kranio-autonomen Symptome

C. Die Episoden erfüllen nicht die ICHD-3 Kriterien für eine andere Kopfschmerzerkrankung

D. Nicht besser erklärt durch eine andere ICHD-3-Diagnose.

\subsection{Münzkopfschmerz}

Früher verwendeter Begriff: Münzförmiger Kopfschmerz.

Beschreibung:

Schmerzen stark variabler Dauer, doch oft chronisch, in einem kleinen, umschriebenen Areal des Kopfes bei fehlender struktureller Läsion der umgebenden Strukturen.

\section{Diagnostische Kriterien:}

A. Kontinuierlich vorhandener oder intermittierender Kopfschmerz, der Kriterium B erfüllt

B. Der Kopfschmerz wird ausschließlich an einer bestimmten Stelle der Kopfhaut wahrgenommen und weist alle der 4 folgenden Merkmale auf:

1. scharf umrissen

2. konstante Größe und Form

3. rund oder elliptisch

4. 1 bis $6 \mathrm{~cm}$ Durchmesser

C. Nicht besser erklärt durch eine andere ICHD-3-Diagnose1. 
Anmerkung:

1. Sonstige Ursachen, insbesondere strukturelle und dermatologische Läsionen, wurden aufgrund der Vorgeschichte, der körperlichen Untersuchung und von sonstigen entsprechenden Untersuchungen ausgeschlossen.

\section{Kommentar:}

Das schmerzhafte Areal kann an jeder Stelle der Kopfhaut lokalisiert sein, es befindet sich am häufigsten jedoch in der Parietalregion. In seltenen Fällen ist der 4.8 Münzkopfschmerz bi- oder multifokal, wobei jeder symptomatische Bereich sämtliche Merkmale eines Münzkopfschmerzes behält.

In der Regel leichte bis mittlere, gelegentlich jedoch starke Schmerzintensität. Spontane oder getriggerte Schmerzexazerbationen können den Grundschmerz überlagern.

Die Schmerzdauer ist hochgradig variabel: in bis zu 75 \% der publizierten Fälle ist die Erkrankung chronisch (seit mehr als 3 Monaten vorliegend), doch wurden auch schon Fälle mit einer Dauer von Sekunden, Minuten, Stunden oder Tagen beschrieben.

Das Schmerzareal kann eine variable Kombination aus Hypästhesie, Dysästhesie, Parästhesie und/oder Schmerzempfindlichkeit aufweisen.

\subsubsection{Wahrscheinlicher Münzkopfschmerz}

Diagnostische Kriterien:

A. Kontinuierlich vorhandener oder intermittierender Kopfschmerz, der Kriterium B erfüllt

B. Der Kopfschmerz wird ausschließlich an einer bestimmten Stelle der Kopfhaut wahrgenommen und weist alle der 4 folgenden Merkmale auf:

1. scharf umrissen

2. konstante Größe und Form

3. rund oder elliptisch

4. 1 bis $6 \mathrm{~cm}$ Durchmesser

C. Die Episoden erfüllen nicht die ICHD-3 Kriterien für eine andere Kopfschmerzerkrankung

D. Nicht besser erklärt durch eine andere ICHD-3-Diagnose.

\subsection{Schlafgebundener Kopfschmerz}

Früher verwendete Begriffe Hypnic-Headache-Syndrom, „alarm clock“ headache-syndrome.

\section{Beschreibung:}

Häufig wiederkehrende Kopfschmerzattacken, die ausschließlich im Schlaf beginnen, den Patienten aufwecken und bis zu 4 Stunden lang anhalten, ohne charakteristische Begleitsymptome und keiner anderen Erkrankung zuzuschreiben.

\section{Diagnostische Kriterien:}

A. Wiederkehrende Kopfschmerzattacken, die die Kriterien B bis D erfüllen

B. Der Kopfschmerz entsteht ausschließlich im Schlaf und weckt den Patienten

C. Die Attacken treten seit $>3$ Monaten an $\geq 10$ Tagen/Monat auf

D. Die Attacken halten 15 Minuten bis 4 Stunden nach dem Aufwachen an
E. Keine kranio-autonomen Symptome und keine Unruhe

F. Nicht besser erklärt durch eine andere ICHD-3-Diagnose1;2.

Anmerkung:

1. Die Unterscheidung von einem der Typen oder Subtypen der 3. trigemino-autonomen Kopfschmerzerkrankungen, insbesondere von einem 3.1 Clusterkopfschmerz, ist für die erfolgreiche Behandlung erforderlich.

2. Andere mögliche Ursachen von im Schlaf entstehenden Kopfschmerzen, die zum Aufwachen führen, sollten ausgeschlossen werden, unter besonderer Berücksichtigung von Schlafapnoe, nächtlicher Hypotonie, Unterzuckerung und Medikamentenübergebrauch; intrakraniellen Erkrankungen müssen ebenfalls ausgeschlossen werden. Das Vorliegen eines Schlafapnoe-Syndroms schließt jedoch nicht unbedingt die Diagnose eines 4.9 schlafgebundenen Kopfschmerzes aus.

\section{Kommentar:}

Eine aktuelle Studie weist darauf hin, dass die hier in der ICHD-3 beta eingeführten Kriterien für einen 4.9 schlafgebundenen Kopfschmerz exakter sind als die in der ICHD-2.

Der 4.9 schlafgebundene Kopfschmerz beginnt meist nach dem 50. Lebensjahr, kann jedoch auch bei jüngeren Menschen auftreten.

Der Schmerz ist meist von leichter bis mittelstarker Intensität, ein Fünftel der Patienten berichtet allerdings von starken Schmerzen. Der Schmerz ist bei etwa zwei Dritteln der Betroffenen bilateral. Die Attacken halten meist 15 bis 180 Minuten an, in Einzelfällen sind auch längere Zeiten beschrieben.

Der Schmerz ist in den meisten Fällen anhaltend, mit täglichen oder beinahe täglichen Kopfschmerzen, doch kann auch ein episodischer Subtyp (an<15 Tagen/Monat) auftreten.

Obwohl man ursprünglich davon ausging, dass die Merkmale eines 4.9 schlafgebundenen Kopfschmerzes im allgemeinen spannungstypartig waren, ergaben neuere Studien, dass Patienten migräneartige Merkmale aufweisen konnten und dass einige während der Kopfschmerzattacken Übelkeit verspürten.

Der Beginn des 4.9 schlafgebundenen Kopfschmerzes weist keinen Zusammenhang mit dem Schlafstadium auf. Eine aktuelle MRT-Untersuchung ergab eine Volumenreduzierung der grauen Substanz im Hypothalamus von Patienten mit 4.9 schlafgebundenem Kopfschmerz.

Lithium, Koffein, Melatonin und Indometacin waren in Einzelfällen wirksam.

\subsubsection{Wahrscheinlicher schlafgebundener Kopf- schmerz}

Diagnostische Kriterien:

A. Wiederkehrende Kopfschmerzattacken, die Kriterium B erfüllen

B. Der Kopfschmerz entsteht ausschließlich im Schlaf und weckt den Patienten

C. Nur zwei Punkte von folgenden sind erfüllt:

1. die Attacken treten seit $>3$ Monaten an $\geq 10$ Tagen/Monat auf

2. die Attacken dauern zwischen 15 Minuten und 4 Stunden nach dem Aufwachen

3. keine kranio-autonomen Symptome oder Unruhe 
D. Die Episoden erfüllen nicht die ICHD-3 Kriterien für eine andere Kopfschmerzerkrankung

E. Nicht besser erklärt durch eine andere ICHD-3-Diagnose.

\subsection{Neu aufgetretener täglicher Kopf- schmerz (NDPH)}

Früher verwendete Begriffe Chronischer Kopfschmerz mit akutem Beginn; chronischer de novo Kopfschmerz.

\section{Beschreibung:}

Ein anhaltender Kopfschmerz, der von Anfang an täglich auftritt, an den eine klare Erinnerung besteht. Dem Schmerz fehlen charakteristische Merkmale. Er kann migräneartig oder spannungstypartig sein oder Elemente von beiden aufweisen.

\section{Diagnostische Kriterien:}

A. Anhaltender Kopfschmerz, der die Kriterien B und C erfüllt

B. Eindeutig und klar erinnertes erstmaliges Auftreten, wobei der Schmerz sich innerhalb von 24 Stunden zum kontinuierlichen Dauerschmerz ohne Remissionen entwickelt

C. Besteht $>3$ Monate

D. Nicht besser erklärt durch eine andere ICHD-3-Diagnose $1 ; 2 ; 3 ; 4$.

\section{Anmerkung:}

Der 4.10 neu aufgetretene tägliche Kopfschmerz ist von daher einmalig, dass er von Anfang an täglich auftritt und sehr schnell keine Remissionen mehr aufweist sowie im Regelfall bei Personen mit keiner Vorgeschichte von Kopfschmerzen auftritt. Patienten mit dieser Erkrankung erinnern sich unweigerlich eindeutig an diese Art von Beginn und können diesen genau beschreiben; ansonsten sollte eine andere Diagnose gestellt werden. Dennoch sind Patienten mit Kopfschmerz in der Vorgeschichte (1. Migräne oder 2. Kopfschmerz vom Spannungstyp) nicht von dieser Diagnose ausgeklammert, sie sollten jedoch keine sich steigernde Kopfschmerzfrequenz vor dessen Beginn beschreiben. Ebenso sollten Patienten mit Kopfschmerzen in der Vorgeschichte keine Verschlechterung in Verbindung mit oder gefolgt von Medikamentenübergebrauch beschreiben.

2. 4.10 Neu aufgetretener täglicher Kopfschmerz kann Merkmale aufweisen, die entweder an 1. Migräne oder 2. Kopfschmerz vom Spannungstyp denken lassen. Obwohl auch Kriterien für eine 1.3 chronische Migräne und/oder einen 2.3 chronischen Kopfschmerz vom Spannungstyp erfüllt sein mögen, lautet die Standarddiagnose 4.10 neu aufgetretener täglicher Kopfschmerz, wann immer die Kriterien für diese Erkrankung erfüllt sind. Sind jedoch sowohl die Kriterien für 4.10 neu aufgetretener täglicher Kopfschmerz und 3.4 Hemicrania continua erfüllt, ist Letzteres die definitive Diagnose.

3. Ein akuter Medikamentengebrauch kann die Grenzen überschreiten, die für einen 8.2 Kopfschmerz zurückzuführen auf einen Medikamentenübergebrauch als ursächlich beschrieben werden. In solchen Fällen kann nicht die Diagnose eines 4.10 neu aufgetretenen täglichen Kopfschmerzes gestellt werden, es sei denn, der Beginn der täglichen Kopfschmerzen liegt zeitlich eindeutig vor dem Medikamentenübergebrauch. Ist das der Fall, sollten beide Diagnosen, 4.10 neu aufgetretener täglicher Kopfschmerz und 8.2 Kopfschmerz zurückzuführen auf einen Medikamentenübergebrauch gestellt werden.

4. In sämtlichen Fällen sollten andere sekundäre Kopfschmerzen wie etwa 5.1 akuter Kopfschmerz zurückzuführen auf eine traumatische Kopfverletzung, 7.1 Kopfschmerz zurückzuführen auf eine Liquordrucksteigerung und 7.2 Kopfschmerz zurückzuführen auf einen Liquorunterdruck durch entsprechende Untersuchungen ausgeschlossen werden.

\section{Kommentar:}

Der 4.10 neu aufgetretene tägliche Kopfschmerz weist zwei Subtypen auf: den einer selbstlimitierenden Erkrankung, die innerhalb von einigen Monaten ohne Behandlung verschwindet, und den einer refraktären Form, die auch aggressiven Therapieschemata gegenüber resistent ist. Diese beiden Verlaufsformen werden nicht eigenständig kodiert.

\subsubsection{Wahrscheinlicher neu aufgetretener täglicher Kopfschmerz}

Diagnostische Kriterien:

A. Anhaltender Kopfschmerz, der die Kriterien B und C erfüllt

B. Eindeutig und klar erinnertes erstmaliges Auftreten, wobei der Schmerz sich innerhalb von 24 Stunden zum kontinuierlichen Dauerschmerz ohne Remissionen entwickelt

C. Besteht > 3 Monate

D. Die Episoden erfüllen nicht die ICHD-3 Kriterien für eine andere Kopfschmerzerkrankung

E. Nicht besser erklärt durch eine andere ICHD-3-Diagnose.

\section{Literatur}

\subsection{Primärer Hustenkopfschmerz}

Chen PK, Fuh JL, Wang SJ. Cough headache: a study of 83 consecutive patients. Cephalalgia 2009; 29: 1079-1085.

Chen YY, Lirng JF, Fuh JL, Chang FC, Cheng HC, Wang SJ. Primary cough headache is associated with posterior fossa crowdedness: A morphometric MRI study. Cephalalgia 2004; 24: 694-699.

Cohen ME, Duffner PK, eds. Brain tumors in children. Principles of diagnosis and treatment. New York: Raven Press 1994.

Cutrer FM, Boes C]. Cough, exertional, and sex headaches. Neurol Clin 2004; 22: 133-149.

Ozge C, Atiş S, Ozge A, Nass Duce M, Saraçoğlu M, Saritaş E. Cough headache: frequency, characteristics and the relationship with the characteristics of cough. Eur J Pain 2005; 9: 383-388.

Pascual J. Primary cough headache. Curr Pain Headache Rep 2005; 9: 272-276.

Pascual J, González-Mandly A, Martín R, Oterino A. Headaches precipitated by cough, prolonged exercise or sexual activity: a prospective etiological and clinical study. J Headache Pain 2008; 9: 259-266.

Pascual J. Iglesias F, Oterino A, Vazques-Barquero A, Berciano J. Cough, exertional, and sexual headaches: an analysis of 72 benign and symptomatic cases. Neurology 1996; 46: 1520-1524.

Perini F, Toso V. Benign cough "cluster" headache. Cephalalgia 1998; 18: 493-494.

Raskin NH. The cough headache syndrome treatment. Neurology 1995; 45 : 1784. 


\subsection{Primärer Anstrengungskopfschmerz}

Buzzi MG, Formisano R, Colonnese C, et al. Chiari-associated exertional, cough and sneeze headache responsive to medical therapy. Headache 2003; 43: 404-406.

Chen SP, Fuh JL, Lu SR, Wang SJ. Exertional headache: a survey of 1963 adolescents. Cephalalgia 2009; 29: 401-407.

Doepp F, Valdueza JM, Schreiber SJ. Incompetence of internal jugular valve in patients with primary exertional headache: a risk factor? Cephalalgia 2008; 28: 182-185.

Edis RH, Silbert PL. Sequential benign sexual headache and exertional headache (letter). Lancet 1988; 30: 993.

Green MW. A spectrum of exertional headaches. Headache 2001; 4: 1085-1092.

Heckmann JG, Hilz MJ, Muck-Weymann M, Neundorfer B. Benign exertional headache/benign sexual headache: a disorder of myogenic cerebrovascular autoregulation? Headache 1997; 37: 597-598.

Lance JW, Lambros J. Unilateral exertional headache as asymptom of cardiac ischemia. Headache 1998; 38: 315-316.

Lipton RB, Lowenkopf T, Bajwa ZH, Leckie RS, Ribeiro S,Newman LC, Greenberg MA. Cardiac cephalgia: a treatable form of exertional headache. Neurology 1997; 49: 813-816.

McCrory P. Recognizing exercise-induced headache. Phys Sports Med 1997; 25: 33-43.

Pascual J, Iglesias F, Oterino A, Vazquez-Barquero A, Berciano]. Cough, exertional, and sexual headaches: an analysis of 72 benign and symptomatic cases. Neurology 1996; 46: 1520-1524.

Silbert PL, Edis RH, Stewart-Wynne EG, Gubbay SS. Benign vascular sexual headache and exertional headache: interrelationships and long term prognosis. J Neurol Neurosurg Psychiat 1991; 54: 417-421.

Sjaastad O, Bakketeig LS. Exertional headache. I. Vågå study of headache epidemiology. Cephalalgia 2002; 22: 784-790.

Sjaastad O, Bakketeig LS. Exertional headache-II. Clinical features Vågå study of headache epidemiology. Cephalalgia 2003; 23: 803-807.

Wang SJ, Fuh JL. The "Other" headaches: Primary cough, exertion, sex, and primary stabbing headaches. Curr Pain Headache Rep 2010; 14: 41-46.

\subsection{Primärer Sexualkopfschmerz}

Biehl K, Evers S, Frese A. Comorbidity of migraine and headache associated with sexual activity. Cephalalgia 2007; 27: 1271-1273.

Chakravarty A. Primary headaches associated with sexual activity - some observations in Indian patients. Cephalalgia 2006; 26: 202-207.

Frese A, Eikermann A, Frese K, Schwaag S, Husstedt IW, Evers S. Headache associated with sexual activity: demography, clinical features, and comorbidity. Neurology 2003; 61: 796-800.

Frese A, Rahmann A, Gregor N, Biehl K, Husstedt IW, Evers S. Headache associated with sexual activity: prognosis and treatment options. Cephalalgia 2007; 27: 1265-1270.

Kumar KL, Reuler JB. Uncommon headaches: diagnosis and treatment. J Gen Int Med 1993; 8: 333-341.

Lance JW. Headaches related to sexual activity. J Neurol Neurosurg Psychiat 1976; 39: 1226-1230.

Landtblom AM, Fridriksson S, Boivie J, Hillman J, Johansson G, Johansson I. Sudden onset headache: a prospective study of features, incidence and causes. Cephalalgia 2002; 22: 354-360.

Lundberg PO, Osterman PO. Intercourse and headache. In: Genazzani AR, Nappi G, Facchinetti F, Martignoni E (eds). Pain and reproduction. Parthenon Publishing 1988: 149-153.

Pascual J, Iglesias F, Oterino A, Vazquez-Barquero A, Berciano J. Cough, exertional, and sexual headaches: an analysis of 72 benign and symptomatic cases. Neurology 1996; 46: 1520-1524.
Pascual J, González-Mandly A, Martín R, Oterino A. Headaches precipitated by cough, prolonged exercise or sexual activity: a prospective etiological and clinical study. J Headache Pain 2008; 9: 259-266.

Yeh YC, Fuh JL, Chen SP, Wang SJ. Clinical features, imaging findings and outcomes of headache associated with sexual activity. Cephalalgia 2010; 30: 1329-1335.

\subsection{Primärer Donnerschlagkopfschmerz}

Bassi P, Bandera R, Loiero M, Tognoni G, Mangoni A. Warning signs in subarachnoid hemorrhage: a cooperative study. Acta Neurol Scand 1991; 84: $277-281$.

Chen SP, Fuh JL, Liang JF, Chang FC, Wang SJ. Recurrent Primärer Donnerschlagkopfschmerz and benign CNS angiopathy: spectra of the same disorder? Neurology 2006; 67: 2164-2169.

Dodick DW, Brown RD, Britton JW, Huston J. Nonaneurysmal thunderclap headache with diffuse, multifocal, segmental and reversible vasospasm. Cephalalgia 1999; 19: 118-123.

Garg RK. Recurrent thunderclap headache associated with reversible vasospasm causing stroke. Cephalalgia 2001; 21: 78-79.

Landtblom AM, Fridriksson S, Boivie J, Hillman J, Johansson G, Johansson I. Sudden onset headache: a prospective study of features incidence and causes. Cephalalgia 2002; 22: 354-360.

Linn FHH, Rinkel GJE, Algra A, van Gijn J. Headache characteristics in subarachnoid haemorrhage and benign thunderclap headache. J Neurol Neurosurg Psychiat 1998; 65: 791-793.

Linn FHH, Rinkel GJE, Algra A, van Gijn J. Follow-up of idiopathic thunderclap headache in general practice. J Neurol 1999; 246: 946-948.

Linn FHH, Wijdicks EFM. Causes and management of thunderclap headache: a comprehensive review. The Neurologist 2002; 8: 279-289.

Lu SR, Liao YC, Fuh JL, Lirng JF, Wang SJ. Nimodipine for treatment of primary thunderclap headache. Neurology 2004;2:414-1416.

Markus HS. A prospective follow-up of thunderclap headache mimicking subarachnoid haemorrhage. J Neurol Neurosurg Psychiatry 1991; 54: 1117-1125.

Mauriño J, Saposnik G, Lepera S, Rey RC, Sica RE. Multiple simultaneous intracerebral haemorrhages. Arch Neurol 2001; 58: 629-632.

Nowak DA, Rodiek SO, Henneken S, Zinner J, Schreiner R, Fuchs H-H, Topka $\mathrm{H}$. Reversible segmental cerebral vasoconstriction (Call-Fleming syndrome): are calcium channel inhibitors a potential treatment option? Cephalalgia 2003; 23: 218-222.

Schwedt TJ, Matharu MS, Dodick DW. Thunderclap headache. Lancet-Neurol. 2006; 5: 621-631.

Slivka A, Philbrook B. Clinical and angiographic features of thunderclap headache. Headache 1995; 35: 1-6.

Sturm JW, Macdonell RAL. Recurrent thunderclap headache associated with reversible intracerebral vasospasm causing stroke. Cephalalgia 2000; 20: 132-135.

Wijdicks EFM, Kerkhoff H, van Gjin J. Cerebral vasospasm and unruptured aneurysm in thunderclap headache. Lancet 1988; ii: 1020.

Witham TF, Kaufmann AM. Unruptured cerebral aneurysm producing a thunderclap headache. Am J Emergency Med 2000; 1: 88-90.

\subsection{Primärer kältebedingter Kopfschmerz}

Bird N, MacGregor A, Wilkinson MIP. Ice-cream headache - site, duration, and relationship to migraine. Headache 1992; 32: 35-38.

Burkhart CG, Burkhart CN. Ice cream headaches with cryotherapy of actinic keratoses. Int J Dermatol 2006; 45: 1116-1117.

Drummond PD, Lance JW.Neurovascular disturbances in headache patients. Clin Exp Neurol 1984; 20: 93-99.

Fasano VA, Broggi G, Lo Russo G, Zeme S. Headache induced by freezing external carotid artery branches. Adv Neurol 1982; 33: 399.

Fuh JL, Wang SJ, Lu SR, Juang KD. Ice-cream headache - a large survey of 8359 adolescents. Cephalalgia 2003; 23: 977-981. 
Kaczorowski M, Kaczorowski J. Ice-cream evoked headaches (ICE-H) study: randomised trial of accelerated versus cautious ice-cream eating regimen. BMJ 2002; 21: 1445-1446.

Mattsson P. Headache caused by drinking cold water is common and related to active migraine. Cephalalgia 2001; 21: 230-235.

Raskin NH, Knittle SC. Ice cream headache and orthostatic symptoms in patients with migraine. Headache 1976; 16: 222-225.

Selekler HM, Erdogan MS, Budak F. Prevalence and clinical characteristics of an experimental model of 'ice-cream headache' in migraine and episodic tension-type headache patients. Cephalalgia 2004; 24: 293-297.

\subsection{Kopfschmerz durch Einwirkung von Druck oder Zug auf den Kopf}

Blau JN. Ponytail headache: a pure extracranial headache. Headache 2004; 44: 411-413.

Krymchantowski AV. Headaches due to external compression. Curr Pain Headache Rep 2010; 14: 321-324.

Pestronk A, Pestronk S. Goggle migraine. N Engl J Med 1983; 308: 226-227.

\subsection{Primärer stechender Kopfschmerz}

Dangond F, Spierings EL. Idiopathic stabbing headaches lasting a few seconds. Headache 1993; 33: 257-258.

Fuh JL, Kuo KH, Wang SJ: Primary stabbing headache in a headache clinic. Cephalalgia 2007; 27: 1005-1009.

Fusco C, Pisani F, Faienza C. Idiopathic stabbing headache: clinical characteristics of children and adolescents. Brain Develop 2003; 25: 237-240.

Lee M, Chu MK, Lee J, Yoo J, Song HK. Field testing primary stabbing headache criteria according to the $3 \mathrm{rd}$ beta edition of International Classification of Headache Disorders: a clinic-based study. J Headache Pain 2016; 17: 21.

Martins IP, Parreira E, Costa I. Extratrigeminal ice-pick status. Headache 1995; 35: 107-110.

Pareja JA, Ruiz J, de Isla C, et al: Idiopathic stabbing headache (jabs and jolts syndrome). Cephalalgia 1996; 16: 93-96.

Raskin NH, Schwartz RK: Icepick-like pain. Neurology 1980; 30: 203-205.

Selekler HM, Budak F: Idiopathic stabbing headache and experimental ice cream headache (short-lived headaches). Eur Neurol 2004; 51: 6-9.

Shin JH, Song HK, Lee JH, Kim WK, Chu MK. Paroxysmal stabbing headache in the multiple dermatomes of the head and neck: a variant of primary stabbing headache or occipital neuralgia? Cephalalgia 2007; 27: 1101-1108.

Sjaastad O, Pettersen H, Bakketeig LS. The Vågå study; epidemiology of headache I: the prevalence of ultrashort paroxysms. Cephalalgia 2001; 21:207-215.

Sjaastad, O, Pettersen, H, Bakketeig, LS: Long-lasting cephalic jabs (?) The Vågå study of headache epidemiology. Cephalalgia 2005; 25: 581-592.

Soriani S, Battistella PA, Arnaldi C, De Carlo L, Cernetti R, Corra S, Tosato G. Juvenile idiopathic stabbing headache. Headache 1996; 36: 565-567.

\subsection{Münzkopfschmerz}

Cuadrado ML, Valle B, Fernández de las Peñas C, Barriga FJ, Pareja JA. Bifocal nummular headache: the first three cases. Cephalalgia 2009; 29 : 583-586.

Cuadrado ML, Valle B, Fernández-de-las-Peñas C et al. Pressure pain sensitivity of the head in patients with nummular headache: a cartographic study. Cephalalgia 2010; 30: 200-206.

Fernández-de-las Peñas C, Cuadrado ML, Barriga FJ, Pareja JA. Local decrease of pressure pain threshold in nummular headache. Headache 2006: 46: 1195-1198.

Fernández-de-las-Peñas C, Cuadrado ML, Barriga FJ, Pareja JA. Pericranial tenderness is not related to nummular headache. Cephalalgia 2007; 27: $182-186$.
Grosberg BM, Solomon S, Lipton RB. Nummular headache. Curr Pain Headache Rep 2007; 11: 310-312.

Guerrero AL, Cortijo E, Herrero-Velázquez S, Mulero P, Miranda S, Peñas ML, Pedraza MI, Fernández R. Nummular headache with and without exacerbations: comparative characteristics in a series of 72 patients. Cephalalgia 2012; 32: 649-653.

Moon J, Ahmed K, Garza I. Case series of sixteen patients with nummular headache. Cephalalgia 2010; 12: 1527-1530.

Pareja JA, Caminero AB, Serra J, Barriga FJ, Dobato JL, Barón M, Vela L, Sánchez del Río M. Numular headache: a coin-shaped cephalgia. Neurology 2002; 58: 1678-1679.

Pareja JA, Cuadrado ML, Fernández de las Peñas C, Nieto C, Sols M, Pinedo M. Nummular headache with trophic changes inside the painful area. Cephalalgia 2008; 28: 186-190.

Pareja JA, Montojo T, Alvarez M. Nummular Headache Update. Curr Neurol Neurosci Rep. 2012; 12: 118-124.

Pareja JA, Pareja J, Barriga FJ, Barón M, Dobato JL, Pardo J. Sánchez C, Vela L. Nummular headache. A prospective series of 14 new cases. Headache 2004; 44: 611-614.

Ruscheweyh R, Buchheister A, Gregor N, Jung A, Evers S. Nummular headache: six new cases and lancinating pain attacks as possible manifestation. Cephalalgia 2010; 30: 249-253.

\subsection{Schlafgebundener Kopfschmerz}

Centonze V, D’Amico D, Usai S, Causarano V, Bassi A, Bussone G. First Italian case of hypnic headache, with literature review and discussion of nosology. Cephalalgia 2001; 21: 71-74.

Dodick DW. Polysomnography in hypnic headache syndrome. Headache 2000; 40: 748-752.

Dodick DW, Jones JM, Capobianco DJ. Hypnic headache: another indomethacin-responsive headache syndrome? Headache 2000; 40: 830-835.

Donnet A, Lantéri-Minet M. A consecutive series of 22 cases of hypnic headache in France. Cephalalgia 2009; 29: 928-934.

Evers S, Goadsby PJ. Hypnic headache: clinical features, pathophysiology, and treatment. Neurology 2003; 60: 905-909.

Gil-Gouveia R, Goadsby PJ. Secondary “hypnic headache". J Neurol 2007; 254: 646-654.

Holle D, Naegel S, Krebs S, Gaul C, Gizewski E, Diener HC, Katsarava Z, Obermann M. Hypothalamic gray matter volume loss in hypnic headache. Ann Neurol 2011; 69: 533-539.

Holle D, Naegel S, Krebs S, Katsarava Z, Diener HC, Gaul C, Obermann M. Clinical characteristics and therapeutic options in hypnic headache. Cephalalgia 2010; 30: 1435-1442.

Holle D, Wessendorf TE, Zaremba S, Naegel S, Diener HC, Katsarava Z, Gaul C, Obermann M. Serial polysomnography in hypnic headache. Cephalalgia 2011; 31: 286-290.

Liang JF, Fuh JL, Yu HY, Hsu CY, Wang SJ. Clinical features, polysomnography and outcome in patients with hypnic headache. Cephalalgia 2008; 28 : 209-215.

Liang JF, Wang SJ. Hypnic headache: A review of clinical features, therapeutic options and outcomes. Cephalalgia 2014; 34: 795-805.

Newman LC, Lipton RB, Solomon S. The hypnic headache syndrome: a benign headache disorder of the elderly. Neurology 1990; 40: 1904-1905.

Peres MF, Masruha MR, Zukerman E, Moreira-Filho CA, Cavalheiro EA. Potential therapeutic use of melatonin in migraine and Andere Kopfschmerzerkrankungen. Expert Opin Investig Drugs 2006; 15: 367-375.

Raskin NH. The hypnic headache syndrome. Headache 1988; 28: 534-536.

\subsection{Neu aufgetretener täglicher Kopfschmerz}

Bigal ME, Lipton RB, Tepper SJ, Rapoport AM, Sheftell FD. Primary chronic daily headache and its subtypes in adolescents and adults. Neurology 2004; 14: 843-847. 
Bigal ME, Rapoport AM, Tepper SJ, Sheftell FD, Lipton RB. The classification of chronic daily headache in adolescents - a comparison between the second edition of the international classification of headache disorders and alternative diagnostic criteria. Headache 2005; 45:582-589.

Castillo J, Munoz P, Guitera V, Pascual J. Epidemiology of chronic daily headache in the general population. Headache 1999; 39: 190-196.

Chakravarty A: Chronic daily headache in children and adolescents: a clinic based study from India. Cephalalgia 2005; 25: 795-800.

Donnet A, Levrier O: A consecutive series of ten cases of new daily persistent headache: clinical presentation and morphology of the venous system. Neurology 2009; 72: A419.

Evans RW: New daily persistent headache. Curr Pain Headache Rep 2003; 7: 303-307.

Evans RW, Rozen TD. Etiology and treatment of new daily persistent headache. Headache 2001; 41: 830-832.

Goadsby PJ, Boes C. New daily persistent headache. J Neurol Neurosurg Psychiat 2002; 72 Suppl 2: ii6-ii9.

Grande RB, Aaseth K, Lundqvist C, et al: Prevalence of new daily persistent headache in the general population. The Akershus study of chronic headache. Cephalalgia 2009; 29: 1149-1155.

Kung E, Tepper SJ, Rapoport AM, Sheftell FD, Bigal ME. New daily persistent headache in the pediatric population. Cephalalgia 2009; 29: 17-22.

Li D, Rozen TD. The clinical characteristics of new daily persistent headache. Cephalalgia 2002; 22: 66-69.

Mack KJ. New daily persistent headache in children and adults. Curr Pain Headache Rep 2009; 13: 47-51.

Mack KJ. What incites new daily persistent headache in children? Pediatr Neurol 2004; 31: 122-125.

Meineri P, Torre E, Rota E, Grasso E. New daily persistent headache: clinical and serological characteristics in a retrospective study. Neurol Sci 2004; 25 Suppl 3: S281-S282.

Peng KP, Fuh JL, Yuan HK, Shia BC, Wang SJ. New daily persistent headache: Should migrainous features be incorporated? Cephalalgia 2011; 31: 1561-1569.

Peres MF, Lucchetti G, Mercante JP, Young WB. New daily persistent headache and panic disorder. Cephalalgia 2011; 31: 250-253.

Prakash S, Shah ND: Postinfectious new daily persistent headache may respond to intravenous methylprednisolone. J Headache Pain 2010; 11: 59-66.

Robbins MS, Grosberg BM, Napchan U, et al. Clinical and prognostic subforms of new daily-persistent headache. Neurology 2010; 74: 1358-1364.

Rozen TD, Roth JM, Denenberg N. Cervical spine joint hypermobility: a possible predisposing factor for new daily persistent headache. Cephalalgia 2006; 26: 1182-1185.

Rozen T, Swidan SZ. Elevation of CSF tumor necrosis factor alpha levels in new daily persistent headache and treatment refractory chronic migraine. Headache 2007; 47: 1050-1055.

Santoni JR, Santoni-Williams CJ. Headache and painful lymphadenopathy in extracranial or systemic infection: etiology of new daily persistent headaches. Intern Med 1993; 32: 530-532.

Silberstein SD, Lipton RB, Solomon S, Mathew NT . Classification of daily and near daily headaches: proposed revisions to the IHS classification. Headache 1994; 34: 1-7.

Takase Y, Nakano M, Tatsumi C, Matsuyama T. Clinical features, effectiveness of drug-based treatment, and prognosis of new daily persistent headache (NDPH): 30 cases in Japan. Cephalalgia 2004; 24: 955-959.

Vanast WJ. New daily persistent headaches: definition of a benign syndrome. Headache 1986; 26: 317.

Young WB, Swanson JW. New daily-persistent headache: The switched-on headache. Neurology 2010; 74: 1338-1339.

\section{TEIL ZWEI}

\section{SEKUNDÄRE KOPFSCHMERZ-ERKRANKUNGEN}

5. Kopfschmerz zurückzuführen auf ein Trauma oder eine Verletzung des Kopfes und/oder der HWS

6. Kopfschmerz zurückzuführen auf Gefäßstörungen im Bereich des Kopfes und/oder des Halses

7. Kopfschmerz zurückzuführen auf eine nichtvaskuläre intrakranielle Störung

8. Kopfschmerz zurückzuführen auf eine Substanz oder deren Entzug

9. Kopfschmerz zurückzuführen auf eine Infektion

10. Kopfschmerz zurückzuführen auf eine Störung der Homöostase

11. Kopf- oder Gesichtsschmerz zurückzuführen auf Erkrankungen des Schädels sowie von Hals, Augen, Ohren, Nase, Nebenhöhlen, Zähnen, Mund oder anderen Gesichts- oder Schädelstrukturen

12. Kopfschmerz zurückzuführen auf psychiatrische Störungen

\section{Einleitung sekundäre Kopfschmerz- erkrankungen}

Wenn bei einem Patienten erstmals Kopfschmerzen oder eine neue Form von Kopfschmerzen zusammen mit einem Hirntumor auftreten, ist es folgerichtig zu schließen, dass die Kopfschmerzen sekundäre Folge des Tumors sind. In diesen Fällen sollte nur die Diagnose 7.4 Kopfschmerz zurückzuführen auf ein intrakranielles Neoplasma (oder eines Subtyps hiervon) vergeben werden, auch wenn die Kopfschmerzen die Phänomenologie einer Migräne, eines Kopfschmerzes vom Spannungstyp oder eines Clusterkopfschmerzes aufweisen. In anderen Worten, ein de novo-Kopfschmerz, der in Zusammenhang mit einer anderen Erkrankung auftritt, von der bekannt ist, dass sie Kopfschmerzen verursachen kann, ist immer sekundärer Natur.

Die Situation stellt sich anders da, wenn ein Patient bereits unter einem primären Kopfschmerz litt, der sich dann in engem zeitlichen Zusammenhang mit einer anderen Erkrankung verschlechtert. Für diese Verschlechterung gibt es drei mögliche Erklärung: dass sie zufällig ist; dass es sich um eine Verschlechterung des primären Kopfschmerzes handelt, die in einem ursächlichen Zusammenhang mit der anderen Erkrankung steht; dass es sich um einen neu aufgetretenen Kopfschmerz handelt, der wiederum in eine ursächlichen Zusammenhang mit der anderen Erkrankung steht. Die in der ICDH-II aufgestellten allgemeinen Zuordnungsregeln ließen unter solchen Umständen eine Diagnose oder zwei Diagnosen zu, was jedoch dem persönlichen Urteil überlassen war. In der ICHD-3 beta wurden sie daraufhin geändert, um weniger Interpretationsspielraum zu geben, und diese Änderungen werden beibehalten.

Tritt ein neuer Kopfschmerz erstmals in engem zeitlichem Zusammenhang mit einer anderen Erkrankung auf, von der bekannt ist, dass sie Kopfschmerzen hervorrufen kann oder dieser erfüllt sonstige Kriterien, die für eine Verursachung durch diese andere Erkrankung sprechen, so sollte der neu aufgetretene Kopfschmerz als sekundärer Kopfschmerz zurückzuführen auf die ursächliche Erkrankung kodiert werden. Dies ist auch der Fall, wenn der Kopf- 
schmerz das klinische Bild eines primären Kopfschmerzes (Migräne, Kopfschmerz vom Spannungstyp, Clusterkopfschmerz oder einer der anderen trigemino-autonomen Kopfschmerzerkrankungen) aufweist.

Wenn ein vorbestehender primärer Kopfschmerz in engem zeitlichem Zusammenhang mit einer solchen ursächlichen Erkrankung chronisch wird oder sich deutlich verschlechtert (was üblicherweise eine mindestens zweifache Erhöhung der Häufigkeit und/oder Intensität bedeutet), sollte der Patient die Diagnose eines primären und eines sekundären Kopfschmerzes erhalten, vorausgesetzt, es bestehen sehr gute Hinweise, dass die betreffende Erkrankung Kopfschmerzen verursachen kann.

In der ICHD-2 wurde das Format der diagnostischen Kriterien für sekundäre Kopfschmerzerkrankungen standardisiert, was jedoch nicht frei von Problemen war. Für die ICHD-3 beta wurde eine Überarbeitung beschlossen, und auch diese wird beibehalten:

Allgemeine diagnostische Kriterien für sekundäre Kopfschmerzerkrankungen:

A. Kopfschmerz, der das Kriterium C erfüllt

B. Es wurde eine andere Erkrankung diagnostiziert, bei der wissenschaftlich belegt ist, dass sie Kopfschmerzen verursachen kann1

C. Ein kausaler Zusammenhang kann durch mindestens zwei der folgenden Kriterien gezeigt werden2:

1. Der Kopfschmerz hat sich in einem zeitlichen Zusammenhang mit dem Beginn der vermuteten ursächlichen Störung entwickelt

2. Einer oder beide der folgenden Punkte sind erfüllt:

- Der Kopfschmerz hat sich gleichzeitig mit der Verschlechterung der angenommenen ursächlichen Störung deutlich verschlechtert

- Der Kopfschmerz hat sich gleichzeitig mit der Besserung der vermuteten ursächlichen Störung deutlich gebessert

3. Der Kopfschmerz weist Charakteristika auf, die typisch für die ursächliche Erkrankung sind3

4. Der ursächliche Zusammenhang ist anderweitig belegt4 D. Nicht besser erklärt durch eine andere ICHD-3-Diagnose.

\section{Anmerkung:}

1. Da die Prävalenz von Kopfschmerzen extrem hoch ist, können diese per Zufall und ohne ursächlichen Zusammenhang gleichzeitig mit einer anderen Erkrankung auftreten. Deshalb kann die Diagnose einer sekundären Kopfschmerzerkrankung nur dann mit Sicherheit gestellt werden, wenn aufgrund publizierter wissenschaftlicher Untersuchungen belastbare Beweise vorliegen, dass die unter Kriterium B angegebene Erkrankung Kopfschmerzen verursachen kann. Der wissenschaftliche Nachweis kann durch großangelegte klinische Studien erbracht werden, bei denen enge zeitliche Beziehungen zwischen der Erkrankung und der weiteren Entwicklung der Kopfschmerzen nach Behandlung dieser Erkrankung nachgewiesen wurden, oder durch kleinere Studien, bei denen fortschrittlichste Scanning-Verfahren, Bluttests oder andere paraklinische Tests zum Einsatz kamen, selbst wenn diese dem diagnosestellenden Arzt, der diese Kriterien anwenden wird, nicht ohne weiteres zur Verfügung stehen. Mit anderen Worten, Untersuchungsmethoden, die beim routinemäßigen Rückgriff auf die diagnostischen Kriterien nichts nutzen, können dennoch nützlich sein, um allgemeine kausale Beziehungen als Grundlage von Kriterium B zu ermitteln. In der gesamten ICHD-3 beschränken sich die diagnostischen Kriterien jedoch auf Informationen, bei denen nach vernünftigem Ermessen davon auszugehen ist, dass sie dem diagnostizierenden Arzt in einer typischen klinischen Situation zur Verfügung stehen.

2. Die allgemeinen Kriterien verlangen - wie dargelegt - das Vorliegen von mindestens zwei eigenständigen als Nachweis tauglichen Merkmalen und erlauben bis zu vier Nachweistypen. Nicht alle dieser vier Typen bieten sich für alle Erkrankungen an, und dementsprechend müssen nicht alle vier zu den spezifischen Kriterien für eine bestimmte sekundäre Kopfschmerzerkrankung gehören. Es gibt einige sekundäre Kopfschmerzerkrankungen, bei denen der Nachweis eines ursächlichen Zusammenhangs sehr stark vom Kopfschmerzbeginn in einem zeitlichen Zusammenhang mit der angenommenen Ursache abhängt. Beispiele hierfür sind die Subtypen des 7.2 Kopfschmerzes zurückzuführen auf Liquorunterdruck, die üblicherweise, aber nicht immer orthostatisch sind, so dass dieses Charakteristikum kein verlässliches diagnostisches Kriterium darstellt. In solchen Fällen ist Kriterium D von besonderer Bedeutung.

3. Ein Beispiel ist ein sehr plötzlich einsetzender (Donnerschlag-) Kopfschmerz bei einem 6.2.2 akuten Kopfschmerz zurückzuführen auf eine nicht-traumatische Subarachnoidalblutung (SAB). Die Charakteristika sind gegebenenfalls für jeden sekundären Kopfschmerz zu spezifizieren.

4. Die Spezifikation muss (gegebenenfalls) für jede sekundäre Kopfschmerzerkrankung erfolgen. Ein Beispiel für diese Art von Evidenz ist die Übereinstimmung zwischen dem Kopfschmerzsitus und der Lokalisation einer vermuteten ursächlichen Erkrankung. Ein weiteres sind übereinstimmend auftretende Schwankungen zwischen den Kopfschmerzmerkmalen (etwa ihrer Intensität) und den Markern für die Aktivität der vermuteten ursächlichen Erkrankung (d.h. Veränderungen in der zerebralen Bildgebung oder in anderen Laborwerten [z. B. der Blutsenkungsgeschwindigkeit bei einem 6.4.1 Kopfschmerz zurückzuführen auf eine Riesenzellarteriitis]). 


\section{Kopfschmerz zurückzuführen auf ein Trauma oder eine Verletzung des Kopfes und/oder der HWS}

5.1 Akuter Kopfschmerz zurückzuführen auf eine traumatische Verletzung des Kopfes

5.1.1 Akuter Kopfschmerz zurückzuführen auf eine mittelschwere oder schwere traumatische Verletzung des Kopfes

5.1.2 Akuter Kopfschmerz zurückzuführen auf eine leichte traumatische Verletzung des Kopfes

5.2 Anhaltender Kopfschmerz zurückzuführen auf eine traumatische Verletzung des Kopfes

5.2.1 Anhaltender Kopfschmerz zurückzuführen auf eine mittelschwere oder schwere traumatische Verletzung des Kopfes

5.2.2 Anhaltender Kopfschmerz zurückzuführen auf eine leichte traumatische Verletzung des Kopfes

5.3 Akuter Kopfschmerz zurückzuführen auf ein HWSBeschleunigungstrauma

5.4 Anhaltender Kopfschmerz zurückzuführen auf ein HWS-Beschleunigungstrauma

5.5 Akuter Kopfschmerz zurückzuführen auf eine Kraniotomie

5.6 Anhaltender Kopfschmerz zurückzuführen auf eine Kraniotomie

An anderer Stelle kodiert Primärer und/oder sekundärer Kopfschmerz?

Für Kapitel 5. Kopfschmerz zurückzuführen auf ein Trauma oder eine Verletzung des Kopfes und/oder der HWS gelten die allgemeinen Regeln für die kausale Zuordnung zu einer anderen Erkrankung.

Tritt ein neuer Kopfschmerz erstmals in engem zeitlichen Zusammenhang mit einem Trauma oder einer Verletzung des Kopfes und/oder der HWS auf, sollte der Kopfschmerz als Kopfschmerz zurückzuführen auf ein Trauma oder eine Verletzung kodiert werden. Dies ist auch der Fall, wenn der Kopfschmerz das klinische Bild eines primären Kopfschmerzes zeigt, der in Teil 1 der ICHD-3 klassifiziert wird.

Wenn aber ein vorbestehender Kopfschmerz mit den Charakteristika eines primären Kopfschmerzes in engem zeitlichen Zusammenhang mit einem solchen Trauma oder einer solchen Verletzung chronisch wird oder sich deutlich verschlechtert (üblicherweise definiert als eine mindestens zweifache Steigerung der Häufigkeit und/oder Schwere), dann sollte sowohl die vorbestehende primäre Kopfschmerzdiagnose als auch die Diagnose 5. Kopfschmerz zurückzuführen auf ein Trauma oder eine Verletzung des Kopfes und/ oder der HWS (oder eines seiner Typen oder Subtypen) vergeben werden, sofern gute Hinweise dafür bestehen, dass die Erkrankung Kopfschmerzen verursachen kann.

\section{Einleitung}

Die Typen des 5. Kopfschmerzes zurückzuführen auf ein Trauma oder eine Verletzung des Kopfes und/oder der HWS gehören zu den häufigsten sekundären Kopfschmerzerkrankungen. Während der ersten 3 Monate ab Kopfschmerzbeginn gelten sie als akut; bestehen sie über diesen Zeitraum hinaus fort, werden sie als anhaltend bezeichnet. Dieser Zeitraum deckt sich mit den diagnos- tischen Kriterien der ICHD-2, obwohl der Begriff anhaltend an die Stelle von chronisch getreten ist.

Es gibt keine spezifischen Kopfschmerzmerkmale, durch die sich die Typen des 5. Kopfschmerzes zurückzuführen auf ein Trauma oder eine Verletzung des Kopfes und/oder der HWS von anderen Kopfschmerzerkrankungen unterscheiden; meist ähneln diese einem 2. Kopfschmerz vom Spannungstyp oder einer 1. Migräne. Demzufolge stützt sich ihre Diagnose größtenteils auf den engen zeitlichen Zusammenhang zwischen dem Kopftrauma oder der Kopfverletzung und dem Kopfschmerzbeginn. Übereinstimmend mit den diagnostischen Kriterien der ICHD-2 erfordern die diagnostischen Kriterien der ICHD-3 für alle Typen des 5. Kopfschmerzes zurückzuführen auf ein Trauma oder eine Verletzung des Kopfes und/oder der HWS, dass die berichteten Kopfschmerzen innerhalb von 7 Tagen nach dem Trauma oder der Verletzung aufgetreten sind bzw. innerhalb von 7 Tagen nach Wiedererlangung des Bewusstseins und/oder 7 Tagen nach der Wiedererlangung der Fähigkeit, Schmerz zu spüren und über ihn zu berichten. Auch wenn dieses 7-Tage-Intervall ein Stück weit willkürlich ist und einige Experten argumentieren, Kopfschmerzen könnten bei einem kleinen Teil der Patienten auch noch nach einem längeren Zeitintervall auftreten, liegen derzeit noch nicht genug stichhaltige Gründe dafür vor, diese Voraussetzung zu ändern. Hier soll zu Untersuchungen angeregt werden, bei denen die diagnostischen Kriterien für einen A5.1.1.1 verzögert auftretenden akuten Kopfschmerz zurückzuführen auf eine mittelschwere oder schwere traumatische Verletzung des Kopfes und eines A5.1.2.1 verzögert auftretenden akuten Kopfschmerzes zurückzuführen auf eine leichte traumatische Verletzung des Kopfes auf den Prüfstand gestellt werden (siehe Anhang).

Kopfschmerz kann als isoliertes Symptom nach einem Trauma oder einer Verletzung des Kopfes auftreten oder auch als eine Konstellation von Symptomen, die in der Regel Schwindel, Müdigkeit, ein vermindertes Konzentrationsvermögen, eine psychomotorische Verlangsamung, leichte Gedächtnisprobleme, Schlafstörungen, Angst, Persönlichkeitsveränderungen und Gereiztheit umfassen. Wenn auf eine Kopfverletzung mehrere dieser Symptome folgen, kann davon ausgegangen werden, dass der Patient ein postkommotionelles Syndrom (Zustand nach Gehirnerschütterung) aufweist.

Die Pathogenese eines 5. Kopfschmerzes zurückzuführen auf ein Trauma oder eine Verletzung des Kopfes und/oder der HWS ist oft unklar. Zu den zahlreichen Faktoren, die zur Kopfschmerzentstehung beitragen können, gehören unter anderem eine axonale Schädigung, Veränderungen des Hirnstoffwechsels, eine Neuroinflammation, Veränderungen der zerebralen Hämodynamik, eine zugrundeliegende genetische Veranlagung, psychopathologische Faktoren und die Erwartung des Patienten, von der Kopfverletzung Kopfschmerzen davonzutragen. Neuere Untersuchungen mit Hilfe fortschrittlicher zerebraler Bildgebungsverfahren lassen auf ein Potenzial für die Aufdeckung struktureller, funktioneller und metabolischer Anomalien im Gehirn nach geringfügigen Traumata schließen, die mit konventionellen Diagnosetests nicht zu eruieren sind. Es ist plausibel, anzunehmen, dass posttraumatische Schlafstörungen, Stimmungsstörungen sowie psychosoziale und sonstige Stressoren die Entstehung und das Fortbestehen von Kopfschmerzen beeinflussen können. Der Übergebrauch nicht anschlagender Kopfschmerzmedikationen kann zum Persistieren von Kopfschmerzen 
nach einer Kopfverletzung durch Auftreten eines 8.2 Kopfschmerzes zurückzuführen auf Medikamentenübergebrauch führen. Kliniker müssen diese Möglichkeit immer dann berücksichtigen, wenn posttraumatische Kopfschmerzen über die erste posttraumatische Phase hinaus anhalten.

Zu den Risikofaktoren, die das Auftreten eines 5. Kopfschmerzes zurückzuführen auf ein Trauma oder eine Verletzung des Kopfes und/oder der HWS begünstigen, können eine Kopfschmerzvorgeschichte, eine weniger schwere Verletzung, die Zugehörigkeit zum weiblichen Geschlecht und das Vorliegen komorbider psychiatrischer Störungen gehören. Der Zusammenhang zwischen wiederholten Kopftraumata und dem Auftreten von Kopfschmerzen sollte noch näher untersucht werden. In welchem Umfang die Erwartung des Patienten, dass nach der Kopfverletzung Kopfschmerzen eintreten werden und Rechtsstreitigkeiten rund um Entschädigungsansprüche in Verbindung mit Kopfschmerzen dieser Art ihr Auftreten und Persistieren begünstigen, wird noch diskutiert. Mehrheitlich verweisen die Belege darauf, dass Simulieren nur bei einer kleinen Minderheit der Patienten eine Rolle spielt.

Es ist anerkannt, dass bei einigen Patienten nach sehr geringfügigen Kopftraumata Kopfschmerzen auftreten - Traumata so geringfügiger Art, dass sie nicht einmal die Kriterien für eine leichte traumatische Hirnverletzung erfüllen. Diese Kopfschmerzen können nach einem einmaligen Trauma beginnen oder nach wiederholten kleineren Aufprallereignissen gegen den Kopf (z. B. bei American-Football- oder Rugbyspielern). Kopfschmerzen infolge sehr kleiner Kopftraumata wurden jedoch noch nicht hinreichend untersucht, daher reicht die Datenlage nicht aus, um diese anzuerkennen und in die ICHD-3 aufzunehmen. Hier sind Untersuchungen zu Kopfschmerz nach sehr geringfügigen Kopftraumata gefordert, vielleicht anhand der diagnostischen Kriterien für einen A5.8 akuten Kopfschmerz zurückzuführen auf ein anderes Kopf- und/oder HWS-Trauma oder eine andere Kopf- und/oder HWS-Verletzung und einen A5.9 anhaltenden Kopfschmerz zurückzuführen auf ein anderes Kopf- und/oder HWS-Trauma oder eine andere Kopf- und/ oder HWS-Verletzung.

Von einem 5. Kopfschmerz zurückzuführen auf ein Trauma oder eine Verletzung des Kopfes und/oder der HWS wird auch bei Kindern berichtet, wenngleich weniger häufig als bei Erwachsenen. Die Charakteristika der Typen sind bei Kindern und Erwachsenen ähnlich, und die diagnostischen Kriterien bei Kindern sind dieselben.

\subsection{Akuter Kopfschmerz zurückzuführen auf eine traumatische Verletzung des Kopfes}

An anderer Stelle kodiert: Ein Trauma infolge von Akzeleration/ Dezelerationsbewegungen des Kopfes, mit Flexion/Extension der HWS, wird als HWS-Beschleunigungstraume klassifiziert. Akut auftretende Kopfschmerzen nach einem solchen Trauma werden unter 5.3 akuter Kopfschmerz zurückzuführen auf ein HWS-Beschleunigungstrauma kodiert. Akute Kopfschmerzen nach einem chirurgischen Eingriff mittels Kraniotomie aus anderen Gründen als aufgrund der traumatischen Kopfverletzung sollten unter 5.5 akuter Kopfschmerz nach Kraniotomie kodiert werden.
Beschreibung:

Kopfschmerz von weniger als 3 Monaten Dauer infolge einer traumatischen Kopfverletzung.

Diagnostische Kriterien:

A. Jeder Kopfschmerz, der die Kriterien C und D erfüllt

B. Es liegt eine traumatische Kopfverletzung1 vor

C. Der Kopfschmerz hat sich laut Bericht innerhalb von 7 Tagen nach einem von Folgendem entwickelt:

1. der Kopfverletzung

2. der Wiedererlangung des Bewusstseins nach Eintritt der Kopfverletzung

3. Absetzen von Medikamenten, welche die Fähigkeit beeinträchtigen, Kopfschmerzen nach der Kopfverletzung zu spüren oder von diesen zu berichten

D. Es ist wenigstens einer der folgenden Punkte erfüllt:

1. der Kopfschmerz ist innerhalb von 3 Monaten nach seinem Beginn verschwunden

2. der Kopfschmerz ist noch nicht verschwunden, aber es sind noch keine 3 Monate nach seinem Beginn verstrichen

E. Nicht besser erklärt durch eine andere ICHD-3-Diagnose.

\section{Anmerkung:}

1. Der Begriff der traumatischen Kopfverletzung bezieht sich auf eine strukturelle oder funktionelle Verletzung infolge der Einwirkung äußerer Kräfte auf den Kopf. Hierzu gehören der Aufprall des Kopfes auf ein Objekt oder umgekehrt, das Eindringen eines Fremdkörpers in den Kopf, die Einwirkung von Kräften, die von Druckwellen oder Explosionen erzeugt werden sowie andere noch zu definierende Krafteinwirkungen.

\section{Kommentar:}

Die Vorgabe, dass der berichtete Kopfschmerz sich innerhalb von 7 Tagen entwickelt haben muss, ist ein Stück weit beliebig (siehe Einleitung oben). Gegenüber längeren Intervallen ergibt ein 7-Tage-Intervall diagnostische Kriterien mit höherer Spezifizität für einen 5.1 akuten Kopfschmerz zurückzuführen auf eine traumatische Verletzung des Kopfes (d. h., belastbarere Hinweise auf einen ursächlichen Zusammenhang), aber einen entsprechenden Sensitivitätsverlust. Weitere Untersuchungen werden erforderlich sein, um zu ermitteln, ob ein anderes Intervall angebrachter sein könnte. Bis dahin können die im Anhang angeführten Kriterien für einen A5.1.1.1 verzögert auftretenden akuten Kopfschmerz zurückzuführen auf eine mittelschwere oder schwere traumatische Verletzung des Kopfes und einen A5.1.2.1 verzögert auftretenden akuten Kopfschmerz zurückzuführen auf eine leichte traumatische Verletzung des Kopfes benutzt werden, wenn das Intervall zwischen Verletzung und Kopfschmerzbeginn länger als 7 Tage ist.

\subsubsection{Akuter Kopfschmerz zurückzuführen auf eine mittelschwere oder schwere traumatische Verletzung des Kopfes}

Diagnostische Kriterien:

A. Kopfschmerz, der die Kriterien für einen 5.1 akuten Kopfschmerz zurückzuführen auf eine traumatische Verletzung des Kopfes erfüllt 
B. Kopftrauma, welches wenigstens einen der folgenden Punkte erfüllt:

1. Bewusstseinsverlust $>30$ Minuten

2. Glasgow Coma Scale (GCS) Wert $<13$

3. posttraumatische Amnesie von $>24$ Stunden Dauer 1

4. Bewusstseinsstörung für $>24$ Stunden

5. Nachweis einer traumatischen Kopfverletzung wie etwa einer Schädelfraktur, intrakraniellen Blutung und/oder Hirnkontusion in der zerebralen Bildgebung.

Anmerkung:

1. Die Dauer der posttraumatischen Amnesie wird definiert als die Zeit zwischen dem Eintritt der Kopfverletzung und der Wiedererlangung der Erinnerung an aktuelle Ereignisse und die in den letzten 24 Stunden.

\subsubsection{Akuter Kopfschmerz zurückzuführen auf eine leichte traumatische Verletzung des Kopfes}

Diagnostische Kriterien:

A. Kopfschmerz, der die Kriterien für einen 5.1 akuten Kopfschmerz zurückzuführen auf eine traumatische Verletzung des Kopfes erfüllt

B. Kopftrauma, welches beide der folgenden Punkte erfüllt:

1. mit keinem der folgenden Aspekte verbunden:

- Bewusstseinsverlust für>30 Minuten

- Glasgow Coma Scale (GCS) Wert $<13$

- posttraumatische Amnesie von>24 Stunden Dauer1

- Bewusstseinsstörung für>24 Stunden

- Nachweis einer traumatischen Kopfverletzung wie etwa einer Schädelfraktur, intrakraniellen Blutung und/oder Hirnkontusion in der zerebralen Bildgebung

2. Tritt in Begleitung von einem oder mehreren der folgenden Symptome und/oder Zeichen auf:

- vorübergehende Verwirrung, Orientierungslosigkeit oder Bewusstseinsbeeinträchtigung

- Verlust der Erinnerung an Ereignisse unmittelbar vor oder nach der Kopfverletzung

- zwei oder mehr der folgenden Symptome, die an eine leichte traumatische Hirnverletzung denken lassen:

i. Übelkeit

ii. Erbrechen

iii. Sehstörungen

iv. Benommenheit und/oder Schwindel

v. unsicheres Gangbild und/oder orthostatische Dysregulation mit Gleichgewichtsstörungen

vi. Beeinträchtigungen des Gedächtnisses und/oder der Konzentration.

Anmerkung:

Die Dauer der posttraumatischen Amnesie wird definiert als die Zeit zwischen dem Eintritt der Kopfverletzung und der Wiedererlangung der Erinnerung an aktuelle Ereignisse und die in den letzten 24 Stunden
Kommentar:

Die diagnostischen Kriterien für eine leichte, mittelschwere oder schwere traumatische Kopfverletzung lassen beträchtlichen Spielraum zu, was die Klassifizierung des Schweregrads der Verletzung anbelangt. Deshalb schlugen einige Experten die Einbeziehung zusätzlicher Kategorien vor: Kopfschmerz zurückzuführen auf eine sehr leichte traumatische Kopfverletzung und Kopfschmerz zurückzuführen auf eine sehr schwere traumatische Kopfverletzung. Derzeit liegen nicht ausreichend Hinweise darauf vor, als dass es geboten wäre, diese Kategorien hinzuzufügen, doch sollten zukünftige Untersuchungen abklären, ob dies zweckmäßig wäre.

\subsection{Anhaltender Kopfschmerz zurückzu- führen auf eine traumatische Verletzung des Kopfes}

An anderer Stelle kodiert: Trauma infolge von Akzeleration/ Dezelerationsbewegungen des Kopfes, mit Flexion/Extension der HWS, wird als HWS-Beschleunigungstraume klassifiziert. Anhaltende Kopfschmerzen nach einem solchen Trauma werden unter 5.4 anhaltender Kopfschmerz zurückzuführen auf ein HWS-Beschleunigungstrauma kodiert. Anhaltende Kopfschmerzen nach einem chirurgischen Eingriff mittels Kraniotomie aus anderen Gründen als aufgrund der traumatischen Kopfverletzung sollten unter 5.6 anhaltender Kopfschmerz zurückzuführen auf eine Kraniotomie kodiert werden.

\section{Beschreibung:}

Kopfschmerz von mehr als 3 Monaten Dauer infolge einer traumatischen Kopfverletzung.

Diagnostische Kriterien:

A. Jeder Kopfschmerz, der die Kriterien C und D erfüllt

B. Zustand nach traumatischer Kopfverletzung1

C. Der Kopfschmerz hat sich laut Bericht innerhalb von 7 Tagen nach einem von Folgendem entwickelt:

1. der Kopfverletzung

2. der Wiedererlangung des Bewusstseins nach Eintritt der Kopfverletzung

3. dem Absetzen von Medikamenten, welche die Fähigkeit beeinträchtigen, Kopfschmerzen nach der Kopfverletzung zu spüren oder von diesen zu berichten

D. Der Kopfschmerz hält>3 Monate nach seinem Beginn an

E. Nicht besser erklärt durch eine andere ICHD-3-Diagnose2.

\section{Anmerkung:}

Der Begriff der traumatischen Kopfverletzung bezieht sich auf eine strukturelle oder funktionelle Verletzung infolge einer Einwirkung äußerer Kräfte auf den Kopf. Hierzu gehören der Aufprall des Kopfes gegen ein Objekt oder umgekehrt, das Eindringen eines Fremdkörpers in den Kopf, die Einwirkung von Kräften, die auf Druckwellen oder Explosionen zurückgehen sowie andere noch zu definierende Krafteinwirkungen.

2. Hält der Kopfschmerz nach der Kopfverletzung an, sollte die Möglichkeit eines 8.2 Kopfschmerzes zurückzuführen auf Medikamentenübergebrauch in Betracht gezogen werden. 


\section{Kommentar:}

Die Vorgabe, dass der berichtete Kopfschmerz sich innerhalb von 7 Tagen entwickelt haben muss, ist ein Stück weit beliebig (siehe Einleitung oben). Gegenüber längeren Intervallen ergibt ein 7-Tage-Intervall diagnostische Kriterien mit höherer Spezifizität für einen 5.2 anhaltender Kopfschmerz zurückzuführen auf eine traumatische Verletzung des Kopfes (d. h. belastbarere Hinweise auf einen ursächlichen Zusammenhang), aber einen entsprechenden Sensitivitätsverlust. Weitere Untersuchungen werden erforderlich sein, um zu ermitteln, ob ein anderes Intervall angebrachter sein könnte. Bis dahin können die im Anhang angeführten Kriterien für einen A5.2.1.1 verzögert auftretenden anhaltenden Kopfschmerz zurückzuführen auf eine mittelschwere oder schwere traumatische Verletzung des Kopfes und einen A5.2.2.1 verzögert auftretenden anhaltenden Kopfschmerz zurückzuführen auf eine leichte traumatische Verletzung des Kopfes benutzt werden, wenn das Zeitintervall zwischen Verletzung und Kopfschmerzbeginn länger als 7 Tage ist.

Im Sinne der Übereinstimmung mit den diagnostischen Kriterien der ICHD-2 für einen chronischen posttraumatischen Kopfschmerz und mit dem bei der Diagnose anderer sekundärer Kopfschmerzerkrankungen angesetzten Zeitintervall ist 3 Monate das Zeitintervall, nach dem ein Kopfschmerz zurückzuführen auf ein Trauma oder eine Verletzung des Kopfes als anhaltend gilt. In weiteren Untersuchungen wird abzuklären sein, ob kürzere oder längere Intervalle zweckmäßiger wären.

\subsubsection{Anhaltender Kopfschmerz zurückzuführen auf eine mittelschwere oder schwere traumatische Verletzung des Kopfes}

\section{Diagnostische Kriterien:}

A. Kopfschmerz, der die Kriterien für einen 5.2 anhaltenden Kopfschmerz zurückzuführen auf eine traumatische Verletzung des Kopfes erfüllt

B. Verletzung des Kopfes begleitet von beiden der folgenden Punkte:

1. Bewusstseinsverlust für $>30$ Minuten

2. Glasgow Coma Scale (GCS) Werte $<13$

3. posttraumatische Amnesie von $>24$ Stunden 1 Dauer

4. Bewusstseinsstörung für $>24$ Stunden

5. Nachweis einer traumatischen Kopfverletzung wie etwa einer Schädelfraktur, intrakraniellen Blutung und/oder Hirnkontusion in der zerebralen Bildgebung

Anmerkung:

Die Dauer der posttraumatischen Amnesie wird definiert als die Zeit zwischen dem Eintritt der Kopfverletzung und der Wiedererlangung der Erinnerung an aktuelle Ereignisse und die in den letzten 24 Stunden

\subsubsection{Anhaltender Kopfschmerz zurückzuführen auf eine leichte traumatische Verletzung des Kopfes}

Diagnostische Kriterien:

A. Kopfschmerz, der die Kriterien für einen 5.2 anhaltenden Kopfschmerz zurückzuführen auf eine traumatische Verletzung des Kopfes erfüllt
B. Kopftrauma, welches beide der folgenden Punkte erfüllt:

1. mit keinem der folgenden Aspekte verbunden:

- Bewusstseinsverlust für >30 Minuten

- Glasgow Coma Scale (GCS) Wert $<13$

- posttraumatische Amnesie von>24 Stunden Dauer1

- Bewusstseinsstörung für $>24$ Stunden

- Nachweis einer traumatischen Kopfverletzung wie etwa einer Schädelfraktur, intrakraniellen Blutung und/oder Hirnkontusion in der zerebralen Bildgebung

2. Tritt in Begleitung von einem oder mehreren der folgenden Symptome und/oder Zeichen auf:

- vorübergehende Verwirrung, Orientierungslosigkeit oder Bewusstseinsbeeinträchtigung

- Verlust der Erinnerung an Ereignisse unmittelbar vor oder nach der Kopfverletzung

- zwei oder mehr der folgenden Symptome, die an eine leichte traumatische Hirnverletzung denken lassen:

i. Übelkeit

ii. Erbrechen

iii. Sehstörungen

iv. Benommenheit und/oder Schwindel

v. unsicheres Gangbild und/oder orthostatische Dysregulation mit Gleichgewichtsstörungen

vi. Beeinträchtigungen des Gedächtnisses und/oder der Konzentration.

\section{Anmerkung:}

Die Dauer der posttraumatischen Amnesie wird definiert als die Zeit zwischen dem Eintritt der Kopfverletzung und der Wiedererlangung der Erinnerung an aktuelle Ereignisse und die in den letzten 24 Stunden

\subsection{Akuter Kopfschmerz zurückzuführen auf ein HWS-Beschleunigungstrauma1}

\section{Beschreibung:}

Kopfschmerz von weniger als 3 Monaten Dauer infolge eines HWS-Beschleunigungstraumas.

Diagnostische Kriterien:

A. Jeder Kopfschmerz, der die Kriterien C und D erfüllt

B. Zustand nach HWS-Beschleunigungstrauma1 bei Beginn von Nacken- und/oder Kopfschmerzen

C. Der Kopfschmerz ist innerhalb von 7 Tagen nach dem HWS-Beschleunigungstrauma aufgetreten

D. Es ist wenigstens einer der folgenden Punkte erfüllt:

1. der Kopfschmerz ist innerhalb von 3 Monaten nach seinem Beginn verschwunden

2. der Kopfschmerz ist noch nicht verschwunden, aber es sind seit seinem Beginn noch keine 3 Monate verstrichen

E. Nicht besser erklärt durch eine andere ICHD-3-Diagnose.

Anmerkung:

1. Der Begriff des HWS-Beschleunigungstraumas bezieht sich auf eine plötzliche und nicht ausreichend gebremste Akzeleration/Dezeleration des Kopfes in Verbindung mit einer Flexion/ 
Extension der HWS. Ein HWS-Beschleunigungstrauma kann nach Einwirkung starker wie auch geringer Kräfte auftreten.

\section{Kommentar:}

Das HWS-Beschleunigungstrauma tritt meist bei Verkehrsunfällen auf.

Ein 5.3 akuter Kopfschmerz zurückzuführen auf ein HWS-Beschleunigungstrauma kann als isoliertes Symptom auftreten oder auch mit einer Konstellation weiterer Symptome, die sowohl von der HWS als auch von extrazervikalen Strukturen herrühren und ebenso Störungen der Neurosensorik, des Verhaltens, der Kognition und/oder der Stimmung. Das HWS-Beschleunigungstrauma als solches kann anhand eines Schemas wie dem von der „Quebec Task Force on Whiplash-Associated Disorders “ vorgestellten nach dem Schweregrad der Charakteristika klassifiziert werden.

\subsection{Anhaltender Kopfschmerz zurückzu- führen auf ein HWS-Beschleunigungs- trauma}

Beschreibung:

Kopfschmerz von mehr als 3 Monaten Dauer infolge eines HWS-Beschleunigungstraumas.

Diagnostische Kriterien:

A. Jeder Kopfschmerz, der die Kriterien C und D erfüllt

B. Zustand nach HWS-Beschleunigungstrauma1 bei Eintritt von Nacken- und/oder Kopfschmerzen

C. Der Kopfschmerz ist innerhalb von 7 Tagen nach dem HWS-Beschleunigungstrauma aufgetreten

D. Der Kopfschmerz hält>3 Monate nach seinem Beginn an

E. Nicht besser erklärt durch eine andere ICHD-3-Diagnose2.

\section{Anmerkung:}

1. Der Begriff des HWS-Beschleunigungstraumas bezieht sich auf eine plötzliche und nicht ausreichend ausgebremste Akzeleration/Dezeleration des Kopfes mit Flexion/Extension der HWS. Ein HWS-Beschleunigungstrauma kann nach Einwirkung starker wie auch geringer Kräfte auftreten.

2. Hält der Kopfschmerz nach dem HWS-Beschleunigungstrauma an, sollte die Möglichkeit eines 8.2 Kopfschmerzes zurückzuführen auf Medikamentenübergebrauch in Betracht gezogen werden.

\subsection{Akuter Kopfschmerz zurückzuführen auf eine Kraniotomie}

Beschreibung:

Kopfschmerz von weniger als 3 Monaten Dauer nach einem chirurgischen Eingriff mittels Kraniotomie.

\section{Diagnostische Kriterien:}

A. Jeder Kopfschmerz, der die Kriterien C und D erfüllt

B. Zustand nach chirurgischem Eingriff mittels Kraniotomie1

C. Der Kopfschmerz ist laut Bericht innerhalb von 7 Tagen nach einem von Folgendem aufgetreten:
1. der Kraniotomie

2. der Wiedererlangung des Bewusstseins nach der Kraniotomie

3. Absetzen von Medikamenten, welche die Fähigkeit beeinträchtigen, Kopfschmerzen nach der Kraniotomie zu spüren oder von diesen zu berichten

D. Es ist wenigstens einer der folgenden Punkte erfüllt:

1. der Kopfschmerz ist innerhalb von 3 Monaten nach seinem Beginn verschwunden

2. der Kopfschmerz ist noch nicht verschwunden, aber es sind seit seinem Beginn noch keine 3 Monate verstrichen

E. Nicht besser erklärt durch eine andere ICHD-3-Diagnose 1;2.

\section{Anmerkung:}

1. Erfolgte die Kraniotomie nach und aufgrund einer Kopfverletzung, erfolgt die Kodierung unter 5.2.1 akuter Kopfschmerz zurückzuführen auf eine mittelschwere oder schwere traumatische Verletzung des Kopfes.

2. Vor Zuweisung der Diagnose 5.5 akuter Kopfschmerz zurückzuführen auf eine Kraniotomie ist ein Ausschluss anderer sekundärer Kopfschmerzerkrankungen, die nach einer Kraniotomie auftreten können, erforderlich. Obwohl Kopfschmerz nach Kraniotomie auf eine ganze Reihe von Ursachen zurückgehen kann, sind u. a. insbesondere zervikogene Kopfschmerzen (aufgrund der Position während der Operation), Kopfschmerzen durch austretenden Liquor, Infektionen, ein Hydrozephalus und eine intrakranielle Blutung in Betracht zu ziehen.

\section{Kommentar:}

Ein 5.5 akuter Kopfschmerz zurückzuführen auf eine Kraniotomie tritt bei einem erheblichen Teil der Patienten auf, bei denen eine Kraniotomie vorgenommen wurde. In der Mehrheit der Fälle beginnt der Kopfschmerz innerhalb der ersten Tage nach dem Eingriff und verschwindet innerhalb des akuten postoperativen Zeitraums. Nach Eingriffen an der Schädelbasis kommt es im Vergleich zu anderen Stellen häufiger zu Kopfschmerzen.

Obwohl das Schmerzmaximum bei einem 5.5 akuter Kopfschmerz zurückzuführen auf eine Kraniotomie an der Stelle der Kraniotomie erlebt wird, kann der Schmerz diffuser sein und einem Kopfschmerz vom Spannungstyp oder einer Migräne ähneln.

\subsection{Anhaltender Kopfschmerz zurückzu- führen auf eine Kraniotomie}

Beschreibung:

Kopfschmerz von mehr als 3 Monaten Dauer nach einem chirurgischen Eingriff mittels Kraniotomie.

Diagnostische Kriterien:

A. Jeder Kopfschmerz, der die Kriterien C und D erfüllt

B. Zustand nach chirurgischem Eingriff mittels Kraniotomie1

C. Der Kopfschmerz ist laut Bericht innerhalb von 7 Tagen nach einem von Folgendem aufgetreten:

1. der Kraniotomie

2. der Wiedererlangung des Bewusstseins nach der Kraniotomie 
3. dem Absetzen von Medikamenten, welche die Fähigkeit beeinträchtigen, Kopfschmerzen nach der Kopfverletzung zu spüren oder von diesen zu berichten

D. Der Kopfschmerz hält>3 Monate nach seinem Beginn an

E. Nicht besser erklärt durch eine andere ICHD-3-Diagnose1;2.

\section{Anmerkung:}

1. Erfolgte die Kraniotomie nach und aufgrund einer Kopfverletzung, erfolgt die Kodierung unter 5.2.1 anhaltender Kopfschmerz zurückzuführen auf eine mittelschwere oder schwere traumatische Verletzung des Kopfes.

2. Hält der Kopfschmerz nach der Kraniotomie an, sollte die Möglichkeit eines 8.2 Kopfschmerzes zurückzuführen auf Medikamentenübergebrauch in Betracht gezogen werden.

\section{Kommentar:}

Etwa ein Viertel der Patienten, die einen 5.5 akuten Kopfschmerz zurückzuführen auf eine Kraniotomie entwickeln, erfahren im Anschluss einen 5.6 anhaltenden Kopfschmerz zurückzuführen auf eine Kraniotomie.

\section{Literatur}

Traumatic Brain Injury (TBI) Task Force for the US Department of the Army. TBI Task Force Report to the Surgeon General. US Army 2008 @ https://www.hsdl.org/?view\&did=482727 (accessed 19.9.17).

\section{Einleitung}

Aoki Y, Inokuchi R, Gunshin M, Yahagi N, Suwa H. Diffusion tensor imaging studies of mild traumatic brain injury: a meta-analysis. J Neurol Neurosurg Psychiat 2012; 83: 870-876.

Chong CD, Schwedt TJ. White matter damage and brain network alterations in concussed patients: a review of recent diffusion tensor imaging and resting-state functional connectivity data. Current Pain and Headache Reports 2015; 19: 485.

Faux S, Sheedy J, A prospective controlled study in the prevalence of posttraumatic headache following mild traumatic brain injury. Pain Med 2008; 9: 1001-1011.

Heyer GL, Young JA, Rose SC, McNally KA, Fischer AN. Post-traumatic headaches correlate with migraine symptoms in youths with concussion. Cephalalgia 2016; 36: 309-316.

Kirk C, Naquib G, Abu-Arafeh I. Chronic post-traumatic headache after head injury in children and adolescents. Dev Med Child Neurol 2008; 50: 422-425.

Kjeldgaard D, Forchhammer H, Teasdale T, Jensen RH. Chronic post-traumatic headache after mild head injury: a descriptive study. Cephalalgia 2014; 34: 191-200.

Lucas S, Hoffman JM, Bell KR, Dikmen S. A prospective study of prevalence and characterization of headache following mild traumatic brain injury. Cephalalgia 2014; 34: 93-102.

Lucas S, Hoffman JM, Bell KR, Walker W, Dikmen S. Characterization of headache after traumatic brain injury. Cephalalgia 2012; 32: 600-606.

Mayer CL, Huber BR, Peskind E. Traumatic brain injury, neuroinflammation, and post-traumatic headaches. Headache 2013; 53: 1523-1530.

Nampiaparampil DE. Prevalence of chronic pain after traumatic brain injury: a systematic review. JAMA 2008; 300: 711-719.

Olesen J. Problem areas in the International Classification of Headache Disorders, 3 rd edition (beta). Cephalalgia 2014; 34: 1193-1199.
Russell MB, Olesen J. Migraine associated with head trauma. Eur J Neurol 1996; 3: 424-428.

Theeler BJ, Flynn FG, Erickson JC. Headaches after concussion in US soldiers returning from Iraq or Afghanistan. Headache 2010; 50: 1262-1272.

Theeler B, Lucas S, Riechers RG, Ruff RL. Post-traumatic headaches in civilians and military personnel: a comparative, clinical review. Headache 2013; 53: 881-900.

Walker WC, Marwitz JH, Wilk AR, Ketchum JM, Hoffman JM, Brown AW, Lucas S. Prediction of headache severity (density and functional impact) after traumatic brain injury: a longitudinal multicentre study. Cephalalgia 2013; 33: 998-1008.

Xu H, Pi H, Ma L, Su X, Wang J. Incidence of headache after traumatic brain injury in China: a large prospective study. World Neurosurgery 2016; 88: 289-296.

\section{1, 5.2 Akuter oder anhaltender Kopfschmerz zurückzuführen auf} eine traumatische Verletzung des Kopfes

Afari N, Harder LH, Madra NJ, Heppner PS, Moeller-Bertram T, King C, Baker DG. PTSD, combat injury, and headache in Veterans Returning from Iraq/Afghanistan. Headache 2009; 49: 1267-1276.

Alfano DP. Emotional and pain-related factors in neuropsychological assessment following mild traumatic brain injury. Brain Cogn 2006; 60:194-196.

Bazarian JJ, Wong T, Harris M, Leahey N, Mookerjee S, Dombovy M. Epidemiology and predictors of post-concussive syndrome after minor head injury in an emergency population. Brain Inj 1999; 13: 173-189.

Bazarian JJ, Zhong J, Blyth B, Zhu T, Kavcic V, Peterson D. Diffusion tensor imaging detects clinically important axonal damage after mild traumatic brain injury: a pilot study. J Neurotrauma 2007; 24: 1447-1459.

Borgaro SR, Prigatano GP, Kwasnica C, Rexer JL. Cognitive and affective sequelae in complicated and uncomplicated mild traumatic brain injury. Brain Inj 2003; 17: 189-198.

Buzzi MG, Bivona U, Matteis M, Spanedda F, Formisano R. Cognitive and psychological patterns in post-traumatic headache following severe traumatic brain injury. Cephalalgia 2003; 23: 672 (P4L22).

Carney N, Ghajar J, Jagoda A, Bedrick S, Davis-O'Reilly C, du Coudray H, et al. Concussion guidelines step 1: systematic review of prevalent indicators. Neurosurgery 2014; 75: S2-S15.

Couch JR, Bearss C. Chronic daily headache in the posttrauma syndrome: relation to extent of head injury. Headache 2001; 41: 559-564.

Couch JR, Lipton RB, Stewart WF, Scher Al. Head or neck injury increases the risk of chronic daily headache: a population-based study. Neurology 2007; 69: 1169-1177.

Couch JR, Lipton R, Stewart WF. Is post-traumatic headache classifiable and does it exist? Eur J Neurol 2009; 16: 12-13.

De Benedittis G, De Santis A. Chronic post-traumatic headache: clinical, psychopathological features and outcome determinants. J Neurosurg Sci 1983; 27: 177-186.

De Kruijk JR, Leffers P, Menheere PP, Meerhoff S, Rutten J, Twijnstra A. Prediction of post-traumatic complaints after mild traumatic brain injury: early symptoms and biochemical markers. J Neurol Neurosurg Psychiat 2002; 73: 727-732.

Evans RW. Post-traumatic headaches. Neurol Clin N Am 2004; 22: 237-249.

Formisano R, Bivona U, Catani S, D’Ippolito M, Buzzi MG. Post-traumatic headache: facts and doubts. J Headache Pain 2009; 10: 145-152.

Gladstone J. From psychoneurosis to ICHD-2: an overview of the state of the art in post-traumatic headache. Headache 2009; 49: 1097-1111.

Jensen OK, Nielsen FF. The influence of sex and pre-traumatic headache on the incidence and severity of headache after head injury. Cephalalgia 1990; 10: 285-293. 
King NS. Emotional, neuropsychological, and organic factors: their use in the prediction of persisting postconcussion symptoms after moderate and mild head injuries. J Neurol Neurosurg Psychiat 1996; 61: 75-81.

King NS, Crawford S, Wenden FJ, Caldwell FE, Wade DT. Early prediction of persisting post-concussion symptoms following mild and moderate head injuries. Br J Clin Psychol 1999; 38 (Pt 1): 15-25.

Lahz S, Bryant RA. Incidence of chronic pain following traumatic brain injury. Arch Phys Med Rehabil 1996; 77: 889-891.

Leininger BE, Gramling SE, Farrell AD, Kreutzer JS, Peck EA, 3 rd. Neuropsychological deficits in symptomatic minor head injury patients after concussion and mild concussion. J Neurol Neurosurg Psychiat 1990; 53: 293-296.

Lenaerts ME. Post-traumatic headache: from classification challenges to biological underpinnings. Cephalalgia 2008; 28 Suppl 1:12-15.

Lew HL, Lin PH, Fuh JL, Wang SJ, Clark DJ, Walker WC. Characteristics and treatment of headache after traumatic brain injury: a focused review. Am J Phys Med Rehabil 2006; 85: 619-627.

Martins HA, Ribas VR, Martins BB, Ribas Rde M, Valenca MM. Post-traumatic headache. Arq Neuropsiquiatr 2009; 67: 43-45.

McAllister TW, Saykin AJ, Flashman LA, Sparling MB, Johnson SC, Guerin SJ, et al. Brain activation during working memory 1 month after mild traumatic brain injury: a functional MRI study. Neurology 1999; 53: 1300-1308.

Metting Z, Rodiger LA, De Keyser J, van der Naalt J. Structural and functional neuroimaging in mild-to-moderate head injury. Lancet-Neurol 2007; 6: 699-710.

Mickeviciene D, Schrader H, Nestvold K, Surkiene D, Kunickas R, Stovner L], Sand T. A controlled historical cohort study on the post-concussion syndrome. Eur J Neurol 2002; 9: 581-587.

Mickeviciene D, Schrader H, Obelieniene D, Surkiene D, Kunickas R, Stovner LJ, Sand T. A controlled prospective inception cohort study on the post-concussion syndrome outside the medicolegal context. Eur ] Neurol 2004; 11: 411-419.

Neely ET, Midgette LA, Scher Al. Clinical review and epidemiology of headache disorders in US service members: with emphasis on post-traumatic headache. Headache 2009; 49: 1089-1096.

Nampiaparampil DE. Prevalence of chronic pain after traumatic brain injury: a systematic review. JAMA 2008; 300: 711-719.

Obermann M, Nebel K, Schumann C, Holle D, Gizewski ER, Maschke M, et al. Gray matter changes related to chronic posttraumatic headache. Neurology 2009; 73: 978-983.

Obermann M, Holle D, Katsarava Z. Post-traumatic headache. Expert Rev Neurother 2009; 9: 1361-1370.

Packard RC. Current concepts in chronic post-traumatic headache. Curr Pain Headache Rep 2005; 9: 59-64.

Packard RC. Posttraumatic headache: permanency and relationship to legal settlement. Headache 1992; 32: 496-500.

Packard RC. Epidemiology and pathogenesis of posttraumatic headache. J Head Trauma Rehabil 1999; 14: 9-21.

Rimel RW, Giordani B, Barth JT, Boll TJ, Jane JA. Disability caused by minor head injury. Neurosurgery 1981; 9: 221-228.

Ruff RL, Ruff SS, Wang XF. Headaches among Operation Iraqi Freedom/ Operation Enduring Freedom veterans with mild traumatic brain injury associated with exposures to explosions. J Rehabil Res Dev 2008; 45: 941-952.

Sarmento E, Moreira P, Brito C, Souza J, Jevoux C, Bigal M. Proton spectroscopy in patients with post-traumatic Kopfschmerz zurückzuführen aufmild head injury. Headache 2009; 49: 1345-1352.

Schaumann-von Stosch R, Schmidt H, Sandor P. Posttraumatic headache - IHS chapter 5. Cephalalgia 2008; 28: 908-909.

Sheedy J, Harvey E, Faux S, Geffen G, Shores EA. Emergency department assessment of mild traumatic brain injury and the prediction of post- concussive symptoms: a 3-month prospective study. J Head Trauma Rehabil 2009; 24: 333-343.

Sheftell FD, Tepper S], Lay CL, Bigal ME. Post-traumatic headache: emphasis on chronic types following mild closed head injury. Neurol Sci 2007; 28: S203-S207.

Solomon S. Post-traumatic headache: commentary: an overview. Headache 2009; 49: 1112-1115.

Stovner LJ, Schrader H, Mickeviciene D, Surkiene D, Sand T. Postconcussion headache: reply to editorial. Eur J Neurol 2009; 16: e14.

Stovner LJ, Schrader H, Mickeviciene D, Surkiene D, Sand T. Headache after concussion. Eur J Neurol 2009; 16: 112-120.

Tatrow K, Blanchard EB, Hickling EJ, Silverman DJ. Posttraumatic headache: biopsychosocial comparisons with multiple control groups. Headache 2003; 43: 755-766.

Theeler BJ, Flynn FG, Erickson JC. Headaches after concussion in US soldiers returning from Iraq or Afghanistan. Headache 2010; 50: 1262-1272.

Theeler B], Erickson JC. Mild head trauma and chronic headaches in returning US soldiers. Headache 2009; 49: 529-334.

Thornhill S, Teasdale GM, Murray GD, McEwen J, Roy CW, Penny KI. Disability in young people and adults one year after head injury: prospective cohort study. BMJ 2000; 320: 1631-1635.

Uomoto JM, Esselman PC. Traumatic brain injury and chronic pain: differential types and rates by head injury severity. Arch Phys Med Rehabil 1993; 74: 61-64.

Walker WC, Seel RT, Curtiss G, Warden DL. Headache after moderate and severe traumatic brain injury: a longitudinal analysis. Arch Phys Med Rehabil 2005; 86: 1793-1800.

Yamaguchi M. Incidence of headache and severity of head injury. Headache 1992; 32: 427-431.

Yang CC, Hua MS, Tu YK, Huang SJ. Early clinical characteristics of patients with persistent post-concussion symptoms: a prospective study. Brain Inj 2009; 23: 299-306.

Yang CC, Tu YK, Hua MS, Huang SJ. The association between the postconcussion symptoms and clinical outcomes for patients with mild traumatic brain injury. J Trauma 2007; 62: 657-663.

Zasler ND. Posttraumatic headache: caveats and controversies. J Head Trauma Rehabil 1999; 14: 1-8.

\section{3, 5.4 Akuter oder anhaltender Kopfschmerz zurückzuführen auf ein} HWS-Beschleunigungstrauma

Obelieniene D, Schrader H, Bovim G, Miseviciene I, Sand T. Pain after whiplash: a prospective controlled inception cohort study. J Neurol Neurosurg Psychiat 1999; 66: 279-283.

Obermann M, Nebel K, Riegel A, Thiemann D, Yoon MS, Keidel M, et al. Incidence and predictors of chronic Kopfschmerz zurückzuführen aufwhiplash injury. Cephalalgia 2010; 30: 528-534.

O’Neill B, Haddon W, Jr., Kelley AB, Sorenson WW. Automobile head restraints - frequency of neck injury claims in relation to the presence of head restraints. Am J Public Health 1972; 62: 399-406.

Richter M, Otte D, Pohlemann T, Krettek C, Blauth M. Whiplash-type neck distortion in restrained car drivers: frequency, causes and long-term results. Eur Spine J 2000; 9: 109-117.

Spitzer WO, Skovron ML, Salmi LR, Cassidy JD, Duranceau J, Suissa S, et. al. Scientific monograph of the Quebec Task Force on Whiplash-Associated Disorders: redefining "whiplash" and its management. Spine 1995; 20 Suppl 8: 1S-73S. 


\section{5, 5.6 Akuter oder anhaltender Kopfschmerz zurückzuführen auf eine Kraniotomie}

De Benedittis G, Lorenzetti A, Spagnoli D, Migliore M, Tiberio F, Villani R. Postoperative pain in neurosurgery: a pilot study in brain surgery. Neurosurgery 1996; 38: 466-470.

De Gray LC, Matta BF. Acute and chronic pain following craniotomy: a review. Anaesthesia 2005; 60: 693-704.

De Oliveira Ribeiro MDC, Pereira CU, Sallum AM, Martins-Filho RP, Desantana JM, da Silva Nunes M, Hora EC. Immediate post-craniotomy headache. Cephalalgia 2013; 33: 897-905.

Gee JR, Ishaq Y, Vijayan N. Post craniotomy headache. Headache 2003; 43 : 276-278.

Harner SG, Beatty CW, Ebersold MJ. Headache after acoustic neuroma excision. Amer J Otology 1993; 14: 552-555.

Kaur A, Selwa L, Fromes G, Ross D. Persistent headache after supratentoria craniotomy. Neurosurgery 2000; 47: 633-636.

Rocha Filho P. Post-craniotomy headache after acoustic neuroma surgery. Cephalalgia 2010; 30: 509-510.

Rocha-Filho PA. Post-craniotomy headache: a clinical view with a focus on the persistent form. Headache 2015; 55: 733-738.

Rocha-Filho PAS, Gherpelli JLD, De Siqueira JTT, Rabello GD. Post-craniotomy headache: characteristics, behavior and effect on quality of life in patients operated for treatment of supratentorial intracranial aneurysms. Cephalalgia 2008; 28: 41-48.

Rocha-Filho PAS, Gherpelli JLD, de Siqueira JTT, Rabello GD. Post-craniotomy headache: a proposed revision of IHS diagnostic criteria. Cephalalgia 2010; 30: 560-566.

Schaller B, Baumann A. Headache after removal of vestibular schwannoma via the retrosigmoid approach: a long-term follow-up study. Otolaryngol Head Neck Surgery 2003; 128: 387-395.

Thibault M, Girard F, Moumdijian R, Chouinard P, Boudreault D, Ruel M. Craniotomy site influences postoperative pain following neurosurgical procedures: a retrospective study. Can J Anesth 2007; 54: 544-548.

Vijayan N. Postoperative headache in acoustic neuroma. Headache 1995; 2: 98-100.

\section{Kopfschmerz zurückzuführen auf Gefäßstörungen im Bereich des Kopfes und/oder des Halses}

\begin{tabular}{|l|l|}
\hline 6.1 & $\begin{array}{l}\text { Kopfschmerz zurückzuführen auf ein zerebrales ischä- } \\
\text { misches Ereignis }\end{array}$ \\
\hline 6.1 .1 & $\begin{array}{l}\text { Kopfschmerz zurückzuführen auf einen ischämischen } \\
\text { Infarkt (zerebraler Infarkt) }\end{array}$ \\
\hline 6.1 .1 .1 & $\begin{array}{l}\text { Akuter Kopfschmerz zurückzuführen auf einen ischä- } \\
\text { mischen Infarkt (zerebraler Infarkt) }\end{array}$ \\
\hline 6.1 .1 .2 & $\begin{array}{l}\text { Anhaltender Kopfschmerz zurückzuführen auf einen } \\
\text { früheren ischämischen Infarkt (zerebraler Infarkt) }\end{array}$ \\
\hline 6.1 .2 & $\begin{array}{l}\text { Kopfschmerz zurückzuführen auf eine transitorische } \\
\text { ischämische Attacke (TIA) }\end{array}$ \\
\hline 6.2 & $\begin{array}{l}\text { Kopfschmerz zurückzuführen auf eine nicht-traumatische } \\
\text { intrakranielle Blutung }\end{array}$ \\
\hline 6.2 .1 & $\begin{array}{l}\text { Akuter Kopfschmerz zurückzuführen auf eine nicht- } \\
\text { traumatische intrazerebrale Blutung }\end{array}$ \\
\hline 6.2 .2 & $\begin{array}{l}\text { Kopfschmerz zurückzuführen auf eine nicht-traumatische } \\
\text { Subarachnoidalblutung (SAB) }\end{array}$ \\
\hline 6.2 .3 & $\begin{array}{l}\text { Akuter Kopfschmerz zurückzuführen auf ein nicht- } \\
\text { traumatisches akutes Subduralhämatom (aSDH) }\end{array}$ \\
\hline
\end{tabular}

\begin{tabular}{|c|c|}
\hline 6.2 .4 & $\begin{array}{l}\text { Anhaltender Kopfschmerz zurückzuführen auf eine } \\
\text { frühere nicht-traumatische intrakranielle Blutung }\end{array}$ \\
\hline 6.3 & $\begin{array}{l}\text { Kopfschmerz zurückzuführen auf eine nicht-rupturierte } \\
\text { Gefäßmissbildung }\end{array}$ \\
\hline 6.3 .1 & $\begin{array}{l}\text { Kopfschmerz zurückzuführen auf ein nicht-rupturiertes } \\
\text { sackförmiges Aneurysma }\end{array}$ \\
\hline 6.3 .2 & $\begin{array}{l}\text { Kopfschmerz zurückzuführen auf eine arterio-venöse } \\
\text { Malformation (AVM) }\end{array}$ \\
\hline 6.3 .3 & $\begin{array}{l}\text { Kopfschmerz zurückzuführen auf eine durale arterio- } \\
\text { venöse Fistel (DAVF) }\end{array}$ \\
\hline 6.3 .4 & Kopfschmerz zurückzuführen auf ein kavernöses Angiom \\
\hline 6.3 .5 & $\begin{array}{l}\text { Kopfschmerz zurückzuführen auf eine enzephalo-trigemi- } \\
\text { nale oder leptomeningeale Angiomatose (Sturge-Weber- } \\
\text { Syndrom) }\end{array}$ \\
\hline 6.4 & Kopfschmerz zurückzuführen auf eine Arteriitis \\
\hline 6.4 .1 & $\begin{array}{l}\text { Kopfschmerz zurückzuführen auf eine Riesenzellarteriitis } \\
\text { (RZA) }\end{array}$ \\
\hline 6.4 .2 & $\begin{array}{l}\text { Kopfschmerz zurückzuführen auf eine primäre Vaskulitis } \\
\text { des ZNS }\end{array}$ \\
\hline 6.4 .3 & $\begin{array}{l}\text { Kopfschmerz zurückzuführen auf eine sekundäre } \\
\text { Vaskulitis des ZNS }\end{array}$ \\
\hline 6.5 & $\begin{array}{l}\text { Kopfschmerz zurückzuführen auf eine Störung der } \\
\text { A. carotis oder A. vertebralis }\end{array}$ \\
\hline 6.5 .1 & $\begin{array}{l}\text { Kopf-, Gesichts- oder Halsschmerz zurückzuführen auf } \\
\text { eine Dissektion der A. carotis oder vertebralis }\end{array}$ \\
\hline 6.5.1.1 & $\begin{array}{l}\text { Akuter Kopf-, Gesichts- oder Halsschmerz zurückzuführen } \\
\text { auf eine arterielle Dissektion }\end{array}$ \\
\hline 6.5.1.2 & $\begin{array}{l}\text { Anhaltender Kopf-, Gesichts- oder Halsschmerz zurück- } \\
\text { zuführen auf eine frühere arterielle Dissektion }\end{array}$ \\
\hline 6.5 .2 & Kopfschmerz zurückzuführen auf eine Endarteriektomie \\
\hline 6.5 .3 & $\begin{array}{l}\text { Kopfschmerz zurückzuführen auf eine Carotis- oder } \\
\text { Vertebralis-Angioplastie oder Stentimplantation }\end{array}$ \\
\hline 6.6 & $\begin{array}{l}\text { Kopfschmerz zurückzuführen auf eine Hirnvenen- } \\
\text { erkrankung }\end{array}$ \\
\hline 6.6 .1 & $\begin{array}{l}\text { Kopfschmerz zurückzuführen auf eine Hirnvenenthrom- } \\
\text { bose }\end{array}$ \\
\hline 6.6 .2 & $\begin{array}{l}\text { Kopfschmerz zurückzuführen auf Stentimplantation eines } \\
\text { venösen Hirnsinus }\end{array}$ \\
\hline 6.7 & $\begin{array}{l}\text { Kopfschmerz zurückzuführen auf eine sonstige akute } \\
\text { intrakranielle Störung }\end{array}$ \\
\hline 6.7 .1 & $\begin{array}{l}\text { Kopfschmerz zurückzuführen auf einen intrakraniellen } \\
\text { endarteriellen Eingriff }\end{array}$ \\
\hline 6.7 .2 & Kopfschmerz zurückzuführen auf eine Angiographie \\
\hline 6.7 .3 & $\begin{array}{l}\text { Kopfschmerz zurückzuführen auf ein reversibles zere- } \\
\text { brales Vasokonstriktionsyndrom (RCVS) }\end{array}$ \\
\hline 6.7.3.1 & $\begin{array}{l}\text { Akuter Kopfschmerz zurückzuführen auf ein reversibles } \\
\text { zerebrales Vasokonstriktionsyndrom (RCVS) }\end{array}$ \\
\hline 6.7.3.2 & $\begin{array}{l}\text { Akuter Kopfschmerz wahrscheinlich zurückzuführen auf } \\
\text { ein reversibles zerebrales Vasokonstriktionsyndrom (RCVS) }\end{array}$ \\
\hline 6.7.3.3 & $\begin{array}{l}\text { Anhaltender Kopfschmerz zurückzuführen auf ein früheres } \\
\text { reversibles zerebrales Vasokonstriktionsyndrom (RCVS) }\end{array}$ \\
\hline 6.7 .4 & $\begin{array}{l}\text { Kopfschmerz zurückzuführen auf eine intrakranielle } \\
\text { arterielle Dissektion }\end{array}$ \\
\hline 6.8 & $\begin{array}{l}\text { Kopfschmerz und/oder migräneartige Aura zurückzu- } \\
\text { führen auf eine chronische intrakranielle Vaskulopathie }\end{array}$ \\
\hline 6.8 .1 & $\begin{array}{l}\text { Kopfschmerz zurückzuführen auf eine zerebrale autoso- } \\
\text { mal dominante Arteriopathie mit subkortikalen Infarkten } \\
\text { und Leukenzephalopathie (CADASIL) }\end{array}$ \\
\hline
\end{tabular}


6.8.2 Kopfschmerz zurückzuführen auf eine mitochondriale Enzephalopathie, Laktatazidose und Schlaganfall-ähnlichen Episoden (MELAS)

6.8.3 Kopfschmerz zurückzuführen auf eine MoyamoyaAngiopathie

6.8.4 Migräneartige Aura zurückzuführen auf eine zerebrale Amyloidangiopathie

6.8.5 Kopfschmerz zurückzuführen auf ein retinales Vaskulopathie-Syndrom mit zerebraler Leukenzephalopathie und systemischen Manifestationen

6.8.6 Kopfschmerz zurückzuführen auf eine sonstige genetisch bedingte Vaskulopathie

6.9 Kopfschmerz zurückzuführen auf einen Hypophyseninfarkt

An anderer Stelle kodiert Primärer und/oder sekundärer Kopfschmerz?

Primärer und/oder sekundärer Kopfschmerz?

Für den 6. Kopfschmerz zurückzuführen auf Gefäßstörungen im Bereich des Kopfes und/oder des Halses gelten die allgemeinen Regeln für die Zuordnung zu einer anderen Erkrankung:

Tritt ein neuer Kopfschmerz erstmals in engem zeitlichen Zusammenhang mit einer kraniellen oder vaskulären Störung auf, sollte der Kopfschmerz als Kopfschmerz zurückzuführen auf eine vaskuläre Störung kodiert werden. Dies ist auch der Fall, wenn der neue Kopfschmerz das klinische Bild einer der in Teil eins der ICDH-3 klassifizierten primären Kopfschmerzerkrankungen aufweist.

Diese Regel gilt analog für neue migräneauraartige Symptome, die erstmals in enger zeitlicher Beziehung zu einer kraniellen oder zervikalen vaskulären Störung auftreten.

Wenn ein vorbestehender Kopfschmerz mit dem klinischen Erscheinungsbild in engem zeitlichen Zusammenhang mit einer kraniellen oder zervikalen Gefäßstörung chronisch wird oder sich deutlich verschlechtert (was üblicherweise eine mindestens zweifache Zunahme der Häufigkeit und/oder Schwere bedeutet), sollte sowohl die Ausgangs-Kopfschmerzdiagnose als auch die Diagnose eines 6. Kopfschmerzes zurückzuführen auf Gefäßstörungen im Bereich des Kopfes und/oder des Halses (bzw. eines Typs oder Subtyps hiervon) gestellt werden, vorausgesetzt, es bestehen gute Hinweise darauf, dass die Störung Kopfschmerzen verursachen kann.

\section{Einleitung}

In der Regel lässt sich ein kausaler Zusammenhang zwischen Kopfschmerzen und den aufgeführten Gefäßstörungen leicht herstellen, da die Kopfschmerzen akut auftreten, mit anderen neurologischen Symptomen verbunden sind und häufig schnell remittieren. Der Nachweis des engen zeitlichen Zusammenhanges zwischen Auftreten der Kopfschmerzen und diesen neurologischen Symptomen ist dabei entscheidend, um den Kausalzusammenhang zu ermitteln.

Bei vielen dieser Störungen, z. B. beim ischämischen bzw. hämorrhagischen Infarkt, werden die Kopfschmerzen durch fokal-neurologische Symptome und/oder Bewusstseinsstörungen überschattet. Bei anderen Störungen stehen die Kopfschmerzen ganz im Vordergrund, wie z. B. bei der Subarachnoidalblutung. Bei einigen Erkrankungen, die sowohl Kopfschmerzen als auch einen
Hirninfarkt hervorrufen können, z. B. bei einer Gefäßdissektion, einer Hirnvenenthrombose, einer Riesenzellarteriitis oder anderen Angiitiden des ZNS, sind Kopfschmerzen oft ein initiales Warnsymptom. Es ist hier von entscheidender Bedeutung, den Zusammenhang zwischen den Kopfschmerzen und der zugrundeliegenden Störung zu erkennen, um die richtige Diagnose stellen und die entsprechende Therapie schnellstmöglich einleiten zu können, um potentiell verheerende neurologische Komplikationen zu verhindern.

Diese Gefäßstörungen können auch bei Patienten auftreten, die bereits unter einem primären Kopfschmerz gelitten haben. Ein entscheidender Hinweis auf eine vaskuläre Genese von Kopfschmerzen ist hier der in der Regel plötzliche Beginn eines neuen Kopfschmerztyps, wie er dem Patienten bislang unbekannt war. In diesen Fällen sollte immer nach vaskulären Ursachen der Kopfschmerzen gefahndet werden.

Die diagnostischen Kriterien der aufgeführten Gefäßstörungen enthalten möglichst folgende Punkte:

A. Kopfschmerz, der das Kriterium C erfüllt

B. Es wurde eine kranielle und/oder zervikale Gefäßstörung nachgewiesen, von der bekannt ist, dass sie Kopfschmerzen verursachen kann

C. Ein kausaler Zusammenhang kann durch mindestens zwei der folgenden Kriterien gezeigt werden:

1. Der Kopfschmerz ist in einem zeitlichen Zusammenhang mit dem Beginn der kraniellen und/oder zervikalen Gefäßstörung aufgetreten

2. Einer oder beide der folgenden Punkte sind erfüllt:

a) der Kopfschmerz hat sich gleichzeitig mit der Verschlechterung der kraniellen und/oder zervikalen Gefäßstörung deutlich verschlechtert

b) der Kopfschmerz hat sich gleichzeitig mit der Besserung der kraniellen und/oder zervikalen Gefäßstörung deutlich gebessert

3. Der Kopfschmerz weist Merkmale auf, die für die kranielle und/oder zervikale Gefäßstörung typisch sind

4. Es existieren weitere Belege für den ursächlichen Zusammenhang

D. Nicht besser erklärt durch eine andere ICHD-3-Diagnose.

\subsection{Kopfschmerz zurückzuführen auf ein zerebrales ischämisches Ereignis}

\subsubsection{KKopfschmerz zurückzuführen auf einen ischämischen Infarkt (zerebraler Infarkt)}

\subsubsection{KAkuter Kopfschmerz zurückzuführen auf einen ischämischen Infarkt (zerebraler Infarkt) \\ Beschreibung:}

Neu aufgetretener und gewöhnlich akut beginnender Kopfschmerz, der durch einen ischämischen Infarkt verursacht wird und von fokalen neurologischen Hinweisen auf den Infarkt begleitet wird. Er ist sehr selten das führende oder prominente Merkmal einer ischämischen Attacke. In der Regel verläuft er selbst-limitierend.

Diagnostische Kriterien:

A. Jeder neue Kopfschmerz, der die Kriterien C und D erfüllt

B. Es wurde ein akuter ischämischer Infarkt diagnostiziert 
C. Ein kausaler Zusammenhang kann durch mindestens eines der folgenden Kriterien gezeigt werden:

1. Der Kopfschmerz ist in sehr engem zeitlichem Zusammenhang mit anderen Symptomen und/oder klinischen Symptome eines ischämischen Infarkts aufgetreten oder war maßgeblich für die Diagnose eines ischämischen Infarkts

2. Der Kopfschmerz hat sich gleichzeitig zur Stabilisierung oder Besserung anderer Symptome bzw. klinischer oder radiologischer Symptome eines ischämischen Infarkts deutlich gebessert

D. Einer der folgenden Punkte ist erfüllt:

1. Der Kopfschmerz ist innerhalb von 3 Monaten verschwunden 1

2. Der Kopfschmerz ist noch nicht verschwunden, aber die 3 Monate sind noch nicht vorbei1

E. Nicht besser erklärt durch eine andere ICHD-3-Diagnose.

Anmerkung:

Die 3 Monate sollten ab Stabilisierung gezählt werden, ob spontan oder durch Behandlung, statt ab Beginn des ischämischen Infarkts.

\section{Kommentar:}

Der 6.1.1.1 akute Kopfschmerz zurückzuführen auf einen ischämischen Infarkt (zerebraler Infarkt) tritt in Begleitung fokaler neurologischer Symptome und/oder Bewusstseinsstörungen auf, was in den meisten Fällen eine problemlose Differenzierung von den primären Kopfschmerzen erlaubt. Er ist üblicherweise von moderater Intensität und hat keine spezifischen Merkmale. Er kann ipsilateral zum Infarkt auftreten oder bilateral. In seltenen Fällen kann sich ein akuter ischämischer Infarkt, vor allem ein embolischer Kleinhirnoder supratentorieller Hirninfarkt, mit einem isolierten plötzlichen (sogar Donnerschlag-) Kopfschmerz vorliegen.

Ein ischämischer Infarkt wird in bis zu einem Drittel der Fälle von Kopfschmerzen begleitet. Kopfschmerzen sind dabei häufiger bei Infarkten im Versorgungsbereich der A. basilaris als im Versorgungsbereich der A. carotis. Die Kopfschmerzen sind bei der ätiologischen Einordnung der Infarkte wenig hilfreich mit Ausnahme davon, dass bei lakunären Infarkten Kopfschmerzen sehr selten sind.

Sehr häufig treten Kopfschmerzen jedoch bei akuten Störungen der Arterienwände auf, die zu einem ischämischen Infarkt führen können, etwa bei einer arteriellen Dissektion oder einem reversiblen zerebralen Vasokonstriktionssyndrom. Bei Letzteren kann der Kopfschmerz unmittelbar durch die Läsionen der Arterienwand ausgelöst werden und einen ischämischen Infarkt ankündigen; dieser Kopfschmerz wäre dann korrekter unter der Störung der Arterienwand zu kodieren.

\subsubsection{Anhaltender Kopfschmerz zurückzuführen auf einen früheren ischämischen Infarkt (zerebraler Infarkt) \\ Beschreibung:}

Ein Kopfschmerz, der von einem ischämischen Infarkt verursacht wurde und mehr als 3 Monate nach Stabilisierung des Infarkts fortbesteht.
Diagnostische Kriterien:

A. Kopfschmerz, der zuvor als 6.1.1.1 akuter Kopfschmerz zurückzuführen auf einen ischämischen Infarkt (zerebraler Infarkt), diagnostiziert wurde und Kriterium C erfüllt

B. Der ischämische Infarkt hat sich stabilisiert, spontan oder durch Behandlung

C. Der Kopfschmerz hält>3 Monate nach Stabilisierung des ischämischen Infarkts an

D. Nicht besser erklärt durch eine andere ICHD-3-Diagnose.

\section{Kommentar:}

In einigen Untersuchungen wurden Kopfschmerzen dokumentiert, die die Kriterien für einen 6.1.1.2 anhaltenden Kopfschmerz zurückzuführen auf einen früheren ischämischen Infarkt (zerebraler Infarkt) erfüllen. Weitere Untersuchungen sind erforderlich, um Risikofaktoren für einen derartigen anhaltenden Kopfschmerz zu ermitteln; eine 1. Migräne in der Vorgeschichte kann eine Rolle spielen, ebenso wie Ängste/Depressionen.

\subsubsection{Kopfschmerz zurückzuführen auf eine transitorische ischämische Attacke (TIA)}

\section{Beschreibung:}

Kopfschmerz, der durch eine transitorische ischämische Attacke (TIA) verursacht wird und von den plötzlich beginnenden transitorischen fokalen Symptomen einer TIA begleitet wird. Er hält weniger als 24 Stunden an.

\section{Diagnostische Kriterien:}

A. Jeder neue Kopfschmerz, der das Kriterium C erfüllt

B. Es wurde eine transitorische ischämische Attacke (TIA) diagnostiziert

C. Ein kausaler Zusammenhang kann durch beide der folgenden Kriterien gezeigt werden:

1. Der Kopfschmerz ist gleichzeitig mit anderen Symptomen und/oder klinischen Symptomen einer TIA aufgetreten

2. Der Kopfschmerz ist innerhalb von 24 Stunden verschwunden

D. Nicht besser erklärt durch eine andere ICHD-3-Diagnose 1;2.

\section{Anmerkung:}

Die Differenzialdiagnose zwischen einem 6.1.2 Kopfschmerz zurückzuführen auf eine transitorische ischämische Attacke und einer Attacke im Sinne einer 1.2 Migräne mit Aura kann besonders schwierig sein. Die Art und Weise des Beginns ist ausschlaggebend: Das fokal-neurologische Defizit beginnt bei der TIA typischerweise plötzlich, während es sich bei der Migräneaura allmählich entwickelt. Darüber hinaus treten „positive“ Phänomene (z. B. ein Flimmerskotom) häufiger bei Migräneauren auf, während bei einer TIA negative Phänomene überwiegen.

2. Das Zusammentreffen einer ansonsten typischen TIA und eines schweren Kopfschmerzes sollte Anlass zu einer Suche nach arteriellen Störungen sein, die unmittelbar schwere Kopfschmerzen induzieren können (u. a. arterielle Dissektion).

\section{Kommentar:}

Eine transitorische ischämische Attacke (TIA) ist eine vorübergehende Episode neurologischer Funktionsstörungen, die durch das 
fokale Gehirn verursacht werden oder eine retinale Ischämie ohne klinischen, bildgebenden oder sonstigen Nachweis eines akuten zerebralen oder retinalen Infarkts. Die TIA-Symptome dauern üblicherweise, doch nicht immer, weniger als eine Stunde.

Kopfschmerzen sind nur selten ein Leitsymptom einer TIA und dann eher im basilären als im Carotis-Stromgebiet.

\subsection{Kopfschmerz zurückzuführen auf eine nicht-traumatische intrakranielle Blutung}

An anderer Stelle kodiert: Kopfschmerzen zurückzuführen auf eine traumatische intrazerebrale und/oder Subarachnoidalblutung oder ein traumatisches intrazerebrales, Subdural- oder Epiduralhämatom werden unter 5.1.1 akuter posttraumatischer Kopfschmerz bei mittlerer oder schwerer Kopfverletzung oder 5.2.1 chronischer posttraumatischer Kopfschmerz bei mittlerer oder schwerer Kopfverletzung kodiert.

\section{Beschreibung:}

Kopfschmerz verursacht durch eine nicht-traumatische intrakranielle Blutung, gewöhnlich mit plötzlichem (sogar Donnerschlagkopfschmerz) Beginn. Je nach Art der Blutung kann dieser isoliert auftreten oder in Begleitung fokaler neurologischer Defizite.

\subsubsection{Akuter Kopfschmerz zurückzuführen auf eine nicht-traumatische intrazerebrale Blutung}

Beschreibung:

Neuer und meist akut beginnender Kopfschmerz infolge einer nicht-traumatischen intrazerebralen Blutung in Begleitung fokaler neurologischer Symptome der intrazerebralen Blutung. Der Kopfschmerz kann in seltenen Fällen das sich manifestierende und führende Symptom einer nicht-traumatischen intrazerebralen Blutung sein.

\section{Diagnostische Kriterien:}

A. Jeder neue Kopfschmerz, der die Kriterien C und D erfüllt

B. Es wurde eine intrazerebrale Blutung (ICB) 1 bei fehlendem Kopftrauma diagnostiziert

C. Ein kausaler Zusammenhang kann durch mindestens zwei der folgenden Kriterien gezeigt werden:

1. Der Kopfschmerz tritt in engem zeitlichem Zusammenhang mit anderen Symptomen und/oder klinischen Symptome einer ICB auf oder war maßgeblich für die Diagnose einer ICB

2. Der Kopfschmerz hat sich gleichzeitig mit der Stabilisierung oder Besserung anderer Symptome bzw. klinischer oder radiologischer Zeichen von ICB deutlich gebessert

3. Der Kopfschmerz weist mindestens eines der folgenden Merkmale auf:

a) plötzlicher Beginn oder Beginn als Donnerschlagkopfschmerz

b) maximale Intensität am Tag des Kopfschmerzbeginns

c) in Übereinstimmung mit dem Situs der Blutung lokalisiert
D. Es ist einer der folgenden Punkte erfüllt:

1. Kopfschmerz ist innerhalb von 3 Monaten verschwunden2

2. Kopfschmerz ist noch nicht verschwunden, aber es sind noch keine 3 Monate verstrichen2

E. Nicht besser erklärt durch eine andere ICHD-3-Diagnose.

Anmerkung:

1. Gemäß des allgemeinen Wortgebrauchs schließt der Begriff intrazerebral in diesem Zusammenhang auch den Begriff intrazerebellär ein.

2. Die 3 Monate sollten ab Stabilisierung gezählt werden, ob spontan oder durch Behandlung, statt ab Beginn der intrazerebralen Blutung.

\section{Kommentar:}

Der 6.2.1 akute Kopfschmerz zurückzuführen auf eine intrazerebrale Blutung wird häufiger durch subarachnoidales Blut oder eine lokale Kompression hervorgerufen als durch eine intrakranielle Hypertension. In Einzelfällen kann sich der Kopfschmerz als Donnerschlagkopfschmerz manifestieren.

Kopfschmerz ist beim hämorrhagischen Infarkt verbreiteter und schwerer als beim ischämischen Infarkt. Bei seinem Auftreten zum Infarktbeginn ist Kopfschmerz bei einer intrazerebralen Blutung mit einem höheren Risiko frühzeitiger Mortalität verbunden, nicht jedoch beim ischämischen Infarkt.

Der Kopfschmerz wird gewöhnlich von fokalen Defiziten oder Koma überschattet, kann jedoch das hervorstechende Frühsymptom einiger intrazerebraler Blutungen, vor allem einer zerebellären Blutung, sein. Hier ist gegebenenfalls eine notfallmäßige chirurgische Entlastung geboten.

\subsubsection{Kopfschmerz zurückzuführen auf eine nicht-traumatische Subarachnoidalblutung (SAB)}

An anderer Stelle kodiert: Es wird zwischen einer nicht-traumatischen Subarachnoidalblutung (SAB) und einer nicht-traumatischen konvexalen Subarachnoidalblutung unterschieden. Letztere kann je nach ihren unterschiedlichen Grundursachen klinisch und radiologisch ein hochgradig variables Erscheinungsbild bieten. Zu diesen Grundursachen gehören ein reversibles zerebrales Vasokonstriktionssyndrom (RCVS), eine zerebrale Amyloidangiopathie, eine Endokarditis und eine Hirnvenenthrombose. Patienten mit auraähnlichen Attacken, konvexaler Subarachnoidalblutung und zerebralen Amyloidangiopathie sollten unter 6.8.4 migräneartige Aura zurückzuführen auf eine zerebrale Amyloidangiopathie kodiert werden. Patienten mit Kopfschmerzen, konvexaler Subarachnoidalblutung und RCVS sollten unter 6.7.3. Kopfschmerz zurückzuführen auf ein reversibles zerebrales Vasokonstriktionsyndrom kodiert werden.

Beschreibung:

Kopfschmerz infolge einer nicht-traumatischen Subarachnoidalblutung (SAB), gewöhnlich mit heftigem und plötzlichem Beginn, wobei die Schmerzintensität innerhalb von Sekunden (Donnerschlagkopfschmerz) oder Minuten ihren Höhepunkt erreicht. Er kann das einzige Symptom einer nicht-traumatischen SAB sein. 
Diagnostische Kriterien:

A. Jeder neue Kopfschmerz, der die Kriterien C und D erfüllt

B. Es wurde eine Subarachnoidalblutung (SAB) bei fehlendem Kopftrauma diagnostiziert

C. Ein kausaler Zusammenhang kann durch mindestens zwei der folgenden Kriterien gezeigt werden:

1. Der Kopfschmerz ist in engem zeitlichem Zusammenhang mit anderen Symptomen und/oder klinischen Zeichen einer SAB aufgetreten oder war maßgeblich für die Diagnose einer $S A B$

2. Der Kopfschmerz hat sich parallel zur Stabilisierung oder Besserung anderer Symptome bzw. klinischer oder radiologischen Zeichen einer SAB deutlich gebessert

3. Der Kopfschmerz beginnt plötzlich oder als Donnerschlagkopfschmerz

D. Einer der folgenden Punkte ist erfüllt:

1. Kopfschmerz ist innerhalb von 3 Monaten verschwunden 1

2. Kopfschmerz ist noch nicht verschwunden, aber es sind noch keine 3 Monate verstrichen 1

E. Nicht besser erklärt durch eine andere ICHD-3-Diagnose2;3.

\section{Anmerkung:}

1. Die 3 Monate sollten ab Stabilisierung gezählt werden, ob spontan oder durch Behandlung, statt ab Beginn der Subarachnoidalblutung $(\mathrm{SAB})$.

2. Die Diagnose SAB kann meist mittels CCT ohne Kontrastmittel bestätigt werden. Die Sensitivität der Bildgebung liegt in den ersten 6 Stunden nach Kopfschmerzbeginn bei fast 99\% nach 12 Stunden bei $93 \%$, und nach 24 Stunden bei $93 \%$ (fällt jedoch nach 7 Tagen auf $50 \%$ ). Sind die CCT-Ergebnisse diagnostisch unzureichend, ist eine Lumbalpunktion unumgänglich: bei allen Fällen von aneurysmaler SAB zeigt sich bei Liquorentnahme zwischen 12 Stunden und 2 Wochen nach Einsetzen der Symptome und deren spektrophotometrischer Analyse eine Liquorxanthochromie. Ein MRT ist als erster diagnostischer Test auf eine SAB nicht indiziert. Vielmehr können FLAIR und T2-gewichtete Gradienten-Echo (GE)-Sequenzen diagnostisch von Nutzen sein, wenn der CCT-Befund unauffällig und der Liquorbefund auffällig ist.

3. Das Vorliegen einer nicht-traumatischen konvexalen Subarachnoidalblutung sowie von vorgerücktem Lebensalter, sensomotorischen Störungen, stereotypen auraähnlichen Anfällen und Fehlen schwererer Kopfschmerzen lässt an eine zerebrale Amyloidangiopathie als Grundursache denken. Ein jüngeres Lebensalter und rezidivierender Donnerschlagkopfschmerz lassen an ein reversibles zerebrales Vasokonstriktionssyndrom denken.

\section{Kommentar:}

Eine nicht-traumatische Subarachnoidblutung $(S A B)$ ist eine der häufigsten Ursachen für einen anhaltenden extremen Kopfschmerz mit abruptem Beginn (Donnerschlagkopfschmerz). Sie ist ein ernstes Krankheitsbild (die Sterblichkeitsrate beträgt $40 \%$ bis $50 \%, 10 \%$ bis $20 \%$ der Patienten sterben, bevor sie das Krankenhaus erreicht haben und $50 \%$ der Überlebenden behalten bleibende Schäden zurück).

Manchmal ist der 6.2.2 akute Kopfschmerz zurückzuführen auf eine nicht-traumatische Subarachnoidalblutung (SAB) aber auch moderat und tritt ohne Zusatzsymptome auf. Der abrupte Beginn ist das Leitsymptom. Jeder Patient mit abrupten Kopfschmerzbeginn oder Donnerschlagkopfschmerz sollte auf eine SAB hin untersucht werden.

Eine verspätete Diagnose hat oft katastrophale Folgen: eine SAB verlangt notfallmäßig nach entsprechenden Neurointerventionen. Allerdings kommt es zunächst bei einem Viertel bis zur Hälfte der Patienten zu einer Fehldiagnose, wobei die häufigste spezifische Fehldiagnose die einer Migräne ist. Zu den gängigsten Gründen für eine Fehldiagnose gehören unterbliebene bildgebende Untersuchungen oder eine Missdeutung von deren Ergebnissen, oder auch eine nicht durchgeführte Lumbalpunktion in Fällen, in denen eine solche erforderlich ist.

Nach der Diagnose einer SAB besteht der nächste dringende Schritt darin, ein geplatztes Aneurysma zu ermitteln (80\% der Fälle von spontaner SAB gehen auf rupturierte sackförmige Aneurysmen zurück). Bei Patienten, die zunächst eine falsche Diagnose erhalten und bei denen eine SAB erst verspätet erkannt wird, lässt sich bei deren Wiedervorstellung einige Tage später oft kein Aneurysma und keine erkennbare Ursache für die SAB ermitteln.

\subsubsection{Akuter Kopfschmerz zurückzuführen auf ein nicht-traumatisches akutes Subduralhämatom (aSDH)}

An anderer Stelle kodiert: In den meisten Fällen tritt ein akutes Subduralhämatom infolge eines Kopftraumas ein; der Kopfschmerz sollte in solchen Fällen entsprechend kodiert werden.

\section{Beschreibung:}

Kopfschmerz aufgrund eines akuten Subduralhämatoms nicht-traumatischer Ursache, gewöhnlich stark und plötzlich eintretend und bienn Sekunden (Donnerschlagkopfschmerz) oder Minuten den Höhepunkt erreichend. Meist wird dieser von fokalen Symptomen und Bewusstseinsstörungen begleitet oder diese treten dabei schnell auf.

\section{Diagnostische Kriterien:}

A. Jeder neue Kopfschmerz, der die Kriterien C und D erfüllt

B. Es wurde ein akutes Subduralhämatom ohne Kopftrauma diagnostiziert

C. Ein kausaler Zusammenhang kann durch mindestens zwei der folgenden Kriterien gezeigt werden:

1. Der Kopfschmerz ist in einem sehr engen zeitlichen Zusammenhang mit anderen Symptomen und/oder klinischen Zeichen eines aSDH aufgetreten oder war maßgeblich für die Diagnose aSDH

2. Einer oder beide der folgenden Punkte sind erfüllt:

a) es trat eine deutliche Kopfschmerzverschlechterung parallel zu der Verschlechterung des aSDH ein

b) es trat eine deutliche Kopfschmerzbesserung parallel zur Besserung anderer Symptome bzw. klinischer oder radiologischer Zeichen von aSDH ein

3. Einer oder beide der folgenden Punkte sind erfüllt:

a) plötzlicher Beginn oder Beginn als Donnerschlagkopfschmerz

b) in Übereinstimmung mit dem Situs der Blutung lokalisiert 
D. Einer der folgenden Punkte ist erfüllt:

1. Der Kopfschmerz ist innerhalb von 3 Monaten verschwunden 1

2. Der Kopfschmerz ist noch nicht verschwunden, aber es sind noch keine 3 Monate seit seinem Beginn verstrichen 1

E. Nicht besser erklärt durch eine andere ICHD-3-Diagnose.

\section{Anmerkung:}

1. Die 3 Monate sollten ab Stabilisierung, sei es spontan oder durch Behandlung, statt ab Beginn des akuten Subduralhämatoms erfolgen.

\section{Kommentar:}

Ein nicht-traumatisches akutes Subduralhämatom (aSDH) ohne sonstige intrakranielle Blutung („,reines aSDH“) ist selten. Es stellt eine lebensbedrohliche Erkrankung dar und ist ein neurochirurgischer Notfall.

Die Blutung kann arteriellen oder venösen Ursprungs sein. Als Ursachen werden unter anderem eine „spontane“ Ruptur einer kortikalen Arterie, die Ruptur eines Aneurysmas, arteriovenöse Gefäßmissbildungen und durale arteriovenöse Fisteln, Tumore oder Metastasen, Störungen der Blutgerinnung, eine Moyamoya-Erkrankung, eine Hirnvenenthrombose und intrakranieller Hypotonie genannt. Vereinzelte Fälle oder kleine Fallserien wurden meist von Neurochirurgen berichtet. Kopfschmerz wird in $25 \%$ bis $100 \%$ der Fälle beschrieben, je nach der Serie und der Grunderkrankung. Ein isolierter Kopfschmerz kann als führendes Symptom auftreten; dieser ist jedoch in der Regel von einer rapiden neurologischen Verschlechterung begleitet oder eine solche folgt ihm.

\subsubsection{Anhaltender Kopfschmerz zurückzuführen auf eine frühere nicht-traumatische intrakranielle Blutung}

Beschreibung:

Kopfschmerz, der durch eine nicht-traumatische intrakranielle Blutung verursacht wird und für mehr als 3 Monate nach Stabilisierung der Blutung fortbesteht.

\section{Diagnostische Kriterien:}

A. Kopfschmerz, der früher als 6.2.1 akuter Kopfschmerz zurückzuführen auf eine nicht-traumatische intrazerebrale Blutung, 6.2.2 akuter Kopfschmerz zurückzuführen auf eine nicht-traumatische Subarachnoidalblutung (SAB) oder 6.2.3 akuter Kopfschmerz zurückzuführen auf ein nicht-traumatisches akutes Subduralhämatom diagnostiziert wurde und das Kriterium Cerfüllt

B. Die intrakranielle Blutung (gleich welchen Typs) hat sich spontan oder durch Behandlung stabilisiert

C. Der Kopfschmerz besteht > 3 Monate nach Stabilisierung der intrakraniellen Blutung fort

D. Nicht besser erklärt durch eine andere ICHD-3-Diagnose.

\section{Kommentar:}

In einigen Untersuchungen wurden Kopfschmerzen dokumentiert, die die Kriterien für 6.2.4 anhaltender Kopfschmerz zurückzuführen auf eine frühere nicht-traumatische intrakranielle Blutung erfüllen. Weitere Untersuchungen sind erforderlich, um Risikofakto- ren für einen derartigen anhaltenden Kopfschmerz zu ermitteln; 1. Migräne in der Vorgeschichte kann eine Rolle spielen, ebenso wie Ängste/Depression.

Kodierbare Subtypen sind 6.2.4.1 anhaltender Kopfschmerz zurückzuführen auf eine nicht-traumatische intrazerebrale Blutung, 6.2.4.2 anhaltender Kopfschmerz zurückzuführen auf eine nicht-traumatische Subarachnoidalblutung (SAB) und 6.2.4.3 anhaltender Kopfschmerz zurückzuführen auf ein nicht-traumatisches akutes Subduralhämatom.

\subsection{Kopfschmerz zurückzuführen auf eine nicht-rupturierte Gefäßfehlbildung}

An anderer Stelle kodiert: Ein neu aufgetretener Kopfschmerz zurückzuführen auf eine nicht rupturierte Gefäßfehlbildung wird unter 6.2.1 akuter Kopfschmerz zurückzuführen auf eine intrazerebrale Blutung, 6.2.2 akuter Kopfschmerz zurückzuführen auf eine nicht-traumatische Subarachnoidalblutung (SAB) oder in seltenen Fällen 6.2.3 akuter Kopfschmerz zurückzuführen auf ein nicht-traumatisches akutes Subduralhämatom kodiert.

\section{Beschreibung:}

Kopfschmerz als Folge einer nicht-rupturierten intrakraniellen Gefäßfehlbildung (ohne Blutung auftretend). Je nach Art der Gefäßfehlbildung kann der Kopfschmerz einen chronischen Verlauf mit rezidivierenden Attacken aufweisen, die episodische primäre Kopfschmerzen imitieren, oder einen akuten und selbstlimitierenden Verlauf nehmen.

\subsubsection{Kopfschmerz zurückzuführen auf ein nicht-rupturiertes sackförmiges Aneurysma}

Diagnostische Kriterien:

A. Jeder neue Kopfschmerz, der das Kriterium C erfüllt

B. Es wurde ein nicht-rupturiertes sackförmiges Aneurysma diagnostiziert

C. Ein kausaler Zusammenhang mit dem nicht-rupturierten sackförmigen Aneurysma kann durch wenigstens zwei der folgenden Kriterien gezeigt werden:

1. Der Kopfschmerz tritt in engem zeitlichem Zusammenhang mit anderen Symptomen und/oder klinischen Zeichen eines nicht-rupturierten sackförmigen Aneurysmas auf oder war maßgeblich für dessen Diagnose

2. Einer oder beide der folgenden Punkte sind erfüllt:

a) der Kopfschmerz hat sich parallel zu anderen Symptomen und/oder klinischen bzw. radiologischen Zeichen eines Wachstums des sackförmigen Aneurysmas deutlich verschlechtert

b) der Kopfschmerz ist nach Behandlung des sackförmigen Aneurysmas verschwunden

3. Einer oder beide der folgenden Punkte sind erfüllt:

a) Kopfschmerz, der plötzlich oder als Donnerschlagkopfschmerz beginnt

b) Kopfschmerz, der in Begleitung einer schmerzhaften Lähmung des 3. Hirnnervs einhergeht

D. Nicht besser erklärt durch eine andere ICHD-3-Diagnose1. 
Anmerkung:

1. Andere Ursachen von Kopfschmerzen, insbesondere eine intrakranielle Blutung und ein reversibles zerebrales Vasokonstriktionssyndrom wurden durch geeignete Untersuchungen ausgeschlossen.

\section{Kommentar:}

Etwa ein Fünftel der Patienten mit einem nicht-rupturierten zerebralen Aneurysma berichtet über Kopfschmerzen, doch ob diese Verbindung zufälliger oder kausaler Natur ist, ist eine ungelöste Frage.

Der 6.3.1 Kopfschmerz zurückzuführen auf ein nicht-rupturiertes sackförmiges Aneurysma weist üblicherweise keine spezifischen Merkmale auf. Jeder neu begonnene Kopfschmerz kann ein Hinweis auf ein symptomatisches, aber nicht-rupturiertes sackförmiges Aneurysma sein. Eine klassische Variante ist eine akute Lähmung des 3. Hirnnervs mit einem retroorbitalen Schmerz und einer erweiterten Pupille als Hinweis auf ein Aneurysma der A. communicans posterior oder des Endabschnittes der A. carotis interna. Eine solche schmerzhafte Lähmung des 3. Hirnnervs ist ein Notfall und ein Hinweis auf eine bevorstehende Ruptur oder eine zunehmende Vergrößerung der arteriellen Gefäßmissbildung.

Mehrere retrospektive Untersuchungen haben gezeigt, dass ungefähr die Hälfte der Patienten mit einer aneurysmalen Subarachnoidalblutung vom Auftreten eines plötzlichen und starken Kopfschmerzes innerhalb der 4 Wochen vor Diagnose einer Aneurysmaruptur berichten. Lässt man eventuelle Erinnerungsverzerrungen außer Acht, lässt dies darauf schließen, dass diese Kopfschmerzen auf eine plötzliche Ausweitung der arteriellen Gefäßmissbildung zurückgehen (Sentinel-Kopfschmerz) oder auf eine nicht als solche diagnostizierte milde Subarachnoidalblutung („Warnleck“). Die Evidenz für die Existenz von Sentinel-Kopfschmerz ist dürftig. Darüber hinaus sollte der Begriff „Warnleck“ nicht verwendet werden, da eine Undichtigkeit auf eine Subarachnoidalblutung verweist. Angesichts der Tatsache, dass bei mindestens jedem 3. Patienten mit aneurysmaler Subarachnoidalblutung zunächst eine Fehldiagnose gestellt wird und der Rezidivblutungsrisiken sollten Patienten mit plötzlichem schwerem Kopfschmerz sich allen erforderlichen Untersuchungen unterziehen, darunter zerebrale Bildgebung, Liquoruntersuchung und zerebrale Angiographie (MRA- oder CT-Angiographie).

\subsubsection{Kopfschmerz zurückzuführen auf eine arterio-venöse Malformation (AVM)}

Diagnostische Kriterien:

A. Kopfschmerz, der das Kriterium C erfüllt

B. Es wurde eine arterio-venöse Malformation (AVM) diagnostiziert

C. Ein kausaler Zusammenhang kann durch mindestens zwei der folgenden Kriterien gezeigt werden:

1. Der Kopfschmerz tritt in engem zeitlichem Zusammenhang mit anderen Symptomen und/oder klinischen Zeichen einer AVM auf oder war maßgeblich für die Entdeckung einer AVM

2. Einer oder beide der folgenden Punkte sind erfüllt: a) der Kopfschmerz hat sich parallel zum Wachstum der AVM deutlich verschlechtert

b) der Kopfschmerz hat sich parallel zu wirksamen Behandlung der AVM deutlich gebessert oder ist verschwunden
3. Der Kopfschmerz ist am Situs der AVM lokalisiert

D. Nicht besser erklärt durch eine andere ICHD-3-Diagnose1.

Anmerkung:

1. Andere Ursachen von Kopfschmerzen, insbesondere eine intrakranielle Blutung, konnten durch geeignete Untersuchungen ausgeschlossen werden.

\section{Kommentar:}

Es gibt Fallberichte über Verbindungen zwischen einer arterio-venösen Malformation (AVM) und einer Vielzahl von Kopfschmerzen wie den 3. trigemino-autonomen Kopfschmerzerkrankungen, darunter 3.1 Clusterkopfschmerz, 3.2.2 chronische paroxysmale Hemikranie und 3.3.1 SUNCT-Syndrom. Allerdings fanden sich bei diesen Fällen jeweils durchweg atypische Details. Es gibt bislang keine eindeutige Evidenz für eine Beziehung zwischen AVM und diesen primären Kopfschmerzerkrankungen.

Bei bis zu 58 \% der Frauen mit einer arterio-venösen Malformation bestand eine 1.2 Migräne mit Aura. Ein gewichtiges Argument zugunsten einer kausalen Beziehung ist hier die eindeutige Korrelation zwischen der Seite, auf der die Kopfschmerzen bzw. die Aura eintreten, und der Seite, auf der die AVM lokalisiert ist. Damit besteht die begründete Vermutung, dass eine AVM Migräneattacken mit Aura verursachen kann (symptomatische Migräne). In großen AVM-Fallserien waren migräneartige Symptome jedoch im Gegensatz zu epileptischen Anfällen oder fokalen Defiziten mit oder ohne Blutungen deutlich seltener.

\subsubsection{Kopfschmerz zurückzuführen auf eine durale arterio-venöse Fistel (dAVF)}

Diagnostische Kriterien:

A. Jeder neue Kopfschmerz, der das Kriterium C erfüllt

B. Es wurde eine durale arterio-venöse Fistel (dAVF) diagnostiziert

C. Ein kausaler Zusammenhang kann durch mindestens zwei der folgenden Kriterien gezeigt werden:

1. Der Kopfschmerz tritt in engem zeitlichem Zusammenhang mit anderen Symptomen und/oder klinischen Zeichen einer dAVF auf oder war maßgeblich für die Diagnose dAVF

2. Einer oder beide der folgenden Punkte sind erfüllt:

a) der Kopfschmerz hat sich parallel zu anderen Symptomen und/oder klinischen bzw. radiologischen Zeichen eines Wachstums der dAVF deutlich verschlechtert

b) der Kopfschmerz hat sich nach wirksamer Behandlung der DAVF deutlich verbessert oder ist verschwunden

3. Es trifft mindestens einer der folgenden Punkte zu:

a) der Kopfschmerz tritt in Begleitung eines pulsierenden Tinnitus auf

b) der Kopfschmerz tritt in Begleitung einer Ophthalmoplegie auf

c) der Kopfschmerz ist progressiv und morgens und/oder beim Husten oder Vornüberbeugen schlimmer

4. Der Kopfschmerz ist am Situs der dAVF lokalisiert D. Nicht besser erklärt durch eine andere ICHD-3-Diagnose1. 
Anmerkung:

1. Andere Ursachen für Kopfschmerzen, insbesondere eine intrazerebrale Blutung und Hirnvenenthrombose, konnten durch geeignete Untersuchungen ausgeschlossen werden.

\section{Kommentar:}

Untersuchungen, die sich dem 6.3.3 Kopfschmerz zurückzuführen auf eine durale arterio-venöse Fistel widmen, fehlen bislang. Ein schmerzhafter, pulsierender Tinnitus kann das führende Symptom sein, ebenso Kopfschmerzen mit anderen Symptomen eines erhöhten Hirndrucks bei vermindertem venösen Abfluss oder einer Sinusvenenthrombose. Fisteln zwischen der A. carotis interna und dem Sinus cavernosus können sich als schmerzhafte Ophthalmoplegie manifestieren.

\subsubsection{Kopfschmerz zurückzuführen auf ein kavernöses Angiom}

An anderer Stelle kodiert: Kopfschmerz zurückzuführen auf eine intrazerebrale Blutung oder auf einen epileptischen Anfall als Folge eines kavernösen Angioms werden unter 6.2.1 akuter Kopfschmerz zurückzuführen auf eine nicht-traumatische intrazerebrale Blutung oder 7.6 Kopfschmerz zurückzuführen auf einen epileptischen Anfall kodiert.

\section{Diagnostische Kriterien:}

A. Jeder neue Kopfschmerz, der das Kriterium C erfüllt

B. Es wurde ein kavernöses Angiom diagnostiziert

C. Ein kausaler Zusammenhang kann durch mindestens zwei der folgenden Kriterien gezeigt werden:

1. Der Kopfschmerz ist in engem zeitlichem Zusammenhang mit anderen Symptomen und/oder klinischen Zeichen eines kavernösen Angioms aufgetreten oder führte zu ihrer Entdeckung

2. Eine oder beide der folgenden Punkte sind erfüllt:

a) der Kopfschmerz hat sich parallel zu anderen Symptomen und/oder klinischen bzw. radiologischen Zeichen eines Wachstums des kavernösen Angioms deutlich verschlechtert

b) der Kopfschmerz hat sich nach Entfernung des kavernösen Angioms deutlich gebessert oder ist verschwunden

3. Der Kopfschmerz tritt am Situs des kavernösen Angioms auf

D. Nicht besser erklärt durch eine andere ICHD-3-Diagnose1.

\section{Anmerkung:}

1. Andere Ursachen für Kopfschmerzen, vor allem eine intrazerebrale Blutung, konnten durch geeignete Untersuchungen ausgeschlossen werden.

\section{Kommentar:}

Kavernöse Angiome werden zunehmend mittels MRT entdeckt. Isolierte Einzelfälle legen nahe, dass einige kavernöse Angiome clusterkopfschmerzartige, SUNCT- oder migräneähnliche Attacken auslösen können. Es gibt jedoch bislang keine guten Untersuchungen, die sich 6.3.4 Kopfschmerz zurückzuführen auf ein kavernöses Angiom widmen.
Von einer Fallserie bestehend aus 126 symptomatischen Patienten mit kavernösen Angiomen und Mutationen am KRIT1-Gen berichteten nur $4 \%$ von Kopfschmerzen als einem vorliegenden Symptom. Vielmehr wird häufig von Kopfschmerzen als Folge zerebraler Blutungen oder als Folge von epileptischen Anfällen berichtet, den beiden Hauptmanifestationen kavernöser Angiome. Derartige Kopfschmerzen sollten entsprechend unter einem dieser beiden Punkte kodiert werden.

\subsubsection{Kopfschmerz zurückzuführen auf eine en- zephalo-trigeminale oder leptomeningeale Angiomatose (Sturge-Weber-Syndrom)}

An anderer Stelle kodiert: Kopfschmerzen zurückzuführen auf einen epileptischen Anfall als Folge eines Sturge-Weber-Syndroms werden kodiert als 7.6 Kopfschmerz zurückzuführen auf einen epileptischen Anfall.

\section{Diagnostische Kriterien:}

A. Jeder neue Kopfschmerz, der das Kriterium C erfüllt

B. Vorliegen eines Gesichtsangioms, zusammen mit Nachweis eines meningealen Angioms ipsilateral zum Gesichtsangiom mittels zerebraler Bildgebung

C. Ein kausaler Zusammenhang kann durch mindestens zwei der folgenden Kriterien gezeigt werden:

1. Der Kopfschmerz ist in engem zeitlichem Zusammenhang mit anderen Symptomen und/oder klinischen Zeichen aufgetreten und/oder es erfolgte der Nachweis des meningealen Angioms mittels zerebraler Bildgebung

2. Der Kopfschmerz hat sich parallel zu anderen Symptomen bzw. klinischen oder radiologischen Zeichen für ein Wachstum des meningealen Angioms deutlich verschlechtert

3. Der Kopfschmerz ist migräneartig, entweder beidseits oder am Situs des Angioms lokalisiert, und tritt in Begleitung einer kontralateralen zum Angiomsitus lokalisierten Aura auf

D. Nicht besser erklärt durch eine andere ICHD-3-Diagnose.

\section{Kommentar:}

Das Sturge-Weber-Syndrom tritt ausschließlich sporadisch auf und resultiert aus einer somatischen Mosaikmutation im GNAQ-Gen (Guaninnukleotid-bindendes Protein, Q-Polypeptid).

Der 6.3.5 Kopfschmerz zurückzuführen auf eine enzephalo-trigeminale oder leptomeningeale Angiomatose (Sturge-Weber-Syndrom) ist nur schlecht dokumentiert. Mehr als $90 \%$ der Patienten mit Sturge-Weber-Syndrom haben epileptische Anfälle, und die Hälfte berichtet von Kopfschmerzen nach diesen Anfällen, die entsprechend kodiert werden sollten. Einzelfälle legen nahe, dass die enzephalo-trigeminale oder leptomeningeale Angiomatose Ursache einer symptomatischen Migräne sein kann, insbesondere von Migräneattacken mit einer prolongierten und/oder motorischen Aura (möglicherweise zurückzuführen auf eine chronische Minderdurchblutung). 


\subsection{Kopfschmerz zurückzuführen auf eine Arteriitis}

Beschreibung:

Kopfschmerz, der durch eine Entzündung der Hals-, Kopf- und/ oder Hirnarterien verursacht wird und für diese symptomatisch ist. Kopfschmerzen können das alleinige Symptom einer Arteriitis darstellen.

\section{Diagnostische Kriterien:}

A. Jeder neue Kopfschmerz, der das Kriterium C erfültt

B. Es wurde eine Arteriitis diagnostiziert

C. Ein kausaler Zusammenhang kann durch eines oder beide der folgenden Kriterien gezeigt werden:

1. Der Kopfschmerz tritt in engem zeitlichem Zusammenhang mit anderen Symptomen und/oder klinischen Zeichen einer beginnenden Arteriitis auf oder hat zu der Diagnose der Arteriitis geführt

2. Einer oder beide der folgenden Punkte ist/sind erfüllt:

a) der Kopfschmerz hat sich parallel zur Verschlechterung der Arteriitis erheblich verschlechtert

b) der Kopfschmerz hat sich parallel zu der Besserung der Arteriitis erheblich gebessert

D. Nicht besser erklärt durch eine andere ICHD-3-Diagnose.

\subsubsection{Kopfschmerz zurückzuführen auf eine Riesen- zellarteriitis (RZA)}

Früher verwendeter Begriff: Arteriitis temporalis.

\section{Beschreibung:}

Kopfschmerz verursacht durch und symptomatisch für eine Riesenzellarteriitis (RZA). Kopfschmerzen können das einzige Symptom einer RZA sein, einer Erkrankung, die am auffallendsten von Kopfschmerzen begleitet ist. Die Merkmale des Kopfschmerzes sind variabel.

\section{Diagnostische Kriterien:}

A. Jeder neue Kopfschmerz, der das Kriterium C erfüllt

B. Es wurde eine Riesenzellarteriitis (RZA) diagnostiziert

C. Ein kausaler Zusammenhang kann durch mindestens zwei der folgenden Kriterien gezeigt werden

1. Der Kopfschmerz tritt in engem zeitlichem Zusammenhang mit anderen Symptomen und/oder klinischen oder biologischen Zeichen für den Beginn einer RZA auf oder hat zur Diagnose einer RZA geführt

2. Einer oder beide der folgenden Punkte ist/sind erfüllt:

a) der Kopfschmerz hat sich parallel zur Verschlechterung der RZA entscheidend verschlechtert

b) der Kopfschmerz hat sich innerhalb von 3 Tagen nach einer Hochdosistherapie mit Kortikoiden entscheidend gebessert oder ist verschwunden

3. Der Kopfschmerz ist mit einer Schmerzempfindlichkeit der Kopfhaut und/oder Einschränkung der Kieferöffnung verbunden

D. Nicht besser erklärt durch eine andere ICHD-3-Diagnose.

\section{Kommentar:}

Von allen Arteriitiden und Kollagenosen ist die Riesenzellarteriitis (RZA) diejenige Erkrankung, die am häufigsten mit Kopfschmerzen einhergeht (aufgrund der Entzündung von Kopfarterien, häufig Ästen der A. carotis externa). Folgende Punkte müssen betont werden:

Die Variabilität der Kopfschmerzcharakteristika eines 6.4.1 Kopfschmerzes zurückzuführen auf eine Riesenzellarteriitis und der Begleitsymptome (Polymyalgia rheumatica, Einschränkung der Kieferöffnung) ist derart groß, dass jeder neu aufgetretene und anhaltende Kopfschmerz bei einer Person über 60 Jahren an eine Riesenzellarteriitis denken lassen und zu geeigneten Untersuchungen Anlass geben sollte.

Ebenfalls suggestiv für eine Riesenzellarteriitis sind in kurzer Folge rezidivierende Attacken einer Amaurosis fugax verbunden mit Kopfschmerzen; sie sollten ebenfalls unmittelbar zu gezielten Untersuchungen Anlass geben. Das Hauptrisiko liegt in einer Erblindung als Folge einer anterioren ischämischen Optikusneuropathie. Dies kann durch eine notfallmäßig eingeleitete Kortikoidtherapie verhindert werden. Der Abstand zwischen der Erblindung eines Auges und des anderen beträgt üblicherweise weniger als 1 Woche. Zusätzlich besteht auch das Risiko des Auftretens zerebraler ischämischer Ereignisse oder einer Demenz.

Die histologische Diagnose kann schwierig sein. Bei der histologischen Untersuchung ist eine langstreckige Aufarbeitung notwendig, weil die A. temporalis häufig unbefallene Areale („skip areas“) aufweist.

\subsubsection{Kopfschmerz zurückzuführen auf eine primäre Vaskulitis des ZNS}

Früher verwendeter Begriff: Kopfschmerz zurückzuführen auf eine isolierte zerebrale Angiitis oder granulomatöse Vaskulitis des ZNS

\section{Beschreibung:}

Kopfschmerz verursacht durch und symptomatisch für eine primäre Vaskulitis des Zentralnervensystems. Kopfschmerzen sind das vorherrschende Symptom dieser Erkrankung, diesen fehlen jedoch spezifische Merkmale.

\section{Diagnostische Kriterien:}

A. Jeder neue Kopfschmerz, der das Kriterium C erfüllt

B. Es wurde eine primäre Vaskulitis des Zentralnervensystems diagnostiziert

C. Ein kausaler Zusammenhang kann durch eines oder beide der folgenden Kriterien gezeigt werden:

1. Der Kopfschmerz tritt in engem zeitlichem Zusammenhang mit anderen Symptomen und/oder klinischen Zeichen für den Beginn einer primären Vaskulitis des ZNS auf oder hat zu Diagnose einer primären Vaskulitis des ZNS geführt

2. Einer oder beide der folgenden Punkte sind erfüllt:

a) der Kopfschmerz hat sich parallel zur Verschlechterung der primären Vaskulitis des ZNS deutlich verschlechtert

b) der Kopfschmerz hat sich parallel zur Besserung der primären Vaskulitis des ZNS infolge der Behandlung mit Kortikoiden und/oder Immunsuppressiva deutlich gebessert

D. Nicht besser erklärt durch eine andere ICHD-3-Diagnose1. 
Anmerkung:

1. Es wurde durch geeignete Untersuchungen, insbesondere eine ZNS-Infektion, eine Neoplasie des ZNS und ein reversibles zerebrales Vasokonstriktionssyndrom ausgeschlossen.

\section{Kommentar:}

Kopfschmerzen sind das Leitsymptom einer primären oder sekundären Vaskulitis des ZNS. Sie sind in 50 bis $80 \%$ der Fälle vorhanden - je nach eingesetztem Diagnoseverfahren, d. h. Angiographie oder Histologie. Die Kopfschmerzen haben kein spezifisches Muster und sind deshalb nur von geringem diagnostischen Nutzen, bis andere Symptome wie fokal-neurologische Defizite, epileptische Anfälle, Beeinträchtigung der kognitiven Leistungsfähigkeit oder Störungen des Bewusstseins auftreten. Das Fehlen von Kopfschmerzen und einer Pleozytose im Liquor macht jedoch die Diagnose einer zerebralen Vaskulitis unwahrscheinlich.

Bei einer primären Vaskulitis des ZNS können angiographische Befunde vorliegen, die recht ähnlich wie die bei einem reversiblen zerebralen Vasokonstriktionssyndrom (RCVS) sind, darunter eine multifokale Stenose der intrakraniellen Arterien; rezidivierende Donnerschlagkopfschmerzen sollten die Diagnose eines RCVS und nicht einer primären Vaskulitis des ZNS nahelegen.

Die Pathogenese eines 6.4.2 Kopfschmerzes zurückzuführen auf eine primäre Vaskulitis des ZNS ist multifaktoriell: Entzündung, Infarkt (ischämisch oder hämorrhagisch), erhöhter intrakranieller Druck und/oder Subarachnoidalblutung kommen infrage.

Der Behandlungseffekt ist weit weniger dramatisch als bei dem 6.4.1 Kopfschmerz zurückzuführen auf eine Riesenzellarteriitis. Eine histologisch nachgewiesene primäre Vaskulitis des ZNS ist eine ernste und nicht selten tödlich verlaufende Erkrankung.

\subsubsection{Kopfschmerz zurückzuführen auf eine sekundäre Vaskulitis des ZNS}

Beschreibung:

Kopfschmerz, der durch eine sekundäre Vaskulitis des Zentralnervensystems verursacht wird und symptomatisch für diese ist. Kopfschmerzen sind das Leitsymptom dieser Erkrankung, diese weisen jedoch keine spezifischen Merkmale auf.

\section{Diagnostische Kriterien:}

A. Jeder neue Kopfschmerz, der das Kriterium C erfüllt

B. Es wurde eine sekundäre Vaskulitis des Zentralnervensystems (ZNS-Angiitis bei Vorliegen einer systemischen Angiitis) diagnostiziert

C. Ein kausaler Zusammenhang kann durch eines oder beide der folgenden Kriterien gezeigt werden:

1. Der Kopfschmerz ist in enger zeitlicher Beziehung zu anderen Symptomen und/oder klinischen Zeichen des Beginns einer ZNS-Vaskulitis aufgetreten

2. Einer oder beide der folgenden Punkte ist/sind erfüllt:

a) der Kopfschmerz hat sich parallel zur Verschlechterung der systemischen Angiitis erheblich verschlechtert

b) der Kopfschmerz hat sich parallel zur Besserung der systemischen Angiitis infolge einer Behandlung mit Kortikoiden und/oder Immunsuppressiva gebessert

D. Nicht besser erklärt durch eine andere ICHD-3-Diagnose.
Kommentar:

Kopfschmerzen sind das Leitsymptom einer primären oder sekundären Vaskulitis des ZNS. Sie sind in 50 bis $80 \%$ der Fälle vorhanden - je nach eingesetztem Diagnoseverfahren, d. h. Angiographie oder Histologie. Die Kopfschmerzen haben kein spezifisches Muster und sind deshalb nur von geringem diagnostischen Nutzen, bis andere Symptome wie fokal-neurologische Defizite, epileptische Anfälle, Beeinträchtigung der kognitiven Leistungsfähigkeit oder Störungen des Bewusstseins auftreten. Das Fehlen von Kopfschmerzen und einer Pleozytose im Liquor macht jedoch die Diagnose einer zerebralen Vaskulitis unwahrscheinlich.

Grundsätzlich können hier in der Praxis zwei Probleme auftreten: 1) Nachweis einer zerebralen Vaskulitis bei einem Patienten, der unter einer der vielen Erkrankungen leidet, die eine Vaskulitis hervorrufen können; 2) bei einem Patienten mit einer nachgewiesenen zerebralen Vaskulitis muss die zugrundeliegende Erkrankung (Entzündung, Infektion, Intoxikation, Malignom) aufgedeckt werden.

Die Pathogenese des 6.4.3 Kopfschmerzes zurückzuführen auf eine sekundäre Vaskulitis des ZNS ist multifaktoriell: Entzündung, Infarkt (ischämisch oder hämorrhagisch), erhöhter intrakranieller Druck und/oder Subarachnoidalblutung kommen infrage.

\subsection{Kopfschmerz zurückzuführen auf eine Störung der A. carotis oder A. vertebralis}

Beschreibung:

Kopf- und/oder Gesichts- und/oder Nackenschmerz durch nicht-entzündliche Läsionen, von denen die Halsschlagadern und/ oder Wirbelsäulenarterien betroffen sind. Der Schmerz beginnt meist plötzlich (eventuell sogar donnerschlagmäßig). Er kann isoliert bleiben oder es kann sich um ein Warnsymptom handeln, das den fokalen Defiziten eines ischämischen Infarkts vorangeht.

\section{Diagnostische Kriterien:}

A. Jeder neu aufgetretene Kopf- und/oder Gesichts- oder Halsschmerz, der das Kriterium C erfüllt

B. Es wurde eine Störung an einer Halsschlagader nachgewiesen, oder es liegt ein Zustand nach einem chirurgischen oder radiologischen Eingriff an einer Halsschlagader vor

C. Ein kausaler Zusammenhang kann durch mindestens zwei der folgenden Kriterien gezeigt werden:

1. Der Schmerz hat sich in enger zeitlicher Beziehung zu anderen lokalen Symptomen einer Störung im Bereich der Halsschlagadern entwickelt oder führte zur Diagnose einer Störung im Bereich der Halsschlagadern

2. Einer oder beide der folgenden Punkte sind erfüllt:

a) der Schmerz hat sich gleichzeitig mit anderen Symptomen der Halsschlagader-Störung erheblich verschlechtert

b) der Schmerz hat sich innerhalb von 1 Monat nach seinem Beginn erheblich gebessert oder ist verschwunden

3. Der Schmerz ist einseitig und ipsilateral zu der betroffenen Halsschlagader

D. Nicht besser erklärt durch eine andere ICHD-3-Diagnose. 


\subsubsection{Kopf-, Gesichts- oder Halsschmerz zurück- zuführen auf eine arterielle Dissektion}

6.5.1.1 Akuter Kopf-, Gesichts- oder Halsschmerz zurückzuführen auf eine arterielle Dissektion

Beschreibung:

Kopf- und/oder Gesichts- und/oder Halsschmerz infolge der Dissektion einer Halsschlagader oder Wirbelsäulenarterie. Der Schmerz ist üblicherweise ipsilateral zum betroffenen Gefäß und beginnt in der Regel plötzlich (bis hin zum Donnerschlagkopfschmerz). Er kann isoliert bestehen bleiben oder es kann sich um ein Warnsymptom handeln, das einem ischämischen Infarkt vorangeht.

\section{Diagnostische Kriterien:}

A. Jeder neu aufgetretene Kopf- und/oder Gesichts- oder Halsschmerz, der die Kriterien C und D erfüllt

B. Es wurde eine Dissektion der A. carotis oder A. vertebralis diagnostiziert

C. Ein kausaler Zusammenhang kann durch wenigstens zwei der folgenden Kriterien gezeigt werden:

1. der Schmerz hat sich in enger zeitlicher Beziehung zu anderen lokalen Symptomen der Halsarterien-Dissektion entwickelt oder zu deren Diagnose geführt

2. Einer oder beide der folgenden Punkte sind erfüllt:

a) der Schmerz hat sich gleichzeitig mit anderen Symptomen der Halsarterien-Dissektion erheblich verschlechtert

b) der Schmerz hat sich innerhalb von 1 Monat nach seinem Beginn erheblich gebessert oder ist verschwunden

3. Einer oder beide der folgenden Punkte sind erfüllt:

a) der Schmerz ist stark und hält über Tage oder länger an

b) der Schmerz geht Symptomen einer akuten retinalen und/oder zerebralen Ischämie voran

4. Der Schmerz ist einseitig und ipsilateral zu der betroffenen Halsarterie lokalisiert

D. Es ist wenigstens einer der folgenden Punkte erfüllt:

1. der Kopfschmerz ist innerhalb von 3 Monaten verschwunden 1

2. der Kopfschmerz ist noch nicht verschwunden, allerdings sind noch keine 3 Monate verstrichen 1

E. Nicht besser erklärt durch eine andere ICHD-3-Diagnose.

\section{Anmerkung:}

1. Die 3 Monate sollten von der Stabilisierung an gezählt werden, sei es spontan oder durch Behandlung, statt ab Beginn der Dissektion einer Halsarterie.

\section{Kommentar:}

Kopfschmerzen mit oder ohne Schmerzen im Halsbereich können die einzige Manifestation der Dissektion einer Halsarterie sein. Sie sind bei weitem das häufigste Symptom ( $55 \%$ bis $100 \%$ der Fälle) und auch das häufigste Erstsymptom (33\% bis $86 \%$ der Fälle).

Der 6.5.1.1 akute Kopf-, Gesichts- oder Halsschmerz zurückzuführen auf eine arterielle Dissektion ist üblicherweise einseitig (ipsilateral zur betroffenen Arterie), von starker Intensität und anhaltend (im Mittel 4 Tage). Er hat kein konstantes spezifisches Muster und ist zum Teil irreführend, weil andere Kopfschmerzen wie 1. Migräne, 3.1 Clusterkopfschmerz oder ein 4.4 primärer Donnerschlag- kopfschmerz nachgeahmt werden. Begleitsymptome in Form von Zeichen einer zerebralen oder retinalen Ischämie und lokale Symptome sind häufig: Insbesondere ein schmerzhaftes Horner-Syndrom, ein schmerzhafter Tinnitus mit plötzlichem Beginn oder eine schmerzhafte Parese des N. hypoglossus sind sehr suggestiv für eine Dissektion der A. carotis.

Die Dissektion einer Halsarterie kann in Begleitung der Dissektion einer intrakraniellen Arterie einhergehen, was eine potenzielle Ursache für eine Subarachnoidalblutung darstellt. 6.7.4 Kopfschmerz zurückzuführen auf eine arterielle Dissektion kann zusätzlich zu einem 6.5.1.1 akuten Kopf-, Gesichts- oder Halsschmerz zurückzuführen auf eine arterielle Dissektion vorliegen.

6.5.1.1 Akuter Kopf-, Gesichts- oder Halsschmerz zurückzuführen auf eine arterielle Dissektion geht in der Regel dem Beginn ischämischer Symptome voran und erfordert deshalb eine frühzeitige Diagnose und Behandlung. Die Diagnosestellung erfolgt auf Grundlage einer zervikalen MRT mit Fettunterdrückung, Duplex-Scan, MRA und/oder CTA sowie in Zweifelsfällen einer konventionellen Angiographie. Es werden gewöhnlich mehrere dieser Untersuchungen benötigt, da sie jeweils normal ausfallen können.

Hinsichtlich der Therapie existieren keine randomisierten Untersuchungen, aber es besteht der Konsens, zunächst zu heparinisieren und dann entsprechend der Gefäßerholung für 3 bis 6 Monate befristet auf eine orale Antikoagulation überzugehen.

\subsubsection{Anhaltender Kopf-, Gesichts- oder Halsschmerz zurückzuführen auf eine frühere arterielle Dissektion}

\section{Beschreibung:}

Kopfschmerz infolge der Dissektion einer Halsschlagader oder Wirbelsäulenarterie, der mehr als 3 Monate nach erfolgter Stabilisierung der Dissektion fortbesteht.

\section{Diagnostische Kriterien:}

A. Kopfschmerz, der zuvor als 6.5.1.1 akuter Kopf-, Gesichtsoder Halsschmerz zurückzuführen auf eine arterielle Dissektion diagnostiziert wurde und der das Kriterium C erfüllt

B. Die Dissektion hat sich stabilisiert, sei es spontan oder durch Behandlung

C. Der Kopfschmerz hält mehr als > 3 Monate nach Stabilisierung der Dissektion an

D. Nicht besser erklärt durch eine andere ICHD-3-Diagnose.

\section{Kommentar:}

In einigen Untersuchungen wurden Kopfschmerzen dokumentiert, die die Kriterien für einen 6.5.1.2 anhaltenden Kopf-, Gesichts- oder Halsschmerz zurückzuführen auf eine frühere arterielle Dissektion erfüllen. Es sind noch weitere Untersuchungen erforderlich, um Risikofaktoren für derartige anhaltende Kopfschmerzen zu ermitteln; eine 1. Migräne in der Vorgeschichte mag eine Rolle spielen, ebenso wie Ängste/Depressionen.

\subsubsection{Kopfschmerz zurückzuführen auf eine Endarteriektomie}

Beschreibung:

Kopfschmerz, der durch den chirurgischen Eingriff bei einer Endarteriektomie ausgelöst wird. Am Schmerzgeschehen können auch 
Hals und Gesicht beteiligt sein. Er kann isoliert bestehen bleiben oder es kann sich um ein Warnsymptom handeln, das den fokalen Defiziten eines (meist hämorrhagischen) Infarkts vorangeht.

Diagnostische Kriterien:

A. Jeder neue Kopfschmerz, der das Kriterium C erfüllt

B. Zustand nach Durchführung einer Endarteriektomie der A. carotis

C. Ein kausaler Zusammenhang kann durch wenigstens zwei der folgenden Kriterien gezeigt werden:

1. Kopfschmerz entwickelt sich innerhalb von 1 Woche nach Carotis-Endarteriektomie

2. Kopfschmerz verschwindet innerhalb von 1 Monat nach Carotis-Endarteriektomie

3. beides von Folgendem:

a) Kopfschmerz ist einseitig, auf der Seite der Carotis-Endarteriektomie

b) Kopfschmerz weist eines der drei folgenden eindeutigen Merkmale auf1:

i. diffuser leichter Schmerz

ii. Clusterkopfschmerz-ähnlicher Schmerz, der 1- bis 2-mal am Tag in Attacken von 2 bis 3 Stunden Dauer auftritt

iii. pulsierender, starker Schmerz

D. Nicht besser erklärt durch eine andere ICHD-3-Diagnose2.

\section{Anmerkung:}

1. Es wurden drei verschiedene Subtypen von 6.5.2 Kopfschmerz zurückzuführen auf eine Endarteriektomie beschrieben; diese werden jedoch nicht separat kodiert:

a) ein isolierter leichter, diffus lokalisierter Kopfschmerz, der innerhalb der ersten Tage nach der Operation auftritt;

b) ein einseitiger Clusterkopfschmerz-ähnlicher Schmerz mit Attacken, die 2 bis 3 Stunden anhalten und 1- bis 2-mal pro Tag auftreten;

c) ein starker einseitiger pulsierender Schmerz, der 3 Tage nach der Operation auftritt.

2. Durch entsprechende Untersuchungen wurde insbesondere eine arterielle Dissektion ausgeschlossen.

\section{Kommentar:}

Von den drei Subtypen des 6.5.2 Kopfschmerzes zurückzuführen auf eine Endarteriektomie ist die erste und häufigste Form (bis zu $60 \%$ der Fälle) eine gutartige, selbstlimitierende Störung, während die zweite (beschrieben in bis zu 38\% der Fälle) innerhalb von etwa 2 Wochen verschwindet. Die dritte Kopfschmerzform ist Teil des seltenen Hyperperfusionssyndroms, das häufig einem Anstieg des Blutdruckes und dem Auftreten von epileptischen Anfällen bzw. von neurologischen Defiziten um den 7. Tag herum vorangeht. Eine notfallmäßige Behandlung ist notwendig, da diese Symptome eine zerebrale Blutung ankündigen können.

\subsubsection{Kopfschmerz zurückzuführen auf Carotis- oder Vertebralis-Angioplastie oder Stent- implantation}

\section{Beschreibung:}

Kopfschmerz, der durch endovaskuläre Eingriffe wie eine zervikale Angioplastie und/oder Stentimplantation ausgelöst wird. Der Schmerz kann auch Hals und Gesicht einbeziehen. Er kann ein Einzelsymptom bleiben oder es kann sich um ein Warnsymptom handeln, das den fokalen Defiziten eines (meist hämorrhagischen) Infarkts vorausgeht.

\section{Diagnostische Kriterien:}

A. Jeder neu aufgetretene Kopfschmerz, der das Kriterium C erfüllt

B. Zustand nach Carotis- oder Vertebralis-Angioplastie und/oder Stentimplantation

C. Ein kausaler Zusammenhang kann durch alle der folgenden Kriterien gezeigt werden:

1. der Kopfschmerz hat sich innerhalb von 1 Woche nach der Angioplastie und/oder Stentimplantation entwickelt

2. der Kopfschmerz ist innerhalb von 1 Monat nach der Angioplastie und/oder Stentimplantation verschwunden

3. der Kopfschmerz tritt auf der gleichen Seite auf, auf der die Angioplastie und/oder Stentimplantation vorgenommen wurde

D. Nicht besser erklärt durch eine andere ICHD-3-Diagnose1.

\section{Anmerkung:}

1. Durch entsprechende Untersuchungen wurde insbesondere eine arterielle Dissektion ausgeschlossen.

\section{Kommentar:}

Eine Carotis- und Vertebralis-Angioplastie und/oder Stentimplantation werden zur Behandlung von Stenosen der Halsschlagader vorgenommen.

Bei 64 untersuchten Patienten mit Carotis-Stentversorgung traten bei einem Drittel Kopfschmerzen auf, gewöhnlich innerhalb von 10 Minuten nach dem Eingriff. Die Kopfschmerzen waren leicht, ipsilateral, frontotemporal und drückender Nature; meist verschwanden sie innerhalb von 10 Minuten. Ansonsten ist die Datenlage zu 6.5.3 Kopfschmerz zurückzuführen auf Carotis- oder Vertebralis-Angioplastie oder Stentimplantation weiterhin dürftig. Kopfschmerzen werden bei größeren Studien, in denen Carotis-Stentimplantation und Endarteriektomie verglichen werden, nicht erwähnt.

Es wurde über 6.5.3 Kopfschmerz zurückzuführen auf Carotisoder Vertebralis-Angioplastie oder Stentimplantation als Teil des seltenen Hyperperfusionssyndroms berichtet. 


\subsection{Kopfschmerz zurückzuführen auf eine Hirnvenenerkrankung}

\subsubsection{Kopfschmerz zurückzuführen auf eine Hirn- venenthrombose}

\section{Beschreibung:}

Kopfschmerz, der durch eine Hirnvenenthrombose ausgelöst wird. Dieser zeigt keine spezifischen Merkmale: meist ist er diffus, fortschreitend und stark; er kann jedoch einseitig und plötzlich auftreten (sogar als Donnerschlagkopfschmerz), oder leicht und mitunter migräneartig sein.

\section{Diagnostische Kriterien:}

A. Jeder neu aufgetretene Kopfschmerz, der das Kriterium C erfüllt

B. Es wurde eine zerebrale Venenthrombose (ZVT) diagnostiziert

C. Ein kausaler Zusammenhang kann durch die beiden folgenden Kriterien gezeigt werden:

1. der Kopfschmerz hat sich in enger zeitlicher Beziehung zu anderen Symptomen und/oder klinischen Zeichen einer ZVT entwickelt oder zur Aufdeckung der ZVT geführt

2. Einer oder beide der folgenden Punkte sind erfüllt:

a) der Kopfschmerz hat sich zeitgleich mit klinischen und radiologischen Hinweise auf eine Ausweitung der ZVT erheblich verschlechtert

b) der Kopfschmerz hat sich nach Besserung der ZVT erheblich gebessert oder ist verschwunden

D. Nicht besser erklärt durch eine andere ICHD-3-Diagnose.

\section{Kommentar:}

Kopfschmerzen sind das bei weitem häufigste Symptom ( 80 \% bis 90 \% der Fälle) einer zerebralen Venenthrombose (ZVT), und auch das häufigste Erstsymptom.

Der 6.6.1 Kopfschmerz zurückzuführen auf eine Hirnvenenthrombose weist keine spezifischen Merkmale auf, ist jedoch meist diffus, fortschreitend und stark sowie von anderen Symptomen einer intrakraniellen Hypertonie begleitet. Er kann auch einseitig und plötzlich auftreten und dabei mitunter auf eine falsche Fährte führen, indem er die Symptome einer 1.1 Migräne ohne Aura, einer 1.2 Migräne mit Aura, eines 3.1 Clusterkopfschmerzes, einer 3.4 Hemicrania continua, eines 4.4 primären Donnerschlagkopfschmerzes, eines 7.2 Kopfschmerzes zurückzuführen auf einen Liquorunterdruck oder eines 6.2.2 Kopfschmerzes zurückzuführen auf eine nicht-traumatische Subarachnoidalblutung (eine ZVT kann eine Ursache einer SAB sein) imitiert.

Kopfschmerz kann die einzige Manifestation einer ZVT sein, doch in über 90 \% der Fälle tritt er in Begleitung fokaler Symptome auf (neurologische Defizite oder epileptische Anfälle) und/oder Symptome eines intrakraniellen Bluthochdrucks, einer subakuten Enzephalopathie oder eines kavernösen Sinussyndroms.

In Anbetracht des Fehlens spezifischer Charakteristika eines 6.6.1 Kopfschmerzes zurückzuführen auf eine Hirnvenenthrombose sollte jeder neu aufgetretene und anhaltende Kopfschmerz verdächtig sein, insbesondere dann, wenn bei einem Patienten ein erhöhtes Thromboserisiko besteht. Die Diagnose stützt sich auf zerebrale Bildgebung (MRT mit T2* -gewichteten Bildern plus MRA, oder CT-Aufnahme plus CT-Angiographie sowie in unklaren Fällen einer intraarteriellen Angiographie). Die Behandlung sollte so früh wie möglich begonnen werden und eine symptomatische Therapie, die Gabe von Heparin gefolgt von einer zumindest 6-monatigen oralen Antikoagulation und - falls indiziert - eine Behandlung der ätiologischen Faktoren beinhalten.

\subsubsection{Kopfschmerz zurückzuführen auf Stentimplan- tation eines venösen Hirnsinus}

Beschreibung:

Einseitiger durch Stentimplantation eines kraniellen venösen Hirnsinus verursachter Kopfschmerz auf der gleichen Seite wie der Stent.

\section{Diagnostische Kriterien:}

A. Neuer einseitiger Kopfschmerz, der das Kriterium C erfüllt

B. Zustand nach Stentversorgung im Bereich der Halsvenen oder Hirnvenen

C. Ein kausaler Zusammenhang kann durch alle der folgenden Kriterien gezeigt werden:

1. Der Kopfschmerz hat sich innerhalb 1 Woche nach Stentimplantation entwickelt

2. Der Kopfschmerz ist innerhalb von 3 Monaten nach Stentimplantation verschwunden

3. Der Kopfschmerz ist ipsilateral zum Stent lokalisiert D. Nicht besser erklärt durch eine andere ICHD-3-Diagnose1.

\section{Anmerkung:}

1. Insbesondere wurde eine venöse Stentthrombose ausgeschlossen.

\section{Kommentar:}

Im Laufe der letzten zehn Jahre wurde eine Stentversorgung bei lateralen Sinusstenosen zur Behandlung von idiopathischer intrakranieller Hypertonie eingesetzt.

Die Datenlage zu 6.6.2 Kopfschmerz zurückzuführen auf Stentimplantation eines venösen Hirnsinus ist mager. In einem Patientenkollektiv von 21 Patienten, die aufgrund einer idiopathischen intrakraniellen Hypertonie mit einem Stent versorgt wurden, zeigten 10 Patienten „Stent-Kopfschmerzen“, die sich von denen unterschieden, die sie vor der Behandlung erfuhren. Diese befanden sich am Situs des Stents, im Mastoidbereich, und hielten etwa 3 Wochen an.

\subsection{Kopfschmerz zurückzuführen auf eine sonstige akute intrakranielle Störung}

\subsubsection{Kopfschmerz zurückzuführen auf einen intra- kraniellen endarteriellen Eingriff}

Beschreibung:

Einseitiger Kopfschmerz, der unmittelbar auf einen intrakraniellen endarteriellen Eingriff zurückgeht, ipsilateral zum Situs des Eingriffs und weniger als 24 Stunden anhaltend.

\section{Diagnostische Kriterien:}

A. Jeder neue Kopfschmerz, der das Kriterium C erfüllt

B. Zustand nach einem intrakraniellen endarteriellen Eingriff1 
C. Ein kausaler Zusammenhang kann durch wenigstens drei der folgenden Kriterien gezeigt werden:

1. Der Kopfschmerz hat sich innerhalb 1 Woche nach dem Eingriff entwickelt

2. Der Kopfschmerz ist innerhalb 1 Monats nach dem Eingriff verschwunden

3. Der Kopfschmerz ist ipsilateral zum Eingriff oder bilateral

4. Der Kopfschmerz zeigt eines der folgenden klinischen Bilder2:

a) stark, plötzlich innerhalb von Sekunden nach dem Eingriff auftretend und $<1$ Stunde anhaltend

b) mittelstark bis stark, innerhalb von Stunden nach dem Eingriff auftretend und $>24$ Stunden anhaltend

c) tritt bei einem Patienten mit 1. Migräne auf und zeigt die Kennzeichen einer 1.1 Migräne ohne Aura oder 1.2 Migräne mit Aura

D. Nicht besser erklärt durch eine andere ICHD-3-Diagnose3.

\section{Anmerkung:}

1. Zum Beispiel Angioplastie, Embolisation oder Stentimplantation.

2. Es gibt drei anerkannte (aber nicht separat kodierte) Subtypen des 6.7.1 Kopfschmerzes zurückzuführen auf intrakranielle Endarteriektomie:

a) ein sehr spezifischer Subtyp, der nach Balloneinlage oder Embolisation einer arteriovenösen Malformation (AVM) oder eines Aneurysmas auftritt: starker Schmerz mit Lokalisation entsprechend der betroffenen Arterie, der plötzlich innerhalb weniger Sekunden nach dem Eingriff entsteht und dann schnell verschwindet;

b) Kopfschmerz, der sich innerhalb von Stunden bis zu einem Tag nach dem Eingriff entwickelt und einige Tage lang anhält;

c) eine Migräneattacke, die bei einer Person auftritt, die unter einer 1. Migräne leidet und durch den intrakraniellen endarteriellen Eingriff ausgelöst wird; mitunter gefolgt von rezidivierenden intermittierenden Kopfschmerzen über mehrere Wochen (in diesen Fällen sollte der Patient beide Diagnosen erhalten: die des entsprechenden Typs oder Subtyps von 1. Migräne und 6.7.1 Kopfschmerz zurückzuführen auf einen intrakraniellen endarteriellen Eingriff).

3. Andere Ursachen für Kopfschmerzen, insbesondere eine arterielle Dissektion und eine Arterienruptur, konnten durch geeignete Untersuchungen ausgeschlossen werden.

\subsubsection{Kopfschmerz zurückzuführen auf Angio- graphie}

Beschreibung:

Unmittelbar durch eine zerebrale Angiographie ausgelöster Kopfschmerz.

\section{Diagnostische Kriterien:}

A. Jeder neu aufgetretene Kopfschmerz, der das Kriterium C erfüllt

B. Zustand nach intraarterieller Angiographie der Aa. carotides oder vertebrales
C. Ein kausaler Zusammenhang kann durch wenigstens zwei der folgenden Kriterien gezeigt werden:

1. der Kopfschmerz hat sich während der Angiographie oder innerhalb von 24 Stunden nach dieser entwickelt

2. der Kopfschmerz ist innerhalb von 72 Stunden nach der Angiographie verschwunden

3. der Kopfschmerz weist eine der folgenden Kombinationen von Merkmalen auf1:

a) entwickelt sich bei der Kontrastmittelinjektion und hält $<1$ Stunde lang an

b) entwickelt sich wenige Stunden nach der Angiographie und hält > 24 Stunden lang an

c) tritt bei einem Patienten mit 1. Migräne auf und zeigt die Kennzeichen einer 1.1 Migräne ohne Aura oder 1.2 Migräne mit Aura

D. Nicht besser erklärt durch eine andere ICHD-3-Diagnose.

Anmerkung:

1. Es gibt drei anerkannte (doch nicht separat kodierte) Subtypen von 6.7.2 Kopfschmerz zurückzuführen auf Angiographie. a) tritt während der Angiographie und in engem zeitlichen Zusammenhang mit der Kontrastmittelinjektion auf;

b) tritt erst nach der Angiographie auf, allerdings innerhalb von 24 Stunden;

(beide Subtypen kommen häufiger bei Patienten mit primären Kopfschmerzen in der Vorgeschichte vor, haben jedoch einen entschieden anderen Charakter als die primären Kopfschmerzen);

c) eine Migräneattacke bei einer Person, die unter 1. Migräne leidet, welche durch eine Angiographie ausgelöst wird (in solchen Fällen, sollten für den Patienten beide Diagnosen gestellt werden: der entsprechende Typ oder Subtyp von 1. Migräne und 6.7.2 Kopfschmerz zurückzuführen auf Angiographie).

\section{Kommentar:}

Eine Angiographie mit Kontrastmittel ist kontraindiziert bei Patienten mit jedwedem Subtyp von 1.2.3 hemiplegischer Migräne, da sie eine lebensbedrohliche Attacke mit prolongierter Hemiplegie und Koma auslösen kann.

\subsubsection{Kopfschmerz zurückzuführen auf ein rever- sibles zerebrales Vasokonstriktionsyndrom (RCVS)}

6.7.3.1 Akuter Kopfschmerz zurückzuführen auf ein reversibles zerebrales Vasokonstriktionsyndrom (RCVS) Beschreibung:

Kopfschmerz, der durch ein reversibles zerebrales Vasokonstriktionssyndrom (RCVS) verursacht wird, in der Regel ein über 1 bis 2 Wochen rezidivierender Donnerschlagkopfschmerz, oft getriggert sexuelle Aktivität, Anstrengung, Valsalva-Manöver und/oder Emotionen. Der Kopfschmerz kann das alleinige Symptom von RCVS bleiben oder es kann sich um ein Warnsymptom handeln, dass einem hämorrhagische oder ischämischen Infarkt vorweggeht. 
Diagnostische Kriterien:

A. Jeder neu aufgetretene Kopfschmerz, der das Kriterium C erfüllt

B. Es wurde ein reversibles zerebrales Vasokonstriktionsyndrom (RCVS) diagnostiziert

C. Ein kausaler Zusammenhang kann durch eines oder beide der folgenden Kriterien gezeigt werden:

1. Der Kopfschmerz, mit oder ohne fokale Defizite und/ oder epileptische Anfälle, hat zu einer Angiographie (mit „strings“- und „Perlenschnur-Zeichen“) und der Diagnose RCVS geführt

2. Der Kopfschmerz weist eines oder mehrere der folgenden Merkmale auf:

a) Beginn als Donnerschlagkopfschmerz

b) getriggert durch sexuelle Aktivität, Anstrengung, Valsalva-Manöver, Emotion, Baden und/oder Duschen

c) während $\leq 1$ Monat nach Kopfschmerzbeginn vorliegend oder wiederkehrend, ohne neue nennenswerte Kopfschmerzen nach > 1 Monat

D. Einer der folgenden Punkte ist erfüllt:

1. der Kopfschmerz hat sich innerhalb von 3 Monaten nach seinem Beginn aufgelöst

2. der Kopfschmerz hat sich noch nicht aufgelöst, aber noch sind keine 3 Monate seit seinem Beginn verstrichen

E. Nicht besser erklärt durch eine andere ICHD-3-Diagnose1.

\section{Anmerkung:}

1. Andere Ursachen für Kopfschmerzen, insbesondere eine aneurysmatische Subarachnoidalblutung, konnten durch geeignete Untersuchungen ausgeschlossen werden.

\section{Kommentar:}

Beim reversiblen zerebralen Vasokonstriktionssyndrom (RCVS) handelt es sich um ein bislang unzureichend verstandenes Krankheitsbild, das klinisch von schwerem diffusem Kopfschmerz gekennzeichnet ist, wie er für Kopfschmerz vom Donnerschlagtyp typisch ist und die Symptome einer aneurysmatischen Subarachnoidalblutung nachahmt.

Das RCVS ist die häufigste Ursache eines im Laufe weniger Tage oder Wochen rezidivierenden Donnerschlagkopfschmerzes. Der 6.7.3.1 akute Kopfschmerz zurückzuführen auf ein reversibles zerebrales Vasokonstriktionsyndrom kann in Ausnahmefällen auch auf andere Weisen beginnen: im Verlauf von Stunden rasant fortschreitend oder sich im Laufe von Tagen langsamer entwickelnd.

Groß angelegte Untersuchungen an Patienten mit bestätigtem RCVS ergaben, dass bis zu 75 \% mit Kopfschmerz als alleinigem Symptom vorstellig werden, doch kann die Erkrankung mit fluktuierenden fokalen neurologischen Defiziten und mitunter epileptische Anfälle in Verbindung stehen. 6.7.3.1 akuter Kopfschmerz zurückzuführen auf ein reversibles zerebrales Vasokonstriktionsyndrom kann ein Warnsymptom darstellen, dass einem hämorrhagischen oder ischämischen Infarkt vorausgeht. Bei einer Minderheit von RCVS-Fällen fehlt Kopfschmerz.

Die Angiographie zeigt bei RCVS per definitionem ein pathologisches Bild mit alternierenden Segmenten einer arteriellen Vasokonstriktion und Vasodilatation („String- und Perlenschnur-Zeichen“ oder das Erscheinungsbild einer „Wurstkette“). Dennoch können die Befunde der MR-, CT- und sogar Katheter-Angiographie in der ersten Woche nach dem klinischen Kopfschmerzbeginn normal sein. Bei Patienten mit rezidivierendem Donnerschlagkopfschmerz und einem normalen Angiogramm, die jedoch alle sonstigen Kriterien für ein RCVS erfüllen, sollte von einem 6.7.3.2 akuten Kopfschmerz wahrscheinlich zurückzuführen auf ein reversibles zerebrales Vasokonstriktionsyndrom ausgegangen werden. Das kranielles MRT ist bei $30 \%$ bis $80 \%$ der Fälle auffällig und zeigt diverse Läsionsmuster, unter anderen intrakranielle Blutungen (Konvexität subarachnoidal, intrazerebral und/oder subdural), zerebrale Infarkte und/oder zerebrale Ödeme, die dem „posterioren reversiblen Enzephalopathiesyndrom“ entsprechen.

Mindestens die Hälfte der RCVS-Fälle ist sekundärer Natur, meist mit Auftreten im Wochenbett und/oder nach Exposition mit vasoaktiven Substanzen wie illegalen Drogen, Alpha-Sympathomimetika und serotoninergen Drogen. Die Erkrankung ist innerhalb von 1 bis 3 Monaten selbst limitierend, bei Verschwinden der arteriellen Auffälligkeiten (daher „reversibel“) und fast immer einer Auflösung des Kopfschmerzes. Ein Hirninfarkt infolge des RCVS kann allerdings eine bleibende Beeinträchtigung hinterlassen.

\subsubsection{Akuter Kopfschmerz wahrscheinlich zurückzu- führen auf ein reversibles zerebrales Vasokonstrik- tionsyndrom (RCVS) \\ Beschreibung:}

Typischer Kopfschmerz für ein reversibles zerebrales Vasokonstriktionssyndrom (RCVS), nämlich ein über 1 bis 2 Wochen rezidivierender und durch sexuelle Aktivität, Anstrengung, ein Valsalva-Manöver und/oder Emotionen getriggerter Donnerschlagkopfschmerz, doch die Angiographie des Gehirns ergab keine der für RCVS charakteristischen intrakraniellen arteriellen fokalen Stenosen („Perlenschnur-Zeichen“).

\section{Diagnostische Kriterien:}

A. Jeder neue Kopfschmerz, der das Kriterium C erfüllt

B. Es besteht der Verdacht auf ein reversibles zerebrales Vasokonstriktionsyndrom (RCVS), doch die Hirnangiographie ist normal

C. Ein kausaler Zusammenhang kann durch alle der folgenden Kriterien gezeigt werden:

1. mindestens zweimal Kopfschmerzen innerhalb 1 Monats, die alle der drei folgenden Merkmale aufweisen:

a) Beginn als Donnerschlagkopfschmerz mit Höhepunkt in $<1$ Minute

b) starke Intensität

c) Dauer $\geq 5$ Minuten

2. mindestens ein Donnerschlagkopfschmerz wurde von einem der folgenden Punkte ausgelöst:

a) sexuelle Aktivität (unmittelbar vor dem oder beim Orgasmus)

b) Anstrengung

c) eine Art von Valsalva-Manöver

d) Emotion

e) Baden und/oder Duschen

f) Bücken

3. >1 Monate nach Kopfschmerzbeginn tritt kein neuer Donnerschlag- oder anderer deutlicher Kopfschmerz auf 
D. Einer der folgenden Punkte ist erfüllt:

1. der Kopfschmerz hat sich innerhalb von 3 Monaten nach seinem Beginn aufgelöst

2. der Kopfschmerz hat sich noch nicht aufgelöst, aber noch sind keine 3 Monate seit seinem Beginn verstrichen

E. Nicht besser erklärt durch eine andere ICHD-3-Diagnose1.

\section{Anmerkung:}

1. Andere Ursachen für Kopfschmerzen, insbesondere eine aneurysmatische Subarachnoidalblutung, konnten durch geeignete Untersuchungen ausgeschlossen werden.

\section{Kommentar:}

Die ICHD-3 schlägt generell keine Kriterien für wahrscheinliche sekundäre Kopfschmerzen vor. Die arteriellen Anomalien bei einem reversiblen zerebralen Vasokonstriktionssyndrom (RCVS) können jedoch schwer nachzuweisen sein. Bei einigen RCVS-Fällen ist während der 2 bis 3 Wochen nach Kopfschmerzbeginn eine wiederholte CT- oder MR-Angiographie erforderlich, bei anderen wird eine invasive konventionelle Angiographie benötigt, um sie aufzudecken. Bei Patienten, die über einen Zeitraum von weniger als einem Monat unter dem rezidivierendem getriggertem Donnerschlagkopfschmerz leiden, der typisch für RCVS ist, doch eine normale anfängliche zerebrale Angiographie und bei denen eine andere Kopfschmerzursache durch entsprechende Untersuchungen ausgeschlossen wurde, kann die vorläufige Diagnose eines 6.7.3.2 Kopfschmerzes wahrscheinlich zurückzuführen auf ein reversibles zerebrales Vasokonstriktionsyndrom (RCVS) gestellt werden.

\subsubsection{Anhaltender Kopfschmerz zurückzuführen auf ein früheres reversibles zerebrales Vasokonstriktion- syndrom (RCVS) \\ Beschreibung:}

Ein Kopfschmerz, der durch ein reversibles zerebrales Vasokonstriktionsyndrom (RCVS) verursacht wird und mehr als 3 Monate nach seinem Beginn anhält.

\section{Diagnostische Kriterien:}

A. Ein Kopfschmerz, der zuvor als 6.7.3.1 akuter Kopfschmerz zurückzuführen auf ein reversibles zerebrales Vasokonstriktionsyndrom (RCVS) diagnostiziert wurde und Kriterium C erfüllt

B. Normalisierung der Hirnarterien, nachgewiesen bei der Verlaufskontrolle durch indirekte oder direkte Angiographie innerhalb von 3 Monaten nach dem Beginn von RCVS

C. Der Kopfschmerz existiert >3 Monate nach seinem Beginn

D. Nicht besser erklärt durch eine andere ICHD-3-Diagnose.

\section{Kommentar:}

In einigen Untersuchungen wurden Kopfschmerzen dokumentiert, die die Kriterien für 6.7.3.3 anhaltender Kopfschmerz zurückzuführen auf ein reversibles zerebrales Vasokonstriktionsyndrom erfüllen. Weitere Untersuchungen sind erforderlich, um Risikofaktoren für einen solchen anhaltenden Kopfschmerz zu ermitteln; 1. Migräne in der Vorgeschichte kann eine Rolle spielen, ebenso wie Angststörungen/Depressionen.

\subsubsection{Kopfschmerz zurückzuführen auf eine intra- kranielle arterielle Dissektion}

Beschreibung:

Kopfschmerz, der auf die Dissektion einer intrakraniellen Arterie zurückgeht. Der Schmerz tritt meist einseitig und ipsilateral zum betroffenen Gefäß auf und setzt in der Regel plötzlich (sogar als Donnerschlagkopfschmerz) ein. Er kann isoliert fortbestehen oder ein Warnsymptom darstellen, das einer Subarachnoidalblurung oder einem Hirninfarkt vorangeht.

\section{Diagnostische Kriterien:}

A. Ein neu aufgetretener Kopfschmerz, der das Kriterium C erfüllt

B. Es wurde eine intrakranielle arterielle Dissektion diagnostiziert

C. Der ursächliche Zusammenhang besteht aufgrund von mindestens zwei der folgenden Punkte:

1. Der Kopfschmerz hat sich in enger zeitlicher Beziehung zu sonstigen Symptomen und/oder klinischen Zeichen einer intrakraniellen arteriellen Dissektion entwickelt oder zu deren Diagnose geführt

2. Der Kopfschmerz verschwindet innerhalb 1 Monats nach seinem Beginn

3. Einer oder beide der folgenden Punkte ist/sind erfüllt:

a) plötzlicher Beginn oder Beginn als Donnerschlagkopfschmerz

b) starke Intensität

4. Der Kopfschmerz ist einseitig und ipsilateral zur Dissektion D. Nicht besser erklärt durch eine andere ICHD-3-Diagnose.

Kommentar:

Die Dissektion kann jede intrakranielle Arterie betreffen und kann eine Subarachnoidalblutung, ischämische Infarkte, eine Kompression benachbarter Strukturen oder, in selteneren Fällen, eine intrazerebrale Blutung induzieren. Bei Asiaten kommt eine intrakranielle arterielle Dissektion häufiger vor als eine Dissektion der Halsschlagader.

Akute Kopfschmerzen sind oft das im Vordergrund stehende Symptom und können das alleinige Symptom dieser Erkrankung sein.

\subsection{Kopfschmerz und/oder migräneartige Aura zurückzuführen auf eine chronische intrakranielle Vaskulopathie}

\subsubsection{Kopfschmerz zurückzuführen auf eine zereb- rale autosomal dominante Arteriopathie mit subkortikalen Infarkten und Leukenzephalopa- thie (CADASIL)}

Beschreibung:

Anfallsweise auftretender Kopfschmerz ähnlich dem bei einer 1.2 Migräne mit Aura, mit Ausnahme einer unüblichen Häufung von prolongierten Auren, verursacht durch eine zerebrale autosomal dominante Arteriopathie mit subkortikalen Infarkten und Leukoenzephalopathie (CADASIL). Tritt in Verbindung mit den ande- 
ren klinischen Merkmalen von CADASIL auf oder ist, und das häufig, das erste Symptom für diese.

\section{Diagnostische Kriterien:}

A. Wiederkehrende Migräneattacken mit typischen, hemiplegischen oder prolongierten Aura, die Kriterium C erfüllen

B. Es wurde eine zerebrale autosomal dominante Arteriopathie mit subkortikalen Infarkten und Leukoenzephalopathie (CADASIL) nachgewiesen 1

C. Einer oder beide der folgenden Punkte sind erfüllt:

1. Migräne mit Aura war die früheste klinische Manifestation von CADASIL

2. Migräneattacken mit Aura verbessern sich oder hören auf, wenn andere Manifestationen von CADASIL (z. B., ischämischer Infarkt, Störungen der Stimmung und/oder kognitive Dysfunktion) auftreten und sich verschlimmern

D. Nicht besser erklärt durch eine andere ICHD-3-Diagnose.

\section{Anmerkung:}

1. Die Diagnose erfolgt über eine einfache Hautbiopsie mit immunologischen Nachweis von NOTCH-3-Antikörpern oder durch Untersuchung auf Ablagerungen von extrazellulärem granulärem osmiophilem Material (GOM) in der arteriellen Media mit Hilfe der Elektronenmikroskopie.

\section{Kommentar:}

Bei der zerebralen autosomal dominanten Arteriopathie mit subkortikalen Infarkten und Leukoenzephalopathie (CADASIL) handelt es sich um eine autosomal dominante Erkrankung, wenn auch vereinzelte sporadische Fälle bekannt sind, die die glatte Muskulatur in der Media der kleinen Hirnarterien betrifft. Sie geht auf Mutationen des NOTCH-3-Gens zurück.

Klinisch ist CADASIL durch rezidivierende kleine tiefgelegene Infarkte, eine subkortikale Demenz, Störungen der Stimmung sowie in einem Drittel der Fälle durch Attacken gekennzeichnet, wie sie für eine 1.2 Migräne mit Aura typisch sind, abgesehen von einer ungewöhnlich oft vorkommenden prolongierten Aura. Diese sind in der Regel das erste Symptom der Erkrankung, die im Mittel im Alter von 30 Jahren beginnt, etwa 15 Jahre vor dem ersten ischämischen Infarkt und 20 bis 30 Jahre vor dem Tod.

Im MRT finden sich in der T2-Wichtung immer auffällige Veränderungen der weißen Substanz.

\subsubsection{Kopfschmerz zurückzuführen auf eine mitochondriale Enzephalopathie, Laktatazi- dose und Schlaganfall-ähnlichen Episoden (MELAS)}

Beschreibung:

Kopfschmerz, der entweder in migräneartigen Attacken wiederkehrt oder eines der Symptome von infarktähnlichen Episoden ist, die von den sonstigen klinischen Merkmalen einer mitochondrialen Enzephalopathie, Laktatazidose und Schlaganfall-ähnlichen Episoden (MELAS) verursacht werden und diese begleiten.

\section{Diagnostische Kriterien:}

A. Immer wiederkehrende Kopfschmerzattacken, die Kriterium C erfüllen
B. Es konnte der Nachweis einer in Begleitung einer mitochondrialen Enzephalopathie, Laktatazidose und Schlaganfall-ähnlichen Episoden (MELAS) auftretenden mitochondrialen genetischen Anomalie erbracht werden

C. Einer oder beide der folgenden Punkte sind erfüllt:

1. wiederkehrende Migräneattacken mit oder ohne Aura

2. akuter Kopfschmerz im Vorfeld oder in Begleitung fokaler neurologischer Defizite und/oder epileptischer Anfälle

D. Nicht besser erklärt durch eine andere ICHD-3-Diagnose.

\section{Kommentar:}

Die mitochondrielle Enzephalopathie, Laktatazidose und Schlaganfall-ähnlichen Episoden (MELAS) ist eine genetisch heterogene mitochondriale Erkrankung mit variablem klinischem Erscheinungsbild, darunter Merkmale einer Beteiligung des ZNS (epileptische Anfälle, Hemiparese, Hemianopie, kortikale Blindheit, sensorineurale Taubheit und/oder episodisches Erbrechen). Kopfschmerzen sind bei MELAS weit verbreitet, sei es als immer wiederkehrende migräneartige Attacken oder als auftretendes Symptom „infarktartiger“ Episoden.

Migräneartige Attacken sind häufig bei MELAS, was zu der Hypothesis führte, dass mitochondriale Mutationen bei der Vererbung der Migräne mit Aura eine Rolle spielen. Allerdings konnte die 3243-Mutation bei 2 Gruppen von Patienten mit 1.2 Migräne mit Aura nicht identifiziert werden. Möglicherweise spielen andere bis jetzt noch nicht identifizierte Mutationen sowohl bei der Migräne als auch dem ischämischen Hirninfarkt eine Rolle, insbesondere weil Migräneattacken, meistens solche mit Aura, auch bei anderen mitochondrialen Erkrankungen vorkommen.

\subsubsection{Kopfschmerz zurückzuführen auf eine Moyamoya-Angiopathie}

Beschreibung:

Chronisch-rezidivierende Kopfschmerzen, die migräneartig sein können und durch die sonstigen klinischen Merkmale der Moyamoya-Angiopathie verursacht werden oder diese begleiten.

\section{Diagnostische Kriterien:}

A. Immer wiederkehrender Kopfschmerz, der das Kriterium C erfüllt

B. Nachweis der Moyamoya-Angiopathie durch bildgebende Verfahren

C. Ursächlicher Nachweis durch die beiden folgenden Punkte:

1. Der Kopfschmerz hat sich in enger zeitlicher Beziehung zu anderen Symptomen und/oder klinischen Zeichen und/ oder Nachweis der Moyamoya-Angiopathie durch bildgebende Verfahren entwickelt oder führte zu ihrer Entdeckung

2. Einer oder beide der folgenden Punkte sind erfüllt:

a) die Schmerzen haben sich gleichzeitig mit anderen Symptomen und/oder klinischen und/oder radiologischen Zeichen einer Verschlechterung der Moyamoya-Angiopathie deutlich verschlechtert

b) die Schmerzen haben sich nach einer operativen Revaskularisierung deutlich gebessert

D. Nicht besser erklärt durch eine andere ICHD-3-Diagnose. 


\section{Kommentar:}

Kennzeichnend für eine Moyamoya-Angiopathie ist eine bilaterale progressive Verengung und ein progressiver Verschluss des intrakraniellen Abschnitts der A. carotis interna, A. cerebri media und A. cerebri anterior. Es wurden mehrere Suszeptibilitätsgene für eine Moyamoya-Angiopathie identifiziert oder lokalisiert. Bei einigen anderen Patienten tritt eine Moyamoya-Angiopathie in Begleitung anderer Erkrankungen auf (u. a. Sichelzellanämie, Down-Syndrom und Strahlentherapie) und wird als Moyamoya-Syndrom bezeichnet.

Die Moyamoya-Angiopathie zeigt sich meist früh, im Kindesoder Jugendalter, mit einem ischämischen oder hämorrhagischen Infarkt, der einen akuten Kopfschmerz auslösen kann. Außerhalb dieser akuten vaskulären Ereignisse kommen Kopfschmerzen bei Kindern wie auch bei Erwachsenen mit Moyamoya-Angiopathie sehr häufig vor, wobei ihr Erscheinungsbild am ehesten einer 1.1 Migräne ohne Aura, 1.2 Migräne mit Aura, 1.2.3 Hemiplegischen Migräne oder einem 2. Kopfschmerz vom Spannungstyp ähnelt; in seltenen Fällen wurde von clusterkopfschmerzartigen Attacken berichtet.

Die operative Revaskularisierung hat wechselnde Auswirkungen auf die Kopfschmerzen bei einer Moyamoya-Angiopathie. Bei einigen Patienten tritt eine Besserung ein, bei anderen hält Kopfschmerz an. Eine weitere Unterkategorie ist ein postoperativ neu einsetzender Kopfschmerz.

\subsubsection{Migräneartige Aura zurückzuführen auf eine zerebrale Amyloidangiopathie}

\section{Beschreibung}

Erst spät einsetzende migräneartige Auraattacken ohne oder mit leichtem Kopfschmerz, auch „amyloidbedingte Anfälle“ („Amyloid Spells“) genannt, verursacht durch und begleitet von den sonstigen klinischen Merkmalen einer zerebralen Amyloidangiopathie, oft im Kontext einer konvexalen Subarachnoidalblutung.

\section{Diagnostische Kriterien:}

A. Neu auftretende Attacken einer migräneartigen Aura, mit oder ohne leichte Kopfschmerzen, die Kriterium C erfüllen

B. Nachweis einer zerebralen Amyloidangiopathie anhand zerebraler bildgebender Verfahren oder einer Biopsie des Gehirns

C. Ursächlicher Nachweis durch einen der beiden folgenden Punkte:

1. Die Aura hat sich in enger zeitlicher Beziehung zu anderen Symptomen und/oder klinischen Zeichen einer zerebralen Amyloidangiopathie entwickelt oder führte zu ihrer Entdeckung

2. Die Aura hat sich gleichzeitig mit klinischen und/oder radiologischen Zeichen einer Verschlechterung der zerebralen Amyloidangiopathie deutlich verschlechtert

3. erstmaliges Auftreten im Alter von über 50

D. Nicht besser erklärt durch eine andere ICHD-3-Diagnose1.

\section{Anmerkung:}

1. Blutsensitive MRT-Sequenzen sind wichtig für die Diagnose einer zerebralen Amyloidangiopathie und sollten bei sämtlichen Patienten mit spät einsetzender migräneartiger Aura durchgeführt werden.
Kommentar:

Die zerebrale Amyloidangiopathie ist eine Erkrankung der kleinen Gefäße in Begleitung einer progressiven Amyloidablagerung in den Wänden der kortikalen und leptomeningealen Gefäße. Sporadische Formen kommen häufiger vor als ererbte familiäre Formen.

Die zerebrale Amyloidangiopathie ist eine Hauptursache einer lobären symptomatischen intrazerebralen Blutung, transienter fokaler neurologischer Episoden bei alten Menschen sowie kognitiven Beeinträchtigungen. Transiente fokale neurologische Episoden beinhalten sowohl positive migräneauraartige (sich ausbreitende Parästhesien und/oder positive visuelle Phänomene) als auch negative TIA-artige neurologische Symptome und können von einer superfiziellen kortikalen Siderose oder konvexalen Subarachnoidalblutung verursacht werden. Diese Episoden gehen mit einem hohen frühen Risiko einer symptomatischen intrazerebralen Blutung einher.

\subsubsection{Kopfschmerz zurückzuführen auf ein retinales Vaskulopathie-Syndrom mit zerebraler Leuken- zephalopathie und systemischen Manifesta- tionen (RVCLSM)}

\section{Beschreibung:}

Als migräneartige Attacken immer wiederkehrender Kopfschmerz, meist ohne Aura, verursacht durch ein retinales Vaskulopathie-Syndrom mit zerebraler Leukenzephalopathie und systemische Manifestationen (RVCLSM). Der Kopfschmerz kann in Begleitung der sonstigen klinischen Merkmale eines RVCLSM auftreten oder dessen früheste klinische Manifestation darstellen.

\section{Diagnostische Kriterien:}

A. Immer wiederkehrende migräneartige Attacken mit oder ohne Aura, die Kriterium C erfüllen

B. Es wurde der Nachweis eines retinalen Vaskulopathie-Syndrom mit zerebraler Leukenzephalopathie und systemischen Manifestationen (RVCLSM) erbracht1

C. Migräneartige Attacken sind sekundäre Folge und Teil der klinischen Manifestationen des Syndroms

D. Nicht besser erklärt durch eine andere ICHD-3-Diagnose.

Anmerkung:

1. Die Diagnose erfolgt durch Gentests auf TREX1-Mutationen.

\section{Kommentar:}

Das retinale Vaskulopathie-Syndrom mit zerebraler Leukenzephalopathie und systemischen Manifestationen (RVCLSM) ist eine autosomal dominante systemische Erkrankung der kleinen Blutgefäße, die durch C-terminale Frameshift-Mutationen in TREX1 verursacht wird. Klinisch ist sie von fokalen neurologischen Defiziten, kognitiven Beeinträchtigungen, psychiatrischen Störungen, epileptischen Anfällen, diversen systemischen Manifestationen sowie in mindestens der Hälfte der Fälle von migräneartigen Attacken gekennzeichnet. Weitere klinische Manifestationen Visusbeeinträchtigung durch vaskuläre Erkrankungen der Retina sowie neurologischer Verfall und vorzeitiger Tod aufgrund fortschreitender kontrastmittelaufnehmender Läsionen in der weißen Hirnsubstanz. Das klinische Spektrum umfasst zudem eine funktionelle Beeinträchtigung von Leber und Niere sowie Anämie, mitunter in Verbindung mit Magen-Darm-Blutungen und Bluthochdruck. Bei jün- 
geren Patienten, bei denen der Befund des Schädel-MRTs normal sein kann. Zu den klinischen Manifestationen gehören leichte Ausprägungen des Raynaud-Phänomens (54\%), Migräne (meist ohne Aura: $42 \%$ ) und psychiatrische Störungen (23\%). Die Verdachtsdiagnose kann in solchen Fällen angesichts der familiären Vorgeschichte naheliegen.

\subsubsection{Kopfschmerz zurückzuführen auf andere intrakranielle Gefäßstörungen}

\section{Beschreibung:}

Migräneartige Attacken mit oder ohne Aura, verursacht durch und auftretend als Bestandteil der klinischen Manifestationen einer genetisch oder nicht-genetisch bedingten chronischen intrakraniellen Gefäßstörung neben den oben beschriebenen.

\section{Diagnostische Kriterien:}

A. Immer wiederkehrende migräneartige Attacken mit oder ohne Aura, die Kriterium C erfüllen

B. Es wurde der Nachweis einer genetisch oder nicht-genetisch bedingten chronische intrakraniellen Gefäßstörung erbracht

C. Migräneartige Attacken treten sekundär zu und als Teil der klinischen Manifestationen der chronischen intrakraniellen Gefäßstörung auf

D. Nicht besser erklärt durch eine andere ICHD-3-Diagnose.

\section{Kommentar:}

Von wiederkehrenden migräneartigen Attacken wurde als Bestandteil der klinischen Manifestationen der autosomal dominanten hereditären infantilen hemiplegischen Zerebralparese, retinalen arteriellen Tortuosität und Leukenzephalopathie (Hereditary Infantile Hemiparesis, Retinal Arterial Tortuosity and Leucoencephalopathy, HIHRATL) berichtet, ein auf COL4A1-Mutationen zurückgehendes Krankheitsbild. Es wurde nur von einigen wenigen Familien mit dieser Erkrankung berichtet. Aufgrund der sonstigen schweren Manifestationen wurden diese migräneartigen Attacken bei HIHRATL nicht systematisch untersucht, sondern scheinen im Großen und Ganzen einer 1.2 Migräne mit Aura zu ähneln.

Alle sonstigen seltenen genetisch und nicht-genetisch bedingten chronischen intrakraniellen Gefäßstörungen können potenziell migräneartige Attacken auslösen.

\subsection{Kopfschmerz zurückzuführen auf einen Hypophyseninfarkt}

\section{Beschreibung:}

Kopfschmerz, der durch einen Hypophyseninfarkt verursacht wird, in der Regel mit plötzlichem Beginn (sogar Donnerschlag) und starker Intensität, von Anfang an oder später in Begleitung visueller Symptome und/oder einer Hypophysenunterfunktion.

\section{Diagnostische Kriterien:}

A. Jeder neu aufgetretene Kopfschmerz, der das Kriterium C erfüllt

B. Es wurde ein akuter hämorrhagischer Hypophyseninfarkt diagnostiziert
C. Nachweis einer Verursachung durch mindestens zwei der folgenden Punkte:

1. Der Kopfschmerz entwickelte sich in enger zeitlicher Beziehung zu anderen Symptomen und/oder klinischen Zeichen eines Hypophyseninfarkts oder war maßgeblich für die Diagnose eines Hypophyseninfarkts

2. Einer oder beide der folgenden Punkte sind erfüllt:

a) deutliche Kopfschmerzverschlechterung gleichzeitig mit anderen Symptomen und/oder klinischen Zeichen eines Hypophyseninfarkts

b) deutliche Kopfschmerzbesserung gleichzeitig mit anderen Symptomen und/oder klinischen Zeichen einer Besserung im Hinblick auf einen Hypophyseninfarkt

3. Kopfschmerz ist stark und tritt plötzlich oder als Donnerschlagkopfschmerz auf

D. Nicht besser erklärt durch eine andere ICHD-3-Diagnose.

\section{Kommentar:}

Der Hypophyseninfarkt ist ein seltenes klinisches Syndrom, das ein akut lebensbedrohliches Krankheitsbild darstellt. Es handelt sich um eine der Ursachen einer nicht-aneurysmatischen Subarachnoidalblutung.

Zudem ist er eine der Ursachen eines Donnerschlagkopfschmerzes. Die meisten Fälle treten als erster Hinweis auf eine rasante Vergrößerung funktionsgestörter Makroadenome der Hypophyse infolge einer Blutung und/oder eines Infarkts auf.

Das MRT ist sensitiver als das CTT in der Aufdeckung von intrasellären Pathologien.

\section{Literatur}

\subsubsection{Kopfschmerz zurückzuführen auf einen ischämischen Infarkt (zerebraler Infarkt)}

Ferro JM, Melo TP, Oliveira V, Salgado AV, Crespo M, Canhao P, Pinto AN. A multivariate study of headache associated with ischemic stroke. Headache 1995; 35: 315-319.

Gorelick PB, Hier DB, Caplan LR, Langenberg D. Headache in acute cerebrovascular disease. Neurology 1986; 36: 1445-1450.

Hansen AP, Marcussen NS, Klit H, Kasch H, Jensen TS, Finnerup NB. Development of persistent headache following stroke: a 3-year follow-up. Cephalalgia 2015; 35: 399-409.

Lopes Azevedo L, Breder R, de A Santos DP, de Freitas GR. Ischemic stroke presenting as thunderclap headache: report of two cases and review of the literature. Eur Neurol 2011; 66: 133-135.

Portenoy RK, Abissi CJ, Lipton RB, Berger AR, Mebler MF, Baglivo J, Solomon S. Headache in cerebrovascular disease. Stroke 1984; 15: 1009-1012.

Schwedt TJ, Dodick DW. Thunderclap stroke: embolic cerebellar infarcts presenting as thunderclap headache. Headache 2006; 46; 520-522.

Verdelho A, Ferro JM, Melo T, Canhao P, Falcao F. Headache in acute stroke. A prospective study in the first 8 days. Cephalalgia 2008; 28; 346-354.

\subsubsection{Kopfschmerz zurückzuführen auf eine transitorische ischämische Attacke (TIA)}

Caplan LR. Migraine and vertebrobasilar ischemia. Neurology 1991; 41: $55-61$.

Ferro JM, Costa I, Melo TP, Canhao P, Oliveira V, Salgado AV, Crespo M, Pinto AN. Headache associated with transient ischemic attacks. Headache 1995; 35: 544-548. 
Fisher CM. Migraine accompaniments versus arteriosclerotic ischemia. Trans Am Neurol Assoc 1968; 93: 211-213.

Fisher CM. Cerebral ischemia: less familiar types. Clin Neurosurg 1971; 18 : 267-336.

Fisher CM. Late-life migraine accompaniements as a cause of unexplained transient ischemic attacks. Can J Med Sci 1980; 7: 9-17.

Martsen BH, Sorensen PS, Marquardsen J. Transient ischemic attacks in young patients: a thromboembolic or migrainous manifestation? A ten-year follow-up of 46 patients. J Neurol Neurosurg Psychiat 1990; 53: 1029-1033.

6.2.1 Akuter Kopfschmerz zurückzuführen auf eine nicht-traumatische intrazerebrale Blutung

Abadie V, Jacquin A, Daubail B, Vialatte AL, Lainay C, Durier J, Osseby GV, Giroud M, Béjot Y. Prevalence and prognostic value of headache on early mortality in acute stroke: the Dijon Stroke Registry. Cephalalgia 2014; 34: 887-894.

Ferro JM, Melo TP, Guerreiro M. Headaches in intracerebral hemorrhage survivors. Neurology 1998; 50: 203-207.

Jensen TS, Gorrelick PB. Headache associated with stroke and intracranial hematoma. In: The Headaches. 2nd edition.J. Olesen, P. Telt-Hansen, KMA Welch eds. Philadelphia: Lippincott Williams and Wilkins 2000, 781-787.

Melo TP, Pinto AN, Ferro JM. Headache in intracerebral hematomas. Neurology 1996; 47: 494-500.

Schuaib A, Metz L, and Hing T. Migraine and intra-cerebral hemorrhage. Cephalalgia 1989; 9: 59-61.

Verdelho A, Ferro JM, Melo T, Canhao P, Falcao F. Headache in acute stroke. A prospective study in the first 8 days. Cephalalgia 2008; 28; 346-354.

\subsubsection{Kopfschmerz zurückzuführen auf eine nicht-traumatische} Subarachnoidalblutung (SAB)

Bassi P, Bandera R, Loiero M, Togoni G, Mangoni A. Warning signs in subarachnoid hemorrhage: a cooperative tudy. Acta Neurol Scand 1991; 84: 277-281.

Edlow JA, Caplan LR. Avoiding pitfalls in the diagnosis of subarachnoid hemorrhage. NEJM 2000; 342: 29-36.

Evans RW. Diagnostic testing for the evaluation of headaches. Neurology Clinics 1996; 14: 1-26.

Graff-Radford J, Fugate JE, Klaas J, Flemming KD, Brown RD, Rabinstein AA. Distinguishing clinical and radiological features of non-traumatic convexal subarachnoid hemorrhage. Eur J Neurol 2016; 23: 839-846.

Linn FHH, Rinkel GJE, Algra A, van Gijn J. Headache characteristics in subarachnoid haemorrhage and benign thunderclap headache. J Neurol Neurosurg Psych 1998; 65: 791-793.

Mayberg MR, Batjer HH, Dacey R, Diringer M, Haley EC, Heros RC, Sternau LL, Torner J, Adams HP, Feinberg W, Thies W. Guidelines for the management of aneurysmal subarachnoid hemorrhage. A statement for healthcare professionals from a special writing group of the Stroke Council of the American Heart Association. Stroke 1994; 25: 2315-2328.

Ramirez-Lassepas M, Espinosa CE, Cicero JJ, Johnston KL, Cipolle RJ, Barber DL. Predictors of intracranial pathologic findings in patients who seek emergency care because of headache. Arch Neurol 1997; 54: 1506-1509.

Rico M, Benavente L, Para M, Santamarta E, Pascual J, Calleja S. Headache as a crucial symptom in the etiology of convexal subarachnoid hemorrhage. Headache 2014; 54: 545-550.

Seymour JJ, Moscati RM, Jehle DV. Response of headaches to non-narcotic analgesics resulting in missed intracranial hemorrhage. Am J Emerg Med 1995; 13: 43-45.
Sidman R, Vconnolly E, Lemke T. Subarachnoid hemorrhage diagnosis : lumbar puncture is still needed when the computed tomography scan is normal. Acad Emerg Med 1996; 3: 827-831.

Van der Wee N, Rinkel GJE, Hasan D, van Gijn J. Detection of subarachnoid hemorrhage on early CT: is lumbar puncture still needed after a negative scan? J Neurol Neurosurg Psychiat 1995; 58: 357-359.

Verweij RD, Wijdicks EFM, van Gijn J. Warning headache in aneurysmal subarachnoid hemorrhage. A case control study. Arch Neurol 1988; 45: $1019-1020$.

Weir B. Diagnostic aspects of SAH. In: Weir B. Subarachnoid hemorrhage: causes and cures. New York: Oxford University Press 1998, 144-176.

6.2.3 Akuter Kopfschmerz zurückzuführen auf ein nicht-traumatisches akutes Subduralhämatom (aSDH)

Chhiber SS, Singh JP. Acute spontaneous subdural hematoma of arterial origin: a report of four cases and review of literature. Neurol India 2010; 58: 654-658.

Depreitere B, Van Calenbergh F, van Loon J. A clinical comparison of non-traumatic acute subdural haematomas either related to coagulopathy or of arterial origin without coagulopathy. Acta Neurochir (Wien) 2003; 145: 541-546

Koerbel A, Ernemann U, Freudenstein D. Acute subdural hematoma without subarachnoid hemorrhage caused by rupture of internal carotid artery bifurcation aneurysm: case report and review of literature. Br J Radiol 2005; 78: 646-650.

Missori P, Fenga L, Maraglino C, Rocchi G, Nardacci B, Calderaro G, Salvati M, Delfini R. Spontaneous acute subdural hematomas. A clinical comparison with traumatic acute subdural hematomas. Acta Neurochir (Wien) 2000; 142: 697-701.

de Noronha RJ, Sharrack B, Hadjivassiliou M, Romanowski CA. Subdural haematoma: a potentially serious consequence of spontaneous intracranial hypotension. J Neurol Neurosurg Psychiatry 2003; 74: 752-755.

Ogawa K, Oishi M, Mizutani T, Maejima S, Mori T. Dural arteriovenous fistula on the convexity presenting with pure acute subdural hematoma. Acta Neurol Belg 2010; 110: 190-192.

Takahashi S, Shinoda J, Hayashi T. Cerebral Venous Sinus Thrombosis in an Adult Patient Presenting as Headache and Acute Subdural Hematoma. J Stroke Cerebrovasc Dis 2010; 21: 338-340.

\subsubsection{Anhaltender Kopfschmerz zurückzuführen auf eine frühere} nicht-traumatische intrakranielle Blutung

Hansen AP, Marcussen NS, Klit H, Kasch H, Jensen TS, Finnerup NB. Development of persistent headache following stroke: a 3-year follow-up. Cephalalgia 2015; 35:399-409.

\subsubsection{Kopfschmerz zurückzuführen auf ein nicht-rupturiertes sack-} förmiges Aneurysma

Byruma EP, McGregor JM, Christoforidisa GA. Thunderclap headache without subarachnoid hemorrhage associated with regrowth of previously coil-occluded aneurysms. Am J Neuroradiol 2009; 30: 1059-1061.

Day JW, Raskin NH. Thunderclap headache: symptom of unruptured cerebral aneurysm. Lancet 1986; 2: 1247-1248.

Linn FHH, Wijdicks EFM, van der Graaf Y, Weerdesteyn-van Vliet FAC, Bartelds A, van Gijn J. Prospective study of sentinel headache in aneurysmal subarachnoid haemorrhage. Lancet 1994; 344: 590-593.

Markus HS. A prospective follow-up of thunderclap headache mimicking subarachnoid haemorrhage. J. Neurol Neurosurg Psychiat 1991; 54: 1117-1125.

Mas JL, Baron JC, Bousser MG, Chiras J. Stroke, migraine and intracrania aneurysm: a case report. Stroke 1986; 17: 1019-1021.

Ostergard JR, Ramadan N. Unruptured vascular malformations and subarachnoid hemorrhage. In J Olesen, P Tfelt-Hansen, KMA Welch eds. The 
Headaches 2nd edition. Philadelphia: Lipincott Williams and Wilkins 2000: 789-796.

Raps EC, Rogers JD, Galetta DL, Solomon RA, Lennihan L, Klebanoff LM, Fink $M E$. The clinical spectrum of unruptured intracranial aneurysms. Arch Neurol 1993; 50: 265-268.

Schievink WI. Intracranial aneurysms. NEJM 1997; 336: 28-40.

Wijdicks EFM, Kerkhoff H, van Gijn J. Long-term follow-up of 71 patients with thunderclap headache mimicking subarachnoid haemorrhage. Lancet 1988; 2: 68-70.

\subsubsection{Kopfschmerz zurückzuführen auf eine arterio-venöse Malforma-} tion (AVM)

Bruyn GW. Intracranial arteriovenous malformation and migraine. Cephalalgia 1984; 4: 191-207.

Haas DC. Arteriovenous malformations and migraine : case reports and an analysis of the relationship. Headache 1991; 31: 509-513.

Nomura M, Mori K, Tamase A, Kamide T, Seki S, lida Y, Kawabata Y, Nakano T, Shima H, Taguchi H. Cavernous sinus dural arteriovenous fistula patients presenting with headache as an initial symptom. J Clin Med Res 2016; 8: 342-345.

Troost BT, Mark LE, Maroon JC. Resolution of classic migraine after removal of an occipital lobe AVM. Ann Neurol 1979; 5: 199-201.

\subsubsection{Kopfschmerz zurückzuführen auf eine durale arterio-venöse Fistel (DAVF)}

Garza I. Images from headache: a "noisy" headache: dural arteriovenous fistula resembling new daily persistent headache. Headache 2008; 48; $1120-1121$.

Malek AM, Halbach VV, Dowd CF, Higashida RT. Diagnosis and treatment of dural arteriovenous fistulas. Neuroimaging Clin N Am 1998; 8: $445-468$.

\subsubsection{Kopfschmerz zurückzuführen auf ein kavernöses Angiom}

Afridi S, Goadsby PJ. New onset migraine with a brain stem cavernous angioma. J Neurol Neurosurg Psychiat 2003;74:680-682.

Bellamio M, Anglani M, Mainardi F, Zanchin G, Maggioni F. Cluster headache: When to worry? Two case reports. Cephalalgia 2017; 37: 491-495.

De Benedittis G. SUNCT syndrome associated with cavernous angioma of the brain stem. Cephalalgia 1996; 16: 503-506.

Denier C, Labauge P, Brunereau L, Cavé-Riant F, Marchelli F, Arnoult M, CecilIon M, Maciazek J, Joutel A, Tournier-Lasserve E. Clinical features of cerebral cavernous malformations patients with krit1 mutations. Ann Neurol 2004; 55: 213-220.

Epstein MA, Beerman PH, Schut L. Cavernous angioma presenting as atypical facial and head pain. J Child Neurol 1990; 5: 27-30.

Kivelev J, Niemela M, Kivisaari R, Hernesniemi J. Intraventricular cerebral cavernomas: a series of 12 patients and review of the literature. J Neurosurg 2010; 112: 140-149.

Robinson JR, Awad IA, Little JR. Natural history of the cavernous angioma. J Neurosurg 1991; 75: 709-714.

\subsubsection{Kopfschmerz zurückzuführen auf eine enzephalo-trigeminale} oder leptomeningeale Angiomatose (Sturge-Weber-Syndrom)

Chabriat H, Pappata S, Traykov L, Kurtz A, Bousser MG. Angiomatose de Sturge Weber responsable d'une hémiplégie sans infarctus cérébral en fin de grossesse. Rev Neurol (Paris) 1996; 152: 536-541.

Klapper J. Headache in Sturge-Weber syndrome. Headache 1994; 34: 521-522.

Lisotto C, Mainardi F, Maggioni F, Zanchin G. Headache in Sturge-Weber syndrome: a case report and review of the Literature. Cephalalgia 2004; 24: 1001-1004
Pascual-Castroviejo I, Pascual-Pascual SI, Velazquez-Fragua R and Viano J. Sturge-Weber syndrome: study of 55 patients. Can J Neurol Sci 2008; 35: 301-307

Planche V, Chassin O, Leduc L, Regnier W, Kelly A, Colamarino R. Sturge-Weber syndrome with late onset hemiplegic migraine-like attacks and progressive unilateral cerebral atrophy.Cephalalgia 2014; 34: 73-77.

Shirley MD, Tang H, Gallione CJ, Baugher JD, Frelin LP, Cohen B, North PE, Marchuk DA, Comi AM, Pevsner J. Sturge-Weber syndrome and port-wine stains caused by somatic mutation in GNAQ. N Engl J Med 2013; 368: 1971-1979.

\subsubsection{Kopfschmerz zurückzuführen auf eine Riesenzellarteriitis (RZA)}

Caselli RJ, Hunder GG. Neurologic aspects of giant cell (temporal) arteritis. Rheum Dis Clin North Am 1993; 19: 941-953.

Gonzalez-Gay MA, Barros S, Lopez-Diaz MJ, Garcia-Porrua C, Sanchez-Andrade $A$ and Llorca J (2005). Giant cell arteritis: disease patterns of clinical presentation in a series of 240 patients. Medicine (Baltimore) 84: 269-276.

Hunder GG. Giant cell (temporal) arteritis. Rheum Dis Clin North Am 1990; 16: 399-409.

Lee AG, Brazis PW. Temporal arteritis : a clinical approach. J Am Geriatr Soc 1999; 47: 1364-1370.

Mukhtyar C, Guillevin L, Cid MC, Dasgupta B, de Groot K, Gross W, Hauser T, Hellmich B, Jayne D, Kallenberg CG, Merkel PA, Raspe H, Salvarani C, Scott DG, Stegeman C, Watts R, Westman K, Witter J, Yazici H and Luqmani R. EULAR recommendations for the management of large vessel vasculitis. Ann Rheum Dis 2009; 68: 318-323.

Solomon S, Cappa KG. The headache of temporal arteritis. J Am Geriatr Soc 1987; 35: 163-165.

Thielen KR, Wydicks EFM, Nichols DA. Giant cell (temporal) arteritis: involvement of the vertebral and internal carotid arteries. Mayo Clin Proc 1998; 73: 444-446.

6.4.2, 6.4.3 Kopfschmerz zurückzuführen auf eine primäre oder sekundäre Vaskulitis des ZNS

Calabrese LH, Furlan AH, Gragg LA, Ropos TH. Primary angiitis of the central nervous system : diagnostic criteria and clinical approach. Cleve J Med 1992: 59: 293-306.

Calabrese LH, Duna GF, Lie JT. Vasculitis in the central nervous system ; Arthritis Rheum 1997; 40: 1189-1201.

Hajj-Ali RA, Singhal AB, Benseler S, Molloy E and Calabrese LH. Primary angiitis of the CNS. Lancet Neurol 2011; 10: 561-572.

Harris KG, Tran DD, Sickels WJ, Cornell SH, Yuh WTC. Diagnosing intracranial vasculitis: the roles or MR and angiography. Am J Neuroradiol 1994; 15: $317-330$.

Kumar R, Wijdicks EFM, Brown RD, Parisi JE, Hammond CA. Isolated angiitis of the CNS presenting as subarachnoid haemorrhage. J Neurol Neurosurg Psych 1997; 62: 649-651.

Lie JT. Primary (granulomatous) angiitis of the central nervous system : a clinicopathologic analysis of 15 new cases and a review of the literature. Hum Pathol 1992; 23: 164-171.

Moore PM. Vasculitis of the central nervous system. Semin Neurol 1994; 14: 313-319.

Salvarani C, Brown RD, Jr., Calamia KT, Christianson TJ, Weigand SD, Miller DV et al. Primary central nervous system vasculitis: analysis of 101 patients. Ann Neurol 2007; 62; 442-451.

Savage COS, Harper L, Cockwell P, Adu D, Howie AJ. ABC of arterial and vascular disease: vasculitis. BMJ 2000; 320: 1325-1328. 
6.5.1 Kopf-, Gesichts- oder Halsschmerz zurückzuführen auf eine arterielle Dissektion

Arnold M, Cumurciuc R, Stapf C, Favrole P, Berthet K and Bousser MG. Pain as the only symptom of cervical artery dissection. J Neurol Neurosurg Psychiat 2006; 77; 1021-1024.

Biousse V, D’Anglejan-Chatillon J, Touboul PJ, Amarenco P, Bousser MG. Time course of symptoms in extracranial carotid artery dissections. A series of 80 patients. Stroke 1995; 26: 235-239.

Debette S, Leys D. Cervical-artery dissections: predisposing factors, diagnosis, and outcome. Lancet-Neurol 2009; 8: 668-678.

Fisher CM. The headache and pain of spontaneous carotid dissection. Headache 1982; 22: 60-65.

Guillon B, Lévy C, Bousser MG.. Internal carotid artery dissection: an update. J Neurol Sci 1998; 153: 146-158.

Nakatomi H, Nagata K, Kawamoto S, Shiokawa Y. Ruptured dissecting aneurysm as a cause of subarachnoid hemorrhage of unverified etiology. Stroke 1997; 28: 1278-1282.

Ramadan NM, Tietjen GE, Levine SR, Welch KMA. Scintillating scotomata associated with internal carotid artery dissection: report of three cases. Neurology 1991; 41: 1084-1087.

Schytz HW, Ashina M, Magyari M, Larsen VA, Olesen J, Iversen HK. Acute headache and persistent Kopfschmerz zurückzuführen aufcervical artery dissection: Field testing of ICHD-III beta. Cephalalgia 2014; 34: 712-716.

Silbert PL, Mokri B, Schievink WI. Headache and neck pain in spontaneous internal carotid and verterbral artery dissections. Neurology 1995; 45 : 1517-1522.

Sturzenegger M. Headache and neck pain. The warning symptoms of vertebral artery dissection. Headache 1994; 34: 187-193.

Tzourio C, Benslamia L, Guillon B, Aïdi S, Bertrand M, Berthet K, Bousser MG. Migraine and the risk of cervical artery dissection: a case-control study. Neurology 2002; 59: 435-437.

\subsubsection{Kopfschmerz zurückzuführen auf eine Endarteriektomie}

Breen JC, Caplan LR, DeWitt LD, Belkin M, Mackey WC, Donnell TP. Brain edema after carotid surgery. Neurology 1996; 46: 175-181.

De Marinis M, Zaccaria A, Faraglia V, Fiorani P, Maira G, Agnoli A. Post endarterectomy headache and the role of the oculo-sympathetic system. J Neurol Neurosurg Psychiat 1991; 54: 314-317.

Ille O, Woimant F, Pruna A, Corabianu O, Idatte JM, Haquenau M. Hypertensive encephalopathy after bilateral carotid endarterectomy. Stroke 1995; 26: 488-491.

Leviton A, Caplan L, Salzman E. Severe headache after carotid endarterectomy. Headache 1975; 15: 207-209.

Tehindrazanarivelo A, Lutz G, Petitjean C, Bousser MG. Headache following carotid endarterectomy: a prospective study. Cephalalgia 1991; 11 suppl 11: 353 .

6.5.3 Kopfschmerz zurückzuführen auf eine Carotis- oder VertebralisAngioplastie oder Stentimplantation

Brott TG, Howard G, Roubin GS, Meschia JF, Mackey A, Brooks W, Moore WS, Hill MD, Mantese VA, Clark WM, Timaran CH, Heck D, Leimgruber PP, Sheffet AJ, Howard V], Chaturvedi S, Lal BK, Voeks JH, Hobson RW 2nd; CREST Investigators. Long-term results of stenting versus endarterectomy for carotid-artery stenosis. N Engl J Med 2016; 374: 1021-1031.

Gündüz A, Göksan B, Koçer N, Karaali-Savrun F. Headache in carotid artery stenting and angiography. Headache 2012; 52: 544-549.

McCabe DJH, Brown MM, Clifton A. Fatal cerebral reperfusion hemorrhage after carotid stenting. Stroke 1999; 30: 2483-2486.

Munari LM, Belloni G, Moschini L, Mauro A, Pezzuoli G, Porta M. Carotid pain during percutaneous angioplasty. Pathophysiology and clinical features. Cephalalgia 1994; 14: 127-131.
Rosenfield K, Matsumura JS, Chaturvedi S, Riles T, Ansel GM, Metzger DC, Wechsler L, Jaff MR, Gray W; ACT I Investigators. Randomized trial of stent versus surgery for asymptomatic carotid stenosis. N Engl J Med 2016; 374: 1011-1020.

Schoser BG, Heesen C, Eckert B, Thie A.. Cerebral hyperperfusion injury after percutaneous transluminal angioplasty of extracranial arteries. J Neurol 1997; 244: 101-104.

\subsubsection{Kopfschmerz zurückzuführen auf eine Hirnvenenthrombose}

Agrawal K, Burger K, Rothrock JF. Cerebral sinus thrombosis. Headache. 2016; 56: 1380-1389.

Aidi S, Chaunu MP, Biousse V, Bousser MG. Changing pattern of headache pointing to cerebral venous thrombosis after lumbar puncture and intra venous high dose cortico-steroids. Headache 1999; 39: 559-564.

Biousse V, Ameri A, Bousser MG. Isolated intracranial hypertension as the only sign of cerebral venous thrombosis. Neurology 1999; 53 : 1537-1542.

Bousser MG, Ferro JM. Cerebral venous thrombosis: an update. Lancet-Neurol 2007; 6; 162-170.

Cumurciuc R, Crassard I, Sarov M, Valade D and Bousser MG. Headache as the only neurological sign of cerebral venous thrombosis: a series of 17 cases. J Neurol Neurosurg Psychiat 2005; 76; 1084-1087.

De Bruijn SFTM, Stam J, Kappelle LJ for CVST study group. Thunderclap headache as first symptom of cerebral venous sinus thrombosis. Lancet 1996; 348: 1623-1625.

Leker RR, Steiner I. Features of dural sinus thrombosis simulating pseudotumor cerebri. Eur J Neurol 1999; 6: 601-604.

Newman DS, Levine SR, Curtis VL, Welch KMA : Migraine like visual phenomena associated with cerebral venous thrombosis. Headache 1989; 29: 82-85.

Wasay M, Kojan S, Dai Al, Bobustuc G and Sheikh Z. Headache in Cerebral Venous Thrombosis: incidence, pattern and location in 200 consecutive patients. J Headache Pain 2011; 11; 137-139.

\subsubsection{Kopfschmerz zurückzuführen auf Stentimplantation eines} venösen Hirnsinus

Lenck S, Vallée F, Labeyrie MA, Touitou V, Saint-Maurice JP, Guillonnet A, Tantot A, Crassard I, Bernat AL, Houdart E. Stenting of the lateral sinus in idiopathic intracranial hypertension according to the type of stenosis. Neurosurgery 2017; 80: 393-400.

\subsubsection{Kopfschmerz zurückzuführen auf einen intrakraniellen end-} arteriellen Eingriff

Beekman R, Nijssen PC, van Rooij WJ, Wijnalda D. Migraine with aura after intracranial endovascular procedures. Headache 2001; 41: 410-413.

Choi KS, Lee JH, Yi HJ, Chun HJ, Lee YJ, Kim DW. Incidence and risk factors of postoperative headache after endovascular coil embolization of unruptured intracranial aneurysms. Acta Neurochir (Wien) 2014; 156: $1281-1287$.

Gil-Gouveia R, Fernandes Sousa R, Lopes L, Campos 」 and Pavao Martins I. Headaches during angiography and endovascular procedures. J Neurol 2007; 254; 591-596.

Martins IP, Baeta E, Paiva T, Campos J, Gomes L. Headaches during intracranial endovascular procedures: a possible model for vascular headache. Headache 1993; 23: 227-233.

Nichols FT, Mawad M, Mohr JP, Hilal S, Adams RJ. Focal headache during balloon inflation in the vertebral and basilar arteries. Headache 1993; 33: 87-89.

Nichols FT, Mawad M, Mohr JP, Stein B, Hilal S, Michelsen W]. Focal headache during balloon inflation in the internal carotid and middle cerebral arteries. Stroke 1990; 21: 555-559. 


\subsubsection{Kopfschmerz zurückzuführen auf Angiographie}

Aktan Ç, Özgür Ö, Sindel T, Dora B. Characteristics of headache during and after digital substraction angiography: A critical re-appraisal of the ICHD-3 criteria. Cephalalgia 2017; 37: 1074-1081.

Gil-Gouveia RS, Sousa RF, Lopes L, Campos J, Martins IP. Post-Kopfschmerz zurückzuführen auf Angiographies. J Headache Pain 2008; 9: 327-330.

Gündüz A, Göksan B, Koçer N, Karaali-Savrun F. Headache in carotid artery stenting and angiography. Headache 2012; 52: 544-549.

Ramadan NM, Gilkey SJ, Mitchell M, Sawaya KL, Mitsias P. PostKopfschmerz zurückzuführen auf Angiographie. Headache 1995;35: 21-24.

Shuaib A, Hachinski VC. Migraine and the risks from angiography. Arch Neurol 1988; 45: 911-912.

6.7.3 Kopfschmerz zurückzuführen auf ein reversibles zerebrales Vasokonstriktionsyndrom (RCVS)

Call GK, Fleming MC, Sealfon S, Levine H, Kistler JP, Fisher CM. Reversible cerebral segmental vasoconstriction. Stroke 1988; 19: 1159-1170.

Calabrese LH, Dodick DW, Schwedt T] and Singhal AB. Narrative review: reversible cerebral vasoconstriction syndromes. Ann Intern Med. 2007; $146 ; 34-44$.

Chen SP, Fuh JL, Lirng JF, Chang FC and Wang SJ. Recurrent primary thunderclap headache and benign CNS angiopathy: spectra of the same disorder? Neurology 2006; 67; 2164-2169.

Chen SP, Fuh JL, Wang SJ, Chang FC, Lirng JF, Fang YC et al. Magnetic resonance angiography in reversible cerebral vasoconstriction syndromes. Ann Neurol 2010; 67; 648-656.

Ducros A, Boukobza M, Porcher R, Sarov M, Valade D and Bousser MG. The clinical and radiological spectrum of reversible cerebral vasoconstriction syndrome. A prospective series of 67 patients. Brain. 2007; 130; 3091-3101.

Ducros A, Bousser MG. Thunderclap headache. BMJ 2012; 345: e8557.

Ducros A, Fiedler U, Porcher R, Boukobza M, Stapf C, Bousser MG. Hemorrhagic manifestations of reversible cerebral vasoconstriction syndrome. Frequency, features, and risk factors. Stroke 2010; 41:2505-2511

Dodick DW, Brown RD, Britton JW, Huston J. Non aneurysmal thunderclap headache with diffuse, multifocal segmental and reversible vasospasm. Cephalalgia 1999; 19: 118-123.

John S, Singhal AB, Calabrese L, Uchino K, Hammad T, Tepper S, Stillman M, Mills B, Thankachan T, Hajj-Ali RA. Long-term outcomes after reversible cerebral vasoconstriction syndrome. Cephalalgia 2016; 36: 387-394.

Singhal AB, Hajj-Ali RA, Topcuoglu MA, Fok J, Bena J, Yang D et al. Reversible cerebral vasoconstriction syndromes: analysis of 139 cases. Arch Neurol 2011; 68; 1005-1012.

\subsubsection{Kopfschmerz zurückzuführen auf eine arterielle Dissektion}

Debette S, Compter A, Labeyrie MA, Uyttenboogaart M, Metso TM, Majersik JJ, et al. Epidemiology, pathophysiology, diagnosis, and management of intracranial artery dissection. Lancet Neurol 2015; 14: 640-654.

Dlamini N, Freeman JL, Mackay MT, Hawkins C, Shroff M, Fullerton HJ et al. Intracranial dissection mimicking transient cerebral arteriopathy in childhood arterial ischemic stroke. J Child Neurol 2011; 26; 12031206.

Kim JG, Choi JY, Kim SU, Jung JM, Kwon DY, Park MH, Oh K. Headache characteristics of uncomplicated intracranial vertebral artery dissection and validation of ICHD-3 beta diagnostic criteria for Kopfschmerz zurückzuführen aufintracranial artery dissection. Cephalalgia 2015; 35: 516-526.

Sharif AA, Remley KB, Clark HB. Middle cerebral artery dissection. A clinicopathologic study. Neurology 1995; 45: 1929-1231.
Szatmary Z, Boukobza M, Vahedi K, Stapf C, Houdart E and Bousser MG. Orgasmic headache and middle cerebral artery dissection. J Neurol Neurosurg Psychiat 2006; 77; 693-694.

6.8.1 Kopfschmerz zurückzuführen auf eine zerebrale autosomal dominante Arteriopathie mit subkortikalen Infarkten und Leukenzephalopathie(CADASIL)

Chabriat H, Vahedi K, Iba-Zizen MT, Joutel A, Nibbio A, Nagy TG, Krebs MO, Julien J, Dubois B, Ducrocq X, Levasseur M, Homeyer P, Mas JL, Lyon-Caen O, Tournier-Lasserve E, Bousser MG. Clinical spectrum of CADASIL: a study of 7 families. Lancet 1995; 346: 934-939.

Choi JC1, Song SK, Lee JS, Kang SY, Kang JH. Headache among CADASIL patients with R544C mutation: prevalence, characteristics, and associations. Cephalalgia 2014; 34: 22-28.

Guey S, Mawet J, Hervé D, Duering M, Godin O, Jouvent E, Opherk C, Alili N, Dichgans M, Chabriat $\mathrm{H}$. Prevalence and characteristics of migraine in CADASIL. Cephalalgia 2016; 36: 1038-1047.

Joutel A, Corpechot C, Ducros A, Katayoun V, Chabriat H, Mouton P, Alamowitch S, Domenga V, Cécillion M, Maréchal E, Maciazek J, Vayssière C, Cruaud C, Cabanis EA, Ruchoux MM, Weissenbach J, Bacxh JF, Bousser MG, Tournier-Lasserve E. "Notch 3" mutations in CADASIL, a hereditary adult-onset condition causing stroke and dementia. Nature 1996; 383: 707-710.

Vahedi K, Chabriat H, Levy C, Joutel A, Tournier-Lasserve E, Bousser MG. Migraine with aura and brain magnetic resonance imaging abnormalities in patients with CADASIL. Arch Neurol 2004; 61: 1237-1240.

6.8.2 Kopfschmerz zurückzuführen auf eine mitochondriale Enzephalopathie, Laktatazidose und Schlaganfall-ähnlichen Episoden (MELAS)

Klopstock A, May P, Siebel E, Papagiannuli E, Diener NC, Heichmann H. Mitochondrial DNA in migraine with aura. Neurology 1996; 46: 1735-1738.

Koo B, Becker L, Chuang S, Merante F, Robinson BH, Mac Gregor D, Tein I, Ho VB, McGreal DA, Wherrett JR, Logan WJ. Mitochondrial encephalomyopathy, lactic acidosis, stroke-like episodes (MELAS): clinical, radiological, pathological and genetic observations. Ann Neurol 1993; 34: 25-32.

Ojaimi J, Katsabanis S, Bower S, Quigley A, Byrne E. Mitochondrial DNA in stroke and migraine with aura. Cerebrovasc Dis 1998; 8: 102-106.

Pavlakis SG, Phillips PC, Di Mauro S, De Vivo DC, Rowland P. Mitochondrial myopathy, encephalopathy, lactic acidosis and stroke-like episodes: a distinct clinical syndrome. Ann Neurol 1984; 16: 481-488.

\subsubsection{Kopfschmerz zurückzuführen auf eine Moyamoya-Angiopathie}

Bohara M, Sugata S, Nishimuta Y, Karki P, Nagayama T, Sakamoto S, Tokimura $\mathrm{H}$, Arita K. Effect of revascularization on headache associated with Moyamoya disease in pediatric patients. Hiroshima J Med Sci 2015; 64: $39-44$.

Kraemer M, Lee SI, Ayzenberg I, Schwitalla JC, DiehI RR, Berlit P, Bosche B, Katsarava Z, Obermann M. Headache in Caucasian patients with Moyamoya angiopathy - a systematic cohort study. Cephalalgia 2017; 37: 496-500.

Seol HJ, Wang KC, Kim SK, Hwang YS, Kim KJ, Cho BK. Headache in pediatric moyamoya disease: review of 204 consecutive cases. J Neurosurg 2005; 103 (5 Suppl): 439-442.

Zach V, Bezov D, Lipton RB, Ashina S. Headache associated with moyamoya disease: a case story and literature review. J Headache Pain 2010; 11: 79-82. 
6.8.4 Migräneartige Aura zurückzuführen auf eine zerebrale Amyloidangiopathie

Charidimou A, Peeters A, Fox Z, et al. Spectrum of transient focal neurological episodes in cerebral amyloid angiopathy: multicentre magnetic resonance imaging cohort study and meta-analysis. Stroke 2012; 43: 2324-2330.

Paterson RW, Uchino K, Emsley HC, et al. Recurrent stereotyped episodes in cerebral amyloid angiopathy: response to migraine prophylaxis in two patients. Cerebrovasc Dis Extra 2013; 3: 81-84.

Samanci B, Coban O, Baykan B. Late onset aura may herald cerebral amyloid angiopathy: A case report. Cephalalgia 2016; 36: 998-1001.

6.8.5 Kopfschmerz zurückzuführen auf ein retinales VaskulopathieSyndrom mit zerebraler Leukenzephalopathie und systemischen Manifestationen (RVCLSM)

Hottenga J], Vanmolkot KR, Kors EE, Kheradmand Kia S, de Jong PT, Haan J, et al. The 3p21.1-p21.3 hereditary vascular retinopathy locus increases the risk for Raynaud's phenomenon and migraine. Cephalalgia 2005; 25: $1168-1172$.

Richards A, van den Maagdenberg AM, Jen JC, Kavanagh D, Bertram P, Spitzer D, et al. C-terminal truncations in human 3'-5' DNA exonuclease TREX1 cause autosomal dominant retinal vasculopathy with cerebral leukodystrophy. Nature Genet 2007; 39: 1068-1070.

Terwindt GM, Haan J, Ophoff RA, Groenen SM, Storimans CW, Lanser JB, et al. Clinical and genetic analysis of a large Dutch family with autosoma dominant vascular retinopathy, migraine and Raynaud's phenomenon. Brain 1998; 121: 303-316.

Stam AH, Kothari PH, Shaikh A, Gschwendter A, Jen JC, Hodgkinson S, et al. Retinal vasculopathy with cerebral leukoencephalopathy and systemic manifestations. Brain 2016; 139: 2909-2922.

\subsubsection{Kopfschmerz zurückzuführen auf andere intrakranielle Gefäß-} störungen

Gould DB, Phalan FC, van Mil SE, Sundberg JP, Vahedi K, Massin P, et al. Role of COL4A1 in small-vessel disease and hemorrhagic stroke. NEJM 2006; 354:1489-1496.

Vahedi K, Boukobza M, Massin P, Gould DB, Tournier-Laserve E, Bousser MG. Clinical and brain MRI follow-up study of a family with COL4A1 mutation. Neurology 2007; 69: 1564-1568.

Vahedi K, Massin P, Guichard JP, Miocque S, Polivka M, Goutières F, et al. Hereditary infantile hemiparesis, retinal arteriolar tortuosity, and leukoencephalopathy. Neurology 2003; 60: 57-63.

\subsection{Kopfschmerz zurückzuführen auf einen Hypophyseninfarkt}

Carral F. Pituitary apoplexy. Arch Neurol 2001; 58: 1143-1144.

Chakeres DW, Curtin A, Ford G. Magnetic resonance imaging of pituitary and parasellar abnormalities. Radiol Clin North Am 1989; 27: 265-281.

Da Motta LA, de Mello PA, de Lacerda CM, Neto AP, Da Motta LD, Filho MF. Pituitary apoplexy. Clinical course, endocrine evaluations and treatment analysis. J Neurosurg Sci 1991; 43: 25-36.

Dodick DW, Wijdicks EFM.. Pituitary apoplexy presenting as thunderclap headache. Neurology 1998; 50: 1510-1511.

Hernandez A, Angeles Del Real M, Aguirre M, Vaamonde J, Gudin M, Ibanez R. Pituitary apoplexy: a transient benign presentation mimicking with subarachnoid hemorrhage with negative angiography. Eur J Neurol 1998; 5: 499-501.

Lee CC, Cho AS, Carter WA. Emergency department presentation of pituitary apoplexy. Am J Emerg Med 2000; 18: 328-331.

McFadzean RM, Doyle D, Rampling R, Teasdale E, Teasdale G. Pituitary apoplexy and its effect on vision. Neurosurgery 1991; 29: 669-675.

\section{Kopfschmerz zurückzuführen auf nichtvaskuläre intrakranielle Störungen}

\begin{tabular}{l|l}
7.1 & Kopfschmerz zurückzuführen auf eine Liquordrucksteigerung
\end{tabular}

7.1.1 Kopfschmerz zurückzuführen auf eine idiopathische intrakranielle Hypertension (IIH)

7.1.2 Kopfschmerz zurückzuführen auf eine sekundäre Liquordrucksteigerung metabolischer, toxischer oder hormoneller Genese

7.1.3 Kopfschmerz zurückzuführen auf eine sekundäre Liquordrucksteigerung infolge einer Chromosomenstörung

7.1.4 Kopfschmerz zurückzuführen auf eine sekundäre Liquordrucksteigerung bei Hydrozephalus

7.2 Kopfschmerz zurückzuführen auf einen Liquorunterdruck

7.2.1 Postpunktioneller Kopfschmerz

7.2.2 Kopfschmerz bei Liquorfistel

7.2.3 Kopfschmerz zurückzuführen auf ein spontanes Liquorunterdrucksyndrom

7.3 Kopfschmerz zurückzuführen auf nicht-infektiöse entzündliche Erkrankungen

7.3.1 Kopfschmerz zurückzuführen auf eine Neurosarkoidose

7.3.2 Kopfschmerz zurückzuführen auf eine aseptische (nicht-infektiöse) Meningitis

7.3.3 Kopfschmerz zurückzuführen auf eine andere nicht-infektiöse entzündliche intrakranielle Erkrankung

7.3.4 Kopfschmerz zurückzuführen auf eine lymphozytäre Hypophysitis

7.3.5 Syndrom der vorübergehenden Kopfschmerzen mit neurologischen Defiziten und Liquorlymphozytose (HaNDL; engl. Transient headache and neurologic deficits with cerebrospinal fluid lymphocytosis)

7.4 Kopfschmerz zurückzuführen auf ein intrakranielles Neoplasma

7.4.1 Kopfschmerz zurückzuführen auf ein intrakranielles Neoplasma

7.4.1.1 Kopfschmerz zurückzuführen auf eine Kolloidzyste des dritten Hirnventrikels

7.4.2 Kopfschmerz zurückzuführen auf eine Meningeosis carcinomatosa

7.4.3 Kopfschmerz zurückzuführen auf eine hypothalamische oder hypophysäre Über- oder Unterfunktion

7.5 Kopfschmerz zurückzuführen auf eine intrathekale Injektion

7.6 Kopfschmerz zurückzuführen auf einen epileptischen Anfall

7.6.1 Iktaler Kopfschmerz bei Epilepsie

7.6.2 Postiktaler Kopfschmerz

7.7 Kopfschmerz zurückzuführen auf eine Chiari-Malformation Typ I(CM1)

\begin{tabular}{l|l}
7.8 & Kopfschmerz zurückzuführen auf eine andere nichtvaskuläre
\end{tabular} intrakranielle Störung

An anderer Stelle kodiert Primärer und/oder sekundärer Kopfschmerz?

Für Kapitel 7. Kopfschmerz zurückzuführen auf nichtvaskuläre intrakranielle Störungen gelten die allgemeinen Regeln für die kausale Zuordnung zu einer anderen Erkrankung.

Tritt ein neuer Kopfschmerz erstmals in engem zeitlichen Zusammenhang mit einer nichtvaskulären Störung auf, sollte der Kopfschmerz als sekundärer Kopfschmerz zurückzuführen auf diese 
intrakranielle Störung kodiert werden. Dies ist auch der Fall, wenn der Kopfschmerz das klinische Bild eines primären Kopfschmerzes zeigt, der in Teil 1 der ICHD-3 klassifiziert wird.

Wenn aber ein vorbestehender Kopfschmerz, der das Bild einer primären Kopfschmerzerkrankung aufweist, in engem zeitlichen Zusammenhang mit einer nichtvaskulären intrakraniellen Störung chronisch wird oder sich deutlich verschlechtert (üblicherweise definiert als eine mindestens zweifache Steigerung der Häufigkeit und/oder Schwere), dann sollte sowohl die vorbestehende primäre Kopfschmerzdiagnose erhalten als auch die Diagnose 7. Kopfschmerz zurückzuführen auf nichtvaskuläre intrakranielle Störungen (oder eines Typs oder Subtyps hiervon) vergeben werden, sofern gute Hinweise dafür bestehen, dass die Erkrankung Kopfschmerzen verursachen kann.

\section{Einleitung}

Dieses Kapitel beschreibt Kopfschmerzen, die durch Veränderungen des intrakraniellen Druckes verursacht werden. Dabei können sowohl ein Liquorüberdruck als auch ein Liquorunterdruck Kopfschmerzen hervorrufen. Andere Kopfschmerzursachen sind nichtinfektiöse entzündliche Erkrankungen, intrakranielle Neoplasmen, epileptische Anfälle und Raritäten wie intrathekale Injektionen, Chiari Malformation Typ I und andere nichtvaskuläre intrakranielle Erkrankungen.

Im Vergleich zu den primären Kopfschmerzen existieren für diese Kopfschmerztypen nur wenige epidemiologische Studien. Kontrollierte Studien zur Therapie fehlen praktisch ganz.

Für Kopfschmerzen, die auf eine der hier aufgeführten nichtvaskulären intrakraniellen Erkrankungen zurückzuführen sind, umfassen die diagnostischen Kriterien nach Möglichkeit folgende Punkte:

A. Kopfschmerz, der Kriterium C erfüllt

B. Es wurde eine nichtvaskuläre intrakranielle Erkrankung diagnostiziert, von der bekannt ist, dass sie Kopfschmerzen auslösen kann

C. Ein kausaler Zusammenhang kann durch mindestens zwei der folgenden Kriterien gezeigt werden:

1. Der Kopfschmerz hat sich in zeitlichem Zusammenhang zum Beginn der nicht-vaskulären intrakraniellen Störung entwickelt oder führte zu ihrer Entdeckung

2. Einer oder beide der folgenden Punkte sind erfüllt:

a) Der Kopfschmerz hat sich gleichzeitig mit der Verschlechterung der nichtvaskulären intrakraniellen Störung deutlich verschlechtert

b) Der Kopfschmerz hat sich gleichzeitig mit der Besserung der nichtvaskulären intrakraniellen Störung deutlich gebessert

3. Der Kopfschmerz weist Merkmale auf, die typisch für die nichtvaskuläre intrakranielle Störung sind

4. Der ursächliche Zusammenhang ist anderweitig belegt D. Nicht besser erklärt durch eine andere ICHD-3-Diagnose. Ein Kopfschmerz, der länger als einen Monat nach erfolgreicher Behandlung oder spontaner Remission einer intrakraniellen Erkrankung fortbesteht, beruht in der Regel auf anderen Mechanismen. Chronische Kopfschmerzen, die über mehr als drei Monate nach Behandlung oder Remission einer intrakraniellen Störung persistieren, werden im Anhang für Forschungszwecke genauer definiert.
Derartige Kopfschmerzen existieren, sind aber bis jetzt nur wenig untersucht und die Aufnahme in den Anhang soll die weitere Erforschung dieser Kopfschmerzen und ihrer Mechanismen stimulieren.

\subsection{Kopfschmerz zurückzuführen auf eine Liquordrucksteigerung}

An anderer Stelle kodiert: Ein Kopfschmerz zurückzuführen auf einen erhöhten intrakraniellen Druck oder einen Hydrozephalus verursacht durch ein intrakranielles Neoplasma wird als 7.4.1 Kopfschmerz zurückzuführen auf ein intrakranielles Neoplasma kodiert.

\section{Beschreibung:}

Kopfschmerz verursacht durch eine Liquordrucksteigerung, üblicherweise in Begleitung anderer Symptome und/oder klinischer Zeichen einer intrakraniellen Druckerhöhung.

\section{Diagnostische Kriterien:}

A. Neu aufgetretener Kopfschmerz oder deutliche Verschlechterung1 eines vorbestehenden Kopfschmerzes, der das Kriterium C erfüllt

B. Es wurde eine intrakranielle Hypertension diagnostiziert, die die beiden folgenden Kriterien erfüllt:

1. Der Liquordruck übersteigt 250 mm H2O (bzw. bei adipösen Kindern 280 mm H2O)2

2. Unauffällige Liquorchemie

C. Ein kausaler Zusammenhang kann durch mindestens zwei der folgenden Kriterien gezeigt werden:

1. Der Kopfschmerz hat sich in einem zeitlichen Zusammenhang zur intrakraniellen Druckerhöhung entwickelt oder führte zu ihrer Entdeckung

2. Der Kopfschmerz verschwindet durch Reduktion der intrakraniellen Druckerhöhung

3. Papillenödem

D. Nicht besser erklärt durch eine andere ICHD-3-Diagnose3.

\section{Anmerkung:}

1. Eine „deutliche Verschlechterung“ impliziert eine mindestens zweifache Steigerung der Häufigkeit und/oder Intensität in Übereinstimmung mit der allgemeinen Regel zur Unterscheidung sekundärer von primären Kopfschmerzen.

2. Zu diagnostischen Zwecken sollte der Liquordruck ohne Behandlung zur Senkung des intrakraniellen Drucks gemessen werden. Der Liquordruck kann durch Lumbalpunktion in Seitenlage ohne Beruhigungsmittel gemessen werden oder durch epidurales oder intraventrikuläres Druckmonitoring. Da der Liquordruck im Tagesverlauf schwankt, sagt eine einmalige Messung am Tag vielleicht nichts über den durchschnittlichen Liquordruck über 24 Stunden aus: zur Diagnosesicherung kann ein längeres lumbales oder intraventrikuläres Druckmonitoring erforderlich sein.

3. Ausschluss eines intrakraniellen Neoplasmas.

\section{Kommentar:}

Ein 7.1 Kopfschmerz zurückzuführen auf eine Liquordrucksteigerung ist eine Kopfschmerzdiagnose. Falls diese Diagnose gestellt 
wird, sollte sie nur vorläufig sein, bis die Ursache für den erhöhten Liquordruck ermittelt ist; der Kopfschmerz sollte dann unter den entsprechenden Subtyp umkodiert werden.

\subsubsection{Kopfschmerz zurückzuführen auf eine idio- pathische intrakranielle Hypertension (IIH)}

Früher verwendete Begriffe Kopfschmerz zurückzuführen auf eine benigne intrakranielle Druckerhöhung (BIH); Pseudotumor cerebri; meningealer Hydrops; seröse Meningitis.

\section{Beschreibung:}

Neu aufgetretener Kopfschmerz oder eine deutliche Verschlechterung eines vorbestehenden Kopfschmerzes, verursacht durch und begleitet von anderen Symptomen und/oder klinischen und/oder in der Bildgebung nachgewiesenen Zeichen einer idiopathischen intrakraniellen Hypertension (IIH), mit typischen auf eine IIH verweisenden Merkmalen.

\section{Diagnostische Kriterien:}

A. Neuer Kopfschmerz oder eine deutliche Verschlechterung1 eines vorbestehenden Kopfschmerzes, der das Kriterium C erfüllt

B. Beide der folgenden Punkte sind erfüllt:

1. Es wurde eine idiopathische intrakranielle Hypertension (IIH) diagnostiziert2

2. Der Liquordruck übersteigt $250 \mathrm{~mm} \mathrm{H2O}$ (oder bei adipösen Kindern 280 mm H2O)3

C. Einer oder beide der folgenden Punkte sind erfüllt:

1. Der Kopfschmerz hat sich in einem zeitlichen Zusammenhang zu der IIH entwickelt oder deutlich verschlechtert1 oder führte zu ihrer Entdeckung

2. Der Kopfschmerz tritt in Begleitung von einem oder beiden der folgenden Punkte auf:

a) pulsierender Tinnitus

b) Papillenödem4

D. Nicht besser erklärt durch eine andere ICHD-3-Diagnose5,6.

Anmerkung:

1. Eine „deutliche Verschlechterung“ impliziert eine mindestens zweifache Steigerung der Häufigkeit und/oder Intensität in Übereinstimmung mit der allgemeinen Regel zur Unterscheidung sekundärer von primären Kopfschmerzen.

2. Eine IIH sollte bei Patienten mit verändertem psychomentalen Status mit Vorsicht diagnostiziert werden.

3. Zu diagnostischen Zwecken sollte der Liquordruck ohne Behandlung zur Senkung des intrakraniellen Drucks gemessen werden. Der Liquordruck kann durch Lumbalpunktion in Seitenlage ohne Beruhigungsmittel gemessen werden oder durch epidurales oder intraventrikuläres Druckmonitoring. Da der Liquordruck im Tagesverlauf schwankt, sagt eine einmalige Messung am Tag vielleicht nichts über den durchschnittlichen Liquordruck über 24 Stunden aus: zur Diagnosesicherung kann ein längeres lumbales oder intraventrikuläres Druckmonitoring erforderlich sein.

4. Ein Papillenödem ist von einem Pseudopapillenödem oder einem Sehnervenkopfödem abzugrenzen. Die Mehrzahl der Patienten mit einer IIH weisen ein Papillenödem auf, und bei
Patienten ohne dieses Zeichen ist bei der Diagnose Vorsicht geboten.

5. Ein 7.1.1 Kopfschmerz zurückzuführen auf eine idiopathische intrakranielle Hypertension kann ähnliche Symptome wie primäre Kopfschmerzen aufweisen, vor allem eine 1.3 chronische Migräne und ein 2.3 chronischer Kopfschmerz vom Spannungstyp; andererseits existieren diese Störungen häufig gleichzeitig mit einer IIH.

6. Ein 8.2 Kopfschmerz zurückzuführen auf einen Medikamentenübergebrauch sollte bei Patienten ohne Papillenödem, Abducensparese oder die charakteristischen in der zerebralen Bildgebung nachgewiesenen Zeichen einer IIH ausgeschlossen werden.

\section{Kommentar:}

Eine idiopathische intrakranielle Hypertension (IIH) kommt am häufigsten bei übergewichtigen Frauen im gebärfähigen Alter vor (bei denen auch eine falsch diagnostizierte IIH am wahrscheinlichsten ist).

Bei einem 7.1.1 Kopfschmerz zurückzuführen auf eine idiopathische intrakranielle Hypertension fehlen spezifische Merkmale und dieser ähnelte im Allgemeinen einer 1. Migräne oder einem 2. Kopfschmerz vom Spannungstyp. Ein tägliches Auftreten ist für die Stellung der Diagnose nicht erforderlich.

Eine Kopfschmerzlinderung nach Ablassen von Liquor unterstützt die Diagnose, hat jedoch keinen eigenständigen diagnostischen Wert: man beobachtet diese auch bei Patienten mit anderen Kopfschmerztypen (Sensitivität $72 \%$ und Spezifizität 77 \% für einen 7.1.1 Kopfschmerz zurückzuführen auf eine idiopathische intrakranielle Hypertension).

Zu den Befunden der zerebralen Bildgebung, die mit der Diagnose einer IIH übereinstimmen, gehören das Empty-Sella-Syndrom (ESS), eine Erweiterung des perioptischen Subarachnoidalraums, eine Abflachung der hinteren Skleren, eine Vorwölbung der Sehnervpapillen in den Glaskörper und eine quer verlaufende zerebrovenöse Sinusstenose.

\subsubsection{Kopfschmerz zurückzuführen auf eine sekun- däre Liquordrucksteigerung metabolischer, toxischer oder hormoneller Genese}

An anderer Stelle kodiert: Ein Kopfschmerz zurückzuführen auf einen erhöhten intrakraniellen Druck als Folge eines Kopftraumas, einer vaskulären Störung oder intrakraniellen Infektion wird entsprechend dieser Erkrankungen kodiert. Ein Kopfschmerz zurückzuführen auf einen erhöhten intrakraniellen Druck als unerwünschte Wirkung einer Medikation wird unter 8.1.10 Kopfschmerz zurückzuführen auf eine nicht gegen Kopfschmerz eingesetzte Dauermedikation kodiert.

\section{Beschreibung:}

Ein Kopfschmerz, der durch eine intrakranielle Hypertension als Folge einer Reihe von systemischen Störungen und begleitet von anderen Symptomen und/oder klinischen und/oder in der zerebralen Bildgebung nachgewiesenen Zeichen sowohl für die intrakranielle Hypertension wie auch für die ursächliche Grunderkrankung hervorgerufen wird. In der Regel verschwindet dieser mit der Beseitigung der systemischen Störung. 
Diagnostische Kriterien:

A. Kopfschmerz, der die Kriterien für einen 7.1 Kopfschmerz zurückzuführen auf eine Liquordrucksteigerung und das Kriterium $C$ unten erfüllt

B. Die intrakranielle Hypertension ist auf eine metabolische, toxische oder hormonelle Störung zurückzuführen1

C. Ein kausaler Zusammenhang kann durch eines oder beide der folgenden Kriterien gezeigt werden:

1. Der Kopfschmerz hat sich in einem zeitlichen Zusammenhang zur Erhöhung des Liquordrucks entwickelt oder führte zu seiner Entdeckung

2. Einer oder beide der folgenden Punkte sind erfüllt:

a) der Kopfschmerz hat sich gleichzeitig mit einer Erhöhung des Liquordrucks deutlich verschlechtert

b) der Kopfschmerz hat sich gleichzeitig mit einer Senkung des Liquordrucks deutlich gebessert

D. Nicht besser erklärt durch eine andere ICHD-3-Diagnose.

\section{Anmerkung:}

1. Zu den potenziellen metabolischen, toxischen oder hormonellen Ursachen einer intrakraniellen Druckerhöhung gehören eine akute Leberinsuffizienz, eine Niereninsuffizienz, eine Hyperkapnie, eine akute hypertensive Krise, ein Reye-Syndrom, eine zerebrale venöse Sinusthrombose, eine Rechtsherzinsuffizienz, eine Reihe von Substanzen (darunter Schilddrüsenhormonersatz bei Kindern, all-trans-Retinsäure, Retinoide, Tetrazykline und Chlordecon), Vitamin-A-Toxizität und Kortikosteroid-Entzug.

\section{Kommentar:}

Die Absetzung der sich entsprechend auswirkenden Substanz oder die Behandlung der ursächlichen Grunderkrankung reicht unter Umständen nicht aus, um den erhöhten Liquordruck zu normalisieren; oft ist eine zusätzliche Behandlung erforderlich, um Kopfschmerzen und andere Symptome zu beseitigen und vor allem einem Sehverlust vorzubeugen.

\subsubsection{Kopfschmerz zurückzuführen auf eine sekundäre Liquordrucksteigerung infolge einer Chromosomenstörung}

\section{Beschreibung:}

Ein neuer Kopfschmerz oder eine deutliche Verschlechterung eines vorbestehenden Kopfschmerzes, der durch eine intrakranielle Hypertension als Folge einer Chromosomenstörung verursacht wird und in Begleitung anderer Symptome und/oder klinischer und/oder in der zerebralen Bildgebung nachgewiesener Zeichen sowohl für die intrakranielle Hypertension als auch für die zugrundeliegende Chromosomenstörung auftritt.

\section{Diagnostische Kriterien:}

A. Neuer Kopfschmerz oder eine deutliche Verschlechterung1 eines vorbestehenden Kopfschmerzes, der die Kriterien für einen 7.1 Kopfschmerz zurückzuführen auf eine Liquordrucksteigerung und Kriterium $C$ unten erfüllt

B. Die intrakranielle Hypertension wurde auf eine Chromosomenstörung zurückgeführt2
C. Ein kausaler Zusammenhang kann durch mindestens zwei der folgenden Kriterien gezeigt werden:

1. Der Kopfschmerz hat sich in einem zeitlichen Zusammenhang mit der intrakraniellen Druckerhöhung entwickelt oder führte zu ihrer Entdeckung

2. Der Kopfschmerz wird durch Reduzierung der intrakraniellen Druckerhöhung gelindert

3. Papillenödem

D. Nicht besser erklärt durch eine andere ICHD-3-Diagnose.

\section{Anmerkung:}

1. „Deutliche Verschlechterung“ impliziert eine mindestens zweifache Steigerung der Häufigkeit oder Intensität.

2. Zu den Chromosomenstörungen, die mit einer intrakraniellen Druckerhöhung assoziiert sind, gehören das Turner-Syndrom und das Down-Syndrom.

\subsubsection{Kopfschmerz zurückzuführen auf eine sekun- däre Liquordrucksteigerung bei Hydrozephalus}

Beschreibung:

Neu aufgetretener Kopfschmerz, der eine deutliche Verschlechterung eines vorbestehenden Kopfschmerzes verursacht durch eine intrakranielle Hypertension als Folge eines Hydrozephalus und in Begleitung anderer Symptome und/oder klinischer Zeichen eines erhöhten Liquordrucks oder eines Hydrozephalus.

\section{Diagnostische Kriterien:}

A. Neu aufgetretener Kopfschmerz oder eine deutliche Verschlechterung1 eines vorbestehenden Kopfschmerzes, der die Kritierien für einen 7.1 Kopfschmerz zurückzuführen auf eine Liquordrucksteigerung und das Kriterium $\mathrm{C}$ unten erfüllt

B. Die intrakranielle Hypertension wurde auf einen Hydrozephalus zurückgeführt

C. Ein kausaler Zusammenhang kann durch eines oder beide der folgenden Kriterien gezeigt werden:

1. Der Kopfschmerz hat sich in einem zeitlichen Zusammenhang mit der Entwicklung oder Verschlechterung der Liquordruckerhöhung entwickelt oder deutlich verschlechtert1 oder führte zu ihrer Entdeckung

2. Einer oder beide der folgenden Punkte sind erfüllt:

a) der Kopfschmerz hat sich gleichzeitig mit der Verschlechterung des Hydrozephalus deutlich verschlechtert

b) der Kopfschmerz hat sich gleichzeitig mit der Besserung des Hydrozephalus deutlich gebessert

D. Nicht besser erklärt durch eine andere ICHD-3-Diagnose.

\section{Anmerkung:}

1. Eine „deutliche Verschlechterung“ impliziert eine mindestens zweifache Steigerung der Häufigkeit und/oder Intensität in Übereinstimmung mit der allgemeinen Regel zur Unterscheidung sekundärer von primären Kopfschmerzen.

\section{Kommentar:}

Ein Normaldruckhydrozephalus verursacht in der Regel keine Kopfschmerzen; gelegentlich wird von einem leichten dumpfen Kopfschmerz berichtet. 


\subsection{Kopfschmerz zurückzuführen auf einen Liquorunterdruck}

\section{Beschreibung:}

Orthostatischer Kopfschmerz verursacht durch einen Liquorunterdruck (spontaner oder sekundärer Art) oder ein Liquorleck, üblicherweise begleitet von Nackenschmerzen, Tinnitus, Veränderungen des Hörens, Photophophie und/oder Übelkeit. Dieser verschwindet nach Normalisierung des Liquordrucks oder erfolgreicher Abdichtung des Liquorlecks.

\section{Diagnostische Kriterien:}

A. Ein beliebiger Kopfschmerz1, der das Kriterium C erfüllt

B. Einer oder beide der folgenden Punkte sind erfüllt:

1. Liquorunterdruck ( $<60 \mathrm{~mm} \mathrm{H2O}$ )

2. Nachweis eines Liquorunterdrucks durch bildgebende Verfahren2

C. Der Kopfschmerz hat sich in zeitlichem Zusammenhang mit dem Liquorunterdruck oder Liquorleck entwickelt oder führte zu seiner Entdeckung3

D. Nicht besser erklärt durch eine andere ICHD-3-Diagnose.

\section{Anmerkung:}

1. Ein 7.2 Kopfschmerz zurückzuführen auf einen Liquorunterdruck ist üblicherweise, aber nicht grundsätzlich orthostatischer Natur. Ein Kopfschmerz, der sich nach Aufsetzen oder Aufstehen deutlich verstärkt und/oder nach Hinlegen bessert, wird wahrscheinlich von einem Liquorunterdruck verursacht, allerdings eignet sich dies nicht als zuverlässiges diagnostisches Kriterium.

2. Die zerebrale Bildgebung zeigt ein Tieferrutschen des Gehirns im knöchernen Schädel („,brain sagging“) oder ein pachymeningeales Enhancement bzw. in der spinalen Bildgebung (spinales MRT oder MRT, CT oder digitale Subtraktionsmyelographie) wird extraduraler Liquor nachgewiesen.

3. Der Nachweis des ursächlichen Zusammenhangs besteht im zeitlichen Zusammenhang des Kopfschmerzbeginns mit der angenommenen Ursache und dem gleichzeitigen Ausschluss anderer Diagnosen.

\subsubsection{Postpunktioneller Kopfschmerz}

Früher verwendeter Begriff: Postpunktioneller Kopfschmerz.

\section{Beschreibung:}

Kopfschmerz, der innerhalb von 5 Tagen nach einer Lumbalpunktion auftritt, verursacht durch ein Liquorleck infolge der duralen Punktion. In der Regel tritt dieser in Begleitung von Nackensteifigkeit und/oder subjektiven Hörsymptomen auf. Er verschwindet spontan innerhalb von 2 Wochen oder nach Verschluss des Liquorlecks durch Setzen eines lumbalen autologen epiduralen Blutpflasters.

\section{Diagnostische Kriterien:}

A. Kopfschmerz, der die Kriterien für einen 7.2 Kopfschmerz zurückzuführen auf einen Liquorunterdruck und das Kriterium C unten erfüllt

B. Zustand nach duraler Punktion
C. Der Kopfschmerz hat sich innerhalb von 5 Tagen nach der duralen Punktion entwickelt

D. Nicht besser erklärt durch eine andere ICHD-3-Diagnose.

Kommentar:

In jüngerer Zeit wurden unabhängige Risikofaktoren für einen 7.2.1 postpunktionellen Kopfschmerz nachgewiesen: weibliches Geschlecht, Alter zwischen 31 und 50 Jahren, ein 7.2.1 postpunktioneller Kopfschmerz in der Vorgeschichte und eine Ausrichtung der abgeschrägten Kante der Kanüle waagerecht zur Längsachse der Wirbelsäule bei der Duralpunktion.

\subsubsection{Kopfschmerz bei Liquorfistel}

\section{Beschreibung:}

Orthostatischer Kopfschmerz, der nach einem Eingriff oder Trauma eintritt, der/das ein persistierendes Liquorleck nach sich zieht, welches zu einem Liquorunterdruck führt. Dieser verschwindet nach erfolgreichem Verschluss des Liquorlecks.

\section{Diagnostische Kriterien:}

A. Kopfschmerz, der die Kriterien für einen 7.2 Kopfschmerz zurückzuführen auf einen Liquorunterdruck und das Kriterium C unten erfüllt

B. Zustand nach Durchführung eines Eingriffs oder Eintritt eines Traumas, von dem bekannt ist, dass es mitunter ein persistierendes Liquorleck verursachen kann (Liquorfistel)

C. Der Kopfschmerz hat sich in zeitlichem Zusammenhang mit dem Eingriff oder Trauma entwickelt

D. Nicht besser erklärt durch eine andere ICHD-3-Diagnose.

\subsubsection{Kopfschmerz zurückzuführen auf ein spontanes Liquorunterdrucksyndrom}

Früher verwendete Begriffe Kopfschmerz zurückzuführen auf ein spontanes Liquorunterdrucksyndrom oder eine primäre intrakranielle Hypotension; Kopfschmerz infolge eines Liquormangels; hypoliquorrhoischer Kopfschmerz.

\section{Beschreibung:}

Orthostatischer Kopfschmerz infolge eines Liquorunterdrucks spontanen Ursprungs. Dieser wird üblicherweise von Nackensteifigkeit und subjektiven Hörsymptomen begleitet. Er verschwindet nach Normalisierung des Liquordrucks.

\section{Diagnostische Kriterien:}

A. Kopfschmerz, der die Kriterien für einen 7.2 Kopfschmerz zurückzuführen auf einen Liquorunterdruck und das Kriterium C unten erfüllt

B. Nichtvorliegen eines Eingriffs oder Traumas, von dem bekannt ist, dass er/es ein Liquorleck nach sich ziehen kann1

C. Der Kopfschmerz hat sich in einem zeitlichen Zusammenhang mit dem Auftreten eines Liquorunterdrucks oder Liquorlecks entwickelt oder führte zu deren Entdeckung2

D. Nicht besser erklärt durch eine andere ICHD-3-Diagnose.

Anmerkung:

1. Ein 7.2.3 Kopfschmerz zurückzuführen auf ein spontanes Liquorunterdrucksyndrom kann nicht bei Patienten diagnosti- 
ziert werden, bei denen innerhalb des Vormonats eine Durapunktion vorgenommen worden ist.

2. Bei Patienten mit positiven Anzeichen eines Liquorlecks im MRT etwa in Form eines duralen Kontrastmittel-Enhancements ist eine Durapunktion zur direkten Messung des Liquordrucks nicht erforderlich.

\section{Kommentar:}

Das Auftreten spontaner Liquorlecks wurde mit erblichen Bindegewebserkrankungen in Verbindung gebracht. Patienten mit einem Liquorleck sollten auf Bindegewebs- und vaskuläre Anomalien überprüft werden.

Zwar besteht bei einem 7.2.3 Kopfschmerz zurückzuführen auf ein spontanes Liquorunterdrucksyndrom in den meisten Fällen eine eindeutige orthostatische Komponente, diese ist vielleicht nicht so dramatisch oder unmittelbar wie bei einem 7.2.1 postpunktionellen Kopfschmerz. Demzufolge kann ein 7.2.3 Kopfschmerz zurückzuführen auf ein spontanes Liquorunterdrucksyndrom unmittelbar auftreten oder innerhalb von Sekunden nach der Einnahme einer aufrechten Haltung und nach dem Hinlegen schnell wieder verschwinden (innerhalb von 1 Minute), ähnlich wie beim 7.2.1 postpunktionellen Kopfschmerz, oder er zeigt eine verzögerte Reaktion auf eine orthostatische Veränderung, indem er sich Minuten oder Stunden nach der aufrechten Haltung verstärkt und sich nach dem Hinlegen innerhalb von Minuten oder Stunden bessert, wenngleich nicht verschwindet. Bei der Anamnese sollte ein orthostatischer Kopfschmerzcharakter beim anfänglichen Auftreten des Kopfschmerzes eruiert werden, da dieses Merkmal im Laufe der Zeit weniger offensichtlich werden kann.

Bei Patienten mit typischem orthostatischem Kopfschmerz ohne offenkundige Ursache sowie nach Ausschluss eines posturalen orthostatischen Tachykardiesyndroms (POTS) bietet sich in der klinischen Praxis die lumbale Versorgung mit einem autologen epiduralen Blutpflaster (EBP) an. Zwar zeigen EBPs häufig Wirkung, wenn es um die Abdichtung von Liquorlecks geht, doch sprechen Patienten vielleicht nicht dauerhaft auf ein einmalig gesetztes EBP an und eine vollständige Symptomfreiheit lässt sich womöglich erst nach dem Setzen von zwei oder noch mehr EBPs erzielen. Von einem gewissen Grad an anhaltender Verbesserung über ein paar Tage hinaus wird jedoch generell ausgegangen. In einigen Fällen lässt sich eine länger anhaltende Verbesserung nicht mit gezielten (am Situs des Liqorlecks) und/oder nicht-gezielten lumbalen EBPs erreichen, und es kann ein chirurgischer Eingriff erforderlich sein.

Es ist nicht eindeutig, dass alle Patienten mit einem 7.2.3 Kopfschmerz zurückzuführen auf ein spontanes Liquorunterdrucksyndrom ein aktives Liquorleck aufweisen, trotz einer schlüssigen entsprechenden Vorgeschichte oder Anzeichen in der zerebralen Bildgebung, die mit einem Liquorleck vereinbar sind. Die zugrunde liegende Störung kann ein Liquormangel sein. Anamnestisch lässt sich häufig eine triviale Liquordruckerhöhung, z. B. durch starkes Husten, erfragen.

Orthostatische Kopfschmerzen sind postkoital beschrieben worden: Diese Kopfschmerzen sollten unter 7.2.3 Kopfschmerz zurückzuführen auf ein spontanes Liquorunterdrucksyndrom kodiert werden, da sie höchst wahrscheinlich auf ein Liquorleck zurückzuführen sind.

\subsection{Kopfschmerz zurückzuführen auf nicht- infektiöse entzündliche Erkrankungen}

Beschreibung:

Kopfschmerz bei Vorliegen einer nicht-infektiösen entzündlichen intrakraniellen Erkrankung, gewöhnlich mit Auftreten einer lymphozytärer Liquorpleozytose. Der Kopfschmerz verschwindet nach Resolution der entzündlichen Erkrankung.

\section{Diagnostische Kriterien:}

A. Kopfschmerz, der das Kriterium C erfüllt

B. Es wurde eine nichtinfektiöse entzündliche Erkrankung diagnostiziert, von der bekannt ist, dass sie Kopfschmerzen verursachen kann

C. Ein kausaler Zusammenhang kann durch eines oder mehrere der folgenden Kriterien gezeigt werden:

1. Der Kopfschmerz hat sich in einem zeitlichen Zusammenhang mit dem Beginn der nichtinfektiösen entzündlichen Erkrankung entwickelt

2. Der Kopfschmerz hat sich gleichzeitig mit der Verschlechterung der nichtinfektiösen entzündlichen Erkrankung deutlich verschlechtert

3. Der Kopfschmerz hat sich gleichzeitig mit der Besserung der nichtinfektiösen entzündlichen Erkrankung deutlich gebessert

D. Nicht besser erklärt durch eine andere ICHD-3-Diagnose.

\subsubsection{Kopfschmerz zurückzuführen auf eine Neurosarkoidose}

Beschreibung:

Kopfschmerz verursacht durch und begleitet von anderen Symptomen und Zeichen einer Neurosarkoidose.

Diagnostische Kriterien:

A. Kopfschmerz, der das Kriterium C erfüllt

B. Es wurde eine Neurosarkoidose diagnostiziert

C. Ein kausaler Zusammenhang kann durch wenigstens zwei der folgenden Kriterien gezeigt werden:

1. Der Kopfschmerz hat sich in einem zeitlichen Zusammenhang mit dem Beginn der Neurosarkoidose entwickelt

2. Einer oder beide der folgenden Punkte sind erfüllt:

a) der Kopfschmerz hat sich gleichzeitig mit einer Verschlechterung der Neurosarkoidose deutlich verschlechtert

b) der Kopfschmerz hat sich gleichzeitig mit einer Besserung der Neurosarkoidose deutlich gebessert

3. Der Kopfschmerz tritt in Begleitung einer oder mehrerer Hirnnervenlähmung(en) auf

D. Nicht besser erklärt durch eine andere ICHD-3-Diagnose.

\section{Kommentar:}

Zu den sonstigen Manifestationen einer Neurosarkoidose gehören eine aseptische Meningitis, Hirnnervenstörungen, (eine) intrakranielle raumfordernde Läsion(en) im Hirn-MRT, periventrikuläre entzündliche fokale Läsionen und/oder großflächige homogen kontrastmittelaufnehmende Läsionen im Hirn- oder spinalen MRT, die durch eine Biopsie als nicht-verkäsende Granulome gesichert sind. 


\subsubsection{Kopfschmerz zurückzuführen auf eine aseptische (nicht-infektiöse) Meningitis}

Beschreibung:

Kopfschmerz, der durch eine aseptische Meningitis verursacht wird, in Begleitung anderer Symptome und/oder klinischer Zeichen einer meningealen Reizung. Er verschwindet nach Verschwinden der Meningitis.

\section{Diagnostische Kriterien:}

A. Kopfschmerz, der das Kriterium C erfüllt

B. Eine aseptische Meningitis wurde anhand der Liquoruntersuchung diagnostiziert1

C. Ein kausaler Zusammenhang kann durch wenigstens zwei der folgenden Kriterien gezeigt werden:

1. Der Kopfschmerz hat sich in einem zeitlichen Zusammenhang mit dem Beginn der aseptischen Meningitis entwickelt oder führte zu ihrer Entdeckung

2. Einer oder beide der folgenden Punkte sind erfüllt:

a) der Kopfschmerz hat sich gleichzeitig mit einer Verschlechterung der aseptischen Meningitis deutlich verschlechtert

b) der Kopfschmerz hat sich gleichzeitig mit einer Besserung der aseptischen Meningitis deutlich gebessert

3. Der Kopfschmerz wird von anderen Symptomen und/oder klinischen Zeichen einer meningealen Entzündung begleitet, darunter Nackensteifigkeit (Meningismus) und/oder eine Photophobie

D. Nicht besser erklärt durch eine andere ICHD-3-Diagnose.

\section{Anmerkung:}

1. In der Liquoruntersuchung von Patienten mit aseptischer Meningitis finden sich eine lymphozytäre Pleozytose, eine diskrete Liquoreiweißerhöhung und normale Glukosewerte bei fehlendem Nachweis von Infektionserregern.

\section{Kommentar:}

Eine aseptische Meningitis kann nach Einwirkung bestimmter Medikamente auftreten, darunter Ibuprofen oder sonstige NSAR, Immunglobuline, Penicillin oder Trimethoprim, intrathekalen Injektionen und/oder Insufflationen.

\subsubsection{Kopfschmerz zurückzuführen auf eine andere nicht-infektiöse entzündliche intrakranielle Erkrankung}

\section{Beschreibung:}

Kopfschmerz verursacht durch eine Reihe von Autoimmunerkrankungen, jedoch ohne üblicherweise ein hervorstechendes oder Leitsymptom zu sein, dabei von anderen Symptomen und/oder klinischen Zeichen der ursächlichen Erkrankung begleitet. Der Kopfschmerz verschwindet nach erfolgreicher Behandlung der Autoimmunerkrankung.

\section{Diagnostische Kriterien:}

A. Kopfschmerz, der das Kriterium C erfüllt

B. Es wurde eine andere als die oben beschriebenen nicht-infektiöse entzündliche Erkrankung diagnostiziert, von der bekannt ist, dass sie Kopfschmerzen verursachen kann1
C. Ein kausaler Zusammenhang kann durch eines oder mehrere der folgenden Kriterien gezeigt werden:

1. Der Kopfschmerz hat sich in einem zeitlichen Zusammenhang zu dem Beginn der nicht-infektiösen entzündlichen Erkrankung entwickelt

2. Der Kopfschmerz hat sich gleichzeitig mit der Verschlechterung der nicht-infektiösen entzündlichen Erkrankung deutlich verschlechtert

3. Der Kopfschmerz hat sich gleichzeitig mit der Besserung der nicht-infektiösen entzündlichen Erkrankung deutlich gebessert

D. Nicht besser erklärt durch eine andere ICHD-3-Diagnose.

\section{Anmerkung:}

1. Der Kopfschmerz kann einen ursächlichen Zusammenhang mit einer akuten demyelinisierenden Enzephalomyelitis (ADEM) aufweisen, einem systemischen Lupus erythematosus (SLE), einem Morbus Behçet und anderen systemischen oder fokalen Autoimmunsyndromen (z. B. limbische Enzephalitis), ist jedoch üblicherweise kein hervorstechendes Symptom oder Leitsymptom hiervon.

\subsubsection{Kopfschmerz zurückzuführen auf eine lympho- zytäre Hypophysitis}

Beschreibung:

Kopfschmerz verursacht durch eine lymphozytäre Hypophysitis, begleitet von einer Vergrößerung der Hypophyse sowie in der Hälfte der Fälle von einer Hyperprolaktinämie. Er verschwindet nach erfolgreicher Behandlung der lymphozytären Hypophysitis.

Diagnostische Kriterien:

A. Kopfschmerz, der das Kriterium C erfüllt

B. Es wurde eine lymphozytäre Hypophysitis diagnostiziert

C. Ein kausaler Zusammenhang kann durch eines oder mehrere der folgenden Kriterien gezeigt werden:

1. Der Kopfschmerz hat sich in einem zeitlichen Zusammenhang zum Beginn der lymphozytären Hypophysitis entwickelt

2. Der Kopfschmerz hat sich gleichzeitig mit der Verschlechterung der lymphozytären Hypophysitis deutlich verschlechtert

3. Der Kopfschmerz hat sich gleichzeitig mit der Besserung der lymphozytären Hypophysitis deutlich gebessert

D. Nicht besser erklärt durch eine andere ICHD-3-Diagnose.

\section{Kommentar:}

Eine lymphozytäre Hypophysitis wird vom Nachweis einer vergröBerten und homogen kontrastmittelaufnehmenden Hypophyse im Hirn-MRT begleitet. Sie tritt in 50 \% der Fälle in Begleitung einer Hyperprolaktinämie bzw. in $20 \%$ der Fälle von Autoantikörpern gegen hypophyseales Zytosolprotein auf.

Diese Erkrankung entwickelt sich typischerweise am Ende einer Schwangerschaft oder im Wochenbett, kann aber auch bei Männern auftreten. 


\subsubsection{Syndrom der vorübergehenden Kopf- schmerzen mit neurologischen Defiziten und Liquorlymphozytose (HaNDL; engl. Transi- ent headache and neurologic deficits with cerebrospinal fluid lymphocytosis)}

Früher verwendete Begriffe Migräne mit Pleozytose; Pseudomigräne mit lymphozytärer Pleozytose.

\section{Beschreibung:}

Migräneartige Kopfschmerzepisoden (typischerweise 1 bis 12) begleitet von neurologischen Defiziten einschließlich Hemiparästhesie, Hemiparese und/oder Dysphasie, visuelle Positivsymptome weniger verbreitet, mehrere Stunden anhaltend. Es besteht eine Liquorpleozytose. Die Störung verschwindet spontan innerhalb von 3 Monaten.

\section{Diagnostische Kriterien:}

A. Migräneartige Kopfschmerzepisoden, die die Kriterien B und C erfüllen 1

B. Beide der folgenden Punkte sind erfüllt:

1. Die Kopfschmerzepisoden werden von mindestens einer der folgenden vorübergehenden $>4$ Stunden anhaltenden neurologischen Defiziten begleitet oder folgen diesen recht unmittelbar:

a) Hemiparästhesie

b) Dysphasie

c) Hemiparese

2. Verbunden mit einer Liquorpleozytose (>15 Leukozyten pro $\mu$ l), Ergebnis der ätiologischen Abklärung negativ

C. Ein kausaler Zusammenhang kann durch eines oder beide der folgenden Kriterien gezeigt werden:

1. Die Kopfschmerzen und vorübergehenden neurologischen Defizite haben sich in einem zeitlichen Zusammenhang mit dem Beginn oder der Verschlechterung der Pleozytose im Liquor entwickelt oder deutlich verschlechtert oder führten zu ihrer Entdeckung

2. Die Kopfschmerzen und vorübergehenden neurologischen Defizite haben sich gleichzeitig mit der Besserung der Liquorpleozytose deutlich gebessert

D. Nicht besser erklärt durch eine andere ICHD-3-Diagnose2.

\section{Anmerkung:}

1. Die meisten Patienten mit diesem Syndrom haben keine Migränevorgeschichte.

2. Zu den anderen Diagnosen, die einige gemeinsame klinische Merkmale aufweisen, gehört die 1.2.3 hemiplegische Migräne, obwohl Mutationen des CACNA1A-Gens, Ursache einer 1.2.3.1.1 familiären hemiplegische Migräne Typ 1 (FHM1), bei diversen Patienten mit einem 7.3.5 Syndrom der vorübergehenden Kopfschmerzen mit neurologischen Defiziten und Liquorlymphozytose (HaNDL) ausgeschlossen wurden. Ebenfalls auszuschließen sind eine Neuroborreliose, Neurosyphilis, Neurobrucellose, Mykoplasmen, eine granulomatöse und neoplastische Arachnoiditis, eine Enzephalitis und eine ZNS-Vaskulitis.

\section{Kommentar:}

Das klinische Bild eines 7.3.5 Syndroms der vorübergehenden Kopfschmerzen mit neurologischen Defiziten und Liquorlymphozytose (HaNDL) besteht aus 1 bis 12 getrennten Episoden vorübergehender neurologischer Defizite, gefolgt oder begleitet von mittelstarken bis starken Kopfschmerzen. Die meisten der Episoden dauern Stunden, einige können jedoch auch mehr als 24 Stunden anhalten. Zu den neurologischen Manifestationen gehören in drei Vierteln der Fälle sensible Symptome, in zwei Dritteln der Fälle Sprachstörungen und in etwas mehr als der Hälfte motorische Defizite. Migräneauraartige visuelle Symptome sind relativ ungewöhnlich (weniger als 20 \% der Fälle). Das Syndrom verschwindet innerhalb von 3 Monaten.

Zusätzlich zu einer Liquorlymphozytose (bis zu 760 Zellen/ $\mu \mathrm{l})$ finden sich bei mehr als 90 \% der Fälle erhöhte Werte für den Gesamteiweißspiegel im Liquor (bis zu $250 \mathrm{mg} / \mathrm{dl}$ ), bei mehr als 50 \% der Fälle ein erhöhter Liquordruck (bis zu 400 mm H2O). Das Vorliegen viraler Vorinfektionen in wenigstens einem Viertel der Fälle hat die Frage einer möglichen Autoimmunpathogenese des 7.3.5 Syndroms der vorübergehenden Kopfschmerzen mit neurologischen Defiziten und Liquorlymphozytose (HaNDL) aufgeworfen. Eine Beschreibung von Antikörpern bei einer Untereinheit des spannungsabhängigen Kalzium-Kanals CACNA1H vom T-Typ in den Seren zweier Patienten mit dieser Erkrankung aus neuerer Zeit stützt diese Ansicht.

Gelegentlich liegt ein Papillenödem vor. Routinemäßige CT- and MRT-Aufnahmen (mit oder ohne intravenöses Kontrastmittel) und die Angiographie sind interiktal durchgängig normal, die zerebrale Bildgebung während einer Episode jedoch kann eine verzögerte Hirndurchblutung ohne erhöhte Veränderungen bei der diffusionsgewichteten Bildgebung zeigen sowie eine Verengung der Hirnarterien. Zudem wurden bei einem einzelnen Patienten ein die graue Substanz betreffendes Ödem und eine Kontrastmittelanreicherung im Sulcus beschrieben. Mikrobiologische Untersuchungen waren einheitlich unauffällig. EEG- und SPECT-Untersuchungen können übereinstimmend mit den fokalen neurologischen Defiziten fokal auffällige Bereiche zeigen.

\subsection{Kopfschmerz zurückzuführen auf ein intrakranielles Neoplasma}

Beschreibung:

Kopfschmerz verursacht durch eine intrakranielle Neoplasie.

\section{Diagnostische Kriterien:}

A. Kopfschmerz, der das Kriterium C erfüllt

B. Es wurde eine intrakranielle Neoplasie diagnostiziert

C. Ein kausaler Zusammenhang kann durch eines oder mehrere der folgenden Kriterien gezeigt werden:

1. Der Kopfschmerz hat sich in einem zeitlichen Zusammenhang zu der intrakraniellen Neoplasie entwickelt oder führte zu ihrer Entdeckung

2. Der Kopfschmerz hat sich gleichzeitig mit der Verschlechterung der intrakraniellen Neoplasie deutlich verschlechtert 
3. Der Kopfschmerz hat sich in einem zeitlichen Zusammenhang zu der erfolgreichen Behandlung der intrakraniellen Neoplasie deutlich gebessert

D. Nicht besser erklärt durch eine andere ICHD-3-Diagnose.

\subsubsection{Kopfschmerz zurückzuführen auf ein intra- kranielles Neoplasma}

Beschreibung:

Kopfschmerz verursacht durch einen oder mehrere raumfordernde intrakranielle Tumore.

Diagnostische Kriterien:

A. Kopfschmerz, der das Kriterium C erfüllt

B. Es wurde ein raumforderndes intrakranielles Neoplasma nachgewiesen

C. Ein kausaler Zusammenhang kann durch mindestens zwei der folgenden Kriterien gezeigt werden:

1. Der Kopfschmerz hat sich in einem zeitlichen Zusammenhang zur Entwicklung des Neoplasmas entwickelt oder führte zu seiner Entdeckung

2. Einer oder beide der folgenden Punkte sind erfüllt:

a) der Kopfschmerz hat sich gleichzeitig mit der Verschlechterung des Neoplasmas deutlich verschlechtert

b) der Kopfschmerz hat sich in einem zeitlichen Zusammenhang zu einer erfolgreichen Behandlung des Neoplasmas deutlich gebessert

3. Der Kopfschmerz weist mindestens eines der folgenden vier Charakteristika auf:

a) fortschreitend

b) morgens und/oder im Liegen schlimmer

c) Verschlimmerung durch Valsalva-Manöver

d) von Übelkeit und/oder Erbrechen begleitet

D. Nicht besser erklärt durch eine andere ICHD-3-Diagnose.

\section{Kommentar:}

Die Prävalenz von Kopfschmerzen bei Patienten mit intrakraniellen Tumoren liegt zwischen 32\% und 71\%. Bei jungen Patienten (Kinder inbegriffen) ist die Kopfschmerzwahrscheinlichkeit größer, ebenso bei Patienten mit primären Kopfschmerzen in der Vorgeschichte und mit raschem Tumorwachstum bzw. oder Schädelgrubenoder Mittellinienlage. Bei Patienten mit aktueller oder in der Vorgeschichte aufgetretener Krebserkrankung sollte die Schwelle für weitere Diagnostik niedrig angesetzt werden.

Es gibt keine pathognomonischen Merkmale eines 7.4.1 Kopfschmerzes zurückzuführen auf ein intrakranielles Neoplasma, obwohl Progression oder Abbau ein zentrales Merkmal darstellen. Die weiteren Symptome, die an etwas Derartiges denken lassen (schwer, morgens schlimmer und in Verbindung mit Übelkeit und Erbrechen auftretend) sind keine klassische Triade; sie gehören eher in den Kontext einer intrakraniellen Druckerhöhung und den von Tumoren der hinteren Schädelgrube.

Der Kopfschmerz sitzt nicht unbedingt ipsilateral zum Tumor. Tendenziell treten eher Massebildungen im Umfeld des Schädels oder der Dura mater in Begleitung von ipsilateralen Kopfschmerzen auf, eine intrakranielle Hypertension jedoch ruft einen diffuseren Kopfschmerz hervor. Der Kopfschmerz, der durch einen Hirntumor verursacht wird, bleibt selten das einzige Symptom: ein isolierter
Kopfschmerz tritt bei $2 \%$ bis $16 \%$ der Patienten auf, neurologische Defizite und epileptische Anfälle sind jedoch sehr verbreitet.

\subsubsection{Kopfschmerz zurückzuführen auf eine Kolloidzyste des dritten Hirnventrikels}

Beschreibung:

Kopfschmerz infolge einer Kolloidzyste des dritten Hirnventrikels, auftretend mit dem sehr charakteristischen klinischen Erscheinungsbild rezidivierender Attacken mit Beginn als Donnerschlagkopfschmerz, oft ausgelöst durch eine Positionsveränderung oder ein Valsalva-ähnliches Manöver, begleitet von einer Bewusstseinstrübung oder Bewusstseinsverlust.

\section{Diagnostische Kriterien:}

A. Kopfschmerz, der Kriterium C erfüllt

B. Es wurde eine Kolloidzyste des dritten Hirnventrikels nachgewiesen

C. Ein kausaler Zusammenhang kann durch die beiden folgenden Kriterien gezeigt werden:

1. Der Kopfschmerz hat sich in einem zeitlichen Zusammenhang mit der Kolloidzyste entwickelt oder war maßgeblich für ihre Entdeckung

2. Einer oder beide der folgenden Punkte sind erfüllt:

a) der Kopfschmerz ist rezidivierend, mit Beginn als Donnerschlagkopfschmerz und in Begleitung einer Bewusstseinstrübung oder Bewusstlosigkeit

b) der Kopfschmerz hat sich in einem zeitlichen Zusammenhang mit der erfolgreichen Behandlung der Kolloidzyste deutlich gebessert oder ist verschwunden

D. Nicht besser erklärt durch eine andere ICHD-3-Diagnose.

Kommentar:

Die überwältigende Mehrheit der Kolloidzysten des dritten Hirnventrikels sind asymptomatisch und daher Zufallsbefunde. Dennoch kann ihre Lage in unmittelbarer Nachbarschaft des Foramen Monroi gelegentlich zu einem plötzlichen okklusiven Hydrozephalus führen und Kopfschmerzen verursachen, die mit einem Donnerschlagkopfschmerz sowie einer Trübung oder einem Verlust des Bewusstseins beginnen. Dieses hochgradig charakteristische klinische Bild sollte zu einer schnellen Diagnose führen.

\subsubsection{Kopfschmerz zurückzuführen auf eine Kolloidzyste des dritten Hirnventrikels signalisiert einen lebens- bedrohlichen Notfall.}

\subsubsection{Kopfschmerz zurückzuführen auf eine Meningeosis carcinomatosa}

Beschreibung:

Kopfschmerz infolge einer Meningeosis carcinomatosa, gewöhnlich begleitet von Symptomen einer Enzephalopathie und/oder Hirnnervenlähmungen.

\section{Diagnostische Kriterien:}

A. Kopfschmerz, der das Kriterium C erfüllt

B. Es wurde eine Meningeosis carcinomatosus (bei Vorliegen systemischer Neoplasmen, die bekannterweise eine Meningeosis carcinomatosus begleiten) nachgewiesen 
C. Ein kausaler Zusammenhang kann durch wenigstens die beiden folgenden Kriterien gezeigt werden:

1. Der Kopfschmerz hat sich in zeitlichem Zusammenhang mit dem Auftreten der Meningeosis carcinomatosus entwickelt

2. Einer oder beide der folgenden Punkte sind erfüllt:

a) der Kopfschmerz hat sich gleichzeitig mit der Verschlechterung der Meningeosis carcinomatosus deutlich verschlechtert

b) der Kopfschmerz hat sich gleichzeitig mit der Verbesserung der Meningeosis carcinomatosus deutlich gebessert

3. Der Kopfschmerz wird von Hirnnervenlähmungen und/ oder einer Enzephalopathie begleitet

D. Nicht besser erklärt durch eine andere ICHD-3-Diagnose.

\subsubsection{Kopfschmerz zurückzuführen auf eine hypo- thalamische oder hypophysäre Über- oder Unterfunktion}

\section{Beschreibung:}

Kopfschmerz infolge eines Hypophysenadenoms und einer hypothalamischen oder hypophysären Über- oder Unterfunktion, in der Regel begleitet von einer gestörten Temperaturregulation, einem veränderten emotionalen Zustand und/oder Veränderungen in Sachen Durst oder Appetit. Nach erfolgreicher Behandlung der Grunderkrankung erfolgt eine Remission.

\section{Diagnostische Kriterien:}

A. Kopfschmerz, der das Kriterium C erfüllt

B. Es wurde eine hypothalamische oder hypophysäre Über- oder Unterfunktion in Begleitung eines Hypophysenadenoms nachgewiesen 1

C. Ein kausaler Zusammenhang kann durch mindestens zwei der folgenden Kriterien gezeigt werden:

1. Der Kopfschmerz ist in einem zeitlichen Zusammenhang mit dem Beginn der hypothalamischen oder hypophysären Über- oder Unterfunktion aufgetreten

2. Einer oder beide der folgenden Punkte sind erfüllt:

a) der Kopfschmerz hat sich gleichzeitig mit der Verschlimmerung der hypothalamischen oder hypophysären Über- oder Unterfunktion deutlich verschlechtert

b) der Kopfschmerz hat sich gleichzeitig mit der Verbesserung der hypothalamischen oder hypophysären Überoder Unterfunktion deutlich gebessert

3. Der Kopfschmerz tritt in Begleitung von mindestens einem der folgenden Punkte auf:

a) gestörte Temperaturregulation

b) anormale emotionale Verfassung

c) veränderter Durst und/oder Appetit

D. Nicht besser erklärt durch eine andere ICHD-3-Diagnose.

\section{Anmerkung:}

1. Darunter Prolaktin, Wachstumshormon und/oder ein Überschuss an adrenokortikotropem Hormon (ACTH).

\subsection{Kopfschmerz zurückzuführen auf eine intrathekale Injektion}

Beschreibung:

Kopfschmerz, der in aufrechter Position wie auch im Liegen erfahren wird, verursacht durch und auftretend innerhalb von 4 Tagen nach einer intrathekalen Injektion und innerhalb von 14 wieder verschwindend.

\section{Diagnostische Kriterien:}

A. Kopfschmerz, der das Kriterium C erfüllt

B. Zustand nach intrathekaler Injektion

C. Ein kausaler Zusammenhang kann durch wenigstens zwei der folgenden Kriterien gezeigt werden:

1. Der Kopfschmerz hat sich innerhalb von 4 Tagen nach der intrathekalen Injektion entwickelt1

2. Der Kopfschmerz hat sich innerhalb von 14 Tagen nach der intrathekalen Injektion2 deutlich gebessert

3. Symptome einer meningealen Reizung

D. Nicht besser erklärt durch eine andere ICHD-3-Diagnose.

\section{Anmerkung:}

1. Kopfschmerz entwickelt sich gewöhnlich innerhalb von 4 Tagen nach der intrathekalen Injektion und ist sowohl in aufrechter Position als auch im Liegen vorhanden.

2. Wenn der Kopfschmerz länger als 14 Tage anhält, sollten alternative Diagnosen in Betracht gezogen werden, etwa 7.2.2 Kopfschmerz bei Liquorfistel, Meningitis oder eine leptomeningealen Erkrankung.

\subsection{Kopfschmerz zurückzuführen auf einen epileptischen Anfall}

An anderer Stelle kodiert: Wenn migräneähnliche oder andere Kopfschmerzen und Epilepsie beide Symptome einer definierten Gehirnerkrankung (z. B. MELAS) sind, sollte der Kopfschmerz unter dieser Erkrankung kodiert werden. Tritt ein epileptischer Anfall während einer Migräneaura oder unmittelbar danach auf, sollte dieser unter 1.4.4 epileptische Anfälle, durch Migräneaura getriggert kodiert werden.

\section{Beschreibung:}

Kopfschmerz infolge eines epileptischen Anfalls, der während des und/oder nach diesem Anfall auftritt und innerhalb von Stunden oder maximal 3 Tagen verschwindet.

\section{Diagnostische Kriterien:}

A. Kopfschmerz, der das Kriterium C erfüllt

B. Der Patient hat oder hatte kurz zuvor einen epileptischen Anfall

C. Ein kausaler Zusammenhang kann durch beide der folgenden Kriterien gezeigt werden:

1. Der Kopfschmerz hat sich gleichzeitig mit dem Beginn des epileptischen Anfalls oder bald danach entwickelt

2. Der Kopfschmerz ist spontan verschwunden, nachdem der epileptische Anfall vorbei war

D. Nicht besser erklärt durch eine andere ICHD-3-Diagnose. 
Kommentar:

Gut dokumentierte Berichte sprechen für die Anerkennung der Subtypen 7.6.1 iktaler Kopfschmerz bei Epilepsie und 7.6.2 postiktaler Kopfschmerz aufgrund ihres zeitlichen Zusammenhangs mit dem epileptischen Anfall.

Auch ein präiktaler Kopfschmerz wurde beschrieben. In einer kleinen Untersuchung an 11 Patienten mit therapierefraktärer fokaler Epilepsie fanden sich frontotemporale Kopfschmerzen bei neun Patienten mit Temporallappenepilepsie (TLE) ipsilateral zum Fokus und bei einem mit TLE sowie einem mit Frontallappenepilepsie jeweils kontralateral. Es werden weitere Untersuchungen benötigt, um die Existenz eines präiktalen Kopfschmerzes nachzuweisen sowie seine Prävalenz und klinischen Merkmale zu ermitteln, bei Patienten mit partieller sowie mit generalisierter Epilepsie. Präiktale Kopfschmerzen müssen zudem von 1.4.4 epileptischen Anfällen, durch Migräneaura getriggert abgegrenzt werden.

\subsubsection{Iktaler Kopfschmerz bei Epilepsie}

Früher verwendeter Begriff: Iktaler Kopfschmerz.

\section{Beschreibung:}

Kopfschmerz, der durch einen partiellen epileptischen Anfall getriggert wird und während diesem auftritt, ipsilateral zur epileptischen Entladung, und sofort oder bald nach Ende des Anfalls verschwindet.

\section{Diagnostische Kriterien:}

A. Kopfschmerz, der das Kriterium C erfüllt

B. Der Patient hat einen partiellen epileptischen Anfall

C. Ein kausaler Zusammenhang kann durch beide der folgenden Kriterien gezeigt werden:

1. Der Kopfschmerz hat sich gleichzeitig mit dem Beginn des partiellen epileptischen Anfalls entwickelt

2. Einer oder beide der folgenden Punkte sind erfüllt:

a) der Kopfschmerz ist ipsilateral zur iktalen Entladung lokalisiert

b) der Kopfschmerz bessert sich deutlich, nachdem der partielle Anfall zu Ende ist

D. Nicht besser erklärt durch eine andere ICHD-3-Diagnose.

\section{Kommentar:}

Einem 7.6.1 iktalen Kopfschmerz bei Epilepsie können andere mit der Epilepsie verbundene Manifestationen folgen (motorisch, sensorisch oder autonom).

Diese Erkrankung sollte von einem „reinen“ oder „isolierten“ iktalen Kopfschmerz bei Epilepsie unterschieden werden, der als alleinige Manifestation einer Epilepsie auftritt und eine Differentialdiagnose zur Abgrenzung von anderen Kopfschmerztypen erforderlich macht.

„Hemicrania epileptica“ (sofern deren Existenz bestätigt wird) ist eine sehr seltene Variante des 7.6.1 iktalen Kopfschmerzes bei Epilepsie. Charakteristisch sind hier die ipsilaterale Lokalisation des Kopfschmerzes und die iktalen Paroxysmen im EEG.

\subsubsection{Postiktaler Kopfschmerz}

\section{Beschreibung:}

Kopfschmerz, der durch einen epileptischen Anfall verursacht wird und innerhalb von 3 Stunden nach einem solchen auftritt sowie innerhalb von 72 Stunden nach dem Ende des epileptischen Anfalls spontan verschwindet.

\section{Diagnostische Kriterien:}

A. Kopfschmerz, der das Kriterium C erfüllt

B. Der Patient hat kurz zuvor einen partiellen oder generalisierten epileptischen Anfall gehabt

C. Ein kausaler Zusammenhang kann durch beide der folgenden Kriterien gezeigt werden:

1. Der Kopfschmerz ist innerhalb von 3 Stunden nach dem Ende des epileptischen Anfalls aufgetreten

2. Der Kopfschmerz ist innerhalb von 72 Stunden nach dem Ende des epileptischen Anfalls verschwunden

D. Nicht besser erklärt durch eine andere ICHD-3-Diagnose.

\section{Kommentar:}

Ein 7.6.2 postiktaler Kopfschmerz tritt bei mehr als $40 \%$ der Patienten mit entweder Temporallappenepilepsie oder Frontallappenepilepsie auf und bei bis zu $60 \%$ der Patienten mit Okzipitallappenepilepsie. Er tritt häufiger nach generalisierten tonisch-klonischen epileptischen Anfällen als nach anderen Anfallstypen auf.

\subsection{Kopfschmerz zurückzuführen auf eine Chiari-Malformation Typ I(CM1)}

\section{Beschreibung:}

Kopfschmerz, der durch eine Chiari-Malformation Typ 1 verursacht wird, meist okzipital oder subokzipital, von kurzer Dauer (weniger als 5 Minuten) und durch Husten oder andere Valsalva-ähnliche Manöver hervorgerufen wird. Er verschwindet nach erfolgreicher Behandlung der Chiari-Malformation.

\section{Diagnostische Kriterien:}

A. Kopfschmerz, der Kriterium C erfüllt

B. Es wurde eine Chiari-Malformation Typ 1 (CM1) nachgewiesen 1

C. Ein kausaler Zusammenhang kann durch mindestens zwei der folgenden Kriterien gezeigt werden:

1. Einer oder beide der folgenden Punkte sind erfüllt:

a) der Kopfschmerz hat sich in einem zeitlichen Zusammenhang mit der CM1 entwickelt oder führte zu deren Entdeckung

b) der Kopfschmerz ist innerhalb von 3 Monaten nach erfolgreicher Behandlung der CM1 verschwunden

2. Der Kopfschmerz weist eines oder mehrere der folgenden drei Merkmale auf:

a) eingeleitet durch Husten oder eine andere Valsalva-ähnliche Manöver

b) okzipital oder subokzipital lokalisiert

c) Dauer $<5$ Minuten

3. Der Kopfschmerz ist von anderen Symptomen und/oder klinischen Zeichen einer Störung des Hirnstamms, Klein- 
hirns, der unteren Hirnnerven und/oder des Halsmarks begleitet2

D. Nicht besser erklärt durch eine andere ICHD-3-Diagnose3.

\section{Anmerkung:}

1. Die Diagnose einer Chiari-Malformation Typ 1 (CM1) mittels MRT erfordert den Nachweis einer Kaudalverlagerung der Kleinhirntonsillen um mindestens 5 mm oder einer Kaudalverlagerung der Kleinhirntonsillen um mindestens $3 \mathrm{~mm}$ plus einer Enge des Subarachnoidalraumes im kraniozervikalen Übergang, nachgewiesen durch eine Kompression der Liquorräume hinter und neben dem Kleinhirn oder eine verringerte Höhe des Supraokziputs, eine Steilstellung des Tentoriums oder ein Kinking der Medulla oblongata.

2. Beinahe alle Patienten mit CM1 (95\%) berichten von einer Konstellation von fünf oder mehr klar umrissenen Symptomen.

3. Bei Patienten mit verändertem Liquordruck, sei es im Sinne eines Überdrucks wie bei einer idiopathischen intrakraniellen Druckerhöhung (IIH), oder eines Unterdrucks wie bei der spontanen intrakraniellen Hypotonie als Folge eines Liquorverlusts, kann im MRT eine sekundäre Verlagerung der Kleinhirntonsillen und CM1 nachgewiesen werden. Diese Patienten können auch Kopfschmerzen in Verbindung mit Husten oder anderen Valsalva-ähnlichen Manöver aufweisen und werden korrekterweise entweder unter 7.1.1 Kopfschmerz zurückzuführen auf eine idiopathische intrakranielle Hypertension oder unter 7.2.3 Kopfschmerz zurückzuführen auf ein spontanes Liquorunterdrucksyndrom kodiert. Vor diesem Hintergrund muss bei allen Patienten mit Kopfschmerz und CM1 ein auffälliger Liquordruck ausgeschlossen werden.

\section{Kommentar:}

7.7 Kopfschmerz zurückzuführen auf eine Chiari-Malformation Typ I (CM1) ähnelt von der Beschreibung her oft Punkt 4.1 primärer Hustenkopfschmerz, bis auf die längere Dauer in manchen Fällen (Minuten statt Sekunden).

Prävalenzstudien zufolge findet sich bei $0,24 \%$ bis 3,6 \% der Population eine tonsilläre Herniation von mindestens $5 \mathrm{~mm}$, wobei die Prävalenz mit steigendem Alter zunimmt.

Der klinische Kontext der CMI ist wichtig, da viele dieser Patienten asymptomatisch sein können. Es liegen widersprüchliche Daten zum Ausmaß der Herniation und zur Schwere des begleitenden Kopfschmerzes und dem Grad der Beeinträchtigung vor: Patienten können bei minimaler tonsillärer Herniation „Chiari-artige“ Symptome aufweisen, während andere bei großen Herniationen durchaus asymptomatisch sein können.

Diese Kriterien für einen 7.7 Kopfschmerz zurückzuführen auf eine Chiari-Malformation Typ I (CM1) harren der Validierung: hier werden prospektive Studien zu langfristigen klinischen Resultaten mit und ohne Operation benötigt. Bis dahin wird beim Abwägen, ob ein chirurgischer Eingriff erfolgen soll, die strenge Einhaltung der klinischen wie auch der radiologischen Kriterien empfohlen, um unnötige Eingriffe mit beträchtlicher potenzieller Operationsmorbidität zu vermeiden. Die derzeitige Datenlage lässt darauf schließen, dass bei sorgfältig ausgewählten Patienten Hustenkopfschmerzen eher als Kopfschmerzen ohne Valsalva-ähnliche Auslöser und okzi- pitale Kopfschmerzen eher als nicht-okzipitale Kopfschmerzen auf eine chirurgische Intervention ansprechen.

Neuere vorliegende Daten weisen auf einen Zusammenhang zwischen Adipositas und der Kopfschmerzwahrscheinlichkeit bei CM1 hin; dieses Ergebnis bedarf weiterer Untersuchungen, vor allem aus der Behandlungsperspektive.

\subsection{Kopfschmerz zurückzuführen auf eine andere nichtvaskuläre intrakranielle Störung \\ Beschreibung:}

Kopfschmerz, der durch eine andere nichtvaskuläre intrakranielle Störung zurückgeht als die oben beschriebenen.

Diagnostische Kriterien:

A. Kopfschmerz, der das Kriterium C erfüllt

B. Es wurde eine andere nichtvaskuläre intrakranielle Störung als die oben beschriebenen nachgewiesen, von der bekannt ist, dass sie Kopfschmerz verursachen kann

C. Ein kausaler Zusammenhang kann durch mindestens zwei der folgenden Kriterien gezeigt werden:

1. der Kopfschmerz hat sich in einem zeitlichen Zusammenhang mit dem Beginn der nichtvaskulären intrakraniellen Störung entwickelt

2. Einer oder beide der folgenden Punkte sind erfüllt: a) der Kopfschmerz hat sich gleichzeitig mit der Verschlechterung der nichtvaskulären intrakraniellen Störung entwickelt oder deutlich verschlechtert

b) der Kopfschmerz hat sich gleichzeitig mit der Besserung der nichtvaskulären intrakraniellen Störung deutlich gebessert

3. Der Kopfschmerz weist Merkmale auf, die typisch für die nichtvaskuläre intrakranielle Störung sind

4. Es existieren sonstige Belege für den ursächlichen Zusammenhang

D. Nicht besser erklärt durch eine andere ICHD-3-Diagnose.

Literatur

7.1.1 Kopfschmerz zurückzuführen auf eine idiopathische intrakranielle Hypertension (IIH)

Avery RA, Shah SS, Licht DJ, et al. Reference range for cerebrospinal fluid opening pressure in children. NEJM 2010; 363: 891-893.

Corbett J], Mehta MP. Cerebrospinal fluid pressure in normal obese subjects and patients with pseudotumor cerebri. Neurology 1983; 33: 1386-1388.

Fisayo A, Bruce BB, Newman NJ, Biousse V. Overdiagnosis of idiopathic intracranial hypertension. Neurology 2016; 86: 341-350.

Friedman DI, Jacobson DM. Idiopathic intracranial hypertension.J Neuroophthalmol 2004; 24: 138-145.

Friedman DI, Liu G, Digre KB. Diagnostic criteria for the pseudotumor cerebri syndrome in adults and children. Neurology 2013; 81: 1159-1165.

Friedman DI, Quiros PA, Subramanian PS, Mejico LJ, Gao S, McDermott M, Wall M, and and the NORDIC IIHTT Study Group. Headache in idiopa- 
thic intracranial hypertension: Findings from the Idiopathic Intracranial Hypertension Trial. Headache 2017; 57: 1195-1205.

Wall M, Kupersmith MJ, Kieburtz KD, et al for the IIHTT Study Group. The Idiopathic Intracranial Hypertension Treatment Trial: Clinical profile at baseline. JAMA Neurol 2014; 71: 693-701.

Whiteley W, Al-Shahi R, Warlow CP, Zeidler M, Lueck CJ. CSF opening pressure: reference interval and the effect of body mass index. Neurology 2006; 67: 1690-1691.

Yri HM, Jensen RH. Idiopathic intracranial hypertension: Clinical nosography and field testing of the ICHD diagnostic criteria. A case-control study. Cephalalgia 2015; 35: 553-562.

\subsubsection{Postpunktioneller Kopfschmerz}

Amorim JA, Gomes de Barros MV, Valenca MM. Post-dural (post-lumbar) puncture headache: Risk factors and clinical features. Cephalalgia 2012; 32: 916-923.

Bezov D, Lipton RB and Ashina S. Post-dural puncture headache:Part I diagnosis, epidemiology,etiology and pathophysiology. Headache 2010; 50: 1144-1152.

\subsubsection{Kopfschmerz zurückzuführen auf ein spontanes Liquor- unterdrucksyndrom}

Mea E, Chiapparini L, Savoiardo M, et al. Application of IHS criteria to Kopfschmerz zurückzuführen aufspontaneous intracranial hypotension in a large population. Cephalalgia 2009; 29: 418-422.

Reinstein E, Pariani M, Bannykh S, Rimoin D, Schievink. Connective tissue spectrum abnormalities associated with spontaneous cerebrospinal fluid leaks: a prospective study. Eur J Hum Genet 2013; 21: 386-390.

Schievink WI. Spontaneous spinal cerebrospinal fluid leaks and intracranial hypotension. JAMA 2006; 295: 2286-2296.

Schievink WI, Dodick DW, Mokri B, Silberstein SD, Bousser MG, Goadsby PJ. Diagnostic criteria for headache due to spontaneous intracranial hypotension: A perspective. Headache 2011; 51: 1442-1444.

Schievink WI, Maya MM,Louy C, Moser FG,Tourje. Diagnostic criteria for spontaneous spinal CSF leaks and intracranial hypotension. Amer J Neuroradiol 2008; 29: 853-856.

Schwedt T], Dodick DW. Spontaneous intracranial hypotension. Curr Pain Headache Rep 2007; 11: 56-61.

Wang YF, Fuh JL, Lirng JF, Chen SP, Hseu SS, Wu JC, Wang SJ. Cerebrospinal fluid leakage and headache after lumbar puncture: a prospective non-invasive imaging study. Brain 2015; 138: 1492-1498.

Wang YF, Lirng JF, Fuh JL, Hseu SS, Wang SJ. Heavily T2-weighted MR myelography vs. CT myelography in spontaneous intracranial hypotension. Neurology 2009; 73: 1892-1898.

Wu JW, Hseu SS, Fuh JL, Lirng JF, Wang YF, Chen WT, Chen SP, Wang SJ. Factors predicting response to the first epidural blood patch in spontaneous intracranial hypotension. Brain 2017; 140: 344-352.

\subsubsection{Kopfschmerz zurückzuführen auf eine aseptische} (nichtinfektiöse) Meningitis

Holle D, Obermann M. Headache in drug-induced aseptic meningitis. Curr Pain Headache Rep 2015; 19: 29.

Morís G, Garcia-Monco JC. The challenge of drug-induced aseptic meningitis. JAMA Internal Med 2014; 174: 1511-1512.

\subsubsection{Syndrom der vorübergehenden Kopfschmerzen mit neuro- logischen Defiziten und Liquorlymphozytose (HaNDL)}

Bartleson JD, Swanson JW, Whisnant JP. A Migrainous syndrome with cerebrospinal fluid pleocytosis. Neurology 1981; 31: 1257-1262.

Berg MJ Williams LS. The transient syndrome of headache with neurologic deficits and CSF lymphocytosis (review). Neurology 1995; 45: 1648-1654.
Chapman KM, Szczygielski BI, Toth C, Woolfenden A, Robinson G, Snutch TP, Spacey SD. Pseudomigraine with lymphocytic pleocytosis: a calcium channelopathy? Clinical description of 10 cases and genetic analysis of the familial hemiplegic migraine gene CACNA1A. Headache 2003; 43: 892-895.

Fuentes B, Diez Tejedor E, Pascual J, Coya J, Quirce R. Cerebral blood flow changes in pseudomigraine with pleocytosis analyzed by single photon emission computed tomography. A spreading depression mechanism? Cephalalgia 1998; 18: 570-573.

Fumal A, Vandenheede M, Coppola G, Di Clemente L, Jacquart J, Gérard P, de Noordhout AM, Schoenen J. The syndrome of transient headache with neurological deficits and CSF lymphocytosis (HaNDL): electrophysiological findings suggesting a migrainous pathophysiology. Cephalalgia 2005; 25: 754-758.

Gomez-Aranda F, Canadillas F, Marti-Masso JF, Diez-Tejedor E et al. Pseudomigraine with temporary neurological symptoms and lymphocytic pleocytosis: A report of fifty cases. Brain 1997; 120: 1105-1113.

Kürtüncü M, Kaya D, Zuliani L, Erdag E, Icoz S, Ugurel E, Cavus F, Aysit N, Birisik O, Vincent A, Eraksoy M, Burcak V, Akman-Demir G, Tuzun E. CACNA1A antibodies associated with headache with neurological deficits and cerebrospinal fluid lymphocytosis (HaNDL). Cephalalgia 2013; 33; 123-129.

Morrison DG, Phuah HK, Reddy AT, Dure LS, Kline LB. Ophthalmologic involvement in the syndrome of headache, neurologic deficits, and cerebrospinal fluid lymphocytosis. Ophthalmology 2003; 110: 115-118.

Parissis D, loannidis P, Balamoutsos G, Karacostas D. Confusional state in the syndrome of HaNDL. Headache 2011; 51: 1285-1288.

\subsubsection{Kopfschmerz zurückzuführen auf einen intrakranielles Neoplasma}

Dowman CE, Smith WA. Intracranial tumors: A review of one hundred verified cases. Arch Neurol Psych 1928; 20: 1312-1329.

Forsyth PA, Posner JB. Headaches in patients with brain tumors: a study of 111 patients. Neurology 1993; 43: 1678-1683.

Hamilton W, Kernick D. Clinical features of primary brain tumours: a case-control study using electronic primary care records. Br J Gen Pract 2007; 57: 695-699.

Iversen $\mathrm{H}$, Strange P, Sommer W, Tjalve E. Brain tumour headache related to tumour size, and location. Cephalalgia 1987; 6 (Suppl 7): 394-395.

Kernick DP, Ahmed F, Bahra A, Dowson A, Elrington G, Fontebasso M, et al. Imaging patients with suspected brain tumour: guidance for primary care. Br J Gen Pract 2008; 58: 880-885.

Kirby S. Headache and brain tumours. Cephalalgia 2010; 30: 387-388.

Kunkle EC, Ray BS, Wolff HG. Studies on headache: The mechanisms and significance of the headache associated with brain tumor. Bull NY Acad Med 1942; 18: 400-422.

Lowry JK, Snyder JJ, Lowry PW. Brain tumors in the elderly: recent trends in a Minnesota cohort study. Arch Neurol 1998; 55: 922-928.

Pfund Z, Szapary L, Jaszberenyi O, Nagy F, Czopf J. Headache in intracranial tumors. Cephalalgia 1999; 19: 787-790; discussion 65.

Rossi LN, Vassella F. Headache in children with brain tumors. Childs Nerv Syst 1989; 5: 307-309.

Schankin C], Ferrari U, Reinisch VM, Birnbaum T, Goldbrunner R, Straube A. Characteristics of brain tumour-associated headache. Cephalalgia 2007; 27: 904-911.

Suwanwela N, Phanthumchinda K, Kaoropthum S. Headache in brain tumor: a cross-sectional study. Headache 1994; 34: 435-438.

Valentinis L, Tuniz F, Valent F, Mucchiut M, Little D, Skrap M, et al. Kopfschmerz zurückzuführen aufintracranial tumours: a prospective cohort study. Cephalalgia 2010; 30: 389-398.

Vazquez-Barquero A, Ibanez FJ, Herrera S, Izquierdo JM, Berciano J, Pascual J. Isolated headache as the presenting clinical manifestation of intracranial tumors: a prospective study. Cephalalgia 1994; 14: 270-272. 
7.4.1.1 Kopfschmerz zurückzuführen auf eine Kolloidzyste des dritten Hirnventrikels

Algin O, Ozmen E, Arslan H. Radiologic manifestations of colloid cysts: a pictorial essay. Can Assoc Radiol J 2013; 64: 56-60.

Brostigen CS, Meling TR, Marthinsen PB, Scheie D, Aarhus M, Helseth E. Surgical management of colloid cyst of the third ventricle. Acta Neurol Scand 2016; 135: 484-487.

Byard RW. Variable presentations of lethal colloid cysts. J Forensic Sci 2016; 61: 1538-1540.

Diyora B, Nayak N, Kukreja S, Sharma A. Hemorrhagic colloid cyst: Case report and review of the literature. Asian J Neurosurg 2013; 8: 162-165.

Jacob MK, Anand SK, George P. Colloid cyst of the third ventricle presenting with features of Terson's syndrome. Middle East Afr J Ophthalmol 2014; 21: 344-346.

Kone L, Chaichana KL, Rincon-Torroella J, Snyman C, Moghekar A, Quiñones-Hinojosa A. The impact of surgical resection on headache disability and quality of life in patients with colloid cyst. Cephalalgia 2016; 37: 442-451.

Lawrence JE, Nadarajah R, Treger TD, Agius M. Neuropsychiatric manifestations of colloid cysts: a review of the literature. Psychiatr Danub 2015; 27 Suppl 1: S315-320.

Mortimer AM, Bradley MD, Stoodley NG, Renowden SA. Thunderclap headache: diagnostic considerations and neuroimaging features. Clin Radiol 2013; 68: e101-113. doi: 10.1016/j.crad.2012.08.032. Epub 2012 Dec 11. Review. PubMed PMID: 23245274.

Ravnik J, Bunc G, Grcar A, Zunic M, Velnar T. Colloid cysts of the third ventricle exhibit various clinical presentation: a review of three cases. Bosn J Basic Med Sci 2014; 14: 132-135.

Ronne-Engström E, Popek E. Symptomatic colloid cysts in the third ventricle of monozygotic twins. Ups J Med Sci 2015; 120: 59-62.

Yadav YR, Yadav N, Parihar V, Kher Y, Ratre S. Management of colloid cyst of third ventricle. Turk Neurosurg 2015; 25: 362-371.

\subsection{Kopfschmerz zurückzuführen auf eine intrathekale Injektion}

Diener HC, Johansson U, Dodick DW. Kopfschmerz zurückzuführen aufnon-vascular intracranial disorder. Handb Clin Neurol 2010; 97: 547-587.

Haché M, Swoboda KJ, Sethna N, Farrow-Gillespie A, Khandji A, Xia S, Bishop KM. Intrathecal injections in children with spinal muscular atrophy: Nusinersen Clinical Trial Experience. J Child Neurol 2016; 31: 899-906.

Obermann M, Holle D, Naegel S, Diener HC. Headache attributable to nonvascular intracranial disorders. Curr Pain Headache Rep 2011; 15: 314-323.

Takagi K, Kato K, Kato Y. Treatment of mild traumatic brain injury by epidural saline and oxygen injection: report of two cases. Acta Neurochir Suppl 2013; 118: 293-296.

\subsection{Kopfschmerz zurückzuführen auf einen epileptischen Anfall}

Cianchetti C, Pruna D, Ledda MG. Epileptic seizures and headache/migraine: A review of types of association and terminology. Seizure 2013; 22: 679-685.

Förderreuther S, Henkel A, Noachtar S, Straube A. Headache associated with epileptic seizures: Epidemiology and clinical characteristics. Headache 2002; 42: 649-655.

Ito M, Adachi N, Nakamura F, et al. Multi-centre study on post-ictal headache in patients with localization-related epilepsy. Psychiatry Clin Neurosci 2002; 53: 385-389.

Ito M, Adachi N, Nakamura F, Koyama T, Okamura T, Kato M, Kanemoto K, Nakano T, Matsuura M., Hara S. Characteristics of postictal headache in patients with partial epilepsy. Cephalalgia 2004; 24:23-28.

Karaali-Savrun F, Göksan B, Yeni SN, Ertan S, Uzun N. Seizure-related headache in patients with epilepsy. Seizure 2002; 11: 67-69.
Kwan P, Man CBL, Leung H, Yu E, Wong KS. Headache in patients with epilepsy: A prospective incidence study. Epilepsia 2008; 49: 1099-1102.

Leniger T, Isbruch K, Von den Driesch S, Diener HC, Hufnagel A. Seizure-associated headache in epilepsy. Epilepsia 2001; 42: 1176-1179.

Schachter SC, Richman K, Loder E, Beluk S. Self-reported characteristics of postictal headaches. J Epilepsy 1995; 8: 41-43.

Schmidt Botha S, Schutte C-M, Olorunju S, Kakaza M. Postictal headache in South African adult patients with generalized epilepsy in a tertiary care setting: A cross-sectional study. Cephalalgia 2012; 30: 1495-1501.

Schon F, Blau JN. Post-epileptic headache and migraine. J Neurol Neurosurg Psychiat 1987; 50: 1148-1152.

Syversten M, Helde G, Stovner LJ, Brodtkorb E. Headache add to the burden of epilepsy. J Headache Pain 2007; 8: 224-230.

Yankovsky AE, Andermann F, Bernasconi A. Characteristics of headache associated with intractable partial epilepsy. Epilepsia 2005; 46: $1241-1245$

7.7 Kopfschmerz zurückzuführen auf eine Chiari-Malformation Typ I(CM1)

Abu-Arafeh I, Campbell E. Headache, chiari malformation type 1 and treatment options. Arch Dis Child 2017; 102: 210-211.

Aiken AH, Hoots JA, Saindane AM, Hudgins PA. Incidence of cerebellar tonsillar ectopia in idiopathic intracranial hypertension: A mimic of the chiari I malformation. Am J Neuroradiol 2012; 33: 1901-1906.

Banik R, Lin D, Miller NR. Prevalence of chiari I malformation and cerebellar ectopia in patients with pseudotumor cerebri. J Neurol Sci 2006; 247: 71-75.

Batzdorf U, McArthur DL, Bentson JR. Surgical treatment of chiari malformation with and without syringomyelia: Experience with 177 adult patients. J Neurosurg 2013; 118: 232-242.

Beretta E, Vetrano IG, Curone M, Chiapparini L, Furlanetto M, Bussone G, Valentini LG. Chiari malformation-related headache: Outcome after surgical treatment. Neurol Sci 2017; 38: 95-98.

Chavez A, Roguski M, Killeen A, Heilman C, Hwang S. Comparison of operative and non-operative outcomes based on surgical selection criteria for patients with chiari I malformations. J Clin Neurosci 2014; 21: 2201-2206.

Chen P-K, Fuh J-L \& Wang S-J. Cough headache: a study of 83 consecutive patients. Cephalalgia 2009; 29: 1079-1085.

Curone M, Valentini LG, Vetrano I, et al. Chiari malformation type 1-related headache: The importance of a multidisciplinary study. Neurol Sci 2017; 38: 91-93.

Grazzi L. Usai S. Headache and Chiari malformation in young age: clinical aspects and differential diagnosis. Neurol Sci 2011; 32 Suppl 3: S299-S301.

Kahn EN, Muraszko KM, Maher CO. Prevalence of chiari I malformation and syringomyelia. Neurosurg Clin N Am 2015; 26: 501-507.

Killeen A, Roguski M, Chavez A, Heilman C, Hwang S. Non-operative outcomes in chiari I malformation patients. J Clin Neurosci 2015; 22: 133-138.

Lam S, Auffinger B, Tormenti M, Bonfield C, Greene S. The relationship between obesity and symptomatic chiari I malformation in the pediatric population. J Pediatr Neurosci 2015; 10: 321-325.

Langridge B, Phillips E, Choi D. Chiari malformation type 1: A systematic review of natural history and conservative management. World Neurosurg 2017; 104: 213-219.

Mea E. Chiapparini L. Leone M. Franzini A. Messina G. Bussone G. Chronic daily headache in the adults: differential diagnosis between symptomatic Chiari I malformation and spontaneous intracranial hypotension. Neurol Sci 2011; 32 Suppl 3: S291-S294. 
Pascual J, Iglesias F, Oterino A, Vazquez-Barquero A, Berciano J. Cough, exertional, and sexual headaches: an analysis of 72 benign and symptomatic cases. Neurology 1996; 46: 1520-1524.

Pascual J, González-Mandly A, Martín R, Oterino A. Headaches precipitated by cough, prolonged exercise or sexual activity: a prospective etiological and clinical study. J Headache Pain 2008; 9: 259-266.

Raza-Knight S, Mankad K, Prabhakar P, Thompson D. Headache outcomes in children undergoing foramen magnum decompression for chiari I malformation. Arch Dis Child 2017; 102: 238-243.

Toldo I, Tangari M, Mardari R, et al. Headache in children with chiari I malformation. Headache 2014; 54: 899-908.

\section{Kopfschmerz zurückzuführen auf eine Substanz oder deren Entzug}

\begin{tabular}{|c|c|}
\hline 8.1 & $\begin{array}{l}\text { Kopfschmerz zurückzuführen auf Substanzgebrauch oder } \\
\text { Substanzexposition }\end{array}$ \\
\hline 8.1 .1 & Kopfschmerz induziert durch Stickoxid (NO)-Donatoren \\
\hline 8.1.1.1 & $\begin{array}{l}\text { Sofortiger Kopfschmerz induziert durch Stickoxid } \\
\text { (NO)-Donatoren }\end{array}$ \\
\hline 8.1 .1 .2 & $\begin{array}{l}\text { Verzögerter Kopfschmerz induziert durch Stickoxid } \\
\text { (NO)-Donatoren }\end{array}$ \\
\hline 8.1 .2 & $\begin{array}{l}\text { Kopfschmerz induziert durch Phosphodiesterase } \\
\text { (PDE)-Hemmer }\end{array}$ \\
\hline 8.1 .3 & Kopfschmerz induziert durch Kohlenmonoxid (CO) \\
\hline 8.1 .4 & Kopfschmerz induziert durch Alkohol \\
\hline 8.1.4.1 & Sofortiger Kopfschmerz induziert durch Alkohol \\
\hline 8.1.4.2 & Verzögerter Kopfschmerz induziert durch Alkohol \\
\hline 8.1 .5 & Kopfschmerz induziert durch Kokain \\
\hline 8.1 .6 & Kopfschmerz induziert durch Histamin \\
\hline 8.1.6.1 & Sofortiger Kopfschmerz induziert durch Histamin \\
\hline 8.1.6.2 & Verzögerter Kopfschmerz induziert durch Histamin \\
\hline 8.1 .7 & $\begin{array}{l}\text { Kopfschmerz induziert durch Calcitonin-Gene-Related } \\
\text { Peptide (CGRP) }\end{array}$ \\
\hline 8.1.7.1 & Sofortiger Kopfschmerz induziert durch CGRP \\
\hline 8.1.7.2 & Verzögerter Kopfschmerz induziert durch CGRP \\
\hline 8.1 .8 & $\begin{array}{l}\text { Kopfschmerz zurückzuführen auf einen akuten Blutdruck- } \\
\text { anstieg durch eine exogene Substanz }\end{array}$ \\
\hline 8.1 .9 & $\begin{array}{l}\text { Kopfschmerz zurückzuführen auf den gelegentlichen } \\
\text { Gebrauch einer nicht gegen Kopfschmerz eingesetzten } \\
\text { Medikation }\end{array}$ \\
\hline 8.1 .10 & $\begin{array}{l}\text { Kopfschmerz zurückzuführen auf eine nicht gegen Kopf- } \\
\text { schmerz eingesetzte Dauermedikation }\end{array}$ \\
\hline 8.1 .11 & $\begin{array}{l}\text { Kopfschmerz zurückzuführen auf Gebrauch oder Exposi- } \\
\text { tion einer anderen Substanz }\end{array}$ \\
\hline 8.2 & $\begin{array}{l}\text { Kopfschmerz zurückzuführen auf einen Medikamenten- } \\
\text { übergebrauch }\end{array}$ \\
\hline 8.2 .1 & $\begin{array}{l}\text { Kopfschmerz zurückzuführen auf einen Ergotaminüber- } \\
\text { gebrauch }\end{array}$ \\
\hline 8.2 .2 & $\begin{array}{l}\text { Kopfschmerz zurückzuführen auf einen Triptanüber- } \\
\text { gebrauch }\end{array}$ \\
\hline 8.2 .3 & $\begin{array}{l}\text { Kopfschmerz zurückzuführen auf einen Übergebrauch von } \\
\text { Nicht-Opioid-Analgetika }\end{array}$ \\
\hline 8.2.3.1 & $\begin{array}{l}\text { Kopfschmerz zurückzuführen auf einen Übergebrauch von } \\
\text { Paracetamol }\end{array}$ \\
\hline
\end{tabular}

\begin{tabular}{|c|c|}
\hline 8.2 .3 .2 & $\begin{array}{l}\text { Kopfschmerz zurückzuführen auf einen Übergebrauch von } \\
\text { nicht-steroidalen Antirheumatika (NSAR) }\end{array}$ \\
\hline 8.2.3.2.1 & $\begin{array}{l}\text { Kopfschmerz zurückzuführen auf einen Übergebrauch von } \\
\text { Acetylsalicylsäure }\end{array}$ \\
\hline 8.2.3.3 & $\begin{array}{l}\text { Kopfschmerz zurückzuführen auf einen Übergebrauch von } \\
\text { anderen Nicht-Opioid-Analgetika }\end{array}$ \\
\hline 8.2 .4 & $\begin{array}{l}\text { Kopfschmerz zurückzuführen auf einen Opioidüber- } \\
\text { gebrauch }\end{array}$ \\
\hline 8.2 .5 & $\begin{array}{l}\text { Kopfschmerz zurückzuführen auf einen Übergebrauch von } \\
\text { Schmerzmittelmischpräparaten }\end{array}$ \\
\hline 8.2 .6 & $\begin{array}{l}\text { Kopfschmerz zurückzuführen auf einen Übergebrauch von } \\
\text { Medikamenten aus mehreren Klassen, ohne Überge- } \\
\text { brauch der Einzelsubstanzen }\end{array}$ \\
\hline 8.2 .7 & $\begin{array}{l}\text { Kopfschmerz zurückzuführen auf einen unspezifiziertem } \\
\text { oder unbestätigtem Übergebrauch von Medikamenten } \\
\text { aus mehreren Klassen }\end{array}$ \\
\hline 8.2 .8 & $\begin{array}{l}\text { Kopfschmerz zurückzuführen auf einen Übergebrauch } \\
\text { einer anderen Medikation }\end{array}$ \\
\hline 8.3 & $\begin{array}{l}\text { Kopfschmerz zurückzuführen auf den Entzug einer } \\
\text { Substanz }\end{array}$ \\
\hline 8.3 .1 & Koffeinentzugskopfschmerz \\
\hline 8.3 .2 & Opioidentzugskopfschmerz \\
\hline 8.3 .3 & Östrogenentzugskopfschmerz \\
\hline 8.3 .4 & $\begin{array}{l}\text { Kopfschmerz zurückzuführen auf den Entzug anderer } \\
\text { dauerhaft eingenommener Substanzen }\end{array}$ \\
\hline
\end{tabular}

An anderer Stelle kodiert: 7.1.2 Kopfschmerz zurückzuführen auf eine sekundäre Liquordrucksteigerung metabolischer, toxischer oder hormoneller Genese; 7.3.2 Kopfschmerz zurückzuführen auf eine aseptische (nichtinfektiöse) Meningitis.

\section{Allgemeiner Kommentar}

Primärer und/oder sekundärer Kopfschmerz?

Für Kapitel 8. Kopfschmerz zurückzuführen auf eine Substanz oder deren Entzug gelten mit einigen Anpassungen die allgemeinen Regeln für die kausale Zuordnung zu einer anderen Erkrankung.

Tritt ein neuer Kopfschmerz erstmals in engem zeitlichen Zusammenhang mit einer Substanzexposition oder dem Entzug einer Substanz auf, wird der Kopfschmerz als sekundärer Kopfschmerz zurückzuführen auf die Exposition gegenüber dieser Substanz oder deren Entzug kodiert. Dies ist auch der Fall, wenn der Kopfschmerz die Charakteristika eines primären Kopfschmerzes zeigt, der in Teil 1 der ICHD-3 klassifiziert ist.

Wenn aber ein vorbestehender Kopfschmerz mit den Charakteristika eines primären Kopfschmerzes in engem zeitlichen Zusammenhang mit einer Substanzexposition oder dem Entzug einer Substanz chronisch wird oder sich deutlich verschlechtert (üblicherweise definiert als eine mindestens zweifache Steigerung der Häufigkeit und/oder Schwere), dann sollte sowohl die vorbestehende primäre Kopfschmerzdiagnose als auch die Diagnose 8. Kopfschmerz zurückzuführen auf eine Substanz oder deren Entzug (oder eines seiner Typen oder Subtypen) vergeben werden, sofern gute Hinweise dafür bestehen, dass die Exposition gegenüber dieser Substanz oder ihr Entzug Kopfschmerzen verursachen kann.

Bestimmte Subtypen von Kopfschmerz zurückzuführen auf die Exposition einer pharmakologisch aktiven Substanz treten einige 
Stunden nach der Exposition auf, und zwar nur bei Patienten mit einer primären Kopfschmerzerkrankung. Sie ähneln dann phänomenologisch dem primären Kopfschmerztyp. Dabei wird ein vom primären Kopfschmerz unterschiedlicher Mechanismus angenommen, nämlich eine Reaktion auf einen nicht physiologischen Reiz, und diese Kopfschmerzen werden daher als sekundär angesehen. Als Diagnose sollte sowohl die primäre Kopfschmerzform als auch der zutreffende Subtyp von 8.1. Kopfschmerz zurückzuführen auf Substanzgebrauch oder Substanzexposition vergeben werden.

\section{Einleitung}

Menschen mit 1. Migräne zeigen eine physiologische und vielleicht auch psychische Überreaktion auf eine Vielzahl innerer und äußerer Reize. Alkohol, Nahrungsmittel und Nahrungszusatzstoffe, sowie Einnahme oder Entzug von Medikamenten und Chemikalien können Berichten zufolge bei empfänglichen Personen Migräne auslösen oder aktivieren.

Die angenommene Beziehung zwischen Kopfschmerz und Substanz basiert dabei häufig auf Einzelfallschilderungen oder Berichten über unerwünschte Wirkungen von Medikamenten. Die Tatsache der Assoziation mit Kopfschmerzen beweist nicht ihre Kausalität und entbindet nicht von der Notwendigkeit, nach anderen Ursachen zu suchen. Da häufige Ereignisse eben häufig sind, kann das Zusammentreffen von Kopfschmerzen und einer Substanzexposition reiner Zufall sein. Kopfschmerzen können zufällig auftreten. Kopfschmerzen können Symptom einer systemischen Erkrankung sein und Medikamente, die zu deren Behandlung eingesetzt werden, werden dann fälschlicherweise mit den Kopfschmerzen in Verbindung gebracht. In den Migräneakutmedikationsstudien sind insbesondere Kopfschmerzen, genauso wie Migräne-Begleitsymptome, als unerwünschte Wirkungen aufgeführt, auch wenn es sich um Symptome der behandelten Erkrankung und nicht um eine Folge der Behandlung handelt. Einige Erkrankungen scheinen zum Auftreten von substanzinduzierten Kopfschmerzen zu prädisponieren: Für sich allein genommen, würde weder die Substanz noch die Erkrankung Kopfschmerzen hervorrufen.

Allgemeine Kriterien für die hier aufgeführten Kopfschmerzerkrankungen sind:

A. Die Kopfschmerzen erfüllen Kriterium C

B. Gebrauch, Exposition oder Entzug einer Substanz, die bekanntermaßen Kopfschmerzen verursachen kann

C. Ein kausaler Zusammenhang kann durch 2 der folgenden Kriterien gezeigt werden:

1. Der Kopfschmerz hat sich in zeitlichem Zusammenhang mit dem Gebrauch, der Exposition oder dem Entzug der Substanz entwickelt

2. Einer der beiden folgenden Punkte ist erfüllt:

a) Der Kopfschmerz hat sich in engem zeitlichen Zusammenhang mit dem Ende des Gebrauchs oder der Exposition der Substanz deutlich gebessert oder ist verschwunden

b) Der Kopfschmerz hat sich innerhalb eines definierten

Zeitraums nach Entzug der Substanz deutlich gebessert

3. Die Kopfschmerz-Charakteristika sind typisch für den Gebrauch, die Exposition oder den Entzug der Substanz
4. Es bestehen sonstige Hinweise auf den ursächlichen Zusammenhang

D. Nicht besser erklärt durch eine andere ICHD-3-Diagnose.

\subsection{Kopfschmerz zurückzuführen auf Substanzgebrauch oder Substanz- exposition \\ Beschreibung:}

Kopfschmerz verursacht durch Substanzgebrauch oder Substanzexposition, mit Beginn sofort oder innerhalb von Stunden.

\section{Kommentar:}

8.1 Kopfschmerz zurückzuführen auf Substanzgebrauch oder Substanzexposition kann durch eine unerwünschte Wirkung einer Substanz im Rahmen des normalen therapeutischen Einsatzes oder in experimentellen Untersuchungen oder durch eine toxische Substanz verursacht werden.

Kopfschmerzen als unerwünschte Wirkung sind für viele Medikamente dokumentiert, wobei dies wahrscheinlich oft nur die ohnehin hohe Prävalenz von Kopfschmerzen widerspiegelt. Nur wenn Kopfschmerzen in doppelblind-kontrollierten Studien häufiger nach Verum als nach Placebo auftreten, können sie als echte unerwünschte Wirkung betrachtet werden. In einem doppelblinden Studiendesign kann die Beziehung zwischen Medikamentenwirkung und Kopfschmerzen auch experimentell untersucht werden. Solche Untersuchungen können, wie z. B. bei den Stickoxid (NO)-Donatoren, zu einem tieferen Verständnis der Beteiligung von Neurotransmittern bei der Entstehung von primären Kopfschmerzen beitragen.

Allgemein sind Menschen mit 1. Migräne sehr viel empfänglicher für die Entwicklung derartiger Kopfschmerzen, und dasselbe gilt möglicherweise auch für Menschen mit 2. Kopfschmerz vom Spannungstyp und 3.1. Clusterkopfschmerz. Eine Reihe von Substanzen wie Stickoxid (NO)-Donatoren oder Histamin lösen sowohl bei Gesunden als auch bei Migränepatienten einen sofortigen Kopfschmerz aus. Inzwischen weiß man, dass Menschen mit primären Kopfschmerzerkrankungen darüber hinaus einen verzögerten Kopfschmerz entwickeln können, der eine Stunde bis mehrere Stunden nach Elimination der Substanz aus dem Blut auftritt.

Im klinischen Alltag verwendete Substanzen mit potentiell kopfschmerzauslösendem Effekt sollten entsprechend gekennzeichnet werden. In manchen Fällen löst auch nur die Kombination von Substanzen, aber nicht die einzelne Substanz einen Kopfschmerz aus, wie z. B. bei der Kombination aus Alkohol und Disulfiram.

Paradoxerweise können Kopfschmerzen nach starkem Alkoholkonsum auch einen positiven Effekt haben, indem sie zukünftigen exzessiven Konsum vermeiden helfen.

Substanzen wie Kohlenmonoxid (CO), die durch ihre toxische Wirkung Kopfschmerzen verursachen, können verständlicherweise nicht experimentell untersucht werden. Die kausale Verknüpfung zwischen Exposition und Kopfschmerzen kann hier nur aus Fallbeschreibungen abgeleitet werden, in denen die Substanzen versehentlich oder in suizidaler Absicht angewendet wurden. 


\subsubsection{Kopfschmerz induziert durch Stickoxid (NO)-Donatoren}

Beschreibung:

Kopfschmerz, der sofort oder verzögert durch akute Exposition gegenüber einem Stickoxid (NO)-Donator verursacht ist. Der Kopfschmerz geht spontan wieder zurück.

\section{Kommentare:}

8.1.1 Kopfschmerz induziert durch Stickoxid (NO)-Donatoren ist typischerweise frontotemporal und pulsierend. Alle NO-Donatoren (z. B. Amylnitrat, Erythrityltetranitrat, Pentaerythrityltetranitrat, Glyzeroltrinitrat, Isosorbidmono- oder -dinitrat, Natriumnitroprussid, Mannitolhexanitrat) können diese Art Kopfschmerz hervorrufen.

Glyzeroltrinitrat (Nitrogylzerin) induziert bei den meisten Menschen einen Sofortkopfschmerz. Bei Patienten mit 1. Migräne kann es einen verzögerten Kopfschmerz hervorrufen, der die diagnostischen Kriterien für eine 1.1 Migräne ohne Aura erfüllt. Bei Patienten mit 2.3 chronischem Kopfschmerz vom Spannungstyp kann Nitroglyzerin einen verzögerten Kopfschmerz induzieren, der das klinische Bild eines 2. Kopfschmerzes vom Spannungstyp aufweist. Dieser Effekt ist bei Patienten mit einem 2.1 selten oder 2.2 häufig auftretendem episodischen Kopfschmerz vom Spannungstyp nicht bekannt. Diese verzögerten Kopfschmerzen treten im Mittel 5-6 Stunden nach der Exposition auf. Menschen mit 3. Clusterkopfschmerz entwickeln einen Nitroglyzerin-induzierten verzögerten Kopfschmerz nur während der Clusterepisoden, üblicherweise 1-2 Stunden nach der Exposition.

Kopfschmerzen sind eine bekannte unerwünschte Wirkung beim therapeutischen Einsatz von Nitroglyzerin. Beim Dauereinsatz entwickelt sich innerhalb einer Woche eine Toleranz, und bei den meisten Betroffenen verschwindet der Nitroglyzerin-induzierte Kopfschmerz nach dieser Zeit. Andere therapeutisch eingesetzte NO-Donatoren können auch Kopfschmerzen induzieren. Isosorbidmononitrat wurde in einer einzelnen doppelblind-kontrollierten Studie untersucht und verursacht einen länger anhaltenden Kopfschmerz als Nitroglyzerin, was auf eine langsamere Freisetzung von Stickoxid zurückzuführen ist.

\subsubsection{Sofortiger Kopfschmerz induziert durch Stickoxid (NO)-Donatoren}

Früher verwendete Begriffe: Nitroglyzerin-Kopfschmerz, Dynamit-Kopfschmerz, Hot-Dog-Kopfschmerz.

\section{Diagnostische Kriterien:}

A. Kopfschmerz, der das Kriterium C erfüllt

B. Aufnahme eines Stickoxid (NO)-Donators

C. Ein kausaler Zusammenhang kann durch alle der folgenden Kriterien gezeigt werden:

1. Der Kopfschmerz hat sich innerhalb von 1 Stunde nach Aufnahme des NO-Donators entwickelt

2. Der Kopfschmerz ist innerhalb 1 Stunde nach Ende der NO-Freisetzung verschwunden

3. Der Kopfschmerz weist zumindest eines der nachfolgenden vier Charakteristika auf:

a) bilateral

b) leichte bis mittlere Schmerzintensität c) pulsierender Charakter

d) Verstärkung durch körperliche Aktivität

D. Nicht besser erklärt durch eine andere ICHD-3-Diagnose.

\subsubsection{Verzögerter Kopfschmerz induziert durch Stick- oxid (NO)-Donatoren}

\section{Diagnostische Kriterien:}

A. Kopfschmerz bei einer Person mit einer primären Kopfschmerzerkrankung, der das klinische Bild des primären Kopfschmerzes und Kriterium C erfüllt

B. Aufnahme eines Stickoxid (NO)-Donators

C. Ein kausaler Zusammenhang kann durch beide der folgenden Kriterien gezeigt werden:

1. Der Kopfschmerz entwickelt sich innerhalb 2 bis 12 Stunden nach der Exposition, und nachdem NO bereits aus dem Körper eliminiert wurde

2. Der Kopfschmerz verschwindet innerhalb von 72 Stunden nach der Exposition

D. Nicht besser erklärt durch eine andere ICHD-3-Diagnose1.

\section{Anmerkung:}

1. Phänomenologisch ähnelt der 8.1.1.2 verzögerte Kopfschmerz induziert durch Stickoxid (NO)-Donatoren dem primären Kopfschmerz des Patienten, aber er wird als sekundärer, auf die Substanz zurückzuführender Kopfschmerz angesehen. Der Patient sollte sowohl die Diagnose der primären Kopfschmerzerkrankung als auch die Diagnose 8.1.1.2 verzögerter Kopfschmerz induziert durch Stickoxid (NO)-Donatoren erhalten.

\section{Kommentar:}

Obwohl 8.1.1.2 verzögerter Kopfschmerz induziert durch Stickoxid (NO)-Donatoren nur bei Personen mit primärer Kopfschmerzerkrankung auftritt und dann phänomenologisch diesem Kopfschmerztyp ähnelt, wird angenommen, dass sein Mechanismus sich von dem des primären Kopfschmerzes unterscheidet.

\subsubsection{Kopfschmerz induziert durch Phosphodie- sterase (PDE)-Hemmer}

Beschreibung:

Kopfschmerz, der durch Aufnahme eines Phosphodiesterase-Hemmers verursacht ist und innerhalb von 72 Stunden spontan wieder zurückgeht.

Diagnostische Kriterien:

A. Kopfschmerz, der das Kriterium C erfüllt

B. Einnahme eines Phosphodiesterase-Inhibitors

C. Ein kausaler Zusammenhang kann durch alle der folgenden Kriterien gezeigt werden:

1. Der Kopfschmerz hat sich innerhalb von 5 Stunden nach Einnahme des Phosphodiesterase-Inhibitors entwickelt

2. Der Kopfschmerz ist innerhalb von 72 Stunden nach Beginn verschwunden

3. Der Kopfschmerz weist zumindest eines der nachfolgenden vier Charakteristika auf:

a) bilateral

b) leichte bis mittlere Schmerzintensität 
c) pulsierender Charakter

d) Verstärkung durch körperliche Aktivität

D. Nicht besser erklärt durch eine andere ICHD-3-Diagnose.

Kommentar:

Phosphodiesterasen sind Enzyme, die die zyklischen Nukleotide cGMP und CAMP abbauen. Die Phosphodiesterase-5-Inhibitoren Sildenafil und Dipyridamol führen zu einem Anstieg von cGMP und/ oder cAMP. Der Kopfschmerz ist meist spannungskopfschmerzartig, bei Personen mit 1. Migräne (die auf diese unerwünschte Wirkung aufmerksam gemacht werden sollten) hat er allerdings das klinische Bild einer 1.1 Migräne ohne Aura.

\subsubsection{Kopfschmerz induziert durch Kohlenmonoxid (CO)}

Früher verwendete Begriffe: Lagerarbeiter-Kopfschmerz.

Beschreibung:

Kopfschmerz, der durch Kohlenmonoxid (CO)-Exposition verursacht ist und innerhalb von 72 Stunden nach Elimination des CO spontan wieder zurückgeht.

\section{Diagnostische Kriterien:}

A. Bilateraler Kopfschmerz, der das Kriterium C erfüllt

B. Kohlenmonoxid (CO)-Exposition

C. Ein kausaler Zusammenhang kann durch alle der folgenden Kriterien gezeigt werden:

1. Der Kopfschmerz hat sich innerhalb von 12 Stunden nach Exposition entwickelt

2. Die Kopfschmerzintensität entspricht dem Schweregrad der CO-Intoxikation

3. Der Kopfschmerz ist innerhalb von 72 Stunden nach CO-Elimination verschwunden

D. Nicht besser erklärt durch eine andere ICHD-3-Diagnose.

\section{Kommentare:}

Typischerweise besteht ein leichter Kopfschmerz ohne gastrointestinale oder neurologische Symptome bei einem Carboxyhämoglobingehalt von $10 \%$ bis $20 \%$, ein mittelstarker pochender Kopfschmerz und eine erhöhte Reizbarkeit bei einem Carboxyhämoglobingehalt von $20 \%$ bis $30 \%$ und ein starker Kopfschmerz mit Übelkeit, Erbrechen und Verschwommensehen bei einem Carboxyhämoglobingehalt von $30 \%$ bis $40 \%$. Bei einem Carboxyhämoglobingehalt über $40 \%$ wird üblicherweise nicht mehr über Kopfschmerzen geklagt, da es zu Bewusstseinsstörungen kommt.

Es existieren keine guten Studien zu Langzeiteffekten einer CO-Intoxikation auf Kopfschmerz, aber es gibt Hinweise darauf, dass ein chronischer Kopfschmerz nach CO-Intoxikation existiert.

\subsubsection{Kopfschmerz induziert durch Alkohol}

\section{Beschreibung:}

Kopfschmerz, der sofort oder verzögert durch Aufnahme von Alkohol (meist in Form alkoholischer Getränke) entsteht. Der Kopfschmerz geht spontan wieder zurück.

\subsubsection{Sofortiger Kopfschmerz induziert durch Alkohol}

Früher verwendete Begriffe: Cocktail-Kopfschmerz.
Diagnostische Kriterien:

A. Kopfschmerz, der das Kriterium C erfüllt

B. Aufnahme von Alkohol

C. Ein kausaler Zusammenhang kann durch alle der folgenden Kriterien gezeigt werden:

1. Der Kopfschmerz hat sich innerhalb von 3 Stunden nach der Alkoholaufnahme entwickelt

2. Der Kopfschmerz ist innerhalb von 72 Stunden nach Beendigung der Alkoholaufnahme verschwunden

3. Der Kopfschmerz weist wenigstens eines der nachfolgenden Charakteristika auf:
a) bilateral
b) pulsierender Charakter
c) Verstärkung durch körperliche Aktivität

D. Nicht besser erklärt durch eine andere ICHD-3-Diagnose.

\section{Kommentar:}

8.1.4.1 sofortiger Kopfschmerz induziert durch Alkohol ist wesentlich seltener als 8.1.4.2 verzögerter Kopfschmerz induziert durch Alkohol. Die Schwellendosis zur Induktion des ersteren ist variabel und kann bei Personen mit einer 1. Migräne zu manchen Zeitpunkten sehr niedrig sein, während sie zu anderen Zeitpunkten Alkohol in ähnlichen Mengen wie Personen ohne Migräne vertragen können.

\subsubsection{Verzögerter Kopfschmerz induziert durch Alkohol}

Früher verwendete Begriffe: Katerkopfschmerz.

\section{Beschreibung:}

Alkohol-induzierter Kopfschmerz, der Stunden nach der Aufnahme von Alkohol (meist in Form alkoholischer Getränke) auftritt und innerhalb von 72 Stunden spontan zurückgeht.

Diagnostische Kriterien:

A. Kopfschmerz, der das Kriterium C erfüllt

B. Aufnahme von Alkohol

C. Ein kausaler Zusammenhang kann durch alle der folgenden Kriterien gezeigt werden:

1. Der Kopfschmerz hat sich 5 bis 12 Stunden nach der Aufnahme von Alkohol entwickelt

2. Der Kopfschmerz ist innerhalb von 72 Stunden nach Beginn verschwunden

3. Der Kopfschmerz weist wenigstens eins der folgenden drei Charakteristika auf:
a) bilateral
b) pulsierender Charakter
c) Verstärkung durch körperliche Aktivität

D. Nicht besser erklärt durch eine andere ICHD-3-Diagnose.

\section{Kommentar:}

8.1.4.2 verzögerter Kopfschmerz induziert durch Alkohol ist eine der häufigsten sekundären Kopfschmerzarten. Es ist unklar, ob als Mechanismus eine verzögerte toxische Wirkung angenommen werden kann, oder ob ähnliche Mechanismen wie bei einem 8.1.1.2 verzögerten Kopfschmerz hervorgerufen durch Stickoxid (NO)-Donatoren involviert sind. 


\subsubsection{Kopfschmerz induziert durch Kokain}

Beschreibung:

Kopfschmerz verursacht durch Kokain, auftretend innerhalb von 1 Stunde nach Aufnahme, unabhängig vom Verabreichungsweg. Der Kopfschmerz geht spontan innerhalb von 72 Stunden zurück. Diagnostische Kriterien:

A. Kopfschmerz, der das Kriterium C erfüllt

B. Aufnahme von Kokain, unabhängig vom Verabreichungsweg

C. Ein kausaler Zusammenhang kann durch alle der folgenden Kriterien gezeigt werden:

1. Der Kopfschmerz hat sich innerhalb von 1 Stunde nach der Aufnahme entwickelt

2. Der Kopfschmerz ist innerhalb von 72 Stunden nach der Aufnahme verschwunden

3. Der Kopfschmerz weist wenigstens eins der folgenden vier Charakteristika auf:

a) bilateral

b) leichte bis mittlere Schmerzintensität

c) pulsierender Charakter

d) Verstärkung durch körperliche Aktivität

D. Nicht besser erklärt durch eine andere ICHD-3-Diagnose.

\section{Kommentar:}

Die Hauptwege der Kokainaufnahme sind oral, intranasal („Schnupfen“, „Ziehen”), intravenös und inhalativ (Rauchen).

\subsubsection{Kopfschmerz induziert durch Histamin}

Beschreibung:

Kopfschmerz, der sofort oder verzögert durch akute Exposition gegenüber Histamin verursacht ist. Der Kopfschmerz geht spontan wieder zurück.

\section{Kommentare:}

Die kopfschmerzinduzierende Wirkung von Histamin wurde nach i.v.-Gabe, subkutaner Anwendung und nach Inhalation getestet. Alle Darreichungsrouten hatten den gleichen Effekt. Die Wirkung ist vornehmlich über den H1-Rezeptor vermittelt, da sie fast komplett durch Mepyramin blockiert werden kann.

Histamin ruft bei den meisten Menschen einen sofortigen Kopfschmerz hervor. Bei Menschen mit 1. Migräne kann es einen verzögerten Kopfschmerz induzieren, der die diagnostischen Kriterien für 1.1. Migräne ohne Aura erfüllt. Bei Menschen mit 2. Kopfschmerz vom Spannungstyp kann Histamin einen verzögerten Kopfschmerz mit den Charakteristika des Kopfschmerzes vom Spannungstyp hervorrufen. Diese verzögerten Kopfschmerzen treten im Mittel 5 bis 6 Stunden nach der Exposition auf. Menschen mit 3. Clusterkopfschmerz entwickeln nur während der Clusterepisoden einen verzögerten Kopfschmerz mit den Charakteristika eines Clusterkopfschmerzes, meist 1 bis 2 Stunden nach der Exposition.

\subsubsection{Sofortiger Kopfschmerz induziert durch Histamin} Diagnostische Kriterien:

A. Kopfschmerz, der das Kriterium C erfüllt

B. Histaminexposition

C. Ein kausaler Zusammenhang kann durch alle der folgenden Kriterien gezeigt werden:
1. Der Kopfschmerz hat sich innerhalb von 1 Stunde nach der Histaminexposition entwickelt

2. Der Kopfschmerz ist innerhalb von 1 Stunde nach Ende der Histaminexposition verschwunden

3. Der Kopfschmerz weist wenigstens eins der folgenden vier Charakteristika auf:

a) bilateral

b) leichte bis mittlere Schmerzintensität

c) pulsierender Charakter

d) Verstärkung durch körperliche Aktivität

D. Nicht besser erklärt durch eine andere ICHD-3-Diagnose.

\subsubsection{Verzögerter Kopfschmerz induziert durch Histamin \\ Diagnostische Kriterien:}

A. Kopfschmerz bei einer Person mit einer primären Kopfschmerzerkrankung, der das klinische Bild des primären Kopfschmerzes und Kriterium C erfüllt

B. Histaminexposition

C. Ein kausaler Zusammenhang kann durch beide der folgenden Kriterien gezeigt werden:

1. Der Kopfschmerz hat sich innerhalb von 2 bis 12 Stunden nach der Histaminexposition entwickelt

2. Der Kopfschmerz ist innerhalb von 72 Stunden nach der Histaminexposition verschwunden

D. Nicht besser erklärt durch eine andere ICHD-3-Diagnose1.

Anmerkung:

1. Phänomenologisch ähnelt 8.1.6.2 verzögerter Kopfschmerz induziert durch Histamin dem primären Kopfschmerz des Patienten, aber er wird als sekundärer, auf die Substanz zurückzuführender Kopfschmerz angesehen. Der Patient sollte sowohl die Diagnose der primären Kopfschmerzerkrankung als auch die Diagnose 8.1.6.2 verzögerter Kopfschmerz induziert durch Histamin erhalten.

\section{Kommentar:}

Obwohl ein 8.1.6.2 verzögerter Kopfschmerz induziert durch Histamin nur bei Personen mit primärer Kopfschmerzerkrankung auftritt und dann phänomenologisch diesem Kopfschmerztyp ähnelt, wird angenommen, dass sein Mechanismus sich von dem des primären Kopfschmerzes unterscheidet.

\subsubsection{Kopfschmerz induziert durch Calcitonin-Gene- Related Peptide (CGRP)}

Beschreibung:

Kopfschmerz, der sofort oder verzögert durch akute Exposition gegenüber Calcitonin-Gene-Related Peptide (CGRP) verursacht ist. Der Kopfschmerz geht spontan wieder zurück.

Kommentar:

Intravenöse Gabe von Calcitonin-Gene-Related Peptide (CGRP) führt zu einem sofortigen Kopfschmerz. Bei Personen mit 1. Migräne kann es 5 bis 6 Stunden nach der Exposition einen verzögerten Kopfschmerz auslösen, der die Kriterien von 1.1 Migräne ohne Aura erfüllt. 
Eine wachsende Anzahl von CGRP-Rezeptor-Antagonisten hat sich als wirksam in der Akutbehandlung der Migräne gezeigt.

\subsubsection{Sofortiger Kopfschmerz induziert durch CGRP} Diagnostische Kriterien:

A. Kopfschmerz, der das Kriterium C erfüllt

B. Aufnahme von Calcitonin-Gene-Related Peptide (CGRP)

C. Ein kausaler Zusammenhang kann durch alle der folgenden Kriterien gezeigt werden:

1. Der Kopfschmerz entwickelt sich innerhalb von 1 Stunde nach der Aufnahme

2. Der Kopfschmerz verschwindet innerhalb von 1 Stunde nach Ende der Aufnahme

3. Der Kopfschmerz weist wenigstens eins der folgenden vier Charakteristika auf:
a) bilateral
b) leichte bis mittlere Schmerzintensität
c) pulsierender Charakter
d) Verstärkung durch körperliche Aktivität

D. Nicht besser erklärt durch eine andere ICHD-3-Diagnose.

\subsubsection{Verzögerter Kopfschmerz induziert durch CGRP} Diagnostische Kriterien:

A. Kopfschmerz bei einer Person mit 1. Migräne, der das klinische Bild dieses Kopfschmerztyps und Kriterium C erfüllt

B. Aufnahme von Calcitonin-Gene-Related Peptide (CGRP)

C. Ein kausaler Zusammenhang kann durch beide der folgenden Kriterien gezeigt werden:

1. Der Kopfschmerz entwickelt sich innerhalb von 2 bis 12 Stunden nach der Aufnahme

2. Der Kopfschmerz verschwindet innerhalb von 72 Stunden nach Ende der Aufnahme

D. Nicht besser erklärt durch eine andere ICHD-3-Diagnose1.

\section{Anmerkung:}

1. Phänomenologisch ähnelt 8.1.7.2 verzögerter Kopfschmerz induziert durch CGRP der Migräne, aber er wird als sekundärer, auf die Substanz zurückzuführender Kopfschmerz angesehen. Der Patient sollte sowohl die Diagnose 1. Migräne (oder des zutreffenden Subtyps) als auch die Diagnose 8.1.7.2 verzögerter Kopfschmerz induziert durch CGRP erhalten.

\section{Kommentar:}

Obwohl 8.1.7.2 verzögerter Kopfschmerz induziert durch CGRP nur bei Personen mit 1. Migräne auftritt und dann phänomenologisch diesem Kopfschmerztyp ähnelt, wird angenommen, dass sein Mechanismus sich von dem der Migräne unterscheidet.

\subsubsection{Kopfschmerz zurückzuführen auf einen akuten Blutdruckanstieg durch eine exogene Substanz Beschreibung: \\ Kopfschmerz auftretend während und induziert durch einen aku- ten Blutdruckanstieg, herbeigeführt durch eine exogene blutdruck- steigernde Substanz.}

Diagnostische Kriterien:

A. Kopfschmerz, der das Kriterium C erfüllt

B. Akuter Blutdruckanstieg nach Aufnahme einer exogenen blutdrucksteigernden Substanz

C. Ein kausaler Zusammenhang kann durch beide der folgenden Kriterien gezeigt werden:

1. Der Kopfschmerz entwickelt sich innerhalb von 1 Stunde nach der Aufnahme

2. Der Kopfschmerz verschwindet innerhalb von 72 Stunden nach Ende der Aufnahme

D. Nicht besser erklärt durch eine andere ICHD-3-Diagnose.

\subsubsection{Kopfschmerz zurückzuführen auf den gelegentlichen Gebrauch einer nicht gegen Kopfschmerz eingesetzten Medikation}

Beschreibung:

Kopfschmerz als akute unerwünschte Wirkung einer nicht gegen Kopfschmerz eingesetzten Medikation bei gelegentlichem Gebrauch dieser Medikation.

\section{Diagnostische Kriterien:}

A. Kopfschmerz, der das Kriterium C erfüllt

B. Eine oder mehrere Dosen eines nicht gegen Kopfschmerz eingesetzten Medikaments wurden eingenommen

C. Ein kausaler Zusammenhang kann durch beide der folgenden Kriterien gezeigt werden:

1. Der Kopfschmerz hat sich innerhalb von Minuten bis zu Stunden nach der Einnahme entwickelt

2. Der Kopfschmerz ist innerhalb von 72 Stunden nach Ende der Einnahme verschwunden

D. Nicht besser erklärt durch eine andere ICHD-3-Diagnose.

\section{Kommentar:}

Zahlreiche Medikamente wurden mit einem 8.1.9 Kopfschmerz zurückzuführen auf den gelegentlichen Gebrauch einer nicht gegen Kopfschmerz eingesetzten Medikation in Zusammenhang gebracht. Die folgenden Substanzen werden am häufigsten genannt: Atropin, Digitalis, Disulfiram, Hydralazin, Imipramin, Nikotin, Nifedipin, Nimodipin, Sildenafil.

Die Kopfschmerzcharakteristika sind in der Literatur nicht gut definiert, und hängen wahrscheinlich vom Medikament ab. Meist sind sie dumpf, kontinuierlich vorhanden, diffus lokalisiert und von mittelstarker bis starker Intensität.

\subsubsection{Kopfschmerz zurückzuführen auf eine nicht gegen Kopfschmerz eingesetzte Dauer- medikation}

An anderer Stelle kodiert: Kopfschmerz als Komplikation eines andauernden Übergebrauchs von Medikamenten zur Akutbehandlung von Kopfschmerz durch eine Person mit einer primären Kopfschmerzerkrankung wird als 8.2 Kopfschmerz zurückzuführen auf einen Medikamentenübergebrauch oder als Subtyp dieser Diagnose kodiert.

Kopfschmerz während der Pillenpause von kombinierten oralen Kontrazeptiva wird als 8.3.3 Östrogenentzugskopfschmerz kodiert. 
Beschreibung:

Der Kopfschmerz entwickelt sich als unerwünschte Wirkung während der Einnahme einer nicht gegen Kopfschmerzen eingesetzten Dauermedikation. Der Kopfschmerz ist nicht immer reversibel.

\section{Diagnostische Kriterien:}

A. Kopfschmerz an $\geq 15$ Tagen pro Monat, der das Kriterium C erfüllt

B. Dauermedikation mit einem nicht gegen Kopfschmerzen eingesetzten Medikament

C. Ein kausaler Zusammenhang kann durch mindestens zwei der folgenden Kriterien gezeigt werden:

1. der Kopfschmerz hat sich in zeitlichem Zusammenhang mit dem Beginn der Medikation entwickelt

2. einer oder mehrere der folgenden Punkte sind erfüllt:

a) Der Kopfschmerz hat nach einer Dosissteigerung deutlich zugenommen

b) Der Kopfschmerz hat sich nach einer Dosisreduktion deutlich gebessert oder ist verschwunden

c) Der Kopfschmerz ist nach Beendigung der Medikamenteneinnahme verschwunden

3. Das Medikament kann bekanntermaßen als Dauermedikation bei manchen Menschen Kopfschmerzen verursachen

D. Nicht besser erklärt durch eine andere ICHD-3-Diagnose.

\section{Kommentar:}

Die Abhängigkeit der Kopfschmerzen von der Dosis und der Dauer der Langzeiteinnahme oder -exposition ist unterschiedlich je nach Medikament. Ebenso variiert die Zeit bis zum Verschwinden des Kopfschmerzes, vorausgesetzt er ist überhaupt reversibel.

Exogene Hormone, typischerweise eingenommen zur Kontrazeption oder Hormonsubstitution, sind eine nicht gegen Kopfschmerz eingesetzte Medikation, daher schließt 8.1.10. Kopfschmerz zurückzuführen auf eine nicht gegen Kopfschmerz eingesetzte Dauermedikation den Kopfschmerz als unerwünschte Wirkung einer Hormontherapie mit ein (vorher kodiert als 8.1.12 Kopfschmerz induziert durch exogene Hormone). Die regelmäßige Einnahme von exogenen Hormonen kann mit einer Frequenzzunahme oder dem neuen Auftreten von migräneartigen oder anderen Kopfschmerzen einhergehen. Als generelle Regel findet Anwendung, dass ein zum ersten Mal in engem zeitlichen Zusammenhang mit einer regelmäßigen Hormoneinnahme auftretender Kopfschmerz als 8.1.10 Kopfschmerz zurückzuführen auf eine nicht gegen Kopfschmerz eingesetzte Dauermedikation kodiert wird. Wenn ein vorbestehender Kopfschmerz mit den Charakteristika einer primären Kopfschmerzerkrankung in engem zeitlichen Zusammenhang mit der regelmäßigen Hormoneinnahme chronisch wird, oder sich deutlich verschlechtert (üblicherweise definiert als mindestens eine zweifache Frequenz oder Schwere), dann soll sowohl die vorbestehende Kopfschmerzdiagnose als auch 8.1.10 Kopfschmerz zurückzuführen auf eine nicht gegen Kopfschmerz eingesetzte Dauermedikation vergeben werden. Dagegen wird ein Kopfschmerz, der nur während der Pillenpause eines kombinierten oralen Kontrazeptivums auftritt als 8.3.3 Östrogenentzugskopfschmerz kodiert.

Davon abgesehen, kann 8.1.10 Kopfschmerz zurückzuführen auf eine nicht gegen Kopfschmerz eingesetzte Dauermedikation
Folge eines direkten pharmakologischen Effekts der Medikation sein, wie maligne Hypertension durch Vasokonstriktion, oder sekundäre Folge einer Medikamentenwirkung wie z. B. einer medikamenteninduzierten intrakraniellen Hypertension. Letztere ist anerkannte Komplikation der Dauereinnahme von anabolen Steroiden, Amiodaron, Lithiumcarbonat, Nalidixinsäure, Schilddrüsenhormonen, Tetrazyklinen oder Minozyklin.

\subsubsection{Kopfschmerz zurückzuführen auf Gebrauch oder Exposition einer anderen Substanz}

Beschreibung:

Kopfschmerz, der während oder kurz nach dem Gebrauch oder der Exposition einer oben nicht beschriebenen Substanz auftritt und durch diese verursacht ist. Mögliche Substanzen schließen pflanzliche, tierische und andere organische oder anorganische Substanzen ein, die von Ärzten oder Laien mit dem Ziel einer medizinischen Wirkung gegeben werden, auch wenn sie nicht als Arzneimittel zugelassen sind.

Diagnostische Kriterien:

A. Kopfschmerz, der das Kriterium C erfüllt

B. Exposition gegenüber einer oben nicht beschriebenen Subs$\operatorname{tanz}$

C. Ein kausaler Zusammenhang kann durch beide der folgenden Kriterien gezeigt werden:

1. Der Kopfschmerz hat sich innerhalb von 12 Stunden nach der Exposition entwickelt

2. Der Kopfschmerz ist innerhalb von 72 Stunden nach der Exposition verschwunden

D. Nicht besser erklärt durch eine andere ICHD-3-Diagnose.

\section{Kommentar:}

8.1.11 Kopfschmerz zurückzuführen auf Gebrauch oder Exposition einer anderen Substanz schließt Kopfschmerzen ein, die durch pflanzliche, tierische und andere organische oder anorganische Substanzen hervorgerufen werden, die von Ärzten oder Laien mit dem Ziel einer medizinischen Wirkung gegeben werden, auch wenn sie nicht als Arzneimittel zugelassen sind. Er wurde mit einer Reihe von organischen und anorganischen Substanzen in Zusammenhang gebracht. Die folgenden Substanzen werden am häufigsten genannt:

Anorganische Verbindungen: Arsen, Borat, Bromat, Chlor, Kupfer, Jod, Blei, Lithium, Quecksilber und Tolazolinhydrochlorid.

Organische Verbindungen: Anilin, Balsam, Kampfer, Kohlenstoffdisulfid, Kohlenstofftetrachlorid, Chlordecon, EDTA, Heptachlor, Hydrogensulfid, Kerosin, Methylalkohol, Methylbromid, Methylchlorid, Methyljod, Naphthalen, organische Phosphorverbindungen (Parathion, Pyrethrum).

Die Kopfschmerzcharakteristika eines 8.1.11. Kopfschmerzes zurückzuführen auf Gebrauch oder Exposition einer anderen Substanz sind in der Literatur nicht hinreichend definiert und sehr wahrscheinlich abhängig von der Substanz. Meist sind sie dumpf, kontinuierlich vorhanden, diffus lokalisiert und von mittelstarker bis starker Intensität. 


\subsection{Kopfschmerz zurückzuführen auf einen Medikamentenübergebrauch}

Früher verwendete Begriffe: Medikamenteninduzierter Kopfschmerz; Kopfschmerz bei Medikamentenmissbrauch; „Rebound“-Kopfschmerz.

An anderer Stelle kodiert: Patienten mit einer vorbestehenden primären Kopfschmerzerkrankung, die während eines Medikamentenübergebrauchs einen neuen Kopfschmerztyp entwickeln oder eine deutliche Verschlechterung ihres vorbestehenden Kopfschmerzes erfahren und die die Kriterien für einen 8.2 Kopfschmerz zurückzuführen auf einen Medikamentenübergebrauch (oder eines Subtyps) erfüllen, sollen sowohl diese Diagnose als auch die Diagnose ihrer vorbestehenden Kopfschmerzen erhalten. Patienten, die die Kriterien sowohl für eine 1.3 chronische Migräne als auch für einen 8.2 Kopfschmerz zurückzuführen auf einen Medikamentenübergebrauch erfüllen, sollen beide Diagnosen erhalten.

\section{Beschreibung:}

Kopfschmerz an 15 oder mehr Tagen/Monat bei einem Patienten mit einer vorbestehenden primären Kopfschmerzerkrankung, der sich als Folge eines regelmäßigen Übergebrauchs von Kopfschmerz-Akutmedikation für mehr als 3 Monate entwickelt. Als Übergebrauch wird, je nach Medikament, die Einnahme an mindestens 10 oder 15 Tagen/Monat bezeichnet. Dieser Kopfschmerz verschwindet meist, aber nicht immer, nach Beendigung des Übergebrauchs.

\section{Diagnostische Kriterien:}

A. Kopfschmerz an $\geq 15$ Tagen/Monat bei einem Patienten mit einer vorbestehenden Kopfschmerzerkrankung

B. Regelmäßiger Übergebrauch für>3 Monate eines oder mehrerer Medikamente, die zur Akuttherapie oder symptomatischen Behandlung von Kopfschmerzen eingesetzt werden können $1 ; 2 ; 3$

C. Nicht besser erklärt durch eine andere ICHD-3-Diagnose.

\section{Anmerkungen:}

Patienten sollten die Diagnose von einem oder mehreren Subtypen von 8.2 Kopfschmerz zurückzuführen auf einen Medikamentenübergebrauch entsprechend der eingenommenen Medikation und der unten beschriebenen Kriterien erhalten. Zum Beispiel sollte ein Patient, der die Kriterien für 8.2.2 Kopfschmerz zurückzuführen auf einen Triptanübergebrauch und die Kriterien eines Subtyps von 8.2.3 Kopfschmerz zurückzuführen auf einen Übergebrauch von Nicht-Opioid-Analgetika erfüllt, beide Diagnosen erhalten. Dagegen werden Patienten, die Kombinationsanalgetika übergebrauchen, unter 8.2.5 Kopfschmerz zurückzuführen auf einen Übergebrauch von Schmerzmittelmischpräparaten kodiert, und nicht entsprechend der in den Kombinationsanalgetika enthaltenen Einzelsubstanzen.

Patienten, die verschiedene Medikamente zur Akuttherapie oder symptomatischen Kopfschmerzbehandlung einnehmen, können insgesamt einen Übergebrauch aufweisen, obwohl keine der Einzelsubstanzen übergebraucht wird. Diese Patienten sollten unter 8.2.6 Kopfschmerz zurückzuführen auf einen Übergebrauch von Medikamenten aus mehreren Klassen, ohne Übergebrauch der Einzelsubstanzen kodiert werden.

Patienten, die eindeutig einen Übergebrauch mehrerer Medikamente zur Akuttherapie oder symptomatischen Behandlung von Kopfschmerzen betreiben, aber keine hinreichenden Angaben zu den verwendeten Medikamenten und/oder der Einnahmehäufigkeit machen können, werden unter 8.2.7 Kopfschmerz zurückzuführen auf einen unspezifiziertem oder unbestätigtem Übergebrauch von Medikamenten aus mehreren Klassen kodiert, bis genauere Information ermittelt worden sind. Diese Situation erfordert fast immer das Führen eines Kopfschmerz- und Medikamentenkalenders.

\section{Kommentare:}

8.2 Kopfschmerz zurückzuführen auf einen Medikamentenübergebrauch ist das Ergebnis einer Interaktion zwischen exzessiv gebrauchten Medikamenten und empfänglichen Patienten. Patienten mit einer primären Kopfschmerzerkrankung haben meist 1. Migräne oder 2. Kopfschmerz vom Spannungstyp (oder beides); nur eine kleine Minderheit hat andere primäre Kopfschmerzerkrankungen wie 3.3 Clusterkopfschmerz oder 4.10 neu aufgetretener täglicher anhaltender Kopfschmerz.

Der Kopfschmerz bei Medikamentenübergebrauch hat sehr große klinische Bedeutung. Epidemiologische Daten aus vielen Ländern zeigen, dass mehr als die Hälfte der Menschen mit Kopfschmerzen an 15 oder mehr Tagen/Monat einen 8.2. Kopfschmerz zurückzuführen auf einen Medikamentenübergebrauch haben. Es ist erwiesen, dass es nach Absetzen der übergebrauchten Medikation bei den meisten Patienten zu einer Besserung der Kopfschmerzen und einem verbesserten Ansprechen auf vorbeugende Medikation kommt. Eine einfache Beratung über die Ursachen und Folgen eines 8.2. Kopfschmerzes zurückzuführen auf einen Medikamentenübergebrauch ist ein entscheidender Baustein seiner Behandlung und kann erfolgreich im Rahmen der hausärztlichen Versorgung eingesetzt werden. Die Mitgabe von Informationsmaterial ist oft ausreichend, um einen Medikamentenübergebrauch zu verhindern oder zu beenden. Die Vorbeugung ist besonders bei Patienten mit häufigen Kopfschmerzen wichtig.

Manche Patienten mit 8.2. Kopfschmerz zurückzuführen auf einen Medikamentenübergebrauch zeigen ein Verhalten wie bei anderen Suchterkrankungen, und hohe Werte auf der Severity of Dependence Scale (SDS) können bei Kopfschmerzpatienten einen Medikamentenübergebrauch vorhersagen.

Die Anzahl der Tage mit Medikamenteneinnahme, die in den untenstehenden Kriterien für die verschiedenen Subtypen als Übergebrauch bezeichnet wird, ist nicht evidenzbasiert, sondern beruht auf Expertenmeinung.

Es ist bekannt, dass populationsbasierte Querschnittsstudien zur Schätzung der Prävalenz von 8.2. Kopfschmerz zurückzuführen auf einen Medikamentenübergebrauch zwar das gleichzeitige Bestehen von Kopfschmerzen an $\geq 15$ Tagen pro Monat und von Medikamentenübergebrauch erfassen können. Jedoch sind selten Informationen über frühere Kopfschmerzen zu erhalten, über die Dauer des aktuellen Kopfschmerzes oder des Medikamentenübergebrauchs und/oder Informationen, die die Annahme eines ursächlichen Zusammenhangs stützen. Daher kommt es vor, dass die Kriterien $A$ und $B$, oder eines der beiden, nicht komplett erfüllt sind. 
Sofern nicht die Kriterien für eine andere ICHD-3-Diagnose erfüllt sind, sollten solche Fälle als wahrscheinlicher Kopfschmerz zurückzuführen auf Medikamentenübergebrauch berichtet werden, obwohl die ICHD-3 keine Diagnose dafür vorsieht.

\subsubsection{Kopfschmerz zurückzuführen auf einen Ergotaminübergebrauch}

Beschreibung:

Kopfschmerz an 15 oder mehr Tagen/Monat bei einem Patienten mit einer vorbestehenden primären Kopfschmerzerkrankung, der sich als Folge eines regelmäßigen Übergebrauchs von Ergotamin an 10 oder mehr Tagen/Monat für mehr als 3 Monate entwickelt. Dieser Kopfschmerz verschwindet meist, aber nicht immer, nach Beendigung des Übergebrauchs.

Diagnostische Kriterien:

A. Kopfschmerz, der die Kriterien für 8.2 Kopfschmerz zurückzuführen auf einen Medikamentenübergebrauch erfüllt

B. Regelmäßige Ergotamineinnahme an $\geq 10$ Tagen/Monat für >3 Monate.

Kommentar:

Die Bioverfügbarkeit von Ergotaminen ist so variabel, dass eine minimale Dosis nicht definiert werden kann.

\subsubsection{Kopfschmerz zurückzuführen auf einen Triptanübergebrauch}

Beschreibung:

Kopfschmerz an 15 oder mehr Tagen/Monat bei einem Patienten mit einer vorbestehenden primären Kopfschmerzerkrankung, der sich als Folge eines regelmäßigen Übergebrauchs von einem oder mehreren Triptanen an 10 oder mehr Tagen/Monat für mehr als 3 Monate entwickelt. Dieser Kopfschmerz verschwindet meist, aber nicht immer, nach Beendigung des Übergebrauchs.

\section{Diagnostische Kriterien:}

A. Kopfschmerz, der die Kriterien für 8.2 Kopfschmerz zurückzuführen auf einen Medikamentenübergebrauch erfüllt

B. Regelmäßige Einnahme eines oder verschiedener Triptane 1 (jede Darreichungsform) an $\geq 10$ Tagen/Monat für $>3$ Monate.

\section{Anmerkung:}

1. Das Triptan/die Triptane wird/werden üblicherweise in Klammern spezifiziert.

\section{Kommentar:}

Triptanübergebrauch bei Personen mit 1.1 Migräne ohne Aura oder 1.2 Migräne mit Aura kann eine Zunahme der Attackenfrequenz bis hin zu einer 1.3 chronischen Migräne verursachen. Es gibt Hinweise, dass dies schneller als bei Ergotaminen geschieht.

8.2.3 Kopfschmerz zurückzuführen auf einen Übergebrauch von Nicht-Opioid-Analgetika

\section{Beschreibung:}

Kopfschmerz an 15 oder mehr Tagen/Monat bei einem Patienten mit einer vorbestehenden primären Kopfschmerzerkrankung, der sich als Folge eines regelmäßigen Übergebrauchs von einem oder mehreren Nicht-Opioid-Analgetika an 15 oder mehr Tagen/Monat für mehr als 3 Monate entwickelt. Dieser Kopfschmerz verschwindet meist, aber nicht immer, nach Beendigung des Übergebrauchs.

\section{Kommentar:}

Ein Patient, der die Kriterien für mehr als einen Subtyp von 8.2.3 Kopfschmerz zurückzuführen auf einen Übergebrauch von Nicht-Opioid-Analgetika erfüllt, sollte alle zutreffenden Diagnosen erhalten.

Viele Patienten nehmen mehr als ein Nicht-Opioid-Analgetikum ein, z. B. Paracetamol und ein nicht-steroidales Antirheumatikum (NSAR). In der ICHD-3 werden die Nicht-Opioid-Analgetika als eine einzelne Klasse angesehen, daher wird ein Patient, der mehrere Nicht-Opioid-Analgetika insgesamt, aber nicht ein einzelnes Medikament an 15 oder mehr Tagen/Monat einnimmt, unter 8.2.3 Kopfschmerz zurückzuführen auf einen Übergebrauch von Nicht-Opioid-Analgetika diagnostiziert (mit Angabe der einzelnen Medikamente in Klammern) und nicht unter 8.2.6 Kopfschmerz zurückzuführen auf einen Übergebrauch von Medikamenten aus mehreren Klassen, ohne Übergebrauch der Einzelsubstanzen.

\subsubsection{Kopfschmerz zurückzuführen auf einen Übergebrauch von Paracetamol}

Diagnostische Kriterien:

A. Kopfschmerz, der die Kriterien für 8.2 Kopfschmerz zurückzuführen auf einen Medikamentenübergebrauch erfüllt

B. Regelmäßige Paracetamoleinnahme an $\geq 15$ Tagen/Monat für $>3$ Monate.

\subsubsection{Kopfschmerz zurückzuführen auf einen Über- gebrauch von nicht-steroidalen Antirheumatika (NSAR)}

An anderer Stelle kodiert: Acetylsalicylsäure ist ein nicht-steroidales Antirheumatikum (NSAR), hat aber andere, spezifische Wirkungen. Daher wird 8.2.3.2.1 Kopfschmerz zurückzuführen auf einen Übergebrauch von Acetylsalicylsäure als eigener Subtyp kodiert.

\section{Diagnostische Kriterien:}

A. Kopfschmerz, der die Kriterien für 8.2 Kopfschmerz zurückzuführen auf einen Medikamentenübergebrauch erfüllt

B. Regelmäßige Einnahme eines oder mehrerer NSAR1 (außer Acetylsalicylsäure) an $\geq 15$ Tagen/Monat für>3 Monate.

Anmerkung:

Das/die NSAR soll(en) in Klammern spezifiziert werden.

\subsection{Kopfschmerz zurückzuführen auf einen Über- gebrauch von Acetylsalicylsäure}

Diagnostische Kriterien:

A. Kopfschmerz, der die Kriterien für 8.2 Kopfschmerz zurückzuführen auf einen Medikamentenübergebrauch erfüllt

B. Regelmäßige Einnahme von Acetylsalicylsäure an $\geq 15$ Tagen/ Monat für>3 Monate.

Kommentar:

Acetylsalicylsäure ist ein nicht-steroidales Antirheumatikum (NSAR), hat aber andere, spezifische Wirkungen. Daher wird 
8.2.3.2.1 Kopfschmerz zurückzuführen auf einen Übergebrauch von Acetylsalicylsäure als eigener Subtyp kodiert.

\subsubsection{Kopfschmerz zurückzuführen auf einen Über- gebrauch von anderen Nicht-Opioid-Analgetika Diagnostische Kriterien:}

A. Kopfschmerz, der die Kriterien für 8.2 Kopfschmerz zurückzuführen auf einen Medikamentenübergebrauch erfüllt

B. Regelmäßige Einnahme eines anderen Nicht-Opioid-Analgetikums (außer Paracetamol oder eines NSAR, inklusive Acetylsalicylsäure) an $\geq 15$ Tagen/Monat für>3 Monate.

\subsubsection{Kopfschmerz zurückzuführen auf einen Opioidübergebrauch \\ Diagnostische Kriterien:}

A. Kopfschmerz, der die Kriterien für 8.2 Kopfschmerz zurückzuführen auf einen Medikamentenübergebrauch erfüllt

B. Regelmäßige Einnahme eines oder mehrerer Opioide1 an $\geq 10$ Tagen/Monat für $>3$ Monate.

\section{Anmerkung:}

1. Das Opioid/die Opioide soll(en) in Klammern spezifiziert werden.

\section{Kommentar:}

Prospektive Studien haben gezeigt, dass Patienten, die Opioide übergebrauchen, die höchste Rückfallrate nach einer Entzugsbehandlung aufweisen.

\subsubsection{Kopfschmerz zurückzuführen auf einen Übergebrauch von Schmerzmittelmisch- präparaten 1 \\ Diagnostische Kriterien:}

A. Kopfschmerz, der die Kriterien für 8.2 Kopfschmerz zurückzuführen auf einen Medikamentenübergebrauch erfüllt

B. Regelmäßige Einnahme eines oder verschiedener Schmerzmittelmischpräparate1,2 an $\geq 10$ Tagen/Monat für > 3 Monate.

\section{Anmerkungen:}

Die Bezeichnung Schmerzmittelmischpräparat wird für Präparate verwendet, die Medikamente aus zwei oder mehr Klassen kombinieren, wobei jedes einen analgetischen (z. B. Paracetamol und Kodein) oder adjuvanten (z. B. Koffein) Effekt hat. Präparate, die zwei Nicht-Opioid-Analgetika ohne zusätzliches Adjuvans kombinieren (z. B. Acetylsalicylsäure und Paracetamol), werden nicht als Schmerzmittelmischpräparate angesehen, da beide Wirkstoffe innerhalb der ICHD-3 derselben Klasse zugeordnet sind.

2. Das/die Schmerzmittelmischpräparat(e) soll(en) in Klammern spezifiziert werden.

Kommentare:

Es befinden sich zahlreiche Schmerzmittelmischpräparate auf dem Markt. Die Verwendung dieser Präparate bei Kopfschmerzen ist weit verbreitet, und bei 8.2 Kopfschmerz zurückzuführen auf einen Medikamentenübergebrauch sehr häufig anzutreffen. Daher ist 8.2.5 Kopfschmerz zurückzuführen auf einen Übergebrauch von Schmerzmittelmischpräparaten als eigene Diagnose aufgeführt.
Die am häufigsten übergebrauchten Schmerzmittelmischpräparate kombinieren Nicht-Opioid-Analgetika mit Opioiden, Butalbital und/oder Koffein.

\subsubsection{Kopfschmerz zurückzuführen auf einen Über- gebrauch von Medikamenten aus mehreren Klassen, ohne Übergebrauch der Einzelsubs- tanzen}

\section{Diagnostische Kriterien:}

A. Kopfschmerz, der die Kriterien für 8.2 Kopfschmerz zurückzuführen auf einen Medikamentenübergebrauch erfüllt

B. Regelmäßige Einnahme von Ergotaminen, Triptanen, Nicht-Opioid-Analgetika, NSAR und/oder Opioiden 1 in beliebiger Kombination an $\geq 10$ Tagen/Monat für $>3$ Monate, ohne Übergebrauch einer Einzelsubstanz oder Substanzklasse2.

\section{Anmerkungen:}

1. Die Medikamente oder Medikamentenklassen sollen in Klammern spezifiziert werden.

2. „Ohne Übergebrauch einer Einzelsubstanz oder einer Substanzklasse“ bedeutet, dass für keine der Diagnosen 8.2.1 bis 8.2.5 das Kriterium B erfüllt ist.

\subsubsection{Kopfschmerz zurückzuführen auf einen unspezifizierten oder unbestätigten Über- gebrauch von Medikamenten aus mehreren Klassen}

\section{Diagnostische Kriterien:}

A. Kopfschmerz, der die Kriterien für 8.2 Kopfschmerz zurückzuführen auf einen Medikamentenübergebrauch erfüllt

B. Beide der folgenden:

- Regelmäßige Einnahme von Ergotaminen, Triptanen, Nicht-Opioid-Analgetika und/oder Opioiden in beliebiger Kombination an $\geq 10$ Tagen/Monat für > 3 Monate.

- Die eingenommenen Substanzen, die Dosierung und/oder das Einnahmemuster können nicht genau festgestellt werden.

\section{Kommentar:}

Patienten, die offensichtlich einen Übergebrauch mehrerer Medikamente zur Akuttherapie oder symptomatischen Kopfschmerzbehandlung betreiben, aber keine genauen Angaben über die eingenommenen Substanzen, die Dosis oder das Einnahmemuster machen können, sind nicht selten. Das Führen eines Kopfschmerztagebuchs über mehrere Wochen könnte Klarheit schaffen, würde aber auch den notwendigen Medikamentenentzug verzögern.

\subsubsection{Kopfschmerz zurückzuführen auf einen Übergebrauch einer anderen Medikation}

Diagnostische Kriterien:

A. Kopfschmerz, der die Kriterien für 8.2 Kopfschmerz zurückzuführen auf einen Medikamentenübergebrauch erfüllt

B. Regelmäßige Einnahme eines oder mehrerer oben nicht aufgeführter Medikamente1 zur Akuttherapie oder symptomatischen Kopfschmerzbehandlung an $\geq 10$ Tagen/Monat für $>3$ Monate. 
Anmerkung:

1. Die Medikation soll in Klammern spezifiziert werden.

8.3 Kopfschmerz zurückzuführen auf den Entzug einer Substanz

Beschreibung:

Kopfschmerz in Folge von und verursacht durch die Unterbrechung einer Wochen bis Monate andauernden Einnahme oder Exposition eines Medikaments oder einer anderen Substanz.

\subsubsection{Koffeinentzugskopfschmerz}

Beschreibung:

Kopfschmerz, der sich innerhalb 24 Stunden nach Unterbrechung eines regelmäßigen Koffeinkonsums von > 200 mg/Tag für $>2$ Wochen entwickelt. Der Kopfschmerz verschwindet innerhalb von 7 Tagen Abstinenz.

Diagnostische Kriterien:

A. Kopfschmerz, der das Kriterium C erfüllt

B. Koffeinkonsum $>200 \mathrm{mg} /$ Tag für $>2$ Wochen, der unterbrochen oder verzögert wurde

C. Ein kausaler Zusammenhang kann durch beide der folgenden Kriterien gezeigt werden:

1. Der Kopfschmerz hat sich innerhalb 24 Stunden nach der letzten Koffeineinnahme entwickelt

2. Eines oder beide der folgenden:

a) Der Kopfschmerz bessert sich innerhalb von 1 Stunde nach Einnahme von $100 \mathrm{mg}$ Koffein

b) Der Kopfschmerz ist innerhalb von 7 Tagen nach komplettem Koffeinentzug verschwunden

D. Nicht besser erklärt durch eine andere ICHD-3-Diagnose.

\subsubsection{Opioidentzugskopfschmerz}

Beschreibung:

Kopfschmerz, der sich innerhalb 24 Stunden nach Unterbrechung einer täglichen Opioideinnahme für > 3 Monate entwickelt. Der Kopfschmerz verschwindet innerhalb von 7 Tagen ohne weitere Opioideinnahme.

\section{Diagnostische Kriterien:}

A. Kopfschmerz, der das Kriterium C erfüllt

B. Tägliche Opioideinnahme für $>3$ Monate, die unterbrochen wurde

C. Ein kausaler Zusammenhang kann durch beide der folgenden Kriterien gezeigt werden:

1. Der Kopfschmerz hat sich innerhalb 24 Stunden nach der letzten Opioideinnahme entwickelt

2. Der Kopfschmerz ist innerhalb von 7 Tagen nach komplettem Opioidentzug verschwunden

D. Nicht besser erklärt durch eine andere ICHD-3-Diagnose.

\subsection{3 Östrogenentzugskopfschmerz}

\section{Beschreibung:}

Kopfschmerzen oder Migräne, die sich innerhalb von 5 Tagen nach Beendigung einer täglichen Einnahme exogener Östrogene für mindestens 3 Wochen entwickelt haben, typischerweise während der Pillenpause bei kombinierten oralen Kontrazeptiva oder nach Ende einer Östrogenersatztherapie. Der Kopfschmerz verschwindet innerhalb von 3 Tagen ohne weitere Östrogeneinnahme.

\section{Diagnostische Kriterien:}

A. Kopfschmerz oder Migräne, die Kriterium C erfüllen

B. Tägliche Einnahme eines exogenen Östrogens für $\geq 3$ Wochen, die unterbrochen wurde

C. Ein kausaler Zusammenhang kann durch beide der folgenden Kriterien gezeigt werden:

1. Der Kopfschmerz oder die Migräne haben sich innerhalb von 5 Tagen nach der letzten Östrogeneinnahme entwickelt

2. Der Kopfschmerz oder die Migräne ist innerhalb von 3 Tagen verschwunden

D. Nicht besser erklärt durch eine andere ICHD-3-Diagnose.

Kommentar:

Ein Östrogenentzug durch Beendigung einer befristeten exogenen Östrogenzufuhr (z. B. in der Pillenpause bei oralen Kombinationskontrazeptiva oder nach Ende einer Östrogenersatztherapie) kann mit Kopfschmerzen oder einer Migräne einhergehen.

\subsubsection{Kopfschmerz zurückzuführen auf den Entzug anderer dauerhaft eingenommener Substanzen}

Beschreibung:

Kopfschmerz in Folge von und verursacht durch die Unterbrechung der Einnahme einer dauerhaft eingenommenen Medikation oder Substanz, die nicht oben bereits beschrieben ist.

Diagnostische Kriterien:

A. Kopfschmerz, der das Kriterium C erfüllt

B. Tägliche Einnahme einer oben nicht aufgeführten Substanz über > 3 Monate, die unterbrochen wurde

C. Ein kausaler Zusammenhang kann durch beide der folgenden Kriterien gezeigt werden:

1. Der Kopfschmerz hat sich in engem zeitlichen Zusammenhang mit dem Entzug der eingenommenen Substanz entwickelt

2. Der Kopfschmerz ist innerhalb von 3 Monaten nach dem vollständigen Entzug verschwunden

D. Nicht besser erklärt durch eine andere ICHD-3-Diagnose.

\section{Kommentare:}

Es gibt Hinweise, jedoch noch ohne ausreichenden wissenschaftlichen Beleg, dass ein Entzug folgender Substanzen Kopfschmerzen verursachen kann: Kortikosteroide, trizyklische Antidepressiva, selektive Serotoninwiederaufnahmehemmer (SSRI) und nichtsteroidale Antiphlogistika.

Es könnte weitere, bisher nicht erkannte Substanzen mit dieser Wirkung geben. 


\subsection{Kopfschmerz zurückzuführen auf Substanzgebrauch oder Substanz-} exposition

Altura BM, Altura BT, Gebrewold A. Alcohol induced spasm of cerebral blood vessels. J Mental Sci 2000; 104: 972-999.

Ashina M, Bendtsen L, Jensen R, Olesen J. Nitric oxide-induced headache in patients with chronic tension-type headache. Brain 2000; 123 : $1830-1837$.

Askmark H, Lundberg PO, Olsson S. Drug related headache. Headache 1989; 29: 441-444.

Beck HG, Schulze WH, Suter GM. Carbon monoxide-a domestic hazard. JAMA 1940; 115: 1.

Birk S, Kruuse C, Petersen KA, Tfelt-Hansen P, Olesen J. The headache-inducing effect of cilostazol in human volunteers. Cephalalgia 2006; 26 : 1304-1309.

Bonnet GF, Nepveux P. Migraine due to tyramine. Sem Hop 1971; 47: 2441-2445.

Brewerton TD, Murphy DL, Lesem MD, Brandt HA, Jimerson DC. Headache responses following $\mathrm{m}$-chlorophenylpiperazine in bulimics and controls. Headache 1992; 32: 217-222.

Cleophas TJ, Niemeyer MG, van der Wall EE, van der Meulen J. Nitrate-induced headache in patients with stable angina pectoris: beneficial effect of starting on a low dose. Angiology 1996; 47: 679-685.

Cregler LL, Mark H. Medical complications of cocaine abuse. NEJM 1986; 315: 1495-1501.

Dhopesh V, Maany I, Herring C. The relationship of cocaine to headache in polysubstance abusers. Headache 1991; 31: 17-19.

Dhuna A, Pascual-Leone A, Belgrade M. Cocaine-related vascular headaches. J Neurol Neurosurg Psychiat 1991; 54: 803-806.

De Marinis M, Janiri L, Agnoli A. Headache in the use and withdrawal of opiates and other associated substances of abuse. Headache 1991; 31 : 159-163.

Ekbom K. Nitroglycerin as a provocative agent in cluster headache. Arch Neurol 1968; 19: 487-493.

el-Mallakh RS. Marijuana and migraine. Headache 1987; 27: 442-443.

el-Mallakh RS, Kranzler HR, Kamanitz JR. Headaches and psychoactive substance use. Headache 1991; 31: 584-587.

Fanciullacci M, Alessandri M, Figini M, Geppetti P, Michelacci S. Increase in plasma calcitonin gene-related peptide from the extracerebral circulation during nitroglycerin-induced cluster headache attack. Pain 1995 ; 60: 119-123.

Forbes HS, Cobb S, Fremont-Smith F. Cerebral edema and headache following carbon monoxide asphyxia. Arch Neurol Psychiatr 1924; 11: 164.

Ghose K, Carrol JD. Mechanisms of tyramine-induced migraine: similarities with dopamine and interactions with disulfiram and propranolol. Neuropsychiobiol 1984; 12: 122-126.

Iversen HK, Nielsen TM, Olesen J, Tfelt-Hansen P. Intravenous nitroglycerin as an experimental model of vascular headache. Basic characteristics. Pain 1989; 38: 17-24.

Krabbe AA, Olesen J. Headache provocation by continuous intravenous infusion of histamine, clinical results and receptor mechanisms. Pain $1980 ; 8: 253-259$.

Kruuse C, Jacobsen TB, Lassen LH, Thomsen LL, Hasselbalch SG, Dige-Petersen $\mathrm{H}$, Olesen J. Dipyridamole dilates large cerebral arteries concomitant to headache induction in healthy subjects. J Cereb Blood Flow Metab 2000; 20: 1372-1379.

Kruuse C, Thomsen LL, Birk S, Olesen J. Migraine can be induced by sildenafil without changes in middle cerebral artery diameter. Brain 2003; 126: 241-247.
Kruuse C, Thomsen LL, Jacobsen TB, Olesen J. The phosphodiesterase 5 inhibitor sildenafil has no effect on cerebral blood flow or blood velocity, but nevertheless induces headache in healthy subjects. J Cereb Blood Flow Metab 2002; 22: 1124-1131.

Lassen LH, Thomsen LL, Olesen J. Histamine induces migraine via the $\mathrm{H}$ receptor. Support for the NO-hypothesis of migraine. Neuroreport 1995; 6: 1475-1479.

Leon AS, Hunninghake DB, Bell C, Rassin DK, Tephly TR. Safety of long-term doses of aspartame. Arch Int Med 1989; 149: 2318-2324.

Leone M, Attanasio A, Croci D, Filippini G, D’Amico D, Grazzi L, Nespolo A, Bussone $G$. The serotonergic agent m-chlorophenylpiperazine induced migraine attacks: a controlled study. Neurology 2000; 55: 136-139.

Lichten E, Lichten J, Whitty A, Pieper D. The confirmation of a biochemical marker for women's hormonal migraine : the depo-oestradiol challenge test. Headache 1996; 36: 367-371.

Lipton RB, Kwong CM, Solomon S. Headaches in hospitalized cocaine users. Headache 1989; 29: 225-228.

Magos AL, Brewster E, Singh R, O’Dowd T, Brincat M, Studd JWW. The effects of norethisterone in postmenopausal women on oestrogen replacement therapy: a model for the premenstrual syndrome. $\mathrm{Br}$ J Obstet Gynaecol 1986; 93: 1290-1296.

Murphree AB, Greenberg LA, Carrol RB. Neuropharmacologic effects of substances other than ethanol in alcoholic beverages. Fed Proc 1967; 26: 1468-1473.

Nappi RE, Cagnacci A, Granella F, Piccinini F, Polatti F, Facchinetti F. Course of primary headaches during hormone replacement therapy. Maturitas 2001; 38: 157-163.

Pepe G, Castelli M, Nazerian P et al. Delayed neuropsychological sequelae after carbon monoxide poisoning: predictive risk factors in the Emergency Department. A retrospective study. Scand J Trauma Resusc Emerg Med 2011; 19: 16

Schiffmann SS, Buckley CE, Sampson HA et al. Aspartame and susceptibility to headache. NEJM 1987; 317: 1181-1185.

Sicuteri F, Bene ED, Poggioni M, Bonazzi A. Unmasking latent dysnociception in healthy subjects. Headache 1987; 27: 180-185.

Thomsen LL, Kruse C, Iversen HK, Olesen J. A Nitric oxide donor triggers genuine migraine attacks. Eur J Neurol 1994; 1: 71-80.

\subsection{Kopfschmerz zurückzuführen auf einen Medikamenten-} übergebrauch

Aaseth K, Grande RB, Šaltytė Bent J, Lundqvist C, Russell MB. 3-years-follow-up of secondary chronic headaches. The Akershus study of chronic headache. Eur J Pain 2011; 15: 186-92.

Ala-Hurula V, Myllyla V, Hokkanen E, Tokola O. Tolfenamic acid and ergotamine abuse. Headache 1981; 21: 240-242.

Andersson PG. Ergotamine headache. Headache 1975; 15: 118-121.

Bigal ME, Rapoport AM, Sheftell FD, Tepper S], Lipton RB. Transformed migraine and Medication overuse in a tertiary headache centre clinical characteristics and treatment outcomes. Cephalalgia 2004; 24 483-490.

Diener HC, Dichgans J, Scholz E, Geiselhart S, Gerber WD, Bille A. Analgesic-induced chronic headache: long-term results of withdrawal therapy. J Neurol 1989; 236: 9-14.

Evers S, Gralow I, Bauer B, Suhr B, Buchheister A, Husstedt IW, Ringelstein EB. Sumatriptan and ergotamine overuse and drug-induced headache: a clinicoepidemiologic study. Clin Neuropharmacol 1999; 22: 201-206.

Find NL, Terlizzi R, Munksgaard SB et al. COMOESTAS Consortium. Medication overuse headache in Europe and Latin America: general demographic and clinical characteristics, referral pathways and national distribution of painkillers in a descriptive, multinational, multicentre study. J Headache Pain 2016; 17: 20. 
Fritsche G, Frettlöh J, Hüppe M et al. Prevention of medication overuse in patients with migraine. Pain 2010; 151: 404-413.

Gaist D, Hallas J, Sindrup SH, Gram LF. Is overuse of sumatriptan a problem? A population-based study. Eur J Clin Pharmacol 1996; 50: 161-165.

Gaist D, Tsiropoulus I, Sindrup SH, Hallas ], Rasmussen BK, Kragstrup J. Inappropriate use of sumatriptan: population based register and interview study. BMJ 1998; 316: 1352-1353.

Gossop M, Darke S, Griffiths P, Hando J, Powis B, Hall W, Strang J. The Severity of Dependence Scale (SDS): psychometric properties of the SDS in English and Australian samples of heroin, cocaine and amphetamine users. Addiction 1995; 90: 607-614.

Grande RB, Aaseth K, Saltyte Benth J, Gulbrandsen P, Russell MB, Lundqvist C. The Severity of Dependence Scale detects people with medication overuse: the Akershus study of chronic headache. J Neurol Neurosurg Psychiat 2009; 80: 784-789.

Grande RB, Aaseth K, Šaltytè Bent J, Lundqvist C, Russell MB. Reduction of medication-overuse headache after modified brief intervention. The Akershus study on chronic headache. Eur J Neurol 2011; 18: 129-37.

Hering R, Steiner TJ. Abrupt outpatient withdrawal from medication in analgesic-abusing migraineurs. Lancet 1991; 337: 1442-1443.

Katsarava Z, Fritsche G, Muessig M, Diener HC, Limmroth V. Clinical features of withdrawal headache following overuse of triptans and other headache drugs. Neurology 2001; 57: 1694-1698.

Kaube H, May A, Diener HC, Pfaffenrath V. Sumatriptan misuse in daily chronic headache. BMJ 1994; 308: 1573.

Kristoffersen ES, Straand J, Vetvik KG, Benth JŠ, Russell MB, Lundqvist C. Brief intervention for medication-overuse headache in primary care. The BIMOH study: a double-blind pragmatic cluster randomised parallel controlled trial. J Neurol Neurosurg Psychiat 2015; 86: 505-512.

Kristoffersen ES, Straand J, Vetvik KG, Benth JŠ, Russell MB, Lundqvist C. Brief intervention by general practitioners for medication-overuse headache, follow-up after 6 months: a pragmatic cluster-randomised controlled trial. J Neurol 2016; 263: 344-353.

Limmroth V, Katsarava Z, Fritsche G, Diener HC. Headache after frequent use of new 5-HT agonists zolmitriptan and naratriptan. Lancet 1999; 353: 378.

Limmroth V, Katsarava Z, Fritsche G, Przywara S, Diener HC. Features of medication overuse headache following overuse of different acute headache drugs. Neurology 2002; 59: 1011-1014.

Lundqvist C, Grande RB, Aaseth K, Russell MB. The severity of dependence scale predicts prognosis of medication overuse headache. The Akershus study of chronic headache. Pain 2012; 153: 682-686.

Nicolodi M, DelBianco PL, Sicuteri F. The way to serotonergic use and abuse in migraine. Int J Clin Pharmacol Res 1997; 17: 79-84.

Rapoport A, Stang P, Gutterman DL, Cady R, Markley H, Weeks R, Saiers J, Fox AW. Analgesic rebound headache in clinical practice: data from a physician survey. Headache 1996; 36: 14-19.

Schnider P, Aull S, Baumgartner C et al. Long-term outcome of patients with headache and drug abuse after inpatient withdrawal: five-year followup. Cephalalgia 1996; 16: 481-485.

Schnider P, Aull S, Feucht M. Use and abuse of analgesics in tension-type headache. Cephalalgia 1994; 14: 162-167.

Seller EM, Busto UE, Kaplan HL, Somer G, Baylon GJ. Comparative abuse liability of codeine and naratriptan. Clin Pharmacol Ther 1998; 63: 121.

Tfelt-Hansen P, Krabbe AA. Ergotamine. Do patients benefit from withdrawal? Cephalalgia 1981; 1: 29-32.

\subsection{Kopfschmerz zurückzuführen auf den Entzug einer Substanz}

Abbott PJ. Caffeine: a toxicological overview. Med J Aust 1986; 145: 518-521.

Epstein MT, Hockaday JM, Hockaday TDR. Migraine and reproductive hormones through the menstrual cycle. Lancet 1975; i: 543-548.

Laska EM, Sunshine A, Mueller F, Elvers WB, Siegel C, Rubin A. Caffeine as an analgesic adjuvant. JAMA 1984; 251: 1711-1718.

Lichten E, Lichten J, Whitty A, Pieper D. The confirmation of a biochemical marker for women's hormonal migraine : the depo-oestradiol challenge test. Headache 1996; 36: 367-371.

Silverman K, Evans SM, Strain EC, Griffiths RR. Withdrawal syndrome after the double-blind cessation of caffeine consumption. NEJM 1992; 327: 1109-1114.

Somerville BW. Estrogen-withdrawal migraine. I. Duration of exposure required and attempted prophylaxis by premenstrual estrogen administration. Neurology 1975; 25: 239-244.

Somerville BW. Estrogen-withdrawal migraine. II. Attempted prophylaxis by continuous estradiol administration. Neurology 1975; 25: 245-250.

Somerville BW. The role of estradiol withdrawal in the etiology of menstrual migraine. Neurology 1972; 22: 355-365.

Somerville BW. The role of progesterone in menstrual migraine. Neurology 1971; 21: 853-859.

van Dusseldorp M, Katan MB. Headache caused by caffeine withdrawal among moderate coffee drinkers switched from ordinary to decaffeinated coffee: a 12 week double blind trial. BM] 1990; 300: 1558-1559.

\section{Kopfschmerz zurückzuführen auf eine Infektion}

9.1

9.1.1 Kopfschmerz zurückzuführen auf eine bakterielle Meningitis oder Meningoenzephalitis

9.1.1.1 Akuter Kopfschmerz zurückzuführen auf eine bakterielle Meningitis oder Meningoenzephalitis

9.1.1.2 Chronischer Kopfschmerz zurückzuführen auf eine bakterielle Meningitis oder Meningoenzephalitis

9.1.1.3 Anhaltender Kopfschmerz zurückzuführen auf eine frühere bakterielle Meningitis oder Meningoenzephalitis

9.1.2 Kopfschmerz zurückzuführen auf eine virale Meningitis oder Enzephalitis

9.1.2.1 Kopfschmerz zurückzuführen auf eine virale Meningitis

9.1.2.2 Kopfschmerz zurückzuführen auf eine virale Enzephalitis

9.1.3 Kopfschmerz zurückzuführen auf eine Pilz- oder andere parasitäre Infektion

9.1.3.1 Akuter Kopfschmerz zurückzuführen auf eine Pilz- oder andere parasitäre Infektion

9.1.3.2 Chronischer Kopfschmerz zurückzuführen auf eine Pilzoder andere parasitäre Infektion

9.1.4 Kopfschmerz zurückzuführen auf eine lokalisierte Gehirninfektion

\begin{tabular}{l|l}
9.2 & Kopfschmerz zurückzuführen auf eine systemische
\end{tabular} Infektion

9.2.1 Kopfschmerz zurückzuführen auf eine bakterielle systemische Infektion

9.2.1.1 Akuter Kopfschmerz zurückzuführen auf eine bakterielle systemische Infektion

9.2.1.2 Chronischer Kopfschmerz zurückzuführen auf eine bakterielle systemische Infektion

\begin{tabular}{l|l}
9.2 .2 & Kopfschmerz zurückzuführen auf eine systemische
\end{tabular} virale Infektion

9.2.2.1 Akuter Kopfschmerz zurückzuführen auf eine systemische virale Infektion 
9.2.2.2 Chronischer Kopfschmerz zurückzuführen auf eine systemische virale Infektion

9.2.3 Kopfschmerz zurückzuführen auf eine andere systemische Infektion

9.2.3.1 Akuter Kopfschmerz zurückzuführen auf eine andere systemische Infektion

9.2.3.2 Chronischer Kopfschmerz zurückzuführen auf eine andere systemische Infektion

An anderer Stelle kodiert: Kopfschmerzerkrankungen zurückzuführen auf extrakranielle Infektionen des Kopfes (wie etwa Infektionen der Augen, Ohren und Nebenhöhlen) sollten als Typen oder Subtypen von 11. Kopf- oder Gesichtsschmerzen zurückzuführen auf Erkrankungen des Schädels sowie von Hals, Augen, Ohren, Nase, Nebenhöhlen, Zähnen, Mund und anderen Gesichts- oder Schädelstrukturen kodiert werden.

\section{Allgemeine Kommentare}

Der Dreiklang von Kopfschmerzen, Fieber und Übelkeit/Erbrechen lässt stark an einen 9. Kopfschmerz zurückzuführen auf eine Infektion denken. Die Wahrscheinlichkeit dafür ist noch höher, wenn auch Antriebslosigkeit oder epileptische Anfälle zum klinischen Bild gehören.

Primärer und/oder sekundärer Kopfschmerz?

Für Kapitel 9. Kopfschmerz zurückzuführen auf eine Infektion gelten die allgemeinen Regeln für die kausale Zuordnung zu einer anderen Erkrankung.

Tritt ein neuer Kopfschmerz erstmals in engem zeitlichen Zusammenhang mit einer Infektion auf, sollte der Kopfschmerz als Kopfschmerz zurückzuführen auf diese Infektion kodiert werden. Dies ist auch der Fall, wenn der Kopfschmerz das klinische Bild eines primären Kopfschmerzes zeigt, der in Teil 1 der ICHD-3 klassifiziert wird.

Wenn aber ein vorbestehender Kopfschmerz mit den Charakteristika eines primären Kopfschmerzes in engem zeitlichen $\mathrm{Zu}$ sammenhang mit einer Infektion chronisch wird oder sich deutlich verschlechtert (üblicherweise definiert als eine mindestens zweifache Steigerung der Häufigkeit und/oder Schwere), dann sollte sowohl die vorbestehende primäre Kopfschmerzdiagnose als auch die Diagnose 9. Kopfschmerz zurückzuführen auf eine Infektion (oder eines ihrer Typen oder Subtypen vergeben werden, sofern gute Hinweise dafür bestehen, dass die Erkrankung Kopfschmerzen verursachen kann

Akut, chronisch oder anhaltend?

9. Kopfschmerz zurückzuführen auf eine Infektion ist gewöhnlich die Folge einer aktiven Infektion und verschwindet innerhalb von 3 Monaten nach Tilgung der Infektion. In einigen Fällen, je nach Krankheitserreger, lässt sich die Infektion nicht wirksam behandeln und bleibt aktiv. In solchen Fällen lässt der Kopfschmerz eventuell nicht nach, da seine Ursache noch vorliegt. Nach 3 Monaten werden die Infektion wie auch der Kopfschmerz als chronisch bezeichnet.

In anderen, selteneren Fällen verschwindet die Infektion oder wird beseitigt, und dennoch verschwindet der Kopfschmerz nicht. Nach 3 Monaten wird ein solcher Kopfschmerz (entsprechend der Handhabung bei anderen sekundären Kopfschmerzen) als anhaltend bezeichnet.
Dementsprechend wurden akute und chronische Subtypen von Kopfschmerz zurückzuführen auf eine aktive oder frische Infektion definiert, in einigen Fällen in Abgrenzung von anhaltenden Subtypen postinfektiöser Kopfschmerzen (siehe zum Beispiel 9.1.1.1 akuter Kopfschmerz zurückzuführen auf eine bakterielle Meningitis oder Meningoenzephalitis, 9.1.1.2 chronischer Kopfschmerz zurückzuführen auf eine bakterielle Meningitis oder Meningoenzephalitis und 9.1.1.3 anhaltender Kopfschmerz zurückzuführen auf eine frühere bakterielle Meningitis oder Meningoenzephalitis). Dies geschieht zu dem Zweck, zwischen zwei wahrscheinlich verschiedenen ursächlichen Mechanismen und Disease-Management-Ansätzen zu unterscheiden und diese nicht zu vermischen.

\section{Einleitung}

Kopfschmerzen sind ein häufiges Begleitsymptom von systemischen viralen Infektionen wie der Influenza. Sie sind auch häufig bei einer Sepsis. Bei anderen systemischen Infektionen findet man sie jedoch seltener.

Kopfschmerzen sind das häufigste und meist auch erste Symptom einer intrakraniellen Infektion. Das Auftreten eines neuen Kopfschmerztyps, der diffus lokalisiert ist, mit fokalen neurologischen Zeichen und/oder Veränderungen der psychischen Verfassung und einem allgemeinen Krankheitsgefühl und/oder Fieber einhergeht, sollte auch bei fehlender Nackensteife an eine intrakranielle Infektion denken lassen.

Bedauerlicherweise existieren keine guten prospektiven Studien zum Kopfschmerz zurückzuführen auf eine intrakranielle Infektion. Mangels ausreichender Evidenz stützen sich die diagnostischen Kriterien für einige der Subtypen von 9.1 Kopfschmerz zurückzuführen auf eine intrakranielle Infektion zumindest in Teilen auf den Konsens in Fachkreisen, unter anderem auf die Ansichten der Experten auf dem Gebiet der Neuroinfektionen.

Die allgemeinen Kriterien für dieses Kapitel, die hier so weit wie möglich eingehalten werden, sind folgende:

A. Kopfschmerz, der Kriterium C erfüllt

B. Es wurde eine Infektion oder Komplikation einer Infektion diagnostiziert, von der bekannt ist, dass sie Kopfschmerzen verursachen kann

C. Ein kausaler Zusammenhang kann durch wenigstens zwei der folgenden Kriterien gezeigt werden:

1. Der Kopfschmerz hat sich in einem zeitlichen Zusammenhang mit dem Beginn der Infektion entwickelt

2. Einer oder beide der folgenden Punkte sind erfüllt:

a) der Kopfschmerz hat sich gleichzeitig mit der Verschlechterung der Infektion deutlich verschlechtert

b) der Kopfschmerz hat sich gleichzeitig mit der Besserung oder dem Verschwinden der Infektion deutlich verbessert oder ist verschwunden

3. Der Kopfschmerz weist Merkmale auf, die für die Infektion typisch sind

D. Nicht besser erklärt durch eine andere ICHD-3-Diagnose. 


\subsection{Kopfschmerz zurückzuführen auf eine intrakranielle Infektion}

Beschreibung:

Kopfschmerz von variabler Dauer, und in seltenen Fällen anhaltend, verursacht durch eine intrakranielle bakterielle, virale, Pilz- oder andere parasitäre Infektion oder durch eine Komplikation einer dieser Infektionen.

\subsubsection{Kopfschmerz zurückzuführen auf eine bakte- rielle Meningitis oder Meningoenzephalitis}

Beschreibung:

Kopfschmerz von variabler Dauer infolge einer bakteriellen Meningitis oder Meningoenzephalitis. Dieser kann sich in Zusammenhang mit leichten grippeähnlichen Symptomen entwickelt. Er ist in der Regel akut und geht mit Nackensteifigkeit, Übelkeit, Fieber und Veränderungen der psychischen Verfassung und/oder anderen neurologischen Symptomen und/oder Zeichen einher. In den meisten Fällen verschwindet er, sobald die Infektion erfolgreich bekämpft wurde, in seltenen Fällen hält er an.

\section{Diagnostische Kriterien:}

A. Kopfschmerz beliebiger Dauer, der Kriterium C erfüllt

B. Es wurde eine bakterielle Meningitis oder Meningoenzephalitis diagnostiziert

C. Ein kausaler Zusammenhang kann durch mindestens zwei der folgenden Kriterien gezeigt werden:

1. Der Kopfschmerz hat sich in einem zeitlichen Zusammenhang mit dem Beginn der bakteriellen Meningitis oder Meningoenzephalitis entwickelt

2. Der Kopfschmerz hat sich gleichzeitig mit der Verschlechterung der bakteriellen Meningitis oder Meningoenzephalitis deutlich verschlechtert

3. Der Kopfschmerz hat sich gleichzeitig mit der Besserung der bakteriellen Meningitis oder Meningoenzephalitis deutlich gebessert

4. Der Kopfschmerz erfüllt einen oder beide der folgenden Punkte:

a) holokraniell

b) in der Nuchalregion lokalisiert und von Nackensteifigkeit begleitet

D. Nicht besser erklärt durch eine andere ICHD-3-Diagnose.

\section{Kommentar:}

Kopfschmerzen sind das häufigste und meist auch das erste Symptom dieser Infektionen. Ein 9.1.1 Kopfschmerz zurückzuführen auf eine bakterielle Meningitis oder Meningoenzephalitis sollte immer dann in Erwägung gezogen werden, wenn Kopfschmerzen in Begleitung von Fieber, Veränderungen der psychischen Verfassung (einschließlich einer herabgesetzten Aufmerksamkeit), fokalen neurologischen Defiziten oder generalisierten epileptischen Anfällen. Im Falle einer Enzephalitis umfassen die hiermit verbundenen Defizite in Form von Sprach- oder Hörstörungen, Doppeltsehen, Gefühlsverlust in einigen Teilen des Körpers, Muskelschwäche, partielle Lähmungen in den Armen und Beinen, Halluzinationen, Persönlichkeitsveränderungen, eingeschränkter Urteilsfähigkeit, Bewusstlosigkeit, plötzliche schwerer Demenz und/oder Gedächtnisverlust.
Dennoch ist es bei intrakraniellen bakteriellen Infektionen meist außerordentlich schwierig, zwischen einer bloßen meningealen Beteiligung und einer bloßen enzephalitischen Beteiligung zu unterscheiden. Abgesehen davon mündet diese Unterscheidung nicht in unterschiedliche Herangehensweisen an die Beurteilung oder die Wahl des Behandlungsansatzes. Aus diesem Grund wird der Kopfschmerz zurückzuführen auf eine bakterielle Meningitis und der Kopfschmerz zurückzuführen auf eine bakterielle Enzephalitis als eine einzige Entität, 9.1.1 Kopfschmerz zurückzuführen auf eine bakterielle Meningitis oder Meningoenzephalitis, zusammengefasst.

Es gibt eine ganze Bandbreite von Bakterien, die eine Meningitis und/oder Enzephalitis verursachen können, darunter Streptococcus pneumoniae, Neisseria meningitidis und Listeria monocytogenes. Der immunologische Hintergrund ist sehr wichtig, da eine Immunsuppression (aufgrund von HIV bzw. nach einer Transplantation oder infolge anderer chronisch immundepressiven Therapien) Auswirkungen auf die Anfälligkeit sowie das klinische und biologische Profil hat.

Der initiale Kopfschmerz wird durch eine direkte Stimulation von sensiblen Nervenendigungen in den Meningen durch die bakterielle Infektion ausgelöst. Bakterienprodukte (Toxine) sowie Entzündungsmediatoren wie Bradykinin, Prostaglandine, Zytokine und andere durch den entzündlichen Prozess freigesetzte Substanzen verursachen nicht nur direkt Schmerzen, sondern führen zu einer Schmerzsensibilisierung und einer Freisetzung von Neuropeptiden. Bei bestehender Enzephalitis kann auch eine intrakranielle Hypertension als Kopfschmerzursache eine Rolle spielen.

In den meisten Fällen verschwindet der Kopfschmerz mit Verschwinden der Infektion. Dennoch kann die Infektion noch für Monate aktiv bleiben und so zu chronischen Kopfschmerz führen. In einer Minderheit der Fälle bleibt der Kopfschmerz noch mehr als 3 Monate nach Verschwinden der ursächlichen Infektion bestehen. Aus diesem Grund werden unten drei eigenständige Subtypen eines 9.1.1 Kopfschmerzes zurückzuführen auf eine bakterielle Meningitis oder Meningoenzephalitis beschrieben, denn ihre Pathophysiologie und Behandlung unterscheiden sich je nachdem, ob die Infektion vollständig ausgemerzt werden konnte oder noch aktiv ist.

\subsubsection{Akuter Kopfschmerz zurückzuführen auf eine bakterielle Meningitis oder Meningoenzephalitis Diagnostische Kriterien:}

A. Kopfschmerz, der die Kriterien für einen 9.1.1 Kopfschmerz zurückzuführen auf eine bakterielle Meningitis oder Meningoenzephalitis sowie Kriterium B unten erfüllt

B. Der Kopfschmerz besteht bereits seit<3 Monaten.

\subsubsection{Chronischer Kopfschmerz zurückzuführen auf eine bakterielle Meningitis oder Meningoenzephalitis} Diagnostische Kriterien:

A. Kopfschmerz, der die Kriterien für einen 9.1.1 Kopfschmerz zurückzuführen auf eine bakterielle Meningitis oder Meningoenzephalitis sowie Kriterium $C$ unten erfüllt

B. Zustand bei noch aktiver1 oder innerhalb der letzten 3 Monate abgeklungener bakterieller Meningitis oder Meningoenzephalitis

C. Der Kopfschmerz besteht bereits seit>3 Monaten. 


\section{Anmerkung:}

1. Nachweis mittels fokaler oder multifokaler Kontrastmittelanreicherung im MRT und/oder Persistieren einer Liquorpleozytose mit oder ohne nachgewiesene Schädigung der Blut-HirnSchranke.

\subsubsection{Anhaltender Kopfschmerz zurückzuführen auf eine frühere bakterielle Meningitis oder Meningoen- zephalitis}

Diagnostische Kriterien:

A. Kopfschmerz, der zuvor die Kriterien für einen 9.1.1 Kopfschmerz zurückzuführen auf eine bakterielle Meningitis oder Meningoenzephalitis erfüllte sowie Kriterium C unten erfüllt

B. Die bakterielle Meningitis oder Meningoenzephalitis ist verschwunden

C. Der Kopfschmerz besteht schon seit>3 Monaten nach Verschwinden der bakteriellen Meningitis oder Meningoenzephalitis fort

D. Nicht besser erklärt durch eine andere ICHD-3-Diagnose.

\subsubsection{Kopfschmerz zurückzuführen auf eine virale Meningitis oder Enzephalitis}

\section{Beschreibung:}

Kopfschmerz infolge einer viralen Meningitis oder Enzephalitis, typischerweise mit Nackensteifigkeit und Fieber und je nach Umfang der Infektion variabel begleitet von neurologischen Symptomen und/oder Zeichen bis hin zu psychomentalen Veränderungen.

\section{Diagnostische Kriterien:}

A. Kopfschmerz, der das Kriterium C erfüllt

B. Es wurde eine virale Meningitis oder Enzephalitis diagnostiziert

C. Ein kausaler Zusammenhang kann durch mindestens zwei der folgenden Kriterien gezeigt werden:

1. Der Kopfschmerz hat sich in einem zeitlichen Zusammenhang mit dem Beginn der viralen Meningitis oder Enzephalitis entwickelt

2. Der Kopfschmerz hat sich gleichzeitig mit einer Verschlechterung der viralen Meningitis oder Enzephalitis deutlich verschlechtert

3. Der Kopfschmerz hat sich gleichzeitig mit einer Besserung der viralen Meningitis oder Enzephalitis deutlich gebessert

4. Der Kopfschmerz erfüllt einen oder beide der folgenden Punkte:

a) holokraniell

b) in der Nuchalregion lokalisiert und von Nackensteifigkeit begleitet

D. Nicht besser erklärt durch eine andere ICHD-3-Diagnose.

\section{Kommentar:}

Der Verdacht auf einen 9.1.2 Kopfschmerz zurückzuführen auf eine virale Meningitis oder Enzephalitis sollte immer dann in Betracht gezogen werden, wenn ein Kopfschmerz in Begleitung von Fieber, Nackensteifigkeit, Lichtempfindlichkeit und Übelkeit und/oder Erbrechen auftritt.

Die Enteroviren spielen bei 9.1.2 Kopfschmerz zurückzuführen auf eine virale Meningitis oder Enzephalitis meist die größte
Rolle, diverse andere virale Erreger können aber auch verantwortlich sein, so u. a. Arboviren, Polioviren, Echoviren, Coxsackie-Viren, Herpes-simplex-Viren, Varicella-zoster-Viren, Adenoviren und Mumpsviren. In den meisten Fällen ist die Identifizierung des verursachenden Virus mit Hilfe der Liquor-Polymerase-Kettenreaktion (PCR) erfolgreich. Ein positiver Liquor-PCR-Befund auf Herpes-simplex-Viren (HSV) vom Typ 1 oder 2 und der serologische Befund für HSV-1 \& 2 DNA führen zur Annahme der Diagnose einer Herpes-simplex-Enzephalitis. In manchen Fällen ist die Liquor-PCR positiv für humane Herpesviren (HHV) Typ 6 oder 7. Es ließ sich dokumentieren, dass die PCR-Sensitivität sich um mehr als die Hälfte reduziert, wenn der Test eine Woche nach Symptombeginn durchgeführt wird, was zu falsch negativen Ergebnissen führt. Fällt die nach einer Woche durchgeführte PCR negativ aus, kann die Diagnose auf Grundlage eines veränderten Liquor/Blut-Antikörper-Verhältnisses gestellt werden.

Wie bei der intrakraniellen bakteriellen Infektion kann es bei einer viralen Infektion schwierig sein, zwischen einer reinen Beteiligung der Meningen und einer reinen Beteiligung des Gehirns zu unterscheiden. Dennoch ist das Treffen und Bewahren dieser Unterscheidung von Bedeutung, da die beiden Erkrankungen prognostisch voneinander abweichen, mit einer ungünstigeren Prognose bei enzephalitischer Beteiligung. Aus diesem Grund werden für 9.1.2.1 Kopfschmerz zurückzuführen auf eine virale Meningitis und 9.1.2.2 Kopfschmerz zurückzuführen auf eine virale Enzephalitis jeweils eigene Kriterien angegeben.

Ebenfalls abweichend von einem 9.1.1 Kopfschmerz zurückzuführen auf eine bakterielle Meningitis oder Meningoenzephalitis wird ein anhaltender postinfektiöser Subtyp eines 9.1.2 Kopfschmerzes zurückzuführen auf eine virale Meningitis oder Enzephalitis nicht belegt und wurde deshalb nicht berücksichtigt.

\subsubsection{Kopfschmerz zurückzuführen auf eine virale Meningitis \\ Diagnostische Kriterien:}

A. Kopfschmerz, der die Kriterien für einen 9.1.2 Kopfschmerz zurückzuführen auf eine virale Meningitis oder Enzephalitis erfüllt.

B. Die zerebrale Bildgebung zeigt ausschließlich eine Kontrastmittelanreicherung der Leptomeningen.

\subsubsection{Kopfschmerz zurückzuführen auf eine virale Enzephalitis \\ Diagnostische Kriterien:}

A. Kopfschmerz, der die Kriterien für einen 9.1.2 Kopfschmerz zurückzuführen auf eine virale Meningitis oder Enzephalitis erfüllt

B. Einer oder beide der folgenden Punkte sind erfüllt:

1. Die zerebrale Bildgebung zeigt diffuse oder multifokal Hirnödeme 1

2. Mindestens eines von Folgendem:
a) psychomentale Veränderungen
b) fokale neurologische Defizite
c) epileptische Anfälle. 
Anmerkung:

1. Es kann zudem begleitend ein leptomeningeales Enhancement auftreten

Kommentar:

Der Schmerz ist in der Regel diffus, mit Fokus in der frontalen und/ oder retroorbitalen Region, heftig oder extrem heftig und pochender oder drückender Natur.

Der Verdacht auf einen 9.1.2.2 Kopfschmerz zurückzuführen auf eine virale Enzephalitis sollte immer dann erwogen werden, wenn Kopfschmerzen von psychomentalen Veränderungen (einschließlich einer herabgesetzten Aufmerksamkeit), von fokalen neurologischen Defiziten und/oder epileptischen Anfällen begleitet werden. Zu den weiteren oft begleitenden neurologischen Defiziten gehören Sprach- oder Hörstörungen, Doppeltsehen, Gefühlsverlust in einigen Teilen des Körpers, Muskelschwäche, partielle Lähmungen in den Armen und Beinen, Ataxie, Halluzinationen, Persönlichkeitsveränderungen, Bewusstlosigkeit und/oder Gedächtnisverlust.

\subsubsection{Kopfschmerz zurückzuführen auf eine Pilz- oder andere parasitäre Infektion}

Beschreibung:

Kopfschmerz von variabler Dauer infolge einer intrakraniellen Pilzoder sonstigen parasitären Infektion. In der Regel wird dieser im Kontext einer kongenitalen oder erworbenen Immunsuppression beobachtet. In den meisten Fällen verschwindet dieser, sobald die Infektion erfolgreich bekämpft ist; in seltenen Fällen hält er an.

\section{Diagnostische Kriterien:}

A. Kopfschmerz, der das Kriterium C erfüllt

B. Es wurde eine intrakranielle Pilz- oder andere parasitäre Infektion diagnostiziert

C. Ein kausaler Zusammenhang kann durch mindestens zwei der folgenden Kriterien gezeigt werden:

1. Der Kopfschmerz hat sich in einem zeitlichen Zusammenhang mit dem Beginn der intrakraniellen Pilz- oder anderen parasitären Infektion entwickelt

2. Der Kopfschmerz hat sich gleichzeitig mit der Verschlechterung der intrakraniellen Pilz- oder anderen parasitären Infektion deutlich verschlechtert

3. Der Kopfschmerz hat sich gleichzeitig mit der Besserung der intrakraniellen Pilz- oder anderen parasitären Infektion deutlich gebessert

4. Der Kopfschmerz entwickelt sich progressiv1 und einer oder beide der folgenden Punkte sind erfüllt:

a) holokraniell

b) in der Nuchalregion lokalisiert und von Nackensteifigkeit begleitet

D. Nicht besser erklärt durch eine andere ICHD-3-Diagnose2.

Anmerkung:

1. Die klinischen Symptome entwickeln sich tendenziell über Wochen, parallel zum Ausmaß der Immunsuppression.

2. Die Früherkennung erfolgt am besten mittels CT oder MRT.
Kommentar:

Dem Verdacht auf einen 9.1.3 Kopfschmerz zurückzuführen auf eine Pilz- oder andere parasitäre Infektion sollte immer dann nachgegangen werden, wenn Kopfschmerz bei Immunsupprimierten in Begleitung von Fieber, zunehmenden psychomentalen Veränderungen (einschließlich einer beeinträchtigten Aufmerksamkeit) und/oder multiplen fokalen neurologischen Defiziten von zunehmendem Schweregrad auftritt und die zerebrale Bildgebung ein leptomeningeales Enhancement und/oder ein diffuses Hirnödem zeigt.

Zu den Pilzen, die eine Meningitis und/oder Enzephalitis verursachen können, gehören Candida, Aspergillus und Cryptococcus neoformans; zu den Parasiten gehört Toxoplasma gondii. Neben Liquorkultur- und PCR-Untersuchungen des Liquors umfassen weitere Liquor- und Blutuntersuchungen den direkten Nachweis (zytologischer Nachweis, mikroskopische Darstellung, Kultur und Identifikation von Pilzelementen in den beobachteten biologischen Materialien) und der indirekte Nachweis des Pathogens (Identifikation eines Antigens oder anderen Elements der Kapsel). Bei der Aspergillose lässt sich das Galaktomannan-Antigen in biologischen Flüssigkeiten (Serum, bronchoalveoläre Waschflüssigkeit oder Liquor) nachweisen. Bei anderen systemischen Pilzinfektionen kann das Serum-1,3- $\beta$-D-Glucan diagnostisch weiterhelfen. Der India-inkTest ermöglicht eine Einfärbung der Kapsel des Cryptococcus neoformans.

Pilz- und parasitäre Infektionen der Meningen oder des Enzephalons werden fast ausschließlich bei immunsupprimierten Patienten oder alten Menschen beobachtet. Konkret besteht ein Risiko bei den folgenden Gruppen:

1) Menschen mit deutlicher Neutropenie (<500 Neutrophilen/ $\mathrm{mm} 3$ );

2) Menschen nach allogenem Stammzellenimplantat;

3) Menschen, die sich einer chronischen Kortikoidtherapie unterziehen (Prednison $0,3 \mathrm{mg} / \mathrm{kg} /$ Tag oder entsprechendes für mehr als 3 Wochen);

4) Menschen mit einer noch laufenden oder kurz zuvor (innerhalb der letzten 90 Tage) abgeschlossenen Behandlung mit Immunsuppressiva (Cyclosporin, TNF-Blocker, monoklonale Antikörper, Nukleosidanaloga);

5) Menschen mit einem schweren primären Immundefekt.

Ein anhaltender postinfektiöser Subtyp eines 9.1.3 Kopfschmerzes zurückzuführen auf eine Pilz- oder andere parasitäre Infektion tritt auf, ist in der Fachliteratur jedoch nicht gut dokumentiert; sie wurde nur als A9.1.3.3 anhaltender Kopfschmerz zurückzuführen auf eine frühere intrakranielle Pilz- oder andere parasitäre Infektion in den Anhang aufgenommen.

\subsubsection{Akuter Kopfschmerz zurückzuführen auf eine Pilz- oder andere parasitäre Infektion \\ Diagnostische Kriterien:}

A. Kopfschmerz, der die Kriterien für einen 9.1.3 Kopfschmerz zurückzuführen auf eine Pilz- oder andere parasitäre Infektion und Kriterium B unten erfüllt

B. Kopfschmerz besteht seit<3 Monaten. 
9.1.3.2 Chronischer Kopfschmerz zurückzuführen auf eine Pilz- oder andere parasitäre Infektion

Diagnostische Kriterien:

A. Kopfschmerz, der die Kriterien für einen 9.1.3 Kopfschmerz zurückzuführen auf eine Pilz- oder andere parasitäre Infektion und Kriterium B unten erfüllt

B. Der Kopfschmerz besteht seit >3 Monaten.

\subsubsection{Kopfschmerz zurückzuführen auf eine lokali- sierte Gehirninfektion}

Beschreibung:

Kopfschmerz infolge eines Hirnabszesses, einer subduralen Eiteransammlung, einem infektiösen Granulom oder einer anderen lokalisierten infektiösen Läsion, meist begleitet von Fieber, (einem) fokalen neurologischen Defizit(en) und/oder psychomentalen Veränderungen (einschließlich einer herabgesetzten Aufmerksamkeit).

Diagnostische Kriterien:

A. Kopfschmerz, der das Kriterium C erfüllt

B. Lokalisierte Gehirninfektion, nachgewiesen anhand von zerebraler Bildgebung und/oder einer Probenanalyse

C. Ein kausaler Zusammenhang kann durch mindestens zwei der folgenden Kriterien gezeigt werden:

1. Der Kopfschmerz hat sich in einem zeitlichen Zusammenhang mit der Entwicklung der lokalisierten Gehirninfektion entwickelt oder führte zu deren Entdeckung

2. Der Kopfschmerz hat sich gleichzeitig mit der Verschlimmerung der lokalisierten Gehirninfektion deutlich verschlechtert, die sich in einem der folgenden Punkte zeigt: a) Verschlechterung anderer Symptome und/oder klinischer Zeichen infolge der lokalisierten Gehirninfektion

b) Nachweis der weiteren Ausdehnung (oder im Falle eines Hirnabszesses Ruptur) der lokalisierten Gehirninfektion

3. Der Kopfschmerz hat sich gleichzeitig mit der Besserung der lokalisierten Gehirninfektion deutlich verbessert

4. Der Kopfschmerz weist wenigstens eines der folgenden Charakteristika auf:

a) Intensität nimmt allmählich zu, über Stunden oder Tage, auf mäßig oder heftig

b) durch Anstrengung oder eine Art von Valsalva-Manöver verschlimmert

c) begleitet von Fieber, Übelkeit und/oder Erbrechen

d) unilateral, dabei ipsilateral zur lokalisierten Gehirninfektion

D. Nicht besser erklärt durch eine andere ICHD-3-Diagnose.

\section{Kommentar:}

Hirnabszesse entstehen in der Regel durch anaerobe oder mitunter gemischte Bakterien, oft einschließlich anaerober Streptokokken oder Bakteroide. Staphylokokken treten häufig nach einem Hirntrauma, einem neurochirurgischen Eingriff oder einer Endokarditis auf. Enterobakterien sind bei chronischen Ohrinfektionen weit verbreitet. Pilze (z. B. Aspergillus) and Einzeller (z. B. Toxoplasma gondii, vor allem bei HIV-infizierten Patienten) können Abszesse verursachen.
Ein subdurales Empyem entsteht häufig sekundär infolge einer Sinusitis oder einer Otitis media. Es kann sich aber auch um eine Komplikation einer Meningitis handeln.

Granulome im Gehirn wurden mit einer Zystizerkose, Sarkoidose, Toxoplasmose und Aspergillose in Verbindung gebracht.

Zu den ursächlichen Mechanismen hinter einem 9.1.4 Kopfschmerz zurückzuführen auf eine lokalisierte Gehirninfektion gehören eine direkte Kompression, eine Irritation meningealer und/ oder arterieller Strukturen, ein erhöhter intrakranieller Druck und Fieber. Kopfschmerz zurückzuführen auf ein subdurales Empyem tritt vor allem in Verbindung mit Fieber und Symptomen und/oder klinischen Zeichen einer meningealen Irritation und eines erhöhten intrakraniellen Drucks auf.

\subsection{Kopfschmerz zurückzuführen auf eine systemische Infektion}

An anderer Stelle kodiert: Ein Kopfschmerz, der auf eine systemische Infektion zurückzuführen ist, die eine Meningitis oder Enzephalitis begleitet, sollte demgemäß unter 9.1 Kopfschmerz zurückzuführen auf eine intrakranielle Infektion kodiert werden.

Beschreibung:

Kopfschmerz von variabler Dauer infolge einer systemischen Infektion, in der Regel in Begleitung anderer Symptome und/oder klinischer Zeichen der Infektion.

\section{Kommentar:}

Kopfschmerzen bei systemischen Infektionen sind ein meist unauffälliges Symptom und diagnostisch wenig hilfreich. Diese Krankheitszustände werden dominiert von Fieber, einem allgemeinen Krankheitsgefühl und systemischen Symptomen. Nichtsdestotrotz finden sich Kopfschmerzen bei einigen systemischen Infektionen, insbesondere bei Influenza, als Leitsymptom zusammen mit Fieber und anderen Symptomen. Wird die systemische Infektion von einer Meningitis oder Enzephalitis begleitet, sollte der Kopfschmerz zurückzuführen auf die Infektion unter diesen Störungen als Subtyp eines 9.1 Kopfschmerzes zurückzuführen auf eine intrakranielle Infektion kodiert werden.

Bei infektiösen Erkrankungen treten Kopfschmerzen meist gemeinsam mit Fieber auf und können darauf beruhen. In einigen Fällen kann Fieber jedoch auch völlig fehlen. Die genaue Natur dieser Mechanismen müssen noch untersucht werden. Derweil verweist die große Variabilität von systemischen Infektionen in ihrer Potenz, Kopfschmerzen hervorzurufen, darauf hin, dass dieser Effekt nicht durch Fieber und exogene oder endogene Pyrogene allein entsteht. Zu den Mechanismen der Kopfschmerzentstehung zählen auch direkt durch die Mikroorganismen selbst hervorgerufene Effekte. Es dürften diverse Zellen beteiligt sein (aktivierte Mikrogliazellen und monozytische Makrophagen, aktivierte Astrozyten sowie die Blut-Hirn-Schranke und Endothelzellen), zusammen mit mehreren immuno-inflammatorischen Mediatoren (Zytokine, Glutamat, COX-2/PGE2-System, NO-iNOS-System und reaktives Sauerstoffspezies-System). 


\subsubsection{Kopfschmerz zurückzuführen auf eine bakte- rielle systemische Infektion}

Beschreibung:

Kopfschmerz bei nicht vorliegender Meningitis oder Meningoenzephalitis, der durch andere Symptome und/oder klinische Zeichen einer systemischen bakteriellen Infektion verursacht wird und in deren Begleitung auftritt.

\section{Diagnostische Kriterien:}

A. Kopfschmerz beliebiger Dauer, der Kriterium C erfülltt

B. Es treffen die beiden folgenden Punkte zu:

1. Es wurde eine systemische bakterielle Infektion diagnostiziert

2. Kein Hinweis auf eine meningitische oder meningoenzephalitische Beteiligung

C. Ein kausaler Zusammenhang kann durch mindestens zwei der folgenden Kriterien gezeigt werden:

1. Der Kopfschmerz hat sich in einem zeitlichen Zusammenhang mit dem Beginn der systemischen bakteriellen Infektion entwickelt

2. Der Kopfschmerz hat sich gleichzeitig mit der Verschlechterung der systemischen bakteriellen Infektion deutlich verschlechtert

3. Der Kopfschmerz hat sich gleichzeitig mit der Besserung oder dem Verschwinden der systemischen bakteriellen Infektion deutlich gebessert oder ist verschwunden

4. Der Kopfschmerz zeigt eines oder beide der folgenden Charakteristika:

a) diffuser Schmerz

b) mäßige oder heftige Intensität

D. Nicht besser erklärt durch eine andere ICHD-3-Diagnose.

\subsubsection{Akuter Kopfschmerz zurückzuführen auf eine bakterielle systemische Infektion}

Diagnostische Kriterien:

A. Kopfschmerz, der die Kriterien für einen 9.2.1 Kopfschmerz zurückzuführen auf eine bakterielle systemische Infektion und Kriterium B unten erfüllt

B. Kopfschmerz besteht seit<3 Monaten.

\subsubsection{Chronischer Kopfschmerz zurückzuführen auf eine bakterielle systemische Infektion}

Diagnostische Kriterien:

A. Kopfschmerz, der die Kriterien für einen 9.2.1 Kopfschmerz zurückzuführen auf eine bakterielle systemische Infektion und Kriterium B unten erfüllt

B. Kopfschmerz besteht sei>3 Monaten.

\subsubsection{Kopfschmerz zurückzuführen auf eine syste- mische virale Infektion}

Beschreibung:

Kopfschmerz infolge einer systemischen viralen Infektion, der in Begleitung andere Symptome und/oder klinischen Zeichen einer solchen auftritt, bei Nichtvorliegen einer Meningitis oder Enzephalitis.
Diagnostische Kriterien:

A. Kopfschmerz von beliebiger Dauer, der Kriterium C erfüllt

B. Die beiden folgenden Punkte treffen zu:

1. Es wurde eine systemische virale Infektion diagnostiziert

2. Kein Hinweis auf eine meningitische oder enzephalitische Beteiligung

C. Ein kausaler Zusammenhang kann durch mindestens zwei der folgenden Kriterien gezeigt werden:

1. Der Kopfschmerz hat sich in einem zeitlichen Zusammenhang mit dem Beginn der systemischen viralen Infektion entwickelt

2. Der Kopfschmerz hat sich gleichzeitig mit der Verschlechterung der systemischen viralen Infektion deutlich verschlechtert

3. Der Kopfschmerz hat sich gleichzeitig mit der Besserung oder dem Verschwinden der systemischen viralen Infektion deutlich verbessert oder ist verschwunden

4. Der Kopfschmerz zeigt eines oder beide der folgenden Charakteristika:

a) diffuser Schmerz

b) mäßige oder heftige Intensität

D. Nicht besser erklärt durch eine andere ICHD-3-Diagnose.

\subsubsection{Akuter Kopfschmerz zurückzuführen auf eine systemische virale Infektion}

Diagnostische Kriterien:

A. Kopfschmerz erfüllt die Kriterien für einen 9.2.2 Kopfschmerz zurückzuführen auf eine systemische virale Infektion und Kriterium B unten

B. Kopfschmerz besteht seit<3 Monaten.

9.2.2.2 Chronischer Kopfschmerz zurückzuführen auf eine systemische virale Infektion

Diagnostische Kriterien:

A. Kopfschmerz erfüllt die Kriterien für einen 9.2.2 Kopfschmerz zurückzuführen auf eine systemische virale Infektion und Kriterium $\mathrm{C}$ unten

B. Die systemische virale Infektion ist noch aktiv oder ist erst innerhalb der letzten 3 Monate abgeklungen

C. Kopfschmerz besteht seit $>3$ Monaten.

\subsubsection{Kopfschmerz zurückzuführen auf eine andere systemische Infektion}

Beschreibung:

Kopfschmerz, der von anderen Symptomen und/oder klinischen Zeichen einer systemischen Pilzinfektion oder Infektion bzw. Befall mit Protozoen oder anderen Parasiten verursacht wird und in deren Begleitung auftritt, bei nicht vorliegender Meningitis oder Meningoenzephalitis.

Diagnostische Kriterien:

A. Kopfschmerz, der das Kriterium C erfüllt

B. Es sind die beiden folgenden Punkte erfüllt:

1. Es wurde eine systemische Pilzinfektion oder ein Befall mit Protozoen oder anderen Parasiten diagnostiziert

2. Kein Hinweis auf eine meningitische oder meningoenzephalitische Beteiligung 
C. Ein kausaler Zusammenhang kann durch mindestens zwei der folgenden Kriterien gezeigt werden:

1. Der Kopfschmerz hat sich in einem zeitlichen Zusammenhang mit dem Beginn der systemischen Infektion oder des Befalls entwickelt

2. Der Kopfschmerz hat sich gleichzeitig mit der Verschlechterung der systemischen Infektion oder des Befalls deutlich verschlechtert

3. Der Kopfschmerz hat sich gleichzeitig mit der Besserung der systemischen Infektion oder des Befalls deutlich gebessert

4. Der Kopfschmerz zeigt eines oder beide der folgenden Charakteristika:

a) diffuser Schmerz

b) mäßige oder heftige Intensität

D. Nicht besser erklärt durch eine andere ICHD-3-Diagnose.

\section{Kommentar:}

Es handelt sich um eine heterogene und unzureichend definierte Gruppe von systemischen Infektionen, die am häufigsten bei immunsupprimierten Patienten oder in bestimmten geografischen Regionen auftreten.

Die am häufigsten beteiligten Pilze sind die pathogenen Pilze (Cryptococcus neoformans, Histoplasma capsulatum und Coccidioides immitis) sowie die opportunistischen Pilze (Candida-Arten, Aspergillus-Arten u. a.). Unter den Protozoen können Infestationen mit Pneumocystis carinii und Toxoplasma gondii mit Kopfschmerzen einhergehen. Kopfschmerzen wurden auch in Verbindung mit dem Fadenwurm Strongyloides stercoralis berichtet.

\subsubsection{Akuter Kopfschmerz zurückzuführen auf eine andere systemische Infektion \\ Diagnostische Kriterien:}

A. Kopfschmerz, der die Kriterien für einen 9.2.3 Kopfschmerz zurückzuführen auf eine andere systemische Infektion und Kriterium B unten erfüllt

B. Kopfschmerz besteht seit $<3$ Monaten.

\subsubsection{Chronischer Kopfschmerz zurückzuführen auf eine andere systemische Infektion}

\section{Diagnostische Kriterien:}

A. Kopfschmerz, der die Kriterien für einen 9.2.3 Kopfschmerz zurückzuführen auf eine andere systemische Infektion und Kriterium B unten erfüllt

B. Kopfschmerz besteht seit>3 Monaten.

\section{Literatur}

\subsection{Kopfschmerz zurückzuführen auf eine intrakranielle Infektion}

Solomon T, Michael BD, Smoth PE et al. Management of suspected viral encephalitis in adults. Association of British Neurologists and British Infection Association National Guidelines. J Infection 2012; 64: 347-373.

\subsubsection{Kopfschmerz zurückzuführen auf eine bakterielle Meningitis oder Meningoenzephalitis}

Bohr V, Hansen B, Kjersen H, Rasmussen N, Johnsen N, Kristensen HS, Jessen $O$. Sequelae from bacterial meningitis and their relation to the clinical condition during acute illness, based on 667 questionnaire returns. Part II of a three part series. J Infection 1983; 7(2): 102-110.

Brooks RG, Licitra CM, Peacock MG. Encephalitis caused by Coxiella burnetii. Ann Neurol 1986; 20: 91-93.

Drexler ED. Severe headache: when to worry, what to do. Postgrad Med J 1990; 87: 164-170, 173-180.

Francke E. The many causes of meningitis. Postgrad Med 1987; 82: 175-178, 181-183, 187-188.

Gedde-Dahl TW, Lettenstrom GS, Bovre K. Coverage for meningococcal disease in the Norwegian morbidity and mortality statistics. NIPH Ann 1980; 3: 31-35.

Helbok R, Broessner G, Pfausler B, Schmutzhard E. Chronic meningitis. J Neurol 2009; 256: 168-175.

Jones HR, Siekert RG. Neurological manifestation of infective endocarditis. Brain 1989; 112: 1295-1315.

Pachner AR, Steere AC. Neurological findings of Lyme disease. Yale Biol Med 1984; 57: 481-483.

Pachner AR, Steere AC. The triad of neurologic manifestations of Lyme disease: meningitis, cranial neuritis, and radiculoneuritis. Neurology 1985; 35: 47-53.

Tonjum T. Nilsson F, Bruun JH, Hanebeg B. The early phase of meningococcal disease. NIPH Ann 1983; 6: 175-181.

van de Beek D, de Gans J, Spanjaard L, Weisfelt M, Reitsma JB, Vermeulen M. Clinical features and prognostic factors in adults with bacterial meningitis. NEJM 2004; 351(18): 1849-1859.

Zhang SR, Zhang YS, Zhao XD. Tuberculous meningitis with hydrocephalus: a clinical and CT study. Chung Hua Nei Ko Tsa Chih 1989; 28: 202-204.

\subsubsection{Kopfschmerz zurückzuführen auf eine virale Meningitis oder Enzephalitis}

Ambrose HE, Granerod J, Clewley JP, Davies NW, Keir G, Cunningham R, Zuckerman M, Mutton KJ, Ward KN, ljaz S, Crowcroft NS, Brown DW. UK Aetiology of Encephalitis Study Group. Diagnostic strategy used to establish etiologies of encephalitis in a prospective cohort of patients in England. J Clin Microbiol 2011; 49: 3576-3583.

Davis LE, McLaren LC. Relapsing herpes simplex encephalitis following antiviral therapy. Ann Neurol 1983; 13: 192-195.

Denes E, Labach C, Durox H, Adoukonou T, Weinbreck P, Magy L, Ranger-Rogez $S$. Intrathecal synthesis of specific antibodies as a marker of herpes simplex encephalitis in patients with negative PCR. Swiss Med Wkly 2010; 140: w13107.

Desmond RA, Accortt NA, Talley L, Villano SA, Soong S-J, Whitley RJ. Enteroviral meningitis: natural history and outcome of pleconaril therapy. Antimicrobial Agents and Chemotherapy 2006; 50: 2409-2414.

Domachowske JB, Cunningham CK, Cummings DL, Crosley CJ, Hannan WP, Weiner LB. Acute manifestations and neurologic sequelae of Epstein-Barr virus encephalitis in children. Pediatr Infect Dis J 1996; 15 : 871-875.

Domingues RB, Kuster GW, Onuki de Castro FL, Souza VA, Levi JE, Pannuti CS. Headache features in patients with dengue virus infection. Cephalalgia 2006; 26: 879-882.

Farazmand P, Woolley PD, Kinghorn GR. Mollaret's meningitis and herpes simplex virus type 2 infections. Int J STD AIDS. 2011; 22: 306-307.

Kennedy PG. Retrospective analsys of 46 cases of simplex encephalitis seen in Glasgow between 1962 and 1985. OJM 1988; 86: 533-540.

Kennedy PG, Adams IH, Graham DI, Clements GB. A clinico-pathological study of herpes simplex encephalitis. Neuropathol Appl Neurobiol 1998; 14: 395-415.

Mutton K, Guiver M. Laboratory techniques for human viral encephalitis diagnosis. Infect Disord Drug Targets 2011; 11(3): 206-234.

Poneprasert B. Japanese encephalitis in children in northern Thailand. Southeast Asian J Trop Med Public health 1989; 20:599-603. 
Poulikakos PJ, Sergi EE, Margaritis AS, Kioumourtzis AG, Kanellopoulos GD, Mallios PK, Dimitrakis DJ, Poulikakos DJ, Aspiotis AA, Deliousis AD, Flevaris CP, Zacharof AK. A case of recurrent benign lymphocytic (Mollaret's) meningitis and review of the literature. I Infect Public Health 2010; 3: 192-195.

Saged JI, Weinstein Mo, Miller DC. Chronic encephalitis possibly due to herpes simplex virus: two cases. Neurology 1985; 35: 1470-1472.

Sauerbrei A, Wutzler P. Laboratory diagnosis of central nervous system infections caused by herpesviruses. J Clin Virol 2002; 25 Suppl 1: S45-S51.

Singer JI, Maur PR, Riley JP, Smith PB. Management of central nervous system infections during an epidemic of enteroviral aseptic meningitis. J Pediatr 1980; 96: 559-563.

Takeuchi S, Takasato Y, Masaoka H, Hayakawa T, Otani N, Yoshino Y, Yatsushige H, Sugawara T. Hemorrhagic encephalitis associated with Epstein-Barr virus infection. J Clin Neurosci 2010; 17: 153-154.

\subsubsection{Kopfschmerz zurückzuführen auf eine Pilz- oder andere parasitäre Infektion}

Cochius JI, Burns RJ, Willoughby JO. CNS cryptococcosis: unusual aspects. Clin Exp Neurol 1989; 26: 183-191.

Contini C. Clinical and diagnostic management of toxoplasmosis in the immunocompromised patient. Parassitologia 2008; 50: 45-50.

Drake KW, Adam RD. Coccidioidal meningitis and brain abscesses: analysis of 71 cases at a referral center. Neurology 2009; 73: 1780-1786.

Onishi A, Sugiyama D, Kogata Y, Saegusa J, Sugimoto T, Kawano S, Morinobu A, Nishimura K, Kumagai S. Diagnostic accuracy of serum 1,3- $\beta$-Dglucan for pneumocystis jiroveci pneumonia, invasive candidiasis, and invasive aspergillosis: systematic review and meta-analysis. J Clin Microbiol 2012; 50: 7-15.

Patil SA, Katyayani S, Arvind N. Significance of antibody detection in the diagnosis of cryptococcal meningitis. J Immunoassay Immunochem 2012; 33: 140-148.

Prandota J. Recurrent headache as the main symptom of acquired cerebral toxoplasmosis in nonhuman immunodeficiency virus-infected subjects with no lymphadenopathy: the parasite may be responsible for the neurogenic inflammation postulated as a cause of different types of headaches. Am J Ther 2007; 14: 63-105.

Singh RR, Chaudhary SK, Bhatta NK, Khanal B, Shah D. Clinical and etiological profile of acute febrile encephalopathy in eastern Nepal. Indian J Pediatr 2009; 76: 1109-1111.

9.1.4 Kopfschmerz zurückzuführen auf eine lokalisierte Gehirninfektion

Chalstrey S, Pfleiderer AG, Moffat DA. Persisting incidence and mortality of sinogenic cerebral abscess: a continuing reflection of late clinical diagnosis. J R Soc Med 1991; 84: 193-195.

Chun CH, Johnson JD, Hofstetter M, Raff MJ. Brain abscess: a study of 45 consecutive cases. Medicine 1986; 65: 415-431.

French H, Schaefer N, Keijzers G, Barison D, Olson S. Intracranial subdural empyema: a 10-year case series. Ochsner J 2014; 14: 188-194.

Harris LF, Maccubbin DA, Triplett JN, Haws FB. Brain abscess: recent experience at a community hospital. South Med J 1985; 78: 704-707.

Hodges J, Anslow P, Gillet G. Subdural empyema: continuing diagnostic problems in the CT scan era. QJM 1986; 59: 387-393.

Kulay A, Ozatik N, Topucu I. Otogenic intracranial abscesses. Acta Neurochir (Wien) 1990; 107: 140-146.

Leotta N, Chaseling R, Duncan G, Isaacs D. Intracranial suppuration. J. Paediatr Child Health 2005; 41: 508-512.

McIntyre PB, Lavercombe PS, Kemp RJ, McCormack JG. Subdural and epidural empyema: diagnostic and therapeutic problems. Med J Aust 1991; 154: 653-657.

Sellik JA. Epidural abscess and subdural empyema. J Am Osteopath Assoc 1989; 89: 806-810.

Seven H, Coskun BU, Calis AB, Sayin I, Turgut S. Intracranial abscesses associated with chronic suppurative otitis media. Eur Arch Otorhinolaryngol 2005; 262: 847-851.

Yen PT, Chan ST, Huang TS. Brain abscess: with special reference to otolaryngologic sources of infection. Otolaryngol Head Neck Surg 1995; 113: $15-22$

Yıldırmak T, Gedik H, Simşek F, Kantürk A. Community-acquired intracranial suppurative infections: A 15-year report. Surg Neurol Int 2014; 5: 142.

\subsection{Kopfschmerz zurückzuführen auf eine systemische Infektion}

Arredondo M, Hackett J, de Bethencourt FR, Trevi A, Escudero D, Collado A, Qiu X, Swanson P, Soriano V, de Mendoza C. Prevalence of XMRV infection in different risk populations in Spain. AIDS Res Hum Retroviruses 2012; 28: 1089-1094.

Capelli E, Zola R, Lorusso L, Venturini L, Sardi F, Ricevuti G. Chronic fatigue syndrome/myalgic encephalomyelitis: an update. Int J Immunopathol Pharmacol 2010; 23(4): 981-989.

De Marinis M, Welch KM, Headache associated with non-cephalic infections: classification and mechanisms. Cephalalgia 1992; 12: 197-201.

Hou CC, Lin H, Chang CP, Huang WT, Lin MT. Oxidative stress and pyrogenic fever pathogenesis. Eur J Pharmacol 2011; 667(1-3): 6-12.

Leligdowicz A, Fischer WA 2nd, Uyeki TM et al. Ebola virus disease and critical illness. Critical Care 2016; 20: 217.

Saiz JC, Vázquez-Calvo Á, Blázquez AB, Merino-Ramos T, Escribano-Romero E, Martín-Acebes MA. Zika Virus: the latest newcomer. Front Microbiol 2016; 7: 496.

\section{Kopfschmerz zurückzuführen auf eine Störung der Homöostase}

10.1

10.1.1

10.1.2

10.1 .3

10.1 .4

10.2

10.3

10.3.1

10.3 .2

10.3 .3

10.3.4

10.3 .5

10.4

10.5

10.6

10.7
Kopfschmerz zurückzuführen auf eine Hypoxie und/oder Hyperkapnie

Höhenkopfschmerz

Kopfschmerz zurückzuführen auf eine Flugreise

Taucherkopfschmerz

Kopfschmerz zurückzuführen auf ein SchlafapnoeSyndrom

Dialysekopfschmerz

Kopfschmerz zurückzuführen auf eine arterielle Hypertonie

Kopfschmerz zurückzuführen auf ein Phäochromozytom Kopfschmerz zurückzuführen auf eine hypertensive Krise ohne hypertensive Enzephalopathie

Kopfschmerz zurückzuführen auf eine hypertensive Enzephalopathie

Kopfschmerz zurückzuführen auf eine Präeklampsie oder Eklampsie

\section{Kopfschmerz zurückzuführen auf eine Dysreflexie}

Kopfschmerz zurückzuführen auf eine Hypothyreose

Kopfschmerz zurückzuführen auf Fasten

Kopfschmerz zurückzuführen auf eine kardiale Erkrankung

Kopfschmerz zurückzuführen auf andere Störungen der Homöostase 
An anderer Stelle kodiert: 7.1.2 Kopfschmerz zurückzuführen auf eine sekundäre Liquordrucksteigerung metabolischer, toxischer oder hormoneller Genese.

An anderer Stelle kodiert

Primärer und/oder sekundärer Kopfschmerz?

Für Kapitel 10. Kopfschmerz zurückzuführen auf eine Störung der Homöostase gelten die allgemeinen Regeln für die kausale Zuordnung zu einer anderen Erkrankung.

Tritt ein neuer Kopfschmerz erstmals in engem zeitlichen Zusammenhang mit einer Störung der Homöostase auf, sollte der Kopfschmerz als Kopfschmerz zurückzuführen auf diese Störung kodiert werden. Dies ist auch der Fall, wenn der Kopfschmerz das klinische Bild eines primären Kopfschmerzes zeigt, der in Teil 1 der ICHD-3 klassifiziert wird.

Wenn aber ein vorbestehender Kopfschmerz mit den Charakteristika eines primären Kopfschmerzes in engem zeitlichen Zusammenhang mit einer Störung der Homöostase chronisch wird oder sich deutlich verschlechtert (üblicherweise definiert als eine mindestens zweifache Steigerung der Häufigkeit und/oder Schwere), dann sollte sowohl die vorbestehende primäre Kopfschmerzdiagnose als auch die Diagnose 10. Kopfschmerz zurückzuführen auf eine Störung der Homöostase (oder eines ihrer Typen oder Subtypen) vergeben werden, sofern gute Hinweise dafür bestehen, dass diese Störung Kopfschmerzen verursachen kann.

\section{Einleitung}

Die ursächlichen Mechanismen hinter den verschiedenen Typen eines 10. Kopfschmerzes zurückzuführen auf eine Störung der Homöostase sind vielfältig. Dennoch ist es möglich, wie folgt allgemeine diagnostische Kriterien darzulegen, die in den meisten Fällen anwendbar sind:

A. Kopfschmerz, der Kriterium C erfüllt

B. Es wurde eine Störung der Homöostase diagnostiziert, die bekanntermaßen Kopfschmerzen verursachen kann

C. Ein kausaler Zusammenhang kann durch mindestens zwei der folgenden Kriterien gezeigt werden:

1. Der Kopfschmerz hat sich in einem zeitlichen Zusammenhang mit der Störung der Homöostase entwickelt

2. Einer oder beide der folgenden Punkte sind erfüllt:

a) der Kopfschmerz hat sich gleichzeitig mit der Verschlechterung der Störung der Homöostase deutlich verschlechtert

b) der Kopfschmerz hat sich nach Verschwinden der Störung der Homöostase deutlich gebessert

3. Der Kopfschmerz weist Charakteristika auf, die typisch für eine Störung der Homöostase sind

D. Nicht besser erklärt durch eine andere ICHD-3-Diagnose.

\subsection{Kopfschmerz zurückzuführen auf eine Hypoxie und/oder Hyperkapnie}

Beschreibung:

Kopfschmerz infolge einer Hypoxie und/oder Hyperkapnie, der unter Bedingungen auftritt, bei denen eine Verbindung mit einem oder beiden dieser Umstände gegeben ist.
Diagnostische Kriterien:

A. Kopfschmerz, der das Kriterium C erfüllt

B. In Verbindung mit Hypoxie und/oder Hyperkapnie

C. Ein kausaler Zusammenhang kann durch mindestens eines der folgenden Kriterien gezeigt werden:

1. Der Kopfschmerz hat sich in einem zeitlichen Zusammenhang mit der Exposition entwickelt

2. Einer oder beide der folgenden Punkte sind erfüllt:

a) Der Kopfschmerz hat sich gleichzeitig mit einer verstärkten Verbindung mit Hypoxie und/oder Hyperkapnie deutlich verschlechtert

b) Der Kopfschmerz hat sich gleichzeitig mit einer Besserung in Sachen Hypoxie und/oder Hyperkapnie deutlich gebessert

D. Nicht besser erklärt durch eine andere ICHD-3-Diagnose.

\subsubsection{Höhenkopfschmerz}

\section{Beschreibung:}

Ein in der Regel bilateraler Kopfschmerz, der sich bei Anstrengung verschlimmert und durch einen Aufstieg auf über 2.500 Meter entsteht. Dieser verschwindet innerhalb von 24 Stunden nach dem Abstieg spontan.

\section{Diagnostische Kriterien:}

A. Kopfschmerz, der Kriterium C erfüllt

B. Es kam zu einem Aufstieg auf eine Höhe von über $2.500 \mathrm{Me}-$ tern

C. Ein kausaler Zusammenhang kann durch mindestens zwei der folgenden Kriterien gezeigt werden:

1. Der Kopfschmerz hat sich in einem zeitlichen Zusammenhang mit dem Aufstieg entwickelt

2. Einer oder beide der folgenden Punkte sind erfüllt:

a) Der Kopfschmerz hat sich gleichzeitig mit dem sich fortsetzenden Aufstieg deutlich verschlechtert

b) Der Kopfschmerz ist sich innerhalb von 24 Stunden nach Abstieg auf unter 2.500 Meter verschwunden

3. Der Kopfschmerz weist wenigstens zwei der folgenden drei Charakteristika auf:

a) bilateral lokalisiert

b) leichte oder mittlere Intensität

c) Verschlimmerung bei Anstrengung, Bewegung, Pressen, Husten und/oder Bücken

D. Nicht besser erklärt durch eine andere ICHD-3-Diagnose.

\section{Kommentar:}

10.1.1 Höhenkopfschmerz ist eine häufige Komplikation beim Aufstieg in größere Höhen; er tritt bei mehr als $30 \%$ der Bergsteiger auf. Zu den Risikofaktoren gehören eine individuelle Vorgeschichte in Sachen 1. Migräne, eine geringe arterielle Sauerstoffsättigung, eine als sehr anstrengend wahrgenommene Aktivität, venöse Abflussbehinderungen und eine geringe Flüssigkeitsaufnahme (<2 Liter in 24 Stunden).

In den meisten Fällen spricht ein 10.1.1 Höhenkopfschmerz auf einfache Analgetika wie Paracetamol (Acetaminophen) oder Ibuprofen an. Die akute Bergkrankheit besteht jedoch aus zumindest mittelstarken Kopfschmerzen in Verbindung mit einer oder meh- 
reren der folgenden Beschwerden: Übelkeit, Appetitlosigkeit Müdigkeit, Photophobie, Schwindel oder Schlafstörungen. Die Einnahme von Acetazolamid ( 2 bis $3 \times 125$ mg/Tag) und Kortikoiden kann die Wahrscheinlichkeit des Auftretens einer akuten Bergkrankheit senken. Zu den vorbeugenden Maßnahmen gehören eine zweitägige Akklimatisationsphase vor der anstrengenden Aktivität in großer Höhe, eine großzügige Flüssigkeitszufuhr und Alkoholkarenz.

Der Aufenthalt auf Höhe von über 1.000 Metern erhöht nicht nur die Prävalenz, sondern auch die Intensität der Symptome einer 1. Migräne. Die Mechanismen sind unbekannt und weisen wahrscheinlich keine Verbindung zu denen bei einem 10.1.1 Höhenkopfschmerz auf.

\subsubsection{Kopfschmerz zurückzuführen auf eine Flugreise}

Beschreibung:

Kopfschmerz, oft schwer, üblicherweise unilateral und periokular und ohne autonome Symptome, der während einer Flugreise auftritt und von dieser verursacht wird. Er verschwindet nach der Landung.

\section{Diagnostische Kriterien:}

A. Mindestens zwei Episoden eines Kopfschmerzes, der Kriterium C erfüllt

B. Der Patient war/ist mit dem Flugzeug unterwegs

C. Ein kausaler Zusammenhang kann durch mindestens zwei der folgenden Kriterien gezeigt werden:

1. Der Kopfschmerz hat sich während des Fluges entwickelt

2. Einer oder beide der folgenden Punkte sind erfüllt:

a) der Kopfschmerz hat sich in einem zeitlichen Zusammenhang mit dem Aufstieg nach dem Start und/oder während des Landeanflugs verschlechtert

b) der Kopfschmerz hat sich innerhalb von 30 Minuten nach Abschluss des Aufstiegs auf Reiseflughöhe oder des Landeanflugs spontan gebessert

3. Der Kopfschmerz ist schwer und erfüllt dabei mindestens zwei der folgenden Charakteristika:

a) unilateral lokalisiert1

b) orbitofrontal lokalisiert2

c) stechender Charakter3

D. Nicht besser erklärt durch eine andere ICHD-3-Diagnose4.

\section{Anmerkung:}

1. In rund $10 \%$ der Fälle tritt zwischen verschiedenen Flügen eine Verlagerung von einer Seite zu anderen ein.

2. Es kann zu einer parietalen Ausdehnung kommen.

3. Es kann ein Pulsieren (Pochen) bemerkt werden.

4. Es sollte eine andere Erkrankung, insbesondere eine Nebenhöhlenerkrankung ausgeschlossen werden.

\section{Kommentar:}

Eine aktuelle skandinavische Befragung ergab, dass bis zu $8,3 \%$ der Flugreisenden 10.1.2 Kopfschmerz zurückzuführen auf eine Flugreise erfahren. Dieser tritt in über $90 \%$ der Fälle während der Landung auf.

In bis zu 30\% der Fälle werden Begleitsymptome berichtet. Am häufigsten sind diese Unruhe und unilaterales Augentränen; ande- re lokalisierte parasympathische Symptome, Übelkeit oder Photo-/ Phonophobie wurden in weniger als $5 \%$ der Fälle beschrieben.

Ein gewisser Teil der Befragten, die von einem 10.1.2 Kopfschmerz zurückzuführen auf eine Flugreise berichteten, schilderten ähnliche Kopfschmerzen beim Schnorcheln und/oder einem schnellen Abstieg aus dem Gebirge, was nahelegt, dass diese Kopfschmerzen mit einem Ungleichgewicht zwischen dem Luftdruck in den Nasennebenhöhlen und außen zusammenhängt.

\subsubsection{Taucherkopfschmerz}

An anderer Stelle kodiert: 1. Migräne, 2. Kopfschmerz vom Spannungstyp, 4.2 primärer Anstrengungskopfschmerz, 4.5 primärer kältebedingter Kopfschmerz, 4.6.1 Kopfschmerz durch äußeren Druck und 11.2.1 zervikogener Kopfschmerz können während eines Tauchganges auftreten. In diesem Fall sollte das Tauchen eher als ein begünstigender Faktor denn als Ursache berücksichtigt werden, und der Kopfschmerz sollte dementsprechend unter diesen Störungen kodiert werden.

Es ist bekannt, dass das Tauchen eine Dissektion der A. carotis interna oder A. vertebralis verursachen kann. Kopfschmerzen, die infolge dessen auftreten, sollten unter 6.5.1. akuter Kopf-, Gesichts- oder Halsschmerz zurückzuführen auf eine arterielle Dissektion kodiert werden.

\section{Beschreibung:}

Kopfschmerz durch Tauchen auf Tiefen von mehr als 10 Metern, oft während des Tauchgangs auftretend, sich jedoch oft beim Wiederauftauchen intensivierend, bei fehlender Dekompressionskrankheit. In der Regel wird dieser Kopfschmerz von Symptomen einer Kohlendioxid-(CO2)-Intoxikation begleitet. Er verschwindet nach Sauerstoffgabe schnell oder, sofern eine solche nicht erfolgt, spontan innerhalb von 3 Tagen nach dem Ende des Tauchens.

Diagnostische Kriterien:

A. Kopfschmerz, der das Kriterium C erfüllt

B. Es treffen beide folgenden Punkte zu:

1. Der Patient unternimmt einen Tauchgang in $>10$ Metern Tiefe

2. Kein Hinweis auf eine Dekompressionskrankheit

C. Ein kausaler Zusammenhang kann durch wenigstens eines der folgenden Kriterien gezeigt werden:

1. Der Kopfschmerz hat sich während des Tauchganges entwickelt

2. Einer oder beide der folgenden Punkte sind erfüllt:

a) Der Kopfschmerz hat sich im weiteren Verlauf des Tauchgangs verschlechtert

b) Es ist wenigstens einer der folgenden Punkte erfüllt:

i. Der Kopfschmerz ist innerhalb von 3 Tagen nach Einstellen des Tauchens spontan verschwunden

ii. Der Kopfschmerz ist innerhalb von 1 Stunden nach einer Behandlung mit $100 \%$ O2 verschwunden

3. Es liegt mindestens eines der folgendem Symptome einer CO2-Intoxikation:

a) Verwirrtheitszustand

b) Benommenheit

c) motorische Koordinationsstörungen

d) Dyspnoe 
e) Gesichtsrötung

D. Nicht besser erklärt durch eine andere ICHD-3-Diagnose.

\section{Kommentar:}

Es gibt Hinweise darauf, dass eine Hyperkapnie ohne gleichzeitige Hypoxie mit Kopfschmerzen assoziiert sein kann. Es ist bekannt, dass eine Hyperkapnie (arterieller pCO2 > $50 \mathrm{mmHg}$ ) zu einer Relaxation der glatten Muskulatur zerebraler Gefäße und damit zu einer Vasodilatation und einer Erhöhung des intrakraniellen Druckes führt. Das beste klinische Beispiel für Kopfschmerzen, die auf eine Hyperkapnie zurückzuführen sind, ist 10.1.3 Taucherkopfschmerz. Kohlendioxid (CO2) kann sich bei einem Taucher akkumulieren, der beim missglückten Versuch, Atemluft zu konservieren, die Luft gezielt intermittierend anhält oder nur oberflächlich atmet, um die Variabilität des Auftriebs in engen Passagen eines Wracks oder eine Höhle zu minimieren. Taucher hyperventilieren aber manchmal auch unbewusst, wenn ein enger Tauchanzug oder eine auftriebsvermindernde Weste die Ausdehnung des Brustkorbes behindern oder die Atmung im Vergleich zur körperlichen Anstrengung nicht ausreichend ist. Eine starke körperliche Anstrengung erhöht die CO2-Produktion um mehr als das 10 fache und führt so zu einer vorübergehenden CO2-Erhöhung auf über $60 \mathrm{mmHg}$.

\subsubsection{Taucherkopfschmerz intensiviert sich gewöhnlich während der Dekompressions- phase des Tauchganges oder beim Wieder- auftauchen.}

\subsubsection{Kopfschmerz zurückzuführen auf ein Schlafapnoe-Syndrom}

Beschreibung:

Morgendlicher Kopfschmerz, in der Regel bilateral und mit einer Dauer von unter 4 Stunden, verursacht durch Schlafapnoe-Syndrom. Die Störung verschwindet nach erfolgreicher Behandlung des Schlafapnoe-Syndroms.

Diagnostische Kriterien:

A. Kopfschmerz, der bei Aufwachen vorhanden ist und Kriterium C erfüllt

B. Es wurde ein Schlafapnoe-Syndrom mit einem Apnoe-Hypopnoe-Index $\geq 5$ diagnostiziert 1

C. Ein kausaler Zusammenhang kann durch mindestens zwei der folgenden Kriterien gezeigt werden:

1. Der Kopfschmerz hat sich in einem zeitlichen Zusammenhang mit dem Beginn des Schlafapnoe-Syndroms entwickelt

2. Einer oder beide der folgenden Punkte sind erfüllt:

a) Der Kopfschmerz hat sich gleichzeitig zu der Verschlechterung der Schlafapnoe verschlechtert

b) Der Kopfschmerz hat sich gleichzeitig mit der Besserung oder dem Verschwinden der Schlafapnoe deutlich gebessert oder ist verschwunden

3. Der Kopfschmerz weist mindestens eines der folgenden 3 Charakteristika auf:

a) kehrt an $\geq 15$ Tagen/Monat wieder

b) alles von folgendem:

i. bilateral lokalisiert

ii. drückender Charakter iii. nicht von Übelkeit, Photophobie oder Phonophobie begleitet

c) verschwindet innerhalb von 4 Stunden

D. Nicht besser erklärt durch eine andere ICHD-3-Diagnose2.

Anmerkung:

1. Der Apnoe-Hypopnoe-Index errechnet sich durch Dividieren der Anzahl apnoeischer Ereignisse durch die Anzahl der Stunden Schlaf (5-15/Std. = mild; 15-30/Std. = mittelgradig; $>30 /$ Std. = schwer).

2. Eine definitive Diagnose erfordert eine Polysomnographie über Nacht.

\section{Kommentar:}

10.1.4 Kopfschmerz zurückzuführen auf ein Schlafapnoe-Syndrom scheint weniger häufig und von längerer Dauer als ursprünglich angenommen. Obwohl morgendliche Kopfschmerzen beim Schlafapnoe-Syndrom bedeutend häufiger als in der Normalbevölkerung auftreten, sind Kopfschmerzen beim Erwachen doch ein unspezifisches Symptom, welches bei einer Vielzahl primärer und sekundärer Kopfschmerzerkrankungen ebenso vorkommt wie bei anderen den Schlaf beeinflussenden respirativen Störungen (neben dem Schlafapnoe-Syndrom z. B. Pickwick-Syndrom, chronisch obstruktive Lungenerkrankung) und bei anderen primären Schlafstörungen wie den periodischen Beinbewegungen im Schlaf.

Es ist unklar, ob der ursächliche Mechanismus bei einem 10.1.4 Kopfschmerz zurückzuführen auf ein Schlafapnoe-Syndrom eine Hypoxie, Hyperkapnie oder die Störung des Schlafes ist.

\subsection{Dialysekopfschmerz}

Beschreibung:

Kopfschmerz ohne spezifischen Charakteristika, der während der Hämodialyse auftritt und von dieser verursacht wird. Er verschwindet spontan innerhalb von 72 Stunden nach der Hämodialysebehandlung.

\section{Diagnostische Kriterien:}

A. Wenigstens 3 akute Kopfschmerzepisoden, die Kriterium C erfüllen

B. Der Patient erhält eine Hämodialysebehandlung

C. Ein kausaler Zusammenhang kann durch mindestens zwei der folgenden Kriterien gezeigt werden:

1. Der Kopfschmerz hat sich jeweils während einer Hämodialysebehandlung entwickelt

2. Einer oder beide der folgenden Punkte sind erfüllt:

a) Der Kopfschmerz hat sich während der Dialysesitzung jedes Mal verschlechtert

b) Der Kopfschmerz ist innerhalb von 72 Stunden nach der Dialysesitzung verschwunden

3. Die Kopfschmerzepisoden hören nach einer erfolgreichen Nierentransplantation und Beendigung der Hämodialysebehandlung ganz auf

D. Nicht besser erklärt durch eine andere ICHD-3-Diagnose1. 
Anmerkung:

1. Da Koffein durch eine Dialyse sehr schnell entfernt wird, sollte daran gedacht werden, dass bei Patienten, die davon größere Mengen konsumieren, ein 8.3.1 Koffeinentzugskopfschmerz auftreten kann.

\section{Kommentar:}

Ein 10.2 Dialysekopfschmerz tritt in Verbindung mit einer Hypotonie und einem Dialyse-Dysäquilibrium-System auf. Das Dysäquilibrium-Syndrom kann mit Kopfschmerzen beginnen und zu Bewusstseinsstörungen bis hin zum Koma mit oder ohne epileptischen Anfall führen. Dieses Syndrom ist relativ selten und kann eventuell durch eine Veränderung der Dialyseparameter verhindert werden.

Abweichungen beim Harnstoff-, Natrium- und Magnesiumspiegels sowie bei Blutdruck und Körpergewicht können Risikofaktoren für die Entwicklung eines 10.2 Dialysekopfschmerzes sein.

\subsection{Kopfschmerz zurückzuführen auf eine arterielle Hypertonie}

\section{Beschreibung:}

Kopfschmerz, oft bilateral und pulsierend, der durch eine arterielle Hypertonie verursacht wird, gewöhnlich bei einem akuten Anstieg des systolischen ( $\geq 180 \mathrm{mmHg}$ ) und/oder diastolischen $(\geq 120 \mathrm{mmHg}$ ) Blutdrucks. Der Kopfschmerz verschwindet nach einer Normalisierung des Blutdrucks.

\section{Diagnostische Kriterien:}

A. Kopfschmerz, der das Kriterium C erfüllt

B. Es wurde eine Hypertonie mit systolischem

Druck $\geq 180 \mathrm{mmHg}$ und/oder diastolischem

Druck $\geq 120 \mathrm{mmHg}$ nachgewiesen

C. Ein kausaler Zusammenhang kann durch eines oder beide der folgenden Kriterien gezeigt werden:

1. Der Kopfschmerz hat sich in einem zeitlichen Zusammenhang mit dem Beginn der Hypertonie entwickelt

2. Einer oder beide der folgenden Punkte sind erfüllt:

a) der Kopfschmerz hat sich gleichzeitig mit einer Verschlechterung der Hypertonie deutlich verschlechtert

b) der Kopfschmerz hat sich gleichzeitig mit einer Besserung der Hypertonie deutlich gebessert

D. Nicht besser erklärt durch eine andere ICHD-3-Diagnose.

\section{Kommentar:}

Eine chronische arterielle Hypertonie, die leicht (140-159/90$99 \mathrm{mmHg}$ ) oder moderat (160-179/100-109 mmHg) ist, scheint keine Kopfschmerzen zu verursachen. Ob eine moderate arterielle Hypertonie aber zu Kopfschmerzen zumindest prädisponiert, wird noch kontrovers diskutiert. Es gibt nur wenig Anhaltspunkte hierfür.

Ambulante Blutdruckkontrollen bei Patienten mit einer milden und moderaten arteriellen Hypertonie konnten keinen überzeugenden Zusammenhang zwischen Fluktuationen des Blutdrucks über 24 Stunden hinweg und dem Vorhandensein oder Nicht-Vorhandensein von Kopfschmerzen aufdecken.

\subsubsection{Kopfschmerz zurückzuführen auf ein Phäochromozytom}

An anderer Stelle kodiert: Wenn eine hypertensive Enzephalopathie vorliegt, sollte eine Kodierung unter 10.3.3 Kopfschmerz zurückzuführen auf eine hypertensive Enzephalopathie erfolgen. Falls die Diagnose eines Phäochromozytoms bisher noch nicht gestellt wurde und eine hypertensive Enzephalopathie fehlt, könnten Patienten die diagnostischen Kriterien für eine 10.3.2 Kopfschmerz zurückzuführen auf eine hypertensive Krise ohne hypertensive Enzephalopathie erfüllen.

\section{Beschreibung:}

Kopfschmerzattacken, meist schwer und von kurzer Dauer (weniger als 1 Stunde) und von Schwitzen, Palpitationen, Blässe und/ oder Angst begleitet, verursacht durch ein Phäochromozytom.

\section{Diagnostische Kriterien:}

A. Wiederkehrende diskrete langanhaltende Kopfschmerzepisoden, die Kriterium C erfüllen

B. Es wurden ein Phäochromozytom nachgewiesen

C. Ein kausaler Zusammenhang kann durch mindestens zwei der folgenden Kriterien gezeigt werden:

1. Die Kopfschmerzepisoden haben in einem zeitlichen Zusammenhang mit der Entwicklung des Phäochromozytoms begonnen oder zu seiner Entdeckung geführt

2. Einer oder beide der folgenden Punkte sind erfüllt:

a) individuelle Kopfschmerzepisoden entwickeln sich in einem zeitlichen Zusammenhang mit plötzlichen Blutdruckanstiegen

b) individuelle Kopfschmerzepisoden verschwinden in einem zeitlichen Zusammenhang mit einer Normalisierung des Blutdrucks

3. Die Kopfschmerzen treten in Begleitung von wenigstens einem der folgenden Symptome auf:
a) Schwitzen
b) Palpitationen
c) Angst
d) Blässe

4. Die Kopfschmerzepisoden verschwinden nach Beseitigung des Phäochromozytoms komplett

D. Nicht besser erklärt durch eine andere ICHD-3-Diagnose1.

Anmerkung:

1. Die Diagnose eines Phäochromozytoms wird gesichert über den Nachweis einer erhöhten Exkretion von Katecholaminen und ihrer Metaboliten. Dieser gelingt mittels Analyse des 24-Stunden-Sammelurins, der in der Zeit gesammelt wird, in der der Patient erhöhte Blutdruckwerte hat oder symptomatisch ist.

\section{Kommentar:}

10.3.1 Kopfschmerz zurückzuführen auf ein Phäochromozytom tritt bei 51 \% bis 80 \% der Patienten mit einem Phäochromozytom als paroxysmaler Kopfschmerz auf.

10.3.1 Kopfschmerz zurückzuführen auf ein Phäochromozytom ist oft schwer, frontal oder okzipital und wird gewöhnlich entweder als von pulsierendem oder konstantem Charakter beschrieben. Ein 
wichtiges Merkmal ist seine kurze Dauer: weniger als 15 Minuten bei $50 \%$ und weniger als 1 Stunde bei $70 \%$ der Patienten. Ein anderes Merkmal sind Befürchtungen und/oder Ängste, die häufig verbunden sind mit dem Gefühl des bevorstehenden Todes, Tremor, Sehstörungen, Bauch- oder Brustschmerzen, Übelkeit, Erbrechen und manchmal mit Parästhesien. Während der Attacke kann das Gesicht blass oder gerötet sein.

10.3.2 Kopfschmerz zurückzuführen auf eine hypertensive Krise ohne hypertensive Enzephalopathie

An anderer Stelle kodiert:

\subsubsection{Kopfschmerz zurückzuführen auf ein Phäochromozytom.}

\section{Beschreibung:}

Kopfschmerz, gewöhnlich bilateral und pulsierend, verursacht durch einen paroxysmalen Anstieg des arteriellen Bluthochdrucks (systolisch $\geq 180 \mathrm{mmHg}$ und/oder diastolisch $\geq 120 \mathrm{mmHg}$ ). Der Kopfschmerz verschwindet nach Normalisierung des Blutdruckes.

\section{Diagnostische Kriterien:}

A. Kopfschmerz, der Kriterium C erfüllt

B. Beide der folgenden Punkte sind erfüllt:

1. Es tritt eine hypertensive Krise 1 auf

2. Keine klinischen Merkmale oder anderen Hinweise auf eine Hochdruckenzephalopathie

C. Ein kausaler Zusammenhang kann durch mindestens zwei der folgenden Kriterien gezeigt werden:

1. Der Kopf hat sich während einer hypertensiven Krise entwickelt

2. Einer oder beide der folgenden Punkte sind erfüllt:

a) Der Kopfschmerz hat sich gleichzeitig mit dem Anstieg der Hypertonie deutlich verschlechtert

b) Der Kopfschmerz hat sich gleichzeitig mit der Besserung oder dem Verschwinden der hypertensiven Krise deutlich verbessert oder ist verschwunden

3. Der Kopfschmerz weist mindestens eines der folgenden Charakteristika auf:

a) bilateral

b) pulsierender Charakter

c) hervorgerufen durch körperliche Aktivität

D. Nicht besser erklärt durch eine andere ICHD-3-Diagnose.

\section{Anmerkung:}

1. Eine hypertensive Krise wird definiert als paroxysmaler Anstieg des systolischen (auf $\geq 180 \mathrm{mmHg}$ ) und/oder diastolischen (auf $\geq 120 \mathrm{mmHg}$ ) Blutdrucks.

\section{Kommentar:}

Paroxysmale Hypertonien können als Folge einer Störung von Barorezeptorreflexen (nach einer Endarterektomie der A. carotis oder in Folge einer Bestrahlung des Nackens) oder bei Patienten mit einem enterochromaffinen Zelltumor auftreten.

\subsubsection{Kopfschmerz zurückzuführen auf eine hyper- tensive Enzephalopathie}

Beschreibung:

Kopfschmerz, in der Regel bilateral und pulsierend, verursacht durch eine anhaltende Blutdruckerhöhung auf $180 / 120 \mathrm{mmHg}$ oder höher und begleitet von Symptomen einer Enzephalopathie wie Verwirrtheitszustand, Antriebslosigkeit, Sehstörungen oder epileptischen Anfällen. Der Kopfschmerz bessert sich nach der Normalisierung des Blutdrucks.

\section{Diagnostische Kriterien:}

A. Kopfschmerz, der Kriterium C erfüllt

B. Es wurde eine hypertensive Enzephalopathie diagnostiziert

C. Ein kausaler Zusammenhang kann durch mindestens zwei der folgenden Kriterien gezeigt werden:

1. Der Kopfschmerz hat sich in einem zeitlichen Zusammenhang mit dem Beginn der hypertensiven Enzephalopathie entwickelt

2. Einer oder beide der folgenden Punkte sind erfüllt:

a) Der Kopfschmerz hat sich gleichzeitig mit der Verschlechterung der hypertensiven Enzephalopathie deutlich verschlechtert

b) Der Kopfschmerz hat sich gleichzeitig mit der Besserung oder dem Verschwinden der hypertensiven Enzephalopathie deutlich gebessert oder ist verschwunden

3. Der Kopfschmerz weist mindestens zwei der folgenden drei Charakteristika auf:

a) diffuser Schmerz

b) pulsierender Charakter

c) Verstärkung durch körperliche Aktivität

D. Nicht besser erklärt durch eine andere ICHD-3-Diagnose.

\section{Kommentar:}

Eine hypertensive Enzephalopathie geht mit einem anhaltenden erhöhten Blutdruck auf $\geq 180 / 120$ mmHg und mindestens zwei Komponenten der Symptome Verwirrtheitszustand, eingeschränkte Bewusstseinslage, Sehstörungen einschließlich Blindheit und epileptischen Anfällen einher. Eine hypertensive Enzephalopathie entsteht, wenn eine zerebrale Hyperperfusion als Folge eines Blutdruckanstiegs nicht länger durch eine kompensatorische zerebrale Vasokonstriktion verhindert werden kann. Mit dem Zusammenbruch der zerebralen Autoregulation zur Steuerung des Blutflusses steigt die endotheliale Permeabilität an und es entwickelt sich ein Hirnödem. Im MRT ist dies vornehmlich in der weißen Substanz der Parietookzipitalregion zu sehen.

Obwohl eine hypertensive Enzephalopathie bei Patienten mit einer chronischen arteriellen Hypertonie üblicherweise mit einem diastolischen Blutdruck von > $120 \mathrm{mmHg}$ und einer Retinopathie Grad 3 oder 4 (Keith-Wagener-Barker-Klassifikation) einhergeht, können auch Patienten mit einem ursprünglich normalen Blutdruck Zeichen einer Enzephalopathie bereits bei Blutdruckwerten, die unterhalb 160/100 mmHg liegen, entwickeln. Eine hypertensive Retinopathie muss zum Zeitpunkt der klinischen Manifestation noch nicht in jedem Fall präsent sein.

Jede Ursache einer arteriellen Hypertonie kann zu einer hypertensiven Enzephalopathie führen. Ein Kopfschmerz zurückzuführen auf eine hypertensive Enzephalopathie sollte unabhängig von 
der Grundursache unter 10.3.3 Kopfschmerz zurückzuführen auf eine hypertensive Enzephalopathie kodiert werden.

\subsubsection{Kopfschmerz zurückzuführen auf eine Prä- eklampsie oder Eklampsie}

Beschreibung:

Kopfschmerz, gewöhnlich bilateral und pulsierend, der bei Schwangeren oder im frühen Wochenbett mit Präeklampsie oder Eklampsie auftritt. Nach dem Verschwinden der Präeklampsie oder Eklampsie kommt es zur Remission des Kopfschmerzes.

\section{Diagnostische Kriterien:}

A. Kopfschmerz bei einer Frau in der Schwangerschaft oder im Wochenbett (bis zu 4 Wochen postpartal), der Kriterium C erfüllt

B. Es wurde eine Präeklampsie oder Eklampsie diagnostiziert

C. Ein kausaler Zusammenhang kann durch mindestens zwei der folgenden Kriterien gezeigt werden:

1. Der Kopfschmerz hat sich in einem zeitlichen Zusammenhang mit dem Beginn der Präeklampsie oder Eklampsie entwickelt

2. Einer oder beide der folgenden Punkte sind erfüllt:

a) der Kopfschmerz hat sich gleichzeitig mit der Verschlechterung der Präeklampsie oder Eklampsie deutlich verschlechtert

b) der Kopfschmerz hat sich gleichzeitig mit der Besserung oder dem Verschwinden der Präeklampsie oder Eklampsie deutlich gebessert oder ist verschwunden

3. Der Kopfschmerz weist mindestens zwei der folgenden drei Charakteristika auf:

a) bilateral lokalisiert

b) pulsierender Charakter

c) Verstärkung durch körperliche Aktivität

D. Nicht besser erklärt durch eine andere ICHD-3-Diagnose.

\section{Kommentar:}

Präeklampsie und Eklampsie scheinen mit einer starken mütterlichen Entzündungsantwort mit einer breiten Aktivierung des Immunsystems einherzugehen. Die Plazenta scheint eine entscheidende Rolle bei deren Entstehung zu spielen, obwohl Fallberichte zeigen, dass eine Eklampsie sowohl während der Schwangerschaft als auch im Wochenbett auftreten können.

Die Präeeklampsie und Eklampsie sind Multisystemerkrankungen in unterschiedlichen Erscheinungsformen. Ihre Diagnose setzt eine arterielle Hypertonie (Blutdruck $>140 / 90 \mathrm{mmHg}$ ) dokumentiert durch 2 Blutdruckmessungen mit einem Abstand von mindestens 4 Stunden voraus oder einen Anstieg des diastolischen Blutdruckes von $\geq 15 \mathrm{mmHg}$ oder des systolischen Blutdruckes von $\geq 30 \mathrm{mmHg}$, gekoppelt mit einer Proteinurie von >0,3 g/24 Stunden. Zusätzlich können Gewebsödeme, eine Thrombozytopenie und Auffälligkeiten bei der Leberfunktion auftreten.

\subsubsection{Kopfschmerz zurückzuführen auf eine Dysreflexie} Beschreibung:

Pochender schwerer Kopfschmerz mit plötzlichem Beginn bei Patienten mit Rückenmarkverletzung und autonomer Dysreflexie. Letztere, die lebensbedrohlich sein kann, manifestiert sich neben anderen Symptomen und klinischen Zeichen als paroxysmaler Blutdruckanstieg und wird oft durch eine Blasen- oder Darmreizung getriggert (durch eine Infektion, Distension oder Koprostase).

Diagnostische Kriterien:

A. Kopfschmerz mit plötzlichem Beginn, der Kriterium C erfüllt

B. Vorliegen einer Rückenmarkverletzung und autonomen Dysreflexie, dokumentiert durch einen paroxysmalen Blutdruckanstieg oberhalb der Grundlinie von $\geq 30 \mathrm{mmHg}$ beim systolischen Blutdruck und/oder von $\geq 20 \mathrm{mmHg}$ beim diastolischen Blutdruck

C. Ein kausaler Zusammenhang kann durch mindestens zwei der folgenden Kriterien gezeigt werden:

1. Der Kopfschmerz hat sich in einem zeitlichen Zusammenhang mit dem Blutdruckanstieg entwickelt

2. Einer oder beide der folgenden Punkte sind erfüllt:

a) Der Kopfschmerz hat sich gleichzeitig mit dem Blutdruckanstieg deutlich verschlechtert

b) Der Kopfschmerz hat sich gleichzeitig mit der Blutdrucksenkung deutlich gebessert

3. Der Kopfschmerz weist mindestens zwei der folgenden vier Charakteristika auf:

a) starke Intensität

b) pochender oder pulsierender Charakter

c) begleitet von einer Diaphorese kraniell zur Höhe der Rückenmarkverletzung

d) durch Blasen- oder Darmreflexe getriggert

D. Nicht besser erklärt durch eine andere ICHD-3-Diagnose.

Kommentar:

Die Zeit bis zum Beginn einer autonomen Dysreflexie nach Rückenmarkverletzung ist variabel. Berichtet wurden Zeitspannen von 4 Tagen bis 15 Jahren.

Angesichts der Tatsache, dass eine autonome Dysreflexie eine lebensbedrohliche Erkrankung darstellen kann, ist ihr rechtzeitiges Erkennen und adäquates Management entscheidend. Üblicherweise ist der 10.3.5 Kopfschmerz zurückzuführen auf eine Dysreflexie ein plötzlich beginnender schwerer Kopfschmerz, der in Begleitung diverser anderer Symptome und klinischer Zeichen auftritt, darunter ein erhöhter Blutdruck, eine veränderte Herzfrequenz und eine Diaphorese kraniell zur Höhe der Rückenmarkverletzung. Diese werden durch schädliche oder nichtschädliche Stimuli getriggert, meist viszeralen Ursprungs (Überdehnung der Blase, Harnwegsinfektion, Distension oder Koprostase des Darmes, urologische Eingriffe, Magengeschwür u. a.), doch mitunter auch somatischer Natur (Dekubitus, eingewachsener Zehennagel, Verbrennungen, ein Trauma oder chirurgische bzw. invasive diagnostische Verfahren).

\subsection{Kopfschmerz zurückzuführen auf eine Hypothyreose}

An anderer Stelle kodiert: Bei Vorliegen einer Hypothyreose kann Kopfschmerz auch eine Manifestation eines Hypophysenadenoms sein, kodiert unter 7.4.3 Kopfschmerz zurückzuführen auf eine hypothalamische oder hypophysäre Über- oder Unterfunktion. 
Beschreibung:

Kopfschmerz, üblicherweise bilateral und nicht pulsierend, bei Patienten mit Hypothyreose, der nach Normalisierung des Schilddrüsenhormonspiegels verschwindet.

\section{Diagnostische Kriterien:}

A. Kopfschmerz, der Kriterium C erfüllt

B. Es wurde eine Hypothyreose nachgewiesen

C. Ein kausaler Zusammenhang kann durch mindestens zwei der folgenden Kriterien gezeigt werden:

1. Der Kopfschmerz hat sich in einem zeitlichen Zusammenhang mit dem Beginn der Hypothyreose entwickelt oder führte zu ihrer Entdeckung

2. Einer oder beide der folgenden Punkte ist/sind erfüllt:

a) der Kopfschmerz hat sich gleichzeitig mit der Verschlechterung der Hypothyreose deutlich verschlechtert

b) der Kopfschmerz hat sich gleichzeitig mit der Besserung oder dem Verschwinden der Hypothyreose deutlich gebessert oder ist verschwunden

3. Der Kopfschmerz erfüllt einen oder beide der folgenden Punkte:

a) bilateral lokalisiert

b) im Zeitablauf kontinuierlich

D. Nicht besser erklärt durch eine andere ICHD-3-Diagnose.

\section{Kommentar:}

Es wird geschätzt, dass etwa $30 \%$ der Patienten mit einer Hypothyreose unter 10.4 Kopfschmerz zurückzuführen auf eine Hypothyreose leiden. Der Mechanismus ist unklar. Frauen sind dabei häufiger betroffen. Oft bestand in der Vorgeschichte eine Migräne.

Zwar geht ein 10.4 Kopfschmerz zurückzuführen auf eine Hypothyreose nach jetzigem Verständnis nicht mit Übelkeit oder Erbrechen einher, doch ergab eine aktuelle Untersuchung, dass Patienten mit einer Hypothyreose unilaterale, episodische, pulsierende Kopfschmerzen aufweisen können, die von Übelkeit und/oder erbrechen begleitet sind. Bei der Hälfte der untersuchten Patienten bestand in der Vorgeschichte eine 1. Migräne, also ist die Bedeutung dieser Ergebnisse unklar und sie verlangen nach einer Bestätigung durch zukünftige Studien.

\subsection{Kopfschmerz zurückzuführen auf Fasten}

An anderer Stelle kodiert: Eine durch Fasten ausgelöste Migräneepisode wird unter 1. Migräne oder einem ihrer Typen kodiert.

\section{Beschreibung:}

Diffuser nicht-pulsierender Kopfschmerz, in der Regel von leichter bis mittelstarker Intensität, der während eines mindestens achtstündigen Fastens auftritt und hiervon verursacht wird. Der Kopfschmerz verschwindet nach Nahrungsaufnahme.

\section{Diagnostische Kriterien:}

A. Diffuser Kopfschmerz, der nicht die Kriterien für eine 1. Migräne oder einen ihrer Typen erfüllt, aber Kriterium C unten erfüllt

B. Der Patient hat für $\geq 8$ Stunden gefastet

C. Ein kausaler Zusammenhang kann durch beide der folgenden Kriterien gezeigt werden:
1. Der Kopfschmerz hat sich während des Fastens entwickelt

2. Der Kopfschmerz hat sich nach Nahrungsaufnahme deutlich gebessert

D. Nicht besser erklärt durch eine andere ICHD-3-Diagnose1.

\section{Anmerkung:}

1. 10.5 Kopfschmerz zurückzuführen auf Fasten ist typischerweise von diffusem, nicht-pulsierendem Charakter und von leichter bis mittelstarker Intensität. Bei Patienten mit 1. Migräne in der Vorgeschichte kann der beim Fasten auftretende Kopfschmerz einer 1.1 Migräne ohne Aura ähneln und sollte entsprechend kodiert werden (da das Fasten hier ein auslösender Faktor sein kann), wenn die Kriterien für diese Erkrankung erfüllt sind.

\section{Kommentar:}

10.5 Kopfschmerz zurückzuführen auf Fasten tritt bei Individuen mit primären Kopfschmerzen in der Vorgeschichte signifikant häufiger auf.

Die Wahrscheinlichkeit des Auftretens von Kopfschmerzen als Resultat des Fastens steigt mit der Dauer des Fastens. 10.5 Kopfschmerz zurückzuführen auf Fasten scheint jedoch unabhängig von der Schlafdauer, einem Koffeinentzug oder einer Hypoglykämie zu sein. Auch wenn Kopfschmerzen unter den Bedingungen einer Hypoglykämie-induzierten Hirndysfunktion auftreten können, gibt es keinen abschließenden Beweis, um eine kausale Beziehung zu belegen. 10.5 Kopfschmerz zurückzuführen auf Fasten kann beim Fasten auch ohne eine Hypoglykämie auftreten; Insulin-induzierte Hypoglykämien rufen bei einem Migränepatienten keine Kopfschmerzen hervor und schließlich sind Kopfschmerzen keine typische Klage von Patienten, die sich mit einer symptomatischen Hypoglykämie in einer Notaufnahme vorstellen.

\subsection{Kopfschmerz zurückzuführen auf eine kardiale Erkrankung}

\section{Beschreibung:}

Migräneartiger Kopfschmerz, in der Regel, jedoch nicht immer durch körperliche Betätigung verschlimmert, der während eines Myokardinfarkts auftritt. Nitroglyzerin wirkt schmerzlösend.

\section{Diagnostische Kriterien:}

A. Kopfschmerz, der das Kriterium C erfüllt

B. Es wurde ein akuter Myokardinfarkt nachgewiesen

C. Ein kausaler Zusammenhang kann durch mindestens zwei der folgenden Kriterien gezeigt werden:

1. Der Kopfschmerz hat sich in einem zeitlichen Zusammenhang mit dem Beginn des akuten Myokardinfarkts entwickelt

2. Einer oder beide der folgenden Punkte sind erfüllt:

a) der Kopfschmerz hat sich gleichzeitig mit der Verschlechterung des Myokardinfarkts deutlich verschlechtert

b) der Kopfschmerz hat sich gleichzeitig mit der Besserung oder dem Verschwinden des Myokardinfarkts deutlich gebessert oder ist verschwunden 
3. Der Kopfschmerz weist mindestens zwei der folgenden vier Charakteristika auf:
a) mittelstarke bis starke Intensität
b) von Übelkeit begleitet
c) nicht von Photophobie oder Phonophobie begleitet
d) Verstärkung durch körperliche Anstrengung

4. Der Kopfschmerz verschwindet durch Behandlung mit Nitroglyzerin oder Nitroglyzerinderivaten

D. Nicht besser erklärt durch eine andere ICHD-3-Diagnose.

\section{Kommentar:}

Die Diagnose setzt eine sorgfältige Dokumentation der Kopfschmerzen und den gleichzeitigen Nachweis einer kardialen Ischämiemittels Belastungs-EKG oder Myokardszintigraphie voraus. Es wurde jedoch auch schon ein im Ruhezustand auftretender 10.6 Kopfschmerz zurückzuführen auf eine kardiale Erkrankung beschrieben.

Ein nicht erkannter und nicht korrekt diagnostizierter 10.6 Kopfschmerz zurückzuführen auf eine kardiale Erkrankung kann schwerwiegende Folgen haben. Die Abgrenzung von einer 1.1 Migräne ohne Aura ist entscheidend, insbesondere da Medikamente mit einer vasokonstriktiven Wirkung (Triptane, Ergotaminderivate) zur Behandlung der Migräne indiziert, aber bei Patienten mit einer ischämischen Herzerkrankung kontraindiziert sind. Beide Erkrankungen können zu starken Kopfschmerzen mit begleitender Übelkeit führen und beide können durch körperliche Anstrengung getriggert werden. Migräne-ähnliche Kopfschmerzen können darüber hinaus durch eine medikamentöse Behandlung einer Angina pectoris z. B. mit Nitroglyzerin ausgelöst werden.

\subsection{Kopfschmerz zurückzuführen auf andere Störungen der Homöostase}

\section{Beschreibung:}

Kopfschmerz aufgrund einer oben nicht beschriebenen Störung der Homöostase.

\section{Diagnostische Kriterien:}

A. Kopfschmerz, der das Kriterium C erfüllt

B. Es wurde eine Störung der Homöoostase diagnostiziert, die oben nicht aufgeführt wurde und von der bekannt ist, dass sie Kopfschmerzen verursachen kann

C. Ein kausaler Zusammenhang kann durch eines oder mehrere der folgenden Kriterien gezeigt werden:

1. Der Kopfschmerz hat sich in einem zeitlichen Zusammenhang mit dem Beginn der Störung der Homöostase entwickelt

2. Der Kopfschmerz hat sich gleichzeitig mit der Verschlechterung der Störung der Homöostase deutlich verschlechtert

3. Der Kopfschmerz hat sich gleichzeitig mit der Besserung oder dem Verschwinden der Störung der Homöostase deutlich gebessert oder ist verschwunden

D. Nicht besser erklärt durch eine andere ICHD-3-Diagnose.
Kommentar:

Obwohl ursächliche Beziehungen zwischen Kopfschmerzen und einer Reihe von systemischen und metabolischen Erkrankungen vorgeschlagen wurden, wurde noch keine systematische Auswertung dieser Beziehungen durchgeführt und sie sind unzureichend belegt, um hierauf praktikable diagnostische Kriterien aufzubauen.

Literatur

Bigal ME, Gladstone J. The metabolic headaches. Current Pain Headache Rep 2008; 12: 292-295.

\subsubsection{Höhenkopfschmerz}

Appenzeller O. Altitude headache. Headache 1972; 12: 126-129.

Arngrim N, Schytz HW, Hauge MK, Ashina M, Olesen J. Carbon monoxide may be an important molecule in migraine and other headaches. Cephalalgia 2014; 34: 1169-1180.

Burtscher M, Mairer K, Wille M, Broessner G. Risk factors for high-altitude headache in mountaineers. Cephalalgia 2011; 31: 706-711.

Clarke C. Acute mountain sickness: medical problems associated with acute and subacute exposure to hypobaric hypoxia. Postgrad Med J 2006; 82: 748-753.

Ginsberg MD. Carbon monoxide intoxication: clinical features, neuropathology and mechanisms of injury. J Toxicol Clin Toxicol 1985; 23 : 281-288.

Heckerling PS, Leikiin JB, Maturen A, Perkins JT. Predictors of occult carbon monoxide poisoning in patients with headache and dizziness. Ann Intern Med 1987; 107: 174-176.

Jafarian S, Gorouhi F, Lotfi J. Reverse association between high-altitude headache and nasal congestion. Cephalalgia 2007; 27: 899-903.

Linde M, Edvinsson L, Manandhar K, Risal A, Steiner TJ. Migraine associated with altitude: results from a population-based study in Nepal. Eur ] Neurol 2017; 24: 1055-1061.

Lipton RB, Mazer C, Newman LC, Solomon S. Sumatriptan relieves migraine-like headaches associated with carbon monoxide exposure. Headache 1997; 37: 392-395.

Porcelli J, Gugelchuk G. A trek to the top: A review of acute mountain sickness. J Amer Osteopath Assoc 1995; 95: 718-720.

Serrano-Dueñas M. High-altitude headache. Expert Rev Neurother 2007; 7: 245-248.

Schoonman GG, Sándor PS, Agosti RM, Sicoli M, et al. Normobaric hypoxia and nitroglycerin as trigger factors for migraine. Cephalalgia 2006; 26 : 816-819.

Silber E, Sonnenberg P, Collier DJ, Pollard A, Murdoch DR, Goadsby PJ. Clinical features of headache at altitude: a prospective study. Neurology 2003; 60: 1167-1171.

Wilson MH, Davagnanam I, Holland G, et al. Cerebral venous system and anatomical predisposition to high-altitude headache. Ann Neurol 2013; 73: 381-389.

Wilson MH, Newman S, Imray CH. The cerebral effects of ascent to high altitudes. Lancet-Neurol 2009; 8: 175-191.

\subsubsection{Kopfschmerz zurückzuführen auf eine Flugreise}

Berilgen MS, Mungen B. Headache associated with airplane travel: report of six cases. Cephalalgia 2006; 26: 707-711.

Berilgen MS, Mungen B. A new type of headache. Headache associated with airplane travel: preliminary diagnostic criteria and possible mechanisms of aetiopathognesis. Cephalalgia 2011; 31: 1266-1273.

Bui SB, Petersen T, Poulsen JN, Gazerani P. Headaches attributed to airplane travel: a Danish survey. J Headache Pain 2016; 17: 33. 
Mainardi F, Lissotto C, Maggioni F, Zanchin G. Kopfschmerz zurückzuführen aufairplane travel ("Airplane headache"). Clinical profile based on a large case series. Cephalalgia 2012; 32: 592-599.

Mainardi F, Maggioni F, Zanchin G. Aeroplane headache, mountain descent headache, diving ascent headache. Three subtypes of Kopfschmerz zurückzuführen aufimbalance between intrasinusal and external air pressure? Cephalalgia 2017. doi: 10.1177/0333102417724154 (Epub ahead of print].

\subsubsection{Taucherkopfschmerz}

Cheshire WP, Ott MC Jr. Headache in divers. Headache 2001; 41: 235-247.

Di Fabio R, Vanacore N, Davassi C, Serrao M, Pierelli F. Scuba diving is not associated with high prevalence of headache: a cross-sectional study in men. Headache 2012; 52: 385-392.

Edmonds RC, Greene ER, Schoene RB, et al. Diving and subaquative medicine. 3 rd Ed. Oxford: Butterworth-Heinemann 1992: 404-406.

Englund M, Risberg J. Self-reported headache during saturation diving. Aviat Space Environ Med 2003; 74: 236-241.

Sliwka U, Kransney JA, Simon SG, et al. Effects of sustained low-level elevations of carbon dioxide on cerebral blood flow and autoregulation of the intracerebral arteries in humans. Aviat Space Environ Med 1998; 69: 299-306.

\subsubsection{Kopfschmerz zurückzuführen auf ein Schlafapnoe-Syndrom}

Aldrich MS, Chauncey JB. Are morning headaches part of obstructive sleep apnea syndrome? Arch Intern Med 1990; 150: 1265-1267.

Alberti A, Mazzotta G, Gallinella E, et al. Headache characteristics in obstructive sleep apnea syndrome and insomnia. Acta Neurol Scand 2005; 111: 309-316.

Chen PK, Fuh JL, Lane HY, Chin PY, Tien HC, Wang SJ. Morning headache in habitual snorers: frequency, characteristics, predictors and impacts. Cephalalgia 2011; 31: 829-836.

Greenough GP, Nowell PD, Sateia MJ. Headache complaints in relation to nocturnal oxygen saturation among patients with sleep apnea syndrome. Sleep Med 2002; 3: 361-364.

Goksan B, Gunduz A, Karadeniz D, et al. Morning headache in sleep apnoea: clinical and polysomnographic evaluation and reponse to nasal continuous positive airway pressure. Cephalalgia 2009; 29: 635-641.

Kristiansen HA, Kvaerner KJ, Akre H, et al. Tension-type headache and sleep apnea in the general population. J Headache Pain 2011; 12: 63-69.

Kristiansen HA, Kvaerner KJ, Akre H, Overland B, Sandvik L, Russell MB. Sleep apnea headache in the general population. Cephalalgia 2012; 32: 451-458.

Loh NK, Dinner DS, Foldvary DO, Skobieranda F, Yew WW. Do patients with obstructive sleep apnea wake up with headaches? Arch Intern Med 1999; 159: 1765-1768.

Luchesi LM, Speciali JG, Santos-Silva R, et al. Nocturnal awakening with headache and its relationship with sleep disorders in a population sample of adult inhabitants of Sao Paulo City, Brazil. Cephalalgia 2010; 30: 1477-1485.

Mitsikostas DD, Vikelis M, Viskos A. Refractory chronic headache associated with obstructive sleep apnoea syndrome. Cephalalgia 2008; 28: 139-143.

Ozge A, Ozge C, Kaleagasi H, et al. Headache in patients with chronic obstructive pulmonary disease: effects of chronic hypoxemia. J Headache Pain 2006; 7: 37-43.

Poceta JS, Dalessio DJ. Identification and treatment of sleep apnea in patients with chronic headache. Headache 1995; 35: 586-589.

Russell MB, Kristiansen HA, Kværner KJ. Headache in sleep apnea syndrome: epidemiology and pathophysiology. Cephalalgia 2014; 34: 752-755.

Suzuki K, Miyamoto M, Miyamoto T, et al. Sleep apnoea headache in obstructive sleep apnoea syndrome patients presenting with morning headache: comparison of the ICDH-2 and ICHD-3 beta criteria. J Headache Pain 2015; 16: 56.

\subsection{Dialysekopfschmerz}

Antoniazzi AL, Bigal ME, Bordini CA, Speciali JG. Headache associated with dialysis. The IHS criteria revisited. Cephalalgia 2003; 23: 146-149

Gksel BK, Torun D, Karaca S, et al. Is low blood magnesium level associated with hemodialysis headache? Headache 2006; 46: 40-45.

Antonazzzi AL, Corrado AP. Dialysis headache. Curr Pain Headache Rep 2007; 11: 297-303.

Jameson MD, Wiegmann TB. Principles, uses, and complications of hemodialysis. Medical Clinics of North America 1990; 74: 945-960.

\subsection{Kopfschmerz zurückzuführen auf eine arterielle Hypertonie}

Dodick DW. Recurrent short-lasting headache associated with paroxysmal hypertension: a clonidine-responsive syndrome. Cephalalgia 2000; 20: 509-514.

Gipponi S, Venturelli E, Rao R, et al. Hypertension is a factor associated with chronic daily headache. Neurol Sci 2010; 31 Suppl 1: 171-173.

Furlan JC. Kopfschmerz zurückzuführen auf eine Dysreflexie. Neurology 2011; 77: 792-798.

Gus M, Fuchs FD, Pimentel M, Rosa D, Melo AG, Moreira LB. Behavior of ambulatory blood pressure surrounding episodes of headache in mildly hypertensive patients. Arch Intern Med 2001; 161: 252-255.

Kruszewski P, Bieniaszewski L, Neubauer J, Krupa-Wojciechowska B. Headache in patients with mild to moderate hypertension is generally not associated with simultaneous blood pressure elevation. J Hypertension 2000; 18: 437-444.

Lance JW, Hinterberger H. Symptom of pheochromocytoma with particular reference to headache, correlated with catecholamine production. Arch Neurol 1976; 33: 281-288.

Land SH, Donovan T. Pre-eclampsia and eclampsia headache: classification recommendation [letter]. Cephalalgia 1999; 19: 67-69.

Loh KC, Shlossberg AH, Abbott EC, Salisbury SR, Tan MH. Phaeochromocytoma: a ten-year survey. Quart J Med 1997; 90: 51-60.

Mannelli M, Ianni L, Cilotti A, Conti A. Pheochromocytoma in Italy: A multicentric retrospective study. Eur J Endocrinol 1999; 141: 619-624.

Sousa Melo E, Carrilho Aguiar F, Sampaio Rocha-Filho PA. Dyalisis headache: a narrative review. Headache 2017; 57: 161-164.

Thomas JE, Rooke ED, Kvale WF. The neurologists experience with pheochromocytoma. JAMA 1966; 197: 754-758.

Vaughan CJ, Delanty N. Hypertensive emergencies. Lancet 2000; 356: 411-417.

Walker JJ. Pre-eclampsia. Lancet 2000; 56: 1260-1265.

Weiss NS. Relation of high blood pressure to headache, epistaxis, and selected other symptoms. The United States Health Examination Survey of Adults. NEJM 1972; 287: 631-633.

Zampaglione B, Pascale C, Marchisio M, Cavallo-Perin P. Hypertensive urgencies and emergencies. Prevalence and clinical presentation. Hypertension 1996; 27: 144-147.

\subsection{Kopfschmerz zurückzuführen auf eine Hypothyreose}

Airaghi L, Catania A. Endocrine headache. In: Seminars in headache management. Neuroendocrinological aspects of headache, vol 4, number 4. BC Decker Inc 1999: 1-15.

Amy JR. Tests of thyroid function in chronic headache patients. Headache 1987; 27: 351-353.

Arafah BM, Prunty D, Ybarra J, Hlavin ML, Selman WR. The dominant role of increased intrasellar pressure in the pathogenesis hypopituitarism, hyperprolactinemia, and headache in patients with pituitary adenomas. Clin Endocrinol Metab 2000; 85: 1789-1793. 
Fenichel NM. Chronic headache due to masked hypothyroidism. Ann Intern Med 1948; 29: 456-460.

Levy MJ, Matharu MS, Meeran K, et al. The clinical characteristics of headache in patients with pituitary tumours. Brain 2005; 128: 1921-1930.

Lima Carvalho MF, de Medeiros JS, Valença MM. Headache in recent onset hypothyroidism: prevalence, characteristics and outcome after treatment with levothyroxine. Cephalalgia 2017; 37: 938-946.

Moreau T. Headache in hypothyroidism. Prevalence and outcome under thyroid hormone therapy. Cephalalgia 1988; 18: 687-689.

\subsection{Kopfschmerz zurückzuführen auf Fasten}

Dalton K. Food intake prior to migraine attacks. Study of 2,313 spontaneous attacks. Headache 1975; 15: 188-193.

Dexter JD, Roberts J, Byer JA. The five hour glucose tolerance test and effect of low sucrose diet in migraine. Headache 1978; 18: 91-94.

Malouf R, Brust JCM. Hypoglycemia: causes, neurological manifestations, and outcome. Ann Neurol 1985; 17: 421-430.

Mosek, AC, Korczyn, A. D. Yom Kippur Headache. Neurology 1995; 45: 1953-1955.

Pearce J. Insulin induced hypoglycaemia in migraine. J Neurol Neurosurg Psychiat 1971; 34: 154-156.

Service FJ. Hypoglycemic disorders. In: Wyngaarden JB, Smith LH, Bennett JC, eds. Cecil's textbook of medicine, 18th ed. Philadelphia: WB Saunders 1992: 1310-1317.

\subsection{Kopfschmerz zurückzuführen auf eine kardiale Erkrankung}

Blacky RA, Rittelmeyer JT, Wallace MR. Headache angina. Am J Cardiol 1987; 60: 730.

Chen SP, Fuh JL, Yu WC, et al. Cardiac cephalalgia: case series and review of the literature with new ICDH-II criteria revisited. Eur Neurol 2004; 24: 231-234.

Bowen J, Oppenheimer G. Headache as a presentation of angina: reproduction of symptoms during angioplasty. Headache 1993; 33: 238-239.

Fleetcroft R, Maddocks JL. Headache due to ischaemic heart disease. J Roy Soc Med 1985; 78: 676.

Grace A, Horgan J, Breathnach K, Staunton H. Anginal headache and its basis. Cephalalgia 1997; 17: 195-196.

Gutiérrez-Morlote J, Pascual J. Cardiac cephalalgia is not necessarily and exertional headache: case report. Cephalalgia 2002; 22: 765-766.

Lefkowitz D, Biller J. Bregmatic headache as a manifestation of myocardial ischemia. Arch Neurol 1982; 39: 130.

Lipton RB, Lowenkopf T, Bajwa ZH, Leckie RS, Ribeiro S, Newman LC, Greenberg MA. Cardiac cephalgia: a treatable form of exertional headache. Neurology 1997; 49: 813-816.

Vernay D, Deffond D, Fraysse P, Dordain G. Walk headache: an unusual manifestation of ischemic heart disease. Headache 1989; 29: 350-351.

Wei JH, Wang HF. Cardiac cephalalgia: case reports and review. Cephalalgia 2008: 28: 892-896.
11. Kopf- oder Gesichtsschmerzen zurückzuführen auf Erkrankungen des Schädels sowie von Hals, Augen, Ohren, Nase, Nebenhöhlen, Zähnen, Mund und anderen Gesichts- oder Schädelstrukturen

\begin{tabular}{|c|c|}
\hline 11.1 & $\begin{array}{l}\text { Kopfschmerz zurückzuführen auf Erkrankungen der } \\
\text { Schädelknochen }\end{array}$ \\
\hline 11.2 & $\begin{array}{l}\text { Kopfschmerz zurückzuführen auf Erkrankungen des } \\
\text { Halses }\end{array}$ \\
\hline 11.2.1 & Zervikogener Kopfschmerz \\
\hline 11.2 .2 & $\begin{array}{l}\text { Kopfschmerz zurückzuführen auf eine retropharyngeale } \\
\text { Tendinitis }\end{array}$ \\
\hline 11.2 .3 & $\begin{array}{l}\text { Kopfschmerz zurückzuführen auf eine kraniozervikale } \\
\text { Dystonie }\end{array}$ \\
\hline 11.3 & $\begin{array}{l}\text { Kopfschmerz zurückzuführen auf Erkrankungen der } \\
\text { Augen }\end{array}$ \\
\hline 11.3.1 & $\begin{array}{l}\text { Kopfschmerz zurückzuführen auf ein akutes Eng- } \\
\text { winkelglaukom }\end{array}$ \\
\hline 11.3 .2 & Kopfschmerz zurückzuführen auf einen Brechungsfehler \\
\hline 11.3 .3 & $\begin{array}{l}\text { Kopfschmerz zurückzuführen auf eine entzündliche } \\
\text { Augenerkrankung }\end{array}$ \\
\hline 11.3.4 & Trochlearis-Kopfschmerz \\
\hline 11.4 & $\begin{array}{l}\text { Kopfschmerz zurückzuführen auf Erkrankungen der } \\
\text { Ohren }\end{array}$ \\
\hline 11.5 & $\begin{array}{l}\text { Kopfschmerz zurückzuführen auf Erkrankungen der } \\
\text { Nase oder Nasennebenhöhlen }\end{array}$ \\
\hline 11.5 .1 & $\begin{array}{l}\text { Kopfschmerz zurückzuführen auf eine akute Rhinosi- } \\
\text { nusitis }\end{array}$ \\
\hline 11.5 .2 & $\begin{array}{l}\text { Kopfschmerz zurückzuführen auf eine chronische oder } \\
\text { rezidivierende Rhinosinusitis }\end{array}$ \\
\hline 11.6 & $\begin{array}{l}\text { Kopfschmerz zurückzuführen auf Erkrankungen der } \\
\text { Zähne }\end{array}$ \\
\hline 11.7 & $\begin{array}{l}\text { Kopfschmerz zurückzuführen auf Erkrankungen des } \\
\text { Kiefergelenkes (TMD) }\end{array}$ \\
\hline 11.8 & $\begin{array}{l}\text { Kopf- oder Gesichtsschmerz zurückzuführen auf Ent- } \\
\text { zündungen des Ligamentum stylohyoideum }\end{array}$ \\
\hline 11.9 & $\begin{array}{l}\text { Kopf- oder Gesichtsschmerz zurückzuführen auf andere } \\
\text { Erkrankungen des Schädels sowie von Hals, Augen, } \\
\text { Ohren, Nase, Nebenhöhlen, Zähnen, Mund oder ande- } \\
\text { ren Gesichts- oder Schädelstrukturen }\end{array}$ \\
\hline
\end{tabular}

An anderer Stelle kodiert: Kopfschmerzen, die von einem Kopfder HWS-Trauma verursacht werden, sind unter 5. Kopfschmerz zurückzuführen auf eine Verletzung oder ein Trauma des Kopfes und/oder der HWS zu klassifizieren. Dies gilt insbesondere für Kopfschmerz nach einem Beschleunigungstrauma, ungeachtet der wahrscheinlichen Möglichkeit, dass diese Kopfschmerzen auf pathologische HWS-Veränderungen zurückzuführen sind.

Neuralgiforme Kopfschmerzen, die sich in Verbindung mit Gesichts-, Nacken- und/oder Kopfschmerzen manifestieren, werden unter 13. Schmerzhafte Läsionen der Hirnnerven und andere Gesichtsschmerzen eingeordnet.

An anderer Stelle kodiert Primärer und/oder sekundärer Kopfschmerz? 
Für Kapitel 11. Kopf- oder Gesichtsschmerzen zurückzuführen auf Erkrankungen des Schädels sowie von Hals, Augen, Ohren, Nase, Nebenhöhlen, Zähnen, Mund und anderen Gesichts- oder Schädelstrukturen gelten die allgemeinen Regeln für die kausale Zuordnung zu einer anderen Erkrankung.

Tritt ein neuer Kopfschmerz erstmals in engem zeitlichen Zusammenhang mit Erkrankungen des Schädels sowie von Hals, Augen, Ohren, Nase, Nebenhöhlen, Zähnen, Mund auf, von denen bekannt ist, dass sie Kopfschmerz verursachen, sollte der Kopfschmerz als Kopfschmerz zurückzuführen auf diese Erkrankung kodiert werden. Dies ist auch der Fall, wenn der Kopfschmerz das klinische Bild eines primären Kopfschmerzes zeigt, der in Teil 1 der ICHD-3 klassifiziert wird.

Wenn aber ein vorbestehender primärer Kopfschmerz in engem zeitlichen Zusammenhang mit Erkrankungen des Schädels sowie von Hals, Augen, Ohren, Nase, Nebenhöhlen, Zähnen, Mund chronisch wird oder sich deutlich verschlechtert (üblicherweise definiert als eine mindestens zweifache Steigerung der Häufigkeit und/oder Schwere), und ein kausaler Zusammenhang bestätigt wird, dann sollten sowohl die ursprüngliche primäre Kopfschmerzdiagnose als auch 11. Kopf- oder Gesichtsschmerzen zurückzuführen auf Erkrankungen des Schädels sowie von Hals, Augen, Ohren, Nase, Nebenhöhlen, Zähnen, Mund und anderen Gesichts- oder Schädelstrukturen (oder eines ihrer Typen oder Subtypen) vergeben werden, sofern gute Hinweise bestehen, dass diese Erkrankung Kopfschmerzen verursachen kann.

\section{Einleitung}

Erkrankungen der Halswirbelsäule und anderer Strukturen des Halses und des Kopfes wurden nicht selten als die häufigste Ursache von Kopfschmerzen überhaupt angesehen, da viele Kopfschmerzen ihren Ursprung in der Hals-, Nacken- oder Okzipitalregion haben oder dort lokalisiert sind. Darüber hinaus finden sich degenerative Veränderungen an der Halswirbelsäule bei praktisch allen über Vierzigjährigen. Umfangreiche kontrollierte Studien konnten jedoch zeigen, dass diese Veränderungen genauso häufig bei Menschen vorkommen, die nicht unter Kopfschmerzen leiden. Spondylose oder Osteochondrose können daher nicht als Ursache von Kopfschmerzen angesehen werden. Ähnliches gilt für andere weit verbreitete Erkrankungen wie chronische Sinusitiden, Kiefergelenkserkrankungen oder Brechungsfehler der Augen.

Ohne spezifische Kriterien könnten praktisch alle Kopfschmerzen als 11. Kopf- oder Gesichtsschmerzen zurückzuführen auf Erkrankungen des Schädels sowie von Hals, Augen, Ohren, Nase, Nebenhöhlen, Zähnen, Mund und anderen Gesichts- oder Schädelstrukturen klassifiziert werden. Es reicht nicht aus, Kopfschmerzmanifestationen einfach aufzulisten, um sie zu definieren, da diese Manifestationen nicht allein ihnen vorbehalten sind. Das Ziel, das mit diesem Kapitel verfolgt wurde, ist nicht Kopfschmerzen in allen ihren Subtypen zu beschreiben, sondern vielmehr gegebenenfalls, die spezifische ursächliche Beziehung zwischen Kopf- und Gesichtsschmerz und Erkrankungen des Schädels sowie von Hals, Augen, Ohren, Nase, Nebenhöhlen, Zähnen, Mund oder anderen Gesichtsoder Schädelstrukturen herauszuarbeiten. Aus diesem Grunde war es notwendig, strikte spezifische operationalisierte Kriterien für den zervikogenen Kopfschmerz und andere Kopfschmerzursachen in diesem Kapitel festzulegen. Diagnostische Testverfahren, die unbestätigt sind oder deren Testqualität bisher nicht überprüft wurde, konnten nicht berücksichtigt werden. Stattdessen soll durch die überarbeiteten Kriterien dazu motiviert werden, zukünftig zuverlässige und valide Testverfahren zu entwickeln, um das Ausmaß des ursächlichen Zusammenhanges zwischen Kopfschmerzen und einer kraniozervikalen Erkrankung zu bestimmen, was heute nur in sehr beschränktem Maße möglich ist.

Aus diesen Gründen und aufgrund der Vielfalt der in diesem Kapitel dargelegten ursächlichen Grunderkrankungen ist es schwer, feste allgemeine Kriterien für auf sie zurückgeführte Kopf- und/ oder Gesichtsschmerzen zu umreißen. In den meisten Fällen findet sich jedoch eine Übereinstimmung mit folgendem:

A. Kopf- oder Gesichtsschmerz, der Kriterium C erfüllt

B. Eine Läsion des Schädels sowie von Hals, Augen, Ohren, Nase, Nebenhöhlen, Zähnen, Mund oder anderen Gesichts- oder Schädelstrukturen, die als valide Ursache von Kopfschmerzen bekannt ist, wurde klinisch, laborchemisch und/oder durch die Bildgebung nachgewiesen

C. Nachweis, dass der Schmerz auf die Erkrankung oder Läsion zurückzuführen ist

D. Nicht besser erklärt durch eine andere ICHD-3-Diagnose.

\subsection{Kopfschmerz zurückzuführen auf Erkrankungen der Schädelknochen}

An anderer Stelle kodiert: Kopfschmerz, der auf ein Schädeltrauma zurückgeht, wird unter 5. Kopfschmerz zurückzuführen auf eine Verletzung oder ein Trauma des Kopfes und/oder der HWS oder einem seiner Typen kodiert.

Beschreibung:

Kopfschmerz, der von einer nichttraumatischen Erkrankung oder Läsion der Schädelknochen verursacht wird.

\section{Diagnostische Kriterien:}

A. Kopfschmerz, der das Kriterium C erfüllt

B. Eine Läsion im Schädelknochen, die als valide Ursache von Kopfschmerzen bekannt ist, wurde klinisch, laborchemisch und/oder mittels Bildgebung nachgewiesen

C. Ein kausaler Zusammenhang kann durch mindestens zwei der folgenden Kriterien gezeigt werden:

1. Der Kopfschmerz hat sich in einem zeitlichen Zusammenhang mit dem Beginn der Erkrankung der Schädelknochen oder des Auftretens der Läsion entwickelt

2. Einer oder beide der folgenden Punkte sind erfüllt:

a) der Kopfschmerz hat sich gleichzeitig mit der Verschlechterung der Erkrankung oder Läsion der Schädelknochen deutlich verschlechtert

b) der Kopfschmerz hat sich gleichzeitig mit der Besserung der Erkrankung oder Läsion der Schädelknochen deutlich verbessert

3. Der Kopfschmerz verschlimmert sich bei Druck auf die Läsion in den Schädelknochen

4. Der Kopfschmerz ist dort lokalisiert, wo sich die Läsion in den Schädelknochen befindet 
D. Nicht besser erklärt durch eine andere ICHD-3-Diagnose.

\section{Kommentar:}

Die meisten Erkrankungen des Schädelknochens - wie kongenitale Fehlbildungen, Frakturen, Tumore oder Metastasen - werden üblicherweise nicht von Kopfschmerzen begleitet. Wichtige Ausnahmen sind Osteomyelitis, Multiples Myelom und M. Paget. Kopfschmerzen können auch durch Läsionen des Mastoids oder durch eine Petrositis hervorgerufen werden.

\subsection{Kopfschmerz zurückzuführen auf Erkrankungen des Halses}

An anderer Stelle kodiert: Kopfschmerz, der durch ein HWS-Trauma verursacht wird, ist unter 5 . Kopfschmerz zurückzuführen auf eine Verletzung oder ein Trauma des Kopfes und/oder der HWS oder einem seiner Typen zu kodieren.

\section{Beschreibung:}

Kopfschmerz, der durch eine nicht-traumatische Störung verursacht wird, bei der eine Struktur im Halsbereich involviert ist, darunter knochige, muskuläre und andere Weichteilelemente.

\subsubsection{Zervikogener Kopfschmerz}

An anderer Stelle kodiert: Kopfschmerzen, die ursächlich mit myofaszialen Triggerpunkten assoziiert sind können, sofern sie andere Kriterien erfüllen, unter 2.1.1 selten auftretender episodischer Kopfschmerz vom Spannungstyp mit perikranieller Schmerzempfindlichkeit, 2.2.1 häufig auftretender episodischer Kopfschmerz vom Spannungstyp mit perikranieller Schmerzempfindlichkeit oder 2.3.1 chronischer Kopfschmerz vom Spannungstyp mit perikranieller Schmerzempfindlichkeit kodiert werden. A11.2.5 Kopfschmerz zurückzuführen auf ein zervikales myofasziales Schmerzsyndrom ist eine im Anhang angeführte Diagnose, bei der noch zu bestätigen gilt, dass dieser Kopfschmerztyp enger mit anderen zervikogenen Kopfschmerzen verwandt ist als mit einem 2. Kopfschmerz vom Spannungstyp. Es gibt eindeutig viele Fälle, in denen sich diese beiden Kategorien überschneiden und in denen die Diagnose schwierig sein kann.

\section{Beschreibung:}

Kopfschmerz, der durch eine Störung in der Halswirbelsäule und der zu ihr gehörigen Knochen-, Wirbel- und/oder Weichteilgewebe-Komponenten verursacht wird, gewöhnlich, jedoch nicht unweigerlich begleitet von Nackenschmerzen.

\section{Diagnostische Kriterien:}

A. Kopfschmerz, der das Kriterium C erfüllt

B. Klinischer Nachweis einer Erkrankung oder Läsion innerhalb der HWS oder des Weichteilgewebes im Halsbereich, die als valide Ursache von Kopfschmerzen bekannt ist2 und/oder deren Nachweis mittels Bildgebung1

C. Ein kausaler Zusammenhang kann durch mindestens zwei der folgenden Kriterien gezeigt werden:
1. Der Kopfschmerz hat sich in einem zeitlichen Zusammenhang mit dem Beginn der zervikalen Erkrankung oder des Auftretens der Läsion entwickelt

2. Der Kopfschmerz hat sich gleichzeitig mit der Besserung oder dem Verschwinden der zervikalen Erkrankung deutlich gebessert oder ist verschwunden

3. Es besteht eine eingeschränkte HWS-Beweglichkeit und der Kopfschmerz verschlechtert sich durch entsprechende kopfschmerzprovozierende Manöver deutlich

4. Beseitigung des Kopfschmerzes nach diagnostischer Blockade einer zervikalen Struktur bzw. des versorgenden Nervs

D. Nicht besser erklärt durch eine andere ICHD-3-Diagnose3;4;5.

\section{Anmerkung:}

1. Klinische Zeichen in der Bildgebung der oberen HWS sind bei Patienten ohne Kopfschmerz weit verbreitet; sie können Merkmale eines zervikogenen Kopfschmerzes sein, beweisen aber keinen ursächlichen Zusammenhang.

2. Tumoren, Frakturen, Infektionen und eine rheumatoide Arthritis der oberen Halswirbelsäule sind formell nicht als Kopfschmerzursachen validiert, werden bei Nachweis im Einzelfall jedoch nichtsdestotrotz als Kriterium B erfüllend akzeptiert. Eine zervikale Spondylose und Osteochondritis können zu den validen Ursachen zählen, die Kriterium B erfüllen oder auch nicht, auch hier wieder abhängig vom Einzelfall.

3. Wenn ein zerviko-myofasziales Schmerzsyndrom die Kopfschmerzursache ist, sollte der Kopfschmerz wahrscheinlich unter 2. Kopfschmerz vom Spannungstyp kodiert werden; bis zur weiteren Abklärung findet sich jedoch die alternative Diagnose eines A11.2.5 Kopfschmerzes zurückzuführen auf ein zerviko-myofasziales Schmerzsyndrom im Anhang.

4. Es wurden Kopfschmerzen postuliert, die durch eine Radikulopathie der oberen HWS verursacht werden, und angesichts der mittlerweile gut verstandenen Konvergenz zwischen der oberen zervikalen und der trigeminalen Nozizeption ist das eine logisch nachvollziehbare Kopfschmerzursache. Bis weitere Nachweise erbracht sind, findet sich diese Diagnose im Anhang als A11.2.4 Kopfschmerz zurückzuführen auf eine Radikulopathie der oberen HWS.

5. Zu den Charakteristika, die tendenziell den 11.2.1 zervikogenen Kopfschmerz von einer 1. Migräne und einem 2. Kopfschmerz vom Spannungstyp unterscheiden, gehören ein streng einseitiger Kopfschmerz, die Provokation der typischen Kopfschmerzen durch Fingerdruck auf die Nackenmuskeln und durch Kopfbewegung und einer Schmerzausstrahlung von hinten nach vorne. Doch obwohl dies Merkmale eines 11.2.1 zervikogenen Kopfschmerzes sein können, sind sie nicht nur diesem vorbehalten und definieren nicht unbedingt kausale Beziehungen. Bei einem 11.2.1 zervikogenen Kopfschmerz können migränöse Charakteristika wie Übelkeit, Erbrechen und Photo-/Phonophobie vorliegen, wenngleich generell in geringerem Umfang als bei 1. Migräne, und diese können in einigen Fällen zu einer Abgrenzung von einem 2. Kopfschmerz vom Spannungstyp beitragen. 


\subsubsection{Kopfschmerz zurückzuführen auf eine retro- pharyngeale Tendinitis}

Beschreibung:

Kopfschmerz, der durch Entzündungen oder Verkalkungen in den retropharyngealen Weichteilen verursacht wird und meist durch Dehnung oder Kompression von prävertebralen Muskeln im oberen Halsbereich entsteht.

\section{Diagnostische Kriterien:}

A. Kopfschmerz, der das Kriterium C erfüllt

B. Durch die Bildgebung wurde eine abnorme Schwellung der prävertebralen Weichteile in Höhe der oberen Halswirbel und damit eine retropharyngeale Tendonitis nachgewiesen

C. Ein kausaler Zusammenhang kann durch mindestens zwei der folgenden Kriterien gezeigt werden:

1. Der Kopfschmerz hat sich in einem zeitlichen Zusammenhang mit dem Beginn der retropharyngealen Tendinitis entwickelt oder führte zu deren Entdeckung

2. Einer oder beide der folgenden Punkte sind erfüllt:

a) der Kopfschmerz hat sich gleichzeitig mit dem Fortschreiten der retropharyngealen Tendinitis deutlich verschlechtert

b) der Kopfschmerz hat sich gleichzeitig mit der Besserung oder dem Verschwinden der retropharyngealen Tendinitis deutlich gebessert oder ist verschwunden

3. Der Kopfschmerz verschlechtert sich deutlich durch Extension des Halses, Rotation des Kopfes und/oder Schlucken1

4. Es kommt zu einer Druckempfindlichkeit über den Dornfortsätzen der drei oberen Halswirbel2

D. Nicht besser erklärt durch eine andere ICHD-3-Diagnose3.

Anmerkung:

1. Obwohl eine Retroflexion des Halses den Schmerz am zuverlässigsten verstärkt, kann dies auch bei Rotationsbewegungen des Kopfes und beim Schlucken geschehen.

2. Die Querfortsätze der oberen drei Halswirbel sind üblicherweise bei Palpation druckempfindlich.

3. Eine hohe Dissektion der A. carotis (oder eine andere Läsion im Bereich der A. carotis) sollte ausgeschlossen werden, bevor die Diagnose eines 11.2.2 Kopfschmerzes zurückzuführen auf eine retropharyngeale Tendinitis bestätigt wird.

\section{Kommentar:}

Körpertemperatur und Blutsenkungsgeschwindigkeit sind bei der retropharyngealen Tendinitis üblicherweise erhöht.

Verkalkungen in den prävertebralen Weichteilen werden am besten mittels CT oder MRT nachgewiesen, können jedoch auch auf konventionellen Röntgenaufnahmen deutlich werden. In mehreren Fällen konnte amorphes kalzifiziertes Material aus dem geschwollenen prävertebralen Gewebe aspiriert werden.

\subsubsection{Kopfschmerz zurückzuführen auf eine kranio- zervikale Dystonie}

\section{Beschreibung:}

Kopfschmerz, der durch eine Dystonie mit Beteiligung der Halsmuskulatur, mit abnormen Bewegungen oder Fehlhaltung des
Halses oder Kopfes zurückzuführen auf eine muskuläre Hyperaktivität verursacht wird.

\section{Diagnostische Kriterien:}

A. Schmerz im Halsbereich und Hinterkopf, der das Kriterium C erfüllt

B. Eine kraniozervikale Dystonie zeigt sich in abnormen Bewegungen oder Fehlhaltungen des Halses und/oder Kopfes infolge einer muskulären Hyperaktivität

C. Ein kausaler Zusammenhang kann durch mindestens zwei der folgenden Kriterien gezeigt werden:

1. Der Schmerz hat sich in einem zeitlichen Zusammenhang mit dem Beginn der kraniozervikalen Dystonie entwickelt

2. Der Schmerz hat sich gleichzeitig mit dem Fortschreiten der kraniozervikalen Dystonie deutlich verschlechtert

3. Der Schmerz hat sich gleichzeitig mit der Besserung oder dem Verschwinden der kraniozervikalen Dystonie deutlich gebessert oder ist verschwunden

4. Die Schmerzlokalisation entspricht der Lokalisation der dystonischen Muskulatur

D. Nicht besser erklärt durch eine andere ICHD-3-Diagnose.

\section{Kommentar:}

Fokale Dystonien des Kopfes und Halses, die von 11.2.3 Kopfschmerz zurückzuführen auf eine kraniozervikale Dystonie begleitet werden, sind die pharyngeale Dystonie, der spasmodische Tortikollis, die mandibuläre Dystonie, die linguale Dystonie und eine Kombination von kraniellen und zervikalen Dystonien (segmentale kraniozervikale Dystonie).

Die Schmerzen werden vermutlich verursacht durch lokale Muskelkontraktionen und sekundäre sensible Veränderungen.

\subsection{Kopfschmerz zurückzuführen auf Erkrankungen der Augen}

\section{Beschreibung:}

Kopfschmerz, der durch eine ein- oder beidseitige Augenerkrankung verursacht wird.

\subsubsection{Kopfschmerz zurückzuführen auf ein akutes Engwinkelglaukom}

Beschreibung:

Kopfschmerz, meist unilateral, der auf ein akutes Engwinkelglaukom zurückzuführen ist und mit anderen Symptomen und klinischen Zeichen dieser Erkrankung verbunden ist.

\section{Diagnostische Kriterien:}

A. Kopfschmerz, der das Kriterium C erfüllt

B. Es wurde ein akutes Engwinkelglaukom diagnostiziert bei nachweislich erhöhtem intraokulärem Druck

C. Ein kausaler Zusammenhang kann durch zwei der folgenden Kriterien gezeigt werden:

1. Der Kopfschmerz hat sich in einem zeitlichen Zusammenhang mit dem Beginn des Glaukoms entwickelt

2. Der Kopfschmerz hat sich gleichzeitig mit dem Fortschreiten des Glaukoms deutlich verschlechtert 
3. Der Kopfschmerz hat sich gleichzeitig mit der Besserung oder dem Verschwinden des Glaukoms signifikant gebessert oder ist verschwunden

4. Die Schmerzlokalisation schließt das betroffene Auge ein D. Nicht besser erklärt durch eine andere ICHD-3-Diagnose.

\section{Kommentar:}

Ein akutes Engwinkelglaukom verursacht in der Regel Augen- und/ oder periorbitale Schmerzen, Sehschärfeverlust (Verschwommensehen), konjunktivale Injektion und Ödembildung, Übelkeit und Erbrechen.

Bei Anstieg des intraokulären Drucks auf mehr als $30 \mathrm{mmHg}$ steigt die Gefahr eines bleibenden Visusverlusts dramatisch, was eine frühzeitige Diagnose grundlegend wichtig macht.

\subsubsection{Kopfschmerz zurückzuführen auf einen Brechungsfehler}

Beschreibung:

Kopfschmerz, der durch (einen) okulare(n) Brechungsfehler verursacht wird, in der Regel symptomatisch nach längerer Anstrengung der Augen.

\section{Diagnostische Kriterien:}

A. Kopfschmerz, der das Kriterium C erfüllt

B. Nicht oder fehlerhaft korrigierte( $r$ ) Brechungsfehler in einem Auge oder in beiden Augen

C. Ein kausaler Zusammenhang kann durch mindestens zwei der folgenden Kriterien gezeigt werden:

1. Der Kopfschmerz hat sich in einem zeitlichen Zusammenhang mit dem Beginn oder der Verschlechterung des/der Brechungsfehler(s) deutlich verschlechtert

2. Der Kopfschmerz hat sich nach Korrektur des/der Brechungsfehler(s) deutlich gebessert

3. Der Kopfschmerz verstärkt sich bei längerem Blick in die Ferne oder in den Winkelbereich, in dem das Sehen gestört ist

4. Der Kopfschmerz bessert sich deutlich, wenn die visuelle Anstrengung eingestellt wird

D. Nicht besser erklärt durch eine andere ICHD-3-Diagnose.

\section{Kommentar:}

Die meisten Patienten mit einem 11.3.2 Kopfschmerz zurückzuführen auf einen Brechungsfehler werden einen Augenarzt hinzuziehen.

Obwohl Brechungsfehler wesentlich seltener Kopfschmerzursache sind als allgemein angenommen, gibt es einige Hinweise auf solche bei Kindern sowie eine Reihe von Fällen bei Erwachsenen, die diese Annahme unterstützen.

\subsubsection{Kopfschmerz zurückzuführen auf eine entzündliche Augenerkrankung}

\section{Beschreibung:}

Kopfschmerz, der durch eine entzündliche Augenerkrankung wie eine Iritis, Uveitis, Skleritis oder Konjunktivitis verursacht wird und mit anderen Symptomen und klinischen Zeichen der Erkrankung einhergeht.
Diagnostische Kriterien:

A. Periorbitaler Kopf- und Augenschmerz, der Kriterium C erfüllt

B. Eine entzündliche Augenerkrankung, die als valide Ursache von Kopfschmerzen bekannt ist, wurde klinisch, laborchemisch und/oder mittels Bildgebung nachgewiesen.1

C. Ein kausaler Zusammenhang kann durch mindestens zwei der folgenden Kriterien gezeigt werden:

1. Der Kopfschmerz hat sich in einem zeitlichen Zusammenhang mit dem Beginn der Augenerkrankung entwickelt

2. Einer oder beide der folgenden Punkte sind erfüllt:

a) der Kopfschmerz hat sich gleichzeitig mit der Verschlechterung der entzündlichen Augenerkrankung deutlich verschlechtert

b) der Kopfschmerz hat sich gleichzeitig mit der Besserung oder dem Verschwinden der entzündlichen Augenerkrankung deutlich gebessert oder ist verschwunden

3. Einer oder beide der folgenden Punkte ist/sind erfüllt:

a) der Kopfschmerz bessert sich bei topischer Anwendung eines lokalen Anästhetikums auf das Auge deutlich

b) der Kopfschmerz verstärkt sich bei Ausübung von Druck auf das Auge

4. Im Falle einer unilateralen entzündlichen Augenerkrankung ist der Kopfschmerz umschrieben und ipsilateral zu dieser2

D. Nicht besser erklärt durch eine andere ICHD-3-Diagnose.

Anmerkung:

1. Entzündliche Augenerkrankungen, die als valide Ursache von Kopfschmerzen bekannt sind, sind unter anderem Iritis, Uveitis, Zyklitis, Skleritis, Chorioiditis, Konjunktivitis und Keratitis.

2. Aufgrund der Überschneidung und Konvergenz der nozizeptiven Felder (die zu einer komplexen Schmerzübertragung führt), kann jede okuläre Schmerzursache Kopfschmerzen in einer beliebigen Region auslösen. Bei einer unilateralen entzündlichen Augenerkrankung jedoch ist der Kopfschmerz wahrscheinlich umschrieben und ipsilateral.

\section{Kommentar:}

Entzündungen des Auges sind vielseitig und können entsprechend der Lokalisation (z. B. Iritis, Zyklitis, Chorioiditis), des Verlaufes (akut, subakut, chronisch), der vermuteten Ursache (endogen oder durch exogene Infektion, mit der Linse zusammenhängend, traumatisch) oder des Entzündungstyps (granulomatös, nicht-granulomatös) eingeteilt werden.

\subsubsection{Trochlearis-Kopfschmerz}

Früher verwendete Begriffe Kopfschmerz zurückzuführen auf eine Trochleitis.

Eine nicht-entzündliche Erkrankung, die mit einer Dysfunktion des N. trochlearis assoziiert ist, primärer Trochlearis-Kopfschmerz genannt, ruft Schmerzen in der trochlearen und temporoparietalen Region hervor, die sich bei Supraduktion des Auges verschlechtern. Ihre Diagnose und Behandlung erfolgt ähnlich wie die einer Trochleitis und wird deshalb unter 11.3.4 Trochlearis-Kopfschmerz aufgenommen. 
Beschreibung:

Kopfschmerz, üblicherweise frontal und/oder periorbital lokalisiert, mit oder ohne Augenschmerzen, verursacht durch eine peritrochleare Entzündung oder Dysfunktion. Oft wird dieser durch Augenbewegungen verstärkt.

\section{Diagnostische Kriterien:}

A. Periorbitaler und/oder frontaler Kopfschmerz, der Kriterium C erfüllt

B. Klinisch und/oder durch die Bildgebung erbrachter Nachweis einer Entzündung oder Dysfunktion einschließlich einer Druckempfindlichkeit bei Palpation der Trochlea im superomedialen Orbit

C. Ein kausaler Zusammenhang kann durch mindestens zwei der folgenden Kriterien gezeigt werden:

1. Unilateraler Augenschmerz

2. Der Kopfschmerz wird durch Bewegungen des Auges verstärkt1

3. Der Kopfschmerz bessert sich nach Injektion eines lokalen Anästhetikums oder Kortikoids in die peritrochleäre Region deutlich

4. Der Kopfschmerz ist umschrieben und ipsilateral zur beteiligten Trochlea

D. Nicht besser erklärt durch eine andere ICHD-3-Diagnose.

\section{Anmerkung:}

1. Vor allem vertikale Bewegungen.

\section{Kommentar:}

Eine Trochleitis, definiert als Entzündung der Trochlea und/oder das Epimysium des M. obliquus superior, kann zu Augenschmerzen und frontalen Kopfschmerzen führen, die durch Bewegungen des Auges, bei denen der M. obliquus superior beteiligt ist, verstärkt werden. Auch wenn sie nicht sehr häufig vorkommt, ist sie doch auch keine seltene Erkrankung und muss bei der Beurteilung eines einseitigen periorbitalen Kopfschmerzes in Betracht gezogen werden.

Eine Trochleitis kann bei Patienten mit einer 1. Migräne auch eine Migräneepisode auslösen, die unter ihrem jeweiligen Typ oder Subtyp kodiert werden sollte.

Ein 11.3.4 Trochlearis-Kopfschmerz kann durch Lesen provoziert werden.

\subsection{Kopfschmerz zurückzuführen auf Erkrankungen der Ohren}

Beschreibung:

Kopfschmerz, der durch eine entzündliche, neoplastische oder sonstige ein- oder beidseitige Erkrankung des Ohres verursacht wird und mit weiteren Symptomen und/oder klinischen Zeichen der Erkrankung einhergeht.

\section{Diagnostische Kriterien:}

A. Kopfschmerz, der das Kriterium C erfüllt

B. Eine infektiöse, neoplastische oder andere irritative Erkrankung oder eine anders gelagerte einseitige oder beidseitige Läsion des Ohres, die als valide Ursache von Kopfschmerzen bekannt ist, wurde klinisch, laborchemisch und/oder mittels Bildgebung nachgewiesen.

C. Ein kausaler Zusammenhang kann durch mindestens zwei der folgenden Kriterien gezeigt werden:

1. Der Kopfschmerz hat sich in einem zeitlichen Zusammenhang mit dem Beginn der Erkrankung des Ohres oder dem Auftreten der Läsion des Ohres entwickelt

2. Einer oder beide der folgenden Punkte sind erfüllt:

a) der Kopfschmerz hat sich gleichzeitig mit der Verschlechterung oder dem Fortschreiten der Erkrankung oder Läsion des Ohres deutlich verschlechtert

b) der Kopfschmerz hat sich gleichzeitig mit der Besserung oder dem Verschwinden der Erkrankung oder Läsion des Ohres deutlich gebessert

3. Der Kopfschmerz verstärkt sich bei Ausübung von Druck auf das/die betroffene(n) Ohr(en) oder periaurikulären Strukturen

4. Im Falle einer einseitigen Erkrankung oder Läsion der Ohren ist der Kopfschmerz umschrieben und ipsilateral zu dieser

D. Nicht besser erklärt durch eine andere ICHD-3-Diagnose.

Kommentar:

Aufgrund der Überschneidung und Konvergenz der nozizeptiven Areale in den nozizeptiven Bahnen des Kopfes und Halses scheint es klar, dass eine schmerzhafte Erkrankung oder Läsion des Ohres zu Kopfschmerzen führen kann. Es ist hochgradig unwahrscheinlich, dass Kopfschmerzen unter solchen Umständen bei nicht vorhandenen Ohrenschmerzen auftreten können, der typischen Manifestation einer otologischen Pathologie.

\subsection{Kopfschmerz zurückzuführen auf Erkrankungen der Nase oder Nasen- nebenhöhlen}

Früher verwendeter Begriff: Der Begriff „Sinuskopfschmerzen“ ist überholt, da dieser sowohl für primäre Kopfschmerzerkrankungen als auch für Kopfschmerzen angewandt wurde, die mutmaßlich auf verschiedene Krankheitsbilder zurückzuführen sind, bei denen Strukturen der Nase oder Nasennebenhöhlen beteiligt sind.

\section{Beschreibung:}

Kopfschmerz, der durch eine Erkrankung der Nase und/oder Nasennebenhöhlen verursacht wird und von weiteren Symptomen und/ oder klinischen Zeichen der Erkrankung begleitet wird.

\subsubsection{Kopfschmerz zurückzuführen auf eine akute Rhinosinusitis}

Beschreibung:

Kopfschmerz, der durch eine akute Rhinosinusitis verursacht wird und von weiteren Symptomen und/oder klinischen Zeichen dieser Erkrankung begleitet wird.

\section{Diagnostische Kriterien:}

A. Kopfschmerz, der das Kriterium C erfüllt

B. Nachweis einer akuten Rhinosinusitis klinisch, mittels nasaler Endoskopie und/oder durch bildgebende Verfahren 
C. Ein kausaler Zusammenhang kann durch mindestens zwei der folgenden Kriterien gezeigt werden:

1. Der Kopfschmerz hat sich in einem zeitlichen Zusammenhang mit dem Beginn der Rhinosinusitis entwickelt

2. Einer oder beide der folgenden Punkte sind erfüllt:

a) Der Kopfschmerz hat sich gleichzeitig mit der Verschlechterung der Rhinosinusitis deutlich verschlechtert

b) Der Kopfschmerz hat sich gleichzeitig mit der Besserung oder dem Verschwinden der Rhinosinusitis deutlich gebessert oder ist verschwunden

3. Der Kopfschmerz verstärkt sich bei Ausübung von Druck auf die Nasennebenhöhlen

4. Im Falle einer unilateralen Rhinosinusitis ist der Kopfschmerz umrissen und ipsilateral zu dieser

D. Nicht besser erklärt durch eine andere ICHD-3-Diagnose1.

Anmerkung:

1. 1. Migräne und 2. Kopfschmerz vom Spannungstyp werden aufgrund der Übereinstimmung der Schmerzlokalisation und im Falle der Migräne aufgrund der gemeinsamen autonomen Begleitsymptome in der Nase oft mit einem 11.5.1 Kopfschmerz zurückzuführen auf eine akute Rhinosinusitis verwechselt. Das Vorliegen oder Nichtvorliegen einer eitrigen Sekretion aus der Nase und/oder sonstiger diagnostischer Merkmale einer akuten Rhinosinusitis hilft bei der Abgrenzung dieser Erkrankungen voneinander.

\section{Kommentar:}

Schmerz infolge eines Krankheitsbildes der Nasenschleimhaut oder verwandter Strukturen wird in der Regel als frontal oder im Gesicht wahrgenommen, kann jedoch nach weiter hinten weitergeleitet werden. Der bloße Nachweis pathologischer Veränderungen im Sinne einer akuten Rhinosinusitis durch die Bildgebung, die mit der Schmerzschilderung des Patienten korrelieren, reicht zur Sicherung der Diagnose eines 11.5.1 Kopfschmerzes zurückzuführen auf eine akute Rhinosinusitis nicht aus. Das Ansprechen auf eine Behandlung mit örtlicher Betäubung ist ein überzeugender Beleg, kann aber unter Umständen auch nicht pathognomonisch sein.

Eine Episode von 1. Migräne kann durch pathologische Veränderungen der Nase oder Nasennebenhöhlen getriggert oder verstärkt werden.

\subsubsection{Kopfschmerz zurückzuführen auf eine chronische oder rezidivierende Rhinosinusitis}

Beschreibung:

Kopfschmerz, der durch eine chronische infektiöse oder entzündliche Erkrankung der Nasennebenhöhlen verursacht wird und von anderen Symptomen und/oder klinischen Zeichen der Erkrankung begleitet ist.

\section{Diagnostische Kriterien:}

A. Kopfschmerz, der das Kriterium C erfüllt

B. Eine frische oder frühere Infektion oder ein anderer entzündlicher Prozess in den Nasennebenhöhlen wurde klinisch, durch nasale Endoskopie und/oder bildgebende Verfahren nachgewiesen
C. Ein kausaler Zusammenhang kann durch mindestens zwei der folgenden Kriterien gezeigt werden:

1. Der Kopfschmerz hat sich in einem zeitlichen Zusammenhang mit dem Beginn der chronischen Rhinosinusitis entwickelt

2. Der Kopfschmerz nimmt gleichzeitig mit dem Ausmaß der Verstopfung der Nasennebenhöhlen und anderen Symptomen der chronischen Rhinosinusitis zu und ab

3. Der Kopfschmerz verstärkt sich bei Ausübung von Druck auf die Nasennebenhöhlen

4. Im Falle einer unilateralen Rhinosinusitis ist der Kopfschmerz umrissen und ipsilateral zu dieser

D. Nicht besser erklärt durch eine andere ICHD-3-Diagnose.

\section{Kommentar:}

Es wurde die Frage aufgeworfen, ob chronische pathologische Veränderungen der Nasennebenhöhlen anhaltende Kopfschmerzen nach sich ziehen können. Aktuelle Studien scheinen für einen solchen ursächlichen Zusammenhang zu sprechen. In der Bildgebung oder Endoskopie zu erkennende pathologische Veränderungen jedoch, die mit der Schmerzschilderung des Patienten korrelieren, reichen für sich genommen nicht aus, um die Diagnose eines 11.5.2 Kopfschmerzes zurückzuführen auf eine chronische oder rezidivierende Rhinosinusitis abzusichern.

\subsection{Kopfschmerz zurückzuführen auf Erkrankungen der Zähne}

Beschreibung:

Kopfschmerz, der durch eine Erkrankung verursacht wird, an der die Zähne beteiligt sind.

Diagnostische Kriterien:

A. Kopfschmerz, der das Kriterium C erfüllt

B. Eine Erkrankung oder Läsion eines Zahnes oder mehrerer Zähne, die als valide Ursache von Kopfschmerzen bekannt ist, wurde klinisch und/oder mittels Bildgebung nachgewiesen

C. Ein kausaler Zusammenhang kann durch mindestens zwei der folgenden Kriterien gezeigt werden:

1. Der Kopfschmerz hat sich in einem zeitlichen Zusammenhang mit dem Beginn der Erkrankung oder dem Auftreten der Läsion entwickelt

2. Einer oder beide der folgenden Punkte sind erfüllt:

a) Der Kopfschmerz hat sich gleichzeitig mit der Verschlechterung oder dem Fortschreiten der Erkrankung oder Läsion deutlich verschlechtert

b) Der Kopfschmerz hat sich gleichzeitig mit der Besserung oder dem Verschwinden der Erkrankung oder Läsion deutlich gebessert oder ist verschwunden

3. Der Kopfschmerz verstärkt sich durch Abtasten oder Sondieren des betroffenen Zahns/der betroffenen Zähne bzw. durch Druck auf diese(n)

4. Im Falle einer unilateralen Erkrankung oder Läsion ist der Kopfschmerz umschrieben und ipsilateral zu dieser D. Nicht besser erklärt durch eine andere ICHD-3-Diagnose. 


\section{Kommentar:}

Erkrankungen der Zähne rufen in der Regel Zahn- und/oder Gesichtsschmerzen hervor, diese können jedoch in den Kopf ausstrahlen. Zu den häufigsten Gründen für einen 11.6 Kopfschmerz zurückzuführen auf Erkrankungen der Zähne gehören eine endodontische oder parodontale Infektion oder ein dortiger Abszess bzw. eine traumatische Irritation wie etwa eine Perikoronitis in der Umgebung eines nur teilweise durchgebrochenen unteren Weisheitszahnes.

\subsection{Kopfschmerz zurückzuführen auf Erkrankungen des Kiefergelenkes (TMD)}

An anderer Stelle kodiert: Andere Kiefererkrankungen als eine Erkrankung des Kiefergelenks, etwa ein bösartiger Kiefertumor, eine Osteomyelitis oder eine Fraktur, erzeugen einen umschriebenen Schmerz, der in das Gesicht und den Kopf ausstrahlen kann, doch verursachen sie selten nur Kopfschmerzen. Wenn in solchen Fällen Kopfschmerzen auftreten, sollten diese unter 11.9 Kopf- oder Gesichtsschmerz zurückzuführen auf andere Erkrankungen des Schädels sowie von Hals, Augen, Ohren, Nase, Nebenhöhlen, Zähnen, Mund oder anderen Gesichts- oder Schädelstrukturen kodiert werden.

\section{Beschreibung:}

Kopfschmerz, der von Erkrankungen verursacht wird, die vom Kiefergelenk oder benachbarten Strukturen ausgehen.

\section{Diagnostische Kriterien:}

A. Jeder Kopfschmerz,1 der Kriterium C erfüllt

B. Ein schmerzhafter pathologischer Prozess, der Elemente des Kiefergelenkes/der Kiefergelenke, der Kaumuskeln und/oder verwandte Strukturen auf nur einer Seite oder beidseits betrifft, wurde klinisch nachgewiesen

C. Ein kausaler Zusammenhang kann durch mindestens zwei der folgenden Kriterien gezeigt werden:

1. Der Kopfschmerz hat sich in einem zeitlichen Zusammenhang mit dem Beginn der Erkrankung des Kiefergelenkes entwickelt oder führte zu ihrer Entdeckung

2. Der Kopfschmerz verstärkt sich durch Kiefergelenksbewegungen, die Ausübung von Funktionen (z. B. Kauen) und/ oder Parafunktionen (z. B. Zähneknirschen) des Kiefergelenks

3. Der Kopfschmerz wird bei körperlicher Untersuchung mittels Palpation des M. temporalis und/oder passive Kieferbewegung provoziert

D. Nicht besser erklärt durch eine andere ICHD-3-Diagnose2.

\section{Anmerkung:}

1. In der Regel temporal lokalisiert, dabei ein- oder beidseitig.

2. Es besteht eine gewisse Überschneidung zwischen einem 11.7 Kopfschmerz zurückzuführen auf Erkrankungen des Kiefergelenkes (TMD), der auf muskuläre Verspannung zurückgeht, und einem 2. Kopfschmerz vom Spannungstyp. Wenn die Diagnose TMD unsicher ist, sollte der Kopfschmerz unter 2. Kopfschmerz vom Spannungstyp oder einem Typ oder
Subtyp hiervon (möglicherweise mit perikraniellen Muskelschmerzen) kodiert werden.

\section{Kommentar:}

11.7 Kopfschmerz zurückzuführen auf Erkrankungen des Kiefergelenkes (TMD) tritt üblicherweise am meisten in der/den Schläfenregion(en), der/den präaurikulären Region(en) des Gesichtes und/ oder dem/den Kaumuskel(n) auf. Er kann unilateral sein, wird aller Wahrscheinlichkeit nach aber bilateral auftreten, wenn die Grunderkrankung beidseits die temporomandibuläre Region betrifft. Eine Schmerzübertragung zum Gesicht kommt häufig vor; nach Zahnschmerzen sind Erkrankungen des Kiefergelenkes die häufigste Ursache von Gesichtsschmerzen.

Zu den Schmerzursachen gehören Bandscheibenvorfälle, Gelenkarthrose, degenerative Erkrankungen und/oder Gelenkhypermobilität und ein regionaler myofaszialer Schmerz.

Die Diagnose einer TMD kann schwierig sein, bei gewissen Kontroversen im Hinblick auf die relative Bedeutung klinischer und röntgenologischer Nachweise. Es wird der Gebrauch der diagnostischen Kriterien empfohlen, die vom International RDC/TMD Consortium Network und der Orofacial Pain Special Interest Group entwickelt wurden.

\subsection{Kopf- oder Gesichtsschmerz zurück- zuführen auf Entzündungen des Liga- mentum stylohyoideum}

Früher verwendeter Begriff: Eagle-Syndrom.

\section{Beschreibung:}

Unilateraler Kopfschmerz mit Nacken, Rachen- und/oder Gesichtsschmerz, verursacht durch eine Entzündung des Ligamentum stylohyoideum und in der Regel durch Kopfdrehen provoziert oder verschlimmert.

\section{Diagnostische Kriterien:}

A. Jeder Kopf-, Nacken-, Rachen- und/oder Gesichtsschmerz, der Kriterium C erfüllt1

B. Radiologischer Nachweis eines kalzifizierten oder elongierten Ligamentum stylohyoideum

C. Ein kausaler Zusammenhang kann durch mindestens zwei der folgenden Kriterien gezeigt werden:

1. Der Schmerz wird durch digitale Palpation des Ligamentum stylohyoideum hervorgerufen oder verschlimmert

2. Der Schmerz wird durch Kopfdrehen hervorgerufen oder verschlimmert

3. Der Schmerz bessert sich deutlich nach Injektion eines örtlichen Betäubungsmittels in das Ligamentum stylohyoideum oder durch eine Styloidektomie

4. Der Schmerz ist ipsilateral zu dem entzündeten Ligamentum stylohyoideum lokalisiert

D. Nicht besser erklärt durch eine andere ICHD-3-Diagnose.

Anmerkung:

1. Ein 11.8 Kopf- oder Gesichtsschmerz zurückzuführen auf eine Entzündung des Ligamentum stylohyoideum wird im 
Allgemeinen im Oropharynx, Hals und/oder Gesicht wahrgenommen, einige Patienten erfahren jedoch eher diffuse Kopfschmerzen.

11.9 Kopf- oder Gesichtsschmerz zurückzuführen auf andere Erkrankungen des Schädels sowie von Hals, Augen, Ohren, Nase, Nebenhöhlen, Zähnen, Mund oder anderen Gesichtsoder zervikalen Strukturen

\section{Beschreibung:}

Kopf- und/oder Gesichtsschmerz, der durch eine Erkrankung des Schädels sowie von Hals, Augen, Ohren, Nase, Nebenhöhlen, Zähnen, Mund oder anderen oben nicht beschriebenen Gesichts- oder zervikalen Strukturen hervorgerufen wird.

\section{Diagnostische Kriterien:}

A. Jeder Kopf- und/oder Gesichtsschmerz, der Kriterium C erfüllt

B. Es wurde eine Erkrankung oder Läsion bezogen auf Schädel, Hals, Augen, Ohren, Nase, Nebenhöhlen, Zähne, Mund oder andere oben nicht beschriebene Gesichts- oder zervikalen Strukturen, von denen jedoch bekannt ist, dass sie Kopfschmerzen verursachen können, diagnostiziert

C. Ein kausaler Zusammenhang kann durch mindestens zwei der folgenden Kriterien gezeigt werden:

1. Der Kopf- und/oder Gesichtsschmerz hat sich in einem zeitlichen Zusammenhang mit dem Beginn der Erkrankung oder dem Auftreten der Läsion entwickelt

2. Einer oder beide der folgenden Punkte sind erfüllt:

a) der Kopf- und/oder Gesichtsschmerz hat sich gleichzeitig mit dem Fortschreiten der Erkrankung oder Läsion deutlich verschlechtert

b) der Kopf- und/oder Gesichtsschmerz hat sich gleichzeitig mit der Besserung oder dem Verschwinden der Erkrankung oder Läsion deutlich gebessert oder ist verschwunden

3. Der Kopf- und/oder Gesichtsschmerz wird durch Druckausübung auf die Läsion verstärkt

4. Der Kopf- und/oder Gesichtsschmerz ist in Übereinstimmung mit dem Situs der Läsion lokalisiert

D. Nicht besser erklärt durch eine andere ICHD-3-Diagnose.

Literatur

\subsection{Kopfschmerz zurückzuführen auf Erkrankungen der Schädel-} knochen

Bhatoe HS, Deshpande GU. Primary cranial Ewing's sarcoma. Br J Neurosurg 1998; 12: 165-169.

Hayashi T, Kuroshima Y, Yoshida K, Kawase T, Ikeda E, Mukai M. Primary osteosarcoma of the sphenoid bone with extensive periosteal extension - case report. Neurol Med Chir (Tokyo) 2000; 40: 419-422.

Scherer A, Engelbrecht V, Nawatny J, Messing-Junger M, Reifenberger $\mathrm{G}$, Modder U. MRI of the cerebellopontine angle in patients with cleidocranial dysostosis. Rofo Fortschr Geb Rontgenstr Neuen Bildgeb Verfahr 2001; 173: 315-318.

\subsubsection{Zervikogener Kopfschmerz}

Antonaci F, Fredriksen TA, Sjaastad O. Cervicogenic headache: clinical presentation, diagnostic criteria, and differential diagnosis. Curr Pain Headache Rep 2001; 5: 387-392.
Antonaci F, Ghirmai S, Bono G, Sandrini G, Nappi G. Cervicogenic headache: evaluation of the original diagnostic criteria. Cephalalgia 2001; 21: 573-583.

Bogduk N, Corrigan B, Kelly P, Schneider G, Farr R. Cervical Headache. Med J Aust 1985; 143: 202-207.

Bogduk N. Cervicogenic headache: anatomic basis and pathophysiologic mechanisms. Curr Pain Headache Rep 2001; 5: 382-386.

Bogduk N: Headache and the neck. In: Goadsby PJ, Silberstein SD (eds): Headache. Boston: Butterworth-Heinemann 1997: 369-381.

Fredriksen TA, Sjaastad O. Cervicogenic headache: current concepts of pathogenesis related to anatomical structure. Clin Exp Rheumatol 2000, 18 (2 Suppl 19): S16-18.

Göbel H, Edmeads JG. Disorders of the skull and cervical spine. In: Olesen J, Tfelt-Hansen P, Welch KMA (eds). The Headaches. 2nd edition. Philadelphia: Lippincott Williams \& Wilkins 2000: 891-898.

Knackstedt, H, Bansevicius, Dalius, Kjersti Aaseth, et al. Cervicogenic headache in the general population: The Akershus study of chronic headache. Cephalalgia 2010; 30: 1468-1476.

Lance JW, Anthony M. Neck-tongue syndrome on sudden turning of the head. J Neurol Neurosurg Psychiat 1980; 43: 97-101.

Leone M, D’Amico D, Grazzi L, Attanasia A, Bussone G: Cervicogenic headache: a critical review of the current diagnostic criteria. Pain 1998; 78: $1-5$.

Leone M, D'Amico D, Moschiano F, Farinotti M, Fillipini G, Bussone G: Possible identification of cervicogenic headache among patients with migraine: an analysis of 374 headaches. Headache 1995; 35: 461-464.

Lord S, Barnsley L, Wallis B, Bogduk N: Third occipital headache: a prevalence study. J Neurol Neurosurg Psychiat 1994; 57: 1187-1190.

Lord SM, Bogduk N: The cervical synovial joints as sources of post-traumatic headache. J Musculoskel Pain 1996; 4: 81-94.

Poughias L, Kruszewski P, Inan L. Cervicogenic headache: a clinical review with special emphasis on therapy. Funct Neurol 1997; 12: 305-317.

Sjaastad O, Fredriksen TA, Stolt-Nielsen A, Salvesen R, Jansen J, Pareja JA, Sjaastad O, Fredriksen TA. Cervicogenic headache: the importance of sticking to the criteria. Funct Neurol 2002; 17: 35-36.

Sjaastad O, Bakketeig LS. Prevalence of cervicogenic headache: Vågå study of headache epidemiology. Acta Neurol Scand 2008; 117: 173-180.

\subsubsection{Kopfschmerz zurückzuführen auf eine retropharyngeale} Tendinitis

Eastwood JD, Hudgins PA, Malone D. Retropharyngeal effusion in acute calcific prevertebral tendonitis: diagnosis with $\mathrm{CT}$ and MR imaging. Am J Neuroradiol 1998; 19: 1789-1792.

Ekbom K, Torhall J, Annell K, Traff J. Magnetic resonance imaging in retropharyngeal tendonitis. Cephalalgia 1994; 14: 266-269.

Pearce JM. Longus cervicis colli “myositis” (syn: retropharyngeal tendonitis). J Neurol Neurosurg Psychiat 1996; 61: 324-329.

Sarkozi J, Fam AG. Acute calcific retropharyngeal tendonitis: an unusual cause of neck pain. Arthritis Rheum 1984; 27: 708-710.

\subsubsection{Kopfschmerz zurückzuführen auf eine kraniozervikale Dystonie}

Csala B, Deuschl G. Craniocervical dystonia. Pragmatic general concept or nosologic entity? Nervenarzt 1994; 65: 75-94.

Friedman J, Standaert DG. Dystonia and its disorders. Neurol Clin 2001; 19: 681-705.

Göbel H, Deuschl G. Dauerkontraktionen kranialer oder zervikaler Muskeln. Münchener Medizinische Wochenschrift 1997; 139: 456-458.

Göbel $\mathrm{H}$, Heinze A, Heinze-Kuhn K, Austermann K. Botulinum toxin A in the treatment of headache syndromes and pericranial pain syndromes. Pain 2001; 91: 195-199.

\subsection{Kopfschmerz zurückzuführen auf Erkrankungen der Augen}

Akinci A, Güven A, Degerliyurt A, Kibar E, Mutlu M, Citirik M. The correlation between headache and refractive errors. J AAPOS 2008; 12: 290-293. 
Daroff RB. Ocular causes of headache. Headache 1998; 38: 661-667.

Daum KM, Good G, Tijerina L. Symptoms in video display terminal operators and the presence of small refractive errors. J Am Optom Assoc 1988; 59: 691-697.

Gerling J, Janknecht P, Kommerell G. Orbital pain in optic neuritis and anterior ischemic optic neuropathy. Neuro-Ophthalmology 1998; 19: 93-99.

Göbel H, Martin TJ. Ocular disorders. In: Olesen J, Tfelt-Hansen P, Welch KMA. The Headaches. 2nd edition. Philadelphia: Lippincott Williams \& Wilkins 2000: 899-904.

Gordon GE, Chronicle EP, Rolan P. Why do we still not know whether refractive error causes headaches? Towards a framework for evidence based practice. Ophthalmic Physiol Opt 2001;21: 45-50.

Lewis J, Fourman S. Subacute angle-closure glaucoma as a cause of headache in the presence of a white eye. Headache 1998; 38: 684-686.

McCluskey PJ, Lightman S, Watson PG et al. Posterior scleritis. Clinical features, systemic associations, and outcome in a large series of patients. Ophthalmology 1999; 106: 2380-2386.

Nesher R, Mimouni MD, Khoury S et al. Delayed diagnosis of subacute angle closure glaucoma in patients presenting with headaches. Acta Neurologica Belgica 2014; 114: 269-272.

Shindler KS, Sankar PS, Volpe N], Piltz-Seymour JR. Intermittent headaches as the presenting sign of subacute angle-closure glaucoma. Neurology 2005; 65: 757-758.

Smith JH, Garrity JA, Boes C]. Clinical features and long-term prognosis of trochlear headaches. Eur J Neurol 2014; 21: 577-585.

Tychsen L, Tse DT, Ossoinig K, Anderson RL. Trochleitis with superior oblique myositis. Ophthalmology 1984; 91: 1075-1079.

Yangüela J, Pareja JA, Lopez N, Sanchez del Rio M. Trochleitis and migraine headache. Neurology 2002; 58: 802-805.

Yangüela J, Sánchez del Rio M, Bueno A, et al. Primary trochlear headache. A new cephalgia generated and modulated on the trochlear region. Neurology 2004; 62: 1134-1140.

Zaragoza-Casares P, Gómez-Fernández T, Gómez de Liaño MA, Zaragoza-Garcia P. Bilateral idiopathic trochleitis as a cause of frontal cephalgia. Headache 2009; 49: 476-477.

\subsection{Kopfschmerz zurückzuführen auf Erkrankungen der Nase oder} Nasennebenhöhlen

Aaseth K, Grande RB, Kvaerner K, Lundqvist C, Russell MB. Chronic rhinosinusitis gives a ninefold increased risk of chronic headache. The Akershus study of chronic headache. Cephalalgia 2010; 30: 152-160.

Abu-Bakra M, Jones NS. Prevalence of nasal mucosal contact points in patients with facial pain compared with patients without facial pain. Laryngol Otol 2001; 115: 629-632.

Blumenthal HJ. Headache and sinus disease. Headache 2001; 41: 883-888.

Cady RK, Dodick DW, Levine HL, et al. Sinus headache: A neurology, otolaryngology, allergy and primary care consensus on diagnosis and treatment. Mayo Clin Proc 2005; 80: 908-916.

Close LG, Aviv J. Headaches and disease of the nose and paranasal sinuses. Semin Neurol 1997; 17: 351-354.

Göbel H, Baloh RW. Disorders of ear, nose, and sinus. In: Olesen J, Tfelt-Hansen P, Welch KMA. The Headaches. 2nd edition. Philadelphia: Lippincott Williams \& Wilkins 2000: 905-912.

Kenny TJ, Duncavage J, Bracikowski J, Yildirim A, Murray J], Tanner SB. Prospective analysis of sinus symptoms and correlation with paranasal computed tomography scan. Otolaryngol Head Neck Surg 2001; 125 : 40-43.

Lam DK, Lawrence HP, Tenenbaum HC. Aural symptoms in temporomandibular disorder patients attending a craniofacial pain unit. J Orofac Pain 2001; 15: 146-157.

Lanza DC, Kennedy DW. Adult rhinosinusitis defined. Report of the Rhinosinusitis Task Force Committee of the American Academy of Otolaryngology Head and Neck Surgery. Otolaryngol Head Neck Surg 1997; 117: S1-S7.
Levine HL. Patients with headache and visual disturbance: a differentiation between migraine and sinus headache. Arch Otolaryngol Head Neck Surg 2000: 126: 234-235.

Pinto A, De Rossi SS, McQuone S, Sollecito TP. Nasal mucosal headache presenting as orofacial pain: a review of the literature and a case report. Oral Surg Oral Med Oral Pathol Oral Radiol Endod 2001; 92: 180-183.

Seiden AM, Martin VT. Headache and the frontal sinus. Otolaryngol Clin North Am 2001; 34: 227-241.

Tosun F, Gerek M, Ozkaptan Y. Nasal surgery for contact point headaches. Headache 2000; 40: 237-240.

West B, Jones NS. Endoscopy-negative, computed tomography-negative facial pain in a nasal clinic. Laryngoscope 2001; 111 (4 Pt 1): 581-586.

Yoon MS, Mueller D, Hansen $\mathrm{N}$ et al. Prevalence of facial pain in migraine: a population-based study. Cephalalgia 2009; 30: 92-96.

11.6 Kopfschmerz zurückzuführen auf Erkrankungen der Zähne

Alonso AA, Nixdorf DR. Case series of four different headache types presenting as tooth pain. J Endodontics 2006; 32: 1110-1113.

Fabri GM, Siqueira SR, Simione C, Nasri C, Teixeira MJ, Siqueira JT. Refractory craniofacial pain: Is there a role of periodontal disease as a comorbidity? Arq Neuro-psiquiatria 2009; 67(2B): 474-479.

Murphy E, Merrill RL. Non-odontogenic toothache. J Ir Dent Assoc 2001; 47: $46-58$.

\subsection{Kopfschmerz zurückzuführen auf Erkrankungen des Kiefer-} gelenkes

Ballegaard V, Thede-Schmidt-Hansen P, Svensson P et al. Are headache and temporomandibular disorders related? A blinded study. Cephalalgia 2008; 28: 832-841.

Egermark I, Carlsson GE, Magnusson T. A 20-year longitudinal study of subjective symptoms of temporomandibular disorders from childhood to adulthood. Acta Odontol Scand 2001; 59: 40-48.

Franco AL, Goncalves DA, Castanharo SM et al. Migraine is the most prevalent primary headache in individuals with temporomandibular disorders. J Orofacial Pain 2010; 24: 287-292.

Glaros AG, Urban D, Locke J. Headache and temporomandibular disorders: evidence for diagnostic and behavioural overlap. Cephalalgia 2007; 27: 542-549.

List T, Wahlund K, Larsson B. Psychosocial functioning and dental factors in adolescents with temporomandibular disorders: a case-control study. J Orofacial Pain 2001; 15: 218-227.

Magnusson T, Egermark I, Carlsson GE. A longitudinal epidemiologic study of signs and symptoms of temporomandibular disorders from 15 to 35 years of age. J Orofacial Pain 2000; 14: 310-319.

Molina OF, dos Santos Junior J, Nelson SJ, Nowlin T. Profile of TMD and bruxer compared to TMD and nonbruxer patients regarding chief complaint, previous consultations, modes of therapy, and chronicity. Cranio 2000; 18: 205-219.

Ogus $\mathrm{H}$. Degenerative disease of the temporomandibular joint and pain-dysfunction syndrome. J Roy Soc Med 1978; 71: 748-754.

Schiffman ES, Ohrbach R, List T et al. Diagnostic criteria for Kopfschmerz zurückzuführen auf Erkrankungen des Kiefergelenkess (TMD). Cephalalgia 2012; 32: 683-692.

Schiffman E, Ohrbach R, Truelove E et al. Diagnostic criteria for temporomandibular disorders (DC/TMD) for clinical and research applications: Recommendations of the International RDC/TMD Consortium Network and Orofacial Pain Special Interest Group. J Orofacial Pain 2014; 28 : $6-27$.

11.8 Kopf- oder Gesichtsschmerz zurückzuführen auf Entzündungen des Ligamentum stylohyoideum

Colby CC, Del Gaudio JM. Stylohyoid complex syndrome: a new diagnostic classification. Arch Otolaryngol Head Neck Surg 2011; 137: 248-252. 


\section{Kopfschmerz zurückzuführen auf psychiatrische Störungen}

12.1 Kopfschmerz zurückzuführen auf eine Somatisierungsstörung

12.2 Kopfschmerz zurückzuführen auf eine psychotische Störung

An anderer Stelle kodiert: Kopfschmerz zurückzuführen auf psychische Störungen durch Substanzgebrauch (z. B. Abhängigkeit), Kopfschmerz bei Entzug einer Substanz, Kopfschmerzen bei akuter Intoxikation durch eine Substanz und Kopfschmerz bei Medikamentenübergebrauch werden als Subtypen von 8. Kopfschmerz zurückzuführen auf eine Substanz oder deren Entzug kodiert.

\section{Allgemeiner Kommentar}

Primärer und/oder sekundärer Kopfschmerz?

Sowohl Kopfschmerzen als auch psychiatrische Erkrankungen sind häufig, so dass eine Komorbidität auch rein zufällig sein kann. Trotzdem kann eine kausale Beziehung zwischen einem neuen oder deutlich verschlechterten Kopfschmerz und einer psychiatrischen Erkrankung bestehen. Für Kapitel 12. Kopfschmerz zurückzuführen auf psychiatrische Störungen gelten mit einigen Anpassungen die allgemeinen Regeln für die kausale Zuordnung zu einer anderen Erkrankung.

1. Tritt ein neuer Kopfschmerz erstmals in engem zeitlichen Zusammenhang zu einer psychiatrischen Störung auf und ein kausaler Zusammenhang wird bestätigt, dann wird der Kopfschmerz als sekundärer Kopfschmerz zurückzuführen auf diese psychiatrische Störung kodiert. Dies ist auch der Fall, wenn der Kopfschmerz das klinische Bild eines in Teil 1 der ICHD-3 kodierten primären Kopfschmerzes aufweist.

2. Wenn sich aber ein vorbestehender primärer Kopfschmerz in engem zeitlichen Zusammenhang mit einer psychiatrischen Störung deutlich verschlechtert (üblicherweise definiert als eine mindestens zweifache Steigerung der Häufigkeit oder Schwere) und ein kausaler Zusammenhang bestätigt wird, dann sollten sowohl die ursprüngliche primäre Kopfschmerzdiagnose als auch 12. Kopfschmerz zurückzuführen auf psychiatrische Störungen (oder eines Subtyps) vergeben werden, sofern gute Hinweise bestehen, dass diese psychiatrische Störung Kopfschmerzen verursachen kann.

3. Wenn in einem dieser Fälle ein kausaler Zusammenhang nicht bestätigt werden kann, dann sollten der vorbestehende primäre Kopfschmerz und die psychiatrische Störung getrennt kodiert werden.

Chronische Kopfschmerzen zurückzuführen auf eine psychiatrische Störung, die nach deren Ende persistieren, sind bislang nicht beschrieben.

\section{Einleitung}

Insgesamt existieren nur begrenzte Hinweise auf psychiatrische Ursachen von Kopfschmerzen. Die diagnostischen Kategorien in dieser Klassifikation sind daher den seltenen Fällen vorbehalten, in denen Kopfschmerzen im Kontext und als direkte Folge einer psy- chiatrischen Erkrankung auftreten, von der bekannt ist, dass sie sich mit Kopfschmerzen manifestieren kann.

Die diagnostischen Kriterien müssen restriktiv genug sein, um keine falsch positiven Fälle einzuschließen, aber gleichzeitig muss die Schwelle niedrig genug sein, um die Mehrzahl der betroffenen Patienten einzuschließen. In der überwiegenden Zahl der Fälle mit 12. Kopfschmerz zurückzuführen auf psychiatrische Störungen basiert die Diagnose auf der persönlichen Bewertung der Krankengeschichte und der körperlichen Untersuchung und nicht auf objektivierbaren diagnostischen Biomarkern.

Kopfschmerzen können auch in Assoziation mit psychiatrischen Störungen auftreten, ohne dass ein kausaler Zusammenhang besteht. Kopfschmerzen können zufällig gleichzeitig mit einer Vielzahl psychiatrischer Störungen bestehen, einschließlich Depressionen (depressive Episode, rezidivierende depressive Störung oder Dysthymie), Angststörungen (Trennungsangst, Panikstörung, soziale Phobie oder generalisierte Angststörung) und Trauma- oder Stress-assoziierte Störungen (reaktive Bindungsstörung, akute Belastungsreaktion, posttraumatische Belastungsstörung und Anpassungsstörung). In diesen Fällen sollte sowohl die Diagnose der primären Kopfschmerzerkrankung als auch die begleitende psychiatrische Diagnose gestellt werden.

Epidemiologische Daten zeigen allerdings, dass Kopfschmerzen und psychiatrische Störungen häufiger komorbid auftreten als rein zufällig zu erwarten wäre. Gemeinsame zugrundeliegende Faktoren können beide Erkrankungen verursachen oder zu ihnen prädisponieren; andererseits können konfundierende Faktoren auch zu einer Überschätzung der Komorbidität beitragen (zum Beispiel könnten Patienten mit einer der beiden Diagnosen häufiger auch noch die andere erhalten, einfach weil sie sich unter engmaschigerer medizinischer Beobachtung befinden). Echte kausale Beziehungen sind auch möglich, wobei der Kopfschmerz die psychiatrische Störung verursachen kann, die psychiatrische Störung den Kopfschmerz verursachen kann, oder es besteht eine wechselseitige (bidirektionale) Beeinflussung zwischen dem Kopfschmerz und der psychiatrischen Störung.

Die klinische Erfahrung nahe legt nahe, dass Kopfschmerzen, die ausschließlich während verbreiteter psychiatrischer Störungen wie Depressionen, Angststörungen oder Trauma- oder Stress-assoziierten Störungen auftreten, in einigen Fällen auf diese psychiatrischen Störungen zurückgeführt werden können. Wegen des unsicheren Kausalzusammenhangs und des Mangels an wissenschaftlichen Ergebnissen zu diesem Thema sind Kriterien für Kopfschmerzen zurückzuführen auf diese und alle anderen bis auf zwei psychiatrische Störungen im Anhang verblieben. Weitere Untersuchungen zu möglichen kausalen Zusammenhängen auf diesem Gebiet sind notwendig.

Es bestehen Hinweise, dass eine psychiatrische Störung den Verlauf einer 1. Migräne und eines 2. Kopfschmerz vom Spannungstyp verschlimmern kann, indem sie die Häufigkeit und die Intensität der Kopfschmerzen erhöht. Auch sprechen die Kopfschmerzen dann häufig schlechter auf die Behandlung an. Somit ist das Erkennen und Behandeln jeder begleitend auftretenden psychiatrischen Störung wichtig für eine erfolgreiche Behandlung von Kopfschmerzen. Bei Kindern und Jugendlichen treten primäre Kopfschmerzen (1. Migräne, 2.2 häufige episodische Kopfschmerzen vom Spannungstyp und vor allem 2.3 chronische Kopfschmerzen vom Spannungstyp) häufig komorbid mit psychiatrischen Störungen auf. Das Vorhan- 
densein von Schlafstörungen, posttraumatischen Belastungsstörungen (PTBS), sozialen Phobien, Schulphobien, dem Aufmerksamkeitsdefizit-/Hyperaktivitätssyndrom, Verhaltensstörungen, Lernstörungen, Enuresis, Enkopresis und Tics sollte sorgfältig beurteilt und die Störung behandelt werden. Zu berücksichtigen sind dabei die mit solchen Erkrankungen einhergehenden Beeinträchtigungen im Alltag und die negativen Auswirkungen auf die Prognose der kindlichen Kopfschmerzen.

Um zu klären, ob ein Kopfschmerz tatsächlich auf eine psychiatrische Störung zurückzuführen ist, muss zuerst die Frage geklärt werden, ob überhaupt eine psychiatrische Störung als Komorbidität vorliegt oder nicht. Es wird empfohlen, alle Kopfschmerzpatienten nach typischen psychiatrischen Symptomen zu fragen, wie sie bei Angststörungen und Depressionen vorkommen. Wenn der Verdacht auf eine psychiatrische Ursache der Kopfschmerzen besteht, dann sollte eine Evaluation durch einen Psychiater oder Psychologen erfolgen.

\subsection{Kopfschmerz zurückzuführen auf eine Somatisierungsstörung}

Beschreibung:

Der Kopfschmerz tritt als Symptom einer Somatisierungsstörung auf.

\section{Diagnostische Kriterien:}

A. Kopfschmerz, der das Kriterium C erfüllt

B. Es wurde eine Somatisierungsstörung1 diagnostiziert, die beide der folgenden Kriterien erfüllt:

1. Es besteht eine Vorgeschichte mit multiplen körperlichen Symptomen mit Beginn vor dem 30. Lebensjahr. Die körperlichen Symptome sind trotz geeigneter Untersuchungen nicht oder nicht vollständig durch eine bekannte körperliche Erkrankung erklärt. Falls eine körperliche Erkrankung bestand, überschreiten die Symptome das aus der Anamnese, körperlichen Untersuchung und Laboruntersuchungen zu erwartende Ausmaß.

2. Im Verlauf der Erkrankung treffen alle der folgenden Kriterien zu:

a) Vier Schmerzsymptome: eine Vorgeschichte von Schmerzsymptomen, die mindestens vier verschiedene Körperbereiche oder Funktionen betreffen (z. B. Kopf, Brust, Rücken, Abdomen, Gelenke, Extremitäten und/ oder Rektum; während der Menstruation, während des Geschlechtsverkehrs und/oder beim Wasserlassen)

b) Zwei gastrointestinale Symptome: eine Vorgeschichte von mindestens zwei gastrointestinalen Symptomen außer Schmerzen (z. B. Übelkeit, Völlegefühl, Erbrechen außer während einer Schwangerschaft, Durchfall, Unverträglichkeit von verschiedenen Speisen)

c) Ein sexuelles Symptom: eine Vorgeschichte von mindestens einem Symptom im Bereich Sexualität oder Fortpflanzung außer Schmerzen (z. B. sexuelle Gleichgültigkeit, Erektions- oder Ejakulationsstörungen, unregelmäßige Menstruationen, sehr starke Menstruationsblutungen, Erbrechen während der gesamten Schwangerschaft) d) Ein pseudoneurologisches Symptom: eine Vorgeschichte von mindestens einem Symptom oder Defizit (nicht begrenzt auf Schmerz), das einen neurologischen Krankheitsfaktor nahe legt (Konversionssymptome wie z. B. Koordinations- oder Gleichgewichtsstörungen, Lähmungen oder lokalisierte Muskelschwäche, Schluckschwierigkeiten oder Kloßgefühl im Hals, Aphonie, Harnverhaltung, Halluzinationen, Verlust der Berührungs- oder Schmerzempfindung, Sehen von Doppelbildern, Blindheit, Taubheit, Anfälle; dissoziative Symptome wie z. B. Amnesie oder Bewusstseinsverluste, jedoch nicht einfache Ohnmacht)

C. Ein kausaler Zusammenhang kann durch mindestens eines der folgenden Kriterien gezeigt werden:

1. Der Kopfschmerz hat sich parallel zur Entwicklung anderer auf die Somatisierungsstörung zurückzuführender somatischer Symptome entwickelt oder deutlich verstärkt

2. Der Kopfschmerz fluktuiert gleichzeitig mit anderen auf die Somatisierungsstörung zurückzuführenden somatischen Symptomen

3. Der Kopfschmerz ist gleichzeitig mit anderen auf die Somatisierungsstörung zurückzuführenden somatischen Symptomen verschwunden

D. Nicht besser erklärt durch eine andere ICHD-3-Diagnose.

\section{Anmerkung:}

Angemerkt sei, dass die Somatisierungsstörung in dieser Form nicht mehr in der fünften Auflage des Diagnostic and Statistical Manual of Mental Disorders (DSM-5), der letzten Revision des diagnostischen Manuals der Amerikanischen Psychiatrischen Gesellschaft von 2013 vorkommt, sie wurde durch die Kategorie Somatic Symptom Disorder (Somatische Belastungsstörung) ersetzt. Diese ist charakterisiert durch ein oder mehrere somatische Symptome, mit übersteigerten und anhaltenden Gedanken, die um die ernste Bedeutung dieser Symptome für die Gesundheit kreisen, einer anhaltend hohen Ängstlichkeit in Bezug auf die eigene Gesundheit und die beschriebenen Symptome, und/oder einem übersteigerten Maß an Zeit und Energie, das diesen Symptomen und Gesundheitssorgen gewidmet wird. Aufgrund der enormen Heterogenität dieser Kategorie (z. B. schließt sie sowohl Patienten mit Kopfschmerzen und übersteigerter Sorge bezüglich der Bedeutung ihrer Kopfschmerzen ein als auch klassische Fälle einer Somatisierungsstörung mit einem Muster hartnäckig fortbestehender multipler somatischer Symptome, darunter auch Kopfschmerz) wurde beschlossen, dass ein Zusammenhang nur dann behauptet werden kann, wenn der Kopfschmerz Teil eines umfassenderen Musters aus multiplen somatischen Beschwerden ist. Daher bezieht sich die vorliegende Klassifikation ICHD-3 weiterhin auf die Definition der Somatisierungsstörung aus dem DSM-IV.

\section{Kommentar:}

Somatisierungsstörungen sind charakterisiert durch eine Kombination vieler beeinträchtigender Symptome und eine überschieBende oder maladaptive Antwort auf diese Symptome oder damit zusammenhängende Gesundheitsbedenken. Zu den möglichen Symptomen gehören gastrointestinale Probleme, Rückenschmerzen, Schmerzen in den Extremitäten oder Gelenken, Kopfschmer- 
zen, Thoraxschmerzen und/oder Dyspnoe, Schwindel, Müdigkeit und Kraftlosigkeit sowie Schlafstörungen. Das Leiden des Patienten ist real, unabhängig davon, ob eine somatische Erklärung gefunden wird. Patienten mit Somatisierungsstörung leiden unter Distress und hoher funktioneller Beeinträchtigung. Die Symptome können, müssen aber nicht begleitend zu diagnostizierten somatischen oder anderen psychiatrischen Erkrankungen auftreten. Oft besteht eine hohe Inanspruchnahme medizinischer Versorgung, meist ohne deutliche Besserung der Beschwerden. Aus Sicht des Klinikers erscheinen viele dieser Patienten als therapierefraktär, und neue Therapieversuche und Interventionen führen oft nur zu einer Verschlechterung der Symptome oder zu unerwünschten Wirkungen und Komplikationen. Ein Teil der Patienten empfindet seine bisherige medizinische Diagnostik und Behandlung als unzureichend.

\subsection{Kopfschmerz zurückzuführen auf eine psychotische Störung}

\section{Beschreibung:}

Kopfschmerz als Ausdruck einer wahnhaften Überzeugung, deren Inhalt für den Patienten die Kopfschmerzen erklärt (z. B. der Kopfschmerz ist verursacht durch ein Gerät, das ihm von Außerirdischen in den Kopf implantiert wurde).

\section{Diagnostische Kriterien:}

A. Kopfschmerz, der das Kriterium C erfüllt

B. Wahnhafte Überzeugung, die einen den Kopfschmerz erklärenden Mechanismus beinhaltet1

C. Ein kausaler Zusammenhang kann durch eines oder beide der folgenden Kriterien gezeigt werden:

1. der Kopfschmerz hat sich mit oder nach dem Beginn der wahnhaften Überzeugung entwickelt oder zu ihrer Diagnose geführt

2. der Kopfschmerz ist nach Ende der wahnhaften Überzeugung verschwunden

D. Nicht besser erklärt durch eine andere ICHD-3-Diagnose2.

\section{Anmerkung:}

1. Zum Beispiel: der Patient glaubt, dass ihm ein Gerät in den Kopf implantiert wurde, das die Kopfschmerzen verursacht, oder dass er einen für die Kopfschmerzen verantwortlichen Hirntumor hat, obwohl dies sicher ausgeschlossen wurde.

2. Wenn ein Patient zunächst einen Kopfschmerz (z. B. eine primäre Kopfschmerzerkrankung wie in Teil 1 der ICHD-3 klassifiziert) und danach eine wahnhafte Erklärung für den Kopfschmerz entwickelt, wie die Überzeugung, dass der Kopfschmerz auf einen Hirntumor zurückzuführen ist, obwohl dafür kein medizinischer Anhalt besteht, dann soll der Kopfschmerz nicht auf die psychiatrische Störung zurückgeführt, sondern als primäre Kopfschmerzerkrankung kodiert werden, und der Patient soll die zusätzliche psychiatrische Diagnose einer Wahnstörung, somatischer Typ erhalten.

\section{Kommentar:}

Wahnhafte Überzeugungen sind unkorrigierbare falsche Überzeugungen, die auf einer falschen Beurteilung der Realität beruhen und an denen trotz Beweis des Gegenteils festgehalten wird. Sie können die Überzeugung einschließen, dass eine ernsthafte $\mathrm{Er}$ krankung vorliegt, die den Kopfschmerz verursacht (z. B. ein Hirntumor oder ein Aneurysma), trotz wiederholter und begründeter Versicherung, dass eine solche Erkrankung nicht besteht. In anderen Fällen ist der Inhalt des Wahnes bizarrer, z. B. dass ein Sender operativ in den Kopf implantiert worden sei und dass dieser Sender der Grund für die Kopfschmerzen sei.

\section{Literatur}

Allet JL, Allet RE. Somatoform disorders in neurological practice. Curr Opin Psychiatry 2006; 19: 413-420.

Borkum JM. Chronic headaches and the neurobiology of somatization. Curr Pain Headache Rep 2010; 14: 55-61.

Canestri P, Galli F, Guidetti V, Tomaciello A. Chronic daily headache in children and adolescents: a two years follow-up. Cephalalgia 2001; 21: 288.

Curioso EP, Young WB, Shecter AL, Kaiser R. Psychiatric comorbidity predicts outcome in chronic daily headache patients. Neurology 1999; 52 (Suppl 2): A471.

Gambini O, Islam L, Demartini B, Scarone S. Psychiatric issues in patients with headaches. Neurol Sci 2010; 31 Suppl 1: S111-S113.

Guidetti V, Galli F, Fabrizi P, Napoli L, Giannantoni AS, Bruni O, Trillo S. Headache and psychiatric comorbidity: clinical aspects and outcome in an 8-year follow-up study. Cephalalgia 1998; 18: 455-462.

Hung Cl, Liu CY, Cheng YT, Wang SJ. Migraine: a missing link between somatic symptoms and major depressive disorder. J Affect Disord 2009; 117(1-2): 108-115.

Lake A. Behavioral and nonpharmacologic treatments of headache. Med Clin North Am 2001; 85: 1055-1075.

Lake AE 3 rd, Rains JC, Penzien DB, Lipchik, GL. Headache and psychiatric comorbidity: historical context, research relevance, and clinical implications. Headache 2005; 45: 493-506.

Maizels M, Burchette R. Somatic symptoms in headache patients: the influence of headache diagnosis, frequency, and comorbidity. Headache 2004; 44: 983-993.

Marazzitti D, Toni C, Pedri S, Bonucelli $U$ et al. Prevalence of headache syndromes in panic disorder. Int Clin Psychopharmacol 1999; 14: 247-251.

Mitsikostas DD, Thomas AM. Comorbidity of headache and depressive disorders. Cephalalgia 1999; 19: 211-217.

Nicholson RA. Chronic headache: the role of the psychologist. Curr Pain Headache Rep 2010; 14: 47-54.

Pakalnis A, Greenberg G, Drake ME, Paolich J. Pediatric migraine prophylaxis with divalproex. J Child Neurol 2001; 16: 731-734.

Radat F, Psychopathology and headache. Rev Neurol 2000; 156 Suppl 4: 4S62-67.

Radat F, Milowska D, Valade D. Headaches secondary to psychiatric disorders (HSPD): a retrospective study of 87 patients. Headache. 2011; 51 : 789-795.

Radat F, Sakh D, Lutz G, el Amrani M, Ferreri M, Bousser MG. Psychiatric comorbidity is related to headache induced by chronic substance use in migraineurs. Headache 1999; 39: 477-480.

Radat F, Swendsen J. Psychiatric comorbidity in migraine: a review. Cephalalgia 2005; 25: 165-178.

Smitherman TA, Baskin SM. Headache secondary to psychiatric disorders. Curr Pain Headache Rep 2008; 12: 305-310.

Voigt K, Nagel A, Meyer B, Langs G, Braukhaus C, Löwe B. Towards positive diagnostic criteria: a systematic review of somatoform disorder diagnoses and suggestions for future classification. J Psychosom Res 2010; 68: 403-414.

Yutzy S. Somatoform disorders. In: Tasman A, Kay J, Lieberman JA (eds). Psychiatry, 2nd ed. Chichester: John Wiley and Sons 2003: 1419-1420. 


\section{TEIL DREI}

\section{Kranielle Neuralgien und andere Gesichts- und Kopfschmerzen}

13. Schmerzhafte Läsionen der Hirnnerven und andere Gesichtsschmerzen

14. Andere Kopfschmerzerkrankungen

\section{Schmerzhafte Läsionen der Hirnnerven und andere Gesichtsschmerzen}

\begin{tabular}{|c|c|}
\hline 13.1 & $\begin{array}{l}\text { Schmerzen zurückzuführen auf eine Läsion oder } \\
\text { Erkrankung des N. trigeminus }\end{array}$ \\
\hline 13.1.1 & Trigeminusneuralgie \\
\hline 13.1.1.1 & Klassische Trigeminusneuralgie \\
\hline 13.1.1.1.1 & Klassische Trigeminusneuralgie, rein paroxysmal \\
\hline 13.1.1.1.2 & Klassische Trigeminusneuralgie mit Dauerschmerz \\
\hline 13.1.1.2 & Sekundäre Trigeminusneuralgie \\
\hline 13.1.1.2.1 & Trigeminusneuralgie zurückzuführen auf Multiple Sklerose \\
\hline 13.1.1.2.2 & $\begin{array}{l}\text { Trigeminusneuralgie zurückzuführen auf eine raum- } \\
\text { fordernde Läsion }\end{array}$ \\
\hline 13.1.1.2.3 & $\begin{array}{l}\text { Trigeminusneuralgie zurückzuführen auf eine andere } \\
\text { Ursache }\end{array}$ \\
\hline 13.1.1.3 & Idiopathische Trigeminusneuralgie \\
\hline 13.1.1.3.1 & Idiopathische Trigeminusneuralgie, rein paroxysmal \\
\hline 13.1.1.3.2 & Idiopathische Trigeminusneuralgie mit Dauerschmerz \\
\hline 13.1 .2 & Schmerzhafte Trigeminusneuropathie \\
\hline 13.1.2.1 & $\begin{array}{l}\text { Schmerzhafte Trigeminusneuropathie zurückzuführen auf } \\
\text { Herpes zoster }\end{array}$ \\
\hline 13.1.2.2 & Post-herpetische Trigeminusneuralgie \\
\hline 13.1.2.3 & Schmerzhafte posttraumatische Trigeminusneuropathie \\
\hline 13.1.2.4 & $\begin{array}{l}\text { Schmerzhafte Trigeminusneuropathie zurückzuführen auf } \\
\text { eine andere Erkrankung }\end{array}$ \\
\hline 13.1.2.5 & Idiopathische schmerzhafte Trigeminusneuropathie \\
\hline 13.2 & $\begin{array}{l}\text { Schmerz zurückzuführen auf eine Läsion oder Erkrankung } \\
\text { des N. glossopharyngeus }\end{array}$ \\
\hline 13.2 .1 & Glossopharyngeusneuralgie \\
\hline 13.2.1.1 & Klassische Glossopharyngeusneuralgie \\
\hline 13.2.1.2 & Sekundäre Glossopharyngeusneuralgie \\
\hline 13.2.1.3 & Idiopathische Glossopharyngeusneuralgie \\
\hline 13.2 .2 & Schmerzhafte Glossopharyngeusneuropathie \\
\hline 13.2.2.1 & $\begin{array}{l}\text { Schmerzhafte Glossopharyngeusneuropathie zurückzu- } \\
\text { führen auf eine bekannte Ursache }\end{array}$ \\
\hline 13.2.2.2 & $\begin{array}{l}\text { Idiopathische schmerzhafte Glossopharyngeusneuro- } \\
\text { pathie }\end{array}$ \\
\hline 13.3 & $\begin{array}{l}\text { Schmerz zurückzuführen auf eine Läsion oder Erkrankung } \\
\text { des N. intermedius }\end{array}$ \\
\hline 13.3 .1 & Intermediusneuralgie \\
\hline 13.3.1.1 & Klassische Intermediusneuralgie \\
\hline 13.3.1.2 & Sekundäre Intermediusneuralgie \\
\hline 13.3.1.3 & Idiopathische Intermediusneuralgie \\
\hline 13.3.2 & Schmerzhafte Intermediusneuropathie \\
\hline
\end{tabular}

\begin{tabular}{|c|c|}
\hline 13.3.2.1 & $\begin{array}{l}\text { Schmerzhafte Intermediusneuropathie zurückzuführen } \\
\text { auf einen Herpes zoster }\end{array}$ \\
\hline 13.3.2.2 & Postherpetische Intermediusneuralgie \\
\hline 13.3.2.3 & $\begin{array}{l}\text { Schmerzhafte Intermediusneuropathie zurückzuführen } \\
\text { auf eine andere Erkrankung }\end{array}$ \\
\hline 13.3.2.4 & Idiopathische schmerzhafte Intermediusneuropathie \\
\hline 13.4 & Okzipitalisneuralgie \\
\hline 13.5 & Nacken-Zungen-Syndrom \\
\hline 13.6 & Schmerzhafte Optikusneuritis \\
\hline 13.7 & $\begin{array}{l}\text { Kopfschmerz zurückzuführen auf eine ischämische } \\
\text { Lähmung des N. oculomotorius }\end{array}$ \\
\hline 13.8 & Tolosa-Hunt-Syndrom \\
\hline 13.9 & Paratrigeminales okulosympathisches (Raeder-) Syndrom \\
\hline 13.10 & $\begin{array}{l}\text { Rezidivierende schmerzhafte ophthalmoplegische Neuro- } \\
\text { pathie }\end{array}$ \\
\hline 13.11 & Syndrom des brennenden Mundes (BMS) \\
\hline 13.12 & Anhaltender idiopathischer Gesichtsschmerz (PIFP) \\
\hline 13.13 & Zentraler neuropathischer Schmerz \\
\hline 13.13 .1 & $\begin{array}{l}\text { Zentraler neuropathischer Schmerz zurückzuführen auf } \\
\text { Multiple Sklerose (MS) }\end{array}$ \\
\hline 13.13 .2 & $\begin{array}{l}\text { Zentraler neuropathischer Schmerz nach Hirninfarkt } \\
\text { (CPSP) }\end{array}$ \\
\hline
\end{tabular}

\section{Einleitung}

Das vorliegende Kapitel legt ein Klassifizierungssystem für schmerzhafte Läsionen der Hirnnerven und andere Gesichtsschmerzen dar basierend auf einem Konsensus zwischen der Internationalen Kopfschmerzgesellschaft (IHS) und der International Association for the Study of Pain (IASP).

Die bestehende Nosologie von Schmerzen im Bereich der Hirnnerven wird den feinen Unterschieden zwischen unterschiedlichen Krankheitsbildern nicht ganz gerecht. Statt viele bewährte diagnostische Begriffe fallenzulassen, behält diese Klassifikation sie bei und bietet detaillierte Definitionen für die Differenzialdiagnose sowie zu ihren Typen und Subtypen.

Afferente Fasern in den Nn. trigeminus, intermedius, glossopharyngeus und vagus übermitteln, zusätzlich zu den oberen zervikalen Nervenwurzeln der Nn. occipitales, nozizeptive Signale in die zentralen Bahnen innerhalb des Hirnstamms sowie zu den Hirnregionen, die nozizeptive Signale und Schmerzen im Kopf und Nacken verarbeiten. Das Gehirn nimmt Schmerz im entsprechenden Versorgungsgebiet wahr.

Der Schmerz kann sich in jeder der vielen klar umrissenen Formen manifestieren, die nach gängiger Auffassung Unterschiede in der neuronalen Pathophysiologie spiegeln, obwohl die Einzelheiten hierzu nicht hinreichend bekannt sind. Bekannt ist jedoch, dass sich neuropathische Gesichtsschmerzen auf der Basis ihrer klar unterscheidbaren klinischen Erscheinungsbilder und Ätiologie klassifizieren lassen. Zentral für dieses Konzept ist die initiale klinische Bestimmung der wesentlichen diagnostischen Gruppe von Schmerzen, in die sich die Schmerzen des Patienten am besten einordnen lassen, gefolgt von ätiologischen Ermittlungen im Sinne der Diagnosetypen und Subtypen sowie der therapeutischen Entscheidungsfindung.

Es gibt mehrere verschiedene Klassifizierungsachsen. 
a) Syndromologie: Neuralgie oder Neuropathie

Die Unterscheidung etwa zwischen einer Trigeminusneuralgie und einer Trigeminusneuropathie sollte als pragmatischer Weg betrachtet werden, zwischen Erkrankungen zu differenzieren, bei denen das klinische Erscheinungsbild und die Behandlungsansätze unterschiedlich sind, die beiden Erkrankungen jedoch auf der Basis der derzeit bekannten Pathologie oder Pathophysiologie nicht klassifiziert werden können. Das Gleiche gilt für Schmerzzustände in Verbindung mit dem N. glossopharyngeus und dem N. intermedius.

Eine wichtige Ursache von Schmerzen im Bereich der Hirnnerven ist ein Herpes zoster. Trotz der Tatsache, dass trigeminale Schmerzen nach Herpes zoster wahrscheinlich zu verschiedenen Typen von pathologischen Veränderungen in den Trigeminalbahnen führen (d. h., einem „leicht erregbaren Nozizeptor“ gegenüber einem „Deafferentierungs“-Typ), sind die verfügbaren Daten zu begrenzt, um diese als eine Neuralgie in Abgrenzung von einer Neuropathie zu klassifizieren. Deshalb wird der gut eingeführte Begriff postherpetische Neuralgie beibehalten.

b) Lokalisation: zentraler oder peripherer neuropathischer

Schmerz

Eine Läsion oder übermäßige Aktivierung dieser Nerven (peripherer neuropathischer Schmerz) oder ihrer zentralen Bahnen (zentraler neuropathischer Schmerz) verursacht neuropathische Schmerzen im Gesicht.

c) Ätiologie: klassisch, idiopathisch oder sekundär

Die Ursache eines neuropathischen Schmerzes kann klar sein, etwa eine Infektion mit einem Varicella-zoster-Virus oder eine strukturelle Anormalie (d. h. Plaques bei Multipler Sklerose), nachgewiesen durch die Bildgebung: ein derartiger Schmerz wird als sekundär bezeichnet und angegeben, auf welche Ursache er zurückzuführen ist. In anderen Fällen ist keine offensichtliche Ursache erkennbar (als idiopathischer Schmerz bezeichnet).

Für die Trigeminus-, Glossopharyngeus- und Intermedius-Neuralgie ist die Bezeichnung klassisch Fällen vorbehalten, in denen die Bildgebung oder ein operativer Eingriff eine vaskuläre Kompression des entsprechenden Nervs gezeigt haben. Streng genommen sind klassische Neuralgien sekundär (Folge der neurovaskulären Kompression), aufgrund der umfassenderen therapeutischen Möglichkeiten und potenzieller nervenpathophysiologischer Unterschiede ist es jedoch von Vorteil, sie von anderen Ursachen zu trennen.

\subsection{Schmerzen zurückzuführen auf eine Läsion oder Erkrankung des} N. trigeminus

\subsubsection{Trigeminusneuralgie}

Beschreibung:

Die Trigeminusneuralgie ist ein wiederkehrender einseitiger Gesichtsschmerz, der durch kurze, stromstoßartige Schmerzattacken gekennzeichnet ist, die auf das Versorgungsgebiet eines einzelnen Astes oder mehrerer Äste des N. trigeminus beschränkt sind. Der Schmerz wird gewöhnlich durch harmlose Reize ausgelöst. Er kann sich ohne offenkundige Ursache entwickeln oder Folge einer anderen diagnostizierten Störung sein. Zusätzlich kann er in Begleitung eines Dauerschmerzes von mittelstarker Intensität im/in den
Versorgungsbereich(en) des betroffenen Nervenastes/der betroffenen Nervenäste auftreten.

Früher verwendete Begriffe Tic douloureux, primäre Trigeminusneuralgie.

\section{Diagnostische Kriterien:}

A. Wiederkehrende paroxysmale unilaterale Gesichtsschmerzattacken im/in den Versorgungsbereich(en), die einen Ast oder mehrere Äste des N. trigeminus betreffen, ohne Ausstrahlung darüberhinaus 1, die die Kriterien B und C erfüllen.

B. Der Schmerz weist alle der folgenden Charakteristika auf:

1. Dauer zwischen einem Sekundenbruchteil bis zu 2 Minuten2

2. starke Intensität3

3. stromstoßartige, einschießende, stechende oder scharfe Qualität

C. Vorangegangene harmlose Reize im betroffenen Versorgungsbereich des N. trigeminus4

D. Nicht besser erklärt durch eine andere ICHD-3-Diagnose.

Anmerkung:

1. Bei einer kleinen Zahl von Patienten kann der Schmerz zu einem anderen Ast ausstrahlen, bleibt jedoch innerhalb der trigeminalen Dermatome.

2. Die Schmerzdauer kann sich im Laufe der Zeit dahingehend ändern, dass die Paroxysmen länger anhalten. Eine Minderheit von Patienten berichtet von Attacken, die meist über $>2 \mathrm{Mi}-$ nuten anhalten.

3. Der Schmerzintensität kann im Laufe der Zeit zunehmen.

4. Einige Attacken können spontan auftreten oder spontan wirken, doch muss in der Vorgeschichte ein von harmlosen Reizen ausgelöster Schmerz aufgetreten oder festgestellt worden sein, damit dieses Kriterium erfüllt ist. Im Idealfall sollte der untersuchende Kliniker die anamnestischen Angaben durch Wiederholung des auslösenden Phänomens zu bestätigen suchen. Dies mag jedoch aufgrund einer entsprechenden Weigerung des Patienten bzw. einer anatomisch ungünstigen Lokalisation des Triggers und/oder anderer Faktoren nicht immer möglich sein.

\section{Kommentar:}

Die Diagnose einer 13.1.1 Trigeminusneuralgie muss klinisch festgestellt werden. Die Untersuchungen zielen darauf ab, eine wahrscheinliche Ursache zu ermitteln.

Unabhängig von dem auslösenden Phänomen sind bei den meisten Patienten mit einer 13.1.1 Trigeminusneuralgie keine sensorischen Anomalien im Versorgungsbereich des N. trigeminus nachweisbar, es sei denn mit Hilfe modernster Verfahren (z. B. einer Quantitativen Sensorischen Testung). Bei einigen Patienten kann die klinische neurologische Untersuchung jedoch sensible Defizite ergeben, die Anlass zu bildgebenden Untersuchungen sein sollten, um die mögliche Ursache zu erkunden. Eine Diagnose von Subtypen wie die einer 13.1.1.1 klassischen Trigeminusneuralgie, 13.1.1.2 sekundären Trigeminusneuralgie oder 13.1.1.3 idiopathischen Trigeminusneuralgie wird so ermöglicht. 
Bei starker Schmerzintensität löst der Schmerz häufig eine Kontraktion der Gesichtsmuskeln auf der betroffenen Seite aus (Tic douloureux).

Es können leichte autonome Symptome wie ein Tränen und/ oder eine Rötung des ipsilateralen Auges vorliegen.

Einem schmerzhaften Paroxysmus folgt gewöhnlich eine refraktäre Phase, in der keine Schmerzen ausgelöst werden können.

\subsubsection{Klassische Trigeminusneuralgie}

Beschreibung:

Eine Trigeminusneuralgie, die sich ohne Beteiligung einer anderen offensichtlichen Ursache als der einer neurovaskulären Kompression entwickelt.

\section{Diagnostische Kriterien:}

A. Wiederkehrende paroxysmale einseitige Gesichtsschmerzattacken, die die Kriterien für eine 13.1.1 Trigeminusneuralgie erfüllen

B. Nachweis einer neurovaskulären Kompression (nicht nur Kontakt) mittels MRT oder während eines chirurgischen Eingriffs, mit morphologischen Veränderungen 1 in der Wurzel des Trigeminusnervs.

\section{Anmerkung:}

1. Üblicherweise Atrophie oder Verdrängung.

\section{Kommentar:}

Eine Atrophie und/oder Verdrängung der Trigeminuswurzel infolge einer neurovaskulären Kompression treten unabhängig in Begleitung der Zeichen und Symptome einer 13.1.1 Trigeminusneuralgie auf. Bei Vorliegen dieser anatomischen Veränderung wird die Erkrankung als 13.1.1.1 klassische Trigeminusneuralgie diagnostiziert.

Üblicher Ort der neurovaskulären Kompression ist die Eintrittszone der Trigeminuswurzel, wobei eine Kompression durch eine Arterie eindeutiger von Symptomen begleitet ist als die Kompression durch eine Vene. Es stehen MRT-Verfahren zur Messung des Volumens und der Querschnittsfläche der Wurzel zur Verfügung. Zu den atrophischen Veränderungen können Demyelinisierung, ein neuronaler Zellverlust, Veränderungen der Mikrogefäße und andere morphologische Veränderungen gehören. Die genauen Mechanismen, wie atrophische Veränderungen des Trigeminusnervs zur Schmerzentstehung beitragen, sind zwar ungeklärt, manches deutet jedoch darauf hin, dass diese, wenn sie vor der Operation vorliegen, ein positives Ergebnis nach einer mikrovaskulären Dekompression erwarten lassen.

Viele Patienten mit einer 13.1.1.1 klassischen Trigeminusneuralgie erinnern sich deutlich an das erstmalige Auftreten des Schmerzes.

Die 13.1.1.1 klassische Trigeminusneuralgie tritt üblicherweise im Versorgungsbereich des 2. oder 3. Astes auf. Der Schmerz tritt in seltenen Fällen bilateral auf, dann aber nacheinander statt gleichzeitig.

Einer 13.1.1.1 klassischen Trigeminusneuralgie kann eine Phase mit atypischen Dauerschmerzen vorangehen, in der Fachliteratur Prä-Trigeminusneuralgie genannt.
In der Regel besteht zwischen den Paroxysmen Beschwerdefreiheit. Bei dem Subtyp 13.1.1.1.2 klassische Trigeminusneuralgie mit Dauerschmerz kommt es allerdings zu einem anhaltenden Hintergrundschmerz in dem betroffenen Areal.

\subsection{Klassische Trigeminusneuralgie, rein paroxysmal \\ Beschreibung:}

Klassische Trigeminusneuralgie ohne anhaltenden Hintergrundgesichtsschmerz.

\section{Diagnostische Kriterien:}

A. Wiederkehrende paroxysmale einseitige Gesichtsschmerzattacken, die die Kriterien für eine 13.1.1.1 klassische Trigeminusneuralgie erfüllen.

B. Schmerzfreiheit im betroffenen Versorgungsbereich des N. trigeminus zwischen den Attacken.

\section{Kommentar:}

Die 13.1.1.1 klassische Trigeminusneuralgie, rein paroxysmal spricht üblicherweise, zumindest initial, auf eine Pharmakotherapie an (insbesondere auf Carbamazepin oder Oxcarbazepin).

\subsection{Klassische Trigeminusneuralgie mit Dauer- schmerz}

Früher verwendete Begriffe Atypische Trigeminusneuralgie; Trigeminusneuralgie Typ 2.

\section{Beschreibung:}

Klassische Trigeminusneuralgie mit anhaltendem Hintergrundgesichtsschmerz.

\section{Diagnostische Kriterien:}

A. Wiederkehrende paroxysmale einseitige Gesichtsschmerzattacken, die die Kriterien für eine 13.1.1.1 klassische Trigeminusneuralgie erfüllen.

B. Begleitende durchgehende oder fast durchgehende Schmerzen im betroffenen Versorgungsbereich des N. trigeminus zwischen den Attacken.

\section{Kommentar:}

Der Dauerschmerz kann auf eine periphere oder zentrale Sensibilisierung zurückgehen.

\subsubsection{Sekundäre Trigeminusneuralgie}

Beschreibung:

Trigeminusneuralgie verursacht durch eine Grunderkrankung. Die klinische Untersuchung ergibt bei einem signifikanten Prozentsatz dieser Patienten sensible Veränderungen.

\section{Diagnostische Kriterien:}

A. Wiederkehrende paroxysmale einseitige Gesichtsschmerzattacken, die die Kriterien für eine 13.1.1 Trigeminusneuralgie erfüllen, sei es rein paroxysmal oder in Begleitung von begleitenden durchgehenden oder beinahe durchgehenden Schmerzen 
B. Es wurde eine Grunderkrankung nachgewiesen, die bekanntermaßen die Neuralgie verursachen kann und erklärt1

C. Nicht besser erklärt durch eine andere ICHD-3-Diagnose2.

Anmerkung:

1. Zu den anerkannten Ursachen zählen ein Tumor im Kleinhirnbrückenwinkel, eine AV-Malformation und Multiple Sklerose.

2. Am besten eignet sich ein MRT, um die Grundursache für eine 13.1.1.2 sekundäre Trigeminusneuralgie aufzudecken. Zu den sonstigen Untersuchungen können eine neurophysiologische Erfassung von Trigeminusreflexen und Trigeminus-evozierten Potentialen gehören, was sich für Patienten eignet, die nicht in der Lage sind, sich einem MRT zu unterziehen.

\subsection{Trigeminusneuralgie zurückzuführen auf Multiple Sklerose}

An anderer Stelle kodiert: 13.12.1 Zentraler neuropathischer Schmerz zurückzuführen auf Multiple Sklerose.

\section{Beschreibung:}

Trigeminusneuralgie verursacht durch (eine) Multiple-Sklerose-(MS)-Plaque oder -Plaques in der Pons oder der Eintrittszone der Trigeminuswurzel und verbunden mit anderen Symptomen und/ oder klinischen Zeichen oder Laborbefunden, die auf MS schlieBen lassen.

\section{Diagnostische Kriterien:}

A. Wiederkehrende einseitige paroxysmale Gesichtsschmerzattacken, die die Kriterien für eine 13.1.1 Trigeminusneuralgie erfüllen.

B. Beide der folgenden Punkte sind erfüllt:

1. Es wurde eine Multiple Sklerose (MS) diagnostiziert

2. Es wurde im MRT eine MS-Plaque an der Eintrittszone der Trigeminuswurzel oder in der Pons diagnostiziert, die Auswirkungen auf die intrapontinen primär-afferenten Fasern hat bzw. auf deren Vorliegen elektrophysiologische Routineuntersuchungen 1 schließen lassen, die eine Beeinträchtigung der trigeminalen Leitungsbahnen zeigen.

C. Nicht besser erklärt durch eine andere ICHD-3-Diagnose.

Anmerkung:

1. Blinkreflex oder trigeminal evozierte Potenziale.

\section{Kommentar:}

Eine 13.1.1.2.1 Trigeminusneuralgie zurückzuführen auf Multiple Sklerose 0 tritt bei $2 \%$ bis $5 \%$ der Patienten mit Multipler Sklerose (MS) auf, mitunter beidseits. Umgekehrt wird bei nur $2 \%$ bis 4 \% der Fälle mit 13.1.1 Trigeminusneuralgie eine Multiple Sklerose entdeckt. Symptome einer Trigeminusneuralgie sind selten ein Leitmerkmal von MS.

Die Läsion in der Pons wirkt sich auf die intrapontinen zentralen Endigungen der trigeminalen afferenten Fasern aus, die zu den trigeminalen Hirnstammkernen projizieren. Pontine Läsionen, die sich auf die Neuronen zweiter Ordnung des trigemino-thalamischen Traktes auswirken, führen üblicherweise zu nicht-paroxysmalen Schmerzattacken und/oder Dysästhesien und sollten unter
13.13.1 zentraler neuropathischer Schmerz zurückzuführen auf Multiple Sklerose klassifiziert werden.

Bei einigen Patienten mit MS findet sich eine neurovaskuläre Kompression der Trigeminuswurzel. Man geht davon aus, dass MS die Anfälligkeit der Nervenwurzel für die Auswirkungen einer Kompression erhöht und so eher zu paroxysmalen Schmerzattacken führt.

Patienten mit 13.1.1.2.1 Trigeminusneuralgie zurückzuführen auf Multiple Sklerose profitieren weniger von pharmakologischen und chirurgischen Interventionen als solche mit einer 13.1.1.1 klassischen Trigeminusneuralgie.

\subsection{Trigeminusneuralgie zurückzuführen auf eine raumfordernde Läsion \\ Beschreibung:}

Trigeminusneuralgie verursacht durch Kontakt zwischen dem betroffenen Trigeminusnerv und einer raumfordernden Läsion.

\section{Diagnostische Kriterien:}

A. Wiederkehrende einseitige paroxysmale Gesichtsschmerzen, die die Kriterien für 13.1.1 Trigeminusneuralgie erfüllen

B. Beide der folgenden Punkte sind erfüllt:

1. Es wurde eine raumfordernde Läsion nachgewiesen, die in Kontakt mit dem betroffenen Trigeminusnerv ist

2. Die Schmerzen haben sich nach Identifikation der Läsion entwickelt oder führten zu deren Entdeckung

C. Nicht besser erklärt durch eine andere ICHD-3-Diagnose.

\section{Kommentar:}

Patienten mit einer 13.1.1.2.2 Trigeminusneuralgie zurückzuführen auf eine raumfordernde Läsion können klinisch nachweisbare sensible Zeichen aufweisen oder nicht, wogegen elektrophysiologische Tests wie die Prüfung der trigeminalen Hirnstammreflexe in nahezu allen Fällen Auffälligkeiten ergibt.

\subsection{Trigeminusneuralgie zurückzuführen auf eine andere Ursache}

Beschreibung:

Trigeminusneuralgie, die durch eine andere Grunderkrankung als die oben beschriebenen verursacht wird.

\section{Diagnostische Kriterien:}

A. Wiederkehrende einseitige paroxysmale Gesichtsschmerzen, die die Kriterien für eine 13.1.1 Trigeminusneuralgie erfüllen, entweder rein paroxysmal oder in Begleitung gleicherzeitiger Dauer- oder annähernder Dauerschmerzen, doch nicht unbedingt einseitig

B. Beide der folgenden Punkte sind gegeben:

1. Eine andere Erkrankung als die oben beschriebenen, die jedoch bekanntermaßen eine Trigeminusneuralgie auslösen kann, wurde diagnostiziert1

2. Der Schmerz hat sich nach dem Beginn der Erkrankungen entwickelt oder führte zu ihrer Entdeckung

C. Nicht besser erklärt durch eine andere ICHD-3-Diagnose. 
Anmerkung:

1. Anerkannte Ursachen sind eine Fehlbildung des Schädelbasisknochens, eine Bindegewebserkrankung, arteriovenöse Malformation, durale arteriovenöse Fistel und genetische Ursachen einer Neuropathie oder neuronalen Übererregbarkeit.

\subsubsection{Idiopathische Trigeminusneuralgie}

Beschreibung:

Trigeminusneuralgie, bei der weder elektrophysiologische Tests noch das MRT signifikante Auffälligkeiten erkennen lassen.

\section{Diagnostische Kriterien:}

A. Wiederkehrende paroxysmale einseitige Gesichtsschmerzattacken, die die Kriterien für eine 13.1.1 Trigeminusneuralgie erfüllen, entweder rein paroxysmal oder in Begleitung gleichzeitiger Dauer- oder annähernder Dauerschmerzen

B. Weder eine 13.1.1.1 klassische Trigeminusneuralgie noch 13.1.1.2 sekundäre Trigeminusneuralgie wurde durch entsprechende Untersuchung einschließlich elektrophysiologischer Tests und MRT bestätigt1

C. Nicht besser erklärt durch eine andere ICHD-3-Diagnose.

\section{Anmerkung:}

1. Ein Kontakt zwischen einem Blutgefäß und dem Trigeminusnerv und/oder der Wurzel des Trigeminusnervs ist bei bildgebenden Untersuchungen an gesunden Probanden ein gängiger Befund. Zeigt sich ein solcher Kontakt bei Vorliegen einer 13.1.1 Trigeminusneuralgie, doch ohne Belege für morphologische Veränderungen (d. h., Atrophie oder Verdrängung) in der Nervenwurzel, so sind die Kriterien für eine 13.1.1.1 klassische Trigeminusneuralgie nicht erfüllt und die Erkrankung wird als idiopathisch betrachtet.

\subsection{Idiopathische Trigeminusneuralgie, rein paroxysmal}

\section{Diagnostische Kriterien:}

A. Wiederkehrende einseitige paroxysmale Gesichtsschmerzattacken, die die Kriterien für eine 13.1.1.3 idiopathische Trigeminusneuralgie erfüllen

B. Es besteht zwischen den Attacken Beschwerdefreiheit im betroffenen Versorgungsbereich des Trigeminusnervs.

\subsection{Idiopathische Trigeminusneuralgie mit Dauer- schmerz \\ Diagnostische Kriterien:}

A. Wiederkehrende einseitige paroxysmale Gesichtsschmerzattacken, die die Kriterien für eine 13.1.1.3 idiopathische Trigeminusneuralgie erfüllen

B. Begleitender Dauerschmerz oder annähernder Dauerschmerz zwischen den Attacken im betroffenen Versorgungsbereich des Trigeminusnervs.

\subsubsection{Schmerzhafte Trigeminusneuropathie}

\section{Beschreibung:}

Gesichtsschmerz im/in den Versorgungsbereich(en) eines einzelnen Astes oder mehrerer Äste des N. trigeminus, der durch eine andere Erkrankung verursacht wird und auf eine neuronale Schä- digung hinweist. Der primäre Schmerz ist üblicherweise ein Dauerschmerz oder annähernder Dauerschmerz und wird in der Regel als brennend, drückend oder nadelstichartig beschrieben. Überlagerte kurze paroxysmale Schmerzattacken können auftreten, doch sind diese nicht der vorherrschende Schmerztyp. Diese Kombination unterscheidet die schmerzhafte Trigeminusneuropathie von den Subtypen der Trigeminusneuralgie. Es finden sich klinisch nachweisbare sensible Defizite innerhalb des Versorgungsbereichs des Trigeminusnervs und mechanische Allodynie und nervöse Übererregbarkeit erfüllen die IASP-Kriterien für neuropathische Schmerzen. Im Regelfall sind allodynische Areale viel größer als die punktförmigen Triggerzonen, die bei einer Trigeminusneuralgie vorliegen.

\subsubsection{Schmerzhafte Trigeminusneuropathie zurück- zuführen auf Herpes zoster \\ Beschreibung:}

Einseitige Gesichtsschmerzen von weniger als 3 Monaten Dauer $\mathrm{im} /$ in den Verteilungsbereich(en) von einem Ast oder mehreren Ästen des N. trigeminus, verursacht durch und begleitet von anderen Symptomen und/oder klinischen Zeichen eines akuten Herpes zoster.

\section{Diagnostische Kriterien:}

A. Einseitiger Gesichtsschmerz im/in den Versorgungsbereich(en) eines Astes oder von Ästen des N. trigeminus mit einer Dauer von $<3$ Monaten

B. Einer oder mehrere von folgenden Punkten treffen zu:

1. Eine Herpeseruption ist im gleichen Versorgungsbereich des N. trigeminus aufgetreten

2. Mittels Polymerase-Kettenreaktion (PCR) wurde ein Varicella-zoster-Virus (VZV) im Liquor gefunden

3. Direkter Immunfluoreszenztest auf VZV-Antigen oder PCRTest auf VZV-DNA in vom Boden der Läsionen gewonnenen Zellen ist positiv

C. Nicht besser erklärt durch eine andere ICHD-3-Diagnose.

\section{Kommentar:}

Herpes zoster zeigt in $10 \%$ bis $15 \%$ der Fälle Auswirkungen auf das Ganglion trigeminale, wobei bei rund $80 \%$ der Patienten der Augenast des N. trigeminus als betroffen ermittelt wurde. Selten folgt dem Schmerz keine Eruption oder keine Effloreszenzen (zoster sine herpete). In solchen Fällen wird die Diagnose mittels einer Polymerase-Kettenreaktion gestellt, die eine Entdeckung von Varicella-zoster-Virus-DNA im Liquor ermöglicht.

Eine 13.1.2.1 schmerzhafte Trigeminusneuropathie zurückzuführen auf Herpes zoster ist gewöhnlich brennend, stechend/einschießend, kribbelnd oder schmerzend und wird von einer kutanen Allodynie begleitet.

Ophthalmischer Herpes kann in Begleitung einer Lähmung des 3., 4. und/oder 6. Hirnnervs auftreten.

Herpes zoster ist bei immungeschwächten Patienten häufig, er tritt bei etwa $10 \%$ von Patienten mit Lymphom und $25 \%$ der Patienten mit M. Hodgkin auf. 


\subsubsection{Post-herpetische Trigeminusneuralgie}

Früher verwendeter Begriff: Postherpetische Trigeminusneuropathie.

\section{Beschreibung:}

Anhaltender oder mindestens 3 Monate lang auftretender einseitiger Gesichtsschmerz im/in den Versorgungsgebiet(en) eines einzelnen Astes oder mehrerer Äste des N. trigeminus, mit variablen sensiblen Veränderungen, verursacht durch Herpes zoster.

\section{Diagnostische Kriterien:}

A. Einseitiger Gesichtsschmerz im/in den Versorgungsgebiet(en) eines Astes oder mehrerer Äste des N. trigeminus, der anhält oder für > 3 Monate wiederkehrt und Kriterium C erfüllt

B. Der Herpes zoster hat denselben Ast/dieselben Äste des N. trigeminus betroffen

C. Der Schmerz hat sich in einem zeitlichen Zusammenhang mit der Herpes-zoster-Infektion entwickelt 1

D. Nicht besser erklärt durch eine andere ICHD-3-Diagnose.

\section{Anmerkung:}

1. Üblicherweise hat sich der Schmerz entwickelt, während die Effloreszenzen noch aktiv waren, gelegentlich jedoch auch später, nach Verheilen der Effloreszenzen. In solchen Fällen können als Folge der Herpeseruption blass- oder hellviolette Narben auftreten.

\section{Kommentar:}

Ungeachtet ihres lange bevorzugten Namens ist die postherpetische Neuralgie eigentlich eine Neuropathie oder Neuronopathie: es wurden signifikante pathoanatomische Veränderungen im Nerv, im Ganglion und in der Nervenwurzel nachgewiesen. Bei einer 13.1.2.2 postherpetischen Trigeminusneuralgie bestehen auch Belege für die Entzündung, die sich bis in den trigeminalen Hirnstammkomplex hinein ausdehnt.

Die postherpetische Neuralgie nach einem akuten Herpes zoster tritt eher mit steigendem Lebensalter auf.

Der erste Ast des N. trigeminus ist bei einer 13.1.2.2 postherpetischen Trigeminusneuralgie am häufigsten betroffen, doch können auch der zweite und dritte Ast involviert sein.

Typischerweise ist der Schmerz bei der postherpetischen Neuralgie brennend und juckend, letzteres mitunter sehr im Vordergrund stehend und außerordentlich störend. Zudem zeigen Patienten mit postherpetischer Neuralgie typischerweise ein eindeutiges sensibles Defizit sowie eine durch leichte Berührung evozierbare mechanische Allodynie im beteiligten Versorgungsgebiet des N. trigeminus. Viele Patienten weisen jedoch nur geringe sensible Verluste auf und zeigen stattdessen erhöhte Reaktionen auf thermische und/oder punktuelle Reize.

\subsubsection{Schmerzhafte posttraumatische Trigeminus- neuropathie}

Früher verwendeter Begriff: Anaesthesia dolorosa.

\section{Beschreibung:}

Ein- oder beidseitige Gesichtsschmerzen oder Schmerzen im Mundraum nach einem sowie verursacht durch ein Trauma des N. trige- minus/der Nn. trigemini, mit weiteren Symptomen und/oder klinischen Zeichen einer funktionellen trigeminalen Störung.

\section{Diagnostische Kriterien:}

A. Gesichtsschmerzen und/oder Schmerzen im Mundraum im/in den Versorgungsgebiet(en) von einem oder beiden Trigeminusnerv(en), die Kriterium C erfüllen

B. Anamnestisch ermittelbares traumatisches Ereignis1 bezogen auf den/die Trigeminusnerv(en) mit klinisch evidenten positiven (Hyperalgesie, Allodynie) und/oder negativen (Hypästhesie, Hypalgesie) Zeichen einer funktionellen trigeminalen Störung

C. Ein kausaler Zusammenhang kann durch beide der folgenden Kriterien gezeigt werden:

1. Schmerzlokalisation im/in den Versorgungsgebiet(en) des/ der von dem traumatischen Ereignis betroffenen N. trigeminus/Nn. trigemini

2. Der Schmerz hat sich < 6 Monate nach dem traumatischen Ereignis entwickelt

D. Nicht besser erklärt durch eine andere ICHD-3-Diagnose.

Anmerkung:

1. Das traumatische Ereignis kann mechanischer, chemischer oder thermischer Art sein oder auf Bestrahlung zurückgehen. Die Anwendung neuroablativer Verfahren auf das Trigeminusganglion oder die Wurzel des N. trigeminus aufgrund einer Trigeminusneuralgie kann zu neuropathischen Schmerzen führen, von denen ein Ast oder mehrere Äste des N. trigeminus betroffen sind; dies sollte als posttraumatisch betrachtet und hierunter kodiert werden.

\section{Kommentar:}

Die Schmerzdauer reicht von paroxysmalen Schmerzattacken bis zu Dauerschmerzen und kann gemischt sein.

Insbesondere nach strahleninduzierten postganglionischen Schäden kann eine Neuropathie nach mehr als 3 Monaten auftreten.

Eine 13.1.2.3 schmerzhafte posttraumatische Trigeminusneuropathie nach Anwendung neuroablativer Verfahren bezogen auf das Trigeminusganglion oder die Trigeminuswurzel kann zusammen mit einer 13.1.1 Trigeminusneuralgie existieren, sofern letztere wiederkehrt.

\subsubsection{Schmerzhafte Trigeminusneuropathie zurück- zuführen auf eine andere Erkrankung \\ Beschreibung:}

Ein- oder beidseitiger Gesichts- oder Mundschmerz im/in den Versorgungsgebiet(en) eines Astes oder mehrerer Äste des N. trigeminus, der durch eine andere Erkrankung als die oben beschriebenen verursacht wird, mit anderen Symptomen und/oder klinischen Zeichen einer funktionalen trigeminalen Störung.

\section{Diagnostische Kriterien:}

A. Ein- oder beidseitiger Gesichtsschmerz im/in den Versorgungsgebiet(en) von einem oder beiden Trigeminusnerv(en), der Kriterium C erfüllt 
B. Eine andere Erkrankung als die oben beschriebenen, die jedoch bekanntermaßen eine schmerzhafte Trigeminusneuropathie verursachen kann verbunden mit klinisch evidenten positiven (Hyperalgesie, Allodynie) und/oder negativen (Hypästhesie, Hypalgesie) Zeichen einer funktionellen Störung des N. trigeminus, die sich auf einen oder beide Trigeminusnerv(en) auswirkt, wurde diagnostiziert

C. Ein kausaler Zusammenhang kann durch die beiden folgenden Kriterien gezeigt werden:

1. Die Schmerzlokalisation im/in den Versorgungsgebiet(en) des von der Erkrankung betroffenen N. trigeminus/der Nn. trigemini

2. Der Schmerz hat sich nach Beginn der Erkrankung entwickelt oder führte zu ihrer Entdeckung

D. Nicht besser erklärt durch eine andere ICHD-3-Diagnose.

\section{Kommentar:}

Eine schmerzhafte Trigeminusneuropathie kann sich als Folge einer Multiplen Sklerose, einer raumfordernden Läsion oder aufgrund von systemischen Erkrankungen entwickeln, wobei nur die klinischen Bilder (Qualität eines Spontanschmerzes, evozierten Schmerzes und Vorliegen sensibler Defizite) die Unterscheidung zwischen einer 13.1.1.2 sekundären Trigeminusneuralgie und einer 13.1.2 schmerzhaften Trigeminusneuropathie ermöglichen.

Eine 13.1.2 schmerzhafte Trigeminusneuropathie verursacht durch eine Bindegewebserkrankung oder durch Erbkrankheiten ist gewöhnlich bilateral lokalisiert, kann jedoch asymmetrisch beginnen und mitunter mit paroxysmalen Schmerzattacken auftreten, die die Hintergrundschmerzen überlagern. Die Patienten entwickeln irgendwann beidseits sensible Defizite und einen Dauerschmerz, wodurch sich die Diagnose klärt. Das MRT ist unauffällig, die Trigeminusreflexe sind jedoch unweigerlich verzögert oder fehlen.

\subsubsection{Idiopathische schmerzhafte Trigeminusneuro- pathie \\ Beschreibung:}

Ein- oder beidseitiger Schmerzen im Versorgungsgebiet von einem Ast oder mehreren Ästen des N. trigeminus/der Nn. trigemini, die auf eine Nervenschädigung hindeuten, deren Ätiologie jedoch unbekannt ist.

\section{Diagnostische Kriterien:}

A. Ein- oder beidseitige Gesichtsschmerzen im/in den Versorgungsgebiet(en) eines Trigeminusnervs oder beider Trigeminusnerven, die Kriterium B erfüllen

B. Klinisch evidente positive (Hyperalgesie, Allodynie) und/oder negative (Hypästhesie, Hypalgesie) Zeichen einer funktionellen trigeminalen Störung

C. Es wurde keine Ursache ermittelt

D. Nicht besser erklärt durch eine andere ICHD-3-Diagnose.

\subsection{Schmerz zurückzuführen auf eine Läsion oder Erkrankung des \\ N. glossopharyngeus}

\subsubsection{Glossopharyngeusneuralgie}

Früher verwendeter Begriff: Vagoglossopharyngeusneuralgie.

\section{Beschreibung:}

Eine Erkrankung, die von einem plötzlich einsetzenden und endenden einseitigen kurzen, stechenden Schmerz nicht nur in den Versorgungsgebieten des N. glossopharyngeus charakterisiert ist, sondern auch in den aurikulären und pharyngealen Ästen des Vagusnervs. Der Schmerz wird im Bereich des Ohres, des Zungengrundes, der Tonsillennische und/oder unterhalb des Kieferwinkels wahrgenommen. Der Schmerz wird üblicherweise ausgelöst durch Schlucken, Sprechen und Husten und kann nach Art der Trigeminusneuralgie remittieren und rezidivieren.

\section{Diagnostische Kriterien:}

A. Wiederkehrende paroxysmale unilaterale Schmerzattacken im Versorgungsgebiet des N. glossopharyngeus1, die das Kriterium B erfüllen

B. Der Schmerz weist alle folgenden Charakteristika auf:

1. Zwischen wenigen Sekunden bis 2 Minuten anhaltend

2. Starke Intensität

3. Von elektrisierender, einschießender, stechender oder scharfer Qualität

4. Auslösung durch Schlucken, Husten, Sprechen oder Gähnen

C. Nicht besser erklärt durch eine andere ICHD-3-Diagnose.

Anmerkung:

1. Lokalisation im hinteren Bereich der Zunge, in der Tonsillennische, im Pharynx oder Kieferwinkel und/oder im Ohr.

\section{Kommentar:}

Eine 13.2.1 Glossopharyngeusneuralgie kann im Verbund mit einer 13.1.1 Trigeminusneuralgie auftreten.

Der N. laryngeus superior ist ein Ast des Vagusnervs. Eine Neuralgie des N. laryngeus superior zeigt sich im Hinblick auf ihre Lokalisation ähnlich wie eine 13.2.1 Glossopharyngeusneuralgie und kann klinisch schwer von einer solchen zu unterscheiden sein.

Die zerebrale Bildgebung kann eine neurovaskuläre Kompression des N. glossopharyngeus zeigen.

Vor der Entwicklung einer 13.2.1 Glossopharyngeusneuralgie können in den betroffenen Arealen für Wochen bis zu mehreren Monaten unangenehme Empfindungen wahrgenommen werden.

Die Schmerzen bei einer 13.2.1 Glossopharyngeusneuralgie können ausstrahlen und Auge, Nase, Kinn oder Schulter beteiligen. Die Schmerzintensität kann so heftig sein, dass es bei Patienten zum Gewichtsverlust kommt. In seltenen Fällen werden Schmerzattacken von vagalen Symptomen wie Husten, Heiserkeit, Synkopen und/oder Bradykardie begleitet. Einige Autoren schlagen vor, zwischen pharyngealen, otalgischen und vagalen Subtypen von Neuralgie zu unterscheidet und haben die Verwendung des Begriffs Vagoglossopharyngeusneuralgie für den Fall ins Spiel 
gebracht, dass der Schmerz von Asystolie, Zuckungen und einer Synkope begleitet wird.

Die klinische Untersuchung ergibt üblicherweise keine sensiblen Veränderungen im Versorgungsgebiet des Nervs, doch falls leichte sensible Defizite entdeckt werden, wird die Diagnose hierdurch nicht entkräftet. Bei größeren Veränderungen oder einem verminderten/fehlenden Würgereflex sollten ätiologische Untersuchungen eingeleitet werden.

Eine 13.2.1 Glossopharyngeusneuralgie spricht üblicherweise zumindest initial auf eine Pharmakotherapie an (insbesondere Carbamazepin oder Oxcarbazepin). Es wird vermutet, dass eine örtliche Betäubung der Rachenmandel und Rachenwand Attacken für einige Stunden vorbeugen kann.

\subsubsection{Klassische Glossopharyngeusneuralgie}

Beschreibung:

Glossopharyngeusneuralgie, die sich ohne andere ersichtliche Ursache als eine neurovaskuläre Kompression entwickelt.

\section{Diagnostische Kriterien:}

A. Wiederkehrende einseitige paroxysmale Schmerzattacken, die die Kriterien für eine 13.2.1 Glossopharyngeusneuralgie erfüllen

B. Nachweis einer neurovaskulären Kompression der Wurzel des N. glossopharyngeus auf dem MRT oder bei einer Operation.

\subsubsection{Sekundäre Glossopharyngeusneuralgie}

Beschreibung:

Glossopharyngeusneuralgie verursacht durch eine Grunderkrankung.

Diagnostische Kriterien:

A. Wiederkehrende einseitige paroxysmale Schmerzattacken, die die Kriterien für eine 13.2.1 Glossopharyngeusneuralgie erfüllen

B. Es wurde eine Grunderkrankung nachgewiesen, die bekanntermaßen die Neuralgie verursachen kann und erklärt1.

Anmerkung:

1. Vereinzelt wurde von einer 13.2.1.2 sekundären Glossopharyngeusneuralgie verursacht durch ein HWS-Trauma, Multiple Sklerose, Tonsillen- oder regionale Karzinome, Kleinhirnbrückentumore und eine Arnold-Chiari-Malformation berichtet.

\subsubsection{Idiopathische Glossopharyngeusneuralgie}

Beschreibung:

Glossopharyngeusneuralgie ohne Nachweis einer neurovaskulären Kompression oder einer ursächlichen Grunderkrankung.

\section{Diagnostische Kriterien:}

A. Wiederkehrende einseitige paroxysmale Schmerzattacken, die die Kriterien für eine 13.2.1 Glossopharyngeusneuralgie erfüllen

B. Untersuchungen haben weder eine neurovaskuläre Kompression noch eine Grunderkrankung ergeben, die bekannterma- ßen eine 13.2.1.2 sekundäre Glossopharyngeusneuralgie verursachen kann

C. Nicht besser erklärt durch eine andere ICHD-3-Diagnose.

\subsubsection{Schmerzhafte Glossopharyngeusneuropathie Beschreibung:}

Schmerz innerhalb des Versorgungsgebiets des N. glossopharyngeus (hinterer Teil der Zunge, Tonsillennische, Rachen und/oder unterhalb des Kieferwinkels). Zudem werden gewöhnlich Schmerzen im ipsilateralen Ohr wahrgenommen. Der primäre Schmerz ist üblicherweise durchgehend oder beinahe durchgehend und wird üblicherweise als brennend, drückend oder nadelstichartig beschrieben. Es können kurze überlagernde Paroxysmen auftreten, sind jedoch nicht der vorherrschende Schmerztyp. Diese Kombination unterscheidet die schmerzhafte Glossopharyngeusneuropathie von den Subtypen einer 13.2.1 Glossopharyngeusneuralgie. Sensible Defizite können im ipsilateralen posterioren Teil der Zunge und Tonsillennische auftreten und der Würgereflex kann schwach sein oder fehlen.

\subsubsection{Schmerzhafte Glossopharyngeusneuropathie zurückzuführen auf eine bekannte Ursache Beschreibung:}

Einseitiger durchgehender oder beinahe durchgehender Schmerz mit oder ohne Überlagerung durch kurze paroxysmale Schmerzattacken im Versorgungsgebiet des N. glossopharyngeus und durch eine andere identifizierte Erkrankung verursacht.

\section{Diagnostische Kriterien:}

A. Einseitiger durchgehender oder beinahe durchgehender Schmerz1 in Versorgungsgebiet des N. glossopharyngeus, der das Kriterium C erfüllt

B. Es wurde eine Erkrankung diagnostiziert, die bekanntermaßen eine schmerzhafte Glossopharyngeusneuropathie verursachen kann2

C. Ein kausaler Zusammenhang kann durch die beiden folgenden Kriterien gezeigt werden:

1. Der Schmerz ist ipsilateral zu dem von der Erkrankung betroffenen N. glossopharyngeus lokalisiert

2. Der Schmerz hat sich nach Beginn der Erkrankung entwickelt oder führte zu deren Entdeckung

D. Nicht besser erklärt durch eine andere ICHD-3-Diagnose.

Anmerkung:

1. Es kann zu überlagernden kurzen Paroxysmen kommen, diese sind jedoch nicht der vorherrschende Schmerztyp.

2. Kleinhirnbrückenwinkeltumore und iatrogene Verletzungen während chirurgischer Eingriffe wurden als Ursache einer schmerzhaften Glossopharyngeusneuropathie berichtet.

\subsubsection{Idiopathische schmerzhafte Glossopharyngeus- neuropathie}

Beschreibung:

Einseitiger durchgehender oder nahezu durchgehender Schmerz unbekannter Ätiologie mit oder ohne überlagernde kurze paroxysmale Schmerzattacken im/in den Versorgungsgebiet(en) des N. glossopharyngeus. 
Diagnostische Kriterien:

A. Einseitiger durchgehender oder nahezu durchgehender Schmerz1 im Versorgungsgebiet des N. glossopharyngeus

B. Es wurde keine Ursache ermittelt

C. Nicht besser erklärt durch eine andere ICHD-3-Diagnose.

Anmerkung:

1. Es kann zu überlagernden kurzen Paroxysmen kommen, diese sind jedoch nicht der vorherrschende Schmerztyp.

\subsection{Schmerz zurückzuführen auf eine Läsion oder Erkrankung des N. intermedius}

\subsubsection{Intermediusneuralgie}

Früher verwendeter Begriff: Neuralgia geniculata.

\section{Beschreibung:}

Seltene Erkrankung, die durch kurze Schmerzparoxysmen in der Tiefe des Gehörganges charakterisiert ist, mitunter in die Scheitel-Hinterhauptregion ausstrahlend. In den allermeisten Fällen findet sich bei Operation eine vaskuläre Kompression, mitunter mit verdickter Arachnoidea, aber der Kopfschmerz kann sich auch ohne offensichtliche Ursache oder als Komplikation eines Herpes zoster entwickeln beziehungsweise (sehr selten) einer Multiplen Sklerose oder eines Tumors. Der Schmerz wird durch Stimulation einer Triggerzone an der Hinterwand des Gehörganges und/oder der Region um die Ohrmuschel.

\section{Diagnostische Kriterien:}

A. Paroxysmale einseitige Schmerzattacken im Versorgungsgebiet des N. intermedius 1, die die Kriterien B und C erfüllen

B. Der Schmerz weist alle der folgenden Charakteristika auf:

1. zwischen wenigen Sekunden bis zu Minuten andauernd

2. schwere Intensität

3. einschießend, stechend oder scharf

4. voraus geht die Stimulation einer Triggerzone an der Hinterwand des Gehörganges und/oder der Region um die Ohrmuschel

C. Nicht besser erklärt durch eine andere ICHD-3-Diagnose2.

\section{Anmerkung:}

1. Der Schmerz ist im Gehörgang, in der Ohrmuschel, um den Warzenfortsatz und gelegentlich im weichen Gaumen lokalisiert und kann manchmal in den Schläfenbereich oder den Unterkieferwinkel ausstrahlen.

2. Angesichts der komplexen und Überschneidungen aufweisenden Innervation des äußeren Ohres, abgeleitet von trigeminalen (aurikulotemporalen) Nerven, Gesichtsnerven (Nervus intermedius), dem N. glossopharyngeus, Vagus- und 2. Hirnnerv, lassen sich Neuralgien in dieser Region unter Umständen nicht ohne weiteres einem einzelnen Nerv zuordnen, wenn ein konkreter neurovaskulärer Kontakt nicht dargestellt werden kann.

\section{Kommentar:}

Störungen der Tränen- oder Speichelsekretion und/oder des Geschmackes können die Schmerzen bei einer 13.3.1 Intermediusneuralgie begleiten.

\subsubsection{Klassische Intermediusneuralgie}

Beschreibung:

Neuralgie des N. intermedius, die sich ohne eine andere offenkundige Ursache als die einer neurovaskulären Kompression entwickelt.

\section{Diagnostische Kriterien:}

A. Wiederkehrende einseitige paroxysmale Schmerzattacken, die die Kriterien für eine 13.3.1 Intermediusneuralgie erfüllen

B. Nachweis einer neurovaskulären Kompression der Nervenwurzel des N. intermedius auf dem MRT oder während einer Operation.

\subsubsection{Sekundäre Intermediusneuralgie}

Beschreibung:

Neuralgie des N. intermedius, die durch eine Grunderkrankung verursacht wird.

\section{Diagnostische Kriterien:}

A. Wiederkehrende einseitige paroxysmale Schmerzattacken, die die Kriterien für eine 13.3.1 Intermediusneuralgie erfüllen

B. Es besteht der Nachweis einer Grunderkrankung, die bekanntermaßen die Neuralgie verursachen und erklären kann 1 .

Anmerkung:

1. Es gibt Einzelberichte zu einer 13.3.1.2 sekundären Intermediusneuralgie infolge einer Multiplen Sklerose oder eines Tumors. Bei Letzterem ist das klinische Erscheinungsbild tendenziell von neurologischen Defiziten durch Schädigung anderer unmittelbar benachbarter Nerven geprägt. Herpes zoster führt typischerweise eher zu einer 13.3.2.1 schmerzhaften Intermediusneuropathie zurückzuführen auf einen Herpes zoster als zu einer 13.3.1.2 sekundären Intermediusneuralgie.

\subsubsection{Idiopathische Intermediusneuralgie} Beschreibung:

Intermediusneuralgie ohne Hinweis auf eine neurovaskuläre Kompression oder eine ursächliche Grunderkrankung.

\section{Diagnostische Kriterien:}

A. Wiederkehrende einseitige paroxysmale Schmerzattacken, die die Kriterien für eine 13.3.1 Intermediusneuralgie erfüllen

B. Untersuchungen haben weder eine neurovaskuläre Kompression ergeben noch eine Grunderkrankung, die bekanntermaßen eine 13.3.1.2 sekundäre Intermediusneuralgie verursachen kann.

C. Nicht besser erklärt durch eine andere ICHD-3-Diagnose.

\subsubsection{Schmerzhafte Intermediusneuropathie Beschreibung:}

Schmerzen im/in den Versorgungsgebiet(en) des N. intermedius/ der Nn. intermedii (Gehörgang, Ohrmuschel oder um den Warzen- 
fortsatz), üblicherweise vom Patienten als dumpf, tief im Ohr und durchgehend oder beinahe durchgehend beschrieben. Überlagerte kurze paroxysmale Schmerzattacken können auftreten, doch sind diese nicht der vorherrschende Schmerztyp. Diese Kombination unterscheidet die schmerzhafte Intermediusneuropathie von den Subtypen einer 13.3.1 Intermediusneuralgie. Im Gehörgang, der Ohrmuschel oder der Haut über dem Warzenfortsatz können sensible Defizite, üblicherweise leichter Natur, zutage treten.

\subsubsection{Schmerzhafte Intermediusneuropathie zurück- zuführen auf einen Herpes zoster}

Früher verwendeter Begriff: Die 13.3.2.1 schmerzhafte Intermediusneuropathie zurückzuführen auf einen Herpes zoster in Begleitung einer Fazialisparese ist unter dem Namen Ramsay-HuntSyndrom bekannt.

\section{Beschreibung:}

Einseitige durchgehende oder beinahe durchgehende Schmerzen mit oder ohne kurze überlagernde Paroxysmen im Versorgungsgebiet des N. intermedius, die in der Tiefe des Gehörganges gespürt werden, auf eine Herpes-zoster-Infektion zurückgehen und meist mit einer Gesichtslähmung und anderen Symptomen und/ oder klinischen Zeichen der Infektion oder ihrer Nachwirkungen einhergehen.

\section{Diagnostische Kriterien:}

A. Einseitige durchgehende oder beinahe durchgehende Schmerzen 1 im Versorgungsgebiet des N. intermedius2, die das Kriterium C erfüllen

B. Einer oder mehrere der folgenden Punkte:

1. Die Herpeseruption ist im Versorgungsgebiet des N. intermedius3 aufgetreten

2. Mit Hilfe der Polymerase-Ketten-Reaktion (PCR) wurde das Varicella-zoster-Virus (VZV) im Liquor nachgewiesen

3. Der direkte Immunfluoreszenztest auf VZV-Antigen oder PCR-Test auf VZV-DNA in aus der Läsion gewonnenen Zellen ist positiv

C. Die Schmerzen haben sich in einer zeitlichen Beziehung zum Herpes zoster entwickelt4

D. Nicht besser erklärt durch eine andere ICHD-3-Diagnose5.

\section{Anmerkung:}

1. Es können Überlagernde kurze paroxysmale Schmerzattacken auftreten, doch sind diese nicht der vorherrschende Schmerztyp.

2. Im Gehörgang, der Ohrmuschel und/oder um den Warzenfortsatz.

3. Aufgrund der Ausbreitung des Virus können auch andere Hirnnerven befallen werden.

4. Der Schmerz kann der Herpeseruption vorangehen.

5. Die klinische Bestätigung der Diagnose in den Akutstadien durch Nachweis von Bläschen auf dem Trommelfell, im Gehörgang, auf der Ohrmuschel und/oder der Haut über dem Warzenfortsatz. Diese können auch im vorderen Zungendrittel zu sehen sein, wohin das Virus über die Chorda tympani gelangt ist, oder auf dem harten Gaumen, versorgt über einen rudimentären Ast des N. facialis (evolutionäres Überbleibsel).
Kommentar:

Auch andere Hirnnerven (der 8., 9., 10.,11.) können betroffen sein, was zu Tinnitus, Hörminderung, Schwindel, Übelkeit, Heiserkeit und Schluckstörungen führt.

Obwohl über den natürlichen Krankheitsverlauf einer 13.3.2.1 schmerzhaften Intermediusneuropathie zurückzuführen auf einen Herpes zoster wenig bekannt ist, kann der Schmerz für mehr als 3 Monate fortbestehen, worauf er unter 13.3.2.2 postherpetische Intermediusneuralgie klassifiziert werden sollte.

\subsubsection{Postherpetische Intermediusneuralgie}

Beschreibung:

Einseitiger anhaltender oder über mindestens 3 Monate wiederkehrender Schmerz im Versorgungsgebiet des $\mathrm{N}$. intermedius, in der Tiefe des Gehörganges verspürt und durch eine intermedius-Herpes-zoster-Infektion verursacht.

Diagnostische Kriterien:

A. Einseitiger Schmerz im Versorgungsgebiet des N. intermedius 1, der anhält oder über > 3 Monate wiederkehrt und das Kriterium C erfüllt

B. Zustand nach intermedius-Herpes-zoster-Infektion

C. Die Schmerzen haben sich in einem zeitlichen Zusammenhang zur Herpes-zoster-Infektion entwickelt2

D. Nicht besser erklärt durch eine andere ICHD-3-Diagnose.

Anmerkung:

1. Im Gehörgang, in der Ohrmuschel und/oder um den Warzenfortsatz.

2. Üblicherweise hat sich der Schmerz entwickelt, während die Infektion noch aktiv war, gelegentlich jedoch auch später.

13.3.2.3 Schmerzhafte Intermediusneuropathie zurückzuführen auf eine andere Erkrankung

\section{Beschreibung:}

Einseitige durchgehende oder beinahe durchgehende Schmerzen mit oder ohne überlagernde kurze Paroxysmen im Versorgungsgebiet des N. intermedius und verursacht durch eine andere Erkrankung als eine Herpes-zoster-Infektion. Es kann andere Symptome und/oder klinische Zeichen der ursächlichen Erkrankung geben.

\section{Diagnostische Kriterien:}

A. Einseitige durchgehende oder beinahe durchgehende Schmerzen $1 \mathrm{im}$ Versorgungsgebiet des N. intermedius2, die das Kritierum C erfüllen

B. Es wurde eine andere Erkrankung als eine Herpes-zoster-Infektion diagnostiziert, die den N. intermedius betrifft und bekanntermaßen eine schmerzhafte Intermediusneuralgie verursachen kann 3

C. Die Schmerzen haben sich nach Beginn der Erkrankung entwickelt oder führten zu ihrer Entdeckung

D. Nicht besser erklärt durch eine andere ICHD-3-Diagnose.

\section{Anmerkung:}

1. Überlagerte kurze paroxysmale Schmerzattacken können auftreten, doch sind diese nicht der vorherrschende Schmerztyp. 
2. Im Gehörgang, in der Ohrmuschel und/oder um den Warzenfortsatz.

3. In seltenen Fällen wurde eine 13.3.2 schmerzhafte Intermediusneuralgie bei Patienten mit Gesichtstumoren oder einer Schädigung des Ganglion genicularis beobachtet.

\subsubsection{Idiopathische schmerzhafte Intermediusneuro- pathie}

Beschreibung:

Einseitige durchgehende oder beinahe durchgehende Schmerzen mit oder ohne überlagerte kurze Paroxysmen im/in den Versorgungsgebieten des N. intermedius und von unbekannter Ätiologie.

\section{Diagnostische Kriterien:}

A. Schmerz im/in den Versorgungsgebiet(en) des N. intermedius1 ein- oder beidseitig

B. Es wurde keine Ursache ermittelt

C. Nicht besser erklärt durch eine andere ICHD-3-Diagnose.

\section{Anmerkung:}

1. Im Gehörgang, in der Ohrmuschel und/oder um den Warzenfortsatz.

13.4 Okzipitalisneuralgie

\section{Beschreibung:}

Ein- oder beidseitige paroxysmale, einschießende oder stechende Schmerzattacken im hinteren Teil des Schädels, im/in den Versorgungsgebiet(en) des N. occipitalis major, minor und/oder tertius, mitunter in Begleitung einer Gefühlsminderung oder Dysästhesie im betroffenen Areal und häufig assoziiert mit einer Druckschmerzhaftigkeit über dem/den betroffenen Nerv(en).

\section{Diagnostische Kriterien:}

A. Ein- oder beidseitige Schmerzen im/in den Versorgungsgebiet(en) des N. occipitalis major, minor und/oder tertius, die die Kriterien B-D erfüllen

B. Der Schmerz weist mindestens zwei der folgenden Charakteristika auf:

1. in paroxysmalen Schmerzattacken rezidivierend, die zwischen wenigen Sekunden bis Minuten dauern

2. schwere Intensität

3. einschießend, stechend oder scharf

C. Der Schmerz wird von den beiden folgenden Punkten begleitet:

1. Dysästhesie und/oder Allodynie zeigen sich bei harmloser Stimulation der Kopfhaut und/oder des Haares

2. Einer oder beide der folgenden Punkte sind erfüllt:

a) Druckschmerzhaftigkeit über den betroffenen Nervenästen

b) Triggerpunkte am Austrittspunkt des N. occipitalis major oder im Versorgungsgebiet von $\mathrm{C} 2$

D. Durch eine Blockade des entsprechenden Nervs/der entsprechenden Nerven lassen sich die Beschwerden zeitweise lindern

E. Nicht besser erklärt durch eine andere ICHD-3-Diagnose.
Kommentar:

Die Schmerzen bei einer 13.4 Okzipitalisneuralgie können die frontoorbitale Region über trigemino-zervikale interneuronale Verbindungen in den Nuclei spinali nervi trigemini erreichen.

Die 13.4 Okzipitalisneuralgie muss von einer okzipitalen Schmerzprojektion aus dem Atlantoaxialgelenk oder den oberen Zygapophysealgelenken sowie von Triggerpunkten in der Halsmuskulatur oder ihren Ansatzstellen abgegrenzt werden.

\subsection{Nacken-Zungen-Syndrom (engl. Neck-tongue-syndrome)}

\section{Beschreibung:}

Plötzliche einsetzender, einseitiger, scharfer oder stechender und in der Regel heftiger Schmerz im Bereich des Hinterkopfs und/ oder der oberen Halsregion ausgelöst durch plötzliches Drehen des Kopfes, begleitet von einer abnormen Wahrnehmung und/oder Stellung der ipsilateralen Zunge.

\section{Diagnostische Kriterien:}

A. Mindestens zwei Episoden, die die Kriterien B bis D erfüllen

B. Scharfer oder stechender einseitiger Schmerz1 in der oberen Hals- und/oder Hinterkopfregion bei gleichzeitiger abnormer Wahrnehmung und/oder Stellung der ipsilateralen Zunge

C. Ausgelöst durch plötzliches Drehen des Kopfes

D. Schmerzdauer von Sekunden bis mehrere Minuten

E. Nicht besser erklärt durch eine andere ICHD-3-Diagnose.

\section{Anmerkung:}

1. Es kann eine gleichzeitige Dysästhesie vorliegen oder auch nicht.

\section{Kommentar:}

In einer neueren Untersuchung wurde diese Erkrankung ausführlich beschrieben, was ihre Aufwertung und Herausnahme aus dem Anhang rechtfertigt (wo sie in der ICHD-3 beta erschien).

\subsection{Schmerzhafte Optikusneuritis}

Früher verwendeter Begriff: Retrobulbärneuritis.

\section{Beschreibung:}

Schmerz hinter einem Auge oder beiden Augen verursacht durch Demyelinisierung des N. opticus/der Nn. optici und begleitet von einer Störung des zentralen Sehens.

\section{Diagnostische Kriterien:}

A. Ein- oder beidseitiger retroorbitaler, orbitaler, frontaler und/ oder temporaler Schmerz, der das Kriterium C erfüllt

B. Klinischer, elektrophysiologischer Nachweis oder Nachweis durch bildgebende Verfahren und/oder im Labor, der eine Optikusneuritis bestätigt1

C. Ein kausaler Zusammenhang kann durch die beiden folgenden Kriterien gezeigt werden:

1. Der Schmerz hat sich in einem zeitlichen Zusammenhang mit der Optikusneuritis entwickelt 
2. Der Schmerz verstärkt sich durch Augenbewegungen

D. Nicht besser erklärt durch eine andere ICHD-3-Diagnose.

\section{Anmerkung:}

1. Das Gadolinium-gestützte MRT zeigt bei $90 \%$ der Fälle von 13.6 schmerzhafter Optikusneuritis eine Kontrastverstärkung des N. opticus.

\section{Kommentar:}

Klinischen Versuchsreihen zufolge liegt die Schmerzprävalenz bei einer Optikusneuritis bei etwa $90 \%$ liegt. Die Schmerzen können der Sehstörung vorangehen.

Die 13.6 schmerzhafte Optikusneuritis ist eine häufige Manifestation einer Multiplen Sklerose.

\subsection{Kopfschmerz zurückzuführen auf eine ischämische Lähmung des \\ N. oculomotorius}

Beschreibung:

Einseitiger frontaler und/oder periorbitaler Schmerz, verursacht durch und begleitet von anderen Symptomen und/oder klinischen Zeichen einer ischämischen Parese des/der ipsilateralen 3., 4. und/ oder 6. Hirnnervs/Hirnnerven.

\section{Diagnostische Kriterien:}

A. Einseitiger frontaler und/oder periorbitaler Kopfschmerz, der Kriterium C erfüllt

B. Klinischer Nachweis sowie Nachweis durch bildgebende Verfahren, der einen Kopfschmerz zurückzuführen auf eine ischämische Lähmung des N. oculomotorius1 bestätigt.

C. Ein kausaler Zusammenhang kann durch die beiden folgenden Kriterien gezeigt werden:

1. Der Kopfschmerz ist ipsilateral zur Lähmung des N. oculomotorius

2. Der Kopfschmerz hat sich in einem zeitlichen Zusammenhang mit der Lähmung des N. oculomotorius entwickelt.

D. Nicht besser erklärt durch eine andere ICHD-3-Diagnose.

\section{Anmerkung:}

1. Ein 13.7 Kopfschmerz zurückzuführen auf eine ischämische Lähmung des N. oculomotorius kann vor dem oder gleichzeitig mit dem Beginn der Diplopie auftreten.

\section{Kommentar:}

Die Mehrheit der Lähmungen des N. oculomotorius ist schmerzhaft, ungeachtet dessen, ob ein Diabetes vorliegt oder nicht. Der Schmerz tritt am häufigsten bei Patienten mit Lähmungen des 3. Hirnnervs auf, weniger bei solchen mit einer Parese des 6. Hirnnervs und am wenigstens im Fall einer Parese des 4 . Hirnnervs.

\subsection{Tolosa-Hunt-Syndrom \\ Beschreibung:}

Einseitiger orbitaler oder periorbitaler Schmerz in Begleitung einer Parese des 3., 4. und/oder 6. Hirnnervs, sei es nur bei einem Hirnnerv oder mehreren hiervon, verursacht durch eine granulomatöse Entzündung im Sinus cavernosus, in der Fissura orbitalis superior oder der Orbita.

Diagnostische Kriterien:

A. Einseitiger orbitaler oder periorbitaler Kopfschmerz, der Kriterium C erfüllt

B. Beide der folgenden Punkte sind erfüllt:

1. Granulomatöse Entzündung des Sinus cavernosus, der Fissura orbitalis superior oder der Orbita nachgewiesen durch MRT oder Biopsie

2. Ipsilaterale Lähmung des 3., 4. und/oder 6. Hirnnerv oder mehrerer dieser Hirnnerven

C. Ein kausaler Zusammenhang kann durch folgendes gezeigt werden:

1. Der Kopfschmerz ist ipsilateral zu der granulomatösen Entzündung lokalisiert

2. Der Kopfschmerz ist der Lähmung des 3., 4. und/oder 6 . Hirnnervs um $\leq 2$ Wochen vorangegangen oder hat sich hiermit entwickelt

D. Nicht besser erklärt durch eine andere ICHD-3-Diagnose.

\section{Kommentar:}

Es gibt Einzelfallberichte über ein 13.8 Tolosa-Hunt-Syndrom mit zusätzlicher Beteiligung des 5. Hirnnervs (üblicherweise des 1. Astes) oder des Nervus opticus, des 7. oder 8. Hirnnervs. Die sympathische Innervation der Pupille ist gelegentlich mit betroffen.

Sorgfältige Nachuntersuchungen sind notwendig, um andere Ursachen einer schmerzhaften Ophthalmoplegie wie Tumore, Vaskulitis, basale Meningitis, Sarcoidose oder Diabetes mellitus.

Schmerz und Parese in Verbindung mit einem 13.8 Tolosa-Hunt-Syndrom verschwinden bei adäquater Behandlung mit Kortikosteroiden.

\subsection{Paratrigeminales okulosympathisches (Raeder-) Syndrom}

Beschreibung:

Konstanter, einseitiger Schmerz im Versorgungsgebiet des ophthalmischen Trigeminusasts, der sich mitunter bis in den maxillären Ast des N. trigeminus ausdehnt, begleitet von einem ipsilateralen Horner-Syndrom und verursacht durch eine Störung in der mittleren Schädelgrube oder der A. carotis.

Diagnostische Kriterien:

A. Konstanter, einseitiger Kopfschmerz, der Kriterium C erfüllt

B. Ipsilaterales Horner-Syndrom mit Nachweis der Grunderkrankung entweder der mittleren Schädelgrube oder der ipsilateralen A. carotis

C. Ein kausaler Zusammenhang kann durch beide der folgenden Kriterien gezeigt werden: 
1. Der Kopfschmerz hat sich in einem zeitlichen Zusammenhang mit dem Beginn der Grunderkrankung entwickelt oder führte zu ihrer Entdeckung

2. Einer oder beide der folgenden Punkte ist/sind erfüllt:

a) Lokalisation im Versorgungsgebiet des ophthalmischen Trigeminusnervasts, mit oder ohne Ausbreitung in den maxillären Ast

b) durch Augenbewegungen verstärkt

D. Nicht besser erklärt durch eine andere ICHD-3-Diagnose.

\section{Kommentar:}

Die ursprüngliche Beschreibung eines 13.9 paratrigeminalen okulosympathischen (Raeder-) Syndroms gilt als ein klassisches Beispiel für die klinisch-anatomische Vorgehensweise Anfang des 20. Jahrhunderts und war nützlich, da die Beteiligung oculopupillärer sympathischer Fasern auf eine Läsion der mittleren Schädelgrube verwies. Ob der Begriff Raeder-Syndrom heute noch benutzt werden sollte, ist heftig umstritten. Einige Autoren jedoch erachten ein schmerzhaftes Horner-Syndrom auch heute noch als diagnostisch nützlichen Hinweis auf eine Läsion der Fossa cranii media oder auf eine Dissektion der A. carotis.

\subsection{Rezidivierende schmerzhafte ophthalmoplegische Neuropathie}

Früher verwendeter Begriff: Ophthalmoplegische Migräne (dieser alte und unpassende Begriff wurde verworfen, da dieses Syndrom nicht migränöser Art ist, sondern es sich um eine rezidivierende schmerzhafte Neuropathie handelt).

\section{Beschreibung:}

Wiederholte Lähmungsattacken an einem oder mehreren okulomotorischen Hirnnerv(en) (üblicherweise am 3. Hirnnerv) mit ipsilateralem Kopfschmerz.

\section{Diagnostische Kriterien:}

A. Mindestens zwei Attacken, die Kriterium B erfüllen

B. Beide der folgenden Punkte sind erfüllt:

1. Unilateraler Kopfschmerz

2. Ipsilaterale Lähmung eines, zweier oder aller drei Augenbewegungsnerven 1

C. Ein parasellärer Prozess bzw. eine Läsion im Bereich der Fossa orbitalis oder der hinteren Schädelgrube konnten durch geeignete Untersuchungen ausgeschlossen werden.

D. Nicht besser erklärt durch eine andere ICHD-3-Diagnose.

\section{Anmerkung:}

1. Einige Daten lassen darauf schließen, dass sich Kopfschmerzen bis zu 14 Tage vor der Okulomotoriusparese entwickeln können.

\section{Kommentar:}

Eine Gadoliniumanreicherung oder Nervenverdickung können im MRT gezeigt werden.

Einige Patienten profitieren von einer Behandlung mit Kortikosteroiden.

\subsection{Syndrom des brennenden Mundes (engl. Burning mouth syndrome)}

Früher verwendete Begriffe Stomatodynie oder Glossodynie, sofern auf die Zunge beschränkt.

Beschreibung:

Intraorale brennende Missempfindung oder Sensibilitätsstörung, die über mehr als 3 Monate für mehr als 2 Stunden/Tag täglich wiederkehrt und für die keine klinisch evidenten ursächlichen Läsionen gefunden werden können.

\section{Diagnostische Kriterien:}

A. Schmerz im Bereich des Mundes1, der die Kriterien B und C erfüllt

B. Täglich für $>3$ Monate für $>2$ Stunden/Tag wiederkehrend

C. Der Schmerz weist beide der folgenden Charakteristika auf:

1. Brennende Qualität2

2. Oberflächlich in der Mundschleimhaut gespürt

D. Die Mundschleimhaut weist ein normales Erscheinungsbild auf und eine klinische Untersuchung einschließlich sensorischer Testung ist unauffällig

E. Nicht besser erklärt durch eine andere ICHD-3-Diagnose.

\section{Anmerkung:}

1. Der Schmerz tritt üblicherweise bilateral auf; häufigster Situs ist die Zungenspitze.

2. Die Schmerzintensität schwankt.

Kommentar:

Es können ein subjektives Gefühl der Mundtrockenheit, Dysästhesien und eine Beeinträchtigung des Geschmackes auftreten.

Es besteht eine hohe Prävalenz bei Frauen in den Wechseljahren, und einige Studien zeigen komorbide psychosoziale und psychiatrische Erkrankungen. Laboruntersuchungen und zerebrale Bildgebung verwiesen auf Veränderungen im Zentral- und peripheren Nervensystem.

Ob ein sekundäres Syndrom des brennenden Mundes zurückzuführen auf eine lokale (Candidiasis, Lichen ruber planus, Hyposalivation) oder systemische Störung (medikamenteninduziert, Anämie, Vitamin-B12- oder Folsäuremangel, Sjögren-Syndrom, Diabetes) als eine eigenständige Entität angesehen werden sollten, ist umstritten. Die aktuellen Erkenntnisse rechtfertigen nicht einmal eine Aufnahme in den Anhang.

\subsection{Anhaltender idiopathischer Gesichtsschmerz} (engl. Persistent idiopathic facial pain)

Früher verwendeter Begriff: Atypischer Gesichtsschmerz.

\section{Beschreibung:}

Anhaltender Gesichtsschmerz und/oder Schmerz im Bereich des Mundes mit unterschiedlichen Erscheinungsbildern, der aber über mehr als 3 Monate für mehr als 2 Stunden/Tag wiederkehrt, bei fehlenden klinischen neurologischen Defiziten. 
Diagnostische Kriterien:

A. Gesichtsschmerz und/oder Schmerz im Mundbereich, der die Kriterien B und C erfüllt

B. Für $>3$ Monate über $>2$ Stunden/Tag täglich wiederkehrend

C. Der Schmerz weist beide der folgenden Charakteristika auf:

1. Schwer zu lokalisieren und nicht dem Versorgungsgebiet eines peripheren Nervs folgend

2. Dumpfe, anhaltende oder bohrende Qualität

D. Die klinische neurologische Untersuchung ist unauffällig

E. Eine dentale Ursache wurde durch entsprechende Untersuchungen ausgeschlossen

F. Nicht besser erklärt durch eine andere ICHD-3-Diagnose.

\section{Kommentar:}

Patienten gebrauchen eine große Bandbreite von Worten, um den Charakter eines 13.12 anhaltenden idiopathischen Gesichtsschmerzes zu beschreiben, am häufigsten wird er aber als dumpf, bohrend oder anhaltend beschrieben, entweder tiefsitzend oder oberflächlich. Es können starke Exacerbationen auftreten und der Schmerz verstärkt sich durch Stress. Im Laufe der Zeit kann er sich über ein größeres Areal der kraniozervikalen Region ausbreiten.

Patienten mit einem 13.12 anhaltenden idiopathischen Gesichtsschmerz sind vorwiegend weiblichen Geschlechts.

Ein 13.12 anhaltender idiopathischer Gesichtsschmerz kann eine Komorbidität mit anderen Schmerzzuständen wie einem generalisierten Schmerzsyndrom und Reizdarmsyndrom aufweisen. Darüber hinaus tritt er in hohem Maße mit psychiatrischer Komorbidität und psychosozialen Behinderungen auf.

Der 13.12 anhaltende idiopathische Gesichtsschmerz kann auf einen kleinen operativen Eingriff oder eine Verletzung des Gesichtes, des Oberkiefers, der Zähne oder des Zahnfleischs zurückgehen, aber nach der Heilung des initialen schädigenden Ereignisses ohne nachweisliche lokale Ursache fortbestehen. Psychophysische oder neurophysiologische Befunde können jedoch sensible Auffälligkeiten zeigen. Es scheint ein Kontinuum von einem 13.12 anhaltenden idiopathischen Gesichtsschmerz induziert durch ein unbedeutendes Trauma zu einer 13.1.2.3 schmerzhaften posttraumatischen Trigeminusneuropathie offenbar verursacht durch signifikanten Insult der peripheren Nerven zu bestehen.

Der Begriff atypische Odontalgie wurde für einen kontinuierlichen Schmerz im Bereich eines Zahns oder mehrerer Zähne oder der Zahnhöhle nach Extraktion verwandt, wenn andere übliche dentale Ursachen fehlen. Sie gilt als ein Subtyp eines 13.12 anhaltenden idiopathischen Gesichtsschmerzes, obwohl er umschriebener ist, das mittlere Alter bei Krankheitsbeginn ist jünger und die Verteilung auf die Geschlechter ausgewogener. Auf Grundlage der Traumavorgeschichte kann eine atypische Odontalgie auch ein Subtyp einer 13.1.2.3 schmerzhaften posttraumatischen Trigeminusneuropathie sein. Diese Subtypen, sofern diese existieren, wurden nicht ausreichend untersucht, um diagnostische Kriterien vorzuschlagen.

\subsection{Zentraler neuropathischer Schmerz}

\section{Beschreibung:}

Ein- oder beidseitiger kraniozervikaler Schmerz zentralen Ursprungs mit wechselndem Erscheinungsbild mit oder ohne sensible Veränderungen. Je nach Ursache kann er konstant oder remittierend und rezidivierend sein.

\subsubsection{Zentraler neuropathischer Schmerz zurück- zuführen auf Multiple Sklerose (MS)}

Beschreibung:

Ein- oder beidseitiger kraniozervikaler Schmerz mit wechselndem Erscheinungsbild, mit oder ohne sensible Veränderungen, zurückzuführen auf eine demyelinisierende Läsion der zentralen aufsteigenden Verbindungen des N. trigeminus bei Personen mit Multipler Sklerose, der üblicherweise remittiert und wieder auftritt.

Diagnostische Kriterien:

A. Gesichts- und/oder Kopfschmerz, der Kriterium C erfüllt1

B. Es wurde eine Multiple Sklerose diagnostiziert, mit Nachweis einer demyelinisierenden Läsion im Hirnstamm oder den aufsteigenden Projektionsbahnen der Nuclei spinales nervi trigemini im MRT

C. Der Schmerz hat sich in einem zeitlichen Zusammenhang mit der demyelinisierenden Läsion entwickelt oder führte zu ihrer Entdeckung

D. Nicht besser erklärt durch eine andere ICHD-3-Diagnose.

Anmerkung:

1. Der Schmerz kann paroxysmal oder kontinuierlich sein.

Kommentar:

Nicht-schmerzhafte sensible Auffälligkeiten (gewöhnlich Dysästhesie, aber auch Hypästhesie, Anästhesie, Hypalgesie, Parästhesie, etc.) können gemeinsam mit einem Schmerz bei 13.13.1 zentralem neuropathischen Schmerz zurückzuführen auf Multiple Sklerose vorkommen.

\subsubsection{Zentraler neuropathischer Schmerz nach Hirninfarkt (engl. Central post stroke pain)}

Beschreibung:

Üblicherweise einseitiger Gesichts- und/oder Kopfschmerz mit unterschiedlichem Erscheinungsbild, der Teile oder die Gesamtheit der kraniozervikalen Region miteinschließt und sensiblen Beeinträchtigungen verbunden ist, innerhalb von 6 Monaten nach einem Hirninfarkt auftritt und von diesem verursacht wird. Er ist nicht durch eine Läsion des peripheren N. trigeminus oder anderer Hirnoder Halsnerven erklärbar.

Diagnostische Kriterien:

A. Gesichts- und/oder Kopfschmerz, der das Kriterium C erfüllt

B. Zustand nach ischämischem oder hämorrhagischem Infarkt

C. Ein kausaler Zusammenhang kann durch beide der folgenden Kriterien gezeigt werden:

1. Der Schmerz hat sich innerhalb von 6 Monaten nach dem Infarkt entwickelt 
2. Die Bildgebung1 konnte eine vaskuläre Läsion an einem entsprechenden Ort nachweisen

D. Nicht besser erklärt durch eine andere ICHD-3-Diagnose. Anmerkung:

1. Üblicherweise MRT.

\section{Kommentar:}

Ein 13.13.2 zentraler neuropathischer Schmerz nach Hirninfarkt wird auf eine Läsion der aufsteigenden Projektionen der Nuclei spinales nervi trigemini zurückgeführt. Auch zervikale spinothalamische Bahnen und die kortikale Verarbeitung können eine wichtige Rolle spielen. Die Symptomatik kann daher auch den Rumpf und/ oder die Extremitäten der betroffenen Seite mit einbeziehen.

Kraniozervikale Schmerzen in Folge einer Thalamusläsion sind normalerweise Teil eines Hemisyndroms. Bei einer lateralen Läsion der Medulla oblongata kann der halbseitige Gesichtsschmerz auch isoliert auftreten, ist aber häufiger verbunden mit einer gekreuzten Hemidysästhesie.

\section{Literatur}

\subsubsection{Trigeminusneuralgie}

Benoliel R, Eliav E, Sharav Y. Self-reports of pain-related awakenings in persistent orofacial pain patients. J Orofac Pain 2009; 23: 330-338.

Cruccu G, Finnerup NB, Jensen TS, Scholz J, Sindou M, Svensson P, Treede R-D, Zakrzweska JM, Nurmikko T. Trigeminal neuralgia: new classification and diagnostic grading for clinical practice and research. Neurology 2016; 87: 220-228.

Di Stefano G, Maarbjerg S, Nurmikko T, Truini A, Cruccu G. Triggering trigeminal neuralgia. Cephalalgia 2017 Jan 1:333102417721677. doi: 10.1177/0333102417721677 [Epub ahead of print].

Drangsholt M, Truelove E. Trigeminal neuralgia mistaken as temporomandibular disorder. J Evid Base Dent Pract 2001; 1: 41-50.

Fromm GH, Graff-Radford SB, Terrence CF, Sweet WH. Pre-trigeminal neuralgia. Neurology 1990; 40: 1493-1495.

Haviv Y, Khan J, Zini A, Almoznino G, Sharav Y, Benoliel R. Trigeminal neuralgia (part I). Revisiting the clinical phenotype. Cephalalgia 2016; 36; 730-746.

Koopman JSHA, Dieleman JP, Huygen FJ, de Mos N, Martin CGM, Sturkenboom MCJM. Incidence of facial pain in the general population. Pain 2009;147:122-7

Mueller D, Obermann M, Yoon MS, et al. Prevalence of trigeminal neuralgia and persistent idiopathic facial pain: A population-based study. Cephalalgia 2011; 31: 1542-1548.

Obermann M, Yoon MS, Ese D et al. Impaired trigeminal nociceptive processing in patients with trigeminal neuralgia. Neurology 2007; 69: 835-841.

Pareja JA, Cuadrado ML, Caminero AB, Barriga FJ, Baron M, Sanchez-del-Rio M. Duration of attacks of first division trigeminal neuralgia. Cephalalgia 2005; 25: 305-308.

Rasmussen P. Facial pain. II. A prospective survey of 1052 patients with a view of: character of the attacks, onset, course, and character of pain. Acta Neurochir (Wien) 1990; 107: 121-128.

Rasmussen P. Facial pain. III. A prospective study of the localization of facial pain in 1052 patients. Acta Neurochir (Wien) 1991; 108: 53-63.

Rasmussen P. Facial pain. IV. A prospective study of 1052 patients with a view of: precipitating factors, associated symptoms, objective psychiatric and neurological symptoms. Acta Neurochir (Wien) 1991; 108: 100-109.

\subsubsection{Klassische Trigeminusneuralgie}

Antonini G, Di Pasquale A, Cruccu G, et al. Magnetic resonance imaging contribution for diagnosing symptomatic neurovascular contact in classical trigeminal neuralgia: a blinded case-control study and meta-analysis. Pain 2014; 155: 1464-1471.

Bowsher D, Miles JB, Haggett CE, Eldridge PR. Trigeminal neuralgia: a quantitative sensory perception threshold study in patients who had not undergone previous invasive procedures. J Neurosurg 1997; 86: 190-192.

Leal PR, Barbier C, Hermier M, Souza MA, Cristino-Filho G, Sindou M. Atrophic changes in the trigeminal nerves of patients with trigeminal neuralgia due to neurovascular compression and their association with the severity of compression and clinical outcomes. J Neurosurg 2014; 120: $1484-1495$.

Maarbjerg S, Wolfram F, Gozalov A, Olesen J, Bendtsen L. Significance of neurovascular contact in classical trigeminal neuralgia. Brain 2015; 138: 311-319.

\subsubsection{Sekundäre Trigeminusneuralgie}

Cheng TM, Cascino TL and Onofrio BM. Comprehensive study of diagnosis and treatment of trigeminal neuralgia secondary to tumors. Neurology 1993; 43: 2298-2302.

Coffey RJ, Fromm GH. Familial trigeminal neuralgia and Charcot-Marie-Tooth neuropathy. Report of two families and review. Surg Neurol 1991; 35 : 49-53.

Cruccu G, Biasiotta A, Di RS, et al. Trigeminal neuralgia and pain related to multiple sclerosis. Pain 2009; 143: 186-191.

De Paula Lucas C, Zabramski JM. Dural arteriovenous fistula of the transverse-sigmoid sinus causing trigeminal neuralgia. Acta Neurochir 2007; 149: 1249-1253.

O'Connor AB, Schwid SR, Herrmann DN, et al. Pain associated with multiple sclerosis: systematic review and proposed classification. Pain 2008; 137: 96-111.

Tanaka BS, Zhao P, Dib-Hajj FB, Morisset V, Tate S, Waxman SG, Dib-Hajj SD. A gain-of-function mutation in Nav1.6 in a case of trigeminal neuralgia. Mol Med 2016; 22: 338-348.

Truini A, Prosperini L, Calistri V, et al. A dual concurrent mechanism explains trigeminal neuralgia in patients with multiple sclerosis. Neurology 2016; 86: 2094-2099.

Wei Y, Zhao W, Pu C, et al. Clinical features and long-term surgical outcomes in 39 patients with tumor-related trigeminal neuralgia compared with 360 patients with idiopathic trigeminal neuralgia. $\mathrm{Br}$ J Neurosurg 2017; 31: 101-106.

Yip V, Michael BD, Nahser HC, Smith D. Arteriovenous malformation: a rare cause of trigeminal neuralgia identified by magnetic resonance imaging with constructive interference in steady state sequences. QJM 2012;105:895-898.

\subsubsection{Idiopathische Trigeminusneuralgie}

Lee A, McCartney S, Burbidge C, Raslan AM, Burchiel KJ. Trigeminal neuralgia occurs and recurs in the absence of neurovascular compression. J Neurosurg 2014;120:1048-1054.

\subsubsection{Schmerzhafte Trigeminusneuropathie zurückzuführen auf einen Herpes zoster}

Dworkin RH, Portenoy RK. Pain and its persistence in herpes zoster. Pain 1996; 67: 241-252.

Haanpää M, Dastidar P, Weinberg A, Levin M, Miettinen A, Lapinlampi A-M, Laippala P, Nurmikko T. Characteristics of cerebrospinal fluid and magnetic resonance imaging findings in patients with acute herpes zoster. Neurology 1998; 51: 1405-1411.

Liesegang TJ. Herpes zoster ophthalmicus. Natural history, risk factors, clinical presentation, and morbidity. Ophthalmology 2008; 115 (2 Suppl): S3-12. 


\subsubsection{Postherpetische Trigeminusneuralgie}

Alvarez FK, de Siqueira SR, Okada M, et al. Evaluation of the sensation in patients with trigeminal post-herpetic neuralgia. J Oral Pathol Med 2007; 36: 347-350.

Truini A, Galeotti F, Haanpää M, et al. Pathophysiology of pain in postherpetic neuralgia: A clinical and neurophysiological study. Pain 2008; 140: 405-410.

Truini A, Haanpää M, Provitera V, et al. Differential myelinated and unmyelinated sensory and autonomic skin nerve fiber involvement in patients with ophthalmic postherpetic neuralgia. Front Neuroanat 2015; 9: 105.

\subsubsection{Schmerzhafte traumatische Trigeminusneuropathie}

Benoliel R, Birenboim R, Regev E, Eliav E. Neurosensory changes in the infraorbital nerve following zygomatic fractures. Oral Surg 2005; 99 : 657-665.

Benoliel R, Zadik Y, Eliav E and Sharav Y. Peripheral painful traumatic trigeminal neuropathy: Clinical features in 91 cases and proposal of novel diagnostic criteria. J Orofac Pain 2012; 26: 49-58.

Jääskeläinen S K, Teerijoki-Oksa T and Forssell H. Neurophysiologic and quantitative sensory testing in the diagnosis of trigeminal neuropathy and neuropathic pain. Pain 2005; 117: 349-357.

Polycarpou N, Ng YL, Canavan D, et al. Prevalence of persistent pain after endodontic treatment and factors affecting its occurrence in cases with complete radiographic healing. Int Endod J 2005; 38: 169-178.

Queral-Godoy E, Figueiredo R, Valmaseda-Castellon E, Berini-Aytes L, Gay-Escoda C. Frequency and evolution of lingual nerve lesions following lower third molar extraction. J Oral Maxillofac Surg 2006; 64 : 402-407.

Renton T and Yilmaz Z. Profiling of patients presenting with posttraumatic neuropathy of the trigeminal nerve. J Orofac Pain 2011; 25: 333-344.

13.1.2.4 Schmerzhafte Trigeminusneuropathie zurückzuführen auf eine andere Erkrankung

Cruccu G, Penisi EM, Antonini G, et al. Trigeminal isolated sensory neuropathy (TISN) and FOSMN syndrome: despite a dissimilar disease course do they share common pathophysiological mechanisms? BMC Neurol 2014; 14: 248 .

Klasser GD, Balasubramaniam R, Epstein J. Topical review-Connective tissue diseases: Orofacial manifestations including pain. J Orofac Pain 2007; 21: $171-184$

\subsubsection{Glossopharyngeusneuralgie}

Blumenfeld A, Nikolskaya G. Glossopharyngeal neuralgia. Curr Pain Headache Rep 2013; 17: 343.

Huynh-Le P, Matsishima T. Hisada K. Matsumoto K. Glossopharyngeal neuralgia due to an epidermoid tumour in the cerebellopontine angle. J Clin Neurosci 2004; 11: 758-760.

Kandan SR, Khan S,. Jeyaretna DS, Lhatoo S, Patel NK, Coakham HB. Neuralgia of the glossopharyngeal and vagal nerves: long-term outcome following surgical treatment and literature review. Br J Neurosurg 2010; 24: 441-446.

Minagar A, Sheremata WA. Glossopharyngeal neuralgia and MS. Neurology 2000; 54: 1368-1370.

Patel A, Kassam A, Horowitz M, Chang YF. Microvascular decompression in the management of glossopharyngeal neuralgia: Analysis of 217 cases. Neurosurgery 2002; 50: 705-710.

Peet MM. Glossopharyngeal neuralgia. Ann Surg 1935; 101: 256-258.

Saman Y, Whitehead D, Gleeson M. Jugular foramen schwannoma presenting with glossopharyngeal neuralgia syncope syndrome. J Laryngol Otol 2010; 124: 1305-1308.
Tanrikulu L, Hastreiter P, Dorfler A, Buchfelder M, Naraghi R. Classification of neurovascular compression in glossophgaryngeal neuralgia: three-dimensional visualiztion of the glossopharyngeal nerve. Surg Neurol Int 2015; 6: 189.

\subsubsection{Schmerzhafte Glossopharyngeusneuropathie}

Bakar B. The jugular foramen schwannomas : Review of the large surgical series. J Kor Neurosurg Soc 2008; 44: 285-294.

Kalladka M, Nasri-Heir C, Eliav E, Ananthan S, Viswanath A, Heir G. Continuous neuropathic pain secondary to endoscopic procedures: report of two cases and review of the literature. Oral Surg Oral Med Oral Pathol Oral Radiol 2016; 122: e55-e59.

Shin HY, Park HJ, Choi YC, Kim SM. Clinical and electromyographic features of radiation-induced lower cranial neuropathy. Clin Neurophysiol 2013; 124: 598-602.

\subsubsection{Intermediusneuralgie}

Pulec JL. Geniculate neuralgia: Long-term results of surgical treatment. Ear Nose Throat ] 2002; 81: 30-33.

Riederer F, Sándor PS, Linnebank M, Ettlin DA. Familial occipital and nervus intermedius neuralgia in a Swiss family. J Headache Pain 2010; 11: 335-338.

Saers SJF, Han KS, de Rue JA. Microvascular decompression may be an effective treatment for nervus intermedius neuralgia. J Laryngol Otol 2011; 125: 520-522.

Tubbs RS, Steck DT, Mortazavi MM, Cohgen-Gadol AA.The nervus intermedius: A review of its anatomy, function, pathology, and role in neurosurgery. World Neurosurg 2013; 79: 763-767.

\subsubsection{Schmerzhafte Intermediusneuralgie}

Günther M, Danckwardt-Lillieström, Gudjonsson O, Nyberg G, Kinnefors A, Rask-Andersen H, Ekvall L. Surgical treatment of patients with facial neuromas. Report of 26 consecutive operations. Otol Neurotol 2010; 31: 1493-1497.

Oldenburg MS, Carlson ML, Van Abel KM, Driscoll CL, Link MJ. Management of geniculate ganglion hemangiomas. Case series and systematic review of the literature. Otol Neurotol 2015; 36: 1735-1740.

Sweeney CJ, Gilden DH. Ramsay Hunt syndrome. J Neurol Neurosurg Psychiatry $2001 ; 71: 149-154$.

\subsection{Okzipitalisneuralgie}

Bartsch T, Goadsby P. Anatomy and physiology of pain referral in primary and cervicogenic headache disorders. Headache Curr 2005; 2: 42-48.

Boes Ch. C2 myelitis presenting with neuralgiform occipital pain. Neurology 2005; 64: 1093-1094.

Bogduk $\mathrm{N}$ et al The anatomy and pathphysiology of neck pain. Phys Med Rehabil Clin North Amer 2005; 14: 455-472.

Ehni G, Benner B. Occipital neuralgia and the C1-C2 arthrosis syndrome. NEJM 1984; 310: 127.

\subsection{Nacken-Zungen-Syndrom}

Bogduk N. An anatomical basis for the neck-tongue syndrome. J Neurol Neurosurg Psychiatry 1981; 44: 202-208.

Elisevich K, Stratford J, Bray G, et al. Neck tongue syndrome: Operative management. J Neurol Neurosurg Psychiatry 1984; 47: 407-409.

Evans RW, Lance JW. Expert opinion: Transient headache with numbness of half of the tongue. Headache 2000; 40: 692-693.

Fortin CJ, Biller J. Neck tongue syndrome. Headache 1985; 25: 255-258.

Gelfand AA, Johnson H, Lenaerts ME, Litwin JR, De Mesa C, Bogduk N, Goadsby PJ. Neck-tongue syndrome: A systematic review. Cephalalgia 2017. doi: $10.1177 / 0333102416681570$ [Epub ahead of print]. 
Lance JW, Anthony M. Neck-tongue syndrome on sudden turning of the head. J Neurol Neurosurg Psychiatry 1980; 43: 97-101.

Lenaerts M, Poblete R. Neck-tongue syndrome and its presentations. Cephalalgia 2015; 35: 143.

Lewis DW, Frank LM, Toor S. Familial neck-tongue syndrome. Headache 2003; 43: 132-134.

Orrell RW, Marsden CD. The neck-tongue syndrome. J Neurol Neurosurg Psychiatry 1994; 57: 348-352.

Sjaastad O, Bakketeig LS. Neck-tongue syndrome and related (?) conditions. Cephalalgia 2006; 26: 233-240.

Webb J, March L, Tyndall A. The neck-tongue syndrome: Occurrence with cervical arthritis as well as normals. J Rheumatol 1984; 11: 530-533.

Wig S, Romanowski C, Akil M. An unusual cause of the neck-tongue syndrome. J Rheumatol 2009; 36: 857-858.

Wong SL, Paviour DC, Clifford-Jones RE. Chiari-1 malformation and the neck-tongue syndrome: cause or coincidence? Cephalalgia 2008; 28: 994-995.

\subsection{Schmerzhafte Optikusneuritis}

Du Y, Yang J, Li JJ, Zhou RW, He JF. Unilateral optic neuritis in a Chinese population in three centers. J Clin Neurosci 2011; 18: 902-904.

Fazzone H E, Lefton DR, Kupersmith MJ. Optic neuritis: correlation of pain and magnetic resonance imaging. Ophthalmology 2003; 110: 1646-1649.

Optic Neuritis Study Group. The clinical profile of optic neuritis. Experience of the Optic Neuritis Treatment Trial. Arch Ophthalmol 1991; 109: $1673-1678$

13.7 Kopfschmerz zurückzuführen auf eine ischämische Lähmung des N. oculomotorius

Kennard C. Disorders of eye movements I. In Swash M, Oxbury J (eds). Clinical neurology. Edinburgh: Churchill Livingstone 1991: 446-447.

Waind APB. Ocular nerve palsy associated with severe headache. BM] 1956; 2: 901-902.

Wilker S, Rucker J, Newman N, Biousse V, Tomsak R. Pain in ischemic ocular motor nerve palsies. Br J Ophthalmology 2009; 93: 1657-1659.

\subsection{Tolosa-Hunt-Syndrom}

Cakirer S. MRI findings in Tolosa-Hunt syndrome before and after systemic corticosteroid therapy. Eur J Radiol 2003; 45: 83-90.

Cohn DF, Carasso R, Streifler M. Painful ophthalmoplegia: the Tolosa-Hunt syndrome. Eur Neurol 1979; 18: 373-381.

de Arcaya AA, Cerezal L, Canga A, Polo JM, Berciano J, Pascual J. Neuroimaging diagnosis of Tolosa-Hunt syndrome: MRI contribution. Headache 1999; 39: 321-325.

Goto Y, Goto I, Hosokawa S. Neurological and radiological studies in painful ophthalmoplegia: Tolosa-Hunt syndrome and orbital pseudotumour. J Neurol 1989; 236: 448-451.

La Mantia L, Curone M, Rapoport AM, Bussone G. Tolosa-Hunt syndrome: critical literature review based on IHS 2004 criteria. Cephalalgia 2006; 26: 772-781.

Odabasi Z, Gokcil Z, Atilla S, Pabuscu Y, Vural O, Yardim M. The value of MRI in a case of Tolosa-Hunt syndrome. Clinical Neurol Neurosurg 1997; 99: 151-154.

Straube A, Bandmann O, Buttner U, Schmidt H. A contrast enhanced lesion of the III nerve on MR of a patient with ophthalmoplegic migraine as evidence for a Tolosa-Hunt syndrome. Headache 1993; 33: 446-448.
13.9 Paratrigeminales okulosympathisches (Raeder-) Syndrom

Goadsby PJ. Raeder's syndrome: paratrigeminal paralysis of the oculopupillary sympathetic system. J Neurol Neurosurg Psychiat 2002; 72: 297-299.

Shoja MM, Tubbs RS, Ghabili K, Loukas M, Oakes W], Cohen-Gadol AA. Johan Georg Raeder and paratrigeminal sympathetic paresis. Childs Nerv Syst 2010; 26: 373-376.

Solomon S. Raeder syndrome. Arch Neurol 2001; 58: 661-662.

13.10 Rezidivierende schmerzhafte ophthalmoplegische Neuropathie

Bharucha DX, Campbell TB, Valencia I, Hardison HH, Kothare SV. MRI findings in pediatric ophthalmoplegic migraine: a case report and literature review. Pediatric Neurol 2007; 37: 59-63.

Doran M, Larner AJ. MRI findings in ophthalmoplegic migraine: nosological implications. J Neurol 2004; 251: 100-101.

Gelfand AA, Gelfand JM, Prabakhar P, Goadsby PJ. Ophthalmoplegic "migraine" or recurrent ophthalmoplegic cranial neuropathy: New cases and a systematic review. J Child Neurol 2012; 27: 759-766.

Lance JW, Zagami AS. Ophthalmoplegic migraine: a recurrent demyelinating neuropathy? Cephalalgia 2001; 21: 84-89.

Weiss AH, Phillips JO. Ophthalmoplegic migraine. Pediatric Neurol 2004; 30: 64-66.

\subsection{Syndrom des brennenden Mundes}

Bergdahl M, Bergdahl J. Burning mouth syndrome: prevalence and associated factors. J Oral Pathol Med 1999; 28: 350-354.

Eliav E, Kamran B, Schaham R, Czerninski R, Gracely RH, Benoliel R. Evidence of chorda tympani dysfunction in patients with burning mouth syndrome. J Am Dent Assoc 2007; 138: 628-633.

Forssell $\mathrm{H}$, Jaaskelainen $\mathrm{S}$, Tenovuo O, Hinkka S. Sensory dysfunction in burning mouth syndrome. Pain 2002; 99: 41-47.

Jaaskelainen SK, Forssell H, Tenovuo O. Abnormalities of the blink reflex in burning mouth syndrome. Pain 1997; 73: 455-460.

Lauria G, Majorana A, Borgna M, Lombardi R, Penza P, Padovani A, et al. Trigeminal small-fiber sensory neuropathy causes burning mouth syndrome. Pain 2005; 115: 332-337.

Patton LL, Siegel MA, Benoliel R, De Laat A. Management of burning mouth syndrome: systematic review and management recommendations. Oral Surg Oral Med Oral Pathol Oral Radiol Endod 2007; 103 Suppl 39 : $1-13$.

Sardella A, Gualerzi A, Lodi G, Sforza C, Carrassi A, Donetti E. Morphological evaluation of tongue mucosa in burning mouth syndrome. Arch Oral Biol 2012; 57: 94-101.

Scala A, Checchi L, Montevecchi M, Marini I, Giamberardino MA. Update on burning mouth syndrome: overview and patient management. Crit Rev Oral Biol Med 2003; 14: 275-291.

Woda A, Pionchon P. A unified concept of idiopathic orofacial pain: clinical features. J Orofac Pain 1999; 13: 172-184.

\subsection{Anhaltender idiopathischer Gesichtsschmerz}

Aggarwal VR, McBeth J, Lunt M, Zakrzewska JM, Macfarlane GJ. Development and validation of classification criteria for idiopathic orofacial pain for use in population-based studies. J Orofac Pain 2007; 21: 203-215.

Aggarwal VR, McBeth J, Lunt M, Zakrzewska J, Macfarlane GJ. Epidemiology of chronic symptoms that are frequently unexplained: do they share common associated factors? Int J Epidemiol 2006; 35; 468-476.

Forssell H, Tenovuo O, Silvoniemi P, Jääskeläinen SK. Differences and similarities between atypical facial pain and trigeminal neuropathic pain. Neurology 2007; 69: 1451-1459.

List T, Leijon G, Svensson P. Somatosensory abnormalities in atypical odontalgia: A case-control study. Pain 2008; 139: 333-341. 
Pfaffenrath V, Rath M, Pollmann W, Keeser W. Atypical facial pain - application of the IHS criteria in a clinical sample. Cephalalgia 1993; 13 Suppl 12: 84-88.

Sardella A, Demarosi F, Barbieri C, Lodi G. An up-to-date view on persistent idiopathic facial pain. Minerva Stomatol 2009; 58: 289-299.

\subsection{Zentraler neuropathischer Schmerz}

Abhinav K, Love S, Kalantzis G, Coakham HB, Patel NK. Clinicopathological review of patients with and without multiple sclerosis treated by partial sensory rhizotomy for medically refractory trigeminal neuralgia: A 12-year retrospective study. Clin Neurol Neurosurg 2012; 114: 361-365.

Cruccu G, Biasiotta A, Di Rezze S, Fiorelli M, Galeotti F, Innocenti P, et al. Trigeminal neuralgia and pain related to multiple sclerosis. Pain 2009; 143: 186-191.

Jensen TS, Rasmussen P, Reske-Nielsen E. Association of trigeminal neuralgia with multiple sclerosis : clinical pathological features. Acta Neurol Scand 1982; 65: 182-189.

Putzki N, Pfriem A, Limmroth V, Yaldizli O, Tettenborn B, Diener HC, et al. Prevalence of migraine, tension-type headache and trigeminal neuralgia in multiple sclerosis. Eur J Neurol 2009; 16: 262-267.

\subsubsection{Zentraler neuropathischer Schmerz zurückzuführen auf Multiple Sklerose}

Mills RJ, Young CA, Smith ET. Central trigeminal involvement in multiple sclerosis using high-resolution MRI at $3 \mathrm{~T}$. Br J Radiol 2010; 83: 493-498.

Osterberg A, Boivie J. Central pain in multiple sclerosis - sensory abnormalities. Eur J Pain 2010; 14: 104-110.

Osterberg A, Boivie J, Thuomas KA. Central pain in multiple sclerosis - prevalence and clinical characteristics. Eur J Pain 2005; 9: 531-542.

\subsubsection{Zentraler neuropathischer Schmerz nach Schlaganfall}

Bowsher D, Leijon G, Thuomas KA. Central poststroke pain. Correlation of MRI with clinical pain characteristics and sensory abnormalities. Neurology 1998; 51: 1352-1358.

Fitzek S, Baumgartner U, Fitzek $C$ et al. Mechanisms and Predictors of chronic facial pain in lateral medullary infarction. Ann Neurol 2001; 49: 493-500.

Hong JH, Bai DS, Jeong JY, Choi BY, Chang CH, Kim SH, et al. Injury of the spino-thalamo-cortical pathway is necessary for central post-stroke pain. Eur Neurol 2010; 64: 163-168.

Kalita J, Kumar B, Misra UK, Pradhan PK. Central post stroke pain: clinical, MRI, and SPECT correlation. Pain Med 2011; 12: 282-288.

Klit H, Finnerup NB, Jensen TS. Central post-stroke pain: clinical characteristics, pathophysiology, and management. Lancet-Neurology 2009; 8: 857-868.

MacGowan DJ, Janal MN, Clark WC, Wharton RN, Lazar RM, Sacco RL, et al. Central poststroke pain and Wallenberg's lateral medullary infarction: frequency, character, and determinants in 63 patients. Neurology 1997; 49: 120-125.

Tuveson B, Leffler AS, Hansson P. Influence of heterotopic noxious conditioning stimulation on spontaneous pain and dynamic mechanical allodynia in central post-stroke pain patients. Pain 2009; 143: 84-91.

\section{Andere Kopfschmerzerkrankungen}

14.1

14.2
Kopfschmerz nicht anderweitig klassifiziert

Kopfschmerz nicht spezifiziert

\section{Einleitung}

Um die vorliegende Klassifikation zu vervollständigen, wurden im Anschluss an zahlreiche Kopfschmerzformen jeweils Unterkategorien für Kopfschmerzzustände angehängt, die alle Kriterien der jeweiligen Kopfschmerzform mit einer Ausnahme erfüllen. Trotzdem dürften Kopfschmerzen existieren, die in keines der aufgeführten Kapitel passen, da sie zum ersten Mal beschrieben werden oder bisher einfach nicht genug Informationen über diese Kopfschmerzen zur Verfügung stehen. Dieses Kapitel ist für diese Kopfschmerztypen und Subtypen gedacht

\subsection{Kopfschmerz nicht anderweitig klassifiziert}

Früher verwendete Begriffe: Nicht klassifizierbarer Kopfschmerz.

\section{Diagnostische Kriterien:}

A. Kopfschmerz mit charakteristischen Merkmalen, die auf eine eigenständige diagnostische Entität schließen lassen

B. Erfüllt nicht die Kriterien einer der in dieser Klassifikation beschriebenen Kopfschmerzerkrankungen.

\section{Kommentar:}

Zwischen dem Erscheinen der ersten Auflage der Internationalen Klassifikation von Kopfschmerzerkrankungen und der jetzigen dritten Auflage wurden zahlreiche neue Kopfschmerzentitäten beschrieben. Es ist vorauszusehen, dass noch mehr Entitäten zu beschreiben sind. Diese Kopfschmerzen können vorerst unter 14.1 Kopfschmerz nicht anderweitig klassifiziert kodiert werden.

\subsection{Kopfschmerz nicht spezifiziert}

Früher verwendete Begriffe: Nicht klassifizierbarer Kopfschmerz.

\section{Diagnostische Kriterien:}

A. Kopfschmerz ist oder war vorhanden

B. Es stehen nicht genug Informationen zur Verfügung, um den Kopfschmerz irgendeiner Ebene dieser Klassifikation zuzuordnen.

\section{Kommentar:}

Es ist offensichtlich, dass bei vielen Patienten Diagnosen gestellt werden müssen, bei denen sehr wenig Informationen zur Verfügung stehen, so dass nur das Vorhandensein von Kopfschmerz, aber nicht die Kopfschmerzart festgestellt werden kann. Diese Patienten werden unter 14.2 Kopfschmerz nicht spezifiziert kodiert. Diese Diagnose sollte aber niemals als Entschuldigung dafür die- 
nen, nicht ausreichend exakte Informationen über Kopfschmerzen zu sammeln, wenn diese verfügbar sind. Sie sollte ausschließlich in Situationen benutzt werden, wenn Informationen nicht erhältlich sind, weil der Patient tot oder kommunikationsunfähig ist oder nicht zur Verfügung steht.

\section{ANHANG}

A1. Migräne

A2. Kopfschmerz vom Spannungstyp (alternative Kriterien)

A3. Trigemino-autonome Kopfschmerzerkrankungen (TAK)

A4. Andere primäre Kopfschmerzen

A5. Kopfschmerz zurückzuführen auf eine Verletzung oder ein Trauma des Kopfes und/oder der HWS

A6. Kopfschmerz zurückzuführen auf Gefäßstörungen im Bereich des Kopfes und/oder des Halses

A7. Kopfschmerz zurückzuführen auf nichtvaskuläre intrakranielle Störungen

A8. Kopfschmerz zurückzuführen auf eine Substanz oder deren Entzug

A9. Kopfschmerz zurückzuführen auf eine Infektion

A10. Kopfschmerz zurückzuführen auf eine Störung der Homöostase

A11. Kopf- oder Gesichtsschmerzen zurückzuführen auf Erkrankungen des Schädels sowie von Hals, Augen, Ohren, Nase, Nebenhöhlen, Zähnen, Mund und anderen Gesichts- oder Schädelstrukturen

A12. Kopfschmerz zurückzuführen auf psychiatrische Störungen

\begin{tabular}{|c|c|}
\hline A1. & Migräne \\
\hline A1.1 & Migräne ohne Aura \\
\hline A1.1.1 & Rein menstruelle Migräne ohne Aura \\
\hline A1.1.2 & Menstruationsassoziierte Migräne ohne Aura \\
\hline A1.1.3 & Nicht menstruationsassoziierte Migräne ohne Aura \\
\hline A1.2 & Migräne mit Aura \\
\hline A1.2.0.1 & Rein menstruelle Migräne mit Aura \\
\hline A1.2.0.2 & Menstruationsassoziierte Migräne mit Aura \\
\hline A1.2.0.3 & Nicht menstruationsassoziierte Migräne mit Aura \\
\hline A1.3 & Chronische Migräne (alternative Kriterien) \\
\hline A1.3.1 & Chronische Migräne mit schmerzfreien Perioden \\
\hline A1.3.2 & Chronische Migräne mit Dauerschmerz \\
\hline A1.4 & Migränekomplikationen \\
\hline A1.4.5 & Migräneaurastatus \\
\hline A1.4.6 & Visual Snow \\
\hline A1.6 & $\begin{array}{l}\text { Episodische Syndrome, die potenziell mit Migräne einher- } \\
\text { gehen }\end{array}$ \\
\hline A1.6.4 & Infantile Koliken \\
\hline A1.6.5 & Alternierende Hemiplegie des Kindesalters \\
\hline A1.6.6 & Vestibuläre Migräne \\
\hline A2. & Kopfschmerz vom Spannungstyp (alternative Kriterien) \\
\hline A3. & Trigemino-autonome Kopfschmerzerkrankungen(TAK) \\
\hline A3.1 & Clusterkopfschmerz (alternative Kriterien) \\
\hline A3.2 & Paroxysmale Hemikranien (alternative Kriterien) \\
\hline
\end{tabular}

\begin{tabular}{|c|c|}
\hline A3.3 & $\begin{array}{l}\text { Short-lasting unilateral neuralgiform headache attacks } \\
\text { (alternative Kriterien) }\end{array}$ \\
\hline A3.4 & Hemicrania continua (alternative Kriterien) \\
\hline A3.6 & $\begin{array}{l}\text { Undifferenzierte trigemino-autonome Kopfschmerz- } \\
\text { erkrankung }\end{array}$ \\
\hline A4. & Andere primäre Kopfschmerzen \\
\hline A4. 11 & Epicrania fugax \\
\hline A5. & $\begin{array}{l}\text { Kopfschmerz zurückzuführen auf eine Verletzung oder ein } \\
\text { Trauma des Kopfes und/oder der HWS }\end{array}$ \\
\hline A5. 1 & $\begin{array}{l}\text { Akuter Kopfschmerz zurückzuführen auf eine trauma- } \\
\text { tische Verletzung des Kopfes }\end{array}$ \\
\hline A5.1.1.1 & $\begin{array}{l}\text { Verzögert auftretender akuter Kopfschmerz zurück- } \\
\text { zuführen auf eine mittlere oder schwere traumatische } \\
\text { Verletzung des Kopfes }\end{array}$ \\
\hline A5.1.2.1 & $\begin{array}{l}\text { Verzögert auftretender akuter Kopfschmerz zurückzu- } \\
\text { führen auf eine leichte traumatische Verletzung des Kopfes }\end{array}$ \\
\hline A5.2 & $\begin{array}{l}\text { Anhaltender Kopfschmerz zurückzuführen auf eine trau- } \\
\text { matische Verletzung des Kopfes }\end{array}$ \\
\hline A5.2.1.1 & $\begin{array}{l}\text { Verzögert auftretender anhaltender Kopfschmerz zurück- } \\
\text { zuführen auf ein mittleres oder schweres Kopftrauma }\end{array}$ \\
\hline A5.2.2.1 & $\begin{array}{l}\text { Verzögert auftretender anhaltender Kopfschmerz zurück- } \\
\text { zuführen auf ein leichtes Kopftrauma }\end{array}$ \\
\hline A5.7 & $\begin{array}{l}\text { Kopfschmerz zurückzuführen auf einen radiochirurgischen } \\
\text { Eingriff am Gehirn }\end{array}$ \\
\hline A5.8 & $\begin{array}{l}\text { Akuter Kopfschmerz zurückzuführen auf ein anderes } \\
\text { Kopf- und/oder HWS-Trauma oder eine andere Kopf- und/ } \\
\text { oder HWS-Verletzung }\end{array}$ \\
\hline A5.9 & $\begin{array}{l}\text { Anhaltender Kopfschmerz zurückzuführen auf ein anderes } \\
\text { Kopf- oder HWS-Trauma oder eine andere Kopf- oder } \\
\text { HWS-Verletzung }\end{array}$ \\
\hline A6. & $\begin{array}{l}\text { Kopfschmerz zurückzuführen auf Gefäßstörungen im } \\
\text { Bereich des Kopfes und/oder des Halses }\end{array}$ \\
\hline A6.10 & $\begin{array}{l}\text { Anhaltender Kopfschmerz zurückzuführen auf frühere } \\
\text { Störungen im Bereich des Kopfes oder des Halses }\end{array}$ \\
\hline A7. & $\begin{array}{l}\text { Kopfschmerz zurückzuführen auf nichtvaskuläre intra- } \\
\text { kranielle Störungen }\end{array}$ \\
\hline A7.6 & $\begin{array}{l}\text { Kopfschmerz zurückzuführen auf einen epileptischen } \\
\text { Anfall }\end{array}$ \\
\hline A7.6.3 & Kopfschmerz nach Elektrokrampftherapie \\
\hline A7.9 & $\begin{array}{l}\text { Anhaltender Kopfschmerz zurückzuführen auf eine } \\
\text { frühere nichtvaskuläre intrakranielle Störung }\end{array}$ \\
\hline A8. & $\begin{array}{l}\text { Kopfschmerz zurückzuführen auf eine Substanz oder } \\
\text { deren Entzug }\end{array}$ \\
\hline A8.4 & $\begin{array}{l}\text { Anhaltender Kopfschmerz zurückzuführen auf früheren } \\
\text { Substanzgebrauch oder frühere Substanzexposition }\end{array}$ \\
\hline A9. & Kopfschmerz zurückzuführen auf eine Infektion \\
\hline A9.1 & $\begin{array}{l}\text { Kopfschmerz zurückzuführen auf eine intrakranielle } \\
\text { Infektion }\end{array}$ \\
\hline A9.1.3.3 & $\begin{array}{l}\text { Anhaltender Kopfschmerz zurückzuführen auf eine frühere } \\
\text { intrakranielle Pilz- oder andere parasitäre Infektion }\end{array}$ \\
\hline A9.1.6 & $\begin{array}{l}\text { Kopfschmerz zurückzuführen auf eine andere raumfor- } \\
\text { dernde infektiöse Läsion }\end{array}$ \\
\hline A9.3 & Kopfschmerz zurückzuführen auf eine HIV-Infektion \\
\hline A10. & $\begin{array}{l}\text { Kopfschmerz zurückzuführen auf eine Störung der } \\
\text { Homöostase }\end{array}$ \\
\hline A10.7 & $\begin{array}{l}\text { Kopf- und/oder Nackenschmerzen zurückzuführen auf } \\
\text { eine orthostatische (posturale) Hypotonie }\end{array}$ \\
\hline
\end{tabular}




\begin{tabular}{|c|c|}
\hline A10.8 & $\begin{array}{l}\text { Kopfschmerz zurückzuführen auf eine andere Störung der } \\
\text { Homöostase }\end{array}$ \\
\hline A10.8.1 & $\begin{array}{l}\text { Kopfschmerz zurückzuführen auf einen Aufenthalt im } \\
\text { Weltraum }\end{array}$ \\
\hline A10.8.2 & $\begin{array}{l}\text { Kopfschmerz zurückzuführen auf andere metabolische } \\
\text { oder systemische Störungen }\end{array}$ \\
\hline A10.9 & $\begin{array}{l}\text { Anhaltender Kopfschmerz zurückzuführen auf eine } \\
\text { frühere Störung der Homöostase }\end{array}$ \\
\hline A11. & $\begin{array}{l}\text { Kopf- oder Gesichtsschmerzen zurückzuführen auf Erkran- } \\
\text { kungen des Schädels sowie von Hals, Augen, Ohren, Nase, } \\
\text { Nebenhöhlen, Zähnen, Mund und anderen Gesichts- oder } \\
\text { Schädelstrukturen }\end{array}$ \\
\hline A11.2 & $\begin{array}{l}\text { Kopfschmerz zurückzuführen auf Erkrankungen des } \\
\text { Halses }\end{array}$ \\
\hline A11.2.4 & $\begin{array}{l}\text { Kopfschmerz zurückzuführen auf eine Radikulopathie der } \\
\text { oberen HWS }\end{array}$ \\
\hline A11.2.5 & $\begin{array}{l}\text { Kopfschmerz zurückzuführen auf ein zervikales myofaszia- } \\
\text { les Schmerzsyndrom }\end{array}$ \\
\hline A11.3 & $\begin{array}{l}\text { Kopfschmerz zurückzuführen auf Erkrankungen der } \\
\text { Augen }\end{array}$ \\
\hline A11.3.5 & $\begin{array}{l}\text { Kopfschmerz zurückzuführen auf eine Heterophorie oder } \\
\text { Heterotropie }\end{array}$ \\
\hline A11.5 & $\begin{array}{l}\text { Kopfschmerz zurückzuführen auf Erkrankungen der Nase } \\
\text { oder Nasennebenhöhlen }\end{array}$ \\
\hline A11.5.3 & $\begin{array}{l}\text { Kopfschmerz zurückzuführen auf Erkrankungen der } \\
\text { Nasenschleimhaut, der Nasenmuscheln oder der Nasen- } \\
\text { scheidewand }\end{array}$ \\
\hline A12. & $\begin{array}{l}\text { Kopfschmerz zurückzuführen auf psychiatrische } \\
\text { Störungen }\end{array}$ \\
\hline A12.3 & Kopfschmerz zurückzuführen auf eine depressive Störung \\
\hline A12.4 & $\begin{array}{l}\text { Kopfschmerz zurückzuführen auf eine Störung mit } \\
\text { Trennungsangst }\end{array}$ \\
\hline A12.5 & Kopfschmerz zurückzuführen auf eine Panikstörung \\
\hline A12.6 & Kopfschmerz zurückzuführen auf eine spezifische Phobie \\
\hline A12.7 & $\begin{array}{l}\text { Kopfschmerz zurückzuführen auf eine soziale Angst- } \\
\text { störung (soziale Phobie) }\end{array}$ \\
\hline A12.8 & $\begin{array}{l}\text { Kopfschmerz zurückzuführen auf eine generalisierte } \\
\text { Angststörung }\end{array}$ \\
\hline A12.9 & $\begin{array}{l}\text { Kopfschmerz zurückzuführen auf eine posttraumatische } \\
\text { Belastungsstörung (PTBS) }\end{array}$ \\
\hline
\end{tabular}

\section{Einleitung}

Der 2. Auflage der Internationalen Klassifikation von Kopfschmerzerkrankungen (ICHD-2) wurde erstmals ein Anhang beigefügt. Dieser sollte gleich mehrere Zwecke erfüllen, was auch für die ICHD-3 seine Gültigkeit behält.

Das primäre Ziel ist es, Forschungskriterien für eine Vielzahl neuer Kopfschmerzentitäten zur Verfügung zu stellen, die bisher durch wissenschaftliche Studien noch nicht ausreichend validiert sind. Sowohl die Erfahrung der Mitglieder des Kopfschmerzklassifikationskomitees als auch Veröffentlichungen unterschiedlicher Qualität legen die Existenz einer Reihe von diagnostischen Entitäten nahe, die als eigenständige Erkrankungen angesehen werden können, bei denen aber weitere wissenschaftlichen Beweise erbracht werden müssen, ehe sie formal akzeptiert werden können. Damit ist, wie bereits zwischen ICHD-2, ICHD-3 beta und ICHD-3 geschehen, zu erwarten, dass ein Teil der diagnostischen Entitäten aus dem Anhang bei der nächsten Revision der Klassifikation in den Hauptteil übernommen werden kann.

An einigen Stellen finden sich im Anhang alternative diagnostische Kriterien zu im Hauptteil der Klassifikation aufgeführten Kopfschmerztypen. Dieses Vorgehen wurde immer dann gewählt, wenn klinische Erfahrungen und eine Reihe von Veröffentlichungen alternative Kriterien sinnvoll erscheinen lassen, die wissenschaftliche Beweislage jedoch als nicht ausreichend angesehen wurde, um eine Änderung im Hauptteil bereits tatsächlich zu vollziehen.

Schließlich soll der Anhang als erster Schritt genutzt werden, um bestehende diagnostische Entitäten zu eliminieren, die lediglich aus traditionellen Gründen in vorherige Auflagen der ICHD aufgenommen worden waren, für deren Existenz ein ausreichender wissenschaftlicher Nachweis aber noch immer nicht erbracht wurde.

\section{A1. Migräne}

\section{A1.1 Migräne ohne Aura}

\section{A1.1.1 Rein menstruelle Migräne ohne Aura Diagnostische Kriterien:}

A. Attacken bei einer menstruierenden Frau1, welche die Kriterien einer 1.1 Migräne ohne Aura und Kriterium B unten erfüllen

B. Die Attacken treten ausschließlich am Tage $1 \pm 2$ (d. h. Tag -2 bis + 3) 2 der Menstruation 1 in mindestens 2 von 3 Menstruationszyklen auf und zu keiner anderen Zeit des Zyklus3.

\section{Anmerkung:}

1. Für die Zwecke der ICHD-3 wird die Menstruation als endometriale Blutung als Folge des normalen endogenen Menstruationszyklusses oder eines Entzuges von externen Gestagenen angesehen, letzteres gilt für kombinierte orale Kontrazeptiva und eine zyklische Hormonersatztherapie.

2. Der erste Tag der Menstruation ist Tag 1, der vorhergehende Tag ist Tag -1; es gibt definitionsgemäß keinen Tag 0 .

3. Zu Forschungszwecken wird das Führen eines prospektiven Tagebuches empfohlen, dies ist jedoch für die klinische Diagnose einer A1.1.1 rein menstruellen Migräne ohne Aura nicht zwingend geboten.

\section{A1.1.2 Menstruationsassoziierte Migräne ohne Aura} Diagnostische Kriterien:

A. Attacken bei einer menstruierenden Frau1, welche die Kriterien einer 1.1 Migräne ohne Aura sowie Kriterium B unten erfüllen

B. Die Attacken treten am Tage $1 \pm 2$ der Menstruation (d. h. Tag -2 bis + 3) 2 der Menstruation 1 in mindestens 2 von 3 Menstruationszyklen auf, zusätzlich aber auch zu anderen Zeiten des Zyklus3.

\section{Anmerkung:}

1. Für die Zwecke der ICHD-3 wird die Menstruation als endometrial Blutung als Folge des normalen endogenen Menstruationszyklusses oder eines Entzuges von externen Gestagenen angesehen - letzteres gilt für kombinierte orale Kontrazeptiva und eine zyklische Hormonersatztherapie.

2. Der erste Tag der Menstruation ist Tag 1, der vorhergehende Tag ist Tag -1; es gibt definitionsgemäß keinen Tag 0 . 
3. Zu Forschungszwecken wird das Führen eines prospektiven Tagebuches empfohlen, dies ist jedoch für die klinische Diagnose einer A1.1.2 menstruationsassoziierten Migräne ohne Aura nicht zwingend geboten.

A1.1.3 Nicht menstruationsassoziierte Migräne ohne Aura Diagnostische Kriterien:

A. Attacken bei einer menstruierenden Frau1, welche die Kriterien einer 1.1 Migräne ohne Aura sowie das Kriterium B unten erfüllen

B. Attacken, welche nicht das Kriterium B für eine A1.1.1 rein menstruelle Migräne ohne Aura oder A1.1.2 menstruationsassoziierte Migräne ohne Aura erfüllen.

\section{Anmerkung:}

Für die Zwecke der ICHD-3 wird die Menstruation als endometriale Blutung als Folge des normalen endogenen Menstruationszyklusses oder eines Entzuges von externen Gestagenen angesehen - letzteres gilt für kombinierte orale Kontrazeptiva und eine zyklische Hormonersatztherapie

\section{Kommentar:}

Diese Subklassifizierung der 1.1 Migräne ohne Aura wird eindeutig nur auf menstruierende Frauen gemäß obiger Definition angewandt.

Menstruelle Migräneattacken verlaufen meist ohne Auren. Die Unterscheidung zwischen einer A1.1.1 rein menstruellen Migräne ohne Aura und einer A1.1.2 menstruationsassoziierten Migräne ohne Aura ist deshalb von Bedeutung, weil eine Hormonprophylaxe bei Letzterer eher wirksam sein dürfte.

Viele Frauen neigen dazu, den Zusammenhang zwischen Menstruation und Attacken überzubewerten; zu Forschungszwecken erfordert die Diagnose den durch Tagebuchaufzeichnungen belegten, prospektiv dokumentierten Nachweis über ein Minimum von drei Zyklen.

Der Mechanismus/die Mechanismen der Migräne unterscheiden sich möglicherweise in Abhängigkeit davon, ob die endometriale Blutung als Folge des normalen endogenen Menstruationszyklusses oder eines Entzuges von externen Gestagenen (wie bei kombinierten oralen Kontrazeptiva und einer zyklischen Hormonersatztherapie) auftritt. So resultiert der endogene Menstruationszyklus aus komplexen hormonellen Veränderungen der Achse von Hypothalamus, Hypophyse und Ovarien, die den Eisprung auslösen, welche wiederum durch Einnahme kombinierter oraler Kontrazeptiva unterdrückt wird. Diese Subpopulationen sollten daher in der Forschung getrennt untersucht werden, obwohl die Diagnosekriterien nicht voneinander abzugrenzen sind. Möglicherweise sollten sich auch die Behandlungsstrategien für die Subpopulationen unterscheiden.

Es gibt Hinweise, dass zumindest bei einigen Frauen menstruelle Migräneattacken durch einen Östrogenentzug ausgelöst werden können, auch wenn möglicherweise andere hormonelle oder biochemische Veränderungen zu diesem Zeitpunkt des Zyklus ebenfalls relevant sein können. Wenn eine rein menstruelle Migräne oder menstruationsassoziierte Migräne mit einem exogenen Östrogenentzug in Zusammenhang stehen, sollten beide Diagnosen, A1.1.1 rein menstruelle Migräne ohne Aura bzw. A1.1.2 menstru- ationsassoziierte Migräne ohne Aura und 8.3.3 Östrogenentzugskopfschmerz vergeben werden.

Der Zusammenhang mit der Menstruation kann sich im Laufe der fortpflanzungsfähigen Lebensspanne einer Frau ändern.

\section{A.1.2 Migräne mit Aura}

\section{A1.2.0.1 Rein menstruelle Migräne mit Aura} Diagnostische Kriterien:

A. Attacken bei einer menstruierenden Frau1, welche die Kriterien einer 1.2 Migräne mit Aura und Kriterium B unten erfüllen

B. Die Attacken treten am Tage $1 \pm 2$ der Menstruation (d. h. Tag -2 bis + 3) 2 der Menstruation 1 in mindestens 2 von 3 Menstruationszyklen auf, zusätzlich aber auch zu anderen Zeiten des Zyklus.3

\section{Anmerkung:}

Für die Zwecke der ICHD-3 wird die Menstruation als endometriale Blutung als Folge des normalen endogenen Menstruationszyklusses oder eines Entzuges von externen Gestagenen angesehen - letzteres gilt für kombinierte orale Kontrazeptiva und eine zyklische Hormonersatztherapie

2. Der erste Tag der Menstruation ist Tag 1, der vorhergehende Tag ist Tag -1; es gibt definitionsgemäß keinen Tag 0 .

3. Zu Forschungszwecken wird das Führen eines prospektiven Tagebuches empfohlen, dies ist jedoch für die klinische Diagnose einer A1.2.0.1 rein menstruellen Migräne mit Aura nicht zwingend geboten.

\section{A1.2.0.2 Menstruationsassoziierte Migräne mit Aura Diagnostische Kriterien:}

Attacken bei einer menstruierenden Frau1, welche die Kriterien einer 1.2 Migräne mit Aura und Kriterium B unten erfüllen

B. Die Attacken treten am Tage $1 \pm 2$ der Menstruation (d. h. Tag -2 bis + 3) 2 der Menstruation 1 in mindestens 2 von 3 Menstruationszyklen auf, zusätzlich aber auch zu anderen Zeiten des Zyklus3.

\section{Anmerkung:}

Für die Zwecke der ICHD-3 wird die Menstruation als endometriale Blutung als Folge des normalen endogenen Menstruationszyklusses oder eines Entzuges von externen Gestagenen angesehen - letzteres gilt für kombinierte orale Kontrazeptiva und eine zyklische Hormonersatztherapie.

2. Der erste Tag der Menstruation ist Tag 1, der vorhergehende Tag ist Tag-1; es gibt definitionsgemäß keinen Tag 0 .

3. Zu Forschungszwecken wird das Führen eines prospektiven Tagebuches empfohlen, dies ist jedoch für die klinische Diagnose einer A1.2.0.2 menstruationsassoziierten Migräne mit Aura nicht zwingend geboten.

A1.2.0.3 Nicht menstruationsassoziierte Migräne mit Aura Diagnostische Kriterien:

A. Attacken bei einer menstruierenden Frau1, welche die Kriterien einer 1.2 Migräne mit Aura und Kriterium B unten erfüllen

B. Attacken, welche nicht das Kriterium B für eine A1.2.0.1 rein menstruelle Migräne mit Aura oder A1.2.0.2 menstruationsassoziierte Migräne mit Aura erfüllen. 
Anmerkung:

Für die Zwecke der ICHD-3 wird die Menstruation als endometrial Blutung als Folge des normalen endogenen Menstruationszyklusses oder eines Entzuges von externen Gestagenen angesehen - letzteres gilt für kombinierte orale Kontrazeptiva und eine zyklische Hormonersatztherapie.

\section{Kommentar:}

Diese Subklassifizierung der 1.2 Migräne mit Aura wird eindeutig nur auf menstruierende Frauen gemäß obiger Definition angewandt.

Menstruelle Migräneattacken verlaufen meist ohne Auren. Diese Kriterien für eine A1.2.0.1 rein menstruelle Migräne mit Aura und eine A1.2.0.2 menstruationsassoziierte Migräne mit Aura werden mit aufgenommen, um eine bessere Charakterisierung dieses ungewöhnlichen Subtyps zu erlauben. Um der Vollständigkeit willen werden die Kriterien für eine A1.2.0.3 nicht menstruationsassoziierte Migräne mit Aura mit aufgenommen.

Viele Frauen neigen dazu, den Zusammenhang zwischen Menstruation und Attacken überzubewerten; zu Forschungszwecken erfordert die Diagnose den durch Tagebuchaufzeichnungen belegten, prospektiv dokumentierten Nachweis über ein Minimum von drei Zyklen.

Der Mechanismus/die Mechanismen der Migräne unterscheiden sich möglicherweise in Abhängigkeit davon, ob die endometriale Blutung als Folge des normalen endogenen Menstruationszyklusses oder eines Entzuges von externen Gestagenen (wie bei kombinierten oralen Kontrazeptiva und einer zyklischen Hormonersatztherapie) auftritt. So resultiert der endogene Menstruationszyklus aus komplexen hormonellen Veränderungen der Achse von Hypothalamus, Hypophyse und Ovarien, die den Eisprung auslösen, welche wiederum durch Einnahme kombinierter oraler Kontrazeptiva unterdrückt wird. Diese Subpopulationen sollten daher in der Forschung getrennt untersucht werden, obwohl die Diagnosekriterien nicht voneinander abzugrenzen sind.

\section{A1.3 Chronische Migräne (alternative Kriterien)}

Alternative diagnostische Kriterien:

A. Kopfschmerz (migräneartig oder spannungstypartig) an $\geq 15$ Tagen/Monaten für $>3$ Monate, welcher die Kriterien B und $C$ erfüllt

B. Der Kopfschmerz tritt bei einem Patienten auf, der mindestens fünf Attacken gehabt hat, welche die Kriterien B bis D für eine 1.1 Migräne ohne Aura und/oder die Kriterien B und C für eine 1.2 Migräne mit Aura erfüllen

C. An $\geq 8$ Tagen/Monat für $>3$ Monate, wobei einer der folgenden Punkte erfüllt ist:

1. Kriterium C und D für eine 1.1 Migräne ohne Aura

2. Kriterium $B$ und $C$ für eine 1.2 Migräne mit Aura

3. Kriterium A und B für eine 1.5 wahrscheinliche Migräne

D. Nicht besser erklärt durch eine andere ICHD-3-Diagnose.
A1.3.1 Chronische Migräne mit schmerzfreien Perioden Diagnostische Kriterien:

A. Kopfschmerz, welcher die Kriterien für eine 1.3 chronische Migräne und Kriterium B unten erfüllt

B. Unterbrochen von schmerzfreien Perioden von $>3$ Stunden an $\geq 5$ Tagen/Monat, die nicht auf medikamentöse Behandlung zurückzuführen sind.

\section{A1.3.2 Chronische Migräne mit Dauerschmerz}

Diagnostische Kriterien:

A. Kopfschmerz, der die Kriterien für eine 1.3 chronische Migräne und Kriterium B unten erfüllt

B. Nicht unterbrochen von schmerzfreien Perioden von $>3$ Stunden an $\geq 5$ Tagen/Monat, es sei denn, diese sind auf medikamentöse Behandlung zurückzuführen.

\section{A1.4 Migränekomplikationen}

A1.4.5 Migräneaurastatus

Diagnostische Kriterien:

A. Migräne, die die Kriterien für eine 1.2 Migräne mit Aura oder eine ihrer Subtypen erfüllt

B. Wenigstens 3 Auren am Tag über einen Zeitraum von 3 Tagen.

\section{Kommentar:}

Andere neurologische Erkrankungen einschließlich eines reversiblen zerebralen Vasokonstriktionssyndroms, eines posterioren reversiblen Enzephalopathiesyndroms und einer arteriellen Dissektion sollten durch entsprechende Untersuchungen ausgeschlossen werden.

\section{A1.4.6 Visual Snow}

Diagnostische Kriterien:

A. Dynamische, kontinuierliche winzige Punkte über das gesamte Gesichtsfeld1, > 3 Monate anhaltend

B. Zusätzliche visuelle Symptome in Form von mindestens 2 der folgenden 4 Typen:

1. Palinopsie2

2. verstärkte entoptische Phänomene3

3. Lichtempfindlichkeit

4. beeinträchtigtes Sehvermögen nachts (Nachtblindheit)

C. Die Symptome decken sich nicht mit denen einer typischen visuellen Migräneaura4

D. Die Symptome lassen sich nicht besser durch eine andere Erkrankung erklären5

Anmerkung:

1. Die Patienten vergleichen Visual Snow mit einem verrauschten Fernsehbild („Bild schneit“). Die Punkte sind vor einem weißen Hintergrund üblicherweise schwarz oder grau, und vor einem schwarzen Hintergrund grau oder weiß; berichtet wurde jedoch auch von durchsichtigen, weiß aufblitzenden und farbigen Punkten.

2. Die Palinopsie kann in visuellen Nachbildern und/oder einem „Nachziehen“ gerade gesehener beweglicher Objekte bestehen. Visuelle Nachbilder unterscheiden sich von Nachbildern auf der Netzhaut, die nur nach längerem Fixieren eines kont- 
rastreichen Lichteindrucks auftreten und die Komplementärfarbe aufweisen.

3. Zu diesen Phänomenen, die auf die Struktur des visuellen Systems als solchem zurückgehen, gehört auch ein übermäßiges Auftreten von Mouches volantes in beiden Augen, ein übermäßiges Auftreten des Scheerer-Phänomens (Blaufeld-Entoptik: unzählige graue/weiße/schwarze Pünktchen oder Ringe, die beim Blick auf eine homogene helle Fläche wie etwa einen blauen Himmel durch das Gesichtsfeld beider Augen schießen), vom Auge selbst hervorgerufene Lichtphänomene (farbige Wellen oder Wolken, die beim Schließen der Augen im Dunkeln gesehen werden) und spontane Photopsie (helle Lichtblitze).

4. Wie unter 1.2.1 Migräne mit typischer Aura beschrieben.

5. Unauffällige ophthalmologische Befunde (korrigierte Sehschärfe, Augenhintergrunduntersuchung bei weit getropfter Pupille, Gesichtsfelduntersuchung und Elektroretinogramm) und keine Einnahme von Psychopharmaka.

\section{Kommentar:}

A1.4.6 Visual Snow wird neu in den Anhang der ICHD-3 aufgenommen. Auch wenn es nicht per se Bestandteil des Migränespektrums sein mag, so scheint es doch epidemiologisch mit der 1.2 Migräne mit Aura assoziiert. Es besteht weiterer Forschungsbedarf, um zu ermitteln, ob diese Störungen gemeinsame pathophysiologische Mechanismen aufweisen, die visuelle Symptome auslösen, bis dahin wird jedoch vermutet, dass eine kortikale Übererregbarkeit bei beidem eine Rolle spielt. Patienten mit einer 1. Migräne weisen eine erhöhte Prävalenz von Palinopsie und Attacken mit erhöhter Empfindlichkeit gegenüber äußeren visuellen Reizen auf: Beim A1.4.6 Visual Snow finden sich sowohl Palinopsie als auch Photophobie. Patienten mit A1.4.6 Visual Snow und einer komorbiden 1. Migräne weisen häufiger Palinopsie, spontane Photopsie, Photophobie, Nyktalopie und Tinnitus auf als solche ohne komorbide Migräne.

Noch zwei weitere Gründe sprechen für eine Aufnahme von A 1.4.6 Visual Snow in die ICHD-3, wobei die Kriterien weitgehend von Schankin et al. übernommen wurden. Erstens bewirkt es eine Sensibilisierung für dieses Krankheitsbild und hilft Medizinern, es zu erkennen. Patienten, die über Visual Snow als Symptom klagen, haben oft (in der Vorgeschichte) eine 1. Migräne; Ärzte ohne Kenntnis des A1.4.6 Visual Snow können seine Symptome fälschlicherweise als anhaltende visuelle Aura deuten. Zweitens werden, einem ähnlichen Argument in Bezug auf die Forschung folgend, für künftige Untersuchungen zu anhaltenden visuellen Symptomen homogene Probandengruppen benötigt; eine Einbeziehung der Kriterien für ein A1.4.6 Visual Snow stellt für Forscher klar, wie diese Erkrankung aktuell definiert wird.

\section{A1.6 Episodische Syndrome, die potenziell mit Migräne einhergehen}

\section{A1.6.4 Infantile Koliken}

Beschreibung:

Exzessives, häufiges Schreien eines ansonsten gesund und wohlgenährt wirkenden Säuglings.

\section{Diagnostische Kriterien:}

A. Wiederkehrende Episoden von Reizbarkeit, Unruhe oder Schreien von der Geburt bis zum Alter von 4 Monaten, die das Kriterium B erfüllen

B. Beides von folgendem trifft zu:

1. Episoden dauern $\geq 3$ Stunden/Tag

2. Episoden treten an $\geq 3$ Tagen/Woche für $\geq 3$ Wochen auf

C. Nicht auf eine andere Erkrankung zurückzuführen 1 .

\section{Anmerkung:}

1. Insbesondere wurde eine Gedeihstörung ausgeschlossen.

\section{Kommentar:}

Infantile Koliken betreffen jeden fünften Säugling.

Säuglinge mit Koliken entwickeln mit höherer Wahrscheinlichkeit in ihrem späteren Leben eine 1.1 Migräne ohne Aura oder 1.2 Migräne mit Aura. Bei Müttern mit 1. Migräne zeigte sich eine 2,5mal höhere Wahrscheinlichkeit, dass diese Säuglinge mit Koliken hatten als bei Müttern ohne eine solche. Bei Vätern mit 1. Migräne verdoppelt sich die Wahrscheinlichkeit, einen Säugling mit Koliken zu haben.

\section{A1.6.5 Alternierende Hemiplegie des Kindesalters}

\section{Beschreibung:}

Infantile hemiplegische Attacken, die abwechselnd einmal die eine, einmal die andere Körperhälfte betreffen, begleitet von einer progressiven Enzephalopathie, anderen paroxysmalen Phänomenen und geistigen Beeinträchtigungen.

\section{Diagnostische Kriterien:}

A. Rezidivierende hemiplegische Attacken, die abwechselnd die beiden Körperhälften betreffen und das Kriterium B erfüllen

B. Beginn vor dem Alter von 18 Monaten

C. Wenigstens ein anderes paroxysmales Phänomen 1 tritt mit den Anfällen der Hemiplegie oder unabhängig davon auf

D. Nachweis eines geistigen und/oder neurologischen Defizits bzw. mehrerer geistiger und/oder neurologischer Defizite

E. Nicht auf eine andere Erkrankung zurückzuführen.

\section{Anmerkung:}

1. Zum Beispiel tonische Anfälle, dystonische Körperhaltung, choreoathetotische Bewegungen, Nystagmus bzw. andere Abnormitäten der Okulomotorik und/oder autonome Störungen.

\section{Kommentar:}

Es handelt sich um eine heterogene neurodegenerative Erkrankung. Ein Zusammenhang mit Migräne wird aus klinischen Gründen vorgeschlagen. Die Möglichkeit, dass es sich um eine ungewöhnliche Form von Epilepsie handelt, ist nicht auszuschließen. Mutationen des Gens ATP1A3 (kodiert die Natrium-Kalium [ $\mathrm{Na}+/ \mathrm{K}+$ ]-ATPase $\alpha 3$ Untereinheit) dürften für mindestens $70 \%$ der Fälle verantwortlich sein. 


\section{A1.6.6 Vestibuläre Migräne}

Früher verwendete Begriffe Migräne-assoziierter Schwindel; migränebedingte Vestibulopathe; migränöser Schwindel.

Diagnostische Kriterien:

A. Mindestens fünf Episoden, die die Kriterien $C$ und D erfüllen

B. Eine aktuelle Geschichte oder Vorgeschichte einer 1.1 Migräne ohne Aura oder 1.2 Migräne mit Aura1

C. Vestibuläre Symptome2 von mittelstarker oder starker Intensität3 mit einer Dauer zwischen 5 Minuten und 72 Stunden 4

D. Mindestens die Hälfte der Episoden tritt in Begleitung von wenigstens einem der folgenden drei migränösen Merkmale5:

1. Kopfschmerz mit mindestens zwei der folgenden vier Charakteristika:

a) einseitig lokalisiert

b) pulsierend

c) mittelschwere oder schwere Intensität

d) Verschlechterung durch körperliche Routineaktivitäten

2. Photophobie und Phonophobie6

3. visuelle Aura7

E. Nicht besser erklärt durch eine andere ICHD-3-Diagnose oder durch eine andere vestibuläre Störung8.

Anmerkung:

1. Auch unter der zugrundeliegenden Migränediagnose zu kodieren.

2. Die vestibulären Symptome umfassen wie in der Klassifikation vestibulärer Symptome durch die Bárány-Gesellschaft definiert und für die Diagnose einer A1.6.6 vestibulären Migräne geeignet:

a) spontaner Schwindel:

i. innerer Schwindel (das unzutreffende Gefühl, in Bewegung zu sein);

ii. äußerer Schwindel (das unzutreffende Gefühl, das visuelle Umfeld würde sich drehen oder fließen);

b) Lagerungsschwindel, auftretend nach Veränderung der Kopfposition;

c) visuell-induzierter Schwindel, ausgelöst durch einen komplexen oder großen sich bewegenden visuellen Stimulus;

d) Durch eine Kopfbewegung induzierter Schwindel, der bei Bewegung des Kopfes ausgelöst wird;

e) Durch eine Kopfbewegung induzierte Benommenheit mit Übelkeit (charakteristisch hierfür ist das Gefühl einer gestörten räumlichen Orientierung; andere Formen von Benommenheit sind aktuell in der Klassifikation der vestibulären Migräne nicht inbegriffen).

3. Vestibuläre Symptome werden als mittelstark eingestuft, wenn sie bei Alltagsaktivitäten behindern, diese aber nicht verhindern, und als stark, wenn den Alltagsaktivitäten nicht länger nachgegangen werden kann.

4. Die Dauer der Episoden variiert stark. Rund $30 \%$ der Patienten haben Episoden, die Minuten dauern; 30 \% haben über Stunden anhaltende Attacken, und weitere $30 \%$ haben Attacken, die sich über mehrere Tage erstrecken. Die restlichen $10 \%$ weisen Attacken auf, die nur Sekunden dauern und die am ehesten wiederholt bei Kopfbewegungen, visueller Stimulation oder nach Veränderungen der Kopfposition eintreten. Bei diesen Patienten wird die Dauer der Episode definiert als der Gesamtzeitraum, in dem kurze Attacken sich wiederholen. Am anderen Ende des Spektrums gibt es Patienten, die womöglich 4 Wochen brauchen, um sich von einer Episode vollständig zu erholen. Die Kernepisode jedoch dauert selten länger als 72 Stunden.

5. Ein Symptom während einer einzelnen Episode reicht aus. Bei unterschiedlichen Episoden können unterschiedliche Symptome auftreten. Begleitsymptome können vor den, während der oder nach den vestibulären Symptomen auftreten.

6. Vorgeschichte und körperliche Untersuchungen lassen nicht auf eine andere vestibuläre Störung schließen oder eine solche Störung wurde in Betracht gezogen, aber durch entsprechende Untersuchungen ausgeschlossen oder eine solche Störung liegt als komorbide Erkrankung vor, aber die Episoden lassen sich eindeutig hiervon abgrenzen. Migräneattacken können durch vestibuläre Stimulation ausgelöst werden. Die Differenzialdiagnose sollte deshalb andere vestibuläre Störungen mit einbeziehen, bei denen Komplikationen durch überlagernde Migräneattacken auftreten.

\section{Kommentar:}

Eine überraschend hohe Prävalenz einer A1.6.6 vestibulären Migräne in Höhe von 10,3\% wurde neulich unter Migränepatienten in chinesischen neurologischen Abteilungen beschrieben.

Sonstige Symptome

Vorübergehende auditive Symptome, Übelkeit, Erbrechen, Prostration und Anfälligkeit für Reisekrankheit können eine A1.6.6 vestibuläre Migräne begleiten. Da diese jedoch auch zusammen mit diversen anderen vestibulären Störungen auftreten, werden sie nicht als diagnostische Kriterien mit aufgenommen.

Beziehung zur Migräneaura und zur Migräne mit Hirnstammaura

Sowohl Migräneaura als auch Migräne mit Hirnstammaura (zuvor: Migräne vom Basilaristyp) sind Begriffe, die in der ICHD3 definiert werden. Nur eine Minderheit der Patienten mit einer A1.6.6 vestibulären Migräne erfährt ihren Schwindel innerhalb des zeitlichen Rahmens von 5 bis 60 Minuten wie für ein Aurasymptom definiert. Noch weniger erleben ihren Schwindel wie für eine 1.2.1.1 typische Aura mit Kopfschmerz erforderlich unmittelbar vor Einsetzen der Kopfschmerzen. Deshalb können Episoden einer A1.6.6 vestibulären Migräne nicht als Migräneauren betrachtet werden.

Obwohl von mehr als 60\% der Patienten mit einer 1.2.2 Migräne mit Hirnstammaura Schwindel berichtet wird, verlangt die ICHD-3 für diese Diagnose zusätzlich zu visuellen, sensiblen oder dysphasischen Aurasymptomen mindestens zwei Hirnstammsymptome. Weniger als $10 \%$ der Patienten mit einer A1.6.6 vestibulären Migräne erfüllen diese Kriterien. Deshalb sind A1.6.6 vestibuläre Migräne und 1.2.2 Migräne mit Hirnstammaura nicht gleichbedeutend, obwohl einzelne Patienten die diagnostischen Kriterien für beide Erkrankungen erfüllen können.

Beziehung zum gutartigen paroxysmalen Schwindel

Die A1.6.6 vestibuläre Migräne kann zwar in jedem Lebensalter beginnen, doch erkennt die ICHD-3 spezifisch eine Störung des Kindesalters an, den 1.6.2 gutartigen paroxysmalen Schwindel. Die Diagnose erfordert fünf Schwindelepisoden, die ohne Vorwarnung auftreten und nach Minuten bis Stunden spontan wieder 
verschwinden. Zwischen den Episoden müssen die Befunde der neurologischen Untersuchung, Audiometrie, vestibulären Funktionen und des EEG unauffällig sein. Ein einseitiger pochender Kopfschmerz kann während der Attacken auftreten, ist jedoch kein unabdingbares Kriterium. Ein 1.6.2 gutartiger paroxysmaler Schwindel gilt als eines der Vorläufersyndrome einer Migräne. Daher sind vorherige Migränekopfschmerzen für die Diagnose nicht erforderlich. Da die Klassifikation als A1.6.6 vestibuläre Migräne nicht mit einer Altersbegrenzung verbunden ist, kann die Diagnose auf Kinder angewandt werden, sofern die entsprechenden Kriterien erfüllt sind, allerdings sollten diese Diagnosen nur für Kinder mit verschiedenen Typen von Schwindelattacken (d. h. kurz andauernde Episoden von weniger als 5 Minuten und länger andauernde von mehr als 5 Minuten) vergeben werden.

Überschneidung mit Morbus Menière

1. Migräne kommt bei Patienten mit M. Menière häufiger vor als bei Mitgliedern gesunder Vergleichsgruppen. Es wurde von vielen Patienten berichtet, die sowohl Kennzeichen von M. Menière als auch die einer A1.6.6 vestibulären Migräne aufwiesen. Tatsache ist, dass Migräne und M. Menière als Symptom-Cluster vererbt werden können. Schwankender Hörverlust, Tinnitus und Ohrendruck können bei einer A1.6.6 vestibulären Migräne auftreten, doch der Hörverlust erreicht im Verlauf kein besonders ausgeprägtes Niveau. Ebenso sind Migränekopfschmerzen, Photophobien und sogar Migräneauren während M. Menière-Attacken häufig. Die pathophysiologische Beziehung zwischen einer A1.6.6 vestibulären Migräne und M. Menière bleibt ungewiss. Im ersten Jahr nach Einsetzen der Symptome kann die Unterscheidung zwischen diesen schwierig sein, da M. Menière monosymptomatisch sein kann, mit nur vestibulären Symptomen in den Frühstadien der Erkrankung.

Sind die Kriterien für einen M. Menière erfüllt, vor allem ein audiometrisch nachgewiesener Hörverlust, sollte ein M. Menière diagnostiziert werden, selbst wenn während der vestibulären Attacken Migränesymptome auftreten. Nur bei Patienten, die zwei unterschiedliche Typen von Attacken aufweisen, von denen einer die Kriterien für eine A1.6.6 vestibuläre Migräne erfüllt und der andere die für M. Menière, sollten beide Erkrankungen diagnostiziert werden. Eine künftige Überarbeitung der ICHD könnte ein Symptom mit Überlappung zwischen vestibulärer Migräne/M. Menière enthalten.

\section{Literatur}

Belcastro V, Cupini LM, Corbelli I, et al. Palinopsia in patients with migraine: A case-control study. Cephalalgia 2011; 31: 999-1004.

Bisdorff A, von Brevern M, Lempert T, Newman-Toker DE (on behalf of the Committee for the Classification of Vestibular Disorders of the Bárány Society). Classification of vestibular symptoms: Towards an international classification of vestibular disorders. J Vest Res 2009; 19: 1-13.

Brantberg K, Baloh RW. Similarity of vertigo attacks due to Meniere's disease and benign recurrent vertigo both with and without migraine. Acta Otolaryngol 2011; 131: 722-727.

Cass SP, Ankerstjerne JKP, Yetiser S, Furman J, Balaban C, Aydogan B. Migraine-related vestibulopathy. Ann Otol Rhinol Laryngol 1997; 106: 182-189.

Cho SJ, Kim BK, Kim BS, Kim JM, Kim SK, Moon HS, Song T], Cha MJ, Park $\mathrm{KY}$, Sohn JH. Vestibular migraine in multicenter neurology clinics according to the Anhang criteria in the third beta edition of the International Classification of Headache Disorders. Cephalalgia 2016; 36: 454-462.

Cutrer FM, Baloh RW. Migraine-associated dizziness. Headache 1992; 32: 300-304.

Dieterich M, Brandt T. Episodic vertigo related to migraine (90 cases): vestibular migraine? J Neurol 1999; 246: 883-892.

Heinzen EL, Swoboda KJ, Hitomi Y, Gurrieri F, Nicole S, de Vries B, et al. De novo mutations in ATP1A3 cause alternating hemiplegia of childhood. Nat Genet 2012; 44: 1030-1034.

Jäger HR, Giffin NJ, Goadsby PJ. Diffusion- and perfusion-weighted MR imaging in persistent migrainous visual disturbances. Cephalalgia 2005; 25: 323-332.

Lempert T, Olesen J, Furman J, Waterston J, Seemungal B, Carey J, Bisdorff A. Versino M, Evers S, Newman-Toker D. Vestibular migraine: diagnostic criteria. Consensus document of the Bárány Society and the International Headache Society. J Vest Res 2012; 22: 167-172.

Li D, Christensen AF, Olesen J. Field-testing of the ICHD-3 beta/proposed ICD-11 diagnostic criteria for migraine with aura. Cephalalgia 2015; 35: 748-756.

Liu GT, Schatz NJ, Galetta SL, et al. Persistent positive visual phenomena in migraine. Neurology 1995; 45: 664-668.

Neff BA, Staab JP, Eggers SD, Carlson ML, Schmitt WR, Van Abel KM, Worthington DK, Beatty CW, Driscoll CL, Shepard NT. Auditory and vestibular symptoms and chronic subjective dizziness in patients with Meniere's disease, vestibular migraine and Meniere's disease with concomitant vestibular migraine. Otol Neurotol 2012; 33: 1235-1244.

Neuhauser H, Leopold M, von Brevern M, Arnold G, Lempert T. The interrelations of migraine, vertigo, and migrainous vertigo. Neurology 2001; 56: 436-441.

Neuhauser H, Radtke A, von Brevern M, Feldmann M, Lezius F, Ziese T, Lempert T. Migrainous vertigo: prevalence and impact on quality of life. Neurology 2006; 67: 1028-1033.

Oh AK, Lee H, Jen JC, Corona S, Jacobson KM, Baloh RW. Familial benign recurrent vertigo. Am J Med Genet 2001; 100: 287-291.

Perenboom M, Zamanipoor Najafabadi A, Zielman R, et al. Visual sensitivity is more enhanced in migraineurs with aura than in migraineurs without aura. Cephalalgia 2015; 35 (suppl): 1224-1226.

Radtke A, Neuhauser H, von Brevern M, Hottenrott T, Lempert T. Vestibular migraine - validity of clinical diagnostic criteria. Cephalalgia 2011; 31: 906-913.

Schankin CJ, Maniyar FH, Digre KB, et al. 'Visual snow' - A disorder distinct from persistent migraine aura. Brain 2014; 137: 1419-1428.

Schankin CJ, Maniyar FH, Springer T et al. The relation between migraine, typical migraine aura and "visual snow". Headache 2014; 54 : 957-966.

Versino M, Sances G. Dizziness and migraine: a causal relationship? Funct Neurol 2003; 18: 97-101.

Vetvik KG, Benth JŠ, MacGregor EA, Lundqvist C, Russell MB. Menstrual versus non-menstrual attacks of migraine without aura in women with and without menstrual migraine. Cephalalgia 2015; 35: 1261-1268.

Vetvik KG, Macgregor EA, Lundqvist C, Russell MB. Prevalence of menstrual migraine: a population-based study. Cephalalgia 2014; 34: 280-288.

Vetvik KG, MacGregor EA, Lundqvist C, Russell MB. A clinical interview versus prospective headache diaries in the diagnosis of menstrual migraine without aura. Cephalalgia 2015; 35: 410-416.

Zhang Y, Kong Q, Chen J, Li L, Wang D, Zhou J. International Classification of Headache Disorders 3 rd edition beta based testing of vestibular migraine in China: Demographic, clinical characteristics, audiometric findings and diagnosis statues. Cephalalgia 2016; 36: 240-248. 


\section{A2. Kopfschmerz vom Spannungstyp (alternative Kriterien)}

Die folgenden alternativen Kriterien können auf den A2.1 selten auftretenden episodischen Kopfschmerz vom Spannungstyp, den A2.2 häufig auftretenden Kopfschmerz vom Spannungstyp und den A2.3 chronischen Kopfschmerz vom Spannungstyp angewandt werden. Sie definieren ein Kernsyndrom des Kopfschmerzes vom Spannungstyp. Mit anderen Worten, diese Kriterien sind sehr spezifisch, haben aber nur eine geringe Sensitivität.

\section{Alternative diagnostische Kriterien:}

Episoden oder Kopfschmerzen, die das Kriterium A für einen [2.1 selten auftretenden episodischen Kopfschmerz vom Spannungstyp bzw. 2.2 häufig auftretenden episodischer Kopfschmerz vom Spannungstyp bzw. 2.3 chronischen Kopfschmerz vom Spannungstyp] und die Kriterien B bis D unten erfüllen

Die Episoden oder Kopfschmerzen erfüllen das Kriterium B für einen 2.1 selten auftretenden episodischen Kopfschmerz vom Spannungstyp, einen 2.2 häufig auftretenden Kopfschmerz vom Spannungstyp oder einen 2.3 chronischen Kopfschmerz vom Spannungstyp.

Der Kopfschmerz weist mindestens 3 der folgenden 4 Charakteristika auf:

- beidseitige Lokalisation

- Schmerzqualität drückend oder beengend, nicht pulsierend

- leichte bis mittlere Schmerzintensität

- keine Verstärkung durch körperliche Routineaktivitäten wie Gehen oder Treppensteigen

Keine Übelkeit, kein Erbrechen, keine Photophobie oder Phonophobie

Nicht besser erklärt durch eine andere ICHD-3-Diagnose.

\section{Literatur}

Chu MK, Cho SJ, Kim JM, Hwang SH. Field testing the alternative criteria for tension-type headache proposed in the third beta edition of the international classification of headache disorders: results from the Korean headache-sleep study. J Headache Pain 2014; 15: 28.

\section{A3. Trigemino-autonome Kopfschmerz- erkrankungen(TAK)}

\section{A3.1 Clusterkopfschmerz (alternative Kriterien)}

Diagnostische Kriterien:

A. Wenigstens 5 Attacken, die die Kriterien B bis D erfüllen

B. Starker oder sehr starker einseitiger orbitaler, supraorbitaler und/oder temporaler Schmerz, der 15 bis 180 Minuten andauert (unbehandelt) 1

C. Einer oder beide der folgenden Punkte sind erfüllt:

1. mindestens eines der folgenden Symptome oder Zeichen, ipsilateral zum Kopfschmerz:

- konjunktivale Injektion und/oder Lakrimation

- nasale Kongestion und/oder Rhinorrhoe
- Lidödem

- vermehrtes Schwitzen im Bereich von Stirn und Gesicht

- Rötung im Bereich von Stirn und Gesicht

- Empfinden eines Völlegefühls im Ohr

- Miosis und/oder Ptosis

2. ein Gefühl der Unruhe oder Erregung

D. Die Attackenfrequenz liegt zwischen $1 x$ jeden 2. Tag und $8 x$ am Tag2

E. Nicht besser erklärt durch eine andere ICHD-3-Diagnose.

Anmerkung:

1. Während eines Teils, jedoch weniger als der Hälfte der Zeit des aktiven zeitlichen Verlaufs beim A3.1 Clusterkopfschmerz können die Schmerzattacken weniger stark und/oder von kürzerer oder längerer Dauer sein.

2. Während eines Teils, jedoch weniger als der Hälfte der Zeit des aktiven zeitlichen Verlaufs beim A3.1 Clusterkopfschmerz können die Schmerzattacken weniger häufig vorkommen.

Kommentar:

Im Hinblick auf die Aufnahme von (e) und (f) in Kriterium C1 sind die Meinungen geteilt. Fachkollegen in der Arbeitsgruppe glauben, dies verbessere die Sensitivität ohne nennenswerte Einbuße an Spezifizität, formale Feldversuche haben dies jedoch nicht bestätigt.

\section{A3.2 Paroxysmale Hemikranie (alternative Kriterien)} Diagnostische Kriterien:

A. Wenigstens 20 Attacken, die die Kriterien B bis E erfüllen

B. Starker einseitiger orbitaler, supraorbitaler und/oder temporaler Schmerz, der 2 bis 30 Minuten andauert

C. Einer oder beide der folgenden Punkte sind erfüllt:

1. mindestens eines der folgenden Symptome oder Zeichen, ipsilateral zum Kopfschmerz:

- konjunktivale Injektion und/oder Lakrimation

- nasale Kongestion und/oder Rhinorrhoe

- Lidödem

- vermehrtes Schwitzen im Bereich von Stirn und Gesicht

- Rötung im Bereich von Stirn und Gesicht

- Empfinden eines Völlegefühls im Ohr

- Miosis und/oder Ptosis

2. ein Gefühl der Unruhe oder Erregung

D. Die Attackenfrequenz liegt bei $>5$ am Tag1

E. Spricht präventiv zuverlässig auf therapeutische Dosen Indometacin2 an

F. Nicht besser erklärt durch eine andere ICHD-3-Diagnose.

Anmerkung:

1. Während eines Teils, jedoch weniger als der Hälfte der Zeit des aktiven zeitlichen Verlaufs bei der A3.2 paroxysmalen Hemikranie können die Attacken weniger häufig vorkommen.

2. Bei Erwachsenen sollte Indometacin anfangs in einer Tagesdosis von mindestens $150 \mathrm{mg}$ oral eingesetzt werden und bei Bedarf auf $\geq 225 \mathrm{mg}$ täglich erhöht werden. Die Dosis für Injektionen beträgt 100 bis $200 \mathrm{mg}$. Als Erhaltungsdosen sind oft jedoch geringere Mengen erforderlich 


\section{Kommentar:}

Im Hinblick auf die Aufnahme von (e) und (f) in Kriterium C1 sind die Meinungen geteilt. Fachkollegen in der Arbeitsgruppe glauben, dies verbessere die Sensitivität ohne nennenswerte Einbuße an Spezifizität, formale Feldversuche, die diese Änderung der Kriterien unterstützen würden, wurden jedoch nicht durchgeführt.

\section{A3.3 Short-lasting unilateral neuralgiform headache attacks (alternative Kriterien)}

Diagnostische Kriterien

A. Wenigstens 20 Attacken, die die Kriterien B-D erfüllen

B. Mittelstarker bis starker einseitiger orbitaler, supraorbitaler, temporaler und/oder im sonstigen Versorgungsgebiet des N. trigeminus auftretende Kopfschmerz, der 1 bis 600 Sekunden anhält und in Form von Einzelstiche, Serien von Stichen oder sägezahnmusterartig auftritt

C. Mindestens eines der folgenden kranio-autonomen Symptome oder Zeichen ipsilateral zum Schmerz:

- konjunktivale Injektion und/oder Lakrimation

- nasale Kongestion und/oder Rhinorrhoe

- Lidödem

- vermehrtes Schwitzen im Bereich von Stirn und Gesicht

- Rötung im Bereich von Stirn und Gesicht

- Empfinden eines Völlegefühls im Ohr

- Miosis und/oder Ptosis

D. Die Attackenfrequenz liegt bei mindestens 1 am Tag1

E. Nicht besser erklärt durch eine andere ICHD-3-Diagnose.

\section{Anmerkung:}

1. Während eines Teils, jedoch weniger als der Hälfte der Zeit des aktiven zeitlichen Verlaufs bei den A3.3 Short-lasting unilateral neuralgiform headache attacks können die Attacken weniger häufig auftreten.

\section{Kommentar:}

Im Hinblick auf die Aufnahme von C5 und C6 sind die Meinungen geteilt. Fachkollegen in der Arbeitsgruppe glauben, dies verbessere die Sensitivität ohne nennenswerte Einbuße an Spezifizität, formale Feldversuche, die diese Änderung der Kriterien unterstützen würden, wurden jedoch nicht durchgeführt.

\section{A3.4 Hemicrania continua (alternative Kriterien)}

Diagnostische Kriterien:

A. Einseitiger Kopfschmerz, der die Kriterien B bis D erfüllt

B. Für >3 Monate vorliegend, mit Verschlechterung von mäßiger oder größerer Intensität

C. Einer oder beide der folgenden Punkte sind erfüllt:

1. Mindestens eines der folgenden Symptome oder Zeichen, ipsilateral zum Kopfschmerz:

- konjunktivale Injektion und/oder Lakrimation

- nasale Kongestion und/oder Rhinorrhoe

- Lidödem

- vermehrtes Schwitzen im Bereich von Stirn und Gesicht

- Rötung im Bereich von Stirn und Gesicht
- Empfinden eines Völlegefühls im Ohr

- Miosis und/oder Ptosis

2. Ein Gefühl der Unruhe oder Erregung oder eine Verschlechterung des Schmerzes durch Bewegung

D. Spricht zuverlässig auf therapeutische Dosen Indometacin an1

E. Nicht besser erklärt durch eine andere ICHD-3-Diagnose.

\section{Anmerkung:}

Bei Erwachsenen sollte Indometacin anfangs in einer Tagesdosis von mindestens $150 \mathrm{mg}$ oral eingesetzt werden und bei Bedarf auf $\geq 225 \mathrm{mg}$ täglich erhöht werden. Die Dosis für Injektionen beträgt 100 bis $200 \mathrm{mg}$. Als Erhaltungsdosen sind oft jedoch geringere Mengen erforderlich

\section{Kommentar:}

Im Hinblick auf die Aufnahme von (e) und (f) in Kriterium C1 sind die Meinungen geteilt. Fachkollegen in der Arbeitsgruppe glauben, dies verbessere die Sensitivität ohne nennenswerte Einbuße an Spezifizität, formale Feldversuche, die diese Änderung der Kriterien unterstützen würden, wurden jedoch nicht durchgeführt

\section{A3.6 Undifferenzierte trigemino-autonome Kopfschmerzerkrankung}

Beschreibung:

Eine bei Kindern und Jugendlichen auftretende Kopfschmerzerkrankung vom Typ einer trigemino-autonomen Kopfschmerzerkrankung mit nicht voll entwickelten Erkrankungsmerkmalen.

\section{Kommentar:}

Eine unvollständige Entwicklung des Gehirns kann das klinische Erscheinungsbild trigemino-autonomer Kopfschmerzerkrankungen (TAK) verändern. Patienten, die unter einer A3.6 undifferenzierten trigemino-autonomen Kopfschmerzerkrankung kodiert werden, wären typischerweise Kinder oder Jugendliche, deren Kopfschmerzen Charakteristika aufweisen, die stark auf TAK hindeuten, aber vermischt und unvollständig sind - z. B. könnten sie lateralisierte 30 Minuten andauernde Kopfschmerzattacken mit autonomen Merkmalen aufweisen, doch ohne das erwartete Ansprechen auf Indometacin, Sauerstoff oder Triptane.

Es werden Langzeituntersuchungen erforderlich sein, um diese Erscheinungsbilder besser zu verstehen und Kriterien für ihre Diagnose vorzuschlagen.

\section{Literatur}

de Coo IF, Wilbrink LA, Haan J, Ferrari MD, Terwindt GM. Evaluation of the new ICHD-III beta cluster headache criteria. Cephalalgia 2016; 36: 547-551. 


\section{A4. Andere primäre Kopfschmerzen}

\section{A4.11 Epicrania fugax}

Beschreibung:

Kurze paroxysmale Kopfschmerzattacken stechender Qualität, die sich in einer geraden Linie oder im Zickzack über eine Kopfhälfte ziehen.

\section{Diagnostische Kriterien:}

A. Wiederkehrende stechende Kopfschmerzattacken, die 1 bis 10 Sekunden andauern und das Kriterium B erfüllen

B. Attackenweiser Kopfschmerz, der sich in einer geraden Linie oder im Zickzack über eine Kopfhälfte zieht und dabei in den Versorgungsgebieten verschiedener Nerven beginnt und endet

C. Nicht besser erklärt durch eine andere ICHD-3-Diagnose1.

\section{Anmerkung:}

1. Eine strukturelle Läsion muss anamnestisch sowie durch ärztliche Untersuchung und gegebenenfalls weitere Abklärung ausgeschlossen werden.

\section{Kommentar:}

Patienten mit einer A4.11 Epicrania fugax beschreiben ihren Kopfschmerz anhand seiner Bahn zwischen zwei weit voneinander entfernten Punkten auf der Kopfoberfläche, wobei der Weg vom Ausgangs- bis zum Endpunkt nur wenige Sekunden in Anspruch nimmt. Eine derart dynamische Topographie ist ein Unterscheidungsmerkmal, das die Abgrenzung einer A4.11 Epicrania fugax von anderen epikraniellen Kopfschmerzen und Neuralgien erlaubt. Ausgangsund Endpunkt bleiben bei jedem Patienten immer gleich, wobei der Schmerz in der Regel von einer Kopfhälfte hinten in Richtung des ipsilateralen Auges oder der ipsilateralen Nase wandert, doch auch eine Ausstrahlung nach hinten von einer frontalen oder periorbitalen Region in Richtung auf die Okzipitalregion ist möglich. In allen Fällen ist der Kopfschmerz streng einseitig, obgleich bei manchen Patienten die Seiten wechseln.

Am Ende der Attacken können ipsilaterale autonome Zeichen wie Lakrimation, konjunktivale Injektion und/oder Rhinorrhoe auftreten.

Obwohl die Attacken meist spontan auftreten, können sie gelegentlich durch eine Berührung am Ausgangspunkt des Kopfschmerzes ausgelöst werden, der zwischen den Attacken druckempfindlich bleiben kann.

Literatur

Cuadrado ML, Gómez-Vicente L, Porta-Etessam J, Marcos-de-Vega MA, Parejo-Carbonell B, Matías-Guiu J. Paroxysmal head pain with backward radiation. Will epicrania fugax go in the opposite direction? J Headache Pain 2010; 11: 75-78.

Cuadrado ML, Guerrero AL, Pareja JA. Epicrania fugax. Curr Pain Headache Rep 2016; 20: 21.

Guerrero AL, Cuadrado ML, Porta-Etessam J, García-Ramos R, Gómez-Vicente L, Herrero S, Peñas ML, Fernández R. Epicrania fugax: ten new cases and therapeutic results. Headache 2010; 50: 451-458.
Herrero-Velázquez S, Guerrero-Peral ÁL, Mulero P, Peñas-Martínez ML, Cortijo E, Miranda S, Rojo-Martínez E, Henao A, Rodríguez R, Pedraza M, Hurtado ML, Marco-Llorente J, Fernández-Buey MN, Fernández R. Epicrania fugax: the clinical characteristics of a series of 18 patients. Rev Neurol 2011; 53: 531-537.

Mulero P, Guerrero AL, Herrero-Velázquez S, Cortijo E, Pedraza M, Peñas ML, Miranda S, Rojo E, Fernández R. Epicrania Fugax with backward radiation. Clinical characteristics of 9 new cases. J Headache Pain 2011; 12: 535-539.

Pareja JA, Alvarez M, Montojo T. Epicrania fugax with backward radiation. J Headache Pain 2012; 13: 175.

Pareja JA, Cuadrado ML, Fernández de las Peñas C. Caminero AB, Nieto C, Sánchez C, Sols M, Porta-Etessam J. Epicrania fugax: an ultrabrief paroxysmal epicranial pain. Cephalalgia 2008; 28: 257-263.

\section{A5. Kopfschmerz zurückzuführen auf eine Verletzung oder ein Trauma des Kopfes und/oder der HWS}

\section{A5.1 Akuter Kopfschmerz zurückzuführen auf eine traumatische Verletzung des Kopfes}

Kommentar:

Die aktuelle Bedingung, dass der Kopfschmerz innerhalb von 7 Tagen nach einem Kopftrauma (oder dem Zeitpunkt, wo ein solches bemerkt wurde) beginnen muss (oder laut Bericht begonnen haben muss), ist etwas willkürlich. Einige Daten lassen darauf schließen, dass der Kopfschmerz nach einem längeren Intervall einsetzen kann. In den im Folgenden vorgeschlagenen diagnostischen Kriterien wird als maximales zeitliches Intervall zwischen Kopftrauma und Kopfschmerzbeginn 3 Monate angesetzt, doch wird angenommen, dass es bei Kopfschmerzen, die zeitlich näher an der Verletzung liegen, wahrscheinlicher ist, dass diese korrekt auf das Kopftrauma zurückgeführt werden. In künftigen Untersuchungen sollte die Nützlichkeit dieser und alternativer diagnostischer Kriterien für einen A5. 1 akuten Kopfschmerz zurückzuführen auf eine traumatische Verletzung des Kopfes weiter untersucht werden, die zulassen, dass der Kopfschmerz mehr als 7 Tage und bis zu 3 Monaten nach dem Kopftrauma beginnt.

\section{A5.1.1.1 Verzögert auftretender akuter Kopfschmerz zurückzuführen auf ein mittleres oder schweres Kopftrauma}

Diagnostische Kriterien:

A. Jeder Kopfschmerz, der die Kriterien C und D erfüllt

B. Kopftrauma, welches wenigstens einen der folgenden Punkte erfüllt:

1. Bewusstseinsverlust für $>30$ Minuten

2. Glasgow Coma Scale (GCS) Werte $<13$

3. posttraumatische Amnesie von $>24$ Stunden 1 Dauer

4. Bewusstseinsstörung für $>24$ Stunden

5. Nachweis einer traumatischen Kopfverletzung wie etwa einer Schädelfraktur, intrakraniellen Blutung und/oder Hirnkontusion in der zerebralen Bildgebung

C. Der Kopfschmerz hat sich laut Bericht zwischen 7 Tagen und 3 Monaten nach Folgendem entwickelt:

1. Der Kopfverletzung 
2. Der Wiedererlangung des Bewusstseins nach der Kopfverletzung (sofern zutreffend)

3. Absetzen von Medikamenten, die die Fähigkeit beeinträchtigen, Kopfschmerzen nach der Kopfverletzung zu spüren oder über diese zu berichten (sofern zutreffend)

D. Es ist wenigstens einer der folgenden Punkte erfüllt:

1. Der Kopfschmerz ist innerhalb von 3 Monaten nach seinem Beginn verschwunden

2. der Kopfschmerz ist noch nicht verschwunden, aber es sind noch keine 3 Monate nach seinem Beginn verstrichen

E. Nicht besser erklärt durch eine andere ICHD-3-Diagnose.

\section{A5.1.2.1 Verzögert auftretender akuter Kopfschmerz zurückzuführen auf ein leichtes Kopftrauma \\ Diagnostische Kriterien:}

A. Jeder Kopfschmerz, welcher die Kriterien C und D erfüllt

B. Kopftrauma, welches beide der folgenden Punkte erfüllt: 1. Mit keinem der folgenden Aspekte verbunden:

a) Bewusstseinsverlust für $>30$ Minuten

b) Glasgow Coma Scale (GCS) Werte $<13$

c) posttraumatische Amnesie von > 24 Stunden 1 Dauer

d) Bewusstseinsstörung für $>24$ Stunden

e) Nachweis einer traumatischen Kopfverletzung wie etwa einer Schädelfraktur, intrakraniellen Blutung und/oder Hirnkontusion in der zerebralen Bildgebung

2. Tritt unmittelbar nach der Kopfverletzung in Begleitung von einem oder mehreren der folgenden Symptome und/ oder Zeichen auf:

- vorübergehende Verwirrung, Orientierungslosigkeit oder Bewusstseinsbeeinträchtigung

- Verlust der Erinnerung an Ereignisse unmittelbar vor oder nach der Kopfverletzung

- zwei oder mehr der folgenden Symptome, die an eine leichte traumatische Hirnverletzung denken lassen:

i. Übelkeit

ii. Erbrechen

iii. Sehstörungen

iv. Benommenheit und/oder Schwindel

v. unsicheres Gangbild und/oder orthostatische Dysregulation mit Gleichgewichtsstörungen

vi. Beeinträchtigungen des Gedächtnisses und/oder der Konzentration.

C. Der Kopfschmerz hat sich laut Bericht zwischen 7 Tagen und 3 Monaten nach allem Folgenden entwickelt:

1. der Kopfverletzung

2. der Wiedererlangung des Bewusstseins nach der Kopfverletzung (sofern zutreffend)

3. dem Absetzen von Medikamenten, die die Fähigkeit beeinträchtigen, Kopfschmerzen nach der Kopfverletzung zu spüren oder über diese zu berichten (sofern zutreffend)

D. Es ist wenigstens einer der folgenden Punkte erfüllt:

1. Der Kopfschmerz ist innerhalb von 3 Monaten nach seinem Beginn verschwunden

2. Der Kopfschmerz ist noch nicht verschwunden, aber es sind noch keine 3 Monate nach seinem Beginn verstrichen E. Nicht besser erklärt durch eine andere ICHD-3-Diagnose.

\section{A5.2 Anhaltender Kopfschmerz zurück- zuführen auf eine traumatische Verletzung des Kopfes}

Kommentar:

Die aktuelle Bedingung, dass der Kopfschmerz innerhalb von 7 Tagen nach einem Kopftrauma (oder dem Zeitpunkt, wo ein solches bemerkt wurde) beginnen muss (oder laut Bericht begonnen haben muss), ist etwas willkürlich. Einige Daten lassen darauf schließen, dass der Kopfschmerz auch nach einem längeren Intervall beginnen kann. In den folgenden vorgeschlagenen diagnostischen Kriterien wird als maximales zeitliches Intervall zwischen Kopftrauma und Kopfschmerzbeginn 3 Monate angesetzt, doch wird angenommen, dass es bei Kopfschmerzen, die zeitlich näher an der Verletzung liegen, wahrscheinlicher ist, dass diese korrekt auf das Kopftrauma zurückgeführt werden. In künftigen Untersuchungen sollte die Nützlichkeit dieser und alternativer diagnostischer Kriterien für einen A5.2 anhaltenden Kopfschmerz zurückzuführen auf eine traumatische Verletzung des Kopfes weiter untersucht werden, die zulassen, dass der Kopfschmerz mehr als 7 Tage und bis zu 3 Monaten nach dem Kopftrauma beginnt.

\section{A5.2.1.1 Verzögert auftretender anhaltender Kopfschmerz zurückzuführen auf ein mittleres oder schweres Kopftrauma}

Diagnostische Kriterien:

A. Jeder Kopfschmerz, welcher die Kriterien C und D erfüllt

B. Es ist ein Kopftrauma eingetreten, begleitet von einem der folgenden Punkte:

1. Bewusstseinsverlust für $>30$ Minuten

2. Glasgow Coma Scale (GCS) Werte $<13$

3. posttraumatische Amnesie von > 24 Stunden 1 Dauer

4. Bewusstseinsstörung für $>24$ Stunden

5. Nachweis einer traumatischen Kopfverletzung wie etwa einer Schädelfraktur, intrakraniellen Blutung und/oder Hirnkontusion in der zerebralen Bildgebung

C. Der Kopfschmerz hat sich laut Bericht zwischen 7 Tagen und 3 Monaten nach allem Folgenden entwickelt:

1. der Kopfverletzung

2. der Wiedererlangung des Bewusstseins nach der Kopfverletzung (sofern zutreffend)

3. dem Absetzen von Medikamenten, die die Fähigkeit beeinträchtigen, Kopfschmerzen nach der Kopfverletzung zu spüren oder über diese zu berichten (sofern zutreffend)

D. Der Kopfschmerz hält für > 3 Monate nach seinem Beginn an

E. Nicht besser erklärt durch eine andere ICHD-3-Diagnose.

\section{A5.2.2.1 Verzögert auftretender anhaltender Kopfschmerz zurückzuführen auf ein leichtes Kopftrauma}

Diagnostische Kriterien:

A. Jeder Kopfschmerz, welcher die Kriterien C und D erfüllt

B. Kopftrauma, welches beide der folgenden Punkte erfüllt:

1. Mit keinem der folgenden Aspekte verbunden:

- Bewusstseinsverlust für > 30 Minuten

- Glasgow Coma Scale (GCS) Werte<13

- posttraumatische Amnesie für die Dauer von>24 Stunden 1 
- Bewusstseinsstörung für > 24 Stunden

- Nachweis einer traumatischen Kopfverletzung wie etwa einer Schädelfraktur, intrakraniellen Blutung und/oder Hirnkontusion in der zerebralen Bildgebung

2. Tritt in Begleitung von einem oder mehreren der folgenden Symptome und/oder Zeichen unmittelbar nach der Kopfverletzung auf:

- vorübergehende Verwirrung, Orientierungslosigkeit oder Bewusstseinsbeeinträchtigung

- Verlust der Erinnerung an Ereignisse unmittelbar vor oder nach der Kopfverletzung

- zwei oder mehr der folgenden Symptome, die an eine leichte traumatische Hirnverletzung denken lassen:

i. Übelkeit

ii. Erbrechen

iii. Sehstörungen

iv. Benommenheit und/oder Schwindel

v. unsicheres Gangbild und/oder orthostatische Dysregulation mit Gleichgewichtsstörungen

vi. Beeinträchtigungen des Gedächtnisses und/oder der Konzentration.

C. Der Kopfschmerz hat sich laut Bericht zwischen 7 Tage und 3 Monaten nach allem Folgenden entwickelt:

1. der Kopfverletzung

2. gfls. der Wiedererlangung des Bewusstseins nach der Kopfverletzung

3. gfls. Absetzung der Medikation(en), welche die Fähigkeit, Kopfschmerzen nach der Kopfverletzung zu spüren oder über sie zu berichten, beeinträchtigt/beeinträchtigen

D. Der Kopfschmerz hält>3 Monate nach seinem Beginn an

E. Nicht besser erklärt durch eine andere ICHD-3-Diagnose.

\section{A5.7 Kopfschmerz zurückzuführen auf einen radiochirurgischen Eingriff am Gehirn}

Diagnostische Kriterien:

A. Jeder neue Kopfschmerz, der das Kriterium C erfüllt

B. Zustand nach radiochirurgischem Eingriff am Gehirn

C. Ein kausaler Zusammenhang kann durch beide der folgenden Kriterien gezeigt werden:

1. Der Kopfschmerz hat sich innerhalb von 7 Tagen nach dem radiochirurgischen Eingriff entwickelt

2. Der Kopfschmerz ist innerhalb von 3 Monaten nach dem radiochirurgischen Eingriff verschwunden

D. Nicht besser erklärt durch eine andere ICHD-3-Diagnose.

Kommentar:

Obwohl das Auftreten neuer Kopfschmerzen im Anschluss an einen radiochirurgischen Eingriff beschrieben wurde, beinhalten die meisten Studien keine detaillierten Beschreibungen der klinischen Charakteristika dieser Kopfschmerzen. Darüber hinaus ist nicht klar, ob es sich in diesen Fällen tatsächlich um einen neuen Kopfschmerz handelt oder doch lediglich um die Exacerbation einer bereits bestehenden Kopfschmerzerkrankung. In den Fällen ohne Kopfschmerzvorgeschichte war das beschriebene Kopfschmerzsyndrom kurzlebig, trat mehr als ein Jahr nach der Behandlung auf und ähnelte einer Migräne oder einem Donnerschlagkopfschmerz. Aus diesem Grund erscheint eine ursächliche Beziehung zwischen den Kopfschmerzen und der Behandlung zumindest zweifelhaft. Weitere sorgfältig durchgeführte prospektive Studien sind notwendig, um endgültig zu klären, ob ein A5.7 Kopfschmerz zurückzuführen auf einen radiochirurgischen Eingriff am Gehirn als Entität vorliegt und, falls dies der Fall sein sollte, wie dieser in Beziehung zu Typ und Lokalisation der bestrahlten Läsion bzw. der eingesetzten Strahlendosis und dem Strahlenfeld steht.

\section{A5.8 Akuter Kopfschmerz zurückzuführen auf ein anderes Kopf- und/oder HWS-Trauma oder eine andere Kopf- und/oder HWS-Verletzung Diagnostische Kriterien:}

A. Jeder Kopfschmerz, der die Kriterien C und D erfüllt

B. Zustand nach Kopf- und/oder HWS-Trauma oder Kopf- und/ oder HWS-Verletzung eines oben nicht bereits beschriebenen Typs

C. Ein kausaler Zusammenhang kann durch eines oder beide der folgenden Kriterien gezeigt werden:

1. Der Kopfschmerz hat sich in engem zeitlichem Zusammenhang mit dem Trauma oder der Verletzung entwickelt

2. Es existieren andere Belege für eine Verursachung durch das Trauma oder die Verletzung

D. Es ist wenigstens einer der folgenden Punkte erfüllt:

1. Der Kopfschmerz ist innerhalb von 3 nach seinem Beginn verschwunden

2. Der Kopfschmerz hält an, aber es sind noch keine 3 Monate seit seinem Beginn verstrichen

E. Nicht besser erklärt durch eine andere ICHD-3-Diagnose.

\section{A5.9 Anhaltender Kopfschmerz zurück- zuführen auf ein anderes Kopf- oder HWS-Trauma oder eine andere Kopf- oder HWS-Verletzung}

\section{Diagnostische Kriterien:}

A. Jeder Kopfschmerz, welcher die Kriterien C und D erfüllt

B. Es hat ein Kopf- und/oder HWS-Trauma oder eine Kopf- und/ oder HWS-Verletzung eines oben nicht beschriebenen Typs stattgefunden

C. Ein kausaler Zusammenhang kann durch eines oder beide der folgenden Kriterien gezeigt werden:

1. Der Kopfschmerz hat sich in einem engen zeitlichen Zusammenhang mit dem Trauma oder der Verletzung entwickelt

2. Es existieren andere Belege für eine Verursachung durch das Trauma oder die Verletzung

D. Der Kopfschmerz hält>3 Monate nach seinem Beginn an

E. Nicht besser erklärt durch eine andere ICHD-3-Diagnose. 
Lucas S, Hoffman JM, Bell KR, Dikmen S. A prospective study of prevalence and characterization of headache following mild traumatic brain injury. Cephalalgia 2014; 34: 93-102.

Lucas S, Hoffman JM, Bell KR, Walker W, Dikmen S. Characterization of headache after traumatic brain injury. Cephalalgia 2012; 32: 600-606.

Olesen J. Problem areas in the International Classification of Headache Disorders, 3 rd edition (beta). Cephalalgia 2014; 34: 1193-1199.

Theeler BJ, Erickson JC. Post-traumatic headaches: time for a revised classification? Cephalalgia 2012; 32: 589-591.

Theeler BJ, Flynn FG, Erickson JC. Headaches after concussion in US soldiers returning from Iraq or Afghanistan. Headache 2010; 50: 1262-1272.

Theeler B, Lucas S, Riechers RG, Ruff RL. Post-traumatic headaches in civilians and military personnel: a comparative, clinical review. Headache 2013; 53: 881-900.

A6. Kopfschmerz zurückzuführen auf Gefäßstörungen im Bereich des Kopfes und/ oder des Halses

\section{A6.10 Anhaltender Kopfschmerz zurückzuführen auf frühere Störungen im Bereich des Kopfes oder des Halses}

A. Kopfschmerz, der zuvor als 6. Kopfschmerz zurückzuführen auf Gefäßstörungen im Bereich des Kopfes und/oder des Halses bzw. einer der Typen oder Subtypen hiervon diagnostiziert wurde und der das Kriterium C erfüllt

B. Die kranielle oder zervikale vaskuläre Störung, die den Kopfschmerz verursacht, wurde wirksam behandelt oder hat sich spontan zurückgebildet

C. Der Kopfschmerz hat nach wirksamer Behandlung oder Spontanremission der vaskulären Erkrankung > 3 Monate anhält

D. Nicht besser erklärt durch eine andere ICHD-3-Diagnose.

Kommentar:

Anhaltender Kopfschmerz zurückzuführen auf frühere Störungen im Bereich des Kopfes oder des Halses ist schlecht dokumentiert; falls er existiert, ist weitere Forschung erforderlich, um bessere Kriterien für den Kausalzusammenhang zu erarbeiten.

\section{A7. Kopfschmerz zurückzuführen auf nicht- vaskuläre intrakranielle Störungen}

\section{A7.6 Kopfschmerz zurückzuführen auf einen epileptischen Anfall}

\section{A7.6.3 Kopfschmerz nach Elektrokrampftherapie} Diagnostische Kriterien:

A. Rezidivierender Kopfschmerz, der Kriterium C erfüllt

B. Zustand nach Elektrokrampftherapie

C. Ein kausaler Zusammenhang kann durch alle der folgenden Kriterien gezeigt werden:

1. Der Kopfschmerz hat sich nach $\geq 50 \%$ der EKT-Sitzungen entwickelt
2. Der Kopfschmerz hat sich jeweils innerhalb von 4 Stunden nach der Elektrokrampftherapie entwickelt

3. Der Kopfschmerz ist jeweils innerhalb von 72 Stunden nach der Elektrokrampftherapie verschwunden

D. Nicht besser erklärt durch eine andere ICHD-3-Diagnose.

\section{Kommentar:}

Eindeutige Beschreibungen von Kopfschmerzen in Verbindung mit einer Elektrokrampftherapie (EKT) sind spärlich. In einer Einzelblind-Vergleichsstudie zu Eletriptan und Paracetamol klagten 20 von 72 Patienten ( $28 \%$ ) über Kopfschmerzen, doch diese wurden nicht genau charakterisiert (es nur wurden nur Sitz und Qualität des Schmerzes beurteilt).

Die veröffentlichten Daten dürften nicht ausreichen, um brauchbare Kriterien für einen A7.6.3 Kopfschmerz nach Elektrokrampftherapie operational zu definieren und es wurden seit der Veröffentlichung von ICHD-3 beta keine Validierungsstudien zu diesen vorgeschlagenen Kriterien durchgeführt.

\section{A7.9 Anhaltender Kopfschmerz zurückzu- führen auf eine frühere nichtvaskuläre intrakranielle Störung \\ Diagnostische Kriterien:}

A. Kopfschmerz, der zuvor als ein 7. Kopfschmerz zurückzuführen auf nichtvaskuläre intrakranielle Störungen bzw. einer der Typen oder Subtypen hiervon diagnostiziert wurde und der das Kriterium C erfüllt

B. Die nichtvaskuläre intrakranielle Störung, die den Kopfschmerz verursacht wurde wirksam behandelt oder hat sich spontan zurückgebildet

C. Der Kopfschmerz hat>3 Monate nach wirksamer Behandlung oder Spontanremission der nichtvaskulären Erkrankung angehalten

D. Nicht besser erklärt durch eine andere ICHD-3-Diagnose.

\section{Kommentar:}

Aus der klinischen Erfahrung ist bekannt, dass anhaltende Kopfschmerzen nach einer früheren (und verschwundenen) nichtvaskulären intrakraniellen Störung auftreten können; in gewissem Umfang wurden derartige Kopfschmerzen nach einem 7.1.1 Kopfschmerz zurückzuführen auf eine idiopathische intrakranielle Hypertension and 7.2.3 Kopfschmerz zurückzuführen auf ein spontanes Liquorunterdrucksyndrom nachgewiesen. Ein A7.9 anhaltender Kopfschmerz zurückzuführen auf eine frühere nichtvaskuläre intrakranielle Störung ist allerdings schlecht dokumentiert. Forschung ist erforderlich, um bessere Kriterien für den Kausalzusammenhang zu erarbeiten.

\section{Literatur}

Belcastro V, Striano P, Kasteleijn-Nolst Trenité DGA, et al. Migralepsy, hemicrania epileptica, post-ictal headache and "ictal epileptic headache": a proposal for terminology and classification revision. J Headache Pain 2011; 12: 289-294. 
Canuet L, Ishii R, Iwase M, Kurimoto R, Ikezawa K, Azechi M, Takahashi H, Nakahachi T, Takeda M. Cephalic auras of supplementary motor area origin: an ictal MEG and SAM(g2) study. Epilepsy Behav 2008; 13: 570-574.

Dinwiddie SH. Huo D. Gottlieb O. The course of myalgia and headache after electroconvulsive therapy. J ECT 2010; 26: 116-120.

Kertesz DP, Trabekin O, Vanetik MS. Headache treatment after electroconvulsive treatment: A single-blinded trial comparator between eletriptan and paracetamol. ECT 2015; 31: 105-109.

Mendez MF, Doss RC, Taylor JL, Arguello R. Relationship of seizure variables to personality disorders in epilepsy. J Neuropsychiatry Clin Neurosci 1993; 5: 283-286.

Parisi P, Striano P, Kasteleijn-Nolst Trenite DGA, Verrotti A, Martelletti P, Pia Villa M, Belcastro V. 'Ictal epileptic headache': Recent concepts for new classification criteria. Cephalalgia 2012; 32: 723-724.

Schweder LJ. Wahlund B. Bergsholm P. Linaker OM. Questionnaire study about the practice of electroconvulsive therapy in Norway. J ECT 2011; 27: 296-299.

Siegel AM, Williamson PD, Roberts DW, Thadani VM, Darcey TM. Localized pain associated with seizures originating in the parietal lobe. Epilepsia 1999; 40: 845-855.

Young GB, Blume WT. Painful epileptic seizures. Brain 1983; 106: 537-554.

\section{A8. Kopfschmerz zurückzuführen auf eine Substanz oder deren Entzug}

\section{A8.4 Anhaltender Kopfschmerz zurückzuführen auf früheren Substanzgebrauch oder frühere Substanzexposition}

An anderer Stelle kodiert: 8.2 Kopfschmerz zurückzuführen auf einen Medikamentenübergebrauch.

\section{Diagnostische Kriterien:}

A. Kopfschmerz zuvor kodiert als 8.1 Kopfschmerz induziert durch akuten Substanzgebrauch oder akute Substanzexposition oder eines Subtyps, der das Kriterium C erfüllt

B. Der Substanzgebrauch oder die Substanzexposition besteht aktuell nicht mehr

C. Der Kopfschmerz besteht > 3 Monate nach Ende der Exposition weiter

D. Nicht besser erklärt durch eine andere ICHD-3-Diagnose.

\section{A9. Kopfschmerz zurückzuführen auf eine Infektion}

\section{A9.1 Kopfschmerz zurückzuführen auf eine intra- kranielle Infektion}

\section{A9.1.3.3 Anhaltender Kopfschmerz zurückzuführen auf} eine frühere intrakranielle Pilz- oder andere parasitäre Infektion

Diagnostische Kriterien:

A. Kopfschmerz, der zuvor die Kriterien für einen 9.1.3 Kopfschmerz zurückzuführen auf eine Pilz- oder andere parasitäre Infektion und das Kriterium C erfüllt

B. Eine intrakranielle Pilz- oder andere parasitäre Infektion ist abgeklungen
C. Der Kopfschmerz hat>3 Monate nach Abklingen der intrakraniellen Pilz- oder anderen parasitären Infektion angehalten

D. Nicht besser erklärt durch eine andere ICHD-3-Diagnose, und ein Hydrozephalus wurde anhand von bildgebenden Verfahren ausgeschlossen.

\section{A9.3 Kopfschmerz zurückzuführen auf eine HIV-Infektion}

An anderer Stelle kodiert: Ein Kopfschmerz, der bei HIV-infizierten Patienten auftritt, aber durch eine spezifische opportunistische Infektion ausgelöst wird, sollte unter Letzterem kodiert werden. Kopfschmerz verursacht durch antiretrovirale Medikamente sollte unter 8.1.10 Kopfschmerz zurückzuführen auf eine nicht gegen Kopfschmerz eingesetzte Dauermedikation kodiert werden.

Diagnostische Kriterien:

A. Kopfschmerz, der das Kriterium C erfüllt

B. Beide der folgenden Punkte sind erfült:

1. Es wurde eine systemische HIV-Infektion nachgewiesen

2. Eine andere ablaufende systemische und/oder intrakranielle Infektion wurde ausgeschlossen

C. Ein kausaler Zusammenhang kann durch mindestens zwei der folgenden Kriterien gezeigt werden:

1. Der Kopfschmerz hat sich in einem zeitlichen Zusammenhang mit dem Beginn der HIV-Infektion entwickelt

2. Der Kopfschmerz hat sich in einem zeitlichen Zusammenhang mit einer Verschlechterung der HIV-Infektion, nachgewiesen durch CD4-Zellzahl und/oder Viruslast, entwickelt oder deutlich verschlechtert

3. Der Kopfschmerz hat sich gleichzeitig mit einer Besserung der HIV-Infektion, nachgewiesen durch CD4-Zellzahl und/ oder Viruslast, deutlich gebessert

D. Nicht besser erklärt durch eine andere ICHD-3-Diagnose.

\section{Kommentar:}

Es gibt drei Gründe dafür, A9.3 Kopfschmerz zurückzuführen auf eine HIV-Infektion von Kopfschmerzen zu trennen, die auf eine andere Erkrankung zurückzuführen sind:

Eine HIV-Infektion ist immer sowohl systemisch als auch innerhalb des Zentralnervensystems zu verstehen;

Die Infektion des Zentralnervensystems kann unabhängig von der systemischen Infektion fortschreiten;

Die HIV-Infektion ist bislang noch nicht heilbar.

Von Kopfschmerzen berichten mehr als die Hälfte der HIV-I AIDS-Infizierten, und Kopfschmerz kann ein Bestandteil der Symptomatik bei akuter wie auch chronischer HIV-Infektion sein (über eine aseptische Meningitis und ähnliche Mechanismen). Dennoch verbleibt der A9.3 Kopfschmerz zurückzuführen auf eine HIV-Infektion im Anhang, da es außerordentlich schwierig ist, Kopfschmerz, der lediglich auf die HIV-Infektion zurückzuführen ist, von den quasi primären Kopfschmerzen zu unterscheiden, von denen die meisten HIV-Patienten berichten. Eine Anwendung dieser Kriterien in prospektiven Untersuchungen könnte schlüssigere Nachweise erbringen. 
In den meisten Fällen ist ein A9.3 Kopfschmerz zurückzuführen auf eine HIV-Infektion dumpf und bilateral oder weist die Merkmale einer primären Kopfschmerzerkrankung auf (1. Migräne oder 2. Kopfschmerz vom Spannungstyp). Die Intensität und Häufigkeit des Kopfschmerzes sowie die auf ihn zurückgeführte Behinderung scheinen mit der durch CD4-Zellzahl und/oder Viruslast nachgewiesenen Schwere der HIV-Infektion assoziiert, nicht jedoch mit der Dauer der HIV-Infektion oder der Anzahl der verschriebenen antiretroviralen Medikamente.

Nur eine Minderheit der HIV-Patienten hat Kopfschmerzen, die auf opportunistische Infektionen zurückzuführen sind, was an der Verfügbarkeit der hochaktiven antiretroviralen Therapie liegen dürfte.

Im Rahmen einer HIV-Infektion kann sich eine sekundäre Meningitis und/oder Enzephalitis in Begleitung opportunistischer Infektionen oder Neoplasmen entwickeln. Die häufigsten intrakraniellen Infektionen in Verbindung mit einer HIV-Infektion, die Kopfschmerzen verursachen können, sind Toxoplasmose und Kryptokokkenmeningitis. Kopfschmerzen, die bei Patienten mit einer HIV-Infektion auftreten, aber auf eine bestimmte opportunistische Infektion zurückgeführt werden, sollten unter dieser Infektion kodiert werden.

Auch antiretrovirale Medikamente können Kopfschmerzen verursachen. In diesen Fällen sollte der Kopfschmerz als ein 8.1.10 Kopfschmerz zurückzuführen auf eine nicht gegen Kopfschmerz eingesetzte Dauermedikation kodiert werden.

\section{Literatur}

Berger JR. Pearls: neurologic complications of HIV/AIDS. Semin Neurol 2010; 30: 66-70.

Brew BJ, Miller J. Human immunodeficiency virus-related headache. Neurology 1993; 43: 1098-1100.

Denning DW. The neurological features of HIV infection. Biomed Pharmacother 1988; 42: 11-14.

Evers S, Wibbeke B, Reichelt D, Suhr B, Brilla R, Husstedt IW. The impact of HIV infection on primary headache. Unexpected findings from retrospective, cross-sectional, and prospective analyses. Pain 2000; 85: 191-200.

Hollander $\mathrm{H}$, Strimgari S. Human immunodeficiency virus-associated meningitis. Clinical course and correlations. Am J Med 1987; 83: 813-816.

Kirkland KE, Kirkland K, Many Jr W], Smitherman TA. Headache among patients with HIV disease: prevalence, characteristics, and associations. Headache 2011; 52: 455-466.

Mirsattari SM, Power C, Nath A. Primary headaches in HIV-infected patients. Headache 1999; 39: 3-10.

Norval DA. Symptoms and sites of pain experienced by AIDS patients. $S \mathrm{Afr}$ Med J 2004; 94: 450-454.

Rinaldi R, Manfredi R, Azzimondi G, et al. Recurrent “migraine-like" episodes in patients with HIV disease. Headache 2007; 37: 443-448.

Valcour V, Chalermchai T, Sailasuta N, Marovich M, Lerdlum S, Suttichom D, Suwanwela NC, Jagodzinski L, Michael N, Spudich S, van Griensven F, de Souza M, Kim J, Ananworanich J; on behalf of the RV254/SEARCH 010 Study Group. Central nervous system viral invasion and inflammation during acute HIV infection. J Infect Dis 2012; 206: 275-282.

Weinke T, Rogler G, Sixt C, et al. Cryptococcosis in AIDS patients: observations concerning CNS involvement. J Neurol 1989; 236: 38-42.

\section{A10. Kopfschmerz zurückzuführen auf eine Störung der Homöostase}

\section{A10.7 Kopf- und/oder Nackenschmerzen zurück- zuführen auf eine orthostatische (posturale) Hypotonie}

\section{Beschreibung:}

Schmerzen meist im Nacken, mitunter jedoch sich nach oben in die Okzipitalregion ausbreitend („Kleiderbügel“-Verteilung), zurückzuführen auf eine orthostatische Hypotonie und nur in aufrechter Körperhaltung entstehend.

\section{Diagnostische Kriterien:}

A. Kopfschmerz, der Kriterium C erfüllt

B. Eine orthostatische (posturale) Hypotonie wurde nachgewiesen

C. Ein kausaler Zusammenhang kann durch wenigstens zwei der folgenden Kriterien gezeigt werden:

1. Der Kopfschmerz entwickelt sich ausschließlich in aufrechter Körperhaltung

2. Der Kopfschmerz bessert sich spontan in liegender Haltung

3. Der Kopfschmerz findet sich größtenteils im Bereich des Nackens, mitunter mit Ausbreitung nach oben in die Hinterhauptsregion („Kleiderbügel“-Verteilung)

D. Nicht besser erklärt durch eine andere ICHD-3-Diagnose.

\section{Kommentar:}

Auf gezielte Nachfrage berichten $75 \%$ der Patienten mit orthostatischer Hypotonie über Nackenschmerzen.

\section{A10.8 Kopfschmerz zurückzuführen auf andere Störungen der Homöostase}

\section{A10.8.1 Kopfschmerz zurückzuführen auf einen Aufenthalt im Weltraum}

Beschreibung:

Nicht-spezifischer Kopfschmerz infolge eines Aufenthalts im Weltraum. Ein Großteil der Kopfschmerzepisoden tritt nicht Begleitung von Symptomen einer Weltraumkrankheit auf.

\section{Diagnostische Kriterien:}

A. Jeder neue Kopfschmerz, der das Kriterium C erfüllt

B. Weltraumaufenthalt des Patienten

C. Ein kausaler Zusammenhang kann durch beide der folgenden Kriterien gezeigt werden:

1. Der Kopfschmerz ist ausschließlich im Weltraum aufgetreten

2. Der Kopfschmerz hat sich bei der Rückkehr zur Erde spontan gebessert

D. Nicht besser erklärt durch eine andere ICHD-3-Diagnose.

\section{Kommentar:}

Von den 16 männlichen Astronauten und einer Astronautin, die an einer Befragung teilnahmen, berichteten 12 (71\%) von mindestens einer im Weltraum erlebten Kopfschmerzepisode, während sie auf der Erde nicht unter Kopfschmerzen litten. 
A10.8.2 Kopfschmerz zurückzuführen auf andere metabolische oder systemische Störungen

Kopfschmerzen, die folgenden Syndromen zugeschrieben werden, sind bislang nicht ausreichend validiert:

Anämie, Hyperkapnie, Nebennierenrindeninsuffizienz, Mangel an Mineralokortikoiden, Hyperaldosteronismus, Polyzythämie, Hyperviskositätssyndrom, thrombotische thrombozytopenische Purpura, Plasmapherese-induzierter Kopfschmerz, Antikardiolipin-Antikörper-Syndrom, M. Cushing, Hyponatriämie, Hyperthyreoidismus, Hyperglykämie, Hyperkalzämie, systemischer Lupus erythematodes, Chronic Fatigue Syndrome, Fibromyalgie.

Kontrollierte, prospektive Untersuchungen werden benötigt, um die Inzidenz und die Merkmale der Kopfschmerzen, die in Verbindung mit diesen Erkrankungen auftreten, eindeutig zu bestimmen. Nur solche Patienten, die die etablierten Kriterien für diese Erkrankungen erfüllen, sollten evaluiert werden.

\section{A10.9 Anhaltender Kopfschmerz zurück- zuführen auf eine frühere Störung der Homöostase}

Diagnostische Kriterien:

A. Kopfschmerz, der zuvor als 10. Kopfschmerz zurückzuführen auf eine Störung der Homöostase diagnostiziert wurde und das Kriterium C erfüllt

B. Die Störung der Homöostase, die den Kopfschmerz verursacht, wurde wirksam behandelt oder hat sich spontan zurückgebildet

C. Der Kopfschmerz hat $>3$ Monate nach wirksamer Behandlung oder Spontanremission der Störung der Homöostase angehalten

D. Nicht besser erklärt durch eine andere ICHD-3-Diagnose.

\section{Literatur}

Cariga P, Ahmed S, Mathias C], Gardner BP. The prevalence and association of neck (coat-hanger) pain and orthostatic (postural) hypotension in human spinal cord injury. Spinal Cord 2002; 40: 77-82.

Mathias C], Mallipeddi R, Bleasdale-Barr K. Symptoms associated with orthostatic hypotension in pure autonomic failure and multiple system atrophy. J Neurol 1999; 246: 893-898.

Vein AA, Koppen H, Haan J, Terwindt GM, Ferrari MD. Space headache: a new secondary headache. Cephalalgia 2009; 29: 683-686.
A11. Kopf- oder Gesichtsschmerzen zurückzuführen auf Erkrankungen des Schädels sowie von Hals, Augen, Ohren, Nase, Nebenhöhlen, Zähnen, Mund und anderen Gesichts- oder Schädelstrukturen

\section{A11.2 Kopfschmerz zurückzuführen auf Erkrankungen des Halses}

A11.2.4 Kopfschmerz zurückzuführen auf eine Radikulopathie der oberen HWS

Diagnostische Kriterien:

A. Kopf- und/oder Nackenschmerz, der das Kriterium C erfüllt

B. Klinischer, elektrodiagnostischer oder radiologischer Nachweis einer C2-oder C3-Radikulopathie

C. Ein kausaler Zusammenhang kann durch beide der folgenden Kriterien gezeigt werden:

1. Wenigstens zwei der folgenden Punkte sind erfüllt:

a) Der Schmerz hat sich in einem zeitlichen Zusammenhang mit dem Beginn der Radikulopathie entwickelt oder führte zu ihrer Entdeckung

b) Der Schmerz hat sich gleichzeitig mit der Besserung oder Verschlechterung der Radikulopathie deutlich gebessert oder verschlechtert

c) Der Schmerz wird durch Anwendung von Lokalanästhetika der entsprechenden Nervenwurzel temporär beseitigt

2. Der Kopfschmerz ist ipsilateral zur Radikulopathie D. Nicht besser erklärt durch eine andere ICHD-3-Diagnose.

Kommentar:

Der Kopfschmerz ist üblicherweise hinten lokalisiert, kann aber in weiter vorne gelegene Regionen ausstrahlen. Oft findet sich ein lanzinierender Schmerz in einem der von den oberen zervikalen Nervenwurzeln versorgten Areale auf einer Seite oder auf zwei Seiten, im Allgemeinen im okzipitalen, retroaurikulären Bereich oder im Bereich der oberen Halswirbel hinten.

\section{A11.2.5 Kopfschmerz zurückzuführen auf ein zervikales myofasziales Schmerzsyndrom}

Diagnostische Kriterien:

A. Kopf und/oder Nackenschmerzen, die das Kriterium C erfüllen

B. Es wurde ein Ursprung myofaszialer Schmerzen in der Nackenmuskulatur einschließlich reproduzierbarer Triggerpunkte nachgewiesen

C. Ein kausaler Zusammenhang kann durch wenigstens zwei der folgenden Kriterien gezeigt werden:

1. Einer oder beide der folgenden Kriterien sind erfüllt:

a) Der Schmerz hat sich in einem zeitlichen Zusammenhang mit dem Beginn des zervikalen myofaszialen Schmerzsyndroms entwickelt

b) Der Schmerz hat sich parallel zur Besserung des zervikalen myofaszialen Schmerzsyndroms deutlich verbessert

2. Durch Druck auf die beteiligten Halsmuskeln wird eine entsprechende Druckschmerzhaftigkeit ausgelöst 
3. Der Schmerz wird durch Injektionen von Lokalanästheti-

ka in Triggerpunkte oder durch Triggerpunktmassage temporär beseitigt.

D. Nicht besser erklärt durch eine andere ICHD-3-Diagnose.

\section{Kommentar:}

Das myofasziale Schmerzsyndrom und seine Beziehung zu so genannten „Triggerpunkten“ ist umstritten. Es ist durchgängig schwierig gewesen, mutmaßliche Triggerpunkte nachzuweisen, und das Ansprechen auf Behandlung variiert.

\section{A11.3 Kopfschmerz zurückzuführen auf Erkrankungen der Augen}

\section{A11.3.5 Kopfschmerz zurückzuführen auf eine Hetero- phorie oder Heterotropie \\ Beschreibung:}

Kopfschmerz verursacht durch einen latenten oder anhaltenden Strabismus (Schielen), üblicherweise nach längerer Anstrengung der Augen auftretend.

\section{Diagnostische Kriterien:}

A. Kopfschmerz, der Kriterium C erfüllt

B. Eine Heterophorie oder Heterotropie wurde ermittelt, mit wenigstens einem der folgenden Symptome:

1. Verschwommensehen

2. Doppeltsehen

3. Schwierigkeiten mit dem Wechsel von Nahsicht zu Fernsicht und/oder umgekehrt

C. Ein kausaler Zusammenhang kann durch wenigstens zwei der folgenden Kriterien gezeigt werden:

1. Der Kopfschmerz hat sich in einem zeitlichen Zusammenhang mit dem Beginn der Heterophorie und/oder Heterotropie entwickelt oder führte zu ihrer Entdeckung

2. Der Kopfschmerz hat sich nach Korrektur der Heterophobie und/oder Heterotropie deutlich gebessert

3. Der Kopfschmerz verschlechtert sich durch länger anhaltende Anstrengung der Augen

4. Der Kopfschmerz wird durch Schließen eines Auges und/ oder Abbruch der Anstrengung der Augen gelindert

D. Nicht besser erklärt durch eine andere ICHD-3-Diagnose.

\section{Kommentar:}

Es gibt eine Reihe von Fällen, die für einen A11.3.5 Kopfschmerz zurückzuführen auf eine Heterophorie oder Heterotropie sprechen, ansonsten gibt es kaum Hinweise für diese Kopfschmerzursache. Deshalb wurde dieser in den Anhang verschoben, bis formellere Untersuchungen durchgeführt wurden.

Patienten mit einem 11.3.5 Kopfschmerz zurückzuführen auf eine Heterophorie oder Heterotropie, wenn diese existiert, dürften aller Wahrscheinlichkeit nach einen Augenarzt um Rat fragen.

\section{A11.5 Kopfschmerz zurückzuführen auf Erkrankungen der Nase oder Nasen- nebenhöhlen}

\section{A11.5.3 Kopfschmerz zurückzuführen auf Erkrankungen der Nasenschleimhaut, der Nasenmuscheln oder der Nasenscheide- wand}

Diagnostische Kriterien:

A. Kopfschmerz, der das Kriterium C erfüllt

B. Ein hypertropher oder entzündlicher Prozess innerhalb der Nasenhöhle wurde klinisch, mit Nasenendoskopie und/oder Bildgebung nachgewiesen 1

C. Ein kausaler Zusammenhang kann durch mindestens zwei der folgenden Kriterien gezeigt werden:

1. Der Kopfschmerz hat sich in einem zeitlichen Zusammenhang mit dem Beginn der intranasalen Läsion entwickelt oder zu ihrer Entdeckung geführt

2. Der Kopfschmerz hat sich gleichzeitig mit der Besserung (mit oder ohne Behandlung) oder Verschlechterung der Läsion an der Nase deutlich gebessert oder verschlechtert

3. Der Kopfschmerz hat sich nach örtlicher Betäubung der Schleimhaut im Umfeld der Läsion deutlich gebessert

4. Der Kopfschmerz ist ipsilateral zum Situs der Läsion lokalisiert

D. Nicht besser erklärt durch eine andere ICHD-3-Diagnose.

Anmerkung:

1. Beispiele sind Concha bullosa und Knochensporn in der Nasenscheidewand.

\section{A12. Kopfschmerz zurückzuführen auf psychiatrische Störungen}

\section{Einleitung}

Obwohl Kopfschmerzen häufig auf verschiedene psychiatrische Störungen zurückgeführt werden, ist die Frage nach einer Kausalität dieser Beziehung (und ihrer Richtung) noch nicht abschließend geklärt. In der überwiegenden Mehrzahl der Fälle dürften Kopfschmerzen, die mit psychiatrischen Störungen assoziiert sind, eher gemeinsame zugrundeliegende Risikofaktoren widerspiegeln, nicht eine Kausalbeziehung. Bevor eine der unten aufgelisteten Diagnosen gestellt wird, sollte unbedingt ein kausaler Zusammenhang zwischen dem Kopfschmerz und der betreffenden psychiatrischen Störung gesichert sein. Das heißt, entweder tritt der Kopfschmerz parallel zur psychiatrischen Störung auf oder es besteht eine klare Verschlechterung des Kopfschmerzes nach dem Auftreten der psychiatrischen Störung.

Definitive Biomarker und klinische Beweise für den Kausalzusammenhang sind kaum zu erhalten, und die Diagnose wird oft eine Ausschlussdiagnose sein. Leidet zum Beispiel ein Kind unter einer emotionalen Störung mit Trennungsangst, sollten eventuell vorhandene Kopfschmerzen nur in dem Fall auf die Trennungsangst zurückgeführt werden, wenn die Kopfschmerzen ausschließlich im 
Kontext mit einer aktuellen oder drohenden Trennung auftreten, ohne Vorliegen einer besseren Erklärung. Auf ähnliche Weise sollten bei einem Erwachsenen mit einer Panikstörung Kopfschmerzen nur dann auf die Panikstörung zurückgeführt werden, wenn die Kopfschmerzen ausschließlich als eines der Symptome einer Panikattacke auftreten.

Die folgenden Kategorien werden als vorläufige Kriteriensätze vorgeschlagen, um die Erforschung eines möglichen Zusammenhanges zwischen bestimmten psychiatrischen Erkrankungen und Kopfschmerzen zu erleichtern. Wir empfehlen aber nicht, diese routinemäßig in der klinischen Praxis anzuwenden, um die Assoziation zwischen Kopfschmerzen und komorbiden psychiatrischen Störungen zu beschreiben.

\section{A12.3 Kopfschmerz zurückzuführen auf eine depressive Störung}

Diagnostische Kriterien:

A. Kopfschmerz der das Kriterium C erfüllt

B. Vorliegen einer Major Depression (einzelne Episode oder rezidivierend) oder einer anhaltenden depressiven Störung nach den Kriterien der DSM-5

C. Der Kopfschmerz tritt ausschließlich während der depressiven Episoden auf

D. Nicht besser erklärt durch eine andere ICHD-3-Diagnose.

Kommentar:

Viele Antidepressiva, v. a. Trizyklika, sind effektiv in der Behandlung von Kopfschmerzerkrankungen, selbst wenn keine Depression vorliegt. Daher ist es schwierig, anhand einer Remission oder Besserung der Kopfschmerzen unter antidepressiver Behandlung mit Trizyklika einen Kausalzusammenhang zwischen den Kopfschmerzen und der Depression festzustellen. Eine Remission der Kopfschmerzen unter Behandlung einer Depression mit Antidepressiva ohne deutliche kopfschmerzlindernde Wirkung spricht dagegen eher für einen Kausalzusammenhang.

\section{A12.4 Kopfschmerz zurückzuführen auf eine Störung mit Trennungsangst}

Diagnostische Kriterien:

A. Kopfschmerz, der das Kriterium C erfüllt

B. Vorliegen einer Störung mit Trennungsangst nach den Kriterien der DSM-5

C. Der Kopfschmerz tritt ausschließlich im Kontext einer aktuellen oder drohenden Trennung von zu Hause oder von wichtigen Bezugspersonen auf

D. Nicht besser erklärt durch eine andere ICHD-3-Diagnose.

\section{Kommentar:}

Die Störung mit Trennungsangst dauert typischerweise mindestens 6 Monate an. Bei akutem Beginn oder Verschlechterung schwerer Symptomatik (z. B. Schulverweigerung oder komplette Unfähigkeit, sich von zu Hause oder von wichtigen Bezugspersonen zu trennen) kann jedoch auch eine kürzere Dauer mit den Kriterien vereinbar sein. Die Störung verursacht klinisch bedeutsames Leiden und/oder Beeinträchtigung in sozialen, schulischen, beruflichen und/oder anderen wichtigen Funktionsbereichen.

\section{A12.5 Kopfschmerz zurückzuführen auf eine Panikstörung}

Diagnostische Kriterien:

A. Kopfschmerz, der das Kriterium C erfüllt

B. Wiederkehrende unerwartete Panikattacken, die die Kriterien der DSM-5 für eine Panikstörung erfüllen

C. Der Kopfschmerz tritt ausschließlich während der Panikattacken auf

D. Nicht besser erklärt durch eine andere ICHD-3-Diagnose. A12.6 Kopfschmerz zurückzuführen auf eine spezifische Phobie

Diagnostische Kriterien:

A. Kopfschmerz, der das Kriterium C erfüllt

B. Vorliegen einer spezifischen Phobie nach den Kriterien der DSM-5

C. Der Kopfschmerz tritt ausschließlich während der Exposition gegenüber dem phobischen Reiz oder der Erwartung einer solchen Exposition auf

D. Nicht besser erklärt durch eine andere ICHD-3-Diagnose.

Kommentar:

Spezifische Phobien dauern üblicherweise sechs Monate oder länger an, und verursachen einen klinisch bedeutsamen Leidensdruck und/oder eine Beeinträchtigung in sozialen, beruflichen und/oder anderen wichtigen Funktionsbereichen.

\section{A12.7 Kopfschmerz zurückzuführen auf eine soziale Angststörung (soziale Phobie)}

Diagnostische Kriterien:

A. Kopfschmerz, der das Kriterium C erfüllt

B. Vorliegen einer sozialen Angststörung (sozialen Phobie) nach den Kriterien der DSM-5

C. Der Kopfschmerz tritt ausschließlich während der Exposition gegenüber der sozialen Situation oder der Erwartung einer solchen Exposition auf

D. Nicht besser erklärt durch eine andere ICHD-3-Diagnose.

\section{Kommentare:}

Bei der sozialen Angststörung (sozialen Phobie) hat die Person ausgeprägte Angst oder Furcht vor einer oder mehreren sozialen Situationen, in denen sie der Beurteilung durch andere ausgesetzt sein könnte. Die Angst oder Furcht ist der durch die soziale Situation bestehenden Bedrohung unangemessen. Beispiele sind soziale Interaktionen (z. B. eine Unterhaltung), Beobachtung durch andere (z. B. beim Essen oder Trinken) oder vor anderen eine Leistung erbringen zu müssen (z. B. eine Rede halten). Die Person befürchtet, dass ihr Verhalten oder ihre Angstsymptome dazu führen werden, dass sie negativ beurteilt (z. B. erniedrigt, beschämt oder zurückgewiesen) wird, oder dass andere durch ihr Benehmen verletzt werden 
könnten. Bei Kindern kann sich die Angst oder Furcht durch Weinen, Schreianfälle, Erstarren, Klammern, Sich-Kleinmachen äußern oder durch die Unfähigkeit, in sozialen Situationen zu sprechen.

Die Störung hält üblicherweise sechs Monate oder länger an.

\section{A12.8 Kopfschmerz zurückzuführen auf eine generalisierte Angststörung}

Diagnostische Kriterien:

A. Kopfschmerz, der das Kriterium C erfüllt

B. Vorliegen einer generalisierten Angststörung nach den Kriterien der DSM-5

C. Der Kopfschmerz tritt ausschließlich während Phasen mit Angst auf

D. Nicht besser erklärt durch eine andere ICHD-3-Diagnose.

\section{Kommentar:}

Patienten mit einer generalisierten Angststörung zeigen für mindestens 3 Monate mehr als die Hälfte der Zeit übermäßige Angst und Sorgen (ängstliche Erwartung) in Bezug auf zwei oder mehr Lebensbereiche oder Ereignisse (z. B. Familie, Gesundheit, Finanzen, Schwierigkeiten in der Schule/bei der Arbeit). Die Symptome können Unruhe, innere Anspannung, Nervosität und erhöhte Muskelanspannung einschließen. Auf der Verhaltensebene finden sich: die Vermeidung von Aktivitäten oder Ereignissen mit der Möglichkeit eines negativen Ausgangs, ein ausgeprägter Zeitaufwand für die Vorbereitung von Aktivitäten oder Ereignissen mit möglichen negativem Ausgang, im Verhalten oder bei Entscheidungen eine ausgeprägte Tendenz zum Aufschieben wegen Bedenken und Sorgen, und das Bedürfnis, sich wegen dieser Sorgen immer wieder Rückversicherung und Bestätigung zu holen.

\section{A12.9 Kopfschmerz zurückzuführen auf eine posttraumatische Belastungsstörung (PTBS)}

Diagnostische Kriterien:

A. Kopfschmerz, der das Kriterium C erfüllt

B. Vorliegen einer posttraumatischen Belastungsstörung nach den Kriterien der DSM-5

C. Der Kopfschmerz hat sich erstmals nach dem traumatischen Erlebnis entwickelt und tritt ausschließlich im Kontext mit anderen Symptomen der posttraumatischen Belastungsstörung auf1

D. Nicht besser erklärt durch eine andere ICHD-3-Diagnose2.

Anmerkungen:

Der Kopfschmerz tritt z. B. im Zusammenhang mit etwas auf, das an das traumatische Erlebnis erinnert.

Insbesondere A12.3 Kopfschmerz zurückzuführen auf eine depressive Störung.

Kommentare:

Es kann eine direkte Exposition mit Erfahrungen wie Tod oder lebensbedrohlichen Situationen, schweren Verletzungen oder Verge- waltigung vorliegen, oder eine indirekte - durch Miterleben, indem jemand erfährt, dass einem nahen Familienmitglied oder Freund derartiges widerfahren ist. Alternativ kann es sich um ein wiederholtes oder extremes Exponiertsein in Bezug auf aversive Details solcher Ereignisse handeln (z. B. Ersthelfer, die Körperteile aufsammeln müssen oder Polizeibeamte, die wiederholt mit Kindesmisshandlung zu tun haben). Die alleinige Exposition durch elektronische Medien, Fernsehen, Film oder Fotos ist nicht ausreichend, es sei denn, diese Exposition ist beruflicher Natur.

Angesichts der hohen Komorbidität zwischen Depression und posttraumatischer Belastungsstörung sollte die Diagnose A12.9 Kopfschmerz zurückzuführen auf eine posttraumatische Belastungsstörung für Patienten reserviert werden, deren Kopfschmerz nicht durch eine begleitende Depression erklärt ist (z. B. Fälle von Kopfschmerz zurückzuführen auf eine posttraumatische Belastungsstörung ohne komorbide Depression).

\section{Glossar}

Allodynie: Ein Unwohlsein oder Schmerzempfinden, das durch Reize ausgelöst wird, die normalerweise nicht ausreichen würden, um diese Wirkung zu haben. Sie wird von einer Hyperalgesie unterschieden.

Anorexie: Appetitmangel und Abneigung gegen Lebensmittel in geringem Maße.

Attackendauer: Zeit vom Beginn bis zum Ende einer Kopfschmerzoder Schmerzattacke, die die Kriterien für einen bestimmten Kopfschmerztyp oder -subtyp erfüllt. Nach einer Migräne oder einer Clusterkopfschmerzattacke kann ein leichter nicht-pulsierender Schmerz ohne Begleitsymptome persistieren. Dieser ist nicht Teil der Attacke und wird zur Bestimmung der Attackendauer nicht herangezogen. Schläft ein Patient während einer Attacke ein und erwacht schmerzfrei, wird die Zeit bis zum Aufwachen zur Attackendauer mitgezählt. Wird eine Migräneattacke durch Medikation erfolgreich kupiert, die Symptome treten aber innerhalb von 48 Stunden wieder auf, kann es sich um eine neue Attacke oder aber um ein Wiederauftreten der alten Attacke handeln. Hier ist ein Abwägen erforderlich, um die Unterscheidung zu treffen (siehe auch Attackenfrequenz).

Aura: Frühe Symptome der Attacke einer Migräne mit Aura, die für eine Manifestation einer fokalen zerebralen Dysfunktion gehalten werden. Die Aura hält typischerweise 20 bis 30 Minuten an und geht dem Kopfschmerz voran. Siehe auch: Fokale Symptome, Prodromi, Vorwarnsymptome und Warnsymptome.

Begleitsymptome: Symptome, die einen Kopfschmerzen typischerweise begleiten, seltener vorangehen oder folgen. Bei der Migräne sind dies in erster Linie Übelkeit, Erbrechen, Licht- und Lärmüberempfindlichkeit.

Chronisch: In der Schmerzterminologie bedeutet chronisch lange anhaltend, oder konkreter ein Anhalten über einen Zeitraum von mindestens 3 Monaten. In der Kopfschmerzterminologie wird diese Bedeutung für die sekundären Kopfschmerzerkrankungen beibehalten (insbesondere für solche, die auf eine Infektion zurückgeführt werden), bei denen die ursächliche Erkrankung als solche chronisch ist. In diesem Wortgebrauch wird zwischen chronisch und anhaltend unterschieden. Bei den primären Kopfschmerzerkrankungen, die häufiger episodisch auf- 
treten, wird der Begriff chronisch immer dann verwandt, wenn Attacken über mindestens 3 Monate an mehr als der Hälfte der Tage auftreten. Eine Ausnahme sind die trigemino-autonomen Kopfschmerzerkrankungen. Hier wird erst von chronischen Verläufen gesprochen, wenn die Erkrankung über mehr als ein Jahr nicht remittiert ist.

Clusterkopfschmerzattacke: Eine Attacke eines kontinuierlichen Schmerzes über 15 bis 180 Minuten.

Clusterepisode: Die Zeit, in der Clusterkopfschmerzattacken regelmäßig mindestens an jedem zweiten Tag auftreten (englisch auch bout genannt).

Clusterremissionsepisode: Die Zeit, in der Clusterkopfschmerzattacken nicht mehr spontan auftreten und auch durch Alkohol oder Nitroglyzerin nicht zu provozieren sind. Von einer Remission wird gesprochen, wenn der schmerzfreie Zeitraum 3 Monate überschreitet.

Drückend/einengend: Schmerz von konstantem Charakter, der häufig mit einem eng sitzenden Band um den Kopf vergleichen wird.

Einschießender Schmerz: Plötzlicher Schmerz, der weniger als eine Minute anhält, meist nur Sekunden oder Sekundenbruchteile.

Enger zeitlicher Zusammenhang: Dieser Begriff wird verwandt, um den Zusammenhang zwischen einer organischen Störung und Kopfschmerzen zu beschreiben. Bei einigen Kopfschmerzen mit akutem Beginn (wenn auch längst nicht bei allen), bei denen eine Kausalität wahrscheinlich ist, ist der genaue zeitliche Zusammenhang bekannt. Bei chronischen Erkrankungen ist der zeitliche Zusammenhang ebenso wie die ursächliche Beziehung häufig schwer zu eruieren.

Entoptische Phänomene: Visuelle Störungen, die auf die Struktur des visuellen Systems selbst zurückgehen, darunter ein übermäßiges Auftreten von Mouches volantes in beiden Augen, ein übermäßiges Auftreten des Scheerer-Phänomens (Blaufeld-Entoptik: unzählige graue/weiße/schwarze Pünktchen oder Ringe, die beim Blick auf eine homogene helle Fläche wie etwa einen blauen Himmel durch das Gesichtsfeld beider Augen schießen), vom Auge selbst hervorgerufene Lichtphänomene (farbige Wellen oder Wolken, die beim Schließen der Augen im Dunkeln gesehen werden) und spontane Photopsie (helle Lichtblitze).

Entzug: Unterbrechung eines/einer wochen- oder monatelangen Medikamenten- oder sonstigen Substanzgebrauchs/Substanzexposition. Der Begriff umfasst auch das therapeutische Absetzen von Medikamenten im Kontext eines Kopfschmerzes zurückzuführen auf Medikamentenübergebrauch.

Episodisch: Wiederkehren und Remittieren von Kopfschmerzoder Schmerzattacken von konstanter oder variierender Dauer in einem regelmäßigen oder unregelmäßigen Zeitmuster. In der Praxis hat dieser Begriff beim episodischen Clusterkopfschmerz eine besondere Bedeutung erfahren. Hier wird mit episodisch nicht das Auftreten von einzelnen Attacken, sondern von Clusterepisoden, die durch Remissionsphasen getrennt sind, bezeichnet. Ähnliches gilt für die paroxysmale Hemikranie und das SUNA-Syndrom.

Fokale neurologische Symptome: Symptome einer fokalen Hirnstörung (in der Regel zerebral), wie sie bei Migräneauren auftreten.
Fortifikationsspektrum: Gezackte, bogenförmige und sich allmählich vergrößernde visuelle Halluzination typisch für eine visuelle Migräneaura, die farbig oder schwarzweiß in Erscheinung treten kann.

Früher verwendete Begriffe: Eine diagnostische Bezeichnung, die früher mit einer ähnlichen oder identischen Bedeutung für den klassifizierten Begriff verwandt wurde. Früher verwendete Begriffe sind häufig mehrdeutig und/oder wurden in verschiedenen Ländern unterschiedlich gebraucht.

Gesichtsschmerz: Schmerz unterhalb der Orbitomeatallinie, oberhalb des Halses und vor der Ohrmuschel.

Heterophorie: Latenter Strabismus (Schielen).

Heterotropie: Manifester Strabismus (Schielen).

Hypalgesie: Herabgesetzte Schmerzempfindung in Reaktion auf einen Reiz, der erwartungsgemäß schmerzhaft wäre.

Hyperalgesie: Gesteigerte Schmerzempfindung in Reaktion auf einen Reiz, der erwartungsgemäß schmerzhaft sein müsste. Hyperalgesie wird von einer Allodynie unterschieden, die durch einen Reiz ausgelöst wird, der erwartungsgemäß nicht schmerzhaft wäre.

Kopfschmerz: Schmerz im Kopf, oberhalb der Orbitomeatallinie und/oder des Nuchalkamms.

Kopfschmerzfrequenz: Die Häufigkeitsrate von Kopfschmerzoder Schmerzattacken in einem bestimmten Zeitraum (meist 1 Monat). Auf eine erfolgreiche medikamentöse Behandlung einer Migräneattacke kann eine Kopfschmerzwiederkehr innerhalb von 48 Stunden folgen. In den IHS-Richtlinien zur Durchführung von kontrollierten Medikamentenstudien, 3. Auflage, wird zur praktischen Lösung dieses Problems, insbesondere zur Unterscheidung von Attacken in über den letzten Monat geführten Kopfschmerztagebüchern empfohlen, als einzelne Attacken nur solche zu werten, die von mindestens 48 kopfschmerzfreien Stunden unterbrochen sind.

Kopfschmerztage: Zahl der Tage in einem bestimmten Zeitraum (meist 1 Monat), an denen Kopfschmerzen auftreten, unabhängig davon, ob der gesamte Tag oder nur Teile betroffen sind.

Kopfschmerz-/Schmerzattacke: Kopfschmerz (oder Schmerz), der sich aufbaut, über Minuten, Stunden oder Tage auf einem bestimmten Niveau verharrt und dann bis zum völligen Verschwinden abklingt.

Lanzinierend: Kurz, stromstoßartig ins Versorgungsgebiet einer Nervenwurzel oder eines Nervs ausstrahlend.

Nachtblindheit: Einschränkung der Sehfähigkeit bei Dämmerlicht. Neu aufgetretener Kopfschmerz: Jede Art von Kopfschmerz, unter der ein Patient zuvor noch nie litt.

Neuralgie: Schmerz im/in den Verteilungsgebiet(en) eines oder mehrerer Nerven, Annahmen zufolge aufgrund einer Dysfunktion oder Verletzung dieser Nervenstrukturen. Im allgemeinen Wortgebrauch wird bei diesem eine paroxysmale oder lanzinierende Qualität impliziert, doch sollte der Begriff Neuralgie nicht paroxysmalen Schmerzen vorbehalten sein.

Neuritis: Ein Sonderfall von Neuropathie; der Begriff ist heute entzündlichen Prozesses vorbehalten, die sich auf Nerven auswirken.

Neuropathie: Eine funktionelle Störung oder pathologische Veränderung in (einem) Nerven (in einem Nerv: Mononeuropathie; in mehreren Nerven: Mononeuropathia multiplex; wenn 
diffus und bilateral: Polyneuropathie). Der Begriff Neuropathie ist nicht dazu gedacht, eine Neurapraxie, Neurotmesis, Axonotmesis, Durchtrennung eines Nerven, Störungen eines Nerven aufgrund einer vorübergehenden Einwirkung wie durch einen Schlag, Dehnen oder epileptische Entladung mit einzuschließen (der Begriff neurogen gilt für Schmerzen zurückzuführen auf derartige temporäre Störungen).

Neuropathische Schmerzen: Schmerz infolge einer Läsion oder Erkrankung des peripheren oder zentralen somatosensorischen Nervensystems.

Nicht ausreichend validiert: Von zweifelhafter Validität als diagnostische Entität aufgrund der Einschätzung des Klassifikationskomittees und/oder der kontroversen Studienlage.

Nuchalregion: Dorsaler (hinterer) Teil des oberen Halses einschließlich der Insertionsregion der Nackenmuskulatur.

Palinopsie: Sehstörungen in Form von Nachbildern und/oder nachgezogene Bilder von beweglichen Objekten (zu differenzieren von Nachbildern auf der Netzhaut, die nach längeren Anblicken eines sehr kontrastreichen Lichteindrucks in der Komplementärfarbe in Erscheinung treten).

Perikranielle Muskeln: Nacken- und Okzipitalmuskulatur, Kaumuskulatur, mimische Muskulatur, Sprechmuskulatur, Muskeln des inneren Ohres (M. tensor tympani, M. stapedius).

Periphere neuropathische Schmerzen: Schmerz, der durch eine Läsion oder Erkrankung des peripheren somatosensorischen Nervensystems verursacht wird (siehe auch Neuropathische Schmerzen).

Anhaltend: Im Kontext bestimmter sekundärer Kopfschmerzen bezeichnet dieser Begriff einen initial akuten und von einer anderen Erkrankung verursachten Kopfschmerz, der nicht innerhalb eines spezifizierten Zeitraums (üblicherweise 3 Monate) nach Genesung von dieser Erkrankung verschwindet. In vielen solcher Fälle wird der Kopfschmerz als ein klar umrissener Subtyp anerkannt, wobei der Nachweis des ursächlichen Zusammenhangs von der früheren Erfüllung der Kriterien für eine Diagnose des akuten Typs abhängt und einem Persistieren desselben Kopfschmerzes.

Phonophobie: Überempfindlichkeit auf Lärm, selbst bei normaler Lautstärke, führt gewöhnlich zur Vermeidung.

Photophobie: Überempfindlichkeit auf Licht, selbst bei normaler Helligkeit; führt gewöhnlich zur Vermeidung.

Pochend: Synonym für „pulsierend“.

Postdromalphase: Eine symptomatische Phase, bis zu 48 Stunden andauernd, im Anschluss an das Verschwinden von Schmerzen bei Migräneattacken mit oder ohne Aura. Zu den gängigen postdromalen Symptomen gehören Abgespanntheit, eine beschwingte oder gedrückte Stimmung und kognitive Schwierigkeiten.

Primäre(r) Kopfschmerz(erkrankung): Kopfschmerz oder Kopfschmerzerkrankung, nicht verursacht durch oder zurückzuführen auf eine andere Störung. Diese(r) wird von einer sekundären Kopfschmerzerkrankung unterschieden.

Prodrom: Eine symptomatische Phase, die der Kopfschmerzphase einer Migräne ohne Aura oder vor der Migräneaura bei einer Migräne mit Aura vorangeht und bis zu 48 Stunden dauert. Die häufigsten prodromalen Symptome sind Müdigkeit, gehobene oder depressive Stimmung, ungewöhnlicher Hunger und Heißhungerattacken auf bestimmte Lebensmittel.

Pulsierend: Synonym für „pochend“.

Punktförmige Reize: Reize, die an genau umrissenen Punkten auf der Haut gesetzt werden.

Refraktärzeit: Die Zeit nach dem Verschwinden/der Resolution einer Schmerzattacke, während der keine weitere Attacke ausgelöst werden kann.

Refraktionsfehler/Brechungsfehler: Myopie, Hypermetropie oder Astigmatismus.

Resolution/Verschwinden: Vollständige Remission aller Symptome und anderer klinischer Hinweise auf eine Erkrankung oder einen Krankheitsprozess (wie etwa eine Kopfschmerzattacke).

Schmerz: Nach IASP-Definition: eine unangenehme sensorische und emotionale Erfahrung in Begleitung einer tatsächlichen oder potenziellen Gewebeschädigung oder im Sinne einer solchen Schädigung beschrieben (siehe auch: neuropathische Schmerzen, zentrale neuropathische Schmerzen und periphere neuropathische Schmerzen).

Schmerzempfindlichkeit: Ein erhöhtes Gefühl des Unbehagens oder Schmerzes ausgelöst durch direkten Druck wie zum Beispiel bei einer Tastuntersuchung

Schmerzintensität: Schmerzgrad, in der Regel auf einer numerischen 4-Punkt-Skala beschrieben ( 0 bis 3), gleichzusetzen mit kein Schmerz, leichter, mittelschwerer und schwerer Schmerz, oder auf einer visuellen Analog-Skala (üblicherweise $10 \mathrm{~cm}$ ). Kann auch gemäß der funktionellen Konsequenzen nach einer verbalen Punkte-Skala bewertet werden: 0 = kein Schmerz; 1 = leichter Schmerz, behindert normale Aktivitäten nicht; 2 = mittelstarker Schmerz, beeinträchtigt zwar normale Aktivitäten, aber verhindert sie nicht; 3 = starker Schmerz, verhindert alle Aktivitäten.

Sekundäre(r) Kopfschmerz(erkrankung): Kopfschmerz, oder eine Kopfschmerzerkrankung, die durch eine andere Grunderkrankung verursacht wird. In der ICHD-3 werden sekundäre Kopfschmerzen als auf die ursächliche Erkrankung zurückzuführen bezeichnet. Sekundäre Kopfschmerzen werden von primären Kopfschmerzen unterschieden. Ein sekundärer Kopfschmerz kann die Merkmale eines primären Kopfschmerzes aufweisen und dennoch die Kriterien für eine Verursachung durch eine andere Erkrankung erfüllen.

Skotom: Verlust eines Teils des Gesichtsfelds eines oder beider Augen. Ein Skotom kann absolut (kein Visus) oder relativ sein (verminderter oder unscharfer Visus).

Strabismus: Ein- oder beidseitige Fehlstellung der Augen (Schielen).

Substanz: Alles von folgendem: eine organische oder anorganische Chemikalie; ein Nahrungsmittel oder Nahrungsmittelzusatz; ein alkoholisches Getränk; ein Gas oder Dampf; eine Droge, ein Medikament oder ein pflanzliches Heilmittel, eine tierische oder anderweitige Substanz, die zu medizinischen Zwecken verabreicht wird, aber nicht als Arzneimittel zugelassen ist.

Szintillationen: Visuelle Halluzinationen, die grell sind und in ihrer Intensität fluktuieren, meist in einer Frequenz von 8 bis 10 Zyklen/Sekunde. Sie sind typisch für eine Migräneaura.

Teichopsie: Synonym für ein Fortifikationsspektrum. 
Übertragener Schmerz: Schmerz, der in einer anderen Körperregion wahrgenommen wird, als in der die Nozizeption stattgefunden hat.

Unilateral: Entweder links- oder rechtsseitig, die Mittellinie nicht überquerend. Ein unilateraler Kopfschmerz muss nicht notwendigerweise die gesamte linke oder rechte Kopfhälfte umfassen, sondern kann auch ausschließlich frontal, temporal oder okzipital lokalisiert sein. Wird der Begriff für sensorische oder motorische Störungen im Rahmen einer Migräneaura benutzt, schließt er sowohl eine komplette als auch eine nur teilweise Halbseitenbeteiligung mit ein.

Vasospasmus: Konstriktion einer Arterie oder einer Arteriole in einem Ausmaß, dass die Gewebsperfusion vermindert ist.

Vorbotensymptome: Dieser Begriff wurde in der Praxis unterschiedlich gebraucht, oft als Synonym für Prodromi, aber auch weniger spezifisch und ein Stück weit mehrdeutig für eine Palette von Symptomen, von die mutmaßlich eine Migräneattacke ankündigen, aber möglicherweise die Anfangsphase einer solchen sind. Der Begriff sollte besser vermieden werden.
Warnsymptome: Früher verwendeter Begriff, um entweder eine Aura oder Vorbotensymptome zu beschreiben. Da der Begriff zweideutig ist, sollte er nicht mehr verwandt werden.

Zentraler neuropathischer Schmerz: Schmerz, der durch eine Läsion oder Erkrankung des zentralen somatosensorischen Nervensystems (siehe auch Neuropathischer Schmerz) verursacht wird.

Zerebrale Bildgebung: Computertomographie (CT), Magnetresonanz-Tomographie (MRT), Positronen-Emissions-Tomographie (PET), Einzel-Photonen-Emissionscomputertomographie (SPECT) oder Szintigraphie in der Regel des Gehirns, gfls. einschließlich funktioneller Modalitäten.

Zickzack-Linien: Synonym für ein Fortifikationsspektrum.

Zurückzuführen auf: Dieser Begriff beschreibt in der ICHD-3 die Beziehung zwischen einem sekundären Kopfschmerz und der Erkrankung, die als dessen Ursache angenommen wird. Voraussetzung hierfür ist, dass die Kriterien erfüllt sind, die hinreichend als Nachweis eines ursächlichen Zusammenhangs anerkannt sind. 